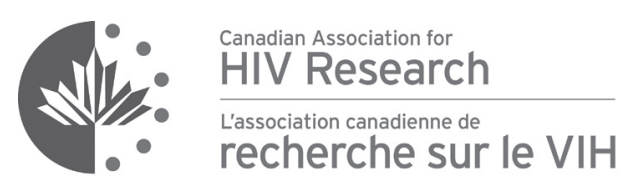

\author{
CAHR 2013 \\ Innovations to Address Complex Challenges \\ $22^{\text {nd }}$ Annual Canadian Conference on HIV/AIDS Research \\ ACRV 2013 \\ Idées nouvelles pour relever les défis complexes \\ $22^{\mathrm{e}}$ Congrès canadien annuel de recherche sur le VIH/sida
}

\title{
ABSTRACTS / RÉSUMÉS
}

CAHR Executive Committee / Comité directeur de I'ACRV

Message from the Co-Chairs of CAHR 2013 / Message des coprésidents du congrès de l'ACRV 2013

Oral Presentations / Exposés oraux

Friday, April 12 / Vendredi 12 avril

Saturday, April 13 / Samedi 13 avril

Poster Presentations / Présentations des affiches

Basic Sciences / Sciences fondamentales

Clinical Sciences / Sciences cliniques

Epidemiology and Public Health Sciences /

Épidémiologie et sciences de la santé publique

Social Sciences / Sciences sociales

Author index / Index des auteurs

\section{Departments}

Calendar of Events 


\title{
$22^{\text {nd }}$ ANNUAL CANADIAN CONFERENCE ON HIV/AIDS RESEARCH \\ $22^{\mathrm{e}}$ CONGRÈS CANADIEN ANNUEL DE RECHERCHE SUR LE VIH/SIDA \\ CAHR COMMITTEES / COMITÉS DE L'ACRV
}

\section{CAHR EXECUTIVE COMMITTEE / COMITÉ DIRECTEUR DE L'ACRV}

President / Président: Dr. Jonathan Angel

President Elect / Président désigné: Dr. Robert Hogg

Past President / Ancien président: Dr. William Cameron

Treasurer / Trésorière: Dr. Curtis Cooper

Secretary / Secrétaire: Dr. Carole Strike

Executive Director / Directeur général: Andrew Matejcic

\section{CAHR COUNCIL MEMBERS / MEMBRES DU CONSEIL DE L'ACRV}

Track A / Volet A : Basic Sciences / Sciences fondamentales: Dr. Matthias Götte

Track B / Volet B : Clinical Sciences / Sciences cliniques: Dr. Stuart Skinner

Track C / Volet C : Epidemiology and Public Health Sciences / Épidémiologie et sciences de la santé publique: Dr. Margaret Millson

Track D / Volet D : Social Sciences / Sciences sociales: Dr. Saara Greene

Community Representative / Représentant communautaire: Terry Howard

\section{CONFERENCE ORGANIZING COMMITTEE / COMITÉ ORGANISATEUR}

Conference Co-Chairs / Coprésidents du congrès: Dr. Robert Hogg, Dr. Mark Hull, Dr. Neora Pick

\section{Track Chairs / Coprésidents des volets}

Track A / Volet A : Basic Sciences / Sciences fondamentales

Dr. Zabrina Brumme

Dr. Keith Fowke

Track B / Volet B : Clinical Sciences / Sciences cliniques

Dr. Deborah Money

Dr. Darrell Tan

Track C / Volet C : Epidemiology and Public Health Sciences / Épidémiologie et sciences de la santé publique

Dr. Ann Burchell

Dr. Mark Gilbert

Track D / Volet D : Social Sciences / Sciences sociales

Dr. Trevor Hart

Dr. Angela Kaida

Dr. Charlotte Reading

Community Representatives / Représentants communautaire

Tracey Conway

Charles Osborne

Kath Webster

Conference Advisory Members / Membres du comité consultatif du congrès :

Dr. Jonathan Angel, Andrew Matejcic, Dr. Marianne Harris

\section{CONFERENCE COORDINATOR / ORGANISATEUR PROFESSIONNEL DU CONGRÈS}

\author{
Sea to Sky Meeting Management Inc. \\ 206, 201 Bewicke Avenue, North Vancouver, British Columbia V7M 3M7, Canada \\ Telephone / Téléphone : 604-984-6455; Fax / Télécopieur : 604-984-6434 \\ www.seatoskymeetings.com; conference@cahr-acrv.ca \\ President / Présidente : Sarah Lowis, CMP, CMM \\ Project Director / Directeur de projet : Ian Holliday \\ Senior Project Manager / Chargée de projet principale : Sandy Becker \\ Registration Manager / Coordonnatrice des inscription : Glenda Freeman
}




\section{Track A / Volet A : Basic Sciences / Sciences fondamentales}

Jonathan Angel

T. Blake Ball

Nicole Bernard

Mark Brockman

James Brooks
Alan Cochrane

Eric Cohen

Matthias Götte

Michael Grant

Naveed Gulzar
Daniel Kaufmann

Mario Ostrowski

Ralph Pantophlet

Art Poon

Ken Rosenthal

\section{Track B / Volet B : Clinical Sciences / Sciences cliniques}

\author{
Ari Bitnun \\ Jason Brophy \\ Ryan Cooper \\ Michelle Foisy \\ Chris Fraser \\ Troy Grennan \\ Mark Hull \\ Yoav Keynan
}

Mona Loutfy

Martin Potter

Stuart Skinner

Marek Smieja
Michel Tremblay

Xiaojian Yao

\section{Track C / Volet C : Epidemiology and Public Health Sciences Epidémiologie et sciences de la santé publique}

$\begin{array}{lll}\text { Chris Archibald } & \text { Thomas Kerr } & \text { Gina Ogilvie } \\ \text { Joe Cox } & \text { Gilles Lambert } & \text { Ralph Pantophlet } \\ \text { Clemon George } & \text { Peggy Millson } & \text { Janet Raboud } \\ \text { Reka Gustafson } & \text { Sharmistha Mishra } & \text { Robert Remis } \\ \text { Jessica Halverson } & \text { David Moore } & \text { Elise Roy } \\ \text { Travis Hottes } & \text { Stephanie Nixon } & \text { Ameeta Singh }\end{array}$

Malcolm Steinberg Hla Hla (Rosie) Thein Mark Tyndall

\section{Track D / Volet D : Social Sciences / Sciences sociales}

\author{
Dan Allman \\ Martin Blais \\ Carrie Bourassa \\ David Brennan \\ Roy Cain \\ Sarah Flicker \\ Jacqueline Gahagan
}

\author{
Dionne Gesink \\ Randy Jackson \\ Thomas Kerr \\ Lynne Leonard \\ Renee Masching \\ Eleanor Maticka-Tyndale \\ Kim McKay-McNabb \\ Ted Myers \\ Earl Nowgesic \\ Kelly O'Brien \\ Joanne Otis \\ Lindsey Richardson \\ Eric Roth \\ Sergio Rueda
}

Cécile Tremblay

Sylvie Trottier

Julie van Schalkwyk

Mark Yudin
Kate Shannon
Jeannie Shoveller
Will Small
Robb Travers
Cathy Worthington 
$\mathrm{W}$

elcome to the 22nd Annual Canadian Conference on HIV/AIDS Research (CAHR 2013).

With a membership of more than 1,000 researchers and others interested in HIV research, the Canadian Association for HIV Research (CAHR) is the leading organization of HIV/AIDS researchers in Canada. The annual CAHR conference is the premier gathering in Canada for those working in the field of HIV, as well as policy makers, persons living with HIV, and other individuals committed to ending the pandemic. It is a chance to assess where we are, evaluate recent scientific developments, and together chart a course forward.

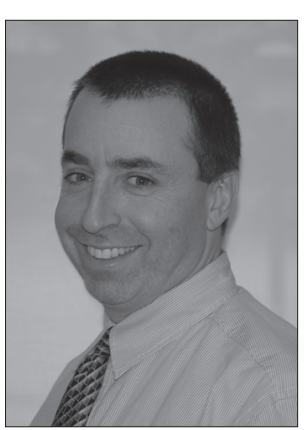

Dr Jonathan Angel
Bienvenue au $22^{\mathrm{e}}$ Congrès canadien annuel de recherche Bsur le VIH/sida (congrès de l'ACRV 2013)!

Comptant plus de 1000 membres - des chercheurs et d'autres personnes s'intéressant à la recherche sur le VIH -, l'Association canadienne de recherche sur le VIH (ACRV) est un organisme canadien de premier plan se consacrant à la recherche sur le VIH/sida. Le congrès annuel de l'ACRV est l'événement le plus important au pays s'adressant aux personnes œuvrant dans le domaine du VIH ainsi qu'aux décideurs, aux personnes vivant avec le VIH et aux autres personnes participant à la lutte contre la pandémie. Il offre une occasion de faire le point sur la situation, d'évaluer les

This year's programme will present new scientific knowledge and offer many opportunities for structured dialogue on the major issues facing the global response to HIV. A variety of sessions such as abstract-driven presentations, symposia, and plenaries will meet the needs of various participants. Other related activities, including ancillary meetings and the New Investigator Workshop, will contribute to an exceptional opportunity for professional development and networking.

CAHR 2013 will be a tremendous opportunity for researchers and community members from coast to coast to share the latest scientific advances in the field, learn from one another's expertise, and develop new ways to treat and prevent HIV. I hope you enjoy the conference, find it to be a worthwhile learning experience, and thank you in advance for your contributions, participation, and continued support. récents progrès scientifiques et d'élaborer un plan d'action.

Le congrès de cette année présentera les nouvelles connaissances scientifiques et permettra de discuter dans un cadre structuré des principaux enjeux liés à la lutte mondiale contre le VIH. Un éventail de séances telles que des présentations d'abrégés, des colloques et des séances plénières répondra aux besoins des différents participants. D'autres activités connexes, dont des réunions auxiliaires et l'atelier des nouveaux chercheurs dans le domaine du VIH, constitueront d'excellentes occasions de perfectionnement professionnel et de réseautage.

À l'occasion du congrès de l'ACRV 2013, les chercheurs et les membres des collectivités d'un océan à l'autre pourront comme jamais échanger sur les plus récentes percées scientifiques, apprendre des autres et établir de nouvelles façons de traiter et de prévenir le VIH. J'espère que le congrès vous plaira et qu'il sera pour vous une expérience d'apprentissage utile. Merci d'avance pour vos contributions, votre participation et votre appui soutenu.

\author{
Dr Jonathan Angel \\ President / Président \\ Canadian Association for HIV Research (CAHR) / Association canadienne de recherche sur le VIH (ACRV)
}


 MESSAGE DES COPRÉSIDENTS DU CONGRÈS DE L'ACRV 2013}

W e are excited to welcome you to Vancouver, to participate in the 22nd Annual Canadian Conference on HIV/AIDS Research (CAHR 2013). Over seventy thousand Canadians are living with HIV, and many more are at risk of being infected. Together with the Organizing Committee, we have chosen the theme of this year's Conference to be "Innovations to Address Complex Challenges" as a deliberate link to CAHR 2012 ("A Turning Point in the Fight Against HIV: Meeting New Challenges"). Turning the tide on the HIV epidemic requires us to consider innovative approaches to reach out to communities and prevent new infections, innovative strategies of expanding testing opportunities, and to build programs to expand access and engage those living with HIV across the country. Despite continued improvements in HIV therapy, complex challenges still exist for people living with HIV, including stigma, criminalization of HIV, co-infections, aging and potential unexpected effects of current therapies.

This year in Vancouver, we have designed the program from over 400 high quality abstracts submitted from across the country that highlight these challenges and describe new interventions to expand and improve care. In addition to evolving knowledge in viral pathogenesis and immunology, updates in epidemiology, and new insights into social constructs of HIV, we have chosen to highlight challenges facing vulnerable populations, including aboriginal communities and men who have sex with men. Challenges unique to delivering care to women and children living with HIV, and uncertainties related to the effects of HIV in aging populations will also be the focus of sessions at this conference.

We hope this conference will offer the opportunity for researchers, clinicians and community members from across the country, and across disciplines to come together to learn, share the latest knowledge, mingle, make new connections and ultimately leave with new ideas and a renewed sense of purpose, as we confront the evolving Canadian HIV epidemic for the years to come.

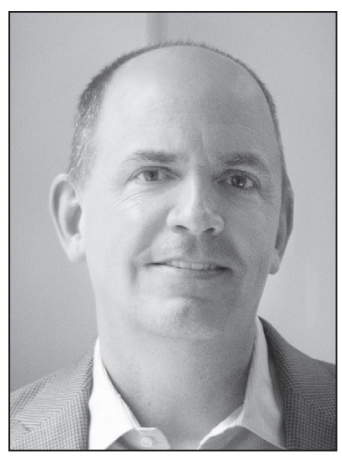

Dr Robert Hogg

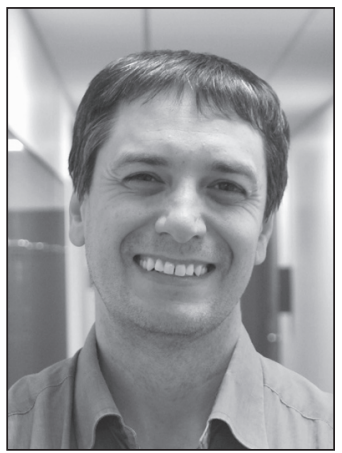

Dr Mark Hull

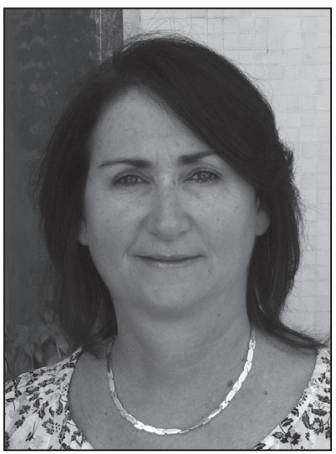

Dr Neora Pick
Tous sommes heureux de vous accueillir à Vancouver à l'occasion du 22e Congrès canadien annuel de recherche sur le $\mathrm{VIH} /$ sida (congrès de l'ACRV 2013). Plus de 70000 Canadiennes et Canadiens vivent actuellement avec le VIH, et beaucoup d'autres sont à risque d'être infectés. En concertation avec le comité organisateur, nous avons intitulé le congrès de cette année Idées nouvelles pour relever les défis complexes, en guise de prolongement du thème du congrès de l'ACRV 2012 (Un point tournant dans la lutte contre le VIH : de nouveaux défis à relever). Pour renverser l'épidémie de VIH, nous devons envisager l'adoption d'approches novatrices pour sensibiliser les collectivités et prévenir les nouvelles infections, l'exécution de stratégies innovantes pour augmenter les occasions de test, et l'élaboration de programmes à plus vaste portée destinés aux personnes vivant avec le VIH au Canada. En dépit des améliorations continues apportées aux traitements contre le VIH, les personnes vivant avec le VIH font toujours face à des défis complexes, notamment la réprobation sociale, la criminalisation du VIH, les co-infections, le vieillissement et les effets possibles inattendus des traitements actuels.

Nous avons élaboré le programme du congrès de cette année à partir de plus de 400 abrégés de grande qualité provenant des quatre coins du pays. Ces abrégés soulignent les défis à relever et décrivent les nouvelles interventions visant à élargir et à améliorer les soins. Outre l'évolution des connaissances sur la pathogénie virale et l'immunologie, les nouveautés en épidémiologie et les nouvelles données sur les constructions sociales du VIH, nous avons choisi de mettre en relief les défis auxquels font face les populations vulnérables, dont les collectivités autochtones et les hommes ayant des relations sexuelles avec d'autres hommes. Les défis particuliers à la prestation de soins aux femmes et aux enfants vivant avec le VIH ainsi que les incertitudes relatives aux effets du VIH dans les populations vieillissantes constitueront aussi des thèmes privilégiés au cours des séances du congrès.

Nous espérons que ce congrès sera pour les chercheurs et cliniciens de différentes disciplines et les membres des collectivités d'un océan à l'autre une occasion de se rassembler pour apprendre, échanger de nouvelles connaissances, établir de nouveaux liens, s'imprégner d'idées nouvelles et renouveler leur mission, dans le cadre de la lutte des prochaines années contre l'épidémie du VIH en évolution au Canada. 


\section{Track A: Basic Sciences: HIV Prevention and Therapy (Preclinical)}

Volet A : Sciences fondamentales : Prévention et traitement (préclinique) du VIH

\section{O001}

ANTIBODY AND T CELL RESPONSE TO THE PROTEASE CLEAVAGE SITES DRIVE EXTENSIVE MUTATIONS AND CORRELATED WITH PROTECTION AGAINST HIGHER DOSE OF SIVMAC239 CHALLENGE AND DISEASE PROGRESSION IN CYNOMOLGUS MACAQUES

Luo, Ma ${ }^{1}$; Tang, David ${ }^{1}$; Capina, Rupert ${ }^{1}$; Yuan, Xin-Yong ${ }^{1}$; Pinto, Jorge $\mathrm{C}^{2}$; Prego, Cecilia ${ }^{2}$; Alonso, Maria ${ }^{2}$; Barry, Christina ${ }^{1}$; Pilon, Richard ${ }^{3}$; La, David'; Daniuk, Christina ${ }^{1}$; Pillet, Stephane'; Bielawny, Thomas ${ }^{1}$; Lacap, Philip1; Czarnecki, Chris ${ }^{1}$; Wong, Gary ${ }^{1}$; Tuff, Jeff'; Tyler, Shaun ${ }^{1}$; Liang, Binhua ${ }^{1}$; Ball, Terry B ${ }^{1}$;

Sandstrom, Paul ${ }^{3}$; Kobinger, Gary ${ }^{1}$; Plummer, Francis A $^{1}$

${ }^{1}$ Winnipeg, MB; ${ }^{2}$ Santiago de Compostela, Spain; ${ }^{3}$ Ottawa, ON An estimated 2.7 million people are newly infected by HIV-1 every year. For every 2 persons starting treatment, 5 become newly infected. A safe and effective preventative HIV-1 vaccine is the only way to stop the new infections and control the pandemic.

The protease of HIV-1 is a small 99-amino acid aspartic enzyme that mediates the cleavage of Gag, Gag-Pol and Nef precursor polyproteins. The process is highly specific, temporally regulated and essential for the production of infectious viral particles. A total of twelve proteolytic reactions are required to generate a viable virion. Therefore, a vaccine targeting the 12 protease cleavage sites of HIV-1 could be effective. Since the protease cleavage sites of HIV-1 are highly conserved among major subtypes of HIV-1, direct immune responses against these cleavage sites would yield two major advantages. First, the host immune response could destroy the virus before its permanent establishment in the host. Second, the vaccine could force the virus to accumulate mutations eliminating the normal function of the HIV protease thus eliminating viable virions. We have conducted a proof of concept study to investigate the feasibility and effectiveness of this approach. The recombinant VSV-peptides were used to immunize cynomolgus macaques and nanopackaged peptides were used to boost the immune response to the peptides overlapping the 12 protease cleavage sites of SIVmac239. The immunized macaques and controls were cumulatively challenged intrarectally with increased dosage of SIVmac239. Results showed that antibody and $\mathrm{T}$ cell responses to the 12 protease cleavage site can protect macaques against higher dosage of SIVmac239 challenge ( $\mathrm{p}=0.005, \mathrm{R}=0.42$ ) and the vaccine group maintains significantly higher $\mathrm{CD}^{+}$counts $(\mathrm{p}=0.0002)$ than the controls weeks after being infected. Viral sequence analysis detected extensive mutations in the PCS and the flanking region. The break-through viruses of the vaccine group have higher mutation rate in PCS regions than that of the control group. The extensive mutations at/around PCS sequences correlated with lower viral load $(\mathrm{P}<0.0001)$.

\section{O002}

\section{HIV RESISTANCE AGAINST THE SECOND-GENERATION INTEGRASE INHIBITOR DOLUTEGRAVIR CORRELATES WITH IMPAIRED VIRAL REPLICATION}

Mesplède, Thibault R; Quashie, Peter K; Han, Yingshan; Osman, Nathan; Wares, Melissa A; Hassounah, Said; Singhroy, Diane N; Wainberg, Mark A

Montréal, QC

No resistance mutation against the strand transfer inhibitor dolutegravir has been observed so far in treatment-naïve patients. In vitro selection studies performed in our laboratory demonstrated that the R263K mutation commonly emerges in integrase in the presence of dolutegravir, often associated with the secondary mutation H51Y. We have shown in subtype B that $\mathrm{R} 263 \mathrm{~K}$ confers resistance to dolutegravir while the addition of H51Y to R263K further decreases HIV susceptibility to this drug. However, resistance correlated with a pronounced decrease in integration and viral replication.

We have further characterized the $\mathrm{R} 263 \mathrm{~K}$ resistance pathway by studying the effect of the H51Y and R263K mutations on HIV CRF02_A/G resistance, protein activity, and viral replication. We also investigated the effect of another secondary mutation, M50I, on viral replication.

Various relevant integrase were cloned into a vector for bacterial expression and mutated by site-directed mutagenesis. Recombinant integrases were purified and tested for strand-transfer activity in cell free assays, and Michaelis-Menten constants and maximal activities were calculated. HIV resistance to dolutegravir and viral replication capacity were measured in cell culture.

Similar to subtype B, the combination of H51Y and R263K in CRF02_A/G confers resistance to dolutegravir while decreasing strand transfer activity and viral replication. Differences between subtype B and CRFO2_A/G were observed in regard to resistance and viral replication capacity that suggest that subtype B may be more susceptible to mutations in the R263K resistance pathway. Additionally, the addition of M50I to the R263K mutation in subtype B virus did not restore viral replication capacity.

Altogether our results suggest that mutations in the R263K resistance pathway impose a profound viral fitness cost to HIV replication that could explain the absence of these mutations in treatment-naïve patients receiving this drug. Since the $\mathrm{R} 263 \mathrm{~K}$ resistance pathway is unique in its ability to confer significant resistance to dolutegravir (more than 5-fold) while being selected de novo by this drug, this suggests that dolutegravir could possess a higher genetic barrier to viral resistance than any compound discovered to date.

\section{O003}

\section{GENE THERAPY BASED ON SECRETED ANTI-HIV PROTEINS TO REPLACE CONTINUOUS DRUG ADMINISTRATION}

Falkenhagen, Alexander; Malm, Maria; Singh, Jastaran; Asad, Sabah; Read, Stanley E; Zúñiga-Pflücker, Juan C; Joshi, Sadhna

\section{Toronto, ON}

Gene therapy has the potential to provide long-term control of HIV replication via a single treatment. Current genetic strategies focus on rendering HIV target cells non-permissive for viral replication, but the clinical success is limited due to the low number of gene-modified target cells that engraft in patients. Modifying cells to secrete anti-HIV proteins that neutralize free virus particles could not only protect gene-modified cells, but also unmodified target cells from HIV infection. We have designed lentiviral vectors for the expression of seven secreted anti-HIV proteins that target the three major steps of HIV entry either individually or as covalently linked bi- or tri-functional fusion proteins to exploit the multistep nature of HIV entry. Soluble CD4 (sCD4), sCD4 fused to the single chain variable fragment $17 \mathrm{~b}$ targeting the co-receptor binding site on HIV Env gp120 (sCD4-scFv17b), sCD4 linked to the fusion inhibitor T45 (sCD4-FIT45), and sCD4 connected to scFv17b and FIT45 (sCD4scFv17b-FIT45) were secreted from gene-modified 293T cells and significantly inhibited entry of primary replication-incompetent HIV strains in the low nanomolar range. The anti-HIV proteins specifically neutralized HIV in a dose-dependent manner. The neutralization potency of sCD4FIT45 was superior to sCD4, FDA-approved FIT20, or non-covalent combinations thereof. sCD4-scFv17b-FIT45 potently blocked HIV entry with an activity comparable to sCD4-scFv17b. When gene-modified cells were co-cultured with unmodified primary peripheral blood mononucleocytes, HIV replication was reduced by over $99 \%$. Vectors optimized for expression in hematopoietic cell types transduced up to $70 \%$ of umbilical cord blood hematopoietic stem/progenitor (HSP) cells without signs of toxicity. The gene-modified HSP cells will be injected into immunecompromised NOD scid gamma mice to allow continuous secretion of the anti-HIV proteins from all HSP progeny cells. In conclusion, secreted anti- 
HIV proteins can advantageously replace administration of purified antiHIV proteins in vitro and may have potential for in vivo applications.

\section{O004}

\section{REACTIVATION OF LATENT VIRUSES BY SUPERINFECTION} IS TAT-DEPENDENT AND CAN LEAD TO THE DEVELOPMENT OF DRUG-RESISTANT RECOMBINANT VIRUSES

\section{Donahue, Daniel A; Sloan, Richard D; Wainberg, Mark A}

Montreal, QC

BACKGROUND: Both wild type and drug-resistant viruses can be archived in latent reservoirs, which persist for decades. Recombinant viruses can result from cellular coinfection or superinfection. It is therefore important to characterize the potential contribution of the latent reservoir to the generation of recombinants.

METHODS: We used a Jurkat model of latency establishment and reactivation. Wild type or drug-resistant latent populations (encoding viral EGFP) were superinfected with wild type or drug-resistant dsRed-expressing viruses, including Nef or Tat mutants, and treated with various antiviral inhibitors. EGFP and dsRed levels were measured by flow cytometry. Unique sequence tags on each resistant virus permitted identification of recombinants.

RESULTS: Latent viruses were reactivated by cellular superinfection. Reactivation required gene expression of the superinfecting virus, since latent viruses were reactivated in the presence of a protease inhibitor, but not an RT or integrase inhibitor. Reactivation occurred following superinfection with Nef-deleted or Tat-attenuated virus, but not with Tatinactive virus. These results rule out latent virus reactivation by superinfecting virus gp120-induced coreceptor signalling or Nef-induced NFkB or NFAT activation, but show a requirement for superinfecting virus Tat activity. Reverse transcriptase (RT) K103N latent populations were reactivated by superinfection with RT M184V virus; the resulting supernatants were used to seed further infections, where FTC+EFV were added. Sequencing and restriction digestion confirmed that recombination routinely occurred, leading to FTC/EFV-resistant virions encoding unique sequence tags derived from both latent and superinfecting viruses. Experiments currently underway aim to confirm these findings in a primary cell latency model.

CONCLUSIONS: Our results suggest that superinfection of latently infected CD4 T-cells can reactivate latent viruses, which can then recombine with the superinfecting virus. This could lead to the development of multi-drug resistance and further diversification of viral quasispecies. Additionally, these newly reactivated viruses may establish additional latent infections if further rounds of replication occur.

\section{O005}

TENOFOVIR ALAFENAMIDE (GS-7340) IS NOT A

SUBSTRATE FOR RENAL ORGANIC ANION TRANSPORTERS (OATS) AND DOES NOT EXHIBIT OAT-DEPENDENT CYTOTOXICITY

Bam, RA2; Yant, SR ${ }^{2}$; Borg, ${ }^{1}$; Cihlar, ${ }^{2}$

${ }^{1}$ Mississauga, ON; ${ }^{2}$ Foster City, California, USA

BACKGROUND: Tenofovir alafenamide (TAF; GS-7340) is a prodrug of tenofovir (TFV) undergoing Phase 3 trials. TAF has shown greater HIV RNA reductions relative to tenofovir DF (TDF) with $>90 \%$ reduced systemic exposure of TFV. Parent TFV released from its prodrugs is actively eliminated via organic anion transporters OAT1 and OAT3, leading to accumulation in proximal renal tubules, which has been the suggested etiology of TDF-associated renal dysfunction. We assessed OAT-mediated transport and cytotoxicity of TAF relative to TFV to evaluate the potential for TAF to accumulate in proximal tubules.

METHODS: Active transport and cytotoxicity were assessed in cells stably and/or transiently expressing the tested OATs and compared with matched control cells. The OAT-dependent uptake was evaluated using radiolabeled TAF and TFV in the presence and absence of the OAT inhibitor probenecid. Cytotoxicity (CC50) of TAF and TFV was determined using a 4-day cell-viability assay.
RESULTS: Compared with control cells, TFV uptake was enhanced 79. and 70-fold in cells stably and transiently expressing OAT1, respectively. Similarly, stable and transient expression of OAT3 increased the intracellular accumulation of TFV 4.2- and 8.2-fold, respectively. In contrast, neither stable nor transient expression of OATs enhanced the intracellular accumulation of TAF $>1.3$-fold over background. In accordance with these results, TAF in vitro cytotoxicity was minimally affected by the over-expression of either renal transporter ( 0.5 to 3.5 -fold change in CC50) whereas parent TFV was $>3.6$ - to $>21$-fold more cytotoxic in OAT expressing cells than controls.

CONCLUSIONS: These in vitro data demonstrate that TAF does not interact with renal transporters OAT1 or OAT3 and no OAT-mediated cytotoxicity of TAF was detected. Thus, in contrast to TFV, TAF is unlikely to accumulate in renal proximal tubules in an OAT-dependent manner, consistent with the improved renal safety profile observed with TAF in combination with emtricitabine and cobicistat/elvitegravir in Phase 2 trials.

\section{6}

\section{IMPACT OF EXPERIMENTAL CONDITIONS ON THE UTILITY OF "PRIMER ID" TAGGING FOR NEXT- GENERATION HIV SEQUENCING}

Liang, Richard H; Harrigan, Richard; Mo, Theresa; Dong, Winnie; Lee, Guinevere; Swenson, Luke; McCloskey, Rosemary; Woods, Conan; Brumme, Chanson; Poon, Art Vancouver, BC

BACKGROUND: Primer IDs (pIDs) are "tags" used in next-generation sequencing: random nucleotides attach to sequencing templates via degenerate primers during reverse transcription of HIV RNA molecules into cDNA. They help track the number of templates in a sample, and allow sequences sharing a pID to be averaged out to correct for sequencing error.

THREE POTENTIAL ISSUES MAY ARISE: multiple cDNAs may share a pID by chance; pIDs with read depth $<3$ must be rejected to allow error correction; and the design of the degenerate primer could create problematic homopolymers. We assess how the utility of pIDs depends on experimental conditions such as the initial number of HIV RNA molecules.

METHODS: Assuming all possible pIDs are equally likely to attach to any given template, we calculated the numbers necessary for a $90 \%$ probability of (1) total coverage of the input templates (i.e. every template is assigned a unique pID) or (2) 95\%-good coverage (95\% uniquely tagged) as functions of template count. We also computed confidence intervals for the number of pIDs represented by $<3$ sequences as a function of the numbers of reads and templates and compared these to data obtained via pyrosequencing of HIV populations using four pID designs (NNNNNNNNN, NNDNNHNNV, NBDHVBDHV, RYRYRYRYR).

RESULTS: The number of pIDs required to reliably achieve good coverage is drastically lower than that required for total coverage. For example, 1,193,698 pIDs are required to assure total coverage of 502 HIV RNA molecules, whereas 6,121 pIDs will assure 95\%-good coverage.

Our model also predicts that discarding pIDs represented by $<3$ sequences rejects $>40 \%$ of reads in samples with 10,000 templates and 20,000 reads. The theoretical predictions appear to fit the empirical data reasonably well. Results were consistent across pID designs.

CONCLUSIONS: While total coverage is unrealistic, achieving good coverage is far more practical. In sequencing samples with high template counts, rejecting pIDs with read depth $<3$ may be inadvisable because it will discard too much data depending on the number of reads. 
Track B: Clinical Sciences: HIV, Aging and Complications

\section{Volet B : Sciences cliniques : VIH, vieillissement et complications}

\section{7}

\section{INEQUITIES IN LIFE EXPECTANCY AMONG PEOPLE INITIATING HAART IN BRITISH COLUMBIA}

Hogg, Robert $\mathrm{S}^{1,2}$; Chan, Keith ${ }^{1}$; Cescon, Angela ${ }^{1}$; Samji, Hasina ${ }^{1}$; Colley, Guillaume ${ }^{1}$; Yip, Benita ${ }^{1}$; Lima, Viviane $D^{1}$;

Montaner, Julio $\mathbf{S}^{1}$

1Vancouver; ${ }^{2}$ Burnaby, BC

BACKGROUND: HAART has increased life expectancy among $\mathrm{HIV}^{+}$ people. However, as life expectancy has increased over time, inequities have emerged. The aim of this study is to characterize these inequities in life expectancy for people on HAART and to compare these against values for the Canadian population as a whole.

METHODS: Our analyses included $\mathrm{HIV}^{+}$persons who had initiated HAART in BC since 2000 and Statistics Canada estimates of survival between the 1991 and 2006 censuses. Abridged life tables were used to estimate life expectancy at age 25 years, to be comparable to inter-censal estimates. We defined life expectancy as the average number of additional years that a person of age 25 will live, if current age-specific mortality rates remain constant. For those on HAART, mortality rates were calculated using participants' person-time until death, loss to follow-up, or administrative censoring. Multivariate survival analysis was used to determine predictors of survival among those initiating HAART.

RESULTS: Since 2000, 3,890 people in BC have initiated HAART. Of these, $3,122(80.3 \%)$ are males, $1,477(38.0 \%)$ have a history of injection drug use and $484(12.4 \%)$ are of Aboriginal ancestry. Those who initiated HAART at a younger age, in 2008-2011, and at CD4s above 350 cells $/ \mathrm{mm}^{3}$ had increased survival, while those diagnosed with AIDS at HAART initiation, were $\mathrm{HCV}$-positive, and who were Aboriginal had poorer survival. Life expectancy at age 25 years for Aboriginal persons on HAART compared to all Aboriginal persons in Canada was an additional 21.0 versus 49.1 years. Canadian men and women also live considerably longer than men and women on HAART (52.6 versus 34.1 years for men and 57.9 versus 27.4 years for women). These differences were reduced when you restricted to those who were not Aboriginal or started HAART in 2008-2011.

CONCLUSION: Considerable gaps in life expectancy still exist in Canada, especially among women and persons of Aboriginal ancestry.

\section{O008}

\section{EZETIMIBE IN ADDITION TO ROSUVASTATIN FOR} IMPROVEMENT OF LIPID PARAMETERS IN HIV-POSITIVE PATIENTS NOT REACHING LIPID TARGETS WITH

\section{ROSUVASTATIN ALONE}

Johns, Kevin W; Bennett, Matthew T; Bondy, Greg P Vancouver, BC

BACKGROUND: HIV-positive patients on highly active antiretroviral therapy are subject lipid elevations, which may persist despite therapy with potent lipid-lowering agents. This prospective pilot study sought to determine whether patients not reaching lipid targets with rosuvastatin $10 \mathrm{mg}$ would show greater improvement in their lipid profile when ezetimibe was added to ongoing rosuvastatin therapy $(\mathrm{R}+\mathrm{E})$ compared to those treated with an increased dose of rosuvastatin (ROSU20). To our knowledge, this is a novel investigation within the HIV-positive population.

METHODS: This study was a 12-week, prospective, randomized, openlabel clinical trial. Subjects were deemed eligible if they had an apolipoprotein $\mathrm{B}(\mathrm{apoB})$ of $>0.80 \mathrm{~g} / \mathrm{L}$ despite therapy with rosuvastatin $10 \mathrm{mg}$ daily for a minimum of three months. The primary endpoint was between-group difference in apoB following 12 weeks of therapy. Secondary outcomes included change from baseline in apoB, betweengroup differences in other lipid measures, and safety parameters. Statistical analyses were performed using SPSS v.19.0.

RESULTS: Forty-three subjects (23 R+E, 20 ROSU20) completed the trial. Baseline characteristics did not differ between groups. More patients in the $\mathrm{R}+\mathrm{E}$ group had a history of hypertension and more were current smokers, whereas more patients in the ROSU20 group self-identified as past smokers. Significant improvements in apoB were seen within both $\mathrm{R}+\mathrm{E}(-0.17 \mathrm{~g} / \mathrm{L}, \mathrm{p}<0.001)$ and ROSU20 $(-0.13 \mathrm{~g} / \mathrm{L}, \mathrm{p}=0.03)$ treatment groups, but these did not differ between groups $(p=0.54)$. Significant between-group differences were observed in total cholesterol $(-1.00 \mathrm{mmol} / \mathrm{L}$ vs. $-0.51 \mathrm{mmol} / \mathrm{L}, \mathrm{p}=0.04)$ and triglycerides $(-0.63 \mathrm{mmol} / \mathrm{L}$ vs. $+0.04 \mathrm{mmol} / \mathrm{L}, \mathrm{p}=0.03)$, both in favour of the $\mathrm{R}+\mathrm{E}$ group. Two patients, both in the Rosu 20 group, experienced mild myalgias, neither discontinued the study medication due to these events.

CONCLUSIONS: This pilot study is the first to investigate the efficacy of rosuvastatin in combination with ezetimibe in HIV-positive patients. Significance of the findings may have been limited by sample size. However, the addition of ezetimibe to ongoing therapy with rosuvastatin was safe and effective at improving certain lipid parameters as compared to rosuvastatin alone.

\section{O009}

\section{BONE HEALTH AND CELLULAR AGING IN A COHORT OF HIV-POSITIVE WOMEN}

Mai, Alice; Sokalski, Kristen; Chu, Jackson; Côté, Helene; Maan, Evelyn J; Albert, Arianne; Murray, Melanie;

Money, Deborah; Pick, Neora; Emerging Team Grant in HIV Therapy and Aging (CARMA) and the CIHR

Vancouver, BC

BACKGROUND: The association between HIV, antiretrovirals, and bone health is controversial. Data in $\mathrm{HIV}^{+}$women is scarce, particularly of pre-menopausal ages. We estimated the prevalence of osteopenia and osteoporosis in $\mathrm{HIV}^{+}$women and explored HIV and cellular aging (leukocyte telomere length (LTL)) factors that may predict osteopenia/ porosis.

METHOD: In this cross-sectional retrospective sub-study, data were obtained through chart review of adult $\mathrm{HIV}^{+}$women enrolled in the CARMA cohort study in Vancouver. Women with at least one available bone mineral density (BMD) measured by DXA (lumbar spine(LS), femoral neck(FN), total hip $(\mathrm{TH})$ ) were included in this analysis Osteopenia and osteoporosis were defined using World Health Organization criteria. Relative LTL was measured by qPCR. Statistical analysis was performed using multivariate logistic regressions.

RESULTS: BMD and LTL data were obtained for 73 women with a mean age of 43 (IQR 34-52) years, 77\% of whom were premenopausal. $90 \%$ percent had CD $4>200,80 \%$ had an undetectable pVL, $31 \%$ had a history of substance use, $23 \%$ had active HCV infection. Among premenopausal women osteopenia was present in $8 / 56(14 \%)$, while osteopenia and osteoporosis were seen in $10 / 17$ (59\%) and $2 / 17(12 \%)$ of postmenopausal women, respectively. Older age was significantly associated with bone abnormalities at all measured sites, while lower BMI was so for the FN and TH sites. Cumulative months on protease inhibitors (PI), but not tenofovir, was a significant factor at the lumbar spine (Odds ratio $[O R]=0.98, p=0.031)$ and total hip $(\mathrm{OR}=0.95, \mathrm{p}=0.003)$. Relationships were also seen between LTL and abnormal BMD at total hip (OR=0.04, $\mathrm{p}=0.026)$.

CONCLUSION: These preliminary results suggest that, while decreased bone health is associated with aging and low BMI. PI use (regardless of type) and longer LTL may predict healthier bones in $\mathrm{HIV}^{+}$women. Tenofovir use did not come up as a significant predictor of reduced bone mass in this analysis. 
0010

ENDOCRINOPATHY AND LEUKOCYTE TELOMERE LENGTH IN HIV ${ }^{+}$INDIVIDUALS IN THE CARMA COHORT

Sokalski, Kristen M; Mai, Alice; Chu, Jackson; Côté, Helene;

Maan, Evelyn J; Pick, Neora; Money, Deborah; Murray, Melanie C Vancouver, BC

INTRODUCTION: HIV infection has been associated with various endocrinopathies. HIV also causes systemic inflammation persisting even with effective HAART. Inflammation-related oxidative stress can damage telomeres potentially promoting cellular aging. In HIV negative individuals, shorter leukocyte telomere length (LTL) is associated with endocrinopathies such as diabetes, ovarian failure and aging. We hypothesized that prevalent endocrinopathy in $\mathrm{HIV}^{+}$persons is associated with shorter LTL.

METHODS: Data were obtained through a retrospective chart review performed on CARMA (Children and Women: AntiRetrovirals and Markers of Aging) cohort participants: $\mathrm{HIV}^{+}$adult ( $\geq 19$ years) women $(\mathrm{N}=192)$ and men $(\mathrm{N}=45)$. Information collected included prevalence of endocrinopathies (diabetes, glucose intolerance, dyslipidemia, thyroid disorders, adrenal insufficiency/excess, hypogonadism, and premature ovarian failure), demographics, HCV status, baseline LTL, HAART exposure, and substance use. Statistical analysis was performed using multivariate logistic or Poisson regressions where appropriate.

RESULTS: Prevalence of endocrinopathy was 45.6\% (dyslipidemia $27 \%$, glucose intolerance/diabetes $10 \%$, thyroid disorders $13.5 \%$ ). Multivariate logistic regression revealed no difference in baseline LTL in relation to number or type of endocrinopathy, after controlling for gender, age, active HCV, and substance use. Presence of any endocrinopathy was associated with increasing body mass index (BMI) $(\mathrm{p}=0.01$, OR 1.06)), $\log _{2}$ cumulative months using either protease inhibitors $(\mathrm{p}=0.03$, OR 1.15 ) or NNRTIs $(p=0.001, O R=1.20)$ but not NRTI. Increasing number of endocrinopathies per individual was associated, with increasing BMI and age (each +1 carried an incident rate ratio [IRR] of 1.04 [p=0.001], and $1.02[\mathrm{p}=0.046]$ respectively), and cumulative months on NRTI (each doubling in months=IRR of 1.18 [p=0.001]). Active HCV infection reduced likelihood of glucose intolerance/diabetes by $87 \%$ $(\mathrm{p}=0.009$, OR 0.13).

CONCLUSION: Increasing BMI and HAART exposure were significantly associated with endocrinopathy prevalence in our cohort, while LTL was not. Further study examining rates of LTL attrition and length exposure to HIV viremia in relation to developing endocrinopathy is ongoing.

\section{O011}

HIV-ASSOCIATED NEUROCOGNITIVE DISORDERS (HAND) IN MEN AND WOMEN ON CART AND SUPPRESSED PLASMA VIRAL LOAD: PROSPECTIVE RESULTS FROM THE OHTN COHORT STUDY

Rourke, Sean B ${ }^{1}$; Gill, John'를 Rachlis, Anita ${ }^{1}$; Carvalhal, Adriana ${ }^{1}$; Collins, Evan'1; Arbess, Gordon'; Brunetta, Jason"1 Pick, Neora3 ; McCombe, Jennifer ${ }^{2}$; Letendre, Scott ${ }^{4}$; Marcotte, Thomas ${ }^{4}$; Bekele, Tsegaye $\mathrm{M}^{1}$; Burchell, Ann N${ }^{1}$; Raboud, Janet ${ }^{1}$; Ibanez-Carrasco, Francisco ${ }^{1}$; Thornton, Allen ${ }^{3}$;

Bourgeois, Claire ${ }^{1,5}$; Rosenes, Ron ${ }^{1}$; Atkinson, Maggie ${ }^{1}$;

Reinhard, Robert ${ }^{1}$; MacPhee, Paul'; McGee, Frank ${ }^{1}$

Centre for Brain Health in HIV/AIDS, The

${ }^{1}$ Toronto, ON; ${ }^{2}$ Calgary, AB; ${ }^{3}$ Vancouver, BC; ${ }^{4}$ San Diego,

California, USA; ${ }^{5}$ Montreal, QC

BACKGROUND: Despite advances in combination antiretroviral therapy (cART), HIV-associated Neurocognitive Disorders (HAND) remain prevalent. This study examined the prevalence and change of HAND over 1 year among people with HIV who are on cART and have suppressed plasma viral load.

METHODS: 375 adults (311 men and 64 women) received a brief battery of neuropsychological (NP) tests assessing learning and memory, psychomotor speed, and attention and working memory. Published age/ gender/education/race norms were used with self-reported complaints to characterize NP impairment status and HAND diagnoses according to Antinori et al., (2007) criteria: NP-normal (NP-N), Asymptomatic Neurocognitive Impairment [ANI], Mild Neurocognitive Disorder (MND), and HIV-associated Dementia (HAD). Changes in NP abilities were also examined.

RESULTS: At baseline, $42 \%$ were NP-Normal $(n=156), 35 \%$ had ANI $(n=133), 13 \%$ had MND $(n=47)$, and 10\% had HAD $(n=39)$. At 1 -year follow up, 66\% remained NP-N or ANI ( $n=249), 11 \%$ improved from MND or HAD to NP-N or ANI ( $n=43), 13 \%$ worsened $(n=47)$, and $10 \%$ $(n=36)$ had either MND or HAD at baseline and remained the same at follow-up. Men were more likely than women to remain NP-N or ANI ( $70 \%$ vs. $49 \%$, respectively); whereas women were more likely than men to experience worsening ( $26 \%$ vs $10 \%$ ) or improvement (19\% vs. $10 \%)$ of HAND. In those who remained MND or HAD, a significant decline was observed in learning and memory ability $(p<0.05)$.

CONCLUSION: Despite being on cART and having suppressed plasma viral load, 1 out of 4 adults with HIV had MND or HAD while $33 \%$ of adults had ANI. Over the follow up period, 13\% experienced a decline in neurocognitive functions (from NP-N/ANI to MND or HAD). This is the first study to characterize the prevalence and changes of HAND in people living with HIV in Canada. Intervention efforts are underway with brain fitness programs to address these neurological complications.

\section{2}

\section{POORER COGNITIVE PERFORMANCE IN HIV ${ }^{+}$WOMEN COINFECTED WITH HEPATITIS C (HCV) RELATIVE TO MONOINFECTED $\left(\right.$ HIV $\left.^{+}\right)$AND HIV NEGATIVE CONTROL WOMEN}

Giesbrecht, Chantelle ${ }^{1}$; Thornton, Allen $\mathrm{E}^{1}$; Hall-Patch, Clare ${ }^{2}$; Maan, Evelyn J²; Graham, Rebecca²; Money, Deborah²; Murray, Melanie'2; Cote, Helene ${ }^{2}$; Pick, Neora ${ }^{2}$ CIHR Team Grant on HIV Therapy and Aging (CARMA) ${ }^{2}$ ${ }^{1}$ Burnaby; ${ }^{2}$ Vancouver; BC

BACKGROUND: Cognitive functioning has not been comprehensively evaluated in $\mathrm{HIV}^{+}$women. Even fewer studies have examined the effect of $\mathrm{HCV}$ infection, despite evidence suggesting adverse impact of $\mathrm{HCV}$ on brain/neurocognitive functioning. We examined neurocognition in $\mathrm{HIV}^{+} / \mathrm{HCV}^{+}$coinfected women, hypothesizing that this group would display poorer cognitive performance relative to $\mathrm{HIV}^{+} / \mathrm{HCV}^{-}$and $\mathrm{HIV}^{-} / \mathrm{HCV}^{-}$women.

METHODS: 126 women were recruited from the Oak Tree Clinic in Vancouver; $64 \% \mathrm{HIV}+$ and $21 \% \mathrm{HCV} \mathrm{PCR}^{+}$. At assessment, mean $\pm \mathrm{SD}$ $\mathrm{CD} 4$ in $\mathrm{HIV}^{+}$was $516 \pm 242$ cells/ $/ \mathrm{l}$ with $76 \%$ having an undetectable HIV viral load. A comprehensive neuropsychology battery was administered that included traditional paper/pencil tests and subtests from a computerized battery (CANTAB). Several cognitive domains were assessed, including fine motor speed and dexterity, learning and memory, psychomotor/processing speed, and executive functions. To control for effects of age and education, each cognitive score was regressed on age and education to generate standardized cognitive residual scores (z-scores). A cognitive composite index (CCI) was computed by averaging $z$-scores across cognitive domains. Analysis of covariance (ANCOVA) was employed to compare groups: $\mathrm{HIV}^{+} / \mathrm{HCV}^{+}(\mathrm{n}=20) ; \mathrm{HIV}^{+} / \mathrm{HCV}^{-}(\mathrm{n}=61)$ and $\mathrm{HIV}^{-} / \mathrm{HCV}^{-}(\mathrm{n}=37)$. The dependent variable was $\mathrm{CCI}$, with depression self-ratings (CES-D) and substance use history as covariates. RESULTS: Groups were well-matched on age and education. There was a significant group difference on cognitive performance $(F[2,117]=4.08$, $\mathrm{p}=0.02$ ). Post hoc comparisons indicated that the $\mathrm{HIV}^{+} / \mathrm{HCV}^{+}$group (mean $\pm \mathrm{SD}-0.51 \pm 1.17$ ) displayed a significantly lower CCI than $\mathrm{HIV}^{+} /$ $\mathrm{HCV}^{-}(0.08 \pm 0.97)$ and $\mathrm{HIV}^{-} / \mathrm{HCV}^{-}(0.14 \pm 0.89)$ groups. $\mathrm{HIV}^{+} / \mathrm{HCV}^{-}$ and $\mathrm{HIV}^{-} / \mathrm{HCV}^{-}$groups were not significantly different.

CONCLUSIONS: Greater cognitive impairment (summarized by CCI) was observed in $\mathrm{HIV}^{+} / \mathrm{HCV}^{+}$compared to $\mathrm{HIV}^{+} / \mathrm{HCV}^{-}$and $\mathrm{HIV}^{-} / \mathrm{HCV}^{-}$ women. These results confirm existing literature suggesting that $\mathrm{HCV}$ infection exerts an additional detrimental impact on neurocognitive performance, and highlight the importance of assessing for other viral infections in $\mathrm{HIV}^{+}$women. 
Track C: Epidemiology and Public Health Sciences: HIV Epidemiology: Exploring Risk and Resiliency

Volet C : Épidémiologie et sciences de la santé publique : Épidémiologie du VIH : examen du risque et de la résilience

\section{O013}

ENHANCED SURVEILLANCE OF HIV AND RISK BEHAVIOURS AMONG THE ABORIGINAL POPULATIONS IN CANADA (A-TRACK) - RESULTS FROM PILOT PHASE OF A-TRACK SURVEY, REGINA, SASKATCHEWAN

Hennink, Maurice ${ }^{1}$; Abbas, Zahid ${ }^{1}$; Lloyd, Kathy ${ }^{1}$; Poitras, Margaret ${ }^{1}$; Ogunnaike-Cooke, Susanna ${ }^{2}$; Tarasuk, Jill ${ }^{2}$; Zylak, Candace ${ }^{2}$; Faye, Rachel ${ }^{1}$; Bourassa, Carrie ${ }^{1}$; Masching, Renee ${ }^{3}$; Archibald, Chris ${ }^{2}$ ${ }^{1}$ Regina, SK; ${ }^{2}$ Ottawa, ON; ${ }^{3}$ Dartmouth, NS

BACKGROUND: A-Track is an enhanced surveillance system designed to investigate trends in HIV infection, behavioural, and socio-demographic risk factors in the Aboriginal population in Canada.

OBJECTIVE: To describe risk behaviours and HIV prevalence among Aboriginal population recruited through selected venues in Regina.

METHODS: From December 5, 2011 to June 15, 2012, self-identified Aboriginal population aged 16-60 years was recruited from selected recruitment venues in Regina. The participants completed a self-administered questionnaire that elicited information on socio-demographics, substance use, injecting and sexual behaviours. Participants provided a dried blood sample (DBS) for HIV, hepatitis C antibodies and syphilis screening. Participants were offered the option of completing the questionnaire without providing a DBS. We performed descriptive statistics of key variables. RESULTS: A total of 1,071 participants were interviewed and 1,064 completed questionnaires were available for analyses. The mean age was 33.9 years, $50.7 \%$ were males, and $40 \%$ had at least a high school education. Among those who had ever injected $(n=532)$, the mean age of injection initiation was 22.1 years. The most frequently injected drugs were cocaine, non-prescribed morphine and Ritalin. Nine percent reported injecting with used needles/syringes and $12.7 \%$ reported passing on used needles/syringes. The proportion of males, who reported consistent condom use in the 12 months prior to the interview with their regular and casual opposite sex partners was $28.3 \%$ and $59.3 \%$ respectively; the corresponding proportion among females was $19.5 \%$ and $56.5 \%$. Overall HIV prevalence was $5.2 \%$ and $53.7 \%$ were aware their HIV-positive status. Among HIV-positive participants, $65.5 \%$ reported currently taking prescribed medication for HIV. Among participants who selfreported as HIV negative or unknown HIV status, $69.6 \%$ reported ever having been tested for HIV.

CONCLUSIONS: The study revealed a high HIV prevalence and notable numbers of individuals unaware of their HIV-positive status signaling significant potential of spread of HIV remains.

\section{O014}

FACTORS ASSOCIATED WITH HIV VULNERABILITY AMONG THE ABORIGINAL POPULATION IN REGINA, SASKATCHEWAN: DESCRIPTIVE RESULTS FROM THE A-TRACK PILOT SURVEY, REGINA, SASKATCHEWAN

Hennink, Maurice ${ }^{1}$; Poitras, Margaret ${ }^{1}$; Abbas, Zahid ${ }^{1}$; Lloyd, Kathy'; Ogunnaike-Cooke, Susanna ${ }^{2}$; Tarasuk, Jill2; Zylak, Candace ${ }^{2}$; Faye, Rachel ${ }^{1}$; Bourassa, Carrie ${ }^{1}$; Masching, Renee ${ }^{3}$; Archibald, Chris ${ }^{2}$ ${ }^{1}$ Regina, SK; ${ }^{2}$ Ottawa, ON; ${ }^{3}$ Dartmouth, NS

BACKGROUND: Aboriginal people continue to be over-represented in the HIV epidemic in Canada with injection drug use as a major exposure category. There is currently insufficient data to measure the full extent of the HIV/AIDS epidemic among First Nations, Inuit and Métis populations in Canada. We analyzed the results of A-Track, a behavioural and biological surveillance survey in Regina, Saskatchewan to provide a socio-demographic profile of survey participants.

METHODS: From December 5, 2011 to June 15, 2012, survey participants were recruited through select community-based venues for an anonymous survey with informed consent. Aboriginal people were identified through self-report by indicating they were First Nations, Métis, Inuit or had Aboriginal ancestors. At selected venues, participants completed a self-administered questionnaire designed to gather information about socio-demographic and behavioural factors.

RESULTS: Of the 1,064 respondents, 89.9\% identified as First Nations, $2.5 \%$ could converse in an Aboriginal language and $60 \%$ had less than high school education. $57 \%$ had history of incarceration and $10.6 \%$ had lived in a correctional facility in the 12 months prior to the interview. More than half had left their community of birth and $89 \%$ of those had done so 5 or more years ago. $43 \%$ had been placed in a foster home; of those, $67 \%$ were placed in more than one foster home and $40 \%$ had a negative or somewhat negative experience. Almost 30\% had been placed in a residential school and of those nearly three-quarters had negative or somewhat negative experience (73\%). Almost 40\% had experienced sexual abuse as a child or as an adolescent and $65 \%$ had witnessed violence between parents and other family members.

CONCLUSIONS: Demographic and socio-economic factors that are risk factors for HIV are prevalent in this urban Aboriginal population. HIV prevention program must continue to focus on these issues to more fully address the situation.

\section{O015}

\section{PERINATAL HIV EXPOSURE: ABORIGINAL MOTHER-} INFANT PAIRS IN WESTERN CANADA

Sauve, Laura J ${ }^{1}$; Forbes, Jack ${ }^{1}$; Brophy, Jason ${ }^{2}$; Alimenti, Ariane ${ }^{1}$; Samson, Lindy ${ }^{2}$; Singer, Joel'; Money, Deborah ${ }^{1}$;

Lapointe, Normand ${ }^{3}$; Lee, Terry $\mathrm{C}^{1}$; Tan, Ben ${ }^{4}$; Lamarre, Valerie ${ }^{3}$; Bitnum, Ari $^{5}$; The Canadian Pediatric AIDS Research Group ${ }^{6}$ ${ }^{1}$ Vancouver, BC; ${ }^{2}$ Ottawa, ON; ${ }^{3}$ Montreal, QC; ${ }^{4}$ Saskatoon, SK; ${ }^{5}$ Toronto, ON; ${ }^{6}$ Canada

OBJECTIVE: To describe the epidemiology of perinatal HIV exposure and vertical transmission (VT) in Aboriginal Canadians using data from the Canadian Perinatal HIV Surveillance Program (CPHSP).

METHODS: CPHSP has collected prospective data on children born to HIV-infected mothers from 22 sites across Canada since 1990; this analysis focused on Aboriginal mother-infant pairs (MIP) identified since 1997 when combination antiretroviral therapy (cART) became available. Data was collected via standardized, web-based remote data entry, with data management by the Canadian HIV Trials Network. Aboriginal status is based on maternal self-report.

RESULTS: Of 2512 MIPs identified, 20\% (508) were Aboriginal. In the Western Provinces (BC, Alberta, Saskatchewan, Manitoba), 476 (47\%) of 1003 MIPs were Aboriginal compared to only 2\% of 1509 in the rest of Canada. In the Western Provinces, IDU was the mode of HIV acquisition for $67 \%$ (302) of Aboriginal mothers, compared to 27\% (129) of nonAboriginals $(\mathrm{p}<0.0001) .57 \%$ (271 of 474$)$ of Aboriginal mothers received $>4$ weeks of cART during pregnancy compared to $71 \%$ (371 of 526) non-Aboriginals $(\mathrm{P}<0.001)$. Increasing proportions of all pregnant women received cART over time; for example, in 1997, 0/17 Aboriginal women did not receive cART, while in 2011 it was $18 \%$ (8/45). The VT rate was $4 \%$ (18 of 467 ) in Aboriginal MIPs versus 1\% (seven of 520 ) in non-Aboriginals. For those women engaged in care, duration of cART and mode of delivery was not different between Aboriginal and nonAboriginal women.

CONCLUSIONS: In Western Canada, a substantial proportion of MIPs are Aboriginal; this group is less likely to have received cART and has higher rates of VT. Additional resources and research are needed to identify HIVinfected pregnant Aboriginal women and engage them in care is needed. 
0016

SPATIALLY-ORIENTED PHYSICAL AND STRUCTURAL FEATURES OF STREET-BASED SEX WORK SPACES: THE ROLE OF BUILT ENVIRONMENT AND POLICE HARASSMENT IN CONFOUNDING NEGOTIATION OF HIV RISKS

Deering, Kathleen N${ }^{1}$; Amram, Ofer ${ }^{2}$; Nguyen, Paul ${ }^{1}$; Feng, Cindy $\mathbf{X}^{\mathbf{1}}$; Chettiar, Jill'; Shannon, Kate ${ }^{1}$

${ }^{1}$ Vancouver; ${ }^{2}$ Burnaby, BC

BACKGROUND: Epidemiological studies have largely failed to capture the dynamic interplay of physical and structural environment in negotiation of HIV risks, and have relied on individual-level variables. Using an innovative approach including neighbourhood environment data, we examined spatially-oriented HIV risks within overlapping street-based sex work and drug scenes.

METHODS: Analyses drew on baseline interview and geographic data (Jan/10-Oct/11) from a large prospective cohort of sex workers (SWs) in Metropolitan Vancouver(AESHA) and external neighborhood environment data. Geographic information systems software was used to create $50 \mathrm{~m}$ buffers surrounding SWs' primary place of public place-based solicitation. Publically available neighborhood environment data (e.g. land use, City of Vancouver) were used to create nine 'environment' variables measuring physical and structural features within buffers (e.g. industrial or commercial zoning, lighting). A series of confounder models, including bivariate and multivariable logistic regression, were used to examine the independent effect of physical and structural features of sex work spaces on HIV risks.

RESULTS: Of 510 SWs, 328 solicited clients in primarily public placebased environments (eg, streets, parks, alleys) and were included in the analyses. In bivariate and multivariable analyses, working within areas with increased commercial zoning, increased lighting and more built environment were associated with reduced likelihood of police harassment without arrest. Working in commercial zones was associated with reduced risk for exchanging sex while high and working in industrial zones was associated with increased risk; however in multivariable analysis, associations between the neighborhood environment and exchanging sex while high were all removed/not significant when adjusting for police harassment

CONCLUSIONS: Our results highlight how neighborhood environment shapes street-based sex work and drug scenes and the critical influence of enforcement-based policing practices in driving HIV risks for SWs, and demonstrate how spatially-oriented and structural responses can improve health, safety and well-being of marginalized SWs within Canada's criminalized prostitution framework.

\section{O017}

FACTORS ASSOCIATED WITH LATE INITIATION OF HAART AMONG YOUNG ADULT HIV-POSITIVE MEN AND WOMEN AGED 18-29 YEARS IN CANADA

Palmer, Alexis $\mathrm{K}^{1,2}$; Chan, Keith ${ }^{1}$; Cescon, Angela ${ }^{1}$;

Cooper, Curtis ${ }^{3}$; Raboud, Janet $\mathbf{M}^{4}$; Miller, Cari²;

Humphreys, Suzanne ${ }^{1}$; Burchell, Ann; N4; Klein, Marina $B^{5}$;

Machouf, Nimaa; Montaner, Julio $\mathrm{S}^{1}$; Tsoukas, Christos ${ }^{5}$;

Hogg, Robert $\mathrm{S}^{1,2}$; Loutfy, Mona $\mathrm{R}^{4}$; Canadian Observational

Cohort, CANOC ${ }^{1}$

${ }^{1}$ Vancouver, BC; ${ }^{2}$ Burnaby; ${ }^{3}$ Ottawa; ${ }^{4}$ Toronto, ON; ${ }^{5}$ Montreal, QC

BACKGROUND: In Canada, from 1998 to 2008, young people made up $21 \%$ to $23 \%$ of HIV-positive tests. People who initiate highly active antiretroviral therapy (HAART) with low CD4 counts or AIDS-defining illnesses (ADI) are at greater risk of treatment failure and mortality. We examined factors associated with late HAART initiation among Canadians 18 to 29 years of age.

METHODS: The Canadian Observational Cohort (CANOC) collaboration is a pan-provincial multi-site cohort study of HIV-positive adults $\geq 18$ years who initiated HAART on or after January 1, 2000 in British Columbia, Ontario, or Quebec. Our analysis was restricted to participants who initiated HAART between the ages of 18 to 29 years. Our outcome of interest was late initiation, defined as beginning HAART with a CD4 count $<200$ cells $/ \mu \mathrm{L}$ and/or having an ADI prior to HAART start. Demographics were compared between late and not late initiators using the Pearson $\chi 2$, Fisher's exact, and Wilcoxon rank-sum tests. Univariate and multivariable logistic regression was used to identify independent correlates of late initiation.

RESULTS: 1026 individuals (British Columbia $=422$, Ontario $=400$, Quebec $=204)$ were included. $340(33 \%)$ were women and approximately one third $(29.3 \%)$ reported a history of injection drug use. At time of first HAART, the median age was 27 (IQR 24 to 28) with 412 (40\%) identified as late initiators. In the multivariable model, late initiation was associated with male sex (aOR 0.74 [95\% CI 0.54 to 1.00$]$ ), being 25 years old or younger at initiation (aOR 1.40 [95\% CI 1.05 to 1.86]), and initiating treatment in an earlier calendar year (aOR 0.88 [95\% CI 0.84 to 0.92]). CONCLUSIONS: HAART initiation in young adults is influenced by a complex interplay of host and non-host factors that requires further study. The high number of young adults in our cohort starting HAART late indicates an important target population for specialized services.

\section{O018}

WAR AND HIV: GENDER DIFFERENCES IN RISK BEHAVIOUR AMONG YOUNG PEOPLE IN POSTCONFLICT GULU DISTRICT, NORTHERN UGANDA

Patel, Sheetal $^{1}$; Schechter, Martin T'1 Sewankambo, Nelson $\mathbf{K}^{2}$; Atim, Stella ${ }^{3}$; Lakor, Sam ${ }^{3}$; Kiwanuka, Noah ${ }^{2}$; Spittal, Patricia $\mathbf{M}^{1}$ ${ }^{1}$ Vancouver, BC; ${ }^{2}$ Kampala; ${ }^{3}$ Gulu, Uganda

OBJECTIVE: Despite growing knowledge of the dynamics of HIV infection during conflict, far less is known about the period that follows cessation of hostilities and its implications for population health. This study sought to fill a lacuna in epidemiological evidence through examining HIV infection and related vulnerabilities of young people living in resource-scarce, post-emergency transit camps that are now home to thousands of IDPs following two decades of war in northern Uganda METHODS: In 2010, a cross-sectional demographic and behavioural survey was conducted with 384 transit camp residents aged 15-29 years residing in Gulu District, northern Uganda. Blood specimens were collected for Rapid HIV-testing in the field and Confirmatory laboratory testing. Multivariable logistic regression models, constructed separately for males and females, identified risk factors independently associated with HIV infection for conflict-affected young men and young women. RESULTS: Of the 384 participants sampled, 192 (50\%) were female and 107 (28\%) were former child soldiers. Overall HIV prevalence was alarmingly high at $12.8 \%$. HIV prevalence among females was $15.6 \% \mathrm{com}$ pared to $9.9 \%$ among males $(p=0.092)$. The strongest predictor of HIV infection among young men was non-consensual sexual debut (adjusted odds ratio [AOR] 3.24; 95\% confidence interval [CI], 1.37-7.67), and the strongest predictor of HIV infection among young women was having practiced dry sex (AOR, 7.62, 95\% CI, 1.56-16.95).

CONCLUSIONS: Conflict-affected young men and women experience vulnerability to HIV infection in different ways than may originally be understood. Post-conflict programme planners must therefore appropriately design and target contextualized, evidence-based responses to HIV that are sensitive to gender and cultural issues. 
Track D: Social Sciences: Men Who Have Sex With Men

Volet D : Sciences sociales : Hommes ayant des relations sexuelles avec d'autres hommes

\section{O019}

'INSIDER' AND ‘OUTSIDER' PERSPECTIVES ON POSITIONALITY AND REFLEXIVITY IN COMMUNITYBASED RESEARCH WITH GAY, BISEXUAL, AND OTHER MEN WHO HAVE SEX WITH MEN

Chown, Sarah A ${ }^{1}$; Lachowsky, Nathan J ${ }^{2}$; Serrano-Sánchez, Angel ${ }^{3}$ ${ }^{1}$ Vancouver, BC; ${ }^{2}$ Guelph; ${ }^{3}$ Windsor, ON

Community-based research (CBR) approaches may be used by researchers working with communities they are not a part of (i.e. 'outsiders'), in order to meaningfully engage people with lived experiences in the community of interest (i.e. 'insiders'). The CBR paradigm is one methodology that encourages researchers to be reflexive about their positionality and its impacts on knowledge production. However, at a time when CBR approaches are becoming increasingly common in HIV research in Canada, there remains a paucity of work that integrates reflexivity into academic writing.

This paper uses narratives from three diversely located people working in gay, bisexual, and other men who have sex with men's HIV research in Canada and an intersectional framework to illustrate the ways identities and social locations impact CBR processes and knowledge production. Intersectionality emphasizes individuals' multiple, simultaneous identities, and the structural forces that dynamically shape identities (Dhamoon, 2011). The narratives are based on a range of research experiences with data collection and analysis in qualitative, quantitative, mixed methods and systematic projects with various groups of MSM.

This paper problematizes current concepts and proposes novel ways of incorporating reflexivity within CBR. It illustrates how dichotomous notions of 'insiders' and 'outsiders' oversimplify the complex, and often overlapping, relationships between researchers and the communities they work with. Further, it interrogates definitions of, and assumptions about, the people who are, or are not, included within certain communities. Lastly, the paper explores how notions of allyship can be incorporated to reframe considerations of 'insiders' and 'outsiders'. These dimensions of reflexivity serve to strengthen CBR practices and our understandings of knowledge production. Encouraging attention to positionality and reflexivity will serve to maximize the value of CBR approaches in addressing Canada's HIV epidemics. Increased consideration of the issues raised in this paper will strengthen ongoing, planned and future CBR in Canada.

\section{O020}

\section{SEROADAPTIVE BEHAVIOURS AS CONTEXT-DEPENDENT} PREVENTION STRATEGIES AMONG HIV-NEGATIVE GAY MEN

Grace, Daniel; Chown, Sarah; Jollimore, Jody; Parry, Robin;

Kwag, Michael; Steinberg, Malcolm; Trussler, Terry;

Rekart, Michael; Gilbert, Mark; CIHR Team in the Study of Acute HIV Infection in Gay Men, The

Vancouver, BC

BACKGROUND: Public health research has adopted the umbrella term "seroadaptive behaviours" to refer to serosorting and other conceptually allied strategies for sexual decision-making that are based on the real or perceived HIV status of sexual partners. However, little qualitative research has been conducted to inductively explore the seroadaptive behaviours of HIV-negative gay men in Canada who practice these strategies.

METHODS: Study recruitment occurred between June 2011 to January 2012 at a community sexual health clinic in Vancouver, British Columbia. All participants received a negative HIV test result and completed a series of sexual networking interviews and self-administered questionnaires over a one-year period $(n=165)$. A sub-sample of the participants who reported recent condomless anal intercourse were recruited into an embedded qualitative study and completed two indepth qualitative interviews $(\mathrm{n}=32)$.

RESULTS: The initial interviews elicited three dynamic narratives relevant to men's everyday practices and rationalities for using different context- or relational-dependent HIV prevention strategies: (1) Many participants describe serosorting with primary sexual partners through the use of partner testing and negotiated safety agreements; (2) Diverse strategies of serosorting and seroguessing are used among participants when having sex with hookups and casual partners; and (3) Those participants who had knowingly had sex with a serodiscordant sexual partner use other seroadaptive behaviors including one or more of seropositioning/ strategic positioning, condom serosorting and viral load sorting.

CONCLUSION: Rather than viewing all recent condomless anal sex as being risky or unprotected, many participants explained the role of seroadaptive behaviours in their decisions to have what they understand to be various forms of safe or protected condomless anal sex. HIV-negative gay men in our sample place a high degree of importance upon remaining seronegative and may benefit from public health guidance regarding the efficacy of different HIV-prevention strategies when having sex with partners of the same, different or unknown serostatus.

\section{O021}

THE COMPLEXITY OF RISK REDUCTION STRATEGIES AMONG MEN WHO HAVE SEX WITH MEN (MSM): DATA FROM SPOT, A COMMUNITY-BASED RAPID HIV TESTING INTERVENTION IN MONTREAL

Blais, Martin; McFadyen, Amélie; Otis, Joanne;

Veillette-Bourbeau, Ludivine; Haig, Thomas; Wainberg, Mark A; Rousseau, Robert; SPOT Study Group, The

Montréal, QC

BACKGROUND: SPOT is an ongoing research-intervention project offering free, anonymous, rapid HIV testing to MSM in Montreal.

OBJECTIVES: 1) To identify patterns of strategic condom-use with potentially at-risk partners and their sociosexual correlates among the SPOT participants; 2) To compare participants' sociosexual profiles according to these patterns.

METHOD: Cross-sectional data from 1184 MSM tested between July 2009 and May 2011 were gathered through structured interviews and selfadministered questionnaires.

RESULTS: Seven patterns of strategic use of condom with potentially at-risk partners were identified: 1 ) No anal intercourse (AI) with partners of unknown or HIV positive status (38.7\%);2) Systematic use of condom for complete or partial AI $(26.7 \%)$; 3 ) No use of condom for both complete and partial AI $(6.3 \%) ; 4)$ Systematic condom use for complete AI but inconsistent use for partial AI (4.6\%); 5) Inconsistent condom use for complete $\mathrm{AI}$ and inconsistent or systematic use a condom for partial AI $(6.2 \%) ; 6)$ Inconsistent condom use for complete AI but no use of condom for partial AI (1.9\%); 7) Combination of seroadaptation and strategic positioning $(15.6 \%)$. Strategic condom-use patterns are contrasted according to the participants' sociosexual profiles such as intentionally unprotected AI, condom-use self-efficacy, age, education.

CONCLUSIONS: Results showed how MSM use condom in various, complex ways, according to different stages of AI, to sexual positioning and to partners' presumed serostatus. Indicators need to be developed by research to capture the diversity of condom-use patterns. Prevention messages and counseling also have to be adapted to this diversity.

\section{O022}

\section{MIGRATION AS A CRITICAL LIFE TRANSITION AND RESILIENCY STRATEGY AMONG ASIAN MSM IN TORONTO}

Utama, Richard B; Li, Alan; Poon, Maurice; Dandal, Alvi;

Hui, Christian

Toronto, ON

BACKGROUND: Literature suggests that migration potentially increases vulnerability to HIV and STI among ethno-racial men who have sex with 
men (MSM) (Myers et al., 2001) (Poon, M.K.L. and Ho, P.T., 2002). However, our current study on Asian MSM shows that migration, as a critical life event, can also foster resiliency and sexual health efficacy.

METHOD: In 2012, Asian Community AIDS Services conducted a research study to explore sexual health challenges and pathways to resiliency among Asian MSM. An advisory committee of community members and service providers was formed to guide the overall research design, data collection, analysis and knowledge translation. The study conducted six focus groups with 50 Asian MSM from diverse ethno-cultural and socioeconomic backgrounds to explore their lived experiences and factors that affect their sexual health and resiliency strategies.

RESULTS: Migration, self-initiated or involuntary, to Toronto from other parts of Canada as well as from foreign countries was identified by participants as one of the most critical life events. Migration was also associated with opportunities to explore gay culture, gay identity development, settlement and adaptation challenges that included service access barriers. However, migration was also attributed as an incentive to seek out and establish new support systems, increased self-efficacy to gain selfconfidence, financial independence, and to deal with family and cultural issues. Insights gained informed the strategies with which participants were able to address other critical life events affecting their sexual and holistic health.

CONCLUSION: Many Asian MSM identified experiences related to migration as a critical life event with major impact on their overall health. Yet, it contributed to resiliency amidst complex life changes. Further in-depth analysis on the pathways through which participants connect with various internal and external support systems to address challenging life events will enable development of interventions to foster resiliency among such marginalized populations.

\section{O023}

MASCULINITY, BODY IMAGE, AND HIV SEXUAL RISK AMONG ETHNORACIALIZED GAY, BISEXUAL AND OTHER MEN WHO HAVE SEX WITH MEN IN TORONTO: RESULTS FROM THE IMAGINE MEN'S HEALTH SURVEY

Brennan, David J'; Souleymanov, Rusty ${ }^{1}$; George, Clemon²; Hart, Trevor $\mathrm{A}^{\mathbf{1}}$; Newman, Peter $\mathrm{A}^{\mathbf{1}}$

${ }^{1}$ Toronto; ${ }^{2}$ Oshawa, ON

BACKGROUND: Previous research on primarily White participants has shown that among MSM, sexual risk has been associated with measures of masculinity and body image. The purpose of this analysis is to examine the relationship between body image, beliefs about body type and masculinity, and HIV risk among ethnoracialized MSM.

METHODS: Utilizing an online survey of 410 ethnoracialized MSM, body image was measured using the Drive for Muscularity scale. Body Type/Masculinity was measured by the Perceived Masculinity Scale which assessed beliefs regarding physical appearance and penis size as indicators of masculinity. HIV risk was measured as reported serodiscordant unprotected anal intercourse (SDUAI; unknown status was considered serodiscordant) in the last 6 months. Relationships between Drive for Muscularity, Body Type/Masculinity, demographics (age, income), sexual orientation, and HIV risk were examined using bivariate tests and logistic regression modeling.

RESULTS: All survey items were completed by 389 men included in the analysis. Participants identified as: South Asian ( $\mathrm{n}=82 ; 21.1 \%)$, Black/ African/Caribbean ( $\mathrm{n}=84 ; 21.6 \%)$, Latino/Brazilian ( $\mathrm{n}=94 ; 24.2 \%)$, East/ Southeast Asian $(n=105 ; 27 \%)$, and mixed race $(n=24 ; 6.2 \%)$. The mean age was 33 years $(\mathrm{SD}=8.6)$. Among respondents, $64(16.9 \%)$ were HIVpositive, $284(75.1 \%)$ were HIV-negative, and 30 (7.9\%) were unsure of their HIV serostatus. The only significant differences in SDUAI were East/Southeast Asian men reported lower rates and those with unsure HIV status reported higher rates than HIV-positive or negative men. Regression analysis indicated Drive for Muscularity (OR=1.31, 95\% $\mathrm{CI}=1.02-1.70)$, and Body Type/Masculinity $(\mathrm{OR}=1.05,95 \% \mathrm{CI}=1.01$ 1.10) significantly predicted HIV risk, after controlling for age, education and sexual orientation.

CONCLUSIONS: Among ethnoracialized MSM, pressures to conform to body image ideals and masculine gender roles in the gay community may be influencing sexual risk. Further research is needed to understand the link between body image and masculinity within the context of preventing HIV risk among ethnoracialized MSM.

\section{O024}

TOWARDS A SOCIAL ECOLOGY OF RECTAL MICROBICIDE ACCEPTABILITY AMONG MEN WHO HAVE SEX WITH MEN AND TRANSGENDER WOMEN IN THAILAND: A COMMUNITY-BASED MIXED METHODS INVESTIGATION

Newman, Peter $A^{1}$; Roungprakhon, Surachet ${ }^{2}$; Tepjan, Suchon ${ }^{1}$ ${ }^{1}$ Toronto, ON; ${ }^{2}$ Bangkok, Thailand

BACKGROUND: With HIV-incidence among men who have sex with men (MSM) in Bangkok among the highest in the world, a topical rectal microbicide would be a tremendous asset to prevention. Yet ubiquitous research-to-practice gaps between clinical trial efficacy and real-world effectiveness of existing interventions highlight the need to anticipate factors that may influence rectal microbicide (RM) effectiveness beyond clinical trials. We explored multi-level factors associated with RM acceptability among MSM and transwomen in Thailand.

METHODS: We conducted a community-based investigation using a mixed methods approach with a concurrent triangulation design. From June-August 2011 we completed 5 focus groups in two Thai cities using a semi-structured interview guide and brief self-administered questionnaire in Thai. Focus groups were recorded, transcribed, coded using thematic analysis and uploaded into a customized Microsoft Access database. We conducted content analysis to quantify theme prevalence and chi-square and Fisher's exact tests to compare themes by sexual orientation/gender expression and age.

RESULTS: Participants $(\mathrm{n}=37)$ mean age was 24.8 years $(\mathrm{SD}=4.2)$; $70.3 \%$ self-identified as gay, $24.3 \%$ transwomen. A social ecology of RM acceptability emerged with product-level themes (e.g., side effects, formulation, efficacy, scent) accounting for $42 \%$, individual (risk compensation, packaging/portability, timing of use) 29\%, interpersonal (trust/ communication, power/negotiation, stealth) $8 \%$ and social-structural (cost, access, community influence, stigma) 21\% of total codes. We found significant differences by sexual orientation/gender identity. Preferences for gel versus suppository and pericoital versus daily use were contingent on interpersonal factors in the context of social-structural constraints due to stigma, access and cost.

CONCLUSIONS: A social ecology of RM acceptability suggests socialstructural interventions to ensure broad access, low cost and to mitigate stigma against gay and other MSM and transwomen in the Thai healthcare system, and targeted educational interventions that acknowledge differences among MSM and transwomen, may support the effectiveness of rectal microbicides in reducing HIV transmission.

\section{Track A: Basic Sciences: HIV Immunology}

\section{Volet A : Sciences fondamentales : Immunologie du VIH}

O025

BY USURPING IL-7 MEDIATED PATHWAYS, THE HIV TAT PROTEIN TARGETS CD127 FOR DEGRADATION AND THUS ATTENUATES CD8 T-CELL SURVIVAL AND FUNCTION

MacPherson, Paul; Ghazawi, Feras; Faller, Elliott; Sugden, Scott; Cherid, Hafsa; Kakal, Juzer; Parmar, Parmvir;

El-Salfiti, Abdulkareem

Ottawa, ON

BACKGROUND: Interleukin (IL)-7 is a pivotal cytokine essential for T-cell survival and function. We previously reported a significant downregulation of the IL-7 receptor alpha-chain (CD127) on CD8 T-cells in 
HIV+ patients and have since shown suppression of CD127 is mediated by soluble HIV Tat protein and IL-7, both of which are elevated during HIV infection. We now elucidate the mechanisms by which IL-7 and Tat suppress CD127 in CD8 T-cells.

METHODS: Primary human CD8 T-cells were isolated from healthy HIV-negative volunteers. Gene transcripts were measured by qPCR. Surface and total protein were examined by flow cytometry and Western respectively while protein-protein interactions were detected by coimmunoprecipitation and confocal microscopy. Where indicated protein function was confirmed by siRNA-mediated knockdowns. Tat protein and deletion mutants were generated as 6xHis-tagged proteins and purified from bacteria or expressed from the pcDNA3.1 eukaryotic expression vector.

RESULTS: Upon binding IL-7, surface CD127 is rapidly internalized while activation of the JAK/STAT5 pathway stimulates expression of the CIS and c-Myb proteins. While c-Myb suppresses CD127 gene transcription, CIS binds directly to phosphorylated CD127 protein and shuttles the receptor from early to late endosomes. Subsequent proteasomal degradation of CD127 and CIS is dependent on an E3 ligase. Acting as an adaptor protein HIV Tat binds via its amino terminal domain to CD127 and appears to recruit CIS to the receptor complex inducing CD127 degradation in the absence of IL-7 and CD127 phosphorylation.

CONCLUSIONS: IL-7 is pivotal to T-cell survival and function and is currently being investigated as a potential therapy in HIV+ individuals with poor immunological response to antiretroviral therapy. We show here that while IL-7 suppresses CD127 both transcriptionally and at the protein level, HIV Tat acts as an adaptor protein to usurp the cellular mechanism and shuttle the receptor to the proteasome for degradation.

\section{O026}

MECHANISMS OF SOLUBLE IL-7 RECEPTOR $\alpha$ RELEASE BY $\mathrm{CD8}^{+}$T CELLS

Davidson, April; Angel, Jonathan B; Crawley, Angela M Ottawa, ON

BACKGROUND: Interleukin-7 (IL-7) regulates T-cell development, proliferation, survival and contributes to anti-viral CD8+ T-cell cytotoxic (CTL) activity. The expression of the IL-7 receptor $\alpha$ (CD127) receptor on CD8+ T-cells is reduced in HIV infection and downregulated by IL-7 in vitro. A soluble form of CD127 (sCD127) alters IL-7 activity in vitro, and sCD127 plasma concentrations are increased in HIV infection. Although sCD127 may play a role in HIV immunopathogenesis, the mechanisms of its production have not been described.

METHODS: Primary human CD8 + T cells were stimulated with IL-2 receptor $\gamma$-chain- $(\gamma \mathrm{C})$ sharing cytokines (IL-2,-4,-7, or -15) with or without T-cell stimulation (anti-CD3/CD28 antibodies or phytohemagglutinin, PHA). The concentration of sCD127 in culture supernatants was measured by a sCD127-specific ELISA after 3, 6, 24, 48 and 72 hours of culture. To determine the potential mechanisms of sCD127 release, the roles of IL-7 signalling (using inhibitors of Jak) and proteolytic cleavage (using serine and cysteine protease inhibitors) were investigated. RESULTS: Although IL- 7 and other $\gamma \mathrm{C}$ cytokines had little effect on sCD127 release by $\mathrm{CD}^{+} \mathrm{T}$ cells, stimulation with IL-7 significantly enhanced anti-CD3/CD28 antibody- or PHA-induced sCD127 release, which was maximal after 72 hours of culture. Inhibition of Jak signalling blocked the expression of sCD127. Despite the presence of multiple potential proteolytic cleavage sites encoded in the CD127 gene, inhibition of serine or cysteine proteases did not alter sCD127 release.

CONCLUSIONS: IL-7 and T-cell stimulation synergistically induce sCD127 release by CD8 ${ }^{+} \mathrm{T}$-cells, and this is dependent on IL-7 signalling. The mechanism by which this occurs is yet to be described and ongoing studies are exploring alternative mechanisms of soluble receptor expression. Increased plasma IL-7 observed in HIV infection, which, in the presence of immune activation (a cardinal feature of HIV infection) may enhance sCD127 release which may have a role in HIV pathogenesis.
O027

PAUCITY OF NAÏVE TH17 PRECURSORS IN HIV-INFECTED INDIVIDUALS: PERSISTENT IMPAIRMENT UNDER ART

Da Fonseca, Sandrina; Cleret-Buhot Aurélie; Gosselin, Annie;

Boulassel, Rachid-Mohamed; Routy, Jean-Pierre; Bernard, Nicole;

Tremblay, Cécile; Ancuta, Petronela

Montréal, QC

BACKGROUND: Th17 cells are preferentially depleted from the gut of HIV-infected individuals and their restoration under viral-suppressive ART is partial. Direct infection is one mechanism for Th17 depletion. Here we investigated the Th17 differentiation potential of naive $\mathrm{CD}^{+}{ }^{+}$ T-cells in HIV-infected individuals in relationship with the frequency of naive Th17 precursors.

METHODS: Leukapheresis from $\mathrm{HIV}^{+}$subjects recently infected untreated (RI, $n=8$, ) and ART-treated (CI/ART, n=9,), and HIVuninfected controls $(n=14)$ were available for this study. The frequency of Th17 precursors $\left(\mathrm{CD} 45 \mathrm{RA}^{+} \mathrm{CCR} 7{ }^{+} \mathrm{CD} 25{ }^{+} \mathrm{CD} 127-\mathrm{FoxP}^{+}\right)$and memory Th17 (CD45RA $\left.{ }^{-} \mathrm{CCR} 6{ }^{+} \mathrm{CD} 26^{+} \mathrm{CD} 161^{+}\right)$was assessed by flow cytometry. Cells were sorted by flow cytometry, cultured under Th17 conditions, and the expression of Th17 cytokines was assessed by intracellular staining. Integrated HIV-DNA levels were quantified by real-time PCR.

RESULTS: Naïve CD4 ${ }^{+}$T-cells from RI and CI/ART HIV-infected individuals versus uninfected controls were significantly impaired in their ability to differentiate into Th17 cells in vitro and this coincided with the paucity of Th17 precursors in HIV-infected subjects ex vivo. Naïve Th17 precursor counts were significantly reduced in RI and CI/ART subjects vs controls, with a significant decrease observed between CI/ART and RI. The frequency of naïve Th17 precursors correlated positively with the frequency of memory Th17 cells and negatively with age. For similarly low Th17 precursor frequency, the median age of RI and CI/ART subjects was significantly lower compared to that of uninfected controls, indicative of premature ageing in HIV-infected individuals. Naïve CD4 ${ }^{+} \mathrm{T}$-cells from CI/ART subjects harbour early reverse transcripts and/or integrated HIV-DNA.

CONCLUSIONS: We provide the first evidence that naive CD4 T-cells from HIV-infected subjects exhibit an altered Th17 differentiation potential, likely caused by the paucity of naïve CD25+CD127FoxP3 $^{+}$Th17 precursors. This impairment is not restored under viral-suppressive ART. Mechanisms responsible for Th17 deficiency at precursor level remain to be elucidated but likely involve permissiveness to abortive/integrative infection.

\section{O028 \\ TH17-POLARIZED CENTRAL MEMORY CELLS CONTRIBUTE TO THE LONG-TERM PERSISTENCE OF HIV-1 RESERVOIRS IN SUBJECTS RECEIVING VIRAL SUPPRESSIVE ART}

Gosselin, Annie; Cleret-Buhot, Aurélie; Monteiro, Patricia; Boulassel, Mohamed-Rachid; Routy, Jean-Pierre;

Ancuta, Petronela

Montreal, QC

BACKGROUND: Persistence of HIV-1 reservoirs in a fraction of longlived central memory $\mathrm{CD}^{+}{ }^{+}$T-cells $(\mathrm{CM})$, with yet unidentified character istics, represents a major barrier to eradication under viral-suppressive ART. We and others demonstrated that HIV targets for infection CD4 T-cells with a Th17 and Th1Th17 polarization profile, specific for C. albicans and M. tuberculosis, respectively. To determine whether Th17 cells contribute to HIV reservoir persistence, we quantified levels of integrated HIVDNA in CM subsets from HIV-infected subjects receiving ART.

METHODS: PBMC from eight ART-treated HIV-infected subjects were included in the study. $\mathrm{CD}^{+}{ }^{+}$-cells with $\mathrm{CM}\left(\mathrm{CD} 45 \mathrm{RA}{ }^{-} \mathrm{CCR} 7^{+}\right)$versus effector memory (EM, CD45RA ${ }^{-} \mathrm{CCR} 7^{-}$) phenotype, expressing or not the Th17 marker CCR6, as well as CM subsets with Th17 (CCR4 ${ }^{+} \mathrm{CCR} 6^{+}$), Th2 (CCR4 ${ }^{+} \mathrm{CCR}^{-}{ }^{-}$, Th1Th17 $\left(\mathrm{CXCR}^{+}{ }^{+} \mathrm{CCR} 6^{+}\right)$, and Th1 $\left(\mathrm{CXCR}^{+} \mathrm{CCR}^{-}{ }^{-}\right.$) polarization profiles, were sorted by flow cytometry. Integrated HIV-DNA levels were quantified by real-time PCR. HIV-p24 
levels were measured by ELISA in supernatants from CM subsets stimulated via $\mathrm{CD} 3 / \mathrm{CD} 28$ and all-trans retinoic acid (ATRA).

RESULTS: CCR6 was a marker for cells harboring the highest levels of integrated HIV-DNA within the CM but not the EM compartment of ART-treated HIV-infected subjects. The size of the HIV reservoir in Th17 and Th1Th17 CM was relatively high in all donors. The lowest levels of HIV-DNA were detected in Th2 CM, consistent with their lack of CCR5 and preserved frequency in vivo. Productive HIV infection was detected in $\mathrm{CCR} 6^{+} \mathrm{CM}$ upon TCR triggering in the presence of ATRA in vitro.

CONCLUSION: We demonstrate for the first time that Th17-polarized CM subsets contribute to the persistence of integrated HIV-DNA in ART-treated subjects and that this viral reservoir is replication competent. The characterization of molecular mechanisms controlling HIV persistence in Th17 CM cells will be critical for the design of new therapeutic strategies aimed at HIV eradication specifically in these cells.

\section{O029}

IN VITRO HIV INFECTION REDUCES IL-17 SECRETION AND STAT3 SIGNALING IN HUMAN TH17 CELLS

Fernandes, Jason; Angel, Jonathan B

Ottawa, ON

BACKGROUND: $\mathrm{T}$ cell dysfunction persists in $\mathrm{HIV}^{+}$individuals despite restoration of $\mathrm{CD}^{+} \mathrm{T}$ cell counts to near normal levels with HAART. Recent analysis of blood and gut Th17 cells from HIV patients have revealed defects in the production of Th17 cytokines including IL-17 which may contribute to microbial translocation and persistent inflammation observed in HIV infection. It is unclear whether this deficiency results from HIV inhibiting Th17 cell differentiation or function. STAT3 and RORC have been identified as master controllers of Th17 differentiation, but the effects of HIV infection on these signaling pathways in Th17 differentiation and function have not been examined. We hypothesize that Th17 differentiation and function is impaired in HIV infection due to altered intracellular signalling.

METHODS: Th17 cells were isolated from peripheral blood by magnetic selection or generated by treating naive blood $\mathrm{CD}^{+} \mathrm{T}$ cells with antibodies against CD3/CD28 in the presence of IL-1B, IL-6, and IL-23 for 12 days. The resulting Th17 cells were cultured with a dual-tropic HIV isolate (CS204) for 24 hours. Expression of IL-17 in response to PMA/ionomycin was examined by flow cytometry. Production of IL-17 in response to anti-CD3/28 and IL-23 was assessd by ELISA, and phosphorylation of STAT3 signalling molecules was assessed by flow cytometry. STAT3 and RORC mRNA induction was measured by quantitative RT-PCR.

RESULTS: Infection with HIV significantly impaired expression of IL-17 in in vitro-generated Th17 cells stimulated with PMA/ionomycin. Secretion of IL-17 by both blood and in vitro-generated Th17 cells stimulated with anti-CD3/28 and IL-23 was significantly reduced in HIVinfected cells. Phosphorylation of STAT3 and induction of STAT3 mRNA was reduced following infection while no changes in RORC mRNA were observed.

CONCLUSIONS: We report that production of IL-17 by blood and in vitro-generated Th17 cells is altered by in vitro HIV infection. We have also identified HIV-induced defects in IL-23-induced STAT3 signaling and transcription. Understanding the mechanisms of this inhibition may provide insight into therapies that will correct the gut-associated immunopathogenesis in HIV infection.
O030

GENOME-WIDE TRANSCRIPTIONAL PROFILING IDENTIFIES RORC, PPARG, AND THE TCR SIGNALING PATHWAY AS MAJOR DETERMINANTS FOR HIV PERMISSIVENESS IN TH17 CELLS

Cleret-Buhot, Aurélie ${ }^{1}$; Goulet, Jean-Philippe ${ }^{1}$; Monteiro, Patricia ${ }^{1}$; Bernier, Annie'; Gosselin, Annie' ${ }^{1}$; Boulassel, Mohamed-Rachid ${ }^{2}$; Routy, Jean-Pierre ${ }^{1}$; Haddad, Elias ${ }^{2}$; Sekaly, Rafick-Pierre ${ }^{2}$; Tremblay, Cécile ${ }^{1}$; Ancuta, Petronela ${ }^{1}$

${ }^{1}$ Montréal, QC; ${ }^{2}$ Port St Lucie, Florida, USA

BACKGROUND: HIV-1 selectively infects specific CD4+ ${ }^{+}$-cell subsets via yet uncharacterized molecular mechanism. We demonstrated that Th17 cells are highly permissive to HIV infection, while Th1 cells are relatively resistant. We hypothesized that Th17 cells express a unique transcriptional profile compatible with HIV permissiveness.

METHODS: Th17 (CCR4 ${ }^{+} \mathrm{CCR}^{+}$phenotype) and Th1 (CXCR3+CCR6- phenotype) subsets were sorted by flow cytometry and stimulated via CD3/CD28 Abs. Total RNA was extracted and the expression of 47,000 probe-sets was tested using the Illumina technology. Cytokine production in proliferating cells was measured by intracellular straining of CFSE-loaded cells and flow cytometry. Levels of HIV-p24 and integrated HIV-DNA were measured by ELISA and real-time PCR, respectively. The Amaxa RNA interference technology was used to evaluate the functional role of top-modulated genes.

RESULTS: Among 2,197 present calls, 1,189 and 872 probe-sets were upregulated and downregulated, respectively, in Th17 vs Th1 cells ( $p$-value $<0.05 ; 1.3$-fold change cutoff). Genes associated with T-cell differentiation (RORC), TCR signaling (ZAP-70, Lck, MAP3K4), activation/apoptosis (PTPN13), and HIV replication (PPARG) were upregulated in Th17 versus Th1 cells. Differential expression of these transcripts was validated by RT-PCR and/or fluorescence microscopy. Functional studies demonstrated that HIV permissiveness was associated with lower TCR activation threshold, increased proliferation potential, and superior NF-kB DNA-binding activity in Th17 versus Th1 cells. RNA interference against RORC and MAP3K4 decreased HIV permis siveness, while PPARG silencing had the opposite effects. Genes downregulated in Th17 versus Th1 cells and previously linked to HIV resistance included CCR5 binding ckemokines and IFN-induced molecules.

CONCLUSIONS: This study reveals the genome-wide transcriptome of CCR $4^{+}$CCR $6{ }^{+}$Th 17 cells and identifies RORC, PPARG, and the TCR signaling pathway as major molecular determinants regulating permissiveness to HIV infection. Novel therapeutic strategies aimed at interfering with Th17-specific transcripts may limit HIV replication, while preserving the beneficial role of Th17 cells in mucosal immunity.

\section{O031}

\section{TIM-3 IS EXPRESSED ON THE SURFACE OF ACTIVATED PLASMACYTOID DENDRITIC CELLS AND CORRELATES WITH CD4 DECLINE DURING CHRONIC HIV-1 INFECTION}

Schwartz, Jordan A; Clayton, Kiera; Mujib, Shariq;

Ostrowski, Mario A

Toronto, ON

A hallmark of late stage HIV-1 infection is the inability of the innate immune system to respond to secondary infections. One innate cell type of importance to normal immune function is the Plasmacytoid Dendritic Cell (pDC). pDCs are highly specialized cells, with the ability to directly sense viral and bacterial nucleotides through endosomal toll-like receptors (TLRs) and respond rapidly by secreting IFN- $\alpha$. However, pDCs decline in HIV-1 patients correlating with CD4 T cell decline and produce less IFN- $\alpha$ when stimulated. We hypothesized that the inflammatory environment present during HIV-1 infection may alter pDC function. For our study we stimulated human pDCs in vitro in the presence of viral or proinflammatory stimuli. We identified Tim-3, a marker of $\mathrm{T}$ cell exhaustion, to be expressed on the surface of $\mathrm{pDC}$ in response to IFN- $\alpha$ receptor stimulation. Tim-3 expressing pDCs do not co-produce IFN- $\alpha$. Addition of the Tim-3 
ligand galectin- 9 decreases the activation state of pDCs as measured by reduction in MFI of CD86 and TRAIL while increasing the frequency of apoptotic pDCs as measured by Live/Dead Violet staining. pDCs in the PBMC of chronically HIV-infected individuals naïve for antiretroviral treatment express high levels of Tim-3 which has a negative correlation with CD4 count. To our knowledge this is the first evidence that Tim-3 is expressed on human pDCs. Additionally, this data suggests that increased IFN- $\alpha$ found during chronic immune activation upregulates Tim-3 on pDCs and may interfere with their ability to respond to secondary infections, contributing to a deficient innate immune response.

\section{O032}

\section{ANTIBODY RECOGNITION OF THE V3 REGION OF HIV-1} GP120 IS MODULATED BY CONSERVED V3 TIP RESIDUES Manhas, Savrina; Clark, Brenda E; Auyeung, Kate; Pantophlet, Ralph

Burnaby, BC

The V3 region on HIV-1 is critically important for virus infectivity and therefore of interest as a vaccine target. However, antibodies to the V3 region of the HIV envelope spike (Env) typically neutralize non-subtype $B$ viruses poorly, an observation that is often attributed to epitope masking. While V3 antibody accessibility has been studied extensively on subtype $\mathrm{B}$ viruses, which tend to be neutralized better by anti-V3 antibodies, V3 exposure and mechanism(s) that restrict V3 exposure on nonsubtype $B$ viruses have yet to be explored in detail. Here we report on the significance of the conserved V3 tip motifs 312GPGR315 (subtype B) and 312GPGQ315 (non-subtype B) for recognition by mAb B4e8, a cross-neutralizing anti-V3 antibody, and a set of additional V3 antibodies. Position 315 in a representative subtype B (SS1196) and two subtype C viruses (ZM249M, CAP45) was mutated to Gln and Arg respectively to assess the effect of the conserved $\mathrm{Arg} / \mathrm{Gln}$ at position 315 on V3-specific neutralization. While both subtype $\mathrm{C}$ viruses were resistant to neutralization by the anti-V3 mAb B4e8, the Q315R mutant viruses were strikingly sensitive to $\mathrm{B} 4 \mathrm{e} 8$ neutralization. The subtype $\mathrm{B}$ virus was, as expected, neutralized by the panel of V3 antibodies (B4e8, 268-D, 2219, 2557, HGN194, 3074) despite having varying fine specificities for the V3 tip. However changing Arg315 to Gln resulted in substantial resistance to neutralization by the aforementioned V3 antibodies, even to those that are not dependent on Arg315 for binding. The results suggest that the tip of V3 is antibody accessible on the surface of at least some nonsubtype $B$ viruses; however, the presence of the Gln at position 315 leads to an antigenic structure that typical V3 antibodies fail to recognize. Our results thus provide insight into how HIV sequence conservation limits antibody recognition of otherwise vulnerable conserved target sites.

\section{Track B: Clinical Sciences: HIV in Women and Children}

Volet B : Sciences cliniques : Le VIH chez les femmes et les enfants

\section{O033}

GENDER INEQUITIES IN QUALITY OF INITIAL HIV CARE (QOC) AMONG HIV-POSITIVE INDIVIDUALS INITIATING ANTIRETROVIRAL TREATMENT IN BRITISH COLUMBIA, CANADA (2000-2010)

Carter, Allison J; Min, Jeong E; Lima, Viviane D; Pick, Neora; Montaner, Julio S; Hogg, Robert S; Kaida, Angela

Vancouver, BC

BACKGROUND: Compliance with HIV clinical care guidelines in the first year after antiretroviral treatment (ART) initiation is a key predictor of health and survival. We measured differences in 'quality of initial HIV care' (QOC) by gender and examined factors associated with poorer QOC.
METHODS: Participants ( $\geq 19$ years) initiated ART between 2000 and 2010 in British Columbia. QOC by gender was estimated using the Programmatic Compliance Score (PCS), a new metric associated with all-cause mortality, which includes six indicators of non-compliance with treatment initiation guidelines at baseline (no resistance testing before treatment initiation; starting a non-recommended ART regimen; and initiating ART at CD4 $<200$ cells $/ \mathrm{mm}^{3}$ ) and during the first year of follow-up (receiving <3 CD4 tests; receiving <3 viral loads (VL); and not achieving viral suppression within six months). Summary scores range from 0-6; higher scores indicate poorer QOC. Ordinal logistic regression was used to explore gender-specific predictors of poorer QOC.

RESULTS: PCS was determined for 3875 participants (20\% women). At baseline, $43 \%$ of women $(36 \%$ of men $(\mathrm{p}<0.001))$ did not receive resistance testing before treatment initiation; $17 \%$ of women ( $9 \%$ of men $(\mathrm{p}<0.001))$ initiated treatment on a non-recommended ART regimen; no gender differences existed by baseline CD4. During follow-up, 35\% of women $(19 \%$ of men $(\mathrm{p}<0.001))$ received $<3$ CD4 tests; $19 \%$ of women $(15 \%$ of men $(p<0.001))$ received $<3 \mathrm{VL}$; and $52 \%$ of women $(44 \%$ of men $(\mathrm{p}<0.001))$ did not achieve viral suppression within 6 months. Overall, mean PCS for women was $2.14(\mathrm{SD}=1.4)$ and $1.72(\mathrm{SD}=1.4)$ for men $(p<0.001)$. Among women, those who were $<50$ years, injection drug users, Aboriginal, from Vancouver Island, and who initiated ART in earlier years (2000-2003) were more likely to have poorer QOC. With the exception of age, similar factors predicted poorer QOC among men. CONCLUSIONS: There remain significant inequities in QOC by gender. Poorer QOC observed among women demands that barriers undermining women's access to high-quality HIV treatment and care must be addressed to improve treatment, health, and survival for women living with HIV.

\section{O034}

INCIDENCE OF CANCER AMONG HIV-POSITIVE WOMEN IN BRITISH COLUMBIA (BC), 1994-2008

Cescon, Angela; Salters, Kate A; Zhang, Wendy; Money, Deborah; Murray, Melanie; Coldman, Andy; Ogilvie, Gina S;

Hamm, Jeremy; Chiu, Connie G; Montaner, Julio S;

Wiseman, Sam M; Hogg, Robert S; Pick, Neora

Vancouver, BC

BACKGROUND: HIV infection has been associated with increased cancer risk but few studies have examined assorted cancer types among women. Using population-based data, we aim to document cancer incidence and correlates from 1994-2008 among HIV-positive women in BC. METHODS: Data were obtained from a retrospective population-based cohort created from linkage of two province-wide databases: the $\mathrm{BC}$ Cancer Registry and the BC Centre for Excellence in HIV/AIDS. This linkage permitted identification of cancer diagnoses among HIV-positive women $\geq 19$ years of age, from the time they engaged in HIV care in BC. Cervical intraepithelial neoplasia cases $(n=32)$ were excluded. Bivariate analysis (Pearson chi-squared, Fisher's exact, or Wilcoxon rank-sum tests) compared women with and without cancer across clinical/socio-demographic variables. Standardized incidence ratios (SIR) were calculated for selected cancers compared to the general population of women in $\mathrm{BC}$ (age-standardized).

RESULTS: Among 2,211 women with 12,529 person-years of follow-up, 78 incident cancers were identified: 47 AIDS-defining malignancies [Kaposi's sarcoma, $\mathrm{n}=5$; non-Hodgkin's lymphoma (NHL), $\mathrm{n}=19$; cervical cancer, $\mathrm{n}=22$ ] and 32 non-AIDS-defining malignancies (NADMs) (overall incidence $=0.62 / 100 \mathrm{PY}$ ) . Of the NADMs, the most common were lung/ bronchus $(n=7,22 \%)$ and breast $(n=7,22 \%)$. The median age and CD4 count at cancer diagnosis were 40 years (IQR: $32-48$ ) and $150 \mathrm{cells} / \mathrm{mm}^{3}$ (IQR: 40-259), respectively. Cancer diagnosis was associated with baseline AIDS-defining illness, lower baseline CD4 count (median 140 vs. 250 cells $/ \mathrm{mm}^{3}$ ), higher baseline and peak viral load, and lower nadir CD4 count (median 50 vs. 150 cells $/ \mathrm{mm}^{3}$ ) (all $\left.\mathrm{p}<0.05\right)$. No differences were noted by age, ethnicity, HCV co-infection, injection drug use history, or years on ART. Based on SIRs, cancer incidence was significantly higher among HIV-positive women than the general population for the following cancers: oral/pharyngeal, genital tract, Hodgkin's lymphoma, NHL, and Kaposi's sarcoma; and lower for breast cancer. 
CONCLUSIONS: HIV-positive women may be at higher risk for a number of cancers, suggesting the importance of targeted screening and detection programs. CD4 count and viral load are important correlates of risk, presenting the possibility to positively impact cancer risk with improved HIV treatment.

\section{O035}

\section{METAGENOMIC CHARACTERIZATION OF THE VAGINAL MICROBIOME IN HIV-POSITIVE WOMEN USING GENE SEQUENCING METHODS}

Mahal, Daljeet $^{1}$; Chaban, Bonnie ${ }^{2}$; Albert Arianne $\mathrm{Y}^{1}$; Vicol, Laura'; Wagner, Emily C1; Hill, Janet ${ }^{2}$; Hemmingsen, Sean ${ }^{2}$; Pick, Neora'; Money, Deborah ${ }^{1}$; Research Group, Vogue ${ }^{1}$

${ }^{1}$ Vancouver, BC; ${ }^{2}$ Saskatoon, SK

BACKGROUND: Imbalances in the vaginal microbiome can lead to negative reproductive health consequences for women, including an increased risk of sexually transmitted infections, pelvic inflammatory disease, and preterm birth. HIV-positive women may be particularly vulnerable to microbiome disruptions. This study's objective was to explore and profile the vaginal microbiome in HIV-positive reproductive-aged women utilizing cpn60 metagenomic profiling.

METHODS: 53 HIV-positive women were recruited from the Oak Tree Clinic (Vancouver,BC). Demographic/clinical information was collected and vaginal gram-stains were assessed by Nugent's score. Total DNA was extracted from vaginal swabs and PCR amplified using cpn60-specific universal primers. Cpn60-sequence libraries were generated with 454-GS-FLX Titanium pyrosequencing. 64 phylotypes were classified based on sequence similarity to known bacterial organisms. Wilcoxon signed-rank tests were utilized to compare bacterial species abundance with demographic/clinical findings, including CD4 count and viral load classifications.

RESULTS: Demographics: mean age $=36.5 y$ (range $=22-49 y$ ); ethnicity= 18 Caucasian, 9 Black, 12 Aboriginal, 4 Asian, 4 South Asian, 6 mixed ethnicities; mean CD4 count $=488$ cells $/ \mathrm{mm}^{3}\left(\right.$ range $\left.=90-930 \mathrm{cells} / \mathrm{mm}^{3}\right)$; mean viral load $=13,392$ copies $/ \mathrm{mL}$ (range $=<40-355,245$ copies $/ \mathrm{mL}$ ); $62 \%$ of women had suppressed viral loads; $85 \%$ of women were on antiretroviral therapy. According to scored vaginal gram stains, 30\% (16/53) of women had bacterial vaginosis and 9\% (5/53) had intermediate scores. Viral loads $<40$ copies/mL significantly correlated with Lactobacillus crispatus and Lactobacillus gasseri while viral loads $>40$ copies $/ \mathrm{mL}$ correlated with Atopobium vaginae, Gardnerella vaginalis 101, Megasphaera sp., and Prevotella amnii. ( $\mathrm{P}<0.03$ for all) CD 4 counts $<350$ cells $/ \mathrm{mm}^{3}$ significantly correlated with Gardnerella vaginalis $\mathrm{N} 153$ and Lactobacillus iners. $(\mathrm{p}<0.04$ for all)

CONCLUSIONS: Cpn60-based sequence data demonstrated substantial variation in the vaginal microbiome among HIV-positive women, with species-specific differences dependent on immune status and uncontrolled HIV replication. Relationships of these shifts with genital tract health need to be explored.

\section{O036}

\section{TIME TO HIV VIRAL LOAD SUPPRESSION WITH COMBINED ANTIRETROVIRAL THERAPY (CART) IN PREGNANCY}

van Schalkwyk, Julianne; Bahlji, Anees; Chaworth-Musters, Tessa; Maan, Evelyn J; Alimenti, Ariane; Alberts, Arianne; Forbes, John; Pick, Neora; Money, Deborah

Vancouver, BC

BACKGROUND: To prevent vertical transmission, the goal of antenatal cART is to achieve viral load suppression before delivery. Given the short duration of pregnancy and challenges in engaging in early prenatal care in this population, we sought to define the duration of treatment required for viral load suppression.

METHODS: A retrospective cohort of a provincial perinatal HIV database from 1997 and 2011, was analysed including only those who had live birth, a detectable viral load in pregnancy and initiated cART in pregnancy. Data on demographics, parity, gestational age at treatment initiation and delivery, medication adherence, history and type of cART, weeks to viral suppression (defined as $<250$ copies/ml), smoking, illicit drug use and hepatitis $\mathrm{C}$ virus antibody positivity were collected. Nonparametric maximum likelihood estimates (NPMLE) for the distribution of suppression times were calculated. Factors associated with time to suppression were assessed using parametric survival analysis with a Weibull distribution.

RESULTS: 161 mother-infant pairs met inclusion criteria and had complete data for modeling covariates. Mean gestational age at delivery was $37.8 \pm 2.47$ (SD) weeks with only 10 women failing to achieve viral suppression by delivery $(6.2 \%)$. The NPMLE revealed that $16.6 \%$ of women were suppressed after 2 weeks, $56.5 \%$ after 4 weeks, $62.7 \%$ after 6 weeks, $76.2 \%$ after 8 weeks, and $79.2 \%$ after 10 weeks of therapy. The median time to suppression was 3.8 weeks. The predicted proportions of women with suppressed viral loads $(<250$ copies $/ \mathrm{mL})$ vs. time since ART initiation revealed $\log _{10}$ viral load $<4.06$, age $\leq 25$ years, excellent and good cART adherence and no past cART use were all associated with shorter treatment durations.

CONCLUSION: Higher maternal viremia and maternal age, prior cART use and poor adherence confers longer treatment time to achieve viral suppression. Consideration for earlier and/or intensified cART therapy and enhanced adherence support may reduce viral suppression failure.

\section{O037}

\section{HIV VIRAL REBOUND NEAR DELIVERY IN PREVIOUSLY} SUPPRESSED, COMBINATION ANTIRETROVIRAL THERAPY (CART) TREATED PREGNANT WOMEN

Money, Deborah M; van Schalkwyk, Julie; Alimenti, Ariane;

Maan, Evelyn J; Albert, Arianne; Wagner, Emily;

Chaworth-Musters, Tessa; Forbes, Jack; Pick, Neora;

Oak Tree Clinic Research Group

Vancouver, BC

BACKGROUND: Preventing HIV vertical transmission is dependent on successful antenatal cART. Protocols to date have included antenatal cART, intrapartum IV zidovudine and infant zidovudine for 6 weeks. US guidelines recently proposed eliminating IV zidovudine for women with suppressed viral load (VL) prior to delivery. We wished to determine the rate of persistent HIV viral suppression through pregnancy and during delivery in women who had achieved virologic suppression in pregnancy. METHODS: Data is from the $\mathrm{BC}$ provincial database of $\mathrm{HIV}^{+}$pregnancies. Pregnancy outcome, HIV status, use of cART and serial VLs were determined. Virologic suppression one month pre-delivery was the dependent variable. Nested mixed-effects logistic regressions were conducted to define relationships between suppression, age, ethnicity, substance use, duration of cART, adherence, timing of HIV pregnancy care initiation.. Relationship significance was determined via Likelihoodratio tests of the model.

RESULTS: From 1997-2011, there were 382 live-births (twins entered once) with 323 women on cART for $>4$ weeks; 297 pregnancies with sufficient VL data, and 252 with at least one suppressed VL reading $(<50$ copies/mL, or $\leq 400$ for $1997 / 98$ ) and 229 with VL data near delivery. $8.7 \%(5.5-13.4 \%, 95 \% \mathrm{CI})$ were not suppressed within 31 days of delivery (50\% within 2 days), with mean VL of 14,810 c/ml [50-232,245]. Univariately, factors significantly associated with viral rebound were: Aboriginal ethnicity, lower gestational age at delivery, $\mathrm{HCV} \mathrm{Ab+,} \mathrm{sub-}$ stance use and poor adherence. Multivariately, adherence and $\mathrm{HCV} \mathrm{Ab}{ }^{+}$ remained significantly associated with viral rebound at delivery. There were no HIV transmissions in this cohort.

CONCLUSIONS: Even women attending for HIV care and achieving viral suppression in pregnancy can experience viral rebound pre-delivery. Attention to all aspects of prevention including mode of delivery, use of intrapartum IV and infant zidovudine remains prudent in maintaining low risk for vertical transmission. 
O038

ADVERSE BIRTH OUTCOMES AFTER COMBINATION ANTIRETROVIRAL THERAPY EXPOSURE MAY BE LINKED TO PROGESTERONE LEVEL CHANGES

Papp, Eszter; Serghides, Lena

Toronto, ON

BACKGROUND: Combination antiretroviral therapy (cART) has been associated with an increased risk for low birth weight (LBW) and preterm delivery. Lower progesterone levels are also associated with LBW and preterm delivery. Protease inhibitors can inhibit enzymes involved in the synthesis of steroid hormones, including progesterone. Our aim was to determine how antiretroviral (ARV) exposure affects progesterone levels and birth outcomes using in vitro and in vivo models.

METHODS: In vitro: Placental cytotrophoblast (BeWo) cells were exposed to ARVs (AZT, 3TC, Atazanavir, Darunavir, Lopinavir and Ritonavir) alone or in combinations for $24 \mathrm{~h}$, and supernatant was collected for progesterone quantification.

In vivo: Pregnant mice were exposed to human equivalent doses of Combivir/Kaletra or water, either for the pre-implantation period (day 1-6), starting post-implantation (day 6-14), or throughout gestation (day 1-18). Pregnancy failure, litter size, fetal viability, fetal and placental weight were recorded. Progesterone levels were quantified from maternal plasma using a commercial ELISA. ANOVA and Pearson's correlation was used for statistical analysis.

RESULTS: BeWo cells exposed to Atazanavir, Lopinavir, or Ritonavir had significantly lower progesterone production. All tested drug combinations decreased progesterone levels.

Mice exposed to Combivir/Kaletra throughout gestation had more pregnancy loss, less viable pups per litter, and lower fetal and placental weights. Progesterone levels were lower in the exposed group, and positively correlated with fetal weight. Pre-implantation exposure was associated with lower progesterone levels. Post-implantation exposure did not affect progesterone levels or fetal weight, but resulted in a decreased number of viable pups.

CONCLUSIONS: Our findings suggest that cART during early pregnancy may result in reduced progesterone levels, and lower fetal weight. Decreased fetal viability appears to be a progesterone-independent effect of cART. Further experiments will clarify the link between progesterone levels and fetal/placental development, advancing our understanding on cART use in pregnancy.

\section{O039}

\section{IMMUNOLOGICAL ABNORMALITIES AMONG HIV-} EXPOSED UNINFECTED INFANTS IN QUEBEC

Kakkar, Fatima; Soudeyns, Hugo; Lamarre, Valerie;

Ducruet, Thierry; Boucher, Marc; Lapointe, Normand

Montreal, QC

OBJECTIVE: The objective of this study was to characterize the immunological status of HIV exposed-uninfected (HEU) infants born to mothers exhibiting different degrees of immune suppression and viral loads at the time of delivery.

METHODS: Study subjects were enrolled prospectively in the Centre Maternel et Infantil sur le Sida (CMIS) mother-child cohort between 1987 and 2010 ( $n=598)$. Infant CD4 ${ }^{+} \mathrm{T}$ cell, CD8 ${ }^{+} \mathrm{T}$ cell, and absolute neutrophil counts were assessed at 2 and 6 months of age among HEU infants.

RESULTS: Mean absolute CD4 ${ }^{+} \mathrm{T}$ cell counts (cells $/ \mathrm{mm}^{3}$ ) at 2 and 6 months of age were significantly lower among HEU infants born to mothers with $\mathrm{CD}^{+} \mathrm{T}$ cell counts $<350$ at the time of delivery compared to those with CD4 ${ }^{+} \mathrm{T}$ cell counts $>500$ ( 2609 vs $2877, \mathrm{p}=0.02$ and 2609 vs $2843, p=0.05$ ), and among infants born to mothers with delivery viral load $>1000$ copies $/ \mathrm{ml}$ compared to $<50$ copies $/ \mathrm{ml}\left(2\right.$ month $\mathrm{CD}^{+} \mathrm{T}$ cell counts: 2430 vs 2842 , p=0.03, 6 months: 2233 vs $2821, p<0.05$ ).

In a multivariate model, maternal viral load was the most significant predictor of infant $\mathrm{CD}^{+} \mathrm{T}$ cell count both at 2 and 6 months of age $(\mathrm{p}=0.02$ and $\mathrm{p}<0.05)$ after adjusting for both maternal and infant antiretroviral use, gestational age, gender and race. In a separate stratified analysis, there was a significant association between maternal viral load and infant CD4 counts at all strata of maternal CD4 counts. There were no differences in infant CD8 or absolute neutrophil counts based on maternal parameters.

CONCLUSIONS: These results suggest that exposure to HIV in utero, even in the absence of perinatal transmission, can affect the infant's developing immune system. In light of reports of increased morbidity and mortality among HEU infants, further work needs to be directed towards understanding the pathogenic mechanisms involved, and clinical implications of these findings.

\section{O040}

VIROLOGICAL FAILURE AND TREATMENT INTERRUPTIONS AMONG CHILDREN INITIATING COMBINATION ANTIRETROVIRAL THERAPY IN QUEBEC

Kakkar, Fatima; Lamarre, Valerie; Ducruet, Thierry; Soudeyns, Hugo; Valois, Sylvie; Lapointe, Normand Montreal, QC

BACKGROUND: The objective of this study is to assess time to virological failure (VF) and treatment interruption (TI) after initiation of combination antiretroviral therapy (cART) among HIV infected children in the province of Quebec.

METHODS: Study subjects were enrolled prospectively in the Centre Maternel et Infantile sur le Sida (CMIS) cohort, CHU Sainte-Justine, Montreal, Canada, between 1997 and 2010. Primary outcome was time to first VF, defined as detectable viremia over two consecutive measurements after an initial response to cART, and TI, defined as cessation of HAART for more than 6 months at any time after initiation.

RESULTS: Among the 45 patients initiated on cART who were followed for a median of 37.8 (IQR 19.2-59.4) months, median time to first VF was 32 months (IQR 14-44); cumulative incidence of VF at 24, 48 and 96 months of follow-up was $28 \%, 51 \%$, and $79 \%$ respectively. Median time to TI was 52.2 months (IQR 24-72 months), cumulative incidence of TI at 24, 48 and 96 months was $11.8 \%, 20.4 \%$ and $51.13 \%$ respectively. Girls were more likely than boys to experience a TI (HR 3.84, 95\% CI $1.38-10.10)$, and this was consistently observed over time ( $\mathrm{p}<0.05$ Lrtest). Children initiated on cART during infancy were more likely to experience VF compared to those initiating treatment after twelve months of age (HR 3.03; 95\% CI 1.38-6.72). Factors not associated with either VF or TI included viral clade (B vs. non B), family structure (mono vs. biparental) or type of regime initiated (PI vs. NNRTI).

CONCLUSIONS: In this cohort of HIV infected children initiating cART in Canada, over 50\% experienced VF within 4 years of cART initiation, and over $50 \%$ had a TI at some point during their follow-up. These results are concerning given recommendations for life-long therapy in children, and the limited therapeutic options available.

Track C: Epidemiology and Public Health Sciences: Men Who Have Sex With Men

Volet $\mathrm{C}$ : Épidémiologie et sciences de la santé publique : Hommes ayant des relations sexuelles avec d'autres hommes

0041

CAUGHT IN A SYNDEMIC: CHARACTERISTICS OF CANADIAN MSM REPORTING MULTIPLE PSYCHOSOCIAL PROBLEMS AND HIV RISK

Ferlatte, Olivier; Salway Hottes, Travis; Trussler, Terry; Marchand, Rick

Vancouver, BC

INTRODUCTION: Syndemics (intertwined epidemics) of psychosocial issues have been shown empirically to increase risk of HIV infection 
among MSM, however, to intervene we must identify those who are most vulnerable. We analyzed data from a national online survey of MSM to assess the risk factors associated with being caught in a syndemic.

METHODS: Analysis was performed on a sample of 8490 MSM. First, multivariable logistic regression was used to examine the association between psychosocial issues (depression, suicidality, binge drinking and drug use) and risk of HIV acquisition (unprotected anal intercourse with unknown status partner). A second multivariable regression model was used to identify marginalization markers and demographic characteristics among those caught in a syndemic of two or more issues.

RESULTS: MSM with two psychosocial issues had two times the odds (95\% CI 1.75-2.32) of reporting unprotected sex with an unknown status partner, while those with 3 or 4 psychosocial issues had 3.2 times the odds (95\% CI 2.50-4.15). Risk factors of being caught in a syndemic included past experiences of harassment (OR 1.7; 95\% CI 1.52-1.99), sexual abuse (OR 1.50; 95\% CI 1.25-1.75), physical violence (OR 1.70; 95\% CI 1.442.00), and career discrimination (OR 1.25 95\% CI 1.07-1.46). The risk of being caught in a syndemic was also higher for gay identified men (OR 1.88 ; $95 \%$ CI 1.64-2.16), under the age of 35 (OR 1.27; 95\% CI 1.111.44 ), with an income less than $\$ 60,000$ (OR $1.42 ; 95 \%$ CI 1.24-1.63), and without a university degree (OR 1.21; 95\% CI 1.07-1.39).

CONCLUSION: Our results provide further evidence that syndemics of psychosocial issues increase risk of HIV infection among MSM and that these syndemics are in part socially produced. Since syndemics are more likely to impact some sub-groups of MSM, interventions to reduce its effects should be targeted.

\section{O042}

GROUP SEX EVENTS FOR MEN WHO HAVE SEX WITH MEN (MSM) ARE ASSOCIATED WITH HIGHER INCOME, SENSATION-SEEKING, AND HIGH-RISK SEXUAL ENCOUNTERS IN VANCOUVER, BRITISH COLUMBIA

Michelow, Warren ${ }^{1}$; Roth, Eric ${ }^{2}$; Forrest, Jamie ${ }^{1}$; Lal, Allan'; Moore, David ${ }^{1}$; Hogg, Robert S1, $^{1}$

${ }^{1}$ Vancouver; ${ }^{2}$ Victoria; ${ }^{3}$ Burnaby, BC

BACKGROUND: The number of new positive HIV tests has not declined among MSM in British Columbia over the past decade. It would be helpful for local HIV prevention efforts to identify subgroups and venues where risk for HIV transmission is especially high. We use data from the ongoing Momentum Health Study on group and anonymous sex events to identify participant characteristics and associated HIV risk practices.

METHODS: Using self-reported data from baseline interviews we provide descriptive statistics on sexual activities and levels of condom use at sex parties, darkrooms and blackout nights. A "darkroom" or "blackout" event is a sex venue or event in a club, bar or bathhouse where one cannot easily see sexual partners, thus facilitating anonymous sexual encounters. Multivariate logistic regression is used to identify characteristics of participants at these venues and events.

RESULTS: Of 235 MSM aged 19 years and older (median age 37 years), 72 (32\%) self-reported as HIV-positive, and 68 (29\%) attended group sex events in the previous 6 months (P6M). Of 42 who attended sex parties, 19 (45\%) reported attending ones specifically designated as 'barebacking' events, and 50\% (21/42) reported having unprotected anal intercourse (UAI). Similarly, of a different 42 who attended darkrooms or blackout events, $22(52 \%)$ reported UAI. In multivariate analysis, higher annual income (adjusted Odds ratio $[A O R]=1.07$ per $\$ 5,000$ increment, $\mathrm{p}<0.05$ ), higher numbers of male anal sex partners $\mathrm{P} 6 \mathrm{M}(\mathrm{AOR}=1.04$ per partner, $\mathrm{p}<0.005$ ), and higher scores on Kalichman's (1995) revised Sexual Sensation-Seeking Scale $(A O R=1.11$ per unit-increase, $\mathrm{p}<0.01$, range 11-44) were significantly associated with attending group sex events. HIV serostatus, sexual orientation and other demographic characteristics were not associated with attendance at these events.

CONCLUSION: The high prevalence of attendance at group sex events with high levels of reported UAI suggests these may be critical sites for HIV transmission among MSM.
0043

NARRATIVES OF SEXUAL HEALTH KNOWLEDGE, ATTITUDES, AND BELIEFS: INFORMING THE MEASUREMENT OF TREATMENT OPTIMISM AMONG GAY AND, BISEXUAL MEN

Rich, Ashleigh ${ }^{1}$; Forrest, Jamie I'; Small, Will1,2; Howard, Terry ${ }^{1}$; Michelow, Warren ${ }^{1}$; Moore, David ${ }^{1}$; Hogg, Robert S1, 2; Roth, Eric $A^{3}$ ${ }^{1}$ Vancouver; ${ }^{2}$ Burnaby; ${ }^{3}$ Victoria, BC

BACKGROUND: With the expansion of access to highly active antiretroviral therapy (HAART) in British Columbia, it is important to understand how knowledge of treatment technologies may impact attitudes towards sexual risk behaviour among gay, bisexual and other men who have sex with men (MSM). This knowledge is increasingly crucial as new HIV treatment technologies, such as pre-exposure prophylaxis $(\mathrm{PrEP})$ and post-exposure prophylaxis (PEP), are introduced.

METHODS: Twenty gay and bisexual identified men of mixed serostatus were recruited from the larger sample of the Momentum Health Study, a five-year bio-behavioural research study, to participate in semi-structured, in-depth interviews. Interviews were recorded and transcribed verbatim. An analysis was conducted using codes based on the study's 12-item HAART Optimism scale and two related questions.

RESULTS: Transcripts were thematically analyzed in terms of emotionally-motivated and treatment knowledge motivated scale items. Treatment-specific knowledge was not widely held among the study participants, and was particularly low among the seronegative group. Among seronegative participants who demonstrated some treatment knowledge, this knowledge was abstract, rather than applied routinely as part of risk reduction strategies.

Risk reduction strategies among participants appeared to be largely emotionally motivated, and not a product of treatment-specific knowledge. Risk reduction decision-making was mainly a function of a general desire to avoid contracting or transmitting HIV. Serosorting and condom use were the strategies most widely reported, among both sero-positive and seronegative participants.

CONCLUSIONS: These data suggest that treatment knowledge may not be widely disseminated among MSM, particularly sero-negative men, in the Greater Vancouver area, and that risk compensation may instead be largely emotionally motivated at this stage. These data aid in understanding the dynamics underlying HAART optimism and risk reduction decision making among MSM, in the context of expanded HAART access in BC.

\section{O044}

\section{IMPACT OF PROSECUTION OF NON-DISCLOSURE OF HIV STATUS ON ATTITUDES AND BEHAVIOUR OF HIV-NEGATIVE AND HIV-POSITIVE MEN WHO HAVE SEX WITH MEN (MSM) IN TORONTO, ONTARIO}

Kesler, Maya A; Kaul, Rupert; Loutfy, Mona; Liu, Juan;

Brunetta, Jason; Rebbapragada, Anu; Halpenny, Roberta;

Robinette, Jennifer; Gamble, Molly; Remis, Robert S

Toronto, ON

BACKGROUND: In Canada, HIV-positive persons have been criminally prosecuted for not disclosing their HIV status prior to sex. The impact of such prosecutions on HIV testing among uninfected MSM, and on disclosure and condom use among HIV-positive MSM is not known. METHODS: Sexually active HIV-positive and HIV-negative MSM were recruited at a Toronto medical clinic in 2010-12. Participants completed a detailed questionnaire using ACASI. HIV-negative men were asked if they were less likely to be HIV tested due to fear of prosecution, and HIVpositive men were asked if they were more likely to disclose or use a condom due to fear of prosecution.

RESULTS: We recruited 150 HIV-negative MSM (median age 44.5 [IQR 37-50]). $7.3 \%$ were less likely or much less likely to have an HIV test due to fear of prosecution. HIV-negative MSM who didn't use a condom at last anal sex with a casual partner indicated they were less likely to have an HIV test due to fear of prosecution (15.0\% vs. $2.3 \%)$. We also recruited $292 \mathrm{HIV}$-positive MSM (median age 45 [IQR 38-50]). 42.9\% 
were more likely to disclose their HIV status and $43.4 \%$ were more likely to use a condom due to fear of prosecution. In a multivariate model, HIVpositive MSM who were younger (OR 2.1), Canadian-born (OR 2.2), never used drugs other than marijuana (OR 2.0), and had no UAI with an HIV-positive partner (OR 2.2) were significantly more likely to use a condom due to fear of prosecution. With respect to disclosure, MSM Canadian-born were significantly more likely to disclose (OR 2.1).

CONCLUSIONS: We found no evidence that fear of prosecution for HIV non-disclosure was associated with negative consequences for prevention in this clinic-based study. Fear of prosecution was infrequently reported as a deterrent to HIV testing among HIV-negative MSM. Furthermore, almost half of HIV-positive MSM reported being more likely to disclose their HIV status or to use a condom due to concerns regarding possible prosecution.

\section{O045}

\section{FACTORS RELATED TO CONDOM USE DURING ANAL} INTERCOURSE WITH CASUAL PARTNERS AMONG YOUNGER GAY AND BISEXUAL MEN IN NEW ZEALAND; RESULTS FROM NATIONAL SOCIOBEHAVIOURAL

\section{SURVEILLANCE (2006-2011)}

Lachowsky, Nathan J1, 2; Saxton, Peter $^{2}$; Dickson, Nigel ${ }^{3}$;

Hughes, Anthony ${ }^{2}$; Dewey, Cat $\mathrm{E}^{1}$; Summerlee, Alastair J1

${ }^{1}$ Guelph, ON; ${ }^{2}$ Auckland; ${ }^{3}$ Dunedin, New Zealand

The objective was to conduct New Zealand's first investigation into condom use with casual partners during receptive anal intercourse (casRAI) and insertive anal intercourse (casIAI) among younger gay, bisexual, and other men who have sex with men aged 16-29 (YMSM). The study included 3,052 YMSM from New Zealand's 2006-2011 sociobehavioural HIV surveillance. Factors associated with high condom use (HCU: "always" or "almost always" used condoms in $<6$ months) for casRAI and casIAI were identified with backward stepwise multivariate logistic regression retaining at $\mathrm{p}<0.05$; [adjusted OR $(95 \% \mathrm{CI})$ ] are presented. Of 1,508 YMSM who reported casRAI, 73.7\% reported HCU. For casRAI, HCU increased for older age [per year: 1.05 (1.00-1.10)], having a tertiary degree or higher [1.79 (1.24-2.58)], and more sexual partners in $<6$ months [e.g. $11-20$ versus one: 3.93 (2.01-7.68)]. HCU decreased for Pacific Islander ethnicities [compared to European/Pākehā ethnicities: 0.35 (0.17-0.71)]. Of 1512 YMSM who reported casIAI, 76.1\% reported HCU. For casIAI, HCU increased for having tested for HIV in $<12$ months [1.64 (1.20-2.24)] and having $2-5$ sexual partners versus one in $<6$ months [1.81 (1.05-3.13)]. HCU decreased for having had sex with a woman in $<6$ months $[0.65$ (0.45-0.94)]. For both casRAI and casIAI, HCU was negatively associated with not having a regular partner (versus reporting a boyfriend) [casRAI: $0.12(0.03-0.48)$ and casIAI: $0.03(0.00-0.29)]$. HCU was also associated with multiple attitude measures on condom use [e.g. disagreement that "I would rather risk HIV than use a condom", for casRAI: 4.04 (2.75-5.93) and for casIAI: 2.94 (2.03-4.26)]. Factors associated with HCU with casual partners differed by anal modality, mostly by behaviours and attitudes, and by select sociodemographics only for casRAI. Future condom use research should investigate behaviours within different relational contexts. More comprehensive understandings of condom use behaviour should inform future health promotion and prevention work with MSM.

\section{O046 \\ AVOIDANCE OF HIV TESTING AMONG GAY AND BISEXUAL MEN DUE TO LACK OF ANONYMOUS TESTING OPTION IN BRITISH COLUMBIA}

Salway Hottes, Travis'; Gilbert, Mark'1; Trussler, Terry'; Marchand, Rick'; Brownrigg, Bobbi ${ }^{1}$; Ogilvie, Gina ${ }^{1}$;

Lester, Richard ${ }^{1}$; Kendall, Perry $\mathbf{R}^{2}$

${ }^{1}$ Vancouver; ${ }^{2}$ Victoria, BC

BACKGROUND: Pervasive HIV stigma continues to make many MSM reluctant to test. In order to investigate the potential for anonymous testing (i.e., no name/identifier linked to test) to improve uptake, we reviewed reasons for avoiding or delaying testing cited by BC gay and bisexual men in the 2011-12 SexNow survey.
METHODS: Participants were recruited through dating/sex-seeking websites, community organizations, and word-of-mouth. Results were restricted to HIV-negative or never-tested BC residents who avoided/ delayed testing within the past 12 months. Participants who cited lack of anonymous testing as a reason for avoiding or delaying testing were further characterized, using logistic regression to control for sociodemographic differences.

RESULTS: Among HIV-negative or never-tested men (87\%, $1567 / 1805), 918$ had not tested $(n=731)$ or delayed testing $(n=187)$ in the past 12 months. Fifteen percent of these participants cited lack of anonymous testing as a reason, $4 \%$ as the sole reason. Subgroups associated with higher likelihood of avoiding/delaying testing because of lack of anonymous option ( $\mathrm{p}<0.05$, univariate) included: non-gayidentified men (19\%), $\geq 45$ yoa (18\%), partnered with women $(21 \%)$, not 'out' to their primary care provider (18\%), not 'out' in general $(21 \%)$, suburban residence $(19 \%)$, recent unprotected anal sex with unknown-status partner (22\%), recent STI diagnosis $(23 \%)$, or selfperceived risk for HIV (25\%). In multivariable analysis, not being 'out' (generally, or to HCP) and self-perceived HIV risk remained associated $(\mathrm{p}<0.05)$

CONCLUSIONS: Fifteen percent of gay and bisexual men who delayed or avoided HIV testing in BC in 2011 cited the lack of anonymous testing as a reason for not testing. In particular, men who are less likely to disclose their sexuality and those at risk of acquiring HIV were more likely to avoid testing for this reason. Offering anonymous testing in BC may increase test uptake and frequency in this population.

\section{O047}

HEPATITIS C VIRUS (HCV) SEROINCIDENCE AMONG HIVPOSITIVE MEN IN ONTARIO WHO HAVE SEX WITH MEN (MSM) AND NO HISTORY OF INJECTION DRUG USE, 2000-2010

Burchell, Ann N; Manno, Michael; Gardner, Sandra;

Mazzulli, Tony; Remis, Robert S; Allen, Vanessa;

Bayoumi, Ahmed M; Kaul, Rupert; McGee, Frank; Millson, Peggy; Raboud, Janet; Rourke, Sean B; The OHTN Cohort Study Team Toronto, ON

BACKGROUND: There is growing evidence that HIV-positive MSM can be infected sexually with HCV. We wished to estimate HCV seroincidence and its risk factors among HIV-positive MSM in Ontario with no known history of injection drug use.

METHODS: We analyzed data from the OHTN Cohort Study, an ongoing cohort of persons in HIV care. Data were obtained from medical charts, interviews, and record linkage with Public Health Ontario Laboratories. We restricted the analysis to 1,534 MSM who: (1) did not report injection drug use; (2) were under follow-up in 2000-2010; and (3) had $2+\mathrm{HCV}$ antibody tests, of which the first was negative. Person-time commenced at the later of the HCV-negative result or HIV diagnosis and ended at the first $\mathrm{HCV}^{+}$or last date of follow-up (median 6.1 personyears (PY) of follow-up; sum 9,987PY). We used Poisson and Cox regression to estimate incidence density and hazard ratios (HR), respectively. RESULTS: We observed $51 \mathrm{HCV}$ seroconversions, for an overall incidence of 0.51 per 100PY (CI: 0.39-0.67). Annual incidence varied from 0.16 to 0.89 per $100 \mathrm{PY}$, with no statistical evidence of a temporal trend. Seroconversion was statistically-significantly associated with acute syphilis infection in the previous 6 months (adjusted $\mathrm{HR}=4.9$, CI 1.2-21) and there was a marginally statistically-significant association for men who had not yet initiated antiretroviral treatment (adjusted HR=1.9, CI 0.914.0). There were no statistically significant effects of age, ethnicity, region, $\mathrm{CD}^{+}{ }^{+}$cell count or viral load.

CONCLUSION: HCV incidence was 10 -fold higher than reported rates for the general male population in Ontario. Sexual behaviour was not measured and we cannot exclude the possibility that some HCV infections were acquired via unreported IDU. Nevertheless, the strong association with recent syphilis suggests that at least some HCV infections may have been sexually acquired. Current guidelines recommend HCV testing at HIV diagnosis. Periodic HCV screening after HIV diagnosis may also be worthwhile. 
0048

EPIDEMIOLOGY OF THE HIV AND INFECTIOUS SYPHILIS SYNDEMIC AMONG GAY AND BISEXUAL MEN IN BRITISH COLUMBIA, 2003-2012

Lindegger, Monika; Consolacion, Theodora B; Salway Hottes, Travis; Achen, Melanie; Doupe, Glenn; Morshed, Muhammad; Lester, Richard; Ogilvie, Gina; Gilbert, Mark

Vancouver, BC

BACKGROUND: In 2012 British Columbia (BC) reported the highest number of infectious syphilis cases in recent decades $(\mathrm{N}=368)$. The proportion of gay, bisexual and other men who have sex with men (MSM) increased from $30 \%$ in 2003 to $82 \%$ of cases in 2012. Given increasing recent trends in infectious syphilis, we sought to describe the epidemiology of cases among MSM in BC, with particular attention to HIV co-infection.

METHODS: All cases of infectious syphilis (primary, secondary or early latent) in self identified MSM diagnosed in BC between August 1st 2003 and June 30th 2012 were examined. Cases were divided according to their documented HIV status at the time of diagnosis. Demographic characteristics, syphilis diagnostic characteristics and risk factors were extracted from the provincial enhanced surveillance database and compared using SAS

RESULTS: $55 \%$ of the 1415 cases were HIV positive. HIV positive individuals were more likely to be diagnosed by private physicians than in public health/community health clinics $(\mathrm{p}<0.05)$ and were more likely to have early latent vs. primary/secondary syphilis $(\mathrm{p}<0.05)$. HIV positive individuals were more likely than HIV negative individuals be named as a contact to syphilis $(\mathrm{p}<0.05) .21 \%$ of HIV positive individuals had more than one syphilis diagnosis during the study period compared to $7 \%$ of HIV negative individuals $(\mathrm{p}<0.05)$.

DISCUSSION: Infectious syphilis rates have risen to high levels in recent time predominantly among MSM, an increasing proportion of whom are HIV positive. HIV co-infected individuals are more likely to have repeat syphilis diagnoses. The data suggests that the recent surge in syphilis rates in BC is concentrated in sexual networks of HIV positive men. Focus on prevention and frequent screening of MSM, particularly those who are HIV positive is important in identifying and treating cases early with the aim of reducing onward transmission.

\section{Track D: Social Sciences: Diversity}

\section{Volet D : Sciences sociales : Diversité}

\section{O049}

THE CEDAR PROJECT: QUALITATIVE NARRATIVES ADDRESSING THE IMPACT OF CHILDHOOD TRAUMA ON STRESS-COPING AND PATHWAYS TO HEALING AMONG YOUNG ABORIGINAL PEOPLE WHO USE DRUGS IN VANCOUVER AND PRINCE GEORGE, BC

Pearce, Margo $\mathrm{E}^{1}$; Christian, Wayne $\mathbf{M}^{2}$; Blair, Alden $\mathrm{H}^{1}$; Schechter, Martin $\mathrm{T}^{1}$; Spittal, Patricia $\mathbf{M}^{1}$

${ }^{1}$ Vancouver; ${ }^{2}$ Enderby, BC

BACKGROUND: Indigenous scholars suggest that understanding childhood trauma, substance use and HIV risk among Aboriginal people requires consideration of intergenerational trauma while acknowledging strength and survival in the face of hardships. However, most HIV research been based on deficit models of health and limited in cultural/ practical relevance to young, at-risk Aboriginal people.

METHODS: Twenty-seven participants (16 women) who are part of the Cedar Project, a cohort study of young Aboriginal people who use drugs in Vancouver and Prince George, BC, had individual in-depth interviews about personal and family history of trauma including residential school or foster care experiences, transitions to drug use and sex work, coping strategies, and opportunities for healing and change. Using phenomenological approaches, researchers transcribed and analysed interview texts.
RESULTS: All participants described complex and multiple occurrences of trauma in childhood. Four major themes emerged from the narratives: (a) The emotional impact of trauma including internalized shame, confusion, and anger, particularly in the aftermath of sexual abuse, and the struggle to 'break the cycle' of family disconnection through child welfare and corrections systems; (c) making sense of trauma, including understandings of the impact of the residential school system; (d) mitigating the trauma effect through stress-coping processes that are negative (initiation of sex work and injection drug use) or positive (becoming resourceful, spirituality, quitting/never injecting drugs); and (e) recovering from addiction to pursue hopes and dreams, including reconnection with children and culture, independence, and healing.

CONCLUSION: The young Aboriginal men and women in this study discussed unbearable emotions and memories associated with having lived through extreme childhood trauma. However, participants' reflections also included the meaning of personal strength and past and present sources of encouragement. Strength-based approaches to health for young Aboriginal people who use drugs must consider historical trauma while supporting access to opportunities for culturally-safe healing.

\section{O050 \\ DISCUSSING ABORIGINAL HIV PREVENTION STRATEGIES: REVIEWING LITERATURE AND RECOMMENDATIONS}

Pooyak, Sherri' ${ }^{1}$; Masching, Renee ${ }^{2}$; Teengs, Doe $^{3}$; Montgomery, Robin ${ }^{3}$

${ }^{1}$ Victoria, BC; ${ }^{2}$ Dartmouth, NS; ${ }^{3}$ Toronto, ON

The CIHR Social Research Centre on HIV \& AIDS Prevention sought to explore existing literature and programming specifically regarding Aboriginal HIV prevention strategies. Rates of HIV within Aboriginal populations continue to increase and as a result, identifying the current status of HIV prevention strategies within Aboriginal communities is a priority. This presentation will summarize discussion paper findings written for the SRC emphasizing prevention themes and key recommendations for future research and programming.

The discussion paper on Aboriginal HIV Prevention Strategies aims to identify existing prevention strategies within the field of Aboriginal HIV and within Aboriginal AIDS service organizations. A thorough literature review was conducted, however it is the themes derived from the review and the recommended outcomes that will be the focus of this presentation. Of particular concern is the increased rate at which HIV is impacting Aboriginal women, youth and people who use injection drugs. Reasons for the high rates of infection are directly correlated to the impact of colonization on Aboriginal people. A review of the literature on Aboriginal people and HIV, found that there is a lack of culturally based prevention programs targeting Aboriginal women, youth and people who use injection drugs. Emergent themes highlight: holistic approaches; arts-based approaches; community-based prevention strategies; collaboration and partnerships; leadership and harm reduction and abstinence-based policies.

The paper's recommendations are meant to provide people working in the field of HIV an opportunity to consider developing or expanding on existing Aboriginal HIV prevention strategies in their work.

Recommendations focus on dedicated funding as a priority; evaluation; research; and exploring the concept of resilience in the context of HIV and Aboriginal People living with HIV \& AIDS. This is where research evidence can lead to action for people working in the field of HIV.

\section{O051}

RELATIONSHIPS TO PLACE AND IDENTITY FOR MÉTIS YOUTH AND WOMEN AS HIV PREVENTION

Konsmo, Erin $\mathbf{M}^{1}$; Danforth, Jessica ${ }^{1}$; Flicker, Sarah ${ }^{1}$;

Thistle, Jesse ${ }^{1}$; Anderson, Kim²; Rankin, Jennifer ${ }^{1}$

${ }^{1}$ Toronto; ${ }^{2}$ Brantford, ON

BACKGROUND: Focusing on 'place' as a social determinant of health, this project worked with two Métis communities: Buffalo Lake Métis Settlement, Alberta and the urban Métis community of Sudbury, 
Ontario. We combined a reproductive and environmental justice framework with CBR methodologies to explore our research questions. In both communities, we hosted a variety of workshops and arts-based activities to explore our themes.

METHODS: In this paper, we will explore results from our Belly Casting activities. We invited women to create, decorate and talk about their belly casts using plaster and paint within the context of the teaching: "women are the first environment". In total, twenty-three women participated and 11 casts were created that explored these relationships. As an arts based methodology, belly casting proved to be a good way to start discussions around Métis health. It provided an embodied experience for women and youth to tell stories about Métis identity, environmental violence, self esteem and connection to land and place. A themed analysis was used to explore the connections between body and environment from an Indigenous understanding of reproductive justice. This understanding takes into account that systemic issues (colonization, poverty, environmental racism, etc.) may impact our bodies and the rates of HIV in our communities.

RESULTS: Results show that most participants were able to see the connections, however there remain gaps in knowledge around connections between land, identity, health and HIV. While images on cast clearly connected to Métis symbolism and Métis pride, many of the casts did not go into depth to explore Métis specific cultural understandings of land and health. The casts (and experience) portrayed strong messaging around Métis bodies being places of identity building, beauty and culture, while resisting colonialism.

\section{O052 \\ THE NEWCOMER SEXUAL HEALTH PROMOTION PROJECT, A POSITIVE MODEL OF STI/HIV PREVENTION AND CARE AMONG ETHNO RACIAL COMMUNITIES IN TORONTO}

Ndayikengurukiye, Godelive; Hui, Christian; Luyombya, Henry; Li, Alan; Owino, Maureen; Bisignano, Alex

Toronto, ON

BACKGROUND/ISSUES: Toronto receives close to 20\% of Canada's population of new immigrants each year. Research has shown that newcomers are at 10 times increased risk for contacting STI and HIV. Due to complex linguistic, social and stigma related barriers that affect access, many newcomers are unable to access timely information on legal and health services or to connect with support. The Committee for Accessible AIDS Treatment developed the Newcomer Sexual Health Promotion Project (NSHP) to address this critical need.

METHODOLOGY/DESCRIPTION: To address the complex challenges related to settlement needs, access barriers, confidentiality concerns and stigma and discrimination, the project developed multi-pronged strategies to reach newcomers at risk and build trusting relationships to enable peer based learning, facilitate disclosure and access to services. Strategies include (1) proactive partnership development and outreach to newcomer service agencies beyond the HIV sector; (2) modular workshops on different topics that can be delivered with flexibility based on audience's needs or schedule requirements; (3) newcomer PHA peer educators delivering the training which facilitates trust building, disclosure and peer based learning; (4) alternative options for participants to follow up separately to access support outside educational forum.

RESULTS: Since its implementation in 2011, the NSHP has reached 1032 newcomers via 90 agencies. $22 \%$ of agencies requested repeated educational workshops based on positive feedback from their clients. 18 newcomer PHAs disclosed through our project and received referrals to AIDS service agencies and went on and attended more in-depth training on HIV and criminalization on non-disclosure. Another 11 newcomer PHAs were connected to ASO and other services upon inquiries made by their agency case workers.

CONCLUSION/RECOMMENDATION: The Newcomer Sexual Health Promotion Project provides an innovative peer based model to effectively reach highly vulnerable populations to facilitate service access, peer support and empowerment. The model is highly replicable in other regions and can be adapted to address various health issues.
0053

REDUCING HIV STIGMA AMONGST ETHNO-RACIAL LEADERS: FINDINGS FROM THE COMMUNITY CHAMPION HIV/AIDS ADVOCATES MOBILIZATION RESEARCH PROJECT (CHAMP)

Li, Alan ${ }^{1}$; Fung, Kenneth ${ }^{1}$; Luyombya, Henry ${ }^{1}$;

Maticka-Tyndale, Eleanor ${ }^{2}$; Wong, Josephine ${ }^{1}$; Bisignano, Ciro ${ }^{1}$; Hui, Christian ${ }^{1}$

${ }^{1}$ Toronto; ${ }^{2}$ Windsor, ON

BACKGROUND: HIV stigma, homophobia and invisibility of community and PHA champions within ethno-racial communities undermine HIV prevention efforts, treatment and care. The CHAMP study (Community Champions HIV/AIDS Advocates Mobilization Project) was developed to evaluate the effectiveness of two interventions in promoting HIV championship among faith, media and social justice leaders and PHAs in African Caribbean, Asian and Latino communities.

METHODS: CHAMP compares two interventions: Acceptance and Commitment Therapy (ACT) combined with Social Justice Capacity Building (SJCB), and SJCB on its own. A total of 35 ethno-racial PHAs and 31 ethno-racial non-PHA participants were recruited and randomly selected to participate in either SJCB training alone, or SJCB and ACT training. All participants took part in a pre-intervention focus group and completed pre- and post-intervention surveys that assessed their perception and experiences with stigma and readiness for engagement on HIV related issues. Monthly activity logs were used to document participants' involvement in HIV related issues for twelve months post-intervention. RESULTS: Preliminary analysis of quantitative data from pre- and postintervention questionnaires showed significant decrease in internalized stigma among PHAs and stigmatizing beliefs among non-PHAs. Readiness to take positive action to address stigma and discrimination was significantly increased in all participant groups.

Results of immediate post-intervention surveys did not show a significant difference between the groups who had SJCB intervention alone versus those who had dual interventions. Further analysis including qualitative data from focus groups and participant activity logs, and end of study questionnaire will enable further assessment of the effects of the different interventions.

CONCLUSIONS: This study demonstrated promising results in stigma reduction among project participants in the short term. Additional data from long term follow up including logged activities is expected to provide further assessment of the impact of the two interventions in addressing stigma and mobilizing communities.

\section{O054}

WHAT IF IT WAS YOU(TH)? THE INTERSECTION OF ARTS AND EVIDENCE-BASED LESSONS LEARNED FROM A CAFÉ SCIENTIFIQUE ON HIV/HCV PREVENTION AND YOUTH IN THE ATLANTIC REGION

Hudson, Pamela; $\mathrm{L}^{1}$; Gahagan, Jacqueline ${ }^{1}$;

Hill-Mann, Alexandra ${ }^{1}$; Ochieng, Cindy ${ }^{1}$; Irving, Anne ${ }^{2}$;

Smith, Danielle ${ }^{1}$; Smith, Victoria ${ }^{1}$; Sweezey, Alisha ${ }^{1}$;

Dykeman, Margaret ${ }^{3}$; Harris, Greg${ }^{4}$; MacDonald, Jo-Ann ${ }^{5}$;

Marshall, Zack ${ }^{4}$; Shea, Rob $^{4}$

${ }^{1}$ Halifax, NS; ${ }^{2}$ Sackville, NB; ${ }^{3}$ Fredericton, NB; ${ }^{4}$ St John's, NL;

${ }^{5}$ Charlottetown, PEI

BACKGROUND: We used the community-based 'Café Scientifique' format of knowledge exchange, as put forth by CIHR, to encourage dialogue between researchers and the broader community regarding health issues relevant to public interest. Our CIHR-funded Café Scientifique brought together community and academic collaborators, youth, and the public in an arts- and evidenced-based dialogue on HIV/HCV prevention efforts aimed at youth in the Atlantic Region.

PROCESS: Youth research advisors played a key role in the planning and execution of the Café Scientifique event, in collaboration with researchers, policy makers, and community members. We used this Café to examine evidence emerging from the NSHRF-funded research study, Our Youth, Our Response: Building Capacity for Effective HIV/HCV 
Policy and Programming Across the Atlantic Region. We also structured our Café to interactively explore and integrate both arts-based and youth-related $\mathrm{HIV} / \mathrm{HCV}$ prevention approaches within research, policy, and programming sectors. For example, a particular component of the Café involved participants documenting their prevention related feedback in the form of artwork which will be displayed and discussed at upcoming knowledge translation events in Atlantic Canada.

FINDINGS: Through modified thematic analysis, the emergent foci provided through participants' feedback were grouped into five key themes: (1) The importance of involving youth in the development and execution of research, policy, and programming initiatives; (2) Relevant means to effectively connect with youth; (3) Differences between mobilizing youth in HIV/HCV related projects versus general health programs; (4) Differences between urban and rural contexts; and (5) General suggestions for research, policy, and programs.

CONCLUSIONS: Implications for future HIV/HCV prevention and youth-related knowledge translation activities were identified. Specifically, there is a need to continue bridging art, accessibility, and evidence into youth-focused prevention efforts to better meet the knowledge translation needs of diverse populations of youth and youth service providers from across the Atlantic Region.

\section{5 \\ "YOU SEE, WHEN THEY DESIGNED THE PROGRAMS THEY DIDN'T THINK PEOPLE WERE GOING TO LIVE THAT LONG ANYWAY": RETHINKING SERVICE PROVISION FOR THE LONG-TERM}

Cattaneo, Jessica S; Carsone, Soo C; Husbands, Winston; Karapita, Stephanie; King, Kenneth; Makoroka, Lydia; Murzin, Kate Toronto, ON

Living with HIV over the Long-term is an exploratory study that examined the priorities and challenges of people who have lived long-term with HIV and the organizations that support them. This communitybased study began in 2011 with the aim of developing a knowledge base related to long-term survivorship of HIV which includes, but is not limited to, issues related to aging and HIV. In this presentation, we examine what we heard from both service providers and people living long-term with HIV about their experiences delivering and accessing programs and services. In particular, we focus on what these experiences suggest for service provision that is appropriate for HIV as a long-term, chronic and episodic illness.

We conducted 4 focus groups with people who self-identified as living long-term with HIV ( $n=31)$, ranging from 24 to 70 years old. We also carried out interviews with a range of service providers $(n=17)$ working in AIDS service organizations and hospitals in Toronto. Our questions centered on: the long-term survivor concept; the needs and priorities of people living long-term with HIV; service provision; and the impact of long-term engagement with programs or organizations. Focus groups and interviews were transcribed and coded through thematic analysis.

In both focus groups and interviews, participants explained that people who have lived long-term with HIV have distinct and pressing needs that are not well addressed in the current service landscape. The accumulated impact of living with HIV for many years shapes experiences of aging, stigma, mental health, social engagement, and poverty in important ways. Participants also identified certain models of service delivery as being conducive to long-term engagement with programs or organizations, emphasizing models that prioritize relationship-building, caring, strengths, and dignity. Our study examines these insights, highlighting potential shifts for service provision as people live longer with HIV.
O056

VIVRE AVEC LE VIH À 50 ANS ET PLUS : UN VIEILLISSEMENT SOCIAL PRÉCOCE?

Wallach, Isabelle; Ducandas, Xuân; Martel, Michel;

Trottier, Benoit; Thomas, Réjean

Montréal, QC

CONTEXTE : Le vieillissement physique prématuré des personnes vivant avec le VIH (PVVIH) est un phénomène de plus en plus documenté. Néanmoins peu de recherches au Canada se sont penchées sur les dimensions sociales du vieillissement des PVVIH.

OBJECTIF : La recherche vise à documenter les répercussions du VIH et du vieillissement sur la vie personnelle et sociale des PVVIH de 50 ans et plus (PVVIH50+).

MÉTHODE : Cette recherche s'appuie sur l'analyse d'entrevues semidirectives réalisées avec un échantillon de 38 PVVIH50+ diversifié selon le genre, l'orientation sexuelle, l'origine ethnique et l'usage ou non de drogues par injection. Les participants ont été recrutés au sein de la clinique médicale l'Actuel à Montréal. L'analyse qualitative, inspirée de la théorisation ancrée, a été réalisée à l'aide du logiciel QDA Miner.

RÉSULTATS : Les résultats de la recherche mettent en évidence que les PVVIH50+ rencontrent de multiples difficultés dans leur existence en lien avec le VIH et le vieillissement. Au niveau de la vie intime, la majorité des participants vivent un ralentissement de leur vie sexuelle et un isolement affectif. Sur le plan de la vie sociale, ils sont nombreux à faire face à l'isolement, l'éloignement et/ou le décès de leurs proches. Beaucoup connaissent également une cessation de leur activité professionnelle et des difficultés financières. Enfin, les participants rapportent un besoin accru de services sociaux et de santé.

DISCUSSION : Les problématiques auxquelles doivent faire face les PVVIH50 + en raison l'intersection du VIH et du vieillissement (détérioration de la vie intime, isolement social, arrêt de la vie professionnelle, difficultés économiques, besoins de services) sont caractéristiques des changements vécus par les personnes âgées à un âge plus avancé (65 ans et plus).

CONCLUSION : Les résultats de cette recherche permettent de faire l'hypothèse qu'au delà d'un vieillissement physique prématuré, les PVVIH50+ connaissent un vieillissement social précoce.

\section{Track A: Basic Sciences: HIV Transmission} and Evolution

\section{Volet A : Sciences fondamentales :} Transmission et évolution du VIH

\section{7}

INCREASED INNATE MUCOSAL FACTOR EXPRESSION IN THE PORTALS OF ENTRY OF HIV-EXPOSED UNINFECTED MEN AND WOMEN

Burgener, Adam ${ }^{1}$; Hasselrot, Klara²; Aboud, Lindsay';

Broliden, Kristina' ${ }^{2}$ Plummer, Francis ${ }^{1}$; Ball, Blake ${ }^{1}$

${ }^{1}$ Winnipeg, MB; ${ }^{2}$ Stockholm, Sweden

The primary receptive portals of entry for HIV are through mucosal surfaces which include the vaginal, rectal, or oral compartments. Mucosal surfaces have many soluble innate components that can neutralize and protect against invading microorganisms. Using proteomics approaches we have identified unique mucosal innate responses in HIV-exposed seronegative (HESN) individuals. In women, specific antiproteases (serpins) have been found to be elevated in the cervical secretions of HIVresistant women from the Pumwani sex worker cohort. We have determined that serpins are influenced by the menstrual cycle and have characterized their expression to reside in the epithelial layer of the female genital tract by immunohistochemical staining. In the oral compartment, we observed an elevation of basic salivary proline-rich proteins in HIV-exposed men who have sex with men (MSM). In the rectal 
mucosa of HESN MSM we also observe elevated innate immune factor expression, with specific clusters of protein expression. Our preliminary analysis shows that many of these yet un-described innate factors can inhibit HIV ( $\mathrm{R}$ and T-tropic strains) in both PBMC and TZM-bl cell lines in vitro, but their complete role HIV pathogenesis is not yet known. This information supports the hypothesis that altered innate immune mechanisms in HESN individuals at the portals of entry for HIV may contribute to reduced susceptibility to infection. Their role in mucosal immunology and HIV-pathogenesis will be discussed. Understanding the role of these factors in mucosal transmission of HIV may help the development of an effective microbicide against HIV.

This work is supported by the Canadian Institute for Health Research, the Public Health Agency of Canada, the Swedish Research Council and the Swedish Physicians against AIDS Foundation.

\section{8}

\section{SPERM MAY ACT AS A VECTOR FOR HIV TRANSMISSION TO THE GENITAL EPITHELIUM}

Young, Charlene D; Fongmoon, Duriya; Kongmanas, Kessiri; Jonathan, Angel; Tanphaichitr, Nongnuj

\section{Ottawa, ON}

BACKGROUND: HIV-1 infection in women occurs primarily through vaginal intercourse. The exact mechanisms of viral transmission from semen through the vaginal mucosa are still poorly understood. Several lines of research studies indicate that gp120 can interact with non-CD4 T-cells through binding sulfoglycolipids, sulfogalactosylceramide (SGC) and sulfogalactosylglycerolipid (SGG), implicating their roles as HIV-1 receptors. Since SGG is abundantly and selectively present on the sperm surface it suggests that sperm may have the ability to capture HIV. We therefore hypothesize that HIV-1 binds to sperm via interaction between gp120 and SGG and act as vectors transferring the captured HIV-1 to genital epithelial cells.

OBJECTIVE: To determine whether 1 . HIV-1 can bind to sperm in vitro. 2. HIV-1 bound to sperm is infectious and 3. Sperm can act as a vector transmitting HIV to vaginal/cervical cells.

METHODS: HIV-1 binding to sperm was shown by co-incubation of HIVcs204 with sperm, followed by p24 ELISA. Infectivity of HIV bound to sperm was demonstrated using TZM-bl reporter cells or by co-culture of HIV bound sperm with PBMCs. V/C cells were exposed to HIV associated to sperm and infection was shown by the presence of HIV-1 DNA in V/C cells.

RESULTS: HIV-1 virions bind to sperm and retain their infectivity. TZM-bl reporter cells showed increase luciferase activity when exposed to sperm which captured HIV. Therefore, HIV virions that were captured by sperm retain their infectivity. In addition HIV virions that were captured by sperm were transmitted to PBMCs and V/C cells.

CONCLUSION: Sperm can capture and transmit HIV to cells of the vaginal mucosa. Understanding alternate mechanisms including sperm associated HIV transmission would allow better and inclusive approaches for preventing transmission. This research could lead to new approaches for microbicides by including dual targets one for preventing cell free HIV and one for sperm associated HIV.

\section{O059}

\section{RECONSTRUCTING TRANSMITTED HIV GENOTYPES BY PHYLOGENETIC ANALYSIS OF SERIAL SAMPLES}

\section{McCloskey, Rosemary $\mathrm{M}^{1}$; Brumme, Zabrina $\mathrm{L}^{2}$;}

Harrigan, $\mathbf{P}$ Richard ${ }^{1}$; Liang, Richard $\mathrm{H}^{1}$; Poon, Art $\mathrm{F}^{1}$

'Vancouver; ${ }^{2}$ Burnaby, BC

BACKGROUND: HIV infection often descends from a single transmitted/founder virus. Understanding the characteristics of founder viruses is critical to vaccine research, however HIV's high mutation rate and delays between infection and diagnosis render this a challenging task. The availability of longitudinal intra-patient HIV clonal sequence data sets provides an opportunity to evaluate the effectiveness of phylogenetic methods for reconstructing transmitted/founder viruses from sequences sampled during later infection.

METHODS: We built a database of 336 published HIV clonal gene sequence data sets (median [IQR] 46 [17-84] sequences/set; median [IQR] baseline timepoint $33[6-70]$ days post infection/seroconversion; range 0.5-12 years follow-up). Majority-rule consensus sequences of baseline samples were defined as the 'true' ancestral sequences. Using all followup samples in each data set, ancestral reconstruction was performed in four stages: (1) multiple sequence alignment by HyPhy and MAFFT; (2) inference of rooted phylogenetic trees under a molecular clock model in BEAST; (3) reconstruction of ancestral codon sequences in HyPhy; (4) reconstruction of ancestral indels by Indelign. Effects of HIV genes and sampling on error rates were evaluated by a generalized linear model.

RESULTS: Reconstructed ancestral sequences possessed a median $1.93 \%$ error rate per codon, representing an almost 20\% improvement over the naive method (median error rate $2.37 \%$ ). Error rates in reconstructing env were significantly greater than nef, gag, or pol (Wilcoxon rank-sum test, $\left.\mathrm{P}<10 \mathrm{e}^{-5}\right)$. Error rates increased significantly with time elapsed between the earliest and first subsequent sample (IQR 47 to 643 days; $\log$ odds $+0.24 /$ year, $\left.\mathrm{P}<10 \mathrm{e}^{-5}\right)$. Within gag, error rates were negatively correlated with the density of CTL epitopes (Spearman's rho = $-0.1, \mathrm{P}=0.05$ ).

CONCLUSIONS: Phylogenetic methods confer a measurable improvement over a naive consensus approach for reconstructing transmitted/ founder viruses from sequences sampled during later infection. Though phylogenetic techniques are increasing in sophistication, they are not yet mature enough to obviate the need to study HIV evolution in patients with acute infection.

\section{O060 \\ NOVEL HLA ASSOCIATIONS WITH HIV CONTROL IN TWO DIFFERENT IMMUNOGENETIC CONTEXTS}

Valenzuela-Ponce, Humberto ${ }^{1}$; Brumme, Chanson J ${ }^{2}$; Ávila-Ríos, Santiago ${ }^{1}$; Brumme, Zabrina L2,3; Garrido-Rodríguez, Daniela1; García-

Téllez, Thalía ${ }^{1}$; García-Morales, Claudia ${ }^{1}$;

Hernández-Juan, Ramón ${ }^{1}$; Carlson, Jonathan $\mathrm{M}^{4}$;

Brockman, Mark ${ }^{2,3}$; Shahid, Aniqa ${ }^{3}$; Reyes-Terán, Gustavo ${ }^{1}$

${ }^{1}$ Mexico City, DF, Mexico; ${ }^{2}$ Vancouver; ${ }^{3}$ Burnaby, BC; ${ }^{4}$ Redmond, Washington, USA

BACKGROUND: Associations between HLA and HIV control have been well characterized. Unique HLA frequency distributions in previously uncharacterized populations represent an opportunity of finding novel protective factors for HIV control. We aimed to study HLA effects on HIV control in a previously uncharacterized cohort comparing with a large Caucasian cohort.

METHODS: HLA-A, - B and -C sequence-based typing was performed on 1187 and 1500 chronically HIV-1 B clade-infected, ART-naïve individuals from Mexico/Guatemala and from the mainly Caucasian British Columbia HOMER cohort. Associations between HIV plasma viral load $(\mathrm{pVL}) / \mathrm{CD}^{+} \mathrm{T}$ cell counts and expression of HLA alleles were evaluated using Mann-Whitney $U$ test and false discovery rate analyses.

RESULTS: The universal associations between $B * 57: 01$ and $B * 27: 05$ and HIV control were evident in both cohorts. As previously described for clade B-infected Caucasian cohorts, $B * 07: 02, B * 08: 01$ and $B * 18: 01$ were associated with higher $p V L$, while $B^{*} 13: 02$ and $B * 14: 01$ showed a protective effect in the HOMER cohort. Interestingly, new associations between $B * 15: 07$ and $B * 07: 05$ and HIV control were observed in the HOMER cohort. New associations between HLA and HIV control were also observed in the Mexican/Guatemalan cohort, including B*39:02, $B * 35: 08, C * 08: 01 / 02$, and $B * 14: 02$, associated with lower pVL and $\mathrm{B} * 35: 12$ associated with higher $\mathrm{pVL}$ (all $\mathrm{p}<0.05, \mathrm{q}<0.15$ ). Remarkably, HIV control by B*39:02, a typically Amerindian HLA allele also associated with autoimmune disease, might be explained by the presence of Glu63 and Ser67 at the peptide binding groove that make this allele different from most other $\mathrm{B} * 39$ molecules. Additionally, the Amerindian haplotype $A * 68: 03 / B * 39: 05 / C * 07: 02$ was associated with low CD4 $T$ cell counts in the Mexican/Guatemalan cohort. Finally, weak HLA frequency-dependent effects were evident in Mexico/Guatemala.

CONCLUSIONS: Our data confirms previous associations between HLA alleles and HIV viral control, but also suggests the existence of new protective and risk associations and mechanisms in the context of a previously uncharacterized population. 
0061

VARIABLE CHANGES IN GAG AND NEF FUNCTION IN CLINICAL SEQUENCES SAMPLED OVER THE COURSE OF THE HIV-1 EPIDEMIC IN NORTH AMERICA

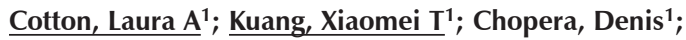
Chan, Benjamin ${ }^{1}$; Carlson, Jonathan ${ }^{2}$; Martin, Eric ${ }^{1}$; Le, Anh ${ }^{1}$; Penney, Kali' ${ }^{1}$; Kelleher, Anthony ${ }^{3}$; Markowitz, Martin ${ }^{4}$; Walker, Bruce ${ }^{5}$; Fuchs, Jonathan ${ }^{6}$; Buchbinder, Susan ${ }^{6}$; Wagner, Theresa ${ }^{6}$; Koblin, Beryl ${ }^{4}$; Mayer, Kenneth ${ }^{7,8}$; Poon, Art ${ }^{1,9}$; Brockman, Mark ${ }^{1,9}$; Brumme, Zabrina ${ }^{1,9}$

${ }^{1}$ Burnaby, BC; ${ }^{2}$ Los Angeles, Californaia; ${ }^{3}$ Sydney, New South Wales, Australia; ${ }^{4}$ New York, New York, USA; ${ }^{5}$ Charlestown, Massachusettes; ${ }^{6}$ San Francisco, California; ${ }^{7}$ Boston, Massachusetts; ${ }^{8}$ Providence, Rhode Island, USA; ${ }^{9}$ Vancouver, BC BACKGROUND: The extent to which HIV fitness and protein activities have changed over the course of the North American epidemic remains unclear. We therefore compared the function of clinical subtype B Gag and Nef sequences sampled from sites in Canada and the USA during historic (1979-1989) and modern (2002-2008) eras.

METHODS: Historic samples were collected from New York, Boston, San Francisco and Vancouver. NL4-3 recombinant viruses expressing clonal plasma RNA Gag sequences $(\mathrm{N}=108$ historic and $\mathrm{N}=58$ modern, one per patient) were constructed and their in vitro replication capacity (RC) was measured using a reporter T-cell assay. Single plasma RNA Nef sequences ( $\mathrm{N}=102$ historic and $\mathrm{N}=52$ modern, one per patient) were cloned into an expression plasmid. Nef-mediated CD4 and HLA-A*02 down-regulation activity was measured by flow cytometry following transfection into CEM T-cells expressing HLA-A*02. An independent panel of subtype B Nef clones from 34 modern-era patients with acute/ early infection was also analyzed.

RESULTS: Recombinant viruses expressing earliest (1979-1982), middle (1983-1985), later (1986-1989) and modern (2002-2008) Gag clonal sequences did not differ significantly in $\mathrm{RC}$ (Kruskal-Wallis $\mathrm{p}=0.6)$. In contrast, the ability of Nef clones to down-regulate CD4 and HLA increased stepwise during these four stages of the epidemic (KruskalWallis $\mathrm{p}<0.005)$. Furthermore, Nef-mediated HLA down-regulation correlated positively with calendar year between 1979-1989 (Spearman $\mathrm{R}=0.19 ; \mathrm{p}=0.056$ ). Nef function did not differ significantly by cohort, geographical area or infection stage $(p>0.1)$, suggesting that results are not confounded by these factors. Amino acids that differed in frequency between earliest and later historic sequences were identified in an exploratory analysis.

CONCLUSION: A modest increase in Nef CD4 and HLA down-regulation functions, but not Gag-mediated RC activity, was observed in patient-derived sequences sampled over the course of the North American HIV epidemic. Potential mechanisms underlying these observations, including HLA-associated immune selection pressure, are under investigation.

\section{2}

DNA SCANNING MECHANISM OF APOBEC3 DEOXYCYTIDINE DEAMINASES AS AN INDICATOR OF HIV-1 MUTAGENESIS POTENTIAL

\section{Love, Robin P; Ara, Anjuman; Chelico, Linda}

Saskatoon, SK

The APOBEC3 enzymes (A, B, C, DE, F, G, and H) can induce mutagenesis of HIV-1 proviral DNA, in the absence of HIV-1 Vif protein, by catalyzing cytosine deaminations to form uracils in (-)DNA. HIV-1 reverse transcriptase copies the altered (-)DNA, which results in guanine to adenine mutations in the (+)DNA that can cause viral inactivation. In cell culture, $\mathrm{APOBEC} 3 \mathrm{G}$ is the most potent restrictor of HIV-1 whereas other APOBEC3 enzymes have been found to restrict HIV-1 replication to lesser degrees. However, the biochemical reason for this was not known. Using in vitro model systems, we have found a correlation between the mechanisms by which APOBEC3 enzymes scan singlestranded DNA in search for cytosines and the ability to cause mutagenesis of HIV-1 proviral DNA. For APOBEC3 enzymes that scan processively
(APOBEC3G, APOBEC3H, and APOBEC3F), we found that an equal balance of sliding and jumping movements are required to most effectively induce mutagenesis. This is due to sliding enabling clustered areas of mutagenesis and jumping movements enabling efficient processive translocations despite the heterogeneous (-)DNA that has RNA segments annealed. Nonprocessive enzymes (APOBEC3A) can repeatedly interact with the (-)DNA and appear to induce more mutations than some processive enzymes that lack a balanced jumping and sliding mechanism. Since the number of mutations induced can be correlated with HIV-1 inactivation, our results indicate that some APOBEC3 enzymes may not induce enough mutations to inactivate HIV-1. Further, it appears that in our model gene for APOBEC3-induced mutagenesis, HIV-1 protease, the deamination motif of APOBEC $3 \mathrm{G}\left(5^{\prime} \mathrm{CCC}\right)$ is more likely to cause gene inactivating mutations than the deamination motif of other APOBEC3 enzymes (5'T/CTC). All together our results indicate that some APOBEC3 enzymes may be predisposed to facilitate HIV-1 evolution rather than HIV-1 inactivation.

\section{Track B: Clinical Sciences: Clinical Trials and Co-infections}

\section{Volet B : Sciences cliniques : Essais cliniques et co-infections}

\section{3}

\section{STAR STUDY: SINGLE-TABLET REGIMEN RILPIVIRINE/ EMTRICITABINE/TENOFOVIR DF IS NON-INFERIOR TO EFAVIRENZ/EMTRICITABINE/TENOFOVIR DF IN ART-NAÏVE HIV-1 INFECTED ADULTS}

Trottier, Benoit' ${ }^{1}$; Brunetta, Jason'2; Rachlis, Anita ${ }^{2}$; Angel, Jonathan $^{3}$; Wohl, David ${ }^{4}$; Henry, Keith ${ }^{5}$; Wang, Hui ${ }^{6}$; Porter, Danielle ${ }^{6}$; De-Oertel, Shampa ${ }^{6}$; Fralich, Todd ${ }^{6}$; Borg, Peter'; Retel, Andre $\mathrm{E}^{7}$

${ }^{1}$ Montreal, QC; ${ }^{2}$ Toronto; ${ }^{3}$ Ottawa, ON; ${ }^{4}$ Chapel Hill, North Carolina; ${ }^{5}$ Minneopolis, Minnesota; ${ }^{6}$ Foster City, California, USA ${ }^{7}$ Vancouver, BC;

Emtricitabine/Rilpivirine/Tenofovir DF (FTC/RPV/TDF) is a well-tolerated, once-daily single-tablet regimen (STR) treatment option. This study evaluates the safety and efficacy of two STRs, Rilpivirine/ Emtricitabine/Tenofovir DF (RPV/FTC/TDF) and Efavirenz/ Emtricitabine/Tenofovir DF (EFV/FTC/TDF) in treatment-naïve adults. $\mathrm{STaR}$ is a randomized, open-label, multi-center, international, 96 week study evaluating the safety and efficacy of the STR RPV/FTC/TDF compared to the STR EFV/FTC/TDF in treatment-naïve HIV-1 infected subjects. Eligibility criteria included screening HIV-1 RNA $\geq 2,500 \mathrm{c} / \mathrm{mL}$, genotypic sensitivity to EFV, FTC, TDF, and RPV, and no prior antiretroviral therapy. Randomization was stratified according to HIV-1 RNA level $(\leq 100,000$ copies $/ \mathrm{mL}$ or $>100,000$ copies $/ \mathrm{mL})$ at screening. The primary endpoint was the proportion of subjects with HIV-1 RNA $<50$ copies $/ \mathrm{mL}$ at Week 48 as determined by the FDA snapshot algorithm (12\% pre-specified non-inferiority margin).

A total of 786 subjects were randomized and received at least one dose of study drug (394 RPV/FTC/TDF; 392 EFV/FTC/TDF). Baseline characteristics were similar in both treatment arms, with a baseline mean HIV-1 RNA of $4.8 \log 10$ copies/mL. RPV/FTC/TDF was non-inferior to EFV/ FTC/TDF ( $86 \%$ vs $82 \%$ ) at Week 48 for HIV RNA $<50$ copies/mL (difference $4.1 \%, 95 \%$ CI [-1.1\%, 9.2\%]) Non-inferior efficacy was demonstrated for baseline HIV-1 RNA $\leq 100,000$ copies/mL $(\mathrm{n}=510), 89 \%$ RPV/FTC/TDF vs $82 \%$ EFV/FTC/TDF (difference $7.2 \%, 95 \%$ CI [1.1\%, $13.4 \%]$ ), and for $>100,000$ copies/mL ( $\mathrm{n}=276$ ), $80 \% \mathrm{RPV} / \mathrm{FTC} / \mathrm{TDF}$ vs 82\% EFV/FTC/TDF (difference -1.8\%, 95\% CI [-11.1\%, 7.5\%]). Overall, virologic failure, defined as HIV-1 RNA $\geq 50$ copies $/ \mathrm{mL}$ at Week 48 , discontinuation due to lack of efficacy per Investigator or discontinuation of study drug for reasons other than an adverse event with HIV RNA $\geq 50$ 
copies/mL was $8 \%$ for RPV/FTC/TDF vs $6 \%$ for EFV/FTC/TDF (difference $2.7 \%, 95 \%$ CI $[-0.9 \%, 6.2 \%])$. There were fewer study drug discontinuations due to adverse events in the FDA snapshot analysis for RPV/ FTC/TDF (3\%) compared to EFV/FTC/TDF (9\%).

The STR RPV/FTC/TDF demonstrated non-inferior efficacy to the STR EFV/FTC/TDF through 48 weeks, with fewer drug discontinuations due to adverse events.

\section{O064 \\ SPIRIT: SWITCHING TO RILPIVIRINE/EMTRICITABINE/ TENOFOVIR DF SINGLE-TABLET REGIMEN FROM BOOSTED PROTEASE INHIBITOR MAINTAINS HIV-1 VIROLOGIC SUPPRESSION THROUGH WEEK 48 IN HIV-1 INFECTED SUBJECTS}

Brunetta, Jason $^{1}$; Rachlis, Anita ${ }^{1}$; Laplante, Francois ${ }^{2}$;

Conway, Brian ${ }^{3}$; Wedgwood, Orson $\mathrm{M}^{4}$; Palella, Frank ${ }^{5}$;

Fisher, Martin ${ }^{6}$; De-Oertel, Shampa ${ }^{7}$; Porter, Danielle ${ }^{7}$;

Fralich, Todd 7 ; Wang, $\mathrm{Hui}^{7}$; Borg, Peter ${ }^{4}$

${ }^{1}$ Toronto, ON; ${ }^{2}$ Montreal, QC; ${ }^{3}$ Vancouver, BC; ${ }^{4}$ Mississauga, ON; ${ }^{5}$ Chicago, Illinois, USA; ${ }^{6}$ Brighton, United Kingdom; ${ }^{7}$ Foster City, California, USA

Rilpivirine/Emtricitabine/Tenofovir DF (RPV/FTC/TDF) is a well-tolerated and efficacious once-daily single-tablet regimen (STR) treatment option. This is the first study to evaluate switching from a boosted protease inhibitor (PI+RTV)-based antiretroviral therapy (ART) to the simplified regimen RPV/FTC/TDF.

SPIRIT is a phase $3 \mathrm{~b}$, randomized, open-label, multi-center, international, 48-week study to evaluate the safety and efficacy of switching from a PI+RTV+2NRTI regimen to RPV/FTC/TDF in virologically-suppressed HIV-1 infected subjects. Subjects were randomized 2:1 to switch to RPV/ FTC/TDF at baseline or maintain their current PI+RTV+2NRTI regimen with a delayed switch to RPV/FTC/TDF at Week 24. The primary endpoint was non-inferiority ( $12 \%$ margin) of RPV/FTC/TDF to $\mathrm{PI}+\mathrm{RTV}+2 \mathrm{NRTI}$ regimens in maintaining plasma HIV-1 RNA $<50$ copies/mL at Week 24 by FDA Snapshot analysis.

A total of 476 subjects were randomized and received at least 1 dose of study drug (317 RPV/FTC/TDF; 159 PI+RTV+2NRTIs). Baseline characteristics were similar across treatment arms. The primary endpoint of non-inferiority at Week 24 was met (HIV-1 RNA $<50$ copies/mL 93.7\% RPV/FTC/TDF vs. $89.9 \%$ PI+RTV+2NRTIs; difference 3.8\%, 95\% CI: -1.6 to 9.1$]$ ). Through Week 48 , virologic suppression (HIV-1<50 copies/ $\mathrm{mL}$ ) was $89.3 \%$ for subjects switching to RPV/FTC/TDF at baseline, and 92.1\% at Week 48 for the 152 subjects that switched to RPV/FTC/TDF at Week 24. Statistically significant favorable changes in lipids were demonstrated at Week 24 for total cholesterol, LDL, and triglycerides for subjects switched to RPV/FTC/TDF at baseline compared to staying on a $\mathrm{PI}+\mathrm{RTV}+2 \mathrm{NRTI}$ regimen $(\mathrm{p}<0.001)$. Additionally, a post-hoc analysis of lipid changes at Week 24 by baseline ART demonstrated statistically significant favorable changes in total cholesterol for subjects switched to RPV/FTC/TDF at baseline compared to staying on a PI+RTV+2NRTI regimen in all the PI+RTV+2NRTI subgroups (LPV, ATV, DRV, other), and in all the NRTI subgroups (FTC/TDF, ABC/3TC, other $[\mathrm{p}<0.05]$ ). Switching to the STR FTC/RPV/TDF from a PI+RTV+2NRTI regimen in virologically-suppressed, HIV-1-infected subjects maintained virologic suppression through 48 weeks, and resulted in favorable lipid changes for total cholesterol, LDL and triglycerides compared to subjects who stayed on their PI+RTV+2NRTI regimen.

\section{5}

A PILOT RANDOMIZED CONTROLLED TRIAL OF VALACYCLOVIR FOR ATTENUATING INFLAMMATION AND IMMUNE ACTIVATION IN HIV, HSV-2 CO-INFECTED ADULTS ON SUPPRESSIVE ANTIRETROVIRAL THERAPY

Yi, Tae Joon; Walmsley, Sharon; Szadkowski, Leah; Raboud, Janet; Kaul, Rupert; Rajwans, Nimerta; Kain, Kevin C; Shannon, Brett; Kumar, Sachin; Tan, Darrell H

Toronto, ON

BACKGROUND: HIV is associated with increased systemic inflammation and immune activation that persist despite suppressive HAART. We conducted a pilot trial of valacyclovir for decreasing T-cell activation and inflammatory markers in HSV-2 co-infected adults on HAART.

METHODS: Sixty HIV, HSV-2 seropositive adults on suppressive HAART (plasma HIV RNA<40 copies/mL) were randomized 1:1:1 to 12 weeks of placebo, low-dose (500mg BID) or high-dose (1g BID) valacyclovir, and underwent study visits at baseline, 6-, 12- and 18-weeks. Co-primary outcome measures were the percentage of activated $\left(\mathrm{CD} 38^{+} \mathrm{HLADR}^{+}\right)$ CD8 T-cells, hsCRP, interleukin-6 and sICAM-1. Secondary outcome measures included immune (CD8 exhaustion, CD4 exhaustion, doublenegative T-cells, regulatory T-cells), inflammatory cytokine (IL-1b, MCP-1, TNF-a), and endothelial activation (sVCAM-1, Ang1/Ang2 ratio) markers. The impact of valacyclovir (both treatment groups combined) on each outcome was estimated using interaction terms between treatment and time in generalized estimating equation regression models.

RESULTS: Participants were mostly Caucasian (75\%), MSM (80\%) and HSV-1 co-infected $(77 \%)$. Median (IQR) age was $51(47,56)$ years, duration of HIV infection $15(8,21)$ years, CD4 count at enrolment 520 (392, $719)$ cells $/ \mathrm{mm} 3$ and nadir CD4 $142(42,240)$ cells $/ \mathrm{mm}^{3}$. No significant differences in demographics or comorbidities were noted between study groups. Valacyclovir was not associated with significant changes in any of the primary or secondary outcome measures in univariate analyses. After adjusting for age, duration of HAART, current CD4 count or nadir CD4 count, valacyclovir was not associated with any of the primary outcomes. Medication adherence was $98 \%$ by self-report, $96 \%$ by pill count and $94 \%$ by urine valacyclovir monitoring.

CONCLUSIONS: Valacyclovir did not decrease systemic immune activation or inflammatory markers in this pilot trial of 60 adults with longstanding well-controlled HIV and HSV-2 co-infection.

Treatment-Time Interaction Estimates From Generalized Estimating Equation Regression Models

\begin{tabular}{|c|c|c|c|c|c|c|}
\hline & \multicolumn{2}{|c|}{ Treatment $\times 6$ Weeks } & \multicolumn{2}{|c|}{ Treatment $x 12$ Weeks } & \multicolumn{2}{|c|}{ Treatment x 18 Weeks } \\
\hline & $\begin{array}{c}\text { Est. } \\
(95 \% \mathrm{Cl})\end{array}$ & $p$ & $\begin{array}{c}\text { Est. } \\
(95 \% \mathrm{Cl})\end{array}$ & $p$ & $\begin{array}{c}\text { Est. } \\
(95 \% \mathrm{Cl})\end{array}$ & $\mathrm{p}$ \\
\hline $\begin{array}{l}\text { CD8 Activation } \\
\text { (Log10) }\end{array}$ & $-0.08(-0.19,0.02)$ & 0.10 & $-0.01(-0.12,0.11)$ & 0.93 & $-0.06(-0.18,0.06)$ & 0.31 \\
\hline hsCRP $(\log 10)$ & $0.06(-0.13,0.26)$ & 0.53 & $-0.16(-0.39,0.07)$ & 0.17 & $-0.11(-0.33,0.11)$ & 0.33 \\
\hline$\| \mathrm{L}-6^{*}$ & $1.26(0.46,3.47)$ & 0.65 & $1.82(0.80,4.17)$ & 0.16 & $0.67(0.39,1.15)$ & 0.15 \\
\hline SICAM & $1.54(-26.4,29.5)$ & 0.91 & $0.23(-26.2,26.7)$ & 0.99 & $-9.61(-35.1,15.9)$ & 0.46 \\
\hline $\begin{array}{l}\text { CD8 Exhaustion } \\
(\log 10)\end{array}$ & $-0.01(-0.10,0.08)$ & 0.82 & $-0.02(-0.12,0.09)$ & 0.78 & $-0.06(-0.17,0.05)$ & 0.32 \\
\hline $\begin{array}{l}\text { CD4 Exhaustion } \\
(\log 10)\end{array}$ & $-0.03(-0.15,0.08)$ & 0.58 & $-0.02(-0.13,0.10)$ & 0.78 & $-0.03(-0.14,0.08)$ & 0.62 \\
\hline $\begin{array}{l}\text { Double Negative T } \\
\text { Cells }\end{array}$ & $-2.82(-10.7,5.07)$ & 0.48 & $-5.56(-13.9,2.77)$ & 0.19 & $-6.62(-14.0,0.78)$ & 0.08 \\
\hline $\begin{array}{l}\text { Regulatory T Cells } \\
\quad(\log 10)\end{array}$ & $0.04(-0.09,0.17)$ & 0.57 & $0.07(-0.07,0.21)$ & 0.35 & $0.00(-0.13,0.14)$ & 0.95 \\
\hline $\mid \mathrm{L}-1 \mathrm{~b}^{*}$ & $0.60(0.05,7.08)$ & 0.68 & $0.60(0.05,7.11)$ & 0.69 & $0.77(0.07,8.76)$ & 0.84 \\
\hline MCP-1* & $1.23(0.55,2.75)$ & 0.62 & $1.26(0.66,2.38)$ & 0.48 & $0.69(0.32,1.50)$ & 0.35 \\
\hline TNFa* & $0.78(0.29,2.08)$ & 0.62 & $1.24(0.82,1.86)$ & 0.31 & $1.59(0.85,2.98)$ & 0.15 \\
\hline sVCAM-1 & $-9.20(-79.1,60.7)$ & 0.80 & $-10.3(-66.3,45.7)$ & 0.72 & $-34.8(-84.9,15.2)$ & 0.17 \\
\hline Ang1/Ang2 (Log10) & $0.14(-0.16,0.44)$ & 0.37 & $0.07(-0.18,0.32)$ & 0.60 & $0.05(-0.22,0.33)$ & 0.69 \\
\hline
\end{tabular}


O066

TREATING HCV-POSITIVE PATIENTS WITH A LOWTHRESHOLD MODEL OF CARE: USING MODIFIED DIRECTLY OBSERVED THERAPY APPEARS HIGHLY BENEFICIAL AND USEFUL, RESULTS FROM THE HEPVIRAC CLINICAL COHORT

Machouf, Nima; Vezina, Sylvie; Thomas, Réjean; Poliquin, Marc;

Gallant, Serge; Huchet, Emmanuelle; Lavoie, Stephane;

Gourde, Danielle; Roy, Marie C; Trottier, Benoit

Montréal, QC

BACKGROUND: HCV-infected patients have been treated at Clinique médicale l'Actuel with a new model of care since 2008 (modified Directly Observed Therapy: mDOT). Under mDOT, patients have weekly visits with medical staff to receive Peg-INF injection, have laboratory tests, and minimize any adverse events. It is a low-threshold model of care so patients do not need to stop injecting drugs in order to be treated. Our objective was to compare the proportion of Sustained Virologic Response (SVR) under mDOT vs. Standard of Care (SOC).

METHODS: This observational study used data from the HepVirAc clinical cohort database. Treatment regimen was mostly Peg-INF and Ribavirine. Data were analyzed using multivariate logistic regression analyses.

RESULTS: Of 296 patient's (344 treatments) analysed, median age was 49 years, $76 \%$ were men, $83 \%$ were intravenous drug users (19\% active IDU) and 23\% were HIV co-infected. HCV-genotypes were types 1,4 in $63 \%$ and types 2,3 in $37 \%$.. Diabetes was present among $9 \%$ and $6 \%$ of patients had liver cirrhosis, and $29 \%$ had liver fibrosis (F2 $=19 \%$, $\mathrm{F} 3=10 \%)$. In intent-to-treat analysis ( $\mathrm{D} / \mathrm{C}=$ failure), overall rate of SVR was $61 \%$. After adjusting for treatment history, genotype, age and gender, patients in the mDOT program had twice the odds of achieving an SVR $(\mathrm{OR}=1.9 ; 95 \%$ C.I: $1.2-3.0)$ when compared with individuals treated with a SOC

\begin{tabular}{llllr} 
Sustained Virologic Response (SVR) & mDOT (n=211) & SOC (n=133) & Total (n=344) \\
\hline \multirow{2}{*}{ GT 1,4 } & HCV mono infected & $53 / 92(58 \%)$ & $26 / 59(44 \%)$ & $79 / 151(52 \%)$ \\
& HIV-HCV co-infected & $16 / 26(62 \%)$ & $13 / 25(52 \%)$ & $29 / 51(57 \%)$ \\
\multirow{2}{*}{ GT 2,3 } & HCV mono infected & $62 / 77(81 \%)$ & $17 / 32(53 \%)$ & $79 / 109(72 \%)$ \\
& HIV-HCV co-infected & $10 / 16(63 \%)$ & $10 / 14(71 \%)$ & $20 / 30(67 \%)$ \\
Total & & $141 / 211(67 \%)$ & $66 / 133(50 \%)$ & $207 / 344(60 \%)$ \\
\hline
\end{tabular}

CONCLUSION: Patients treated with mDOT model had greater odds of treatment success compared with patients treated using SOC. These findings indicate mDOT particular approach may address any adverse effects rapidly and reinforce adherence strategy.

\section{7 \\ OVERCOMING HEPATITIS C: CHANGES IN QUALITY OF LIFE, HEALTHCARE USE, AND SUBSTANCE USE IN HIV- COINFECTED PATIENTS AFTER HCV THERAPY}

Yeung, Man Wah'; ${ }^{1}$ Rollet, Kathleen ${ }^{1}$; Cooper, Curtis ${ }^{2}$; Cox, Joseph ${ }^{1}$; Pick, Neora ${ }^{3}$; Hull, Mark ${ }^{3}$; Rachlis, Anita ${ }^{4}$;

Tyndall, Mark ${ }^{2}$; Walmsley, Sharon ${ }^{5}$; Klein, Marina B1

${ }^{1}$ Montreal; ${ }^{2}$ Ottawa, ON; ${ }^{3}$ Vancouver, BC; ${ }^{4}$ Toronto, ON

For chronic hepatitis $\mathrm{C}$ virus (HCV)-infected patients, the goal of antiviral treatment is a sustained virologic response (SVR) which yields measurable health benefits in $\mathrm{HCV}$-monoinfection. Whether such benefits exist for HIV-coinfected patients who have competing health issues is unclear. We examined changes in quality of life $(\mathrm{QoL})$, health service use and substance use over time among SVR-achievers and non-achievers in the Canadian Co-infection Cohort.

METHODS: HCV RNA+ HIV/HCV co-infected patients without prior HCV treatment were selected and followed every six months. Patients self-assessed QoL from 0 (worst health) to 100 (best) using the visual analog scale of EuroQol-5D questionnaires. We examined median QoL scores, health service incidence rates, and substance use frequency before and after HCV therapy.
RESULTS: Of 646 chronic HCV patients, 125(19\%) received treatment - 65(52\%) achieved SVR, 25(20\%) did not, 29(23\%) had ongoing treatment and 6(5\%) had unknown outcomes. For SVR-achievers, QoL scores were better than non-achievers pre-treatment (median: 70 vs. 65 ) and increased six months-post (median score: 80) before plateauing above baseline at one year (median score: 75). Non-achievers followed a similar trajectory but returned to pre-treatment values after one year (67.5). SVR-achievers used fewer health services than non-achievers overall, particularly emergency visits and hospitalizations (incidence rates, IR, six months-post: 0.28 vs. 1.01 events/person-year and 0.15 vs. 1.14 events/person-year, respectively). One exception was specialist visits (IR six months-post: 4.80 events/person-year vs. 1.01 events/personyear). Patients did not change injection drug use behaviours, but alcohol consumption increased post-treatment among SVR-achievers (from 34\% to $58 \%$ ).

CONCLUSIONS: SVR can have multidimensional effects on QoL, healthcare use and substance use. SVR-achievers report better health and use fewer health services. The implication of increased alcohol consumption after SVR requires investigation as it may counteract benefits of $\mathrm{HCV}$ treatment. Longer follow-up is required to determine the sustainability of health benefits of SVR in co-infection.

\section{8}

\section{HPV ANTIBODY RESPONSE IN HIV POSITIVE WOMEN} VACCINATED WITH AN HPV QUADRAVALENT 6/11/16/18 VACCINE: UPDATED RESULTS

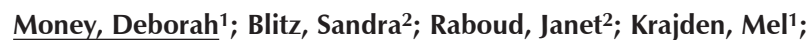
Coutlee, Francois ${ }^{3}$; Walmsley, Sharon²; Loutfy, Mona²; Trottier, Sylvie ${ }^{4}$; Smaill, Fiona ${ }^{5}$; Bitnun, Sean A $^{2}$; Singer, Joel ${ }^{1}$ ${ }^{1}$ Vancouver, BC; ${ }^{2}$ Toronto, ON; ${ }^{3}$ Montreal; ${ }^{4}$ Quebec, QC; ${ }^{5}$ Hamilton, ON

BACKGROUND: HPV vaccines are immunogenic in preventing HPV infection and cervical dysplasia in HIV- women, but data is limited in $\mathrm{HIV}+$ women. This study assesses seroresponsiveness of qHPV vaccine in HIV+ females.

METHODS: An open labeled, multi-centered study of seroresponsiveness of qHPV in HIV+females completed enrollment in December 2012. Genotype specific HPV infection was determined by Linear Array, and antibody levels pre vaccine and 1 month post 3rd dose, by Merck cLIA assay to HPV $6,11,16,18$. Results were grouped by age $(<18$ or $>18)$ and by HPV type specific serostatus.

RESULTS: 407 women were enrolled, with post-vaccine serologic data available on 193. Mean age 36 yrs (range: 9-60); 20.2\% < 18 yrs; $32 \%$ white, $45 \%$ black and $11 \%$ aboriginal; $84 \%$ on ARV's; median (IQR) baseline CD4 was 520/mm3 (399-700); 71\% had HIV RNA < 50/ml. All but 1 female under 19 was seronegative at baseline for all HPV types and women over age 19 had baseline seropositivity of $12-40 \%$ for any type. Seroconversion rates for genotypes $6,11,16 \& 18$ at 7 months were $99.5 \%, 99.0 \%, 99.5 \%, 94.8 \%$, respectively.

\begin{tabular}{|c|c|c|c|c|c|c|c|}
\hline $\begin{array}{l}\text { HPV } \\
\text { Type }\end{array}$ & $\begin{array}{l}\text { Months } \\
\text { from 1st } \\
\text { Vaccine }\end{array}$ & Age $<$ & $\begin{array}{l}\text { 18, Seronegative at } \\
\text { Baseline }\end{array}$ & Age $>$ & $\begin{array}{l}\text { 18, Seronegative at } \\
\text { Baseline }\end{array}$ & & $\begin{array}{l}\text { 18, Seropositive at } \\
\text { Baseline }\end{array}$ \\
\hline \multirow{3}{*}{6} & & $\mathrm{~N}$ & GMT $(95 \% \mathrm{Cl})$ & $\mathrm{N}$ & GMT $(95 \% \mathrm{Cl})$ & $\mathrm{N}$ & GMT $(95 \%$ Cl) \\
\hline & 7 & 39 & $783(552-1110)$ & 93 & $398(315-502)$ & 61 & $721(529-984)$ \\
\hline & 12 & 34 & $173(105-285)$ & 79 & $173(134-223)$ & 43 & $494(347-702)$ \\
\hline \multirow[t]{2}{*}{11} & 7 & 39 & $839(599-1175)$ & 127 & $504(417-609)$ & 27 & $870(563-1343)$ \\
\hline & 12 & 34 & $187(120-291)$ & 107 & $161(130-198)$ & 15 & $509(305-847)$ \\
\hline \multirow[t]{2}{*}{16} & 7 & 39 & $4128(2914-5847)$ & 97 & $1620(1239-2118)$ & 57 & $2167(1648-2850)$ \\
\hline & 12 & 34 & $950(577-1564)$ & 86 & $564(417-763)$ & 36 & $1236(877-1742)$ \\
\hline \multirow[t]{2}{*}{18} & 7 & 39 & 439 (272-709) & 135 & $245(194-310)$ & 19 & 637 (351-1159) \\
\hline & 12 & 34 & $81(44-150)$ & 111 & $68(52-90)$ & 11 & $342(165-710)$ \\
\hline
\end{tabular}

CONCLUSIONS: qHPV vaccinated HIV+ females, show high seroconversion rates, however, the response to HPV 18 was lower than reported for HPV naïve, HIV uninfected females. GMTs at month 12 fell substantially from those at month 7 . 
Track C: Epidemiology and Public Health Sciences: Evaluation of Programs and Interventions

Volet C : Épidémiologie et sciences de la santé publique : Évaluation des programmes et des interventions

\section{O069}

\section{INTERRUPTING THE SOCIAL PROCESSES IMPLICATED IN} THE INJECTION INITIATION PROCESS

Strike, Carol ${ }^{1}$; Rotondi, Michael ${ }^{1}$; Kolla, Gillian ${ }^{1}$; Penn, Rebecca ${ }^{1}$; Silver, Rey (Butch) ${ }^{1}$; Balian, Raffi ${ }^{1}$; Guimond, Tim ${ }^{1}$; Roy, Élise ${ }^{2}$; Millson, Peggy ${ }^{1}$; Altenberg, Jason ${ }^{1}$; Rotondi, Nooshin $\mathbf{K}^{1}$; Katherine, Rudzinski ${ }^{1}$; Hunt, $\mathrm{Neil}^{3}$

\section{${ }^{1}$ Toronto, ON; ${ }^{2}$ Longueuil, QC; ${ }^{3}$ Kent, United Kingdom}

BACKGROUND: We assessed the effects of Change the Cycle (CTC), a peer delivered intervention for current injection drug users (IDUs) designed to the interrupt social processes influencing injection initiation (i.e., helping someone else inject for the first time).

METHODS: Using respondent driven sampling (RDS), we recruited current IDUs in Toronto to complete baseline (T1; $\mathrm{n}=98)$ and six-month post-intervention $(T 2 ; n=86)$ interviews. Participants were asked about demographic characteristics, initiation experiences and behaviours that may influence non-injectors' desire to start injecting. Data were RDSweighted. Multiple imputation was used for missing follow-up data. Analyses include population proportions over time, the proportion of individuals who changed their behaviours, and 95\% confidence intervals.

RESULTS: The proportion of initiators at T1 was $6.23 \%$ (95\% CI: 2.14 , 11.26 ) and at T2 was 3.46\% (95\% CI: 0.8, 7.13). Paired analyses showed a modification of initiation behaviour for $72.71 \%$ (95\% CI:47.66, 83.12) of baseline initiators. The proportion of IDUs who offered to initiate a non-injector at T1 was $8.35 \%(95 \% \mathrm{CI}: 2.53,15.93)$ and at T2 was 1.49 \% (95\% CI: $0.40,3.71)$. Among those who offered to initiate a noninjector at T1, paired analyses showed a $97.72 \%$ (95\% CI: 94.86, 99.62) reduction in this behaviour at T2. The prevalence of speaking positively about injection to non-injectors and encouraging non-injectors to inject also decreased significantly in the paired analyses. A non-significant increase in the proportion of people who showed or explained how to inject was also observed.

CONCLUSIONS: This is the first-ever longitudinal follow-up evaluating a peer-delivered initiation intervention aimed at reducing initiation to injection drug use. Analyses showed a reduction in initiation and potential initiation-provoking behaviours. Scale-up of CTC and evaluation of different modalities (e.g., one-on-one versus group; crack smokers) is needed to fully determine the potential impact of this intervention.

\section{O070}

\section{AN HIV/STI PREVENTION INTERVENTION WITH} INTERNALLY DISPLACED WOMEN IN LEOGANE, HAITI: RESULTS FROM A GRAND CHALLENGES CANADA RISING STAR IN GLOBAL HEALTH PILOT STUDY

\section{Logie, Carmen $\mathbf{H}^{1}$; Daniel, CarolAnn ${ }^{2}$; Newman, Peter $\mathrm{A}^{3}$;} Loutfy, Mona $\mathrm{R}^{3}$

${ }^{1}$ Calgary, AB; ${ }^{2}$ New York, New York, USA; ${ }^{3}$ Toronto, ON

BACKGROUND: Haiti has the highest HIV infection rate in the Western hemisphere. The January 2010 earthquake led to the collapse of Haiti's social, economic and health infrastructure, exacerbating social and structural HIV risk factors. Internally displaced (ID) women are particularly at high risk for HIV infection due to breakdown of community networks, increased poverty and sexual violence. The objective was to develop and pilot-test a community-health worker (CHW) delivered psycho-educational HIV/STI prevention intervention with ID women in Leogane, Haiti.

METHODS: This was a single-centre pragmatic N-of-1 pilot study. We recruited 200 participants (ID women) using purposive peer-driven recruitment methods. ID women were trained as $\mathrm{CHW}$ to deliver the intervention. Participants conducted a pretest that involved an individual HIV/STI educational video followed by a 6- week group program of women's health meetings that addressed HIV, sexually transmitted infections (STI), healthy relationships, communication, and mental health. Repeated-measures MANOVA was used to compare pre-test and posttest differences across primary (HIV knowledge) and secondary (STI knowledge, condom use, social support, resilient coping, depression, relationship control) outcome variables.

RESULTS: The majority (90.3\%) of participants ( $n=200)$ were unemployed; the mean monthly income was $\$ 3.87$ USD (SD: 9.5). The mean age was 36.5 years (SD: 13.5$)$ and two-thirds of participants had primary school education or less $(63.7 \%)$. One-third $(35.3 \%)$ perceived their partner, and one-quarter (25.0\%) themselves, at medium-high risk for HIV infection. In comparison with pre-intervention scores, MANOVA analyses indicated significantly higher HIV knowledge, STI knowledge, consistent condom use and significantly lower depression scores postintervention.

CONCLUSIONS: This was the first trial to develop and evaluate a CHW delivered psycho-educational HIV/STI prevention intervention among ID women in post-earthquake Haiti. Intervention participants reported significantly increased HIV/STI knowledge, consistent condom use and reduced depression. Study results may help to inform HIV prevention interventions among ID women in Haiti and can be tested for applicability with ID women globally.

\section{O071}

INTEGRATING SPECIALIZED HIV PROGRAMMING FOR SEX WORKERS INTO THE POST-CONFLICT TRANSITION: THE HIV TESTING EXPERIENCES OF YOUNG WOMEN SEX WORKERS IN NORTHERN UGANDA

Muldoon, Katherine; $\mathrm{A}^{1}$; Akello, Monica ${ }^{2}$; Dobrer, Sabina ${ }^{1}$;

Muzaaya, Godfrey ${ }^{2}$; Birungi, Josephine ${ }^{2}$; Shannon, Kate ${ }^{1}$

${ }^{1}$ Vancouver, BC; ${ }^{2}$ Kampala, Uganda

OBJECTIVES: Post-conflict transitions are both a period of heightened vulnerability to HIV transmission and a neglected component of HIV testing, treatment and care. In synergy with a criminalized environment, specialized services designed to reach at risk-groups, including sex workers (SWs) evade current programming. The objective of this analysis was to identify the strongest associations of HIV testing among SWs in Gulu, northern Uganda.

METHODS: Through peer/sexwork-led outreach and partnership with HIV service organization (TASO), a cross-sectional sample of 400 young SWs was recruited from bars, truck stops and lodges from May-December 2011. Analysis examined recent HIV testing experiences (last 6 months) among HIV seronegative and newly diagnosed HIV positive women. All known HIV-positive women $(\mathrm{n}=101)$ were excluded from this analysis. Bivariable and multivariable logistic regression were used to examine associations with accessing recent HIV testing.

RESULTS: Of 299 SWs, the median age was 20 years (interquartile range: $18-24), 65.5 \%$ had lived in an internally displaced persons camp, and $29.1 \%$ were surviving abductees from the Lord's Resistance Army. The 6-month period prevalence of HIV testing was 63.5\%, 14.1\% were newly diagnosed with HIV during the study. Previous STI testing was independently associated with accessing HIV testing (aOR: 2.67, 95\% CI:1.56-4.57) while having a newly diagnosed HIV infection was negatively associated with recent HIV testing (aOR:0.41, 95\% CI:0.20-0.83). Being an LRA abductee and migrant to Gulu were both marginally associated with accessing HIV testing.

DISCUSSION: The high levels of recent infection, despite close to twothirds accessing HIV testing within the last 6-month, reflects the immediate gap in responding to HIV during post-conflict transition. Further consideration of the structural barriers to HIV testing for at-risk women 
in post-conflict and criminalized environments in sub-Saharan African is warranted. Transitioning from emergency assistance to societal reconstruction are key uptake opportunities for improved engagement in HIV services.

\section{O072 \\ INTENSIVE CASE MANAGEMENT IN VANCOUVER INCREASES ENGAGEMENT TO CARE AND IMPROVES HIV OUTCOMES FOR MARGINALIZED PEOPLE LIVING WITH HIV}

Tu, David; Thumath, Meaghan A; Demlow, Ellen; Chu, Tim; Heath, Kate; Yip, Benita; Compton, Miranda; Colley, Guillaume; Gustafson, Reka; Sandhu, Jat

Vancouver, BC

BACKGROUND: For many reasons, socially marginalized people who become HIV positive are less likely to optimally engage in HIV care, and more likely to become lost to follow up. Lack of engagement to care and effective antiretroviral treatment (ART) increases the risk of HIV transmission and more rapid disease progression. Most clinics lack the resources, beyond letters or phone calls, to address barriers to engagement and retention in care. Here we evaluate the effects of intensive case management on engagement in care and treatment outcomes.

METHODS: As part of the Seek and Treat to Optimally Prevent (STOP) HIV project, an outreach interdisciplinary (nurses, social workers, outreach workers, peers and a physician) intensive case management (ICM) team was created to improve patient care. Community clinics or hospitals referred patients to ICM if they had not returned for care in > 12 months or were failing to effectively engage in HIV treatment. We conducted a retrospective "pre and post" intervention analysis of patients referred to ICM.

RESULTS: Between January 2011 and July 2012, 404 patients (30\% female, 20\% Aboriginal, mean age 41 years, mean CD4 399 cells $/ \mathrm{ml}$ ) were referred for ICM. At the time of their HIV diagnosis, $45 \%$ were living in Vancouver's inner-city, with 47\% identifying IDU and 25\% MSM as their routes of HIV transmission. Post referral, engagement in care (defined as > two visits in the past 12 months) increased by $17 \%$ ( $76 \%$ vs. $93 \%, \mathrm{p}<0.001)$ and ART uptake increased by $27 \%$ (43\% vs. $70 \%$, $\mathrm{P}<0.001)$. Among those on ART rates of viral load suppression $(\mathrm{vl}<400)$ increased from $71 \%$ to $85 \%$ (p 0.044).

CONCLUSIONS: Intensive outreach case management targeting those struggling with or lost to care is an effective way of increasing engagement in care and improving HIV treatment outcomes.

\section{O073}

\section{ECONOMIC EVALUATION OF COMMUNITY-BASED HIV} PREVENTION PROGRAMS IN ONTARIO: EVIDENCE OF EFFECTIVENESS TO REDUCE HIV INFECTION CASES AND SAVE COSTS TO MEDICAL SYSTEM

Choi, Stephanie; Kennedy, Rick; McGee, Frank; Lush, Joanne;

Bacon, Jean; Rourke, Sean B

Toronto, ON

BACKGROUND: Community-based HIV prevention programs over the past two decades are known to have an impact on the epidemic, although this has never been subjected to a systematic evaluation.

METHODS: We conducted an economic evaluation at the macro-level to examine whether providing community-based HIV prevention programs (focus on education and outreach) in Ontario produced an overall cost savings in Ontario's medical care system from 2001-2009 and reduced HIV infections. A payer's perspective (i.e. MOHLTC-AIDS Bureau and PHAC's AIDS Community Action Program [PHACACAP]) and multiple data sources (surveillance data, budgetary data, and prior literatures) were used in the analyses. The analyses were modeled based on methodologies that guide decision-making in HIV prevention activities at the CDC in the US.

RESULTS: Over the past six years, approximately $\$ 80$ million was invested by MOHLTC-AIDS Bureau and PHAC-ACAP in Ontario in community-based HIV prevention programs with $\sim 160,000$ encounters by people with HIV and people at-risk. To justify that these investments were well spent, minimum of 40-70 HIV infection cases need to be averted by these program per year. Our analyses showed that these programs have made significant impacts in HIV prevention: From 20012009, these programs helped avert approximately 12,673 HIV infection cases $(95 \%$ Confidence Intervals[CI]: 5,690-19,182) and saved the medical system a total of $\$ 3.5$ billion (CI: $\$ 1.6 \mathrm{~B}-\$ 5.8 \mathrm{~B}$ ). Given these benefits generated, the rate of return of the investments made was approximately $\$ 51$ dollars per each dollar invested. Our analysis also showed that funding cuts in these programs would have negative impacts: e.g., funding cut of $10 \%$ would result in medical costs of $\$ 29$ million per year.

CONCLUSIONS: Investments made in community-based HIV prevention program in Ontario by the MOHLTC-AIDS Bureau and PHACACAP in the past decade are effective in reducing HIV infections and saving health care costs, and an effective public health policy.

\section{4}

THE COST-EFFECTIVENESS OF POPULATION-LEVEL HAART EXPANSION IN BRITISH COLUMBIA: 1996-2010

Nosyk, Bohdan; Lima, Viviane D; Yip, Benita; Min, Jeong;

Hogg, Robert S; Montaner, Julio S

Vancouver, BC

BACKGROUND: The secondary preventive benefit of HAART has been demonstrated at the population level in British Columbia (BC), and elsewhere; these results suggest that the cost-effectiveness of HAART scale-up has been understated. Our objective is to determine the costeffectiveness of HAART scale-up observed in BC from 1996-2010 compared to hypothetical scenarios characterized by lesser expansion of HAART coverage, resulting in $75 \%$ and $50 \%$ of the total number of individuals on HAART during the study period.

METHODS: We used population-level data on disease progression and HAART use from the BC HIV Drug Treatment Program, supplemented with other published data sources to construct a deterministic, compartmental mathematical model to simulate population-level dynamics in the HIV epidemic in BC. HIV transmission, costs (HAART and nonHAART direct medical costs, presented in $2010 \$ C D N)$ and qualityadjusted life years (QALYs) were estimated as a function of disease progression, as observed at the population level during the study period. Evolving IAS-USA guidelines on HAART initiation were accounted for in the simulation model. Incremental cost-effectiveness ratios (ICERs) were calculated to compare actual practice to scenarios of slower expansion $(50 \%, 75 \%$ of increase in treatment uptake).

RESULTS: BC increased the number of individuals in HAART from 837 in 1996 to 5413 in 2010. During this period, the number of new HIV cases diagnosed fell from 702 in 1996 to 301 in 2010. Actual practice was costlier than scenarios of reduced treatment expansion, but led to substantially greater QALY gains, resulting in ICERs of \$2442/QALY compared to $75 \%$ expansion, and \$2464/QALY compared to $50 \%$ expansion.

CONCLUSION: The expansion of HAART in BC has resulted in substantial decreases in morbidity and mortality as well as a reduction in new HIV diagnoses. Resulting ICERs were well within the range of societal willingness to pay for an incremental QALY gain.

\section{Track D: Social Sciences: Gender}

\section{Volet D : Sciences sociales : Genre}

\section{O075}

BLACK SUBJECTS: RACE, MASCULINITY AND HIV PREVENTION

Oakes, Wesley; Husbands, Winston C

Toronto, ON

In North America, Black men and women account for a disproportionately large share of the number of people with heterosexually acquired 
HIV. Black men's role in HIV transmission is commonly explained with reference to ideologies of "Black masculinity" that endorse dangerous anti-social behaviours with grave consequences for Black communities. Moreover, Canadian media coverage (and even informed opinion) of criminal prosecutions for non-disclosure of HIV sometimes script Black men as dangerous, if not criminal. Of course, it is important to recognize that Black men may endorse anti-social and risky behaviors, and that Black men are complicit in the spread of HIV in Black communities. However, perspectives that overemphasize Black men's behaviours as inherently Black, inherently risky and solely responsible for HIV in Black communities may be premised on gross stereotypes. At best, prevailing interpretations of masculinity (even among Black men themselves) often treat Black men's behaviour as primordial, self-explanatory and emptied of racialized relations of power.

Drawing on critical race theory, we explore Black masculinity as constituted by tensions rooted in racial inequality. We look at how Black men acknowledge and productively engage their health-related vulnerabilities by using a range of strategies and resources available to them in different situations. This is crucial, considering that Black men's anti-social behaviours emerge in complex circumstances that may not accommodate productive engagement with vulnerability. Furthermore, we argue in favour of an approach to HIV prevention that moves beyond its focus on correcting, controlling, and containing Black (male) bodies. We support our claims with evidence from iSpeak, a community-based research study that in 2012 included focus groups with heterosexually-identified men from African, Caribbean and Black communities in Ontario, and service providers who work with those communities.

\section{O076}

TRANSGENDER, TRANSSEXUAL AND TWO-SPIRIT INDIVIDUALS WHO USE DRUGS: STIGMA, VIOLENCE, AND HIV VULNERABILITIES

Lyons, Tara $^{1}$; Small, Will1,2; Shannon, Kate ${ }^{1}$; Kerr, Thomas ${ }^{1}$

${ }^{1}$ Vancouver; ${ }^{2}$ Burnaby, BC

OBJECTIVES: Transgender, transsexual and two-spirit persons (TT2) often experience marginalization, discrimination and violence based on their gender identity. TT2 individuals who use drugs face multiple stigmas associated with gender and drug use and may be at greater risk for violence, which is associated with elevated rates of HIV incidence. Therefore we sought to qualitatively investigate the intersections of stigma, violence and HIV to gain a better understanding of the unique experiences of TT2 individuals who use drugs.

METHODS: In-depth semi-structured interviews were conducted with 19 individuals who identified as transgender, transsexual or two-spirit. Participants were recruited from 3 open prospective cohorts of individuals who use drugs in Vancouver, Canada. Interviews were transcribed verbatim and imported into ATLAS.ti qualitative data analysis software where codes and themes were developed.

RESULTS: In our sample, 21\% (4) identified as Caucasian and $79 \%$ (15) identified as Aboriginal. Sixteen participants (84\%) were HIVpositive and the majority of participants had current or past involvement in sex work. Participants reported high levels of violence and in some cases this violence was framed by participants as acts that they deserved. Participants reported that their gender identity was accepted while accessing health services in their neighbourhood, yet they experienced stigma in different areas and arenas within the city. In addiction treatment programs, both stigma towards and acceptance of gender was reported, however, experiences of stigma often led to premature withdrawal from treatment.

DISCUSSION: TT2 participants in our study reported high levels of HIV infection, violence, and experiences of both stigma and acceptance of their gender identity. The findings illustrate the need for policies and programs to address the unique needs of this population, such as addiction treatment and HIV prevention interventions tailored to TT2 individuals, particularly TT2 persons of Aboriginal ancestry.
O077

COMMUNITY BASED RESEARCH FROM COAST TO COAST? CHIWOS AND THE FOOD SECURITY STUDY: LESSONS LEARNED TO IMPROVE FURTHER COLLABORATIONS

Bernier, Mélina ${ }^{1}$; $\mathrm{O}^{\prime}$ Brien, Nadia ${ }^{1}$; de Pokomandy, Alexandra ${ }^{1}$; Kaida, Angela ${ }^{2}$; Loutfy, Mona $\mathbf{R}^{3}$; Monteith, Ken ${ }^{1}$; Rourke, Sean $\mathbf{B}^{3}$ ${ }^{1}$ Montreal, QC; ${ }^{2}$ Burnaby, BC; ${ }^{3}$ Toronto, ON

BACKGROUND: Community Based Research (CBR) methodologies are now a well-established part of the Canadian HIV response. Key tenets of CBR include a focus on community input and on shared ownership. As such, the process of the research, much like the results, belongs equally to researchers and collaborators. Despite the wide recognition of these fundamental principles, challenges emerge when translating these principles into practice within national CBR projects where Francophone and Anglophone realities must be navigated.

OBJECTIVES: 1 ) To present challenges to collaborations in the context of national, community based, HIV research projects; 2) to further our understanding of common pitfalls drawing on two Quebec research coordinators' experiences from two ongoing CBR projects; the Canadian HIV Women's Sexual and Reproductive Health Cohort Study (CHIWOS) and the Food Security Study, both of which are conducted in Quebec, Ontario and British Columbia; 3) to elicit a critical reflection of CBR practices to improve further collaborations.

LESSONS LEARNED: Drawing lessons from both national CBR studies allowed us to observe similarities in challenges faced and successes achieved. These challenges included; 1) a tendency towards homogenization of methods and centralization of power and funds; 2) insufficient anticipation of cultural and linguistic differences; 3 ) insufficient resources dedicated to translation and cultural adaptation; 4) and disparities in allotting the burden of translation and related human resources. Successful strategies included: 1) establishing protocols for translation from project inception; 2) building these steps into study timelines; 3 ) allotting sufficient central funds for translation; 4) promoting local autonomy and leadership; 5) and importantly, acknowledging that successfully navigating French and English realities is crucial to national CBR work.

RECOMMENDATIONS: The expansive use of CBR strategies has meant the institutionalization and nationalization of participatory research strategies. However, in nationalizing CBR, new challenges have emerged. Experiences from the CHIWOS and the Food Security Project offer helpful lessons for future projects: in order to truly democratize research, we must also reinforce the capacity of local communities to change the face of HIV research in Canada.

\section{O078 \\ CORRELATES OF HIV RISK AMONG BLACK WOMEN FROM AFRICAN AND CARIBBEAN COMMUNITIES IN THE GREATER TORONTO AREA: "SISTERS, MOTHERS, DAUGHTERS AND AUNTIES"}

Weaver, James; Rwigema, MJ; Williams, Charmaine;

Massaquoi, Notisha; Brown, Marsha; Newman, Peter

Toronto, ON

INTRODUCTION: Black women in Canada are at disproportionately high risk for HIV; however Black women are a diverse population that may reflect different risks and determinants of risk. We explored HIV risk among Black women from African and Caribbean communities in the Greater Toronto Area (GTA) and implications for prevention.

METHODS: Sisters, Mothers, Daughters and Aunties was a communitybased collaboration with women from African and Caribbean communities engaged across all stages of survey design and implementation. Participants were recruited across diverse community organizations. We compared demographic correlates of number of sexual partners, frequency of condom (past 12 months); a risk index, and perceived personal and community risk. We tested associations between perceived HIV risk and reported risk behaviors.

RESULTS: Participants $(\mathrm{n}=127$ ) (mean age $=35$ years, income $=\$ 1,683$ ) were equally divided by Caribbean and African ethnicity. $81 \%$ were born 
outside Canada, among those averaging $17.8(\mathrm{SD}=9.5)$ years in Canada. Half completed college, $58 \%$ ever married, two-thirds worked part/fulltime. Average number of partners (past 12 months) was $1.27(\mathrm{SD}=1.50$, range $0-11$ ). One-fourth reported never using condoms, one-fifth most/all of the time; mean risk index $=0.67(\mathrm{SD}=1.00$, range $0-4)$. Mean perceived personal HIV risk was $8.06(\mathrm{SD}=6.23$, range $0-18)$, perceived community risk $9.44(\mathrm{SD}=6.46$, range $0-35)$. Younger and Canadian born women reported significantly more partners and more consistent condom use. Caribbean and ever-married women reported significantly less condom use. Younger and Canadian born women, and those living longer in Canada reported significantly more risk behaviors. Perceived community HIV risk was positively associated with number of sexual partners and perceived personal HIV risk.

CONCLUSIONS: Different HIV risk behaviors by African/Caribbean ethnicity, age, marital status and years in Canada suggest the importance of tailored preventive interventions for Black women. Women more acculturated to Canada may engage in higher risk behaviors, suggesting some traditional practices may be protective against HIV risk. Further exploration of the importance of perceived community risk is indicated.

\section{O079 \\ OH BABY! EVALUATION OF A PRENATAL GROUP FOR WOMEN LIVING WITH HIV}

\section{Shindler, Simone; Ringlein, Maureen}

Toronto, ON

INTRODUCTION: In light of the growing number of women in Canada who are living with HIV and are having children, as well as data indicating low engagement with prenatal programs not specific to women living with HIV, The Teresa Group initiated an evaluation of their prenatal group. The study focused on the following research questions: What barriers exist for women in accessing prenatal care, and what are their motivations for attending a prenatal group? What has been the impact of prenatal programming on their lives? Has the group reached its target outcomes to enhance engagement with medical practitioners, improve mental health, social supports, and drug adherence?

The study was initiated in response to a significant gap in program evaluation or best practices literature in regards to prenatal group programming for mothers living with HIV. A community-based participatory research model was adopted for this study. Data collection tools included focus groups with program participants, attendance data (2004-2011), participant feedback surveys $(\mathrm{N}=190)$; and semi-structured interviews with program implementers including. All survey respondents from 2004 to 2010 who responded to the question "How would you rate the group" $(\mathrm{N}=190)$ provided an overwhelmingly positive review of the program. $81.6 \%$ rated the group as "excellent" and 18.4\% reported "good". $80 \%$ indicated that they "definitely" received the information they needed. Qualitative data generated further insight into what features of the group contributed towards the mother's improved mental and physical health. The evaluation provided clear direction for enhanced prenatal group support for women living with HIV, and generated evidence of improved health outcomes if women are given a space where they feel emotionally supported and where confidentiality is respected. The group was critical in allowing women to address and support each other through the complex psychosocial dimensions of HIV and pregnancy. A short video was produced documenting the evaluation results and the experiences of pregnant women living with HIV.

\section{O080}

CONTRACEPTIVE USE AND METHOD PREFERENCE AMONG HARDER-TO-REACH WOMEN ON ANTIRETROVIRAL THERAPY (ART) LIVING WITH HIV IN BRITISH COLUMBIA (BC)

Patterson, Sophie $^{1}$; Salters, Kate $\mathrm{A}^{2}$; Zhang, Wendy ${ }^{2}$; Chen, Yalin ${ }^{2}$; Money, Deborah'²; Hogg, Robert ${ }^{2}$; Kaida, Angela ${ }^{1}$

${ }^{1}$ Burnaby; ${ }^{2}$ Vancouver, BC

BACKGROUND: Contraceptive use among HIV-positive women prevents unwanted pregnancy and reduces HIV transmission. Recent studies link hormonal contraception to increased risk of HIV transmission, consequently WHO guidelines recommend dual protection for HIVpositive women. This study sought to measure contraceptive prevalence and preferences among women on ART.

METHODS: The Longitudinal Investigation into Supportive and Ancillary health services (LISA) cohort is a prospective study of harderto-reach HIV-positive individuals accessing ART in BC. Intervieweradministered surveys collected information on socio-demographic, behavioral and healthcare factors, linked with clinical variables through the Drug Treatment Program at BC Centre for Excellence in HIV/AIDS. This analysis included female participants aged 18 to 49 years, not currently pregnant. The outcome variable of interest was self-reported current contraceptive use. Multivariable logistic regression identified independent covariates.

RESULTS: This analysis included 166 women with median age 38 years (IQR 33 to 48). Current intravenous drug use was reported by $25 \%$, and $51 \%$ identified as Aboriginal. Median time on ART was 71 months (IQR 25 to 117 months) and $36 \%$ demonstrated adherence $\geq 95 \%$ in the year before interview. In the six months before interview $57 \%$ demonstrated viral suppression ( $\mathrm{VL}<50$ copies), and median CD4 was 280 (IQR 170 to 490). Prevalence of contraceptive use was $66 \%$. Among women reporting vaginal sexual intercourse in the six months before interview (104/166), contraceptive methods included: permanent methods (tubal ligation/ hysterectomy) (10\%), hormonal methods (nuvaring/intrauterine device/ oral/injectable) (9\%), barrier methods (condoms) (30\%), dual methods (barrier method plus hormonal/permanent method) (27\%), no method (24\%). No measured clinical or socio-demographic covariates showed independent association with contraceptive use.

CONCLUSIONS: Most sexually active women use contraceptive methods, however, few practice dual protection. Contraceptive users rely on barrier methods, with the minority using hormonal methods. Clinically, this cohort demonstrates poor adherence to ART and treatment response, despite long-term therapy. Women-centered integrated care facilities (WCC) combine HIV and sexual/reproductive-health services to improve health outcomes for HIV-positive women, specifically influencing contraceptive preferences and utilization, and improving health-service quality and access by marginalized populations. Promoting access to WCC in this cohort may improve reproductive and HIV-related outcomes.

\section{Track A: Basic Sciences: HIV Virology and} Host Factors

Volet A : Sciences fondamentales : Virologie
et facteurs de l'hôte du VIH

\section{O081}

THE IMPACT OF HIV ON INDUCED REGULATORY T CELL DIFFERENTIATION AND FUNCTION

Dave, Chintan; Fernandes, Jason R; Angel, Jonathan B

Ottawa, ON

BACKGROUND: Regulatory $T$ cells (Tregs) are essential for maintenance of normal immunological self-tolerance and immune homeostasis. Tregs modulate the properties of other immune cells through a variety of mechanisms, including suppression of proliferation and/or function. The effect of HIV infection on Tregs has not been clearly established; some studies indicate a decrease in Treg numbers and function in HIV infection, while others show increased immune activation via Tregs. The effect of HIV-1 envelope protein gp120 has been shown to increase the suppressive function of naturally-occurring Tregs (nTregs), however similar studies involving the effects of gp120 on induced Tregs (iTregs) are sparse. We hypothesize that gp120 increases the immunosuppressive function of iTregs.

METHODS: iTregs were generated from naïve $\mathrm{CD} 4{ }^{+} \mathrm{T}$ cells by stimulation with anti-CD3 antibody for 5 days, followed by culture in IL-2 containing RPMI-1640 medium as previously described. The suppressive activity of iTregs was measured by determining their effect on peripheral 
blood mononuclear cell (PBMC) proliferation using a CFSE dilution assay. iTregs were incubated with gp120 $(1 \mathrm{ug} / \mathrm{mL})$ for up to 12 hours to investigate the effect of gp120 on suppressive activity of differentiated iTregs. Separate experiments were conducted to test the effect of gp120 pre-treatment on the differentiation of naïve $\mathrm{CD}^{+}{ }^{+} \mathrm{T}$ cells into iTregs. RESULTS: Untreated iTregs suppressed the proliferation of autologous PBMC in a dose-dependent manner. Treatment of differentiated iTregs with gp120 for 12 hours reduced their ability to suppress proliferation of PBMC. Treating naïve $\mathrm{CD}^{+} \mathrm{T}$ cells with gp 120 for 4 or 8 hours prior to differentiation resulted in iTregs with increased suppressive activity, while treatment for more than 8 hours generated iTregs with suppressive activity similar to untreated cells.

CONCLUSION: Preliminary results indicate that gp120 reduces the suppressive function of differentiated iTregs. In contrast, we report that treatment of naïve $\mathrm{CD} 4^{+} \mathrm{T}$ cells prior to differentiation may yield iTregs with enhanced immunosuppressive function. These results support a role for gp120 in causing regulatory $\mathrm{T}$ cell dysfunction, and may provide insight into immunopathogenesis in HIV infection.

\section{O082}

\section{IL-18 BINDING PROTEIN: A CORRELATE OF PROTECTION} IN HIV-INFECTED LONG-TERM NON-PROGRESSORS?

\section{Allam, Ossama; Jenabian, Mohammad A; Samarani, Suzanne;} Tremblay, Cécile; Routy, Jean-Pierre; Ahmed, Ali

Montreal, QC

BACKGROUND: HIV-infected individuals are characterized by an aberrant immune activation. This immune activation results, at least in part, from a dysregulated production of cytokines. We have previously reported increased concentrations of IL-18, a pro-inflammatory cytokine of the IL-1 family, in HIV-infected individuals. The cytokine, as a negative feedback mechanism, induces expression of its own antagonist, IL-18 Binding Protein (IL-18BP). We investigated the functioning of this negative feed-back mechanism in the course of HIV infection.

METHODS: Human monocyte-derived macrophages (MDM) were infected in vitro with M-tropic and dual tropic HIV strains. The effects of the infections on the production of IL-18 and its antagonist were determined. The concentrations of these two soluble mediators were also measured in the circulation of 32 healthy controls and 104 HIV-infected individuals in different categories i.e., primary infection, treatment-naïve viremic chronic infection, chronic HAART-treated aviremic, and longterm non-progressors (LTNP). Non-parametric methods (Kruskal-Wallis and Dunn's multiple comparison tests) were used to compare medians. RESULTS: A severe imbalance in the production of IL-18 and its antagonist was found in HIV-infected persons: the concentrations of IL-18 increased but those of IL-18BP decreased in their circulation. Due to this imbalance, the concentrations of the free, biologically active IL-18 increased in the circulation of the infected individuals. Surprisingly, the IL-18BP levels remained close to physiological levels in the LTNP. These results show for the first time that the protective negative feedback mechanism for IL-18 becomes defective in HIV-infected individuals, but remains operative in the LTNP. We provide evidence that HIV directly contributes to this imbalance, as it increases IL-18 production, but decreases that of its antagonist in human monocyte-derived macrophages.

CONCLUSIONS: HIV infection induces a sever imbalance in the production of IL-18 and its natural antagonist in humans. The LTNP, however, do not develop this imbalance. Increased IL-18 biological activity enhances HIV replication and causes aberrant immune activation. Furthermore, IL-18BP could be used to neutralize IL-18 and attenuate immune activation in HIV infections.
0083

INTERFERON-INDUCED HERC5 IS A NOVEL ANTIVIRAL FACTOR THAT INHIBITS HIV-1 REPLICATION BY TWO NOVEL MECHANISMS

Woods, Matthew W; Barr, Stephen D

London, ON

The emergence of drug-resistant HIV-1 strains and the lack of an effective HIV-1 vaccine have emphasized the importance of developing novel, non-toxic HIV-1 therapy. One exciting prospect currently being explored is the anti-HIV-1 activity of cellular HIV-1 restriction factors. Cellular HIV-1 restriction factors are defined as cellular proteins that inhibit (or "restrict") HIV-1 replication. We present here the identification of HECT domain and RCC1-like domain-containing protein 5 (HERC5) as a potential new interferon-induced cellular restriction factor that blocks HIV-1 particle production. HERC5 protein is comprised of a $\mathrm{N}$-terminal Regulator of Chromosome Condensation 1-like domain (RLD), followed by a unique Spacer region that does not share homology with any known protein, and a C-terminal Homologous to the E6-AP Carboxyl Terminus (HECT) domain. HERC5 is the main E3 ligase that conjugates the ubiquitin-like protein called ISG15 to target proteins in human cells. We recently identified two novel and independent mechanisms of antiviral activity for HERC5 that provides cells with a potent defense against HIV-1 replication. One function of HERC5 serves to inhibit HIV-1 particle assembly and is dependent on the E3 ligase activity of HERC5. Inhibition correlated with the post-translational modification of Gag with ISG15 and electron microscopy revealed that Gag particle production was arrested at an early stage of assembly at the plasma membrane. A second function serves to inhibit the intracellular accumulation of late stage HIV-1 proteins. This inhibition was independent of the E3 ligase activity of HERC5 and dependent on the HERC5 RLD. Inhibition of late stage HIV-1 proteins was not a result of reduced intracellular levels of HIV-1 mRNA nor mediated by proteasomal or lysosomal protein degradation. These data identify two novel pathways by which Type I interferons can restrict HIV-1 replication and identifies a potential new avenue for HIV/AIDS therapy.

\section{O084}

INTERFERON INDUCED TRANSMEMBRANE PROTEINS INHIBIT HIV-1 INFECTION

Ding, Shilei; Qian, Jin; Liang, Chen

Montreal, QC

HIV-1 infection is subject to inhibition by interferons. A number of interferon-stimulated genes have been reported to potently restrict HIV-1 infection via distinct mechanisms. Examples include tetherin that blocks the release of nascent virus particles from the surface of infected cells, SAMHD1 that impairs viral reverse transcription by depleting cellular DNA pool, SLFN11 that suppresses the translation of viral proteins, and TRIM22 that disrupts virus assembly. APOBEC3G and TRIM5alpha, another two potent restriction factors, are constitutively expressed in cells, and their levels also increase with IFN treatment. Recent studies led to the identification of a small protein family named interferon induced transmembrane (IFITM) proteins that inhibit an array of different viruses by impeding virus entry, a restriction mechanism that is distinct from those of the other known restriction factors. Here, we report that the three human IFITM proteins, IFITM1, 2 and 3, inhibit different steps of HIV-1 infection, with IFITM2 and 3 diminishing HIV-1 entry and IFITM1 suppressing viral gene expression. In addition, IFITM2 and 3 also potently inhibit the entry of certain SIV strains. Our data support the idea that IFITM proteins inhibit the entry of both $\mathrm{pH}$-dependent viruses such as influenza virus as well as $\mathrm{pH}$-independent viruses such as HIV and SIV. 


\section{5}

\section{TRIM5 $\alpha$-MEDIATED RESTRICTION EXPLOITS PARTS OF} THE CYTOSKELETON

\section{Pawlica, Paulina; Berthoux, Lionel}

Trois-Rivières, QC

TRIM5 $\alpha$ is a protein naturally occurring in some mammals and is known to be a factor providing resistance to HIV infection in non-human primates. It acts in the cytoplasm shortly after retrovirus entry into the cell and inhibits viral replication by several mechanisms. TRIM $5 \alpha$ decreases stability of the viral core, diminishes reverse transcription products, impairs transport to the nucleus and mediates antiviral signaling.

This study focuses on the role of certain cytoskeletal components, such as microtubules and the dynein complex, in the TRIM5 $\alpha$-mediated restriction of retroviruses. Dynein complexes are molecular motors involved in microtubule-dependent transport. Here, we demonstrate that the ability of TRIM5 $\alpha$ to restrict HIV is dependent on (i) an intact microtubules network and (ii) functional dynein complexes. By analyzing viral core integrity as well as viral DNA synthesis and nuclear transport, we were able to assess which of the TRIM5 $\alpha$ effector functions are hindered after disruption of the cytoskeleton. We also performed co-immunostaining experiments of the viral capsid, TRIM5 $\alpha$ and cytoskeleton components. Summing up our observation we conclude that microtubules are important for the dynamics of TRIM $5 \alpha$ cytoplasmic bodies and in supporting TRIM5 $\alpha$-mediated inhibition of the retroviral transport to the nucleus. On the other hand, dynein complexes seem to be important for the initial contact between TRIM5 $\alpha$ and its viral target, probably by transporting the virus itself

\section{O086}

\section{ASSESSMENT OF EARLY HIV-1 NEF FUNCTION IN} INDIVIDUALS WHO SUBSEQUENTLY CONTROL VIREMIA TO LESS THAN 2000 RNA COPIES PER ML

Kuang, Xiaomei T1'; Le, Anh'; Mwimanzi, Philip ${ }^{1}$; Markle, Tristan ${ }^{1}$; Danroth, Ryan ${ }^{1}$; Ueno, Takamasa ${ }^{2}$; Miura, Toshiyuki ${ }^{3}$;

Connick, Elizabeth ${ }^{4}$; Daar, Eric $\mathrm{S}^{5}$; Jessen, Heiko ${ }^{6}$;

Kelleher, Anthony ${ }^{7}$; Little, Susan ${ }^{8}$; Markowitz, Martin ${ }^{9}$;

Pereyra, Florencia ${ }^{10}$; Rosenberg, Eric ${ }^{10}$; Walker, Bruce ${ }^{10}$;

Brumme, Zabrina ${ }^{1,11}$; Brockman, Mark ${ }^{1,11}$

${ }^{1}$ Burnaby, BC; ${ }^{2}$ Kumamoto, Japan; ${ }^{3}$ Nagasaki, Japan; ${ }^{4}$ Denver,

Colorado; ${ }^{5}$ Los Angeles, Californaia USA; ${ }^{6}$ Berlin, Germany;

${ }^{7}$ Kensington, New South Wales, Australia; ${ }^{8}$ San Diego, California;

${ }^{9}$ New York, New York; ${ }^{10}$ Boston, Massachusetts, USA; ${ }^{11}$ Vancouver, BC

BACKGROUND: Nef clones derived from HIV-1 controllers display reduced activity compared to those from individuals with chronic progressive infection; however, the contribution of viral and host factors to this observation remains unclear. To address this, we assessed the CD4 and HLA class I down-regulation activities of acute/early Nef clones derived from individuals who subsequently controlled plasma viremia to $<2000$ RNA copies/mL (Acute Controllers [AC]) compared to those who did not (Acute Progressors [AP]).

METHODS: Nef sequences were cloned from $\mathrm{N}=13 \mathrm{AC}$ (median [IQR] 86 [65-127.5] days post-infection [DPI]) and $\mathrm{N}=67 \mathrm{AP}(58$ [36-73] DPI). Clones were transfected into CEM T cells expressing HLA-A*02, and surface $\mathrm{CD} 4$ and $\mathrm{A} * 02$ protein levels were quantified using flow cytometry. The ability of each Nef to down-regulate CD4 and HLA was normalized to SF2 control.

RESULTS: Nef sequences from AC and AP displayed no significant phylogenetic clustering or imbalance in amino acid frequency. Nevertheless, Nef clones from AC displayed reduced ability to downregulate CD4 (0.94 [0.88-0.97]) compared to AP (1.00 [0.97-1.02]), $p=0.0027$. Similarly, HLA down-regulation activity was reduced in AC (0.84 [0.76-1.00]) compared to AP (1.00 [0.93-1.06]), $\mathrm{p}=0.0033$. Differences remained significant after restricting analyses to samples derived from specimens with plasma HIV RNA $<10 \wedge 5$ copies $/ \mathrm{mL}$, or from specimens derived from time points $<180$ DPI. The frequency of protective HLA class I alleles (B*13, B*1516, B*1517, B*27, B*51, B*57 and $B * 5801)$ was comparable between cohorts (7/13 [54\%] vs. 21/67 [31\%], $\mathrm{p}=0.20$ ); however, Nef clones from AC with protective alleles displayed further reduced CD4 down-regulation function compared to those from AC lacking protective alleles $(p=0.05)$.

CONCLUSIONS: HIV-1 Nef proteins derived from AC displayed reduced CD4 and HLA down-regulation activities. Further impairment of CD4 down-regulation observed in AC expressing protective HLA alleles supports a combination of host and viral factors contributing to this Nef phenotype in HIV controllers.

\section{O087}

INTERSUBTYPE VARIATION IN THE ABILITY OF HIV-1 NEF TO MODULATE CD4 AND HLA CLASS I

Mann, Jaclyn²; Mwimanzi, Philipㅜ; Byakwaga, Hellen ${ }^{3}$;

Kuang, Xiaomei $\mathrm{T}^{1}$; Le, Anh $\mathrm{Q}^{1}$; Omarjee, Saleha ${ }^{2}$;

Brumme, Chanson J'; Lee, Guinevere $\mathrm{Q}^{4}$; Martin, Eric ${ }^{1}$;

Baraki, Bemulu1; Danroth, Ryan ${ }^{1}$; McCloskey, Rosemary ${ }^{1}$;

Muzoora, Conrad ${ }^{3}$; Kelleher, Anthony ${ }^{6}$; Jessen, Heiko ${ }^{7}$;

Markowitz, Martin ${ }^{9}$; Bangsberg, David R ${ }^{5}$; Martin, Jeff N8;

Hunt, Peter $\mathbf{W}^{8}$; Harrigan, Richard ${ }^{4}$; Walker, Bruce $\mathrm{D}^{5}$;

Ndung' $\mathbf{u}$, Thumbi ${ }^{2}$; Brockman, Mark $\mathrm{A}^{1,4}$; Brumme, Zabrina $\mathrm{L}^{1,4}$

${ }^{1}$ Burnaby, BC; ${ }^{2}$ Durban, South Africa; ${ }^{3}$ Mbarara, Uganda;

${ }^{4}$ Vancouver, BC; ${ }^{5}$ Boston, Massachusetts, USA; ${ }^{6}$ Sydney, New

South Wales, Australia; ${ }^{7}$ Berlin, Germany; ${ }^{8}$ San Fransisco,

California; ${ }^{9}$ New York, New York, USA

BACKGROUND: Nef enhances HIV pathogenesis through many mechanisms, including down-regulation of CD4 and HLA class I (HLAI). We examined intersubtype diversity of these Nef activities in a large panel of patient-derived clones representing subtypes A, B, C, and D. METHODS: Single plasma HIV RNA Nef clones from 96 (subtype A), 93 (B), 85 (C), and 86 (D) antiretroviral-naïve chronic patients from the AMU/UARTO (A/D), BC HOMER (B) and Sinikithemba (C) cohorts were cloned into an expression plasmid. CD4 and HLA-I surface expression was measured by flow cytometry following transfection of CEM T-cells expressing HLA-A*02 and results were normalized to Nef-SF2 control. Nef expression was verified by Western blot; $\mathrm{N}=21$ clones with poor expression/function were excluded from analysis. An independent panel of subtype B Nef clones from 67 early (median [IQR] 59 [37-73] days post-infection) and 52 chronic patients were also analyzed.

RESULTS: While Nef-mediated CD4 down-regulation was marginally higher in subtype B clones compared to others (Kruskal-Wallis, $\mathrm{p}=0.06$ ), median [IQR] Nef-mediated HLA-I down-regulation differed strikingly among subtypes: B $(0.95[0.90-0.99]>$ D 0.85 [0.77-0.89] > A 0.84 [0.77$0.90]>\mathrm{C} 0.80$ [0.59-0.89], $\mathrm{p}<0.0001)$. CD4 and HLA-I activities correlated positively for all subtypes (Spearman $R=0.30-0.39, p \leq 0.005$ ). Intersubtype differences in HLA-I down-regulation remained consistent when a subset of $\mathrm{N}=120 \mathrm{Nefs}$ (30/subtype) matched for $\mathrm{pVL}$ and CD4 counts were compared $(p<0.0001)$. No significant differences in CD4 or HLA-I down-regulation were observed in an independent panel of subtype B Nef clones from early vs. chronic patients $(p>0.1)$, indicating that clinical status was not a confounder. Synthetic Nef subtype $\mathrm{C}$ consensus displayed 5.4\% lower HLA-I down-regulation activity than B consensus, supporting inherent differences between clades.

CONCLUSION: Intersubtype differences were observed in the ability of Nef to down-regulate HLA-I. The mechanisms underlying these differences and their contribution to viral pathogenicity require further investigation.

\section{O088}

TARGETING HIV NEF TO ENHANCE IMMUNE RECOGNITION AND KILLING OF HIV INFECTED CELLS

Mujib, Shariq; Bozorgzad, Ardalan; Ostrowski, Mario Toronto, ON

The HIV accessory protein Nef plays a key role in immune evasion of HIV infected cells, CD4 T cells and macrophages, from HIV specific CD8 $\mathrm{T}$ cells by downregulating MHC class I (MHC-I) among other targets. MHC-I is critical for the recognition of infected cells by HIV 
specific CD8 T cells and downmodulation of this molecule presents a challenge for CD8 T cells to target and kill infected cells due to reduced HIV epitope density on the infected cell's surface. Nef mRNA transcripts are particularly enriched early during HIV infection and replication. Nef downregulates MHC-I by binding to and activating the SH3 domain of Src family kinases (SFK) such as Hck and Lyn in infected target cells via a proline rich motif (PXXP75). This activation of the kinase triggers a cascade of events that eventually brings about the endocytosis of surface MHC-I via the PI3K pathway. We have recently acquired novel drugs that inhibit this Nef-SFK mediated effect. We propose that by preventing MHC-I downregulation an infected cell would more readily be recognized and killed by an HIV-specific CD8 T cell. In our studies here, we show, for the first time, that these drugs effectively prevent MHC-I downmodulation on primary CD4 T cells following HIV infection with no or limited toxicity. Furthermore, in a coculture assay with primary infected CD4 $\mathrm{T}$ cells in the presence of these drugs, HIV-specific CD8 T cell responses were improved as measured by IFN $-\gamma$ production. These results implicate HIV Nef as a viable drug target to improve the immune system's response mounted against HIV-infected cells. In a continuation of this work we aim to test these drugs in the setting of HIV latency to enhance the detection and elimination of the HIV latent reservoir.

Track B: Clinical Sciences: Pharmacology, Resistance and Pre-exposure Prophylaxis

Volet B : Sciences cliniques :
Pharmacologie, résistance et prophylaxie
pré-exposition

\section{O089}

\section{EARLY TREATMENT DISCONTINUATION IS NOT PREDICTED BY PROPOSED HOST PHARMACOGENETIC POLYMORPHISMS IN PATIENTS RECEIVING HAART IN \\ BRITISH COLUMBIA}

Brumme, Chanson J; Chui, Celia K; Min, Jeong Eun;

Dong, Weiyan; Colley, Guillaume; Yip, Benita; Harrigan, P Richard Vancouver, BC

BACKGROUND: Host genetic polymorphisms may predict HIV therapy outcome or laboratory abnormalities; however, screening for pharmacogenetic markers is not routinely used in HIV care. We sought to validate proposed associations between a panel of host polymorphisms and early treatment discontinuation.

METHODS: We investigated 12 host polymorphisms previously reported to influence discontinuation of three antiretroviral drugs: for tenofovir (TDF), three polymorphisms in ABCC2 and ABCC4; for efavirenz (EFV), six in CYP2A6, CYP2B6, and CYP3A4; and for atazanavir (ATV), three in ABCB1, NR112, and UGT1A1. Polymorphisms were sequenced in 747 consenting patients from BC. Patients treated with TDF (N=475), EFV $(\mathrm{N}=264)$, and/or ATV $(\mathrm{N}=271)$ were followed for 15 months. Rates of discontinuation in the first year of treatment (defined as stopping a study drug for $\geq 3$ months) were compared between individuals with and without polymorphisms using multivariable Cox proportional hazard models adjusted for age, sex, ethnicity, CD4 count, and plasma viral load. Secondary endpoints corresponding to drug-specific toxicities (serum creatinine [TDF], plasma EFV, bilirubin levels [ATV]) were also investigated. RESULTS: During the first year of treatment, 81 (17\%), 81 (31\%), and 55 (20\%) patients discontinued TDF, EFV, and ATV, respectively. Patients homozygous for the minor allele of rs1045642 (ABCB1) had significantly higher bilirubin levels in the first 6 months of ATV therapy than individuals carrying one or no copies $(\mathrm{p}=0.02)$. These patients were more likely to discontinue ATV early (adjusted HR [95\% CI]: 2.6 [1.16.1], $\mathrm{p}=0.03$ ). Carriage of minor variants in CYP2A6, CYP2B6, and CYP3A4 was associated with elevated plasma EFV levels in the first 6 months of therapy $(p<0.0001)$, but not earlier EFV discontinuation.
CONCLUSION:Screening for host polymorphisms may predict laboratory abnormalities, but not HAART discontinuation in this cohort. Currently we do not have sufficient data to recommend expanded pharmacogenetic screening as a method for routinely identifying patients at risk of early treatment discontinuation in BC.

\section{O090}

TISSUE DISTRIBUTION OF ATAZANAVIR AT SANCTUARY SITES OF HIV-1 INFECTION: POTENTIAL ROLE OF EFFLUX TRANSPORTERS

Robillard, Kevin R${ }^{1}$; Hoque, Tozammel ${ }^{1}$; Zhang, Guijin ${ }^{2}$; Cameron, Bill $^{2}$; la Porte, Charles ${ }^{2}$; Bendayan, Reina ${ }^{1}$

${ }^{1}$ Toronto; ${ }^{2} \mathrm{Ottawa}, \mathrm{ON}$

INTRODUCTION: The blood-testis barrier (BTB) and the bloodbrain barrier are responsible for protecting male genital tract and CNS from xenobiotic exposure. Clinical studies in HIV-treated patients have reported low concentrations of antiretroviral drugs (ARVs) in cerebrospinal fluid and seminal fluid. One mechanism that may contribute to reduced concentrations in tissues is the expression of ATP-binding cassette $(\mathrm{ABC})$ drug efflux transporters i.e., $\mathrm{P}$-glycoprotein $(\mathrm{P}$-gp) and breast cancer resistance protein (BCRP). The objectives of this study were to investigate in vivo, the tissue distribution of atazanavir (ATV) in wild type mice (WT), P-gp/Bcrp knockout (abcb1a/1 $\mathrm{b}^{-}$, abcg $2^{-}$) mice (TKO) and Cyp3a $\mathrm{a}^{-\%}$ knockout mice (CYP).

METHODS: ATV (10 mg/kg) was administered I.V. to 9-12 week old WT and TKO. WT were pre-treated with a P-gp/Bcrp inhibitor Elacridar $(5 \mathrm{mg} / \mathrm{kg})$ while CYP were pre-treated with ritonavir (RTV) $(2 \mathrm{mg} / \mathrm{kg}$ I.V.). Animals were sacrificed at various time points (up to $24 \mathrm{~h}$ ). Blood, brain and testes were collected and ATV concentrations were quantified using liquid chromatography/tandem mass spectrometry.

RESULTS: In the absence of P-gp and Bcrp (TKO), we observed a significant increase in ATV brain to plasma concentration ratios (5.3-fold) and testes to plasma concentration ratios (4.6-fold) compared to WT group $(\mathrm{p}<0.05)$. WT pre-treated with Elacridar showed a significant increase in ATV brain: plasma concentration ratios (12.3-fold) and testes to plasma concentration ratios (13.5-fold) compared to WT at 0.25 and $1 \mathrm{hr}$, respectively. In CYP pre-treated with RTV, a modest increase (2.6fold) in ATV testes to plasma concentrations ratio was detected at $1 \mathrm{hr}$. DISCUSSION/CONCLUSION: These data suggest that ABC drug efflux transporters are involved in limiting the permeability of ATV in rodent brain and genital tract tissues. Since these transport proteins are also known to be expressed in humans, they could contribute to the low CSF and seminal fluid ARVs concentrations reported in the clinic. (Supported by OHTN)

\section{O091}

\section{RALTEGRAVIR PERMEABILITY ACROSS BLOOD-TISSUE BARRIERS: POTENTIAL ROLE OF DRUG EFFLUX TRANSPORTERS}

Hoque, Tozammel; Kis, Olena; De Rosa, Maria Fabiana; Bendayan, Reina

Toronto, ON

BACKGROUND: Drug efflux transporters, P-glycoprotein (P-gp), Breast Cancer Resistant Protein (BCRP) and Multidrug ResistanceAssociated Proteins (MRPs) could alter the efficacy of antiretroviral drugs (ARVs) by preventing their permeability across blood-tissue barriers. In this study, we investigated the interaction of raltegravir, an HIV integrase inhibitor, with drug efflux transporters using in vitro and in situ cell/organ systems, representative of the blood-brain, blood-testicular and blood-intestinal barriers.

METHODS: Cellular accumulation of [3H]-raltegravir by MDA-MDR1, HEK-ABCG2, HeLa-MRP1, HEK-MRP4 overexpressing cells, human brain microvessel endothelial (hCMEC/D3) and rat testicular Sertoli TM4 cells was examined in the absence or presence of P-gp inhibitors: PSC833, cyclosporine A, quinidine; BCRP inhibitors: Ko143, FTC; and MRPs inhibitor: MK571. [3H]-Raltegravir permeability across intestinal segments was assessed in situ by single-pass perfusion of rat intestine in 
the absence or presence of PSC 833 or Ko143 or both.

RESULTS: Compared to control, [3H]-Raltegravir accumulation was significantly inhibited in the presence of P-gp inhibitors: PSC833 (241\%), cyclosporine A (283\%), quinidine (182\%) and BCRP inhibitors: Ko143 (190\%), FTC (185\%) in P-gp and BCRP overexpressing MDA-MDR1 and HEK-ABCG2 cells respectively, while no inhibition could be detected in HeLa-MRP1 and HEK-MRP4 cells. [3H]-Raltegravir accumulation was significantly inhibited by PSC 833 or Ko143 in hCMEC/D3 and TM4 cells respectively, suggesting that it is a substrate of both transporters. In addition, in situ, [3H]-Raltegravir permeability across rat intestinal segments was significantly inhibited by concurrent administration of PSC833 and Ko143, while such effect was not observed when these inhibitors were used individually.

CONCLUSION: Our results indicate that raltegravir can serve as a substrate of drug efflux transporters, P-gp and BCRP but not of MRP1 or MRP4. Taken together, these data further suggest that drug efflux transporters, P-gp and BCRP could play a role in limiting raltegravir permeability across the intestinal, blood-brain and blood-testicular barrier. (Supported by Merck Canada Inc.)

\section{2}

\section{INDEPENDENT REPLICATION SHOWS HIGH INTER-} LABORATORY CONCORDANCE IN NEXT-GENERATION SEQUENCING FOR HIV MINORITY SPECIES DETECTION

Swenson, Luke $\mathrm{C}^{1}$; Brumme, Chanson J'; Kagan, Ron $\mathrm{M}^{2}$; Harrigan, $\mathrm{P}$ Richard ${ }^{1}$

${ }^{1}$ Vancouver, BC; ${ }^{2}$ San Juan Capistrano, California, USA

BACKGROUND: Next-generation sequencing (NGS) methods are increasingly common in HIV research. However, there is little data on the reproducibility of NGS. Here we assess concordance between 2 independent laboratories performing NGS of patient samples.

METHODS: 310 plasma screening samples from maraviroc clinical trials were sequenced at 2 laboratories (Vancouver, BC [BC] and Quest Diagnostics, CA [CA]). RNA extraction, RT-PCR of HIV envelope, and NGS were performed independently at each site. BC used a 454 GS-FLX instrument ("Standard" chemistry); CA used a GS-FLX or GS-JR ("Titanium" chemistry). Both laboratories used different data-processing systems. HIV tropism was inferred using geno2pheno. Samples were classified as $\mathrm{X} 4$ if $\geq 2 \%$ of their sequence reads had a geno2pheno value $\leq 3.5$. Samples also underwent Sanger sequencing.

RESULTS: Inter-laboratory concordance with NGS was excellent (Kappa $=0.84)$. Of 310 samples, $286(92 \%)$ received the same tropism classification by both laboratories. The X4 prevalence per sample was highly correlated (Pearson's $\mathrm{r}=0.92$ ), with a median difference of only $0.3 \%$ (interquartile range $[\mathrm{IQR}] \mathrm{O}-3 \%$ )

Most samples with discordant NGS results had low X4 prevalence: median $6 \%$ or $4 \% \mathrm{X} 4$ in samples classified as X4 by $\mathrm{BC}(\mathrm{N}=10)$ or $\mathrm{CA}$ $(\mathrm{N}=14)$ only. $\mathrm{X} 4$ prevalence was much higher in concordant $\mathrm{X} 4$ samples (median 37\% [IQR 13-70\%] by BC, 34\% [IQR 12-75\%] by CA). Concordant R5 samples had a median X4 prevalence of $0 \%$.

Inter-laboratory concordance was lower $(88 \%)$ by Sanger sequencing, consistent with its lower reproducibility for detecting minority variants. Additionally, $84 \%$ of the samples with discordant Sanger sequencing results gave concordant NGS results. Both NGS methods predicted maraviroc responses equally well.

CONCLUSIONS: Independent replication demonstrates high inter-laboratory concordance in next-generation sequencing, surpassing that of conventional sequencing. Estimates of minority species prevalence in clinical specimens appear to be highly reproducible and robust, despite differences in sequencing platforms, chemistry, and data processing methods.
0093

FOLLOW-UP INVESTIGATION OF A CLUSTER OF TREATMENT-NAÏVE HIV-INFECTED PATIENTS WITH MULTI-DRUG RESISTANCE IN SUDBURY, ONTARIO

Sullivan, Ashleigh ${ }^{1}$; Sutcliffe, Penny ${ }^{2}$; Sandre, Roger ${ }^{2}$;

Harrigan, P Richard ${ }^{3}$; Archibald, Chris ${ }^{4}$; Halverson, Jessica ${ }^{4}$;

Rank, Claudia ${ }^{4}$; Brooks, James ${ }^{4}$; Wu, Keyi ${ }^{5}$;

Dowdall-Smith, Shannon'; ' Latendre-Paquette, Judy²; Remis, Robert S

${ }^{1}$ Toronto; ${ }^{2}$ Sudbury, ON; ${ }^{3}$ Vancouver, BC; ${ }^{4}$ Ottawa; ${ }^{5}$ Etobicoke, ON

INTRODUCTION: After identifying a cluster of HIV-infected patients with multi-drug resistance (MDR) in Sudbury, Ontario, we examined access to HIV care and further characterized patterns of HIV resistance. METHODS: We analyzed HIV cases diagnosed among Sudbury residents from January 1, 2005 to June 30, 2012 using data from the Sudbury District Health Unit, HAVEN Program (which provides care for HIVinfected patients in Sudbury), Ontario HIV Laboratory, and Laboratory Enhancement Program. Clinical drug resistance testing results for treatment-naïve first genotypes were obtained from the BC Centre for Excellence in HIV/AIDS and, if unavailable, from the Strain and Drug Resistance Program, Public Health Agency of Canada. We examined the proportion who attended the HAVEN Program at least once and the characteristics those with MDR HIV infection (NRTI and NNRTI).

RESULTS: 85 patients were included in the analysis. The majority $(64.7 \%)$ were male; the median age at diagnosis was 35.9 years (IQR 28.7, 43.7). Most $(63.1 \%)$ patients were IDU followed by MSM (16.7\%). Aboriginal patients comprised $27.4 \%$; the cumulative HIV diagnosis rate among Aboriginal persons was 3.74 times higher than the rest of the population. $89.4 \%$ of patients were seen at the HAVEN clinic; this proportion did not vary by sex, age, exposure category, or race/ethnicity. Overall, $51.5 \%$ of patients were infected with transmitted MDR. MDR was dramatically higher among IDU $(72.1 \%$ vs. $15.0 \%$ in other exposure categories). Resistance was also higher among women (69.0\%) and Aboriginal persons (65.0\%); however, IDU was more common in these populations and entirely accounted for the higher resistance observed. DISCUSSION: HIV infection among IDU in Sudbury represents an important public health problem; Aboriginal persons and women are overrepresented. Access to care was, in general, excellent and did not vary significantly by any of the covariates examined. The high level of MDR HIV infections, especially among IDU, is of great potential concern.

\section{O094}

MENS' BELIEFS AND INTENTS TOWARDS TREATMENT AS PREVENTION: MALE CALL CANADA'S FINDINGS WITH REGARDS PRE- AND POST-EXPOSURE PROPHYLAXIS

Allman, Dan ${ }^{1}$; Myers, Ted ${ }^{1}$; Alexander, Stephen ${ }^{2}$; Remis, Robert $\mathbf{S}^{1}$; Blais, Martin ${ }^{3}$; Maxwell, John'; Calzavara, Liviana ${ }^{1}$; Marchand, Rick ${ }^{4}$; Adam, Barry $\mathbf{D}^{5}$; Marlatt, Faria ${ }^{1}$;

Docter, Hamal' ${ }^{1}$, Vernich, Lee $^{1}$

${ }^{1}$ Toronto, ON; ${ }^{2}$ Fredericton, NB; ${ }^{3}$ Montréal, QC; ${ }^{4}$ Vancouver, BC; 5 Windsor, ON

OBJECTIVES: To examine the beliefs and intents of gay men and other men who have sex with men in Canada towards the effectiveness and future use of Pre- and Post-Exposure Prophylaxis (PrEP and PEP).

METHODS: Male Call Canada (MCC), a bilingual, nation-wide, tollfree telephone survey carried out in 2011-12, gathered self-report information from men 16 years-of-age or older on knowledge and attitudes related to HIV and health and wellbeing. For this analysis, bivariate statistics were computed using SAS.

RESULTS: 1,235 men completed the telephone interview. Respondents were asked how effective they thought PrEP was in reducing the risk of HIV. Of the 932 men who responded to the question, $28.5 \% \quad(n=266)$ thought PrEP was effective (20.4\% did not know) and $33.9 \% \quad(n=316)$ thought PEP was effective (18.5\% did not know). When asked how effective a pill would have to be in preventing HIV infection for the respondent to use it prior to unprotected insertive anal intercourse, $67.6 \%$ $(n=329)$ indicated "always effective". Asked the same question with respect to unprotected receptive anal intercourse, the proportion 
indicating "always effective" was 78.9\% ( $\mathrm{n}=359)$. Bivariate analyses revealed only an HIV positive status to be associated with attitudes towards the effectiveness of PrEP ( $\mathrm{p}<0.008)$. Men from BC and Quebec relative to other regions $(\mathrm{p}<0.008)$; being single or partnered with a man rather than a woman $(\mathrm{p}<0.007)$; having ever received money in exchange for sex $(p<0.05)$ and an HIV positive status $(p<0001$ were all significantly and positively associated with beliefs in PEP's effectiveness.

CONCLUSIONS: The geographic breadth of MCC allowed the inclusion of men in rural and remote areas who may have had limited opportunity to participate in HIV prevention research previously. Our findings reflect attitudes which, while recognizing the benefits of pre-and postexposure prophylaxis, were arguably realistic about the real-world application. These findings can inform future research and programs with regard to the beliefs and intentions of men towards the use of PrEP and $\mathrm{PEP}$ as components of a treatment as prevention approach.

\section{O095}

CANADIAN PHYSICIANS' PERCEPTIONS OF HIV PREEXPOSURE PROPHYLAXIS: NOT READY FOR PRIME TIME?

Sharma, Malika; Senn, Heather; Wilton, James; Fowler, Shawn;

Tan, Darrell

Toronto, ON

OBJECTIVE: Daily tenofovir/emtricitabine (Truvada®) for HIV preexposure prophylaxis (PrEP) has recently been approved in the United States. However, concerns persist around effectiveness, potential for adverse events, and risk-taking behaviour. Little is known about Canadian physician knowledge, understanding of, and willingness to prescribe PrEP.

METHODS: We disseminated an online survey about readiness for PrEP implementation to Canadian family physicians, infectious disease practitioners, internists, and public health practitioners between OctoberDecember 2012. The primary objective was to determine physician willingness to prescribe PrEP. Chi-square tests and univariate logistic regression models were used to identify correlates of physicians' willingness to prescribe. Preliminary data are presented here.

RESULTS: 56 surveys were analyzed from across Canada. $57.1 \%$ of respondents identified as HIV specialists, $75 \%$ worked in academia, and $50.9 \%$ of participants felt "very familiar" with PrEP. 52.7\% felt PrEP should be approved by Health Canada. $42.9 \%$ of respondents were willing to prescribe PrEP, while $51.8 \%$ were unsure and $5.4 \%$ were unwilling. Being a self-described HIV expert $(\mathrm{OR}=3.9,95 \% \mathrm{CI}=1.2-12.3)$, high baseline familiarity with $\operatorname{PrEP}(\mathrm{OR}=6.6,95 \% \mathrm{CI}=2.0-21.7)$ and having been asked by patients about $\operatorname{PrEP}(\mathrm{OR}=4.6,95 \% \mathrm{CI}=1.3-15.9)$ were associated with willingness to prescribe PrEP on univariate analysis. $28.6 \%$ had been asked about PrEP in the past year, and $12.7 \%$ had prescribed it. Unclear real-world effectiveness, development of resistance, and adverse events were considered primary issues in the implementation of PrEP. Principal barriers to implementation included: unfamiliarity with PrEP, drug costs, and insufficient data to prove effectiveness $(70.4 \%$, $89.2 \%, 76.0 \%$ respectively). $74.5 \%$ of participants did not feel that information about PrEP had been adequately disseminated among physicians. CONCLUSIONS: Canadian physicians demonstrate moderate support for PrEP and several have already prescribed it. Further research on realworld effectiveness, continuing medical education, and clinical support is needed to prepare physicians for this HIV prevention strategy.

\section{O096}

A RANDOMIZED TRIAL OF TIME-LIMITED ANTIRETROVIRAL THERAPY (ART) IN ACUTE AND EARLY HIV INFECTION (CTN214)

Tossonian, Harout ${ }^{1}$; Conway, Brian ${ }^{1}$; Apuzzo, Linda ${ }^{2}$; Singer, Joel ${ }^{1}$; Fraser, Chris ${ }^{3}$; Loutfy, Mona ${ }^{4}$; Rachlis, Anita ${ }^{4}$; Kasper, Ken ${ }^{5}$;

El-Helou, Philippe ${ }^{6}$; Margolick, Joe ${ }^{2}$

${ }^{1}$ Vancouver, BC; ${ }^{3}$ Baltimore, Maryland USA; ${ }^{3}$ Victoria, BC;

${ }^{4}$ Toronto, ON; ${ }^{5}$ Winnipeg, $\mathrm{MB}$; ${ }^{6}$ Hamilton, ON

BACKGROUND: Some randomized clinical trials (RCT) suggest that limited course of ART during acute or early HIV infection confers a temporary virologic and/or clinical benefit. Thus, we conducted an RCT of 12 months of ART vs. deferred treatment in people with acute or early HIV infection.

METHODS: Subjects with HIV infection for $<1$ yr were randomized to receive immediate ( 12 months) or deferred ART. Subjects were followed monthly for 1 year, then quarterly, and permanent ART was started per contemporary treatment guidelines, most commonly CD4 cells $<350 / \mathrm{ul}$. The study endpoint was the total amount of ART-free time before initiation of permanent ART; the arms were compared using survival analysis methods.

RESULTS: 113 patients were enrolled: 57 to immediate ART, 56 to deferred ART. The arms were similar in proportion of acute infection, male sex, race, transmission route, age, baseline CD4 cell count and plasma HIV RNA. Of those randomized to immediate ART and remaining on-study after 12 months, 36/37 achieved full viral suppression. Loss to follow-up by month 12 was 6 subjects in each arm. After excluding those in the immediate treatment arm who did not complete 12 months of ART ( $n=5)$ or chose not to stop ART at 12 months $(n=9)$, the median ART-free time from study entry to initiation of permanent ART was 18 months for both arms. In multivariate analysis, this time was not significantly related to treatment group, sex, duration of infection, or age, but was related to baseline viral load $(\mathrm{p}<.001)$ and injection drug use $(p=0.049)$. Of those on-study at month $12,9 / 37(24 \%)$ in the immediate arm vs $7 / 50(14 \%)$ in the deferred arm remained off ART with HIV RNA $<104 \mathrm{c} / \mathrm{ml}$ at month $24(\mathrm{p}=0.27)$.

CONCLUSIONS: In this RCT, 12 months of ART during acute or early HIV infection did not prolong total ART-free time until permanent initiation of ART according to the standard of care.

Track C: Epidemiology and Public Health

Sciences: Testing, Linkage to Care and

Treatment

Volet C : Épidémiologie et sciences de la santé publique : Tests, lien avec les soins et traitement

\section{O097}

MEASURING THE IMPACT OF ENHANCED TESTING AND TREATMENT ON THE HIV EPIDEMIC IN VANCOUVER, CANADA

Sandhu, Jat; Chu, Tim; Demlow, Ellen; MacDonald, Lauren; Heath, Kate; Yip, Benita; Chau, William; Colley, Guillaume; Chinski, Logan; Thumath, Meaghan; Nathoo, Afshan; Barrios, Rolando; Gustafson, Réka

Vancouver, BC

BACKGROUND: A three year government-funded pilot project (STOP HIV/AIDS) was launched in March 2010 with the goal to reduce new HIV infections in British Columbia through expanding HIV-related services. A broad range of initiatives with the aim of improving early detection of HIV and timely engagement in care were conducted in Vancouver. This paper reports on the monitoring of population-level indicators to evaluate progress towards established goals.

METHODS: Public health surveillance, HIV laboratory testing and near real-time clinical monitoring were used to determine appropriate indicators representing the entire patient journey. Regular sanctioned linkages of clinical and public health datasets allowed for assessment of these indicators to inform progress in comparison to historical trends. In addition, analyses of pertinent sociodemographic, epidemiological and clinical factors provided a more complete picture of the impact of this project.

RESULTS: A combination of targeted testing strategies and the introduction of a routine testing strategy accounted for increases in HIV lab testing volumes by $31 \%$ overall since launch. In the past 12 months testing has increased further to $53 \%$, with significant uptake in lower risk 
neighbourhoods. Greater than $80 \%$ of new HIV diagnoses are in men, with the highest percent positivity $(0.82 \%)$ among men aged $30-49$. Enhanced public health follow-up of partners identified more new HIV positives ( 8 per 100 contacts tested) than population-based testing across Vancouver. Patients actively engaged in treatment increased by $39 \%$, with mean community viral load levels declining significantly.

CONCLUSIONS: These findings provide early insight into the impact of effective screening and early detection of HIV together with timely access and engagement in treatment can reduce community viral load with consequent reduction in HIV transmission risk. While encouraging further innovative strategies to enhance testing, increasing effectiveness of public health follow-up, and reducing the significant socioeconomic barriers to adherence in treatment are required.

\section{O098 \\ MISSED OPPORTUNITIES FOR HIV DIAGNOSES IN VANCOUVER}

Demlow, Ellen; MacDonald, Lauren; Nathoo, Afshan; Chu, Tim; Sandhu, Jat; Gustafson, Reka

Vancouver, BC

BACKGROUND: Vancouver has the highest prevalence and incidence of HIV in British Columbia with sixty-five percent of newly diagnosed individuals having CD4 counts less than 500 . Understanding the extent of missed opportunities for diagnosis is fundamental to developing effective testing strategies. This paper reports on the first known assessment of this kind in Canada.

METHODS: A retrospective, electronic chart review of new HIV cases in Vancouver from 2009-2011 was conducted to estimate the proportion of HIV cases with missed opportunities for earlier diagnosis. Data from hospitals, emergency departments, outpatient clinics and hospital laboratories prior to diagnosis were reviewed as potential encounters of missed opportunities. Visits to family practice physicians or nurse practitioners are not included, and data from outpatient laboratories are pending. Hence, the review is likely to underestimate the total number of missed opportunities for diagnosis.

RESULTS: Total number of missed opportunities for 506 cases is 777 , with a mean of 1.53 missed opportunities per case and a maximum of 38 . $168(33.1 \%)$ patients had at least one missed opportunity, with a mean of 4.7 encounters. Of 177 patients diagnosed with a CD4 count of $<500$ cells $/ \mathrm{ml}, 55 \%$ had at least one missed opportunity; of those with a CD4 count of $<350$ cells $/ \mathrm{ml}$ at diagnosis, $60 \%$ did.

CONCLUSION: Multiple missed opportunities for earlier diagnosis in patients diagnosed late provides further rationale that routine testing in general medical settings has the potential to significantly contribute to earlier diagnosis of HIV.

Percent \& proportion of new HIV diagnoses with $\geq 1$ Outpatient, Lab, ER or Inpatient encounter, by CD4 count

\begin{tabular}{cc} 
CD4 Count $^{*}$ & $\geq 1$ Prior Encounter \\
\hline$<200$ & $58 \%(30 / 52)$ \\
$<350$ & $60 \%(64 / 107)$ \\
$<500$ & $55 \%(97 / 177)$ \\
\hline
\end{tabular}

* Only 57.5\% (291/506) of new HIV Dx had a CD4 count on record at time of Dx

\section{O099}

\section{BC'S PROVINCIAL POINT OF CARE "RAPID" HIV TESTING PROGRAM: A QUALITATIVE EVALUATION}

Fielden, Sarah; Pedersen, Heather; Ogilvie, Gina; Krajden, Mel; McAloney, Coleen; Gilbert, Mark

\section{Vancouver, BC}

OBJECTIVES: Point-of-care (POC) "rapid" HIV tests are gaining global popularity as screening tools that provide results within minutes. In April 2011, the BC Centre for Disease Control implemented a province-wide, centralized model for POC testing in healthcare settings throughout $\mathrm{BC}$. The objective of the evaluation is to capture program successes and challenges.

METHODS: We present preliminary evaluation findings from the pilot phase of the Provincial POC HIV Testing Program, guided by a comprehensive program logic model. Semi-structured key stakeholder interviews were conducted with managers and front line nurses in each of the five regional health authorities in the province. Key stakeholders $(n=20)$ were selected purposively. Interviews were transcribed verbatim and managed using NVivo software. Analysis was guided by Goffman's Frame approach using interpretive qualitative inquiry and thematic comparative coding techniques.

RESULTS: Participants viewed the centralized model of stewardship as facilitating the delivery of HIV testing to some of the most culturally and economically marginalized populations in the province. Consistent with health promotion models, emergent themes were classified into macro, meso, and micro domains of structural, organizational, and client-related factors. Successful elements included: program responsiveness; costeffectiveness for sites; and province-level standardized training and resources. Challenges included: stigma, limited reach into rural/remote communities, data collection, and inter-organizational variations in service delivery. Accessibility and acceptability of the new POC HIV testing technology featured prominently in the narratives of the participants as both program successes and challenges. Results illustrated ongoing policy-practice tensions and the oft-competing priorities of resource allocation, provider comfort, test quality, and client-centred care.

CONCLUSIONS: Results suggest that the success of the Provincial POC HIV Testing Program is facilitated by sociocultural factors including provider and patient attitudes, isolation, and organizational contexts. The influence these may have on client HIV testing access and subsequent follow-up care and treatment requires further consideration.

\section{0}

\section{EVALUATING LONGITUDINAL PATTERNS IN THE CONTINUUM OF HIV CARE IN BRITISH COLUMBIA: 1996-2009}

Nosyk, Bohdan; Montaner, Julio S; Colley, Guillaume; Chan, Keith; Heath, Kate V; Yip, Benita; Samji, Hasina; Gilbert, Mark; Barrios, Rolando; Gustafson, Reka; Lima, Viviane D; Hogg, Robert S

Vancouver, BC

BACKGROUND: The cascade of HIV care has become a focal point for implementation efforts to maximize the impact of HIV treatment at the individual and societal levels. A thorough understanding of the leakage within the cascade of care can inform the optimal allocation of resources. Our objective was to characterize the evolution of engagement within the cascade of care among persons living with HIV in the province of British Columbia (BC), Canada longitudinally, throughout the HAART era.

METHODS: Provincial HIV prevalence estimates from the Public Health Agency of Canada supplemented linked population-level data from the BC Centre for Disease Control, the BC Centre for Excellence in HIV/AIDS HIV registry and the BC Medical Services Plan databases to define, longitudinally, the numbers of individuals in each of the eight stages of the cascade of HIV care in BC from 1996-2009. Supplemental analyses were executed to define aggregate levels of HIV-1-RNA and identify determinants of time-to-retention in HIV care and the probability of accessing HAART.

RESULTS: A total of 12,349 individuals were identified as HIV-positive in $\mathrm{BC}$ during the study period. The percentage of unidentified HIVpositive individuals decreased from 50\% (range: 42\%-63\%) in 1996 to $14 \%(0 \%-28 \%)$ in 2009, while the percentage of HIV-positive individuals with virologic suppression reached 35\% (30\%-40\%) in 2009 . Supplemental analyses identified steadily improving time-to retention in HIV care controlling for covariates, increased access to HAART and steadily higher aggregate levels of virologic suppression among those in HIV care.

CONCLUSIONS: Careful mapping of the cascade of care is critical to understanding what further efforts need to be deployed to maximize the impact of currently available interventions, and as a result, to inform efforts to contain the spread of HIV/AIDS. A high quality HIV surveillance system actively linked to relevant administrative health records is an essential pre-requisite in this regard. 
0101

LATE INITIATION OF HIV TREATMENT IN CANADA: A NEED FOR EXPANSION OF HIV TESTING AND LINKAGE TO CARE

Chan, Keith ${ }^{1}$; Cescon, Angela ${ }^{1}$; Loutfy, Mona R ${ }^{2}$; Burchell, Ann N2; Cooper, Curtis ${ }^{2}$; Hosein, Sean $\mathbf{R}^{2}$; Klein, Marina $\mathbf{B}^{3}$;

Machouf, Nima ${ }^{3}$; Raboud, Janet $\mathbf{M}^{2}$; Rachlis, Anita²; Rourke, Sean $\mathrm{B}^{2}$; Tsoukas, Christos ${ }^{3}$; Hogg, Robert $\mathrm{S}^{\mathbf{1}, 4}$; Montaner, Julio S ${ }^{1}$;

Canadian Observational Cohort, CANOC ${ }^{1}$

${ }^{1}$ Vancouver, BC; ${ }^{2}$ Toronto, ON; ${ }^{3}$ Montreal, QC; ${ }^{4}$ Burnaby, BC

BACKGROUND: Combination antiretroviral therapy (ART) significantly decreases morbidity and mortality, as well as HIV transmission. Using a multi-site Canadian cohort we aim to (1) characterize the timing of ART initiation over time, and (2) determine factors associated with late initiation of treatment.

METHODS: Participants from the Canadian Observational Cohort (CANOC), a cohort of HIV-positive individuals 18+ years initiating ART after 2000 in British Columbia, Ontario, and Quebec, were included. Late initiation was defined as baseline CD4 count $<200$ cells/ $\mathrm{mm} 3$ or a baseline AIDS-defining illness. Temporal trends were assessed using the Cochran-Armitage test and negative binomial regression, and factors independently associated with late initiation were determined using logistic regression.

RESULTS: 7,638 participants (19\% female) were included, of median age 40 years (IQR 34-46). The median baseline CD4 count increased from 190 cells/mm3 (IQR 80-330) in 2000 to 300 cells/mm3 (IQR 200400 ) in 2010 (see table) $(\mathrm{p}<0.001)$. In 2010, 27\% of patients started with CD $4<200$ cells $/ \mathrm{mm} 3$, and $63 \%$ with CD $4<350$ cells $/ \mathrm{mm} 3$. In multivariable analysis, late initiation was more likely among: British Columbians $(\mathrm{AHR}=1.50,95 \% \mathrm{CI}=1.30-1.74)$ and Ontarians $(\mathrm{AHR}=1.30,95 \%$ $\mathrm{CI}=1.13-1.49)$; persons with injection drug use history $(\mathrm{AHR}=1.34,95 \%$ $\mathrm{CI}=1.18-1.53$ ); older individuals ( $\mathrm{AHR}=1.14$ per decade, $95 \% \mathrm{CI}=1.09$. 1.20); and individuals starting ART in earlier calendar years (AHR $=1.16$ per year, $95 \% \mathrm{CI}=1.14-1.17$ ). No differences were observed by sex.

\begin{tabular}{ccc} 
Year of ART initiation & Median baseline CD4 count (IQR) & $\%$ with baseline CD4 <200 cells $/ \mathrm{mm}^{3}$ \\
\hline 2000 & $190(80-330)$ & $55 \%$ \\
2001 & $160(58-270)$ & $62 \%$ \\
2002 & $170(80-250)$ & $64 \%$ \\
2003 & $160(70-240)$ & $67 \%$ \\
2004 & $180(90-250)$ & $59 \%$ \\
2005 & $180(100-260)$ & $62 \%$ \\
2006 & $200(120-260)$ & $55 \%$ \\
2007 & $210(130-280)$ & $49 \%$ \\
2008 & $230(148-300)$ & $41 \%$ \\
2009 & $270(167-350)$ & $33 \%$ \\
2010 & $300(200-400)$ & $27 \%$ \\
\hline
\end{tabular}

CONCLUSIONS: Although improving, CD4 count at first initiation of ART in CANOC remains below treatment guidelines. There is a need to expand HIV testing and subsequent linkage to care, to decrease morbidity and mortality, as well as HIV transmission in Canada.

\section{2}

\section{COMPLIANCE TO TREATMENT GUIDELINES AT THE} PROGRAMMATIC LEVEL IN CANADA: EXTENSION OF A COMPOSITE ASSESSMENT METRIC FOR HIV THERAPY

Cescon, Angela ${ }^{1}$; Min, Jeong ${ }^{1}$; Colley, Guillaume ${ }^{1}$; Hosein, Sean $\mathbf{R}^{2}$; Machouf, Nima ${ }^{3}$; Samji, Hasina ${ }^{1}$; Burchell, Ann N2;

Cooper, Curtis ${ }^{4}$; Klein, Marina $B^{3}$; Loutfy, Mona $\mathbf{R}^{2}$;

Montaner, Julio $\mathrm{S}^{1}$; Raboud, Janet $\mathbf{M}^{2}$; Rachlis, Anita ${ }^{2}$;

Tsoukas, Christos ${ }^{3}$; Hogg, Robert $\mathbf{S}^{1,5}$; Lima, Viviane $\mathrm{D}^{1}$; Canadian Observational Cohort, CANOC ${ }^{1}$

${ }^{1}$ Vancouver, BC; ${ }^{2}$ Toronto, ON; ${ }^{3}$ Montreal, QC; ${ }^{4}$ Ottawa, ON;

${ }^{5}$ Burnaby, BC

BACKGROUND: Guidelines for treating and monitoring HIV-positive individuals have greatly evolved since 1996; however, it remains unclear which metric(s) should be used to monitor and evaluate compliance to guidelines at the programmatic level. A recently developed and validated index, the Programmatic Compliance Score (PCS), includes six indicators of "quality of initial HIV care" based on IAS guidelines from 20002010. We aim to document PCS scores in the Canadian Observational Cohort (CANOC); to explore the PCS' association with all-cause mortality; and, to identify PCS correlates.

METHODS: Participants in CANOC, a cohort of HIV-positive persons $18+$ years who initiated combination antiretroviral therapy (ART) after 2000 in British Columbia, Ontario, and Quebec, were included. PCS indicators include: (1) having $<3$ CD4 count tests in the first year of ART; (2) having <3 viral loads in the first year of ART; (3) not having resistance testing prior to starting ART; (4) starting on a non-recommended ART regimen; (5) starting therapy with CD4 $<200$ cells/mm3; and (6) not achieving viral suppression within 6 months. Summed indicators determine a score from 0-6; higher scores indicate poorer performance. Logistic regression assessed the association between PCS and mortality, and ordinal logistic regression explored PCS correlates.

RESULTS: Of 6348 participants ( $18 \%$ female), the median score was 2 (IQR: $1-3$ ), $19 \%$ scored 0 , and $12 \%$ scored $\geq 4$. In multivariable analysis, compared to score $=0$, poorer PCS was significantly associated with mortality $[$ score $=1, \mathrm{AOR}=1.33(95 \% \mathrm{CI}=0.85-2.07)$; score $=2, \mathrm{AOR}=1.77$ (95\% CI=1.14-2.73); score=3, AOR=2.18 (95\% CI=1.38-3.45); score $\geq 4, \mathrm{AOR}=2.23(95 \% \mathrm{CI}=1.40-3.55)]$, after adjusting for age, sex, province, Aboriginal ancestry, baseline viral load, hepatitis C (HCV) coinfection, ART start year, and follow-up time. Based on estimated model-based probabilities of each PCS score, women, HCV-positive persons, and individuals starting ART in earlier calendar years (2000-2003) were more likely to have poorer scores.

CONCLUSIONS: Our findings lend further validation to the IAS treatment guidelines. However, disparities were identified, and further efforts are thus needed to maximize the benefits of modern ART and ensure care is equitably accessible.

\section{3}

\section{A SIGNIFICANT REDUCTION IN THE PREVALENCE OF HIV-1 DRUG RESISTANCE IN QUEBEC FROM 2001 TO 2011 IS ASSOCIATED WITH A DIMINUTION IN THE MONITORED VIRAL LOAD}

Charest, Hugues'; Cantin, Régis'; Doualla-Bell, Florence'; Lemieux, Linda'; Murphy, Donald G'; Brenner, Bluma ${ }^{2}$; Hardy, Isabelle²; Roger, Michel'2; Moisi, Daniela ${ }^{2}$; Lo, Ernest ${ }^{1}$; Baril, Jean-Guy²; Wainberg, Mark' ${ }^{2}$ Tremblay, Cécile ${ }^{1,2}$ ${ }^{1}$ Sainte-Anne-de-Bellevue; ${ }^{2}$ Montréal, QC

BACKGROUND: As part of our provincial surveillance programs, we evaluated temporal changes in the prevalence of drug resistance mutations and concomitant monitored viral load (VL) in treatment-naïve and treatment-experienced individuals.

METHODS: HIV-1 pol gene sequences ( $\mathrm{n}=9198$ ) obtained for each individual tested by the Quebec HIV genotyping program between 2001 and 2011 were analyzed for drug resistance mutations using the Stanford University algorithms. Population VL data were obtained from the HIV VL provincial program and the Montreal HIV Cohort. Data on antiretroviral prescriptions were obtained from IMS-Brogan Inc. Changes over time in the resistance prevalence were analysed using a chi-square test for trends. A multivariate logistic regression analysis was used to compare trend in the $\mathrm{M} 184 \mathrm{~V} / \mathrm{I}$ mutation prevalence and antiretroviral prescriptions.

RESULTS: The proportion of individuals with undetectable viral load (HIV-1 RNA < 50 copies $/ \mathrm{mL}$ ) increased linearly since 2001 (from $42.1 \%$ individuals in 2001 to $75.9 \%$ in 2011 ; odds ratio $(\mathrm{OR})=1.17 ; \mathrm{P}<0.001$ ). Treatment-experienced patients exhibited a significant decrease in the prevalence of any resistance mutations associated to nucleoside/nucleotide reverse transcriptase inhibitors (from 78\% in 2001-2003 to 37\% in 2011; $\mathrm{P}<0.001$ ), protease inhibitors (from $54 \%$ in $2001-2003$ to $16 \%$ in 2011; $\mathrm{P}<0.001$ ) and nonnucleoside reverse transcriptase inhibitors (from $44 \%$ in $2001-2003$ to $31 \%$ in $2011 ; \mathrm{P}<0.001$ ) over the years. In treatment-naïve patients (newly diagnosed and untreated individuals) the 
prevalence of transmitted drug resistance, which remained stable at $13 \%$ up to 2008 , showed a net $27 \%$ decrease since 2009 . The increased use of the co-formulated emtricitabine and tenofovir was associated to a $50 \%$ decrease $(61 \%$ to $31 \%$ ) in the prevalence of M184V/I mutation ( $r=-0.99$; $\mathrm{P}=0.0004$ )

CONCLUSIONS: Optimization of ARV regimens has significantly reduced circulating drug resistance. This reduction is reflected by a net decrease in the monitored VL.

\section{4}

WOMEN'S HEALTH CARE UTILIZATION AMONG HARDER-TO-REACH HIV-INFECTED WOMEN EVER ON ANTIRETROVIRAL THERAPY IN BRITISH COLUMBIA

Wang, Xuetao'; Salters, Kate A $^{2}$; Zhang, Wendy ${ }^{2}$;

McCandless, Lawrence ${ }^{1}$; Money, Deborah'2; Pick, Neora ${ }^{2}$;

Montaner, Julio S; Hogg, Robert $\mathrm{S}^{2}$; Kaida, Angela ${ }^{1}$

${ }^{1}$ Burnaby; ${ }^{2}$ Vancouver, BC

BACKGROUND: The growing feminization of the HIV epidemic means that women-specific health issues need to be addressed and delivered alongside HIV care. This study investigates the uptake of women's health care (WHC) among a sample of harder-to-reach and treatment experienced HIV-infected women in British Columbia (BC) and the factors associated with utilization.

METHODS: The Longitudinal Investigation into Supportive and Ancillary health services (LISA) study is a prospective study of harder-toreach people living with HIV who are over the age of 19 and have accessed antiretroviral therapy in BC. Participants completed interviewer-administered surveys, which included information on sociodemographic and service utilization factors. Clinical variables, including CD4 cell count and viral load, were obtained through linkages with the Drug Treatment Program database at the BC Centre for Excellence in HIV/AIDS. The outcome variable of interest was self-reported regular use of WHC, which included gynecological, sexual, and reproductive health care and/or counseling. Covariates of interest included socio-demographic, psychosocial, behavioural, individual health status, structural factors, and HIV clinical variables. Logistic regression was used to generate adjusted estimates of associations between use of WHC and covariates of interest.

RESULTS: Of the 231 women, 179 (77\%) reported regularly utilizing WHC. Median age was 41 years (interquartile range (IQR): 34-46) and $114(49 \%)$ reported being of Aboriginal ancestry. WHC utilization varied significantly by region of residence with women living in Vancouver Island Health Authority reporting lowest utilization of WHC (Adjusted Odds Ratio $(A O R)=0.12$, 95\%CI: 0.04-0.37). Additionally, lower use of WHC was associated with an annual income $<\$ 15,000$ (AOR $=0.14$, 95\% CI: 0.04-0.54), current use of illicit drugs $(\mathrm{AOR}=0.42,95 \% \mathrm{CI}$ : 0.19-0.92) and lower provider trust (AOR =0.97, 95\% CI: 0.95-0.99). CONCLUSION: A health service gap for WHC exists along geographical and social axes for harder-to-reach HIV-infected women. The integration of WHC with HIV-specific care, which acknowledges women's social and structural barriers to care, may support a more comprehensive and holistic approach to health for women living with HIV.

\section{Track D: Social Sciences: Harm Reduction}

Volet D : Sciences sociales: Réduction des méfaits

\section{5 \\ HEALTHIER CELLS: LITIGATING FOR PRISON-BASED NEEDLE AND SYRINGE PROGRAMS}

Chu, Sandra K; Elliott, Richard; Symington, Alison;

Kazatchkine, Cecile

Toronto, ON

OBJECTIVES: Almost 20 years after an expert committee acknowl-

edged the need for prison-based needle and syringe programs (PNSPs) and despite mounting evidence in support of such programs and numerous requests from community organizations, sterile injection equipment is not available in Canada's prisons. Yet rates of HIV and hepatitis $\mathrm{C}$ virus $(\mathrm{HCV})$ are escalating among prisoners, an epidemic that is principally fuelled by the sharing of drug injection equipment. Not only is this a public health concern, but a violation of prisoners' constitutional rights. METHODS: Over the years, numerous prisoners' rights, human rights and HIV organizations have advocated for PNSPs, with little success, before parliamentary committees, with public health and correctional officials, and through public education and media campaigns.

RESULTS: The Canadian government has repeatedly denounced PNSPs and clung to a "zero tolerance policy" on drugs in prison. But significant investments in drug interdiction initiatives have yielded little success and rates of drug use remain steady behind bars. In September 2012, a constitutional challenge was initiated by a former prisoner and four HIV organizations. The lawsuit is based on the Charter of Rights and Freedoms, which protects prisoners' rights to life, liberty and security of the person and to equal treatment before and under the law. To raise public awareness of the lawsuit and the rationale for it, a complementary website was launched featuring a series of video clips of prisoners, former prisoners and their loved ones, describing the pressing need for PNSPs. CONCLUSION: Various forms of advocacy undertaken before the launch of the constitutional challenge were instrumental in building public support for PNSPs and helped to identify an applicant who was infected with HCV while incarcerated. Ultimately, the applicants seek an order directing Canada's correctional service to ensure the implementation of PNSPs in federal correctional institutions, in accordance with professionally accepted standards.

\section{6}

\section{LAW AND POLITICS OF SUPERVISED INJECTION FACILITY SCALE-UP IN CANADA}

Hyshka, Elaine; Bubela, Tania; Wild, TC

Edmonton, AB

BACKGROUND: Supervised injection facilities (SIFs) are increasingly recognized as important evidence-based interventions for mitigating risk of HIV/AIDS, overdose and other harms associated with injection drug use. However, Canada has been slow to adopt this public health innovation, with the exception of Vancouver, which established the country's only SIF - Insite - in 2003. Since then, the facility has faced significant political and legal opposition, which culminated in a 2011 Supreme Court of Canada ruling ordering the federal Minister of Health to allow Insite's continued operation. Supporters and opponents alike have speculated that this ruling paves the way for new facilities in other parts of Canada, and several Canadian cities are undertaking SIF preparations. However, others have suggested a more cautious interpretation of the ruling.

METHODS: This policy analysis examines relevant jurisprudence, statutes, academic research, gray literature and media texts to describe the current legal and political context of SIFs in Canada, assess the likelihood that new SIFs will be established, and outline legislative reforms that would facilitate future SIF expansion.

FINDINGS: The Supreme Court's decision does not pave the way for new SIFs in Canada, but it does not pose an insurmountable hurdle either. Health Canada's current SIF regulatory process also creates barriers for new applicants. Whether new SIFs will be established depends mainly on how the federal Minister of Health chooses to interpret the Supreme Court's decision and whether proposed SIFs can meet requisite application criteria. Overall, additional legal action may be required before other Canadian jurisdictions are able to establish SIFs. Legislative reform would provide a more stable legal and policy basis for SIFs in Canada.

CONCLUSION: SIF proponents' continued political advocacy efforts are necessary to ensure scale-up of this evidence-based intervention for reducing injection drug use-related morbidity and mortality in Canada. 
0107

IMPACT OF COMPREHENSIVE HARM REDUCTION SERVICES ON ACCESS TO AND ENGAGEMENT WITH CARE AT A RESIDENTIAL PALLIATIVE AND SUPPORTIVE CARE PROGRAM FOR PEOPLE LIVING WITH HIV/AIDS: A QUALITATIVE STUDY

Dilley, Laura' $^{1}$; McNeil, Ryan²; Guirguis-Younger, Manal ${ }^{3}$;

Hwang, Stephen $W^{4}$; Small, Will1,2

${ }^{1}$ Burnaby; ${ }^{2}$ Vancouver, BC; ${ }^{3}$ Ottawa; ${ }^{4}$ Toronto, ON

Improvements in the availability and effectiveness of highly active antiretroviral therapy (HAART) have prolonged the lives of people living with HIV/AIDS (PLWHA). However, mortality rates have remained high among populations that encounter barriers to accessing and adhering to HAART, notably people who use drugs. This population subsequently has a high burden of illness and complex palliative and supportive care needs but is typically unable to access these services due to anti-drug policies in palliative and supportive care settings. In Vancouver, Canada, the Dr. Peter Centre (DPC), which operates a 24-bed residential palliative and supportive care program for PLWHA, has sought to improve access to care by adopting a comprehensive harm reduction strategy, including nurse-supervised injection services. We undertook this study to explore how the integration of comprehensive harm reduction services into this setting shape access to and engagement with palliative and supportive care. Qualitative interviews were conducted with 13 DPC residents between November 2010 and August 2011. Interviews were facilitated using an interview topic guide exploring how the DPC Residence's model of care: a) shaped health care access; b) influenced healthcare interactions; and, c) impacted drug use practices and overall health. Interview transcripts were analyzed thematically. Participant accounts highlight how the harm reduction policy altered the structuralenvironmental context of health care services and thus mediated access to palliative and supportive care services. Furthermore, this approach fostered an atmosphere in which drug use could be discussed without the risk of punitive action, and thus increased openness between residents and staff. Finally, participants reported that the environmental supports provided by the DPC Residence decreased drug-related risks and improved health outcomes, including HAART adherence and survival. This study highlights how adopting a comprehensive harm reduction services can serve to improve access and equity in palliative and supportive care for drug-using populations.

\section{O108}

BARRIERS AND FACILITATORS OF ADHERENCE TO HIGHLY ACTIVE ANTIRETROVIRAL THERAPY (HAART) AMONG INJECTION DRUG USERS WHO HAVE ACHIEVED LONG-TERM ADHERENCE

Small, Will'1,2; Milloy, M-J'; Montaner, Julio'1; Wood, Evan'1; Kerr, Thomas ${ }^{1}$

${ }^{1}$ Vancouver; ${ }^{2}$ Burnaby, BC

INTRODUCTION: While suboptimal adherence to highly active antiretroviral therapy (HAART) is common among injection drug users (IDU), many IDU attain high levels of adherence. However, the experiences of IDUs achieving treatment success have not been thoroughly investigated. We examined circumstances surrounding prolonged periods of high adherence among IDU, to identify barriers and facilitators of adherence.

METHODS: Using comprehensive clinical monitoring records linked to a community-recruited prospective cohort of HIV-positive IDU (ACCESS), we identified individuals who had achieved $>95 \%$ adherence over a period of 24 months. We conducted 35 in-depth qualitative interviews with 21 male and 14 female participants. Interviews explored participants' experiences receiving HAART, and a thematic analysis was conducted to identify barriers and facilitators of adherence.

RESULTS: Supportive dispensing arrangements, including MAT/DOT programs and daily pick-up or delivery of HIV medications, were common among participants and were perceived to ensure adherence and reduce missed doses of medications. Many participants were dispensed daily doses of methadone and HIV medications together, which addressed barriers to adherence stemming from opiate dependence. Stable housing was an important facilitator of adherence and few participants had been homeless, while housing models incorporating support staff were reported to enhance adherence. Illicit drug use was the primary barrier to adherence among participants, although some reported that they were able to adhere while actively using drugs. Binge drug use was detrimental to adherence and commonly resulted in brief episodes of non-adherence. CONCLUSION: This analysis indicates that supportive dispensing arrangements effectively promoted adherence among this population by addressing the negative impact of illicit drug use. While active drug use did not uniformly hinder adherence among IDU, binge drug use may pose the most significant barrier to adherence. These findings highlight the potential of co-dispensation of methadone and HAART, supportive dispensing arrangements and supportive housing, to address barriers to adherence among IDU.

\section{9}

"DOWN THERE" AND “OVER HERE": NEGOTIATING PLACE, RISK AND HARM REDUCTION IN CANADA'S LARGEST OPEN DRUG SCENE

McNeil, Ryan ${ }^{1}$; Small, Will1,2; Shaver, Laura ${ }^{1}$; Shannon, Kate'; Kerr, Thomas ${ }^{1}$

${ }^{1}$ Vancouver; ${ }^{2}$ Burnaby, BC

BACKGROUND: Vancouver's Downtown Eastside (DTES) is home to Canada's largest open drug scene and only supervised injection facility (SIF). Despite growing evidence of the critical role of place in shaping drug-related harms, including HIV transmission, little attention has been paid to the social geography of drug use within the DTES. This study explores the everyday geographies of people who use drugs (PWUD) within this neighbourhood, and how these shape drug-related risks and access to the SIF.

METHODS: Semi-structured qualitative interviews were conducted with 23 PWUD recruited through the Vancouver Area Network of Drug Users (VANDU) and were accompanied by a mapping exercise. Interview transcripts and maps were analyzed thematically, with an emphasis on how social, structural, and environmental factors shaped participants' everyday geographies and SIF access.

RESULTS: Almost all participants reported that they restricted their activities to particular areas within the DTES that they perceived to be "safe"; areas where they felt less vulnerable to violence, exploitation, and policing and had access to positive peer and social support. These areas were separated by clear boundaries, which participants crossed only when absolutely necessary (eg, when unable to buy drugs from a regular source). Those participants disproportionately impacted by violence within the local drug scene (eg, women and people with disabilities) restricted their movements the most. These restrictions constrained access to harm reduction services, with approximately half of participants reporting that they avoided the area where the SIF is located. As a result, these participants reported increased HIV risk behaviours and experienced diverse drug-related harms.

CONCLUSION: Findings highlight the need to expand the distribution of supervised injection services, and thus mitigate spatial barriers to this form of intervention. In Vancouver and elsewhere, greater attention to the existing sociospatial relations of PWUD is needed in the design and siting of harm reduction interventions.

\section{O110}

TRANSITIONS INTO AND OUT OF HOMELESSNESS AMONG STREET-INVOLVED YOUTH WHO USE ILLICIT DRUGS

Cheng, Tessa K ${ }^{1,2}$; DeBeck, Kora ${ }^{1,2}$; Feng, Cindy ${ }^{3}$; Mathias, Steve ${ }^{1}$; Montaner, Julio' ${ }^{1}$; Kerr, Thomas ${ }^{1}$; Wood, Evan ${ }^{1}$

${ }^{1}$ Vancouver; ${ }^{2}$ Burnaby, BC; ${ }^{3}$ Saskatoon, SK

BACKGROUND: Homelessness among youth who use illicit drugs is associated with a number of health-related harms including increased risk of initiating injection drug use. Although the positive influence of stable 
housing on health and HIV prevention is well established, factors that facilitate housing transitions among vulnerable youth are not well understood. To address this gap and inform policy interventions, we sought to identify factors associated with transitions into and out of homelessness among a cohort of drug using street-involved youth.

METHODS: This longitudinal study was based on data from a prospective cohort of street-involved youth aged 14-26 in Vancouver, Canada. Using data derived from semi-annual study follow-up visits, variables associated with transitions into and out of homelessness were identified using a generalized linear mixed effects model.

RESULTS: Among 685 street-involved youth who were followed for a median of 25 months, 213 (31\%) participants made at least one transition into homelessness and 386 (56\%) made at least one transition out of homelessness between September 2005 and May 2012. In multivariate analysis, factors that were positively associated with transitions into homelessness and negatively associated with transitions out of homelessness included: frequent alcohol use (adjusted odds ratios $[\mathrm{AOR}]=2.33$ and 0.76 respectively), daily heroin use ( $A O R s=1.74$ and 0.66 ), difficulty accessing addiction treatment $(\mathrm{AORs}=1.91$ and 0.53$)$ and incarceration $(\mathrm{AORs}=2.00$ and 0.68$),($ all $\mathrm{p}<0.05)$.

CONCLUSION: High rates of transitions in housing status were observed in this study and incarceration, alcohol use, frequent heroin use, and difficulty accessing addiction treatment were all associated with worsening housing conditions among our sample of street involved youth. These findings suggest that removing barriers to addiction treatment and reducing youth exposure to the criminal justice system may have the greatest impact on addressing youth homelessness and associated harms including HIV risk behaviour.

\section{O111}

\section{THE CEDAR PROJECT: EXPLORING THE ROLE OF} RESIDENTIAL TRANSIENCE IN HIV VULNERABILITY AMONG YOUNG ABORIGINAL PEOPLE WHO USE ILLICIT DRUGS

Jongbloed, Kate ${ }^{1}$; Thomas, Vicky²; Hongbin, Zhang1;

Oviedo-Joekes, Eugenia ${ }^{1}$; Schechter, Martin ${ }^{1}$; Spittal, Patricia $\mathbf{M}^{1}$ ${ }^{1}$ Vancouver; ${ }^{2}$ Prince George, BC

BACKGROUND: Ensuring young Aboriginal people who use illicit drugs have access to safe spaces, including places to live, work and inject, is a fundamental part of dismantling structural injustices that lead to elevated vulnerability to HIV infection. Residential transience as a structural factor in HIV vulnerability has often been overlooked.

METHODS: The Cedar Project is a prospective cohort of young Aboriginal people who use illicit drugs in Vancouver and Prince George, $\mathrm{BC}$. High residential transience was defined as having stayed in 6 or more places in the prior six months. Generalized linear mixed models using data collected semi-annually between 2005-2010 identified associations between transience and sex-and drug-related HIV vulnerabilities over time.

RESULTS: At baseline, nearly 20\% of participants reported high residential transience in the previous six months. Prince George participants were more likely than those in Vancouver to report being transient. Being transient in the prior six months was independently associated with participation in sex work (AOR: 3.52, 95\%CI: 2.06, 6.05) and recent sexual assault (AOR: 2.48, 95\%CI: 1.26, 4.86). Further, transience was independently associated with injection drug use (AOR: 4.54, 95\%CI: 2.71, 7.61), high frequency cocaine injection (AOR: 2.16, $95 \% \mathrm{CI}: 1.26,3.72$ ), and injecting in public (AOR: $2.87,95 \% \mathrm{CI}: 1.65$, 5.00). Stratifying by gender revealed that the association between transience and sexual vulnerability was significant among young Aboriginal women but not among men.

CONCLUSIONS: Research has focused on the material qualities of housing in relation to HIV vulnerability. However, our findings reveal that the spatial dimensions of housing are also closely linked to HIV vulnerability among young Aboriginal people. In particular, the links between gender, transience, and sexual vulnerability indicate that ensuring that young Aboriginal women who use drugs have safe places to work, live, and inject may be a crucial component of reducing vulnerability to HIV infection.
0112

MUNICIPAL LICENSING AND POLICING OF INDOOR SEX WORK ESTABLISHMENTS IN THE GREATER VANCOUVER AREA AND THEIR MICRO-HIV RISK ENVIRONMENTS: NARRATIVES OF SEX WORKERS, MANAGERS AND BUSINESS OWNERS

$\underline{\text { Anderson, Solanna }}^{1}$; Jia, Jessica ${ }^{1}$; Homer, Julia ${ }^{1}$; Chettiar, Jill ${ }^{1}$; Maher, Lisa ${ }^{2}$; Krusi, Andrea ${ }^{1}$; Shannon, Kate ${ }^{1}$ ${ }^{1}$ Vancouver, BC; ${ }^{2}$ Sydney, New South Wales, Australia

BACKGROUND: Despite the quasi-criminalized nature of sex work in Canada, licensed indoor sex work establishments have long existed in the form of massage parlours, beauty salons and body rub parlours. To date, there is little research on the various municipal licensing and policing practices (e.g. checks, raids) of indoor commercial sex venues. We compared licensing and policing practices of indoor sex work establishments across municipalities to investigate how macro-level licensing polices and policing practices shape the micro-level HIV risk environments among off-street sex workers. METHODS: Within a larger ethnographic and qualitative investigation of the physical, social and structural HIV risk environment of the sex industry, this study draws on 46 in-depth interviews conducted with sex workers, managers and business owners of licensed indoor sex work establishments and micro-brothels in 2011 in the Greater Vancouver Area. Interviews were conducted in English or Mandarin and audio-taped, transcribed verbatim, translated, coded and analysed for thematic content.

RESULTS: Analyses suggests that current municipal licensing often includes physical stipulations that increase the risk of theft and violence and make condom negotiation more difficult. Licensing regimes can also enable police to identify and raid businesses. Police raids lead to decreased income, increased stress and may displace sex workers to more dangerous work venues, such as micro-brothels. Finally, owners are often reluctant to provide workers with sexual health and HIV education and harm reduction supplies, out of fear these actions could implicate owners and lead to criminal charges, fines or a loss of their business license.

CONCLUSIONS: Within the context of anticipated socio-legal policy reforms on sex work legislation in Canada, this study suggests the critical need to consider how regulations and macro-municipal licensing can better support sex workers, business owners and managers in promoting HIV risk reduction and safety in the sex industry. 
BASIC SCIENCES SCIENCES FONDAMENTALES

\section{P001}

\section{REGIONS OF THE CD127 CYTOPLASMIC DOMAIN} NECESSARY FOR TAT-INDUCED INTERNALIZATION Cherid, Hafsa; MacPherson, Paul; Ghazawi, Feras Ottawa, ON

BACKGROUND: Impaired cell mediated immunity is the clinical hallmark of HIV infection yet the manner in which CD8 T-cells are disabled is not yet fully understood. IL-7 signalling is essential for normal CD8 T-cell development and function. Our lab has previously shown decreased expression of the IL-7 receptor a-chain (CD127) on circulating CD8 T-cells in HIV+ patients is mediated by the HIV Tat protein which results in poor CD8 T-cell function. Soluble Tat protein is secreted by infected CD4 T-cells and taken up by neighbouring uninfected CD8 T-cells through endocytosis. Once in the cytoplasm, Tat translocates to the inner leaflet of the cell membrane where it binds directly to the cytoplasmic tail of CD127 inducing receptor aggregation, internalization, and degradation by the proteasome.

OBJECTIVE: To demonstrate which region on the CD127 receptor is required for Tat binding.

METHODS: Using a pCMV6-CD127 plasmid, I will construct mutants removing or replacing each of the four identified regions: a membraneproximal basic region rich in lysine residues, an acidic region followed by a serine-rich region and a $\mathrm{C}$-terminal tail containing three tyrosine residues, and transfect these into Jurkat cells which do not express the endogenous CD127 gene. Stable Jurkat clones each expressing a mutated version of CD127 will then be incubated with soluble Tat protein and CD127 expression will be monitored by flow cytometry, and physical interaction of the CD127 mutants with Tat will be assessed by co-immunoprecipitation.

RESULTS: We have successfully generated mutants of the CD127 cytoplasmic tail which have been transfected and expressed into Jurkat cells. We also successfully produced recombinant HIV-1 Tat protein to be used in the subsequent analyses.

CONCLUSIONS: This work will solve an important part of the puzzle in understanding how Tat down regulates the IL-7 receptor. This work also has important therapeutic implications. If the interaction between Tat and CD127 can be disrupted and IL-7 signalling restored in CD8 T-cells, we may improve cell mediated immunity in HIV+ patients and potentially establish immunologic control of HIV replication.

\section{P002}

\section{IMMUNOSUPPRESSIVE ROLE OF PLASMA SOLUBLE CD40} LIGAND (SCD40L) IN HIV-INFECTED PATIENTS

Patel, Mital'; Lenabian, Mohammad-Ali'; ${ }^{1}$ Kema, Ido P2; Kanagaratham, Cynthia ${ }^{1}$; Radzioch, Danuta ${ }^{1}$; Thébault, Paméla ${ }^{1}$; Lapointe, Réjean ${ }^{1}$; Tremblay, Cécile ${ }^{1}$; Gilmore, Norbert ${ }^{1}$; Ancuta, Petronela ${ }^{1}$; Routy, Jean-Pierre ${ }^{1}$ ${ }^{1}$ Montreal, QC; ${ }^{2}$ Groningen, Netherlands

BACKGROUND: CD40/CD40-ligand (CD40L) signaling is an important co-stimulatory pathway which triggers the tryptophan (Trp) catabolizing enzyme IDO in dendritic cells and is immunosuppressive in cancer. Higher IDO activity and Trp catabolism was reported in ART-Naïve patients in association with the imbalance between regulatory T-cells (Tregs) and Th17 cells, contributing to microbial translocation measured by sCD14. We assessed the link between sCD40L, Tregs and IDO activity in HIV infection.
METHODS: Levels of plasmatic sCD40L were measured in ART-naive, ART-successfully treated (ST), elite controllers (EC) and healthy controls (HS) ( $\mathrm{n}=14$ per group). The plasma levels of Trp and its metabolite Kynurenine (Kyn) were measured by isotope dilution tandem mass spectrometry and sCD14, the microbial translocation marker was assessed by ELISA. The in vitro functional assay of sCD40L on Tregs induction and T-cell activation (CD38/HLADR/PD-1) were assessed on PBMCs from HS $(n=10)$. Statistical analyses were performed using Wilcoxon matched pairs and Pearson tests.

RESULTS: sCD40L levels in ART-Naïve subjects were significantly higher compared to ST and HS (1305 \pm 876 vs $503 \pm 626$ and $442 \pm 270$ $\mathrm{pg} / \mathrm{mL} ; \mathrm{P}=0.002$ for both comparisons) whereas EC showed a minor increase $(969 \pm 944 \mathrm{pg} / \mathrm{mL}, \mathrm{P}>0.05)$. In ART-Naïve patients, $\mathrm{sCD} 40 \mathrm{~L}$ was negatively correlated with CD4 $\mathrm{T}$-cell counts $(\mathrm{r}=-0.6132, \mathrm{P}=0.02)$ but not with viral load. sCD40L was positively correlated with IDO activity defined by $\mathrm{Kyn} / \mathrm{Trp}$ ratio $(\mathrm{r}=0.4323, \mathrm{P}=0.004)$ and plasmatic sCD14 levels $(\mathrm{r}=0.3161, \mathrm{P}=0.04)$ in all $\mathrm{HIV}$-infected patients. In vitro functional sCD40L stimulation induced expansion of Tregs $(3.5 \pm 1.9$ vs $4.1 \pm 2.1 \%$, $\mathrm{P}=0.04)$ and favoured CD4 $\mathrm{T}$-cell differentiation by reducing central memory cells $(54.9 \pm 20.3$ vs $47.2 \pm 19.9 \%, P=0.025)$ and increasing terminal effector cells $(0.9 \pm 1.1$ vs $4.7 \pm 4.1 \%, \mathrm{P}=0.006)$ amongst naïve, central, effector and terminal effector memory subsets without impacting T-cell activation (CD38/HLADR/PD-1).

CONCLUSION: These results suggest that higher levels of sCD40L may be immunosuppressive in HIV infection in association with IDO-induced Trp catabolism, microbial translocation and T-cell differentiation.

\section{P003}

\section{EXPRESSION OF IL-7 RECEPTOR $\alpha$ (CD127) IN HCV MONO- AND HIV-HCV CO-INFECTIONS}

Carrasco-Medina, Lorna; Cooper, Curtis L; Crawley, Angela M Ottawa, ON

BACKGROUND: Infection with HIV is initially suppressed by a strong virus-specific cytotoxic CD8+ T-cell (CTL) response but viral clearance is incomplete and chronic infection with impaired CTL function ensues. Interleukin-7 (IL-7) mediates T-cell development, survival and CTL function. Decreased membrane IL-7 receptor $\alpha$ (mCD127) expression on CD8 + T cells in HIV infection may contribute to impaired IL-7 activity and therefore altered immune function. Approximately 30\% of HIV individuals are $\mathrm{HCV}$ co-infected which contributes to more rapid $\mathrm{HCV}$ and HIV disease progression. The role of IL-7/CD127 in HCV mono- and HIV-HCV co-infections has not been determined.

METHODS: To investigate the expression of mCD127 on T-cells in chronic HCV infection, whole blood samples were collected from chronically-infected HCV mono-infected individuals (treatment naïve) and HIV-HCV co-infected individuals (chronic HCV infection, HCV treatment-naïve, receiving highly active anti-retroviral therapy, HAART, for $>1 \mathrm{yr}$ ). Expression of mCD127 on total lymphocytes, CD4 ${ }^{+}$and CD8 ${ }^{+}$ T-cells was analyzed by flow cytometry.

RESULTS: The expression of mCD127 is not significantly reduced on $\mathrm{CD}^{+}$or $\mathrm{CD}^{+}{ }^{+}$-cells in chronic HCV mono-infection compared to matched controls. In HIV-HCV co-infection, where HIV infection is optimally treated, there is a trend towards a decrease in mCD127 expression on all T-cells.

CONCLUSIONS: Expression of mCD127 is reduced in HIV-HCV coinfection, supporting findings that mCD127 expression does not recover to baseline levels in HAART-treated HIV mono-infection. In chronic HCV mono-infection, altered mCD127 expression is less evident, in contrast to the significant reduction of mCD127 expression in chronic HIV infection. It is not known whether IL-7 activity is impaired in chronic HCV infection, and if so, if it is associated with inherent cellular defects. Future research will contribute to the understanding of observed CTL dysfunction in HIV and HCV disease and aid in the development of novel immunotherapeutics. 
P004

CD16 $^{+}$MONOCYTES DIFFERENTIATE INTO DENDRITIC CELLS WITH UNIQUE IMMUNOGENIC AND HIV TRANSINFECTION POTENTIAL: IMPLICATIONS FOR DC-BASED VACCINES IN HIV-INFECTED SUBJECTS

Wacleche, Vanessa S ${ }^{1}$; Gosselin, Annie ${ }^{1}$; Gaudreau, Marie-Claude ${ }^{2}$; Cleret, Aurélie $^{1}$; Tremblay, Cecile ${ }^{1,3}$; Routy, Jean-Pierre' ${ }^{1}$; Ancuta, Petronela ${ }^{1}$

${ }^{1}$ Montreal, QC; ${ }^{2}$ Charleston, South Carolina, USA; ${ }^{3}$ Sainte-Annede-Bellevue, QC

BACKGROUND: Monocytes are dendritic cell (DC) precursors used in cell-based vaccination strategies, including HIV-1 infection. Monocytes are highly heterogeneous in phenotype and function, with the proinflammatory $\mathrm{CD}^{+} 6^{+}$subset being expanded during HIV infection ( $>50 \%$ in AIDS). Here we investigated the immunogenic and HIV transinfection potential of $\mathrm{CD} 16^{+} / \mathrm{CD} 16^{-}$monocyte-derived DC (MDDC). METHODS: Monocyte subsets were isolated from PBMC of HIVuninfected subjects by flow cytometry and differentiated into DC by culture in the presence of GM-CSF and IL-4. Cytokines were quantified in supernatants by ELISA. The immunogenic potential was tested by coculture of MDDC pulsed in Th17 (C. albicans; S. aureus) and Th1 (CMV) antigens with CFSE-loaded CD4 ${ }^{+}$T-cells. The HIV trans-infection potential was measured by coculture of HIV-exposed MDDC with autologous CD4+ T-cells. Integrated HIV-DNA and HIV-p24 levels were quantified by real-time PCR and ELISA.

RESULTS: Both $\mathrm{CD}^{+} 6^{+}$and $\mathrm{CD} 16^{-}$monocytes differentiated into immature MDDC $\left(\mathrm{CD} 14^{-} \mathrm{CD} 1 \mathrm{a}^{+} \mathrm{CD} 1 \mathrm{c}^{+} \mathrm{DC}-\mathrm{SIGN}^{+}\right)$and acquired a mature phenotype in vitro $\left(\mathrm{CD} 83^{+} \mathrm{CCR} 7^{+}\right)$. Despite these similarities, CD16+ versus CD16- MDDC produced higher levels of CCL2 and CCL22, suggesting their superior ability to attract HIV permissive $\mathrm{CCR}^{+} / \mathrm{Th} 17$ cells. Consistent with this prediction, levels of HIV replication and HIV-DNA integration were higher when T cells were cocultured with HIV-exposed CD16 ${ }^{+}$versus CD16- MDDC. At the opposite, CD16 - versus CD16+ MDDC produced superior levels of Th1 (IL-12) and Th17 (IL-23) polarizing cytokines. Finally, T-cells produced higher levels of IFN-gamma and IL-17 when co-cultured with C. albicans and S. aureus-loaded $\mathrm{CD}_{16}{ }^{-}$versus $\mathrm{CD} 16+\mathrm{MDDC}$.

CONCLUSION: These results provide evidence that $\mathrm{CD} 16^{+} \mathrm{MDDC}$ are precursors for a unique DC subset promoting HIV dissemination, while CD16- MDDC may have a superior ability to trigger/sustain Th1/ Th17 polarization in vivo. Our findings suggest that DC-based anti-HIV vaccines should use $\mathrm{CD} 16^{-}$but not $\mathrm{CD} 16^{+} \mathrm{MDDC}$ in order to promote potent antiviral responses while limiting HIV dissemination in HIVinfected subjects.

\section{P005 \\ SIV-SERONEGATIVE MACAQUES ELICIT MUCOSAL IMMUNE RESPONSES FOLLOWING SERIAL LOW-DOSE SIV MUCOSAL CHALLENGES}

Haq, Kamran ${ }^{1}$; Willer, David $\mathrm{O}^{1}$; Chan, Jacqueline $\mathrm{K}^{1}$; Iwajomo, Oluwadamilola H¹; Pilon, Richard ${ }^{2}$; Sandstrom, Paul'; MacDonald, Kelly $\mathbf{S}^{1}$

${ }^{1}$ Toronto; ${ }^{2}$ Ottawa, ON

Very little is known about the effects of sub-infectious HIV/SIV doses on mucosal and systemic immunity. The capacity and mechanism of how these responses may impact acquisition and subsequent disease course are also unknown. To address these questions, we examined a cohort of cynolmolgus macaques in the context of a vaccine trial, for their ability to generate mucosal and systemic immune responses following a schedule of repeated ultra low-dose mucosal SIVmac239 challenges. Animals were challenged intra-rectally on a weekly basis following a 24-week doseescalation schedule. Peripheral blood was obtained weekly, and rectal biopsies were obtained after 24 SIV challenges and analyzed by flow cytometry for anti-SIV T-cell responses. Intracellular cytokine responses (IFN- $\gamma$, IL-2, TNF $\alpha$ and CD107a) were measured following antigenic stimulation using multicolor flow cytometry.
Our results indicate measurable mucosal $\mathrm{CD} 8^{+}$and $\mathrm{CD} 4^{+} \mathrm{T}$-cell responses following multiple sub-infectious challenges. Total $\mathrm{CD}^{+}{ }^{+}$responses against two distinct antigenic targets correlated with apparent resistance to infection as measured by the number of challenges, total dose and infecting dose, moreover, CD8+ responses were also predictive of susceptibility to infection. Poly-functional capacities of $\mathrm{CD}^{+}{ }^{+}$and $\mathrm{CD} 8^{+} \mathrm{T}$ - cell responses were analyzed, however, it was not predictive of outcome with respect to set-point viral loads. Multiple sub-infectious SIV challenges did not induce humoral responses in the blood, as determined by western blot assay.

As part of this on-going vaccine trial, it is anticipated that further studies may reveal how immune responses generated as a consequence of lowdose challenges may affect disease course/pathogenesis following SIV infection.

\section{P006}

\section{CALPROTECTIN AND S10012 PROTEINS AS} INFLAMMATORY MARKERS IN HIV PATHOGENESIS

Subra, Caroline ${ }^{1}$; Proulx, Sophie ${ }^{1}$; Jenabian, Mohammad-Ali²; Routy, Jean-Pierre ${ }^{2}$; Tessier, Philippe ${ }^{1}$; Gilbert, Caroline ${ }^{1}$

${ }^{1}$ Québec; ${ }^{2}$ Montreal, QC

Despite effective antiretroviral treatments that have improved the life expectancy of HIV-1-infected patients, many observations indicate that chronic inflammation of the immune system persists. S100A8, S100A9 and S100A12 are a subgroup of S100 proteins named calgranulins, secreted by neutrophil and monocytes in response to trauma or infection, to mediate inflammation and antimicrobial activity. Elevated serum levels of the heterocomplex of S100A8/A9 known as calprotectin, and S100A12, are indicative of systemic inflammation in several diseases. Preliminary studies demonstrated in vitro increased HIV-1 replication with calprotectin and an enhanced serum calprotectin level in advanced HIV-1-infected patients. In this cross-sectional study, we used an ELISA to measure plasma level of both calprotectin and S100A12 in treatmentnaïve HIV-infected patients $(n=20)$, in patients successfully treated with ART $(n=21)$, elite controllers ( $n=15)$, and age-matched healthy subjects $(n=31)$. Serum calprotectin and S100A12 levels were lower in naïve subjects compared to healthy subjects or elite controllers, while antiretroviral treatment allowed calprotectin and S100A12 protein recovery. Calprotectin and S100A12 levels were significantly correlated with neutrophil and circulating monocyte counts, both of which were reduced in ART-naïve HIV-1-infected patients. In contrast, they tended to be inversely correlated with viral load. Calprotectin and duration of infection tended to correlate negatively in treatment-naïve patients, reaching significance in ART- treated cases. However, calprotectin level and duration of infection tended to be correlated positively in elite controllers. These results suggest a protective effect of serum calprotectin, which is restored by ART or naturally maintained in elite controllers and reflect a likely beneficial inflammation participating to the control of viral load.

\section{P007 \\ CHARACTERIZATION OF T CELL RECEPTOR INTERACTIONS WITH HLA-A*02/GAG FK10 USING AN IN VITRO REPORTER CELL ASSAY}

Anmole, Gursev' ${ }^{1}$; Martin, Eric ${ }^{1}$; Tse, Kinson ${ }^{1}$; Jones, Brad ${ }^{2}$;

Wei, Zhan'; Ostrowski, Mario²; Brockman, Mark ${ }^{1,3}$

${ }^{1}$ Burnaby, BC; ${ }^{2}$ Toronto, ON; ${ }^{3}$ Vancouver, BC

BACKGROUND: $\mathrm{CD}^{+}$cytotoxic $\mathrm{T}$ lymphocytes (CTL) suppress HIV-1 replication. CTL recognize infected cells through highly diverse antigen-specific T-cell receptors (TCR). Studying the sequence and function of TCR may provide new insights into CTL-mediated control; however, such analyses using CTL clones are hindered by non-TCR differences between cell lines. We have therefore implemented an in vitro reporter cell assay to measure TCR activity in response to peptide-HLA ligands.

METHODS: Full-length TCR $\alpha / \beta$ cDNAs were isolated from a CTL clone specific for the HLA-A*02:01-restricted HIV-1 Gag peptide FLGKIWPSHK (FK10), sequenced, and re-expressed as mRNA. TCR 
mRNA, CD8 $\alpha$ expression plasmid, and NFAT driven luciferase reporter plasmid were co-transfected into Jurkat T-cells. These cells were co-cultured with $\mathrm{A} * 02+\mathrm{CEM}$ cells pulsed with FK10 or peptide variants, and TCR signaling was measured by luminescence. TCR $\alpha$ and $\beta$ CDR3 sequences were altered by alanine-scanning mutagenesis. HLA-A*02 binding affinity for FK10 and peptide variants was predicted using netMHCpan.

RESULTS: FK10 alanine variants indicated that positions 1, 2, 4, 6, and 7 were critical for TCR signaling. Positions 1, 2, and 6 were predicted to disrupt HLA binding; positions 4 and 7 were thus identified as putative TCR contact sites. A natural polymorphism at position 4 (Gag K436R) abrogated TCR activity, while variants at position 9 (H441X) had no effect. Alanine substitutions in TCR CDR3 motifs revealed residues required for receptor function. An electrostatic interaction between TCR $\alpha$ CDR3 (Glutamic acid) and FK10 position 4 (Lysine) is postulated based on these results and structural models.

CONCLUSIONS: This in vitro method is sensitive, scalable, and amendable to molecular manipulation. Results provide new details of the tri-partite interaction between this TCR, HLA-A*02, and FK10 necessary for $\mathrm{T}$ cell activation. Analysis of other FK10-specific TCRs will be necessary to examine the functional diversity of responses against this epitope, which may contribute to vaccine discovery efforts.

\section{P008}

\section{SOLUBLE TOLL-LIKE RECEPTOR 2 (STLR2) SIGNIFICANTLY} INHIBITS HIV-1 INFECTION AND INFLAMMATION

Henrick, Bethany M; Yao, Xiaodan; Rosenthal, Kenneth L

\section{Hamilton, ON}

The majority of infants who breastfeed from their HIV-positive mothers remain uninfected despite constant and repeated exposure to virus over weeks to years. This phenomenon is not fully understood but has been closely linked to innate factors in breast milk (BM). We previously identified multiple forms of BM soluble TLR2 (sTLR2) that significantly inhibited bacteria-induced cellular activation and HIV-1 infection in vitro. However, BM is a highly complex mucosal fluid abundant with innate and adaptive immune components, many of which have not had their functions fully elucidated. Additionally, immunodepletion methods used in our previous data did not completely remove all sTLR2 from BM, therefore we could not rule out the possibility that undiscovered immunological factors might have been playing a role in the significant inhibition of cell-free HIV-1 infection in vitro. Here we extend from previous findings to show that sTLR2 produced from stably transfected cells significantly inhibited HIV-1 infection in vitro. This inhibition could be neutralized with TLR2 specific antibody. Further, sTLR2 significantly reduced cellular activation induced by HIV-1 exposure. Importantly, we identified, using a dot-blot method, strong protein-to-protein interaction between sTLR2 and specific recombinant HIV-1 structural proteins indicating a mechanism by which sTLR2 inhibits viral-induced cellular activation and HIV infection. Additionally, sTLR2 levels were significantly increased in HIV-infected samples and positively correlated with p24 levels in BM. Evidence presented here confirms previous observations that link sTLR2 with decreased pro-inflammatory responses and inhibition of HIV infection. Thus, strongly indicating that sTLR2 may be critical to infant health and prove beneficial in decreasing vertical HIV transmission to infants.

\section{P009}

\section{SEROLOGICAL PROFILING OF ANTIBODY RESPONSES DURING HIV INFECTION}

Henry, Kevin A ${ }^{1}$; Wang, Xin'; Wang, Chao' ${ }^{1}$; Wu, Sampson';

Kingsley, Lawrence ${ }^{2}$; Rinaldo Jr, Charles $\mathbf{R}^{1}$; Richman, Douglas ${ }^{3}$;

Silverman, Gregg J ${ }^{4}$; Scott, Jamie $\mathbf{K}^{1}$

${ }^{1}$ Burnaby, BC; ${ }^{2}$ Pittsburgh, Pennsylvania; ${ }^{3}$ San Diego, California;

${ }^{4}$ New York, New York, USA

A major goal in HIV-1 vaccine research is to elicit broadly-neutralizing $(\mathrm{bNt})$ antibody $(\mathrm{Ab})$ responses capable of preventing viral transmission.

However, the immunological factors that give rise to these types of responses remain poorly understood. Here, we investigated whether HIV clinical status or serum neutralization breadth was associated with specific serum reactivity patterns against envelope glycoprotein epitopes, autoantigens and non-specific antigens, and the usage of the VH1-69 gene in serum Abs. A panel of 150 sera from $28 \mathrm{HIV}+$ individuals was grouped according to neutralization breadth (bNt vs. non-bNt); non-bNt sera were stratified by disease progression (slow, normal or fast progression to AIDS). Using ELISA, we showed that anti-HIV-1 Ab responses became stronger and broader in specificity during the first year after seroconversion; thereafter, $\mathrm{Ab}$ responses were relatively stable over time in all groups, with rapid progressors manifesting the broadest reactivity against all antigens. Using autoantigen microarrays, we observed that autoreactivity of HIV serum $\mathrm{Ab}$ responses was greatest in rapid progressors, and still insignificant compared to SLE sera. Surprisingly, all of the HIV sera, but not SLE sera, contained high levels of the IGHV1-69. encoded idiotype, as defined by the murine MAb G6; this idiotype appeared during the first year of infection. High levels of polyreactive serum binding were observed to multiple antigens, an effect that was apparent only when assays were conducted with no BSA blocking agent. We also found that bNt sera bore low self-reactivity and restricted reactivity in general, as compared to non- $\mathrm{Nt}$ sera, and that increased polyand autoreactivity appear to be implicated in rapid progression.

\section{P010}

\section{A SYSTEMS IMMUNOLOGY APPROACH TO INVESTIGATE THE ANTIBODY RESPONSE DURING HIV INFECTION}

Murira, Armstrong1; Henry, Kevin ${ }^{1}$; Aghaeepour, Nima ${ }^{2}$; Brinkman, Ryan ${ }^{2}$; Moir, Susan ${ }^{3}$; Scott, Jamie ${ }^{1}$

${ }^{1}$ Burnaby; ${ }^{2}$ Vancouver, BC; ${ }^{3}$ Bethesda, Maryland, USA

Broadly-neutralizing antibodies (bNt Abs) against HIV are rarely produced in natural infection, yet their elicitation is a central requirement for a vaccine. Our previous work has shown that the characteristics of the HIV bNt Abs (long CDR-H3s, high levels of somatic mutation, preference for $\mathrm{VH}$ gene usage) are present in Abs elicited during HIV and chronic viral infection in general. The emergence of such Abs may also coincide with phenotypic and functional perturbations occurring in peripheral B-cell populations during chronic infection. Our goal is to characterize the $\mathrm{B}$-cell subsets, their $\mathrm{Ab}$ repertoires and accompanying serum reactivities occuring in response to HIV infection, and to compare them to those in systemic autoimmune disease. Peripheral B cells from HIV+ individuals, from SLE patients and from matched healthy controls were phenotyped and sorted into 6 subsets using a six-marker panel comprising CD10, CD19, CD20, CD21, CD27 and IgG. A computational pipeline, FlowMeans, was used to analyze differences in subpopulations; our preliminary results confirmed previously reported aberrations in HIV compared to healthy controls (low naïve B-cells; expanded plasmablasts and tissue-like memory cells), and identified increased transitional B-cell subsets in HIV. Preliminary results of serum analyses to detect the presence of expressed Abs encoded by the IGHV1-69 gene revealed that all of the HIV sera, but none of the SLE sera, bore this idiotype. 454 pyrosequencing of $\mathrm{VH}$ genes expressed in the $6 \mathrm{~B}$-cell subsets from each individual is currently underway, and may reveal the subsets responsible for the IGHV1-69 idiotype we observed in HIV sera. Our novel approach to characterizing the phenotypic, immunogenetic and serological features of the B-cell response during HIV infection may further reveal the biological milieu required for the elicitation of bNt Abs. 


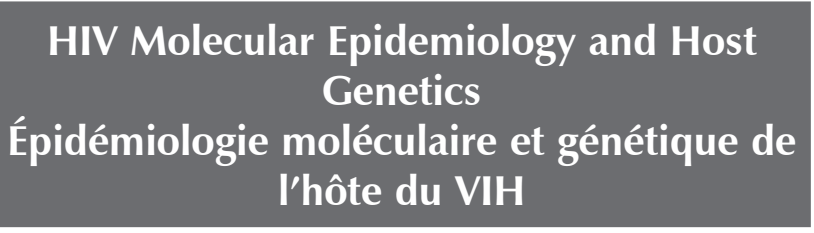

P011

\section{A METHOD FOR KILLER-CELL IMMUNOGLOBULIN-LIKE RECEPTOR (KIR) 3DS1/3DL1 SEQUENCE-BASED TYPING USING DNA RECOVERED FROM FROZEN PLASMA}

Shahid, Aniqa ${ }^{1}$; Chopera, Denis ${ }^{1}$; Martin, Eric ${ }^{1}$; Penney, Kali ${ }^{1}$; Milloy, M-J1,2; Brumme, Zabrina ${ }^{1,2}$

${ }^{1}$ Burnaby; ${ }^{2}$ Vancouver, BC

BACKGROUND: Polymorphisms in the KIR region, notably those at the 3DS1-3DL1 (3D) locus, may influence HIV susceptibility and disease progression. KIR 3D typing is routinely performed using genomic DNA extracted from PBMCs, however methods capable of using low-concentration/low-quality DNA from other sources offers an alternative if PBMCs are unavailable. We developed a novel sequence-based KIR 3D typing method that utilizes DNA recovered from frozen plasma.

METHODS: The method was first validated against two independent genomic DNA reference panels. To validate the approach for plasmaderived DNA, total nucleic acids were extracted from 69 paired frozen $\left(-80^{\circ} \mathrm{C}\right)$ PBMC $(\sim 2.5$ million $)$ and plasma $(500 \mu l)$ specimens representing all common KIR 3D types (3DS1/3DS1, 3DS1/3DL1, 3DL1/3DL1) plus rare allele 3DL1_054 in a blinded fashion. Independent amplification of KIR 3D exon 4 (265 basepairs), and if required exon 3 (231 basepairs), was performed via nested PCR using universal sequence-specific primers. Amplicons were bidirectionally sequenced using the same primers used for 2 nd round PCR. Chromatograms were analyzed using the free, webbased semi-automated base calling software RECall (http://pssm.cfenet. ubc.ca/). KIR types were interpreted using an in-house web-based algorithm KIR 3D, that assigns types based on two key nucleotide polymorphisms in exon 4 and, if required, up to two additional key nucleotides in exon 3. The lower limit of detection was estimated using limiting dilutions of plasma-derived extracts for ten samples.

RESULTS: Extractions from plasma yielded median [IQR] nucleic acid concentrations of 0.9 [0-2.45] ng/ $\mathrm{\mu l}$. PCR was successful for $100 \%$ of Exon 4 (69/69) and Exon 3 (29/29) plasma amplifications and yielded high quality chromatograms. Concordance between PBMC and plasmaderived types was $100 \%$. The estimated lower limit of input DNA required for reliable typing is $0.01 \mathrm{ng} / \mathrm{\mu l}$ DNA.

CONCLUSION: This method provides reliable and accurate KIR 3D typing when PBMCs (or equivalent sources of high-quality genomic DNA) are unavailable.

\section{P013}

\section{IMPACT OF THE HETEROZYGOUS CCR5WT/ 32 GENOTYPE ON RISK OF HIV-1 ACQUISITION IN A COHORT OF INJECTION DRUG USERS}

Davis, Jack ${ }^{1}$; Danroth, Ryan ${ }^{1}$; Simo, Annick ${ }^{2}$; Le, Anh ${ }^{1}$; Johnston, Caitlin¹,2; Milloy, M-J2; Kerr, Thomas²; Wood, Evan²; Brumme, Zabrina ${ }^{1,2}$; Vancouver Injection Drug Users Study (VIDUS), The ${ }^{1}$

${ }^{1}$ Burnaby; ${ }^{2}$ Vancouver, BC

BACKGROUND: Heterozygosity for the CCR $5 \Delta 32$ mutation (CCR5wt/ 32 ) is observed at $10-15 \%$ frequency in Caucasian individuals and is associated with slower HIV disease progression compared to CCR $5 \mathrm{wt} / \mathrm{wt}$. However, its influence on risk of HIV acquisition remains unclear, in part due to lack of seroconverter cohorts with documented risk behaviour. Thus, we sought to assess the impact of the CCR $5 \mathrm{wt} / \Delta 32$ genotype on risk of HIV infection among 495 individuals who use injection drugs participating in the Vancouver Injection Drug Users Study. METHODS: Sequence-based CCR $5 \Delta 32$ genotyping was undertaken for 140 HIV seroconverters, and 355 high-risk seronegative (HRSN) individuals who reported repeated borrowing of used syringes (median [IQR]
30 [10-63] total borrowing events/individual) but remained HIV-negative during followup. Overall follow-up time was 1463.5 person-years. A Cox Proportional Hazards model was constructed to compare the relative time from cohort entry until infection in CCR $5 \mathrm{wt} / \Delta 32$ vs. wt/wt individuals. A measure of HIV exposure risk, quantified in "exposure units", was defined as the estimated mean frequency of rig sharing in the last six months multiplied by the mean number of reported HIV infections in British Columbia during that period.

RESULTS: Among seroconverters, CCR $5 \mathrm{wt} / \Delta 32$ prevalence was $12 / 140$ $(9 \%)$ and 59/355 (17\%) among HRSN. Relative Hazard of HIV infection per exposure unit among CCR $5 \mathrm{wt} / \Delta 32$ individuals was 0.561 compared to $\mathrm{wt} / \mathrm{wt}$ individuals (95\% confidence interval $0.375-1.014$, one sided $\mathrm{p}$-value $=0.028$ ). This difference persisted, although did not remain statistically significant, when the analysis was restricted to individuals with self-reported Caucasian ethnicity $(\mathrm{N}=318 ; 11 / 74[14.8 \%]$ in seroconverters and 53/244 [21.7\%] in HRSN; Relative Hazard $=0.711,95 \%$ confidence interval $0.375-1.350$, one sided $\mathrm{p}$-value $=0.15)$. Results were consistent following adjustment for cohort entry and injection debut dates and when analyses were restricted to individuals who reported used syringe borrowing in at least four bi-annual surveys.

CONCLUSION: The CCR $5 \mathrm{wt} / \Delta 32$ genotype may confer marginal, albeit not statistically significant, protective effects against infection with HIV via injection drug use.

\section{HIV Prevention/Treatment (Preclinical), and HIV Resistance \\ Prévention et traitement (préclinique) du VIH et résistance au VIH}

\section{P014}

\section{ASTRINGIN: AN INHIBITOR POTENTIALLY EFFECTIVE AGAINST HIV-1 INFECTION}

Haidara, Alpha ${ }^{1}$; Wacleche, Vanessa; ${ }^{2}$; Petronela, Ancuta ${ }^{2}$; Legault, Jean ${ }^{3}$; Pichette, André3; Gilbert, Caroline ${ }^{1}$

${ }^{1}$ Quebec; ${ }^{2}$ Montreal; ${ }^{3}$ Chicoutimi, QC

Since the discovery of HIV-1 in 1983, 25 molecules have been approved for use in various combinations to treat infected patients. However, it has become clear that these treatments do not cure HIV-1 infection. Emergence of viral resistance plus the toxicity and inability of most of these molecules to gain access to the latent virus are some of the factors reducing therapeutic possibilities. In the absence of a vaccine, the development of new and more effective anti-retroviral compounds must continue. Plants could provide an alternative approach in the discovery of new active agents against HIV-1. Several anticancer drugs derived from plants, such as vinblastine, vincristine and paclitaxel, have already been approved and used in therapy. In this study, we evaluated the anti-HIV-1 activity of Astringin, a stilbene glycoside isolated from Picea abies. Using cytofluorometry, spectrophometry and ELISA, the immunological safety and anti-HIV-1 activity of Astringin pre-treatment were evaluated. The results show that Astringin does not affect acquired immune response functions such as CD4 and CD8 T lymphocyte antigen-specific proliferation, dendritic cell differentiation or maturation, or cytokine production (IL1- $\beta$ and TNF- $\alpha$ ). Innate responses such as neutrophil degranulation and IL- 8 production were also unaffected by Astringin. Finally, evaluation of the limitation of HIV-1 infection by Astringin showed that the drug effectively blocked infection of dendritic cells and more importantly of human tonsil in a 3D physiological tissue model. To understand the mechanism involved in this inhibitory effect, the impact of Astringin was evaluated in the Raji-CD4 DCIR-positive cell line, which indicated that Astringin targets DCIR. The absence of immunological side effects and the significant effect in a complex model of infection appear promising. Furthermore, limiting infection by targeting DCIR appears to be a potentially useful preventive strategy against the development of AIDS. 
P015

\section{SCREENING NATURAL MUCOSAL FACTORS FOR ANTI-} HIV ACTIVITY

Aboud, Lindsay G; Ramdahin, Sue; Plummer, Frank; Ball, Blake; Burgener, Adam Winnipeg, MB

Greater than $70 \%$ of the current 33 million people living with HIV-1, are young women (aged 15-24 years of age) who have acquired the virus through heterosexual contact. The risk of becoming infected is significantly influenced by innate and adaptive immune factors that are naturally present within the cervix/vagina, the first portal of entry for the HIV-1 virus. A group of HIV-Exposed Sero-Negative (HESN) women within a sex worker cohort in Nairobi, Kenya have shown numerous differences in their immune responses and protein production, in the genital mucosal compartment, compared to women who are susceptible to infection. Immune factors that are up-regulated within the cervicovaginal compartment of HESN women include HIV-1 specific cellular CD4+ and CD8+ T cell responses, HIV-specific IgA, anti-HIV factors such as RANTES, ccchemokines, SLPI and MIP- $1 \alpha / \beta$, as well as various cysteine and serine antiproteases, such as Cystatin A and Serpin A1, respectively.

We hypothesize that the up-regulated innate factors present within the genital secretions of HESN women, play a critical role in their resistance to HIV-1 infection by protecting against mucosal inflammation and/or HIV-1 infection.

Preliminary experiments employed a TZM-bl reporter cell line as well as peripheral blood mononuclear cell (PBMCs), with both an R-topic and $\mathrm{X}$-tropic virus. Results confirm that specific antiproteases, including serpin $\mathrm{A} 1$ and $\mathrm{C} 1$ demonstrate inhibitory activity against HIV-1 infectivity in both cell lines. Preliminary results also uncovered strong inhibitory activity ( $80 \%$ inhibition), for two additional innate factors, which have not previously been described in literature. Future experiments will attempt to uncover the mechanism responsible for the observed inhibitory effect for both factors.

All innate factors used in this study are natural mucosal factors, thus potentially making them novel, effective candidates for use in future microbicides.

\section{P016}

\section{DEVELOPMENT OF A PASSIVE IMMUNIZATION COCKTAIL} FOR HIV USING PLANT-BASED ANTIBODIES

Cheung, Wing-Fai' ${ }^{1}$ Gilbert, Erin' ${ }^{1}$; Teat, John'; Rouleau, Jessica ${ }^{1}$;

Veldhuis, Linda ${ }^{1}$; Liu, Fang'; LeBlanc, Zacharie'; Wang, Haifeng';

Marit, Michael R${ }^{1}$; McLean, Michael $\mathrm{D}^{1}$; Willer, David ${ }^{2}$;

MacDonald, Kelly $\mathrm{S}^{2}$; Hall, J Christopher ${ }^{1}$

${ }^{1}$ Guelph; ${ }^{2}$ Toronto, ON

Since its discovery in the 1980s, human immunodeficiency virus (HIV) has caused millions of deaths, untold human suffering and tremendous economic burden on global medicine. This pathogen is notorious for its ability to mutate and change surface epitopes thereby evading detection and elimination by the immune system. Much effort has been spent towards development of a vaccine for HIV; however, there is currently no effective vaccine against it.

PlantForm Corp. has been awarded a grant from Canada's National Research Council Industrial Research Assistance Program (NRC-IRAP) through a program that is part of the Canadian HIV Vaccine Initiative (CHVI), and a collaborative effort between the Government of Canada and the Bill \& Melinda Gates Foundation. PlantForm is producing antibodies for the treatment of human immunodeficiency virus (HIV) with a focus on producing antibodies that fight infection by disrupting the virus'method of entry into human CD4 ${ }^{+}$T-cell lymphocytes.

One class of anti-HIV antibodies, known as broadly neutralizing antibodies (bNAb), effectively bind and neutralize HIV serotypes from divergent viral clades. PlantForm Corp. has begun to produce bNAbs using a therapeutic protein production platform developed by the company based on transient expression in Nicotiana benthamiana plants. These plant-produced bNAbs will be compared in vitro with their counterparts produced through mammalian cell-culture for their ability to neutralize viral isolates from each of the major HIV clades at the Center for HIV/AIDS
Vaccine Immunology and Immunogen Discovery (www.cavd.org). This center, housed at Duke University (Raleigh, NC, USA), is recognized internationally for performing standardized and rigorous HIV neutralization assays. Should PlantForm's anti-HIV mAbs prove to have HIV neutralization bioequivalence with their mammalian counterparts in vitro, they will subsequently proceed toward testing in an animal model of HIV infection.

The intent of this research pathway is to determine whether passive immunization with a plant-produced $\mathrm{mAb}$ or cocktail of plant-produced mAbs will have true viral neutralization efficacy. This plant-based platform will significantly reduce the costs of production and treatment.

\section{P017}

\section{THE DEVELOPMENT OF AN HIV-1 SPECIFIC MICROBICIDE USING THE S-LAYER MEDIATED DISPLAY CAPABILITIES OF CAULOBACTER CRESCENTUS}

Farr, Christina; Nomellini, John F; Smit, John; Horwitz, Marc S Vancouver, BC

Human Immunodeficiency Virus (HIV) infects over 2.7 million people annually. More than half of the new infections occur in women, and the majority in women in developing countries, where societal norms may prevent women from insisting on condom use. As such, new prevention options need to be investigated. We previously proposed the development of an HIV-1 specific microbicide using the S-layer mediated display capabilities of the non-pathogenic freshwater bacterium Caulobacter crescentus. We have successfully expressed many diverse anti-HIV proteins, including CD4 decoy receptor, fusion inhibitors and mannosebinding lectins on the surface of C. crescentus. Using an in vitro viral blocking assay, we have demonstrated that each of the recombinant $\mathrm{C}$. crescentus are able to inhibit infection of TZM-bl cells from HIV-1 pseudoviruses representing viral clades $\mathrm{B}$ and $\mathrm{C}$. The mannose-binding lectins were most effective, reducing HIV-1 infection by $74 \%$, while the fusion inhibitors provide $50 \%$ protection from infection. Preliminary work investigating combinations of different recombinants shows promise, as protection levels of $97 \%$ have been observed with a CD4-MIP1 $\alpha$ combination. We have begun work with live HIV-1 and are beginning in vivo studies. Initial studies with immune-competent mice indicate that C. crescentus does not induce the production of inflammatory cytokines, suggesting the bacterium are safe for topical application. Further studies will be carried out using the humanized bone marrow-liver-thymus (BLT) mouse model. BLT mice have a fully functional human immune system and are susceptible to intravaginal infection with HIV-1, recapitulating the virus pathogenesis observed in humans. We will utilize the BLT mice to determine if the recombinant C. crescentus can prevent HIV-1 infection in vivo, and to study the initial events of HIV-1 infection, dissemination and host-virus interactions.

\section{P018}

DNA VACCINES THAT OPTIMALLY PRESENT

NEUTRALIZING EPITOPES WITHIN THE MEMBRANE PROXIMAL EXTERNAL REGION OF HIV-1 GP41

Gulzar, Naveed ${ }^{1}$; Klaric, Kristina-Ana ${ }^{1}$; Parfyonov, Maksim¹;

Montero, Marinieve ${ }^{1}$; Hannigan, Brett ${ }^{2}$; Ulas, Gozde ${ }^{2}$;

McCully, Michelle ${ }^{2}$; Wang, Shixia ${ }^{3}$; Huarte, Nerea ${ }^{4}$;

Appellaniz, Beatriz ${ }^{4}$; Nieva, Jose ${ }^{4}$; Lu, Shan ${ }^{3}$; DeGrado, William²; Scott, Jamie $\mathrm{K}^{1}$

${ }^{1}$ Burnaby, BC; ${ }^{2}$ San Francisco, California; ${ }^{3}$ Worcester, Massachusetts, USA; ${ }^{4}$ Bilbao, Spain

The highly conserved membrane proximal external region of HIV-1 gp41 (MPER) is an attractive vaccine target, being the target of three broadly neutralizing (Nt) monoclonal (M) antibodies (Abs): 2F5, 4E10 and 10E8. An obstacle to the successful development of a MPER-based HIV vaccine has been our lack of understanding of the neutralization competent structure (NCS) of the MPER. Previously, we developed DNA vaccines that present the MPER in the context of the plasma membrane, and gauged their ability to recapitulate the NCS as defined by their ability to be bound by $2 \mathrm{~F} 5$ and $4 \mathrm{E} 10$, but not by their mutant counterparts, which 
have lost the ability to neutralize, but can bind peptides bearing MPER sequences. While one of our first-generation MPER vaccines passed this test, it did not elicit MPER-specific Abs; another vaccine elicited MPERspecific 2F5-like Abs, but did not fully expose the 4E10 epitope, suggesting that further engineering of the transmembrane domain (TMD) may optimize epitope exposure. Here, we describe the characterization of second-generation of DNA vaccines that encode a fragment of the C-terminal heptad repeat plus the MPER, fused to engineered TMDs. As immunogenicity was previously enhanced by $\mathrm{N}$-terminal fusion of the TLR ligand, flagellin, we fused it to the $\mathrm{N}$-terminus of our vaccine encoding the TMD of the platelet derived growth factor receptor (MPERPDGFR). Alternatively, to test the hypothesis that a trimeric structure enhances exposure of the MPER, we engineered an $\mathrm{N}$-terminal coiledcoil fused to the MPER, and tethered to the membrane spanning region of beta-alanine (MPER-MS1). Lead candidates for immunization studies, chosen based on assay with 2F5, 4E10 and their non-Nt counterparts, are being tested in a DNA-prime, liposome-boost immunization strategy using liposomes bearing optimized peptide fragments of the gp41 MPER+TMD. By this incremental approach, we hope to produce hightiter, MPER-specific Abs that neutralize HIV infectivity.

\section{P019}

\section{MOLECULAR CHARACTERIZATION OF HIV GROUP O INTEGRASE}

Depatureaux, Agnes ${ }^{1}$; Mesplede, Thibault ${ }^{1}$; Quashie, Peter K ${ }^{1}$; Moisi, Daniela ${ }^{1}$; Oliveira, Maureen ${ }^{1}$; Brenner, Bluma $\mathbf{G}^{1}$; Plantier, Jean-Christophe ${ }^{2}$; Wainberg, Mark A $^{1}$

${ }^{1}$ Montreal, QC; ${ }^{2}$ Rouen, France

HIV-1 group O (HIV-O) is characterized by an important genetic polymorphism, in particular in regions encoding proteins targeted by antiretroviral drugs (ARVs). The impact of this polymorphism on responsiveness to ARVs remains largely unknown and genotypic prediction algorithms suggest a decrease in HIV-O susceptibility to NNRTIs, PIs (tipranavir, saquinavir) and enfuvirtide (fusion inhibitor), compared to HIV-1 group M.

For integrase inhibitors (IIs), a clinical response was obtained with heavily pretreated patients receiving raltegravir (Depatureaux et al., 2012) but the impact of genetic polymorphisms on enzymatic function is unknown.

We analyzed genetic polymorphisms in integrase from representative HIV-O clade A, B, C, and divergent viruses to study their impact on enzymatic function, in comparison with HIV-1 group M.

We finalized a specific PCR to amplify and sequence the entire region of integrase from HIV-O. Genotypic resistance interpretation was performed using Stanford and ANRS algorithms (latest version). E. coli strain XL-Gold ultracompetent cells were used for plasmid production and BL21 (DE3) cells for protein expression. Enzymatic function study was performed by strand transfer activity (cell-free assay).

Sequencing integrase region reveals a strong polymorphism with on average 15 amino acid changes compared with the HIV-M consensus sequence, including changes at positions previously associated with resistance, such as S153A and G163Q.

Genotypic resistance interpretation predicts susceptibility to raltegravir, elvitegravir and dolutegravir. However, the presence of an atypical mutation at position 153 could compromise susceptibility to dolutegravir.

Here we report the successful cloning HIV-O integrases and their enzymatic characterization by calculating their maximum activity ( $\mathrm{Vmax}$ ) and Michaelis-Menten constant $(\mathrm{Km})$.

To our knowledge, this is the first report to study the molecular characteristics of HIV-O integrase from different clades in vitro. These data will be supplemented by phenotypic studies that show the impact of polymorphisms on susceptibility to IIs and the possible emergence of drug resistance.
P020

\section{THE FATE OF HIV-1 CLONES HARBOURING MUTATIONS IMPLICATED IN DOLUTEGRAVIR RESISTANCE WHEN SUBJECTED TO DRUGS OF DIFFERENT CLASSES POSSESSING LOW AND HIGH GENETIC BARRIERS TO DRUG RESISTANCE}

Oliveira, Maureen; Mesplede, Thibault; Moisi, Daniela; Ibanescu, Ilinca; Spira, Bonnie; Quashie, Peter; Brenner, Bluma; Wainberg, Mark

Montreal, QC

BACKGROUND: In recent clinical trials, dolutegravir (DTG) has continued to display formidable skill in avoiding therapy failure. Our laboratory has identified several pathways with the potential to lead to dolutegravir resistance. The replicative detriment incurred by these changes may discourage their occurrence in the clinical setting.

METHODS: To investigate the robustness of the cloned mutants and to discern their potential interaction with drugs of other classes, primary cells were infected with recombinant pNL4.3 viruses bearing single and double site-directed mutations (H51Y, R263K, G118R, H51Y+R263K, and H51Y+G118R). The cultures were treated with two drugs with low genetic barriers, nevirapine and lamivudine, and two drugs with high genetic barriers, rilpivirine and dolutegravir. Drug concentrations were increased gradually to favor the emergence of resistant variants. Virus growth was monitored by weekly determinations of reverse transcriptase (RT) activity. Viral RNA was extracted from tissue culture supernatants and sequenced to identify changes in the RT and integrase regions.

RESULTS: The mutant viruses displayed varying abilities to overcome increasing drug pressure. Even drugs with low barriers stymied the survival of some viral cultures, characterized by a slower rise in drug increments and a delayed appearance of newly-acquired resistance mutations. The viruses were also evaluated for adaptations and reversions in their integrase profiles, with and without drug pressure.

CONCLUSION: In our tissue culture model, the G118R mutation alone and in combination with H51Y had the most impaired growth, coupled with higher sensitivity to drug pressure, thus hindering the development of resistance to drugs of different classes and genetic barriers. The other viruses showed similar but less substantial effects. Should these resistance pathways ultimately emerge in patients on regimens including DTG, the resulting hyper-susceptibility to drugs of other classes may, in fact, be a deterrent to the emergence of treatment failures.

\section{Antiviral Mechanisms \\ Virologie et pathogénie du VIH, y compris les mécanismes antiviraux}

\section{P021}

ONCOLYTIC VIRUSES AS A POTENTIAL APPROACH TO ELIMINATE THE HIV RESERVOIR

Costiniuk, Cecilia T; Cote, Sandra C; Al-Ghazawi, Feras M; Carrasco-Medina, Lorna; Young, Charlene D; Angel, Jonathan B Ottawa, ON

OBJECTIVE: Similar to cancer cells, HIV-infected cells differ from HIV-uninfected cells in that they have altered interferon signaling pathways, the apparent reason for the selectivity of certain oncolytic viruses (OVs). The objective of this study was to determine whether the OV recombinant Maraba virus (MG1) would have a greater propensity to target and kill HIV-infected cells compared to non-infected cells.

METHODS: U1, ACH-2, OM-10 and J1.1 cells, harbouring 1-2 copies of integrated proviral DNA per cell, were infected with varying multiplicities of infection (MOI) of green fluorescent protein (GFP)-encoded MG1. Controls included HIV-uninfected U937, A301, HL60 and Jurkat parent lines. $\mathrm{CD}^{+}{ }^{+} \mathrm{CD} 25^{-} \mathrm{HLADR}^{-}$cells from $20 \mathrm{HIV}$-infected individuals on antiretroviral therapy were infected with MG1 and flow cytometry and MTT assays were performed to quantify MG1 infection and cell viability, respectively. PCR for total HIV DNA in cells, in addition to 
RT-PCR for total HIV RNA and ELISAs for p24 antigen on cell-free supernatants, were performed after a 2-week stimulation period.

RESULTS: MG1 infected and killed a greater proportion of U1 than U937 cells at most MOIs tested but this was not observed in the other cell lines. With the initial experimental approach, MG1 did not appear to infect $\mathrm{CD}^{+}{ }^{+} \mathrm{CD} 25^{-} \mathrm{HLADR}^{-}$cells and viability appeared preserved. Similarly, we were unable to detect any effect of MG1 on quantities of total HIV DNA in cells, or total HIV RNA or p24 antigen levels in supernatants.

CONCLUSIONS: MG1 infects and kills latently HIV-infected U1 cells to a greater degree than the HIV-uninfected parent U937 cells and may be a promising model to facilitate further studies of MG1 as a potential therapy for the eradication of latently HIV-infected cells. Further optimization of the experimental approach for primary cell experiments is required in order to determine the effect of MG1 on cells which constitute the HIV reservoir.

\section{P022}

\section{SELECTION PRESSURE TO MAINTAIN APOBEC3H DOES} NOT CORRELATE WITH HIV-1 RESTRICTION EFFICIENCY Love, Robin P; Baig, Tayyba; Chelico, Linda

\section{Saskatoon, SK}

The APOBEC3 family of single stranded (ss) DNA cytosine deaminases have demonstrated varied activity against HIV-1. APOBEC3G targets HIV-1 reverse transcription by catalysing deaminations of cytosine to uracil on nascently synthesized (-)DNA. This results in guanine to adenine mutations in the (+)DNA strand when reverse transcriptase uses uracils as a template. HIV-1 encodes the Vif (viral infectivity factor) protein that counteracts $\mathrm{APOBEC} 3 \mathrm{G}$ by inducing its degradation by ubiquitination. Of the seven APOBEC 3 family members, APOBEC $3 \mathrm{G}$ is most effective at restricting HIV-1 but the mechanistic reason for this advantage is not fully understood. We hypothesized that the differences in effectiveness between APOBEC3 enzymes are due to different mechanisms of scanning ssDNA in search for cytosines. Here we investigate the deamination capabilities of APOBEC3H in comparison to APOBEC3G. We show on synthetic ssDNA substrates that APOBEC3H, like APOBEC3G, is a processive enzyme able to catalyze multiple deaminations during a single encounter with a substrate DNA. APOBEC3H is also capable of inducing mutagenesis of a model provirus synthesized in vitro. However, fewer deaminations were catalyzed by APOBEC3H compared to $\mathrm{APOBEC} 3 \mathrm{G}$ due to differences in their processive movements on DNA. Furthermore, the APOBEC3H-induced mutations on the model provirus, which included the protease active site, did not perturb protease activity as often as APOBEC3G. That APOBEC3H deaminates 5'CTC and APOBEC3G deaminates 5'CCC motifs provides reasoning for the observed difference. We further investigated APOBEC3H sensitivity to both HIV-1 VifIIIB and VifHXB2 determining that indeed APOBEC3H is sensitive to Vif degradation indicating an evolutionary competition between HIV-1 and APOBEC3H. Despite APOBEC3H being less effective than APOBEC3G at inactivating HIV-1 proviral DNA in our model system, it is maintained as a restriction factor suggesting that APOBEC3 enzymes work in concert to restrict HIV-1 replication.

\section{P023}

\section{HIV-1 RESTRICTION FACTORS APOBEC3F AND APOBEC3G ARE DISTINCTIVELY PROCESSIVE}

\section{Ara, Anjuman; Chelico, Linda}

Saskatoon, SK

The members of the APOBEC3 deoxycytidine deaminase family are intracellularly produced by leukocytes and play crucial roles in various cellular functions and host defences by deaminating cytosine residues to uracil in single-stranded DNA. Two family members, APOBEC3F and APOBEC3G are encapsidated into nascent HIV-1 virions during budding, and upon viral infection of new target cells, APOBEC3 deamination activity leads to hypermutation of the viral genome. Although this activity is largely suppressed by HIV-1 Vif (viral infectivity factor) protein, understanding the biochemical mechanisms by which APOBEC3 enzymes are able to lethally hypermutate HIV-1 proviral DNA has potential to lead to development of novel HIV-1 therapeutics. The biochemical nature of APOBEC3G has been extensively studied with respect to HIV-1 restriction. However, the biochemical nature of APOBEC3F has been elusive. In this study, we found that like APOBEC3G, APOBEC3F is a processive enzyme, that is, it can deaminate at least two cytosine residues before completely dissociating from the DNA substrate. Interestingly, the modus operandi of APOBEC3F and APOBEC $3 \mathrm{G}$ processive DNA scanning as determined on synthetic single-stranded DNA substrates is quite distinct. $\mathrm{APOBEC} 3 \mathrm{G}$ uses a balance of sliding and jumping motions while scanning a DNA substrate to find its target 5'CCC deamination motif. In contrast, $\mathrm{APOBEC} 3 \mathrm{~F}$ uses jumping motions that confer processivity over large distances, but fails to use sliding to enable deamination of closely spaced 5'TTC target motifs. Due to these differences in processive movement, we find that in an in vitro model HIV-1 replication assay, $\mathrm{APOBEC} 3 \mathrm{G}$ is more efficient in inducing high numbers of clustered mutations than APOBEC3F, which induces low numbers of scattered mutations. From these results, it appears that the processive DNA scanning mechanism of an APOBEC3 enzyme can be used to predict whether it will be able to cause extensive mutagenesis of HIV-1 proviral DNA.

\section{P024 \\ HIV-1 VIF ALTERS PROCESSIVE SINGLE-STRANDED DNA SCANNING OF THE RETROVIRAL RESTRICTION FACTOR APOBEC3G}

Feng, Yuqing; Chelico, Linda

Saskatoon, SK

APOBEC3G is a host retroviral restriction factor that can inhibit the replication of HIV-1 in the absence of the viral infectivity factor (Vif) protein. Virion encapsidated APOBEC3G can deaminate cytosine to uracil in viral (-)DNA and leads to hypermutation and inactivation of the provirus. APOBEC3G catalyzes these deaminations processively on single-stranded DNA using sliding and jumping movements. HIV-1 accessory protein Vif employs a multifaceted mechanism to counteract the antiviral restriction function of APOBEC3G. Vif is thought to primarily overcome APOBEC $3 \mathrm{G}$ through an interaction that mediates APOBEC3G ubiquitination and results in its proteasomal degradation. Additionally, Vif is able to inhibit APOBEC3G mRNA translation and virion encapsidation. For those APOBEC $3 \mathrm{G}$ molecules that have escaped Vif inhibition and co-package into the nascent virion with Vif, Vif can further inhibit APOBEC3G by dampening its deamination activity in the viral target cell. Here we applied two commonly used Vif variants, VifIIIB and VifHXB2, to determine the molecular mechanism of Vif-mediated inhibition of APOBEC3G deamination activity. Biochemical assays using a model HIV-1 replication assay and synthetic single-stranded or partially double-stranded DNA substrates demonstrate that APOBEC3G has an altered processive DNA scanning mechanism in the presence of Vif. Specifically, VifHXB2 inhibited the jumping and VifIIIB inhibited the sliding movements of APOBEC3G. The absence of such an effect by Vif on degradation-resistant APOBEC3G D128K, a mutant that has an abrogated physical interaction with Vif, indicates that a Vif-APOBEC3G interaction mediates the change in processivity. That the partially processive APOBEC $3 \mathrm{G}$ was less effective at inducing mutagenesis in a model HIV-1 replication assay suggests that Vif co-encapsidation with APOBEC3G can promote sublethal mutagenesis of HIV-1 proviral DNA. Our investigation provides insights for APOBEC3G based HIV-1 therapeutic intervention.

\section{P025 \\ PKR AND THE VIRAL PROTEIN TAT STIMULATE THE ACTIVITY OF THE IRES OF HIV-1}

Charbonneau, Johanie; Gendron, Karine; Cadar, Alexandra E; Ferbeyre, Gerardo; Brakier-Gingras, Léa

Montréal, QC

HIV-1 full-length mRNA, which expresses the structural proteins and enzymes of the virus, contains an internal ribosome entry site (IRES) in 
its 5'untranslated region (5'UTR). This mRNA is translated via a capdependent mode but is hypothesized to use an IRES-dependent mode when cap-dependent translation is impaired. We investigated the effect of the viral protein Tat and that of the double-stranded RNA-dependent kinase PKR on HIV-1 IRES activity. These proteins have opposite effects on cap-dependent translation, PKR impairing this mode of translation by phosphorylating the translation initiation factor eIF2 and Tat stimulating this mode by inhibiting PKR effect. Jurkat T cells were co-transfected with a dual-luciferase reporter containing the 5'UTR of HIV-1 full-length mRNA between the Renilla (Rluc) and the firefly luciferase (Fluc) coding sequences, such that expression of Fluc depends upon HIV-1 IRES, and with a plasmid expressing PKR or the different isoforms of Tat. The IRES activity was assessed by determining the ratio of Fluc activity to the amount of reporter mRNA coding for Rluc and Fluc, the latter being measured by RT-qPCR. We found that the 86 -amino acid Tat, but not mutants (C31S, K41A, K51A), whose structural flexibility is likely altered, increases HIV-1 IRES activity. The 72-amino acid Tat induces a smaller increase while the 101-amino acid Tat does not affect the IRES activity. PKR wild-type or a mutant that does not phosphorylate eIF2 stimulates HIV-1 IRES activity, but a catalytically inactive mutant does not. This relates the effect of PKR to the phosphorylation of a substrate other than eIF2. The effect of Tat on HIV-1 IRES activity was independent of PKR since it is not affected by the presence of the catalytically inactive mutant. We present a model accounting for the effect of Tat and PKR on HIV-1 IRES in relationship with the virus replication cycle (supported by CIHR).

\section{P026 \\ QUANTIFYING THE RELATIONSHIP BETWEEN HIV-1'S MUTATION RATE AND CLINICAL MARKERS OF DISEASE PROGRESSION}

\section{Adams, Andrew E; Rutherford, Alexander; Wittenberg, Ralf} Burnaby, BC

BACKGROUND: Rapid mutation of the HIV-1 genome is thought to be a critical component in HIV-1 disease progression, and is a unique hurdle in developing better vaccines and antiretroviral regimens. Dynamic mathematical models are ideal tools to incorporate the effect of HIV-1 mutation to explain the characteristics of the acute and latent phase. However there has been a deficit in applying these models to quantify differences seen in clinical data.

METHOD: A model describing the acute to latent phase transition was developed using a branching system of differential equations to incorporate the effect of HIV-1 mutation. CD4 ${ }^{+}$and HIV-1 interactions were modeled by describing the physical mechanisms of their production, infection, and relationship to the immune system. Published rates for the binding of HIV-1 to CD4 ${ }^{+}$and the viral mutation rate were used to fit the infection to available clinical data by allowing a variable immune response.

RESULTS: The model reproduces the viral load peak of 5.8log10 HIV-1 RNA copies/ml over 14 days during the acute phase, as described in the literature. Simulations produced a mean of $180 \pm 46 \mathrm{CD} 4^{+}$cells $/ \mathrm{mm}^{3}$ and $268,000 \pm 80,000$ RNA copies/ml, with $\mathrm{CD}^{+}{ }^{+}$counts declining to AIDS levels after 10-15 years of infection. These results agree with published baseline data on latent phase viral load and $\mathrm{CD}^{+}$profiles for untreated individuals.

CONCLUSION: We developed a validated mathematical model of the progression of HIV-1 infection and the human immune response within the host. This model is able to quantify differences observed in the immune response, such as of long-term survivors, by including the adapting structure of HIV-1. By calibrating our model using past data from untreated individuals we can incorporate treatment to utilize the vast resource of data from clinical researchers. This allows our model to be potentially utilized in the development of treatment regimens, vaccines or a cure.
P027

EVIDENCE FOR THE IMPORTANCE OF THE INTEGRASE MUTATION G118R IN SUBTYPES C AND CRF02_A/G

Quashie, Peter K ${ }^{1}$; Mesplede, Thibault ${ }^{1}$; Veres, $\operatorname{Tamar}^{1}$;

Hassounah, Said ${ }^{1}$; Han, Yingshan ${ }^{1}$; Huang, Wei $^{2}$;

Petropoulos, Chris J ${ }^{2}$; Wainberg, Mark $\mathrm{A}^{1}$

${ }^{1}$ Montreal, QC; ${ }^{2}$ South San Francisco, California, USA

BACKGROUND: The integrase mutation G118R, in a patient undergoing therapy, conferred resistance to raltegravir (RAL). In a subtype C, but not subtype B background, G118R was selected in culture with the integrase inhibitor (INI) MK-2048. Dolutegravir (DTG) also selected G118R in subtype C and CRF02_A/G viruses, but not in subtype B. Our objective was to understand the effect of G118R on the activity of integrase and resistance to INSTIs, particularly RAL, elvitegravir (EVG) and DTG, and the preferential selection of G118R in CRFO2_ A/G and subtype $\mathrm{C}$ over subtype $\mathrm{B}$ and the effect of the secondary mutation E138K. METHODS: Strand-transfer and 3' processing activities as well as INSTI inhibitory constants were measured with purified recombinant HIV-1 subtype AG, B, and C enzymes harboring the G118K mutations using micro-titer plate assays. Drug dose-dependent anti-viral phenotypic assays were performed with pNL-43 virus containing either wild-type IN or mutant IN using the Monogram PhenoSense ${ }^{\circledR}$ Integrase phenotypic assay. Data were processed accordingly using GraphPad Prism5. Modeling of HIV-1 IN intasomes based on the published structures of prototype foamy virus integrase informed on mechanism.

RESULTS: In both subtype B and CRF_A/G, G118R resulted in severely restricted integration activity but not in subtype C. In the three subtypes resistance to DTG was in the order CRF02_A/G> subtype B> subtype C and in subtypes $B$ and $C$ cases resistance was increased in the presence of E138K. In CRF02_A/G integrase the presence of G118R in isolation is sufficient to cause greater than $23 \mathrm{FC}$ in resistance to RAL.

CONCLUSION: In the context of ongoing drug therapy, G118R may be clinically relevant in patients harboring subtype $\mathrm{C}$ and CRF02_A/G viruses and who are undergoing therapy with RAL or DTG. This may be further elucidated as data from DTG usage to treat non-B subtypes becomes more available.

\section{P028}

\section{CRF01_AE SUBTYPE IS ASSOCIATED WITH HIGHER FREQUENCY OF X4 TROPISM AND FASTER DISEASE PROGRESSION IN CHINESE HIV-1 SEXUALLY INFECTED PATIENTS}

Li, Taisheng'; Li, Yijia'; Han, Yang ${ }^{1}$; Xie, Jing ${ }^{1}$; Gu, Lijun² Routy, Jean-Pierre ${ }^{3}$; Wang, Huanling ${ }^{1}$; Ishida, Takaomi ${ }^{1,2}$; Iwamoto, Aikichi ${ }^{1,2}$

${ }^{1}$ Beijing, China; ${ }^{2}$ Tokyo, Japan; ${ }^{3}$ Montreal, QC

BACKGROUND: Molecular epidemiology of HIV-1 CRF01_AE subtype and risk factors for fast HIV-1 progression remain poorly understood in China. METHODS: We did preliminary analysis on viral tropism by utilizing 119 samples available from our multicenter cohort. Tropism was determined by V3 loop sequence and Geno2pheno algorithm. Data from 235 treatment-naïve patients (including the 119 patients in tropism analysis) in this cohort infected sexually with date of estimated seroconversion (EDS) were also retrospectively evaluated. Median time from EDS to AIDS was analyzed with Kaplan-Meier test. Hazard ratio was determined with Cox proportional model.

RESULTS: CRF01_AE subtype was predominant (47.5\%), especially in men having sex with men (MSM) group. We discovered that CRF01_AE subtype was associated with higher frequency of $\mathrm{X} 4$ tropism (shown in Table 1; CRF01_AE, 42.2\%; C/CRF07_BC/CRF08_BC, 0\%; B, 6.3\%; $\mathrm{p}<0.001)$, the tropism associated with accelerated disease progression. Then we probe the association between CRF01_AE subtype and HIV progression and discovered that CRF01_AE subtype was associated with faster progression from EDS to AIDS (4.7 vs. 6.4 years, $p=0.009$ ) compared with non-CRF01_AE subtypes. In multivariate model, the adjusted hazard ratio (aHR) of CRF01_AE was 1.48 (95\% CI, 1.03-2.12, p=0.033), independent of HIV-1 viral load. 


\begin{tabular}{lccc} 
& R5 tropism & R5/X4 dual tropism & X4 tropism \\
\hline CRF01_AE [n (\%)] & $23(35.9)$ & $14(21.9)$ & $27(42.2)$ \\
C/CRF07_BC/CRF08_BC [n (\%)] & $38(97.4)$ & $1(2.6)$ & $0(0)$ \\
B/B' [n(\%)] & $13(81.3)$ & $2(12.5)$ & $1(6.2)$ \\
\hline
\end{tabular}

CONCLUSIONS: CRF01_AE, a predominant HIV-1 subtype in Chinese HIV-1 sexually infected patients, is associated with fast progression to AIDS, which might be ascribed to high frequency of X4 tropism.

CLINICAL SCIENCES / SCIENCES CLINIQUES

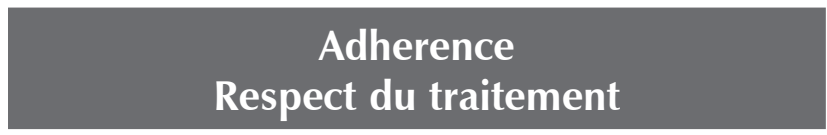

P029

\section{ANTIRETROVIRAL ADHERENCE IN AN OUTPATIENT HIV} CLINIC

Giguere, Pierre; Robinson, Jessica

Ottawa, ON

BACKGROUND: Adherence to antiretroviral (ARV) medication is important for viral suppression and to avoid the development of resistance in patients with HIV.

OBJECTIVES: The primary objective was to describe and compare ARV adherence using two methods: patient reported and provincial claims database information. Secondary objectives were to identify patient characteristics associated with ARV adherence rate, to characterize factors that lead to discrepancies between patient reported and provincial claims database adherence and to identify associations between adherence and virologic response.

METHODS: All consecutive patients attending an appointment at The Ottawa Hospital (TOH) Immunodeficiency Clinic from February 13 until March 9, 2012 were approached for enrolment. 210 patients were enrolled and each patient completed the MASRI, a self-reported adherence questionnaire. Adherence was also calculated for each patient using the Ontario Drug Benefit (ODB) database.

RESULTS: Adherence results were heavily skewed in favour of excellent adherence. The median MASRI reported adherence was $100 \%$, and the median ODB reported adherence was $98 \%$. When adherence data was organized in $25 \%$ interval groups, MASRI and ODB adherence weakly correlated $(\mathrm{r}=0.188, \mathrm{p}=0.042)$. Patient characteristics associated with ARV adherence of $<75 \%$ were female gender (OR 2.73, 95\% CI 1.62-4.61, p=0.005) and non-MSM (OR 1.96, 95\% CI 1.712.25, $\mathrm{p}=0.004$ ). A factor leading to a discrepancy between MASRI and ODB adherence was missed appointments (OR 2.56, CI 1.18-5.56, $\mathrm{p}=0.017$ ). No characteristics were found to correlate with virologic failure and adherence, because of the limited number of therapeutic failures $(n=3)$. However, past treatment failure was found to correlate with a viral load greater than 40 copies/mL (OR 2.79, CI 1.20-6.51, $\mathrm{p}=0.03$ )

CONCLUSION: Patients at the TOH Immunodeficiency Clinic had excellent adherence when measured by the MASRI and ODB database. MASRI and ODB adherence were weakly correlated. Interventions towards improving adherence should target women living with HIV.

\section{P030}

\section{TAKING IT TO HAART: ADHERENCE AND BARRIERS TO} ADHERENCE IN SASKATOON, SASKATCHEWAN

Wilmot, Jillian $\mathbf{M}^{1}{ }^{12}$; Hagen, Briana $\mathbf{N}^{1}$; Skinner, Stuart $\mathbf{J}^{1}$;

Stone, Shannon ${ }^{1}$; Larkan, Fiona ${ }^{2}$

${ }^{1}$ Saskatoon, SK; ${ }^{2}$ Dublin, Ireland

Medication adherence is essential for HIV management; however, there is little agreement on factors affecting adherence. The HIV population in Saskatchewan is unique, meriting local research to inform local clinical practice. In order to develop initiatives to improve adherence in Saskatoon it is imperative to understand the current levels of adherence and what factors contribute to non-adherence.

Data was collected using an interview tool and a calculation of adherence based on prescription refill history. Participants were HIV patients seen within Saskatoon Health Region. The interview included a self-report adherence measure, and used structured and open-ended questions to examine barriers to adherence. The Medication Refill Adherence calculation generated an estimate of adherence based on refill history from the 6 months prior.

Fifty-nine percent of participants $(n=100)$ self-identified as being of Aboriginal descent, 44\% completed less than high school level education, $59 \%$ indicated a current or remote history of injection drug use, and $28 \%$ were engaged with methadone maintenance therapy. Median adherence was $99.0 \%$ (self-report) and $98.9 \%$ (refill). Based on definitions of optimal adherence; $64.0 \%$ and $87.2 \%$ of participants were achieving adherence rates $\geq 95 \%$ and $80 \%$, respectively. Twenty-two percent of participants disclosed missing $\geq 1$ dose in the previous week. The most commonly indicated barriers were forgetting to take doses, being away from home, being too busy, or being too drunk or high. There was a statistically significant negative correlation between adherence and the quantity of barriers participants experienced.

While adherence rates established by this study are encouraging, a proportion of individuals are not achieving the necessary levels of adherence to experience full treatment benefits from HAART. Low levels of formal education and high rates of substance use reflect the challenges in engagement and adherence. Interventions should focus on support to mitigate the effects of barriers to adherence experienced by this population.

\section{P031}

FACTORS ASSOCIATED WITH VARYING LEVELS OF HAART PRESCRIPTION REFILL ADHERENCE IN BRITISH COLUMBIA, CANADA

Lourenco, Lillian' ${ }^{\text {; }}$ Ding, Erin ${ }^{1}$; Shurgold, Susan';

Colley, Guillaume ${ }^{1}$; Yip, Benita ${ }^{1}$; Lima, Viviane'; Samji, Hasina1;

Barrios, Rolando ${ }^{1}$; Montaner, Julio $\mathrm{S}^{1}$; Hogg, Robert $\mathbf{S}^{1,2}$;

Moore, David

${ }^{1}$ Vancouver; ${ }^{2}$ Burnaby, BC

BACKGROUND: Benefits of HAART are compromised both in the presence of imperfect adherence and treatment interruption. We studied levels of prescription refill adherence and characteristics associated with them.

METHODS: We analyzed data from BC Drug Treatment Program (DTP) participants ( $\geq 19$ years) who initiated HAART prior to 2011 and completed one year of treatment follow-up in 2011. We compared characteristics of participants across four levels of prescription refill adherence in 2011: $\geq 95 \%, 80 \%-<95 \%, 40 \%-<80 \%$ and $<40 \%$. Adherence groupings were validated against immunologic, resistance and mortality outcomes in previous analyses. We used the Pearson Chi-square test to compare categorical variables and the Kruskal Wallis test to compare continuous variables across all adherence levels.

RESULTS: A total of 3864 individuals ( $81 \%$ male, median age: 48 (IQR: 41-55)), met inclusion criteria. Of these, 2592(67\%), 574(15\%), $464(12 \%)$ and $234(6 \%)$ achieved $\geq 95 \%, 80 \%-<95 \%, 40 \%-<80 \%$ and $<40 \%$ prescription refill adherence in 2011 , respectively. Individuals with $<40 \%$ prescription refill adherence were more likely to be female, younger, have a history of IDU, be of Aboriginal descent, ever tested positive for $\mathrm{HCV}$, and had a higher median CD4 cell count at baseline (all $\mathrm{p}<0.001)$ (Table 1$)$. Seeing a specialist was also associated with low refill adherence $(\mathrm{p}=0.004)$; however, it is likely this is because sicker people tend to be seen by specialists. Time on HAART was not significantly associated with poorer prescription refill adherence $(\mathrm{p}=0.529)$.

CONCLUSIONS: Our analysis found reducing levels of adherence with increasing levels of marginalization (as marked by Aboriginals, IDUs and females). Additional supports for adherence should be targeted towards individuals with these characteristics or service providers who care for them. 
Table 1. Characteristics Associated with Four Levels of HAART Prescription Refill Adherence in 2011 in British Columbia

\begin{tabular}{|c|c|c|c|c|c|}
\hline & \multicolumn{4}{|c|}{ Prescription Refill Adherence } & \multirow[b]{2}{*}{ p-value } \\
\hline & $\geq 95 \%$ & $<95 \%-80 \%$ & $<80 \%-40 \%$ & $<40 \%$ & \\
\hline$\overline{\text { Total }}$ & $2592(67 \%)$ & $574(15 \%)$ & $464(12 \%)$ & $234(6 \%)$ & \\
\hline \multicolumn{6}{|l|}{ Gender, $n(\%)$} \\
\hline Female & $387(15 \%)$ & $117(20 \%)$ & $148(32 \%)$ & $84(36 \%)$ & $<0.001$ \\
\hline Male & $2205(85 \%)$ & $457(80 \%)$ & $316(68 \%)$ & $150(64 \%)$ & \\
\hline History of IDU, n(\%) & & & & & $<0.001$ \\
\hline No & $1381(53 \%)$ & $211(37 \%)$ & $167(36 \%)$ & $75(32 \%)$ & \\
\hline Yes & $790(30 \%)$ & $288(50 \%)$ & $255(55 \%)$ & $133(57 \%)$ & \\
\hline Unknown & $421(16 \%)$ & $75(13 \%)$ & $42(9 \%)$ & $26(11 \%)$ & \\
\hline Aboriginal Ancestry, $n(\%)$ & & & & & $<0.001$ \\
\hline No & $1199(46 \%)$ & $228(40 \%)$ & $181(39 \%)$ & $78(33 \%)$ & \\
\hline Yes & $225(9 \%)$ & $101(18 \%)$ & $99(21 \%)$ & $46(20 \%)$ & \\
\hline Unknown & $1168(45 \%)$ & $245(43 \%)$ & $184(40 \%)$ & $110(47 \%)$ & \\
\hline Hepatitis C, n(\%) & & & & & $<0.001$ \\
\hline Negative & $1614(62 \%)$ & $251(44 \%)$ & $181(39 \%)$ & $85(36 \%)$ & \\
\hline Positive & $817(32 \%)$ & $294(51 \%)$ & $254(55 \%)$ & $135(58 \%)$ & \\
\hline Unknown & $161(6 \%)$ & $29(5 \%)$ & $29(6 \%)$ & $14(6 \%)$ & \\
\hline Type of follow-up physician, $n$ & & & & & 0.004 \\
\hline Non-specialist & $2097(81 \%)$ & $436(76 \%)$ & $369(80 \%)$ & $91(71 \%)$ & \\
\hline Specialist & $495(19 \%)$ & $138(24 \%)$ & $95(20 \%)$ & $37(29 \%)$ & \\
\hline $\begin{array}{l}\text { Age in } 2011 \text { (years), median } \\
\text { (IQR) }\end{array}$ & $49(42-55)$ & $48(41-54)$ & $45(39-52)$ & $44(39-50)$ & $<0.001$ \\
\hline $\begin{array}{l}\text { Baseline CD4 (cells/mm3), } \\
\text { median (IQR) }\end{array}$ & $220(120-330)$ & $200(110-310)$ & $210(110-350)$ & $250(140-465)$ & $<0.001$ \\
\hline $\begin{array}{l}\text { Baseline viral load }(\log 10 \\
\text { copies/mL), median (IQR) }\end{array}$ & $4.95(4.44-5.00)$ & $4.94(4.44-5.00)$ & $4.80(4.28-5.00)$ & $4.73(3.98-5.00)$ & $<0.001$ \\
\hline $\begin{array}{l}\text { Physician Experience, } \\
\text { median (IQR) }\end{array}$ & $209(49-474)$ & $166(32-376)$ & 207 (36-324) & 212 (77-352) & 0.002 \\
\hline $\begin{array}{l}\text { Duration of ARV use } \\
\text { (months), median (IQR) }\end{array}$ & $72(39-128)$ & 73 (39-138) & $76(41-127)$ & $81(40-137)$ & 0.529 \\
\hline
\end{tabular}

\section{P032}

\section{SUB-OPTIMAL STUDY DRUG ADHERENCE DESPITE DETECTABLE URINE DRUG LEVELS IN A RANDOMIZED CONTROLLED TRIAL}

Szadkowski, Leah M; Raboud, Janet M; Pequeno, Priscila; Walmsley, Sharon L; Tan, Darrell H

\section{Toronto, ON}

BACKGROUND: Biological measures of drug adherence, such as drug detection in urine or plasma, are often considered more accurate than self-report or pill count measures. However, urine drug levels depend on drug half-life, dosing times, and other factors, and can produce detectable drug levels despite recent non-adherence. We assessed self-reported and pill count measures of adherence among patients with detectable urine levels of valacyclovir in the VALIANT pilot randomized controlled trial (RCT).

METHODS: Sixty HIV, HSV-2 co-infected adults on suppressive antiretroviral therapy were randomized 1:1:1 to receive 12 weeks of placebo, low dose (500mg BID), or high dose (1g BID) valacyclovir. At 6- and 12-week visits, returned pills were counted, urine samples were taken, and patients completed a modified version of the ACTG Adherence Questionnaire. Questionnaire responses and pill count results were summarized for patients with detectable urine valacyclovir levels at each visit.

RESULTS: Of 40 participants randomized to receive active drug, 38 and 37 had detectable urine valacyclovir levels at the 6 and 12-week visits respectively. However, self-reported and pill count measures for these participants revealed considerable non-adherence, and are summarized in the Table. No placebo participants had detectable drug levels. All participants maintained HIV virologic suppression.
Table: Measures of Adherence among Patients with Valacyclovir detectable in urine

Low Dos

High Dose

Week $6(n=19) \quad$ Week $12(n=19)$ Week $6(n=19)$ Week $12(n=18)$

Proportion of participants who self-reported:

\begin{tabular}{lcccc} 
Missing $\geq 1$ dose in last 4 days & $2(11 \%)$ & $3(16 \%)$ & $4(21 \%)$ & $3(17 \%)$ \\
Missing $\geq 1$ dose in the last week & $4(21 \%)$ & $3(16 \%)$ & $5(26 \%)$ & $4(22 \%)$ \\
Missing $\geq 1$ dose last weekend & $3(16 \%)$ & $4(21 \%)$ & $5(26 \%)$ & $2(11 \%)$ \\
$\begin{array}{l}\text { Missing } \geq 1 \text { dose on a weekend } \\
\text { in last 3 mo. }\end{array}$ & $6(32 \%)$ & $6(32 \%)$ & $7(37 \%)$ & $7(39 \%)$ \\
$\begin{array}{l}\text { Missing } \geq 1 \text { dose during study } \\
\text { period }\end{array}$ & $11(58 \%)$ & $11(58 \%)$ & $11(58 \%)$ & $13(72 \%)$ \\
$\begin{array}{l}\text { Proportion of pills consumed, by pill count } \\
\text { Median }\end{array}$ & $99 \%$ & $98 \%$ & $95 \%$ & $95 \%$ \\
Interquartile range & $92-101 \%$ & $91-100 \%$ & $80-99 \%$ & $82-99 \%$ \\
Range & $52-107 \%$ & $13-106 \%$ & $40-100 \%$ & $49-103 \%$ \\
\hline
\end{tabular}

CONCLUSIONS: In this 12 -week RCT among patients on suppressive antiretroviral therapy, valacyclovir was detectable in the urine of almost all participants randomized to receive active drug, despite a high frequency of suboptimal adherence as measured by questionnaire and pill counts. Urine tests alone may be an insensitive measure of valacyclovir non-adherence.

\section{P033}

VIRAL SUPPRESSION IN HIV+ PATIENTS WITH SUBOPTIMAL ADHERENCE TO HAART

Mah Ming, Jinell; Lee, Kathy; Krentz, Hartmut B; Gill, MJ

\section{Calgary, $\mathrm{AB}$}

OBJECTIVE: Established antiretroviral (ARV) adherence rates of $>95 \%$ have been the standard measure for best outcomes in HIV disease management. However, current ARV regimens are reputed to have increased potency allowing for some nonadherence although how much has not yet been determined. Our objective was to observe the patient characteristics of those patients that remain undetectable despite long periods of suboptimal adherence

METHODS: Retrospective chart review, database analysis, and medication dispensing date/quantity monitoring was completed at the Southern Alberta Clinic for virally suppressed patients with suboptimal adherence (i.e. $<85 \%$ ) while on ARV therapy for $>12$ months. CD4, viral load, AIDS, ARV exposure (including mono/dual therapy, adherence rate, and regimen type) were collected

RESULTS: We found 17 patients who remained virally suppressed (i.e. $\mathrm{VL}<40 \mathrm{c} / \mathrm{mL}$ ) with no 'blips' for a median of 1053 days (total ARV exposure for a median of 4315 days) on their current HAART regimen despite a median adherence rate of $72 \%$. Two naïve patients were suppressed on their first ARV regimen; the remaining 15 patients were suppressed on their second regimen. HAART regimens consist of $76 \%$ protease inhibitor based (Lopinavir/ritonavir or Daurunavir) or 24\% nonnucleoside reverse transcriptase inhibitor based (Efavirenz or Etravirine). Patients were switched to a median of 4 different regimens. Median current CD4 was $472 / \mathrm{mm} 3$; median nadir CD4 was $184 / \mathrm{mm} 3.11 \%$ of patients had experienced AIDS, 23\% genotypic resistance, $47 \%$ past mono or dual ARV therapy, and $52 \%$ had co-morbidities. No language barriers, mental health, IVDU, or homelessness in this population

CONCLUSION: Certain patients were identified to remain virally suppressed for long periods of time despite major suboptimal adherence to HAART. The majority of patients were using a regimen containing a protease inhibitor 
P034

HIGH-INTENSITY CANNABIS USE AND ADHERENCE TO HIGHLY-ACTIVE ANTIRETROVIRAL THERAPY (HAART) AMONG ILLICIT DRUG USERS IN VANCOUVER, CANADA

Slawson, Greg; Milloy, M-J; Wood, Evan; Balneaves, Lynda; Simo, Annick; Guillemi, Sylvia; Hogg, Robert S; Montaner, Julio; Kerr, Thomas

Vancouver, BC

BACKGROUND: Cannabis is increasingly prescribed clinically and utilized by people living with HIV/AIDS (PLWHA) to address symptoms of HIV disease and to manage side effects of highly-active antiretroviral therapy (HAART). In light of concerns about the possibly deleterious effect of psychoactive drug use on adherence to HAART, we sought to determine the relationship between high-intensity cannabis use and adherence to HAART among a community-recruited cohort of HIVpositive illicit drug users.

METHODS: We used data from the ACCESS study, an ongoing prospective cohort study of HIV-seropositive illicit drug users linked to comprehensive HAART dispensation records in a setting of universal no-cost HIV care. We estimated the association between at least daily cannabis use in the last six months, measured longitudinally, and the likelihood of optimal adherence to HAART during the same period, using a multivariate linear mixed-effects model accounting for relevant socio-demographic, behavioral, clinical and structural factors.

RESULTS: From May 2005 to May 2012, 523 HIV-positive illicit drug users were recruited and contributed 1215 person-years of observation. At baseline, $121(23.1 \%)$ participants reported at least daily cannabis use. In bivariate and multivariate analyses we did not observe an association between using cannabis at least daily and optimal adherence to prescribed HAART (Adjusted Odds Ratio $=1.12$, 95\% Confidence Interval $[95 \%$ CI]: $0.76-1.64$, p-value $=0.555$.)

CONCLUSIONS: High-intensity cannabis use was not associated with adherence to HAART. These findings suggest cannabis may be utilized by PLWHA for medicinal and recreational purposes without compromising effective adherence to HAART.

\section{P035}

WELTEL BC1: A PILOT STUDY EXPLORING FEASIBILITY AND ACCEPTABILITY OF A MHEALTH INTERVENTION TO IMPROVE CARE FOR HIV POSITIVE PATIENTS ON HIGHLY ACTIVE ANTIRETROVIRAL THERAPY (HAART) IN BRITISH COLUMBIA (BC), CANADA

Murray, Melanie C; Borek, Natasha V; Graham, Rebecca;

Maan, Evelyn J; van der Kop, Mia L; Friesen, Karen; Levine, Sarah; Abaki, Joshua; Pick, Neora; Maginley, Juanita; Ogilvie, Gina;

Money, Deborah M; Lester, Richard

Vancouver, BC

INTRODUCTION: HAART improves health and survival of HIV+ individuals. However, engagement in care and medication adherence are essential to prevent resistance, morbidity and mortality. In an RCT in Kenya, WelTelKenya1, a weekly mHealth (mobile phone technology for health care) intervention improved HAART adherence and viral suppression. We conducted a six-month pilot study of a weekly interactive text-messaging intervention using the WelTel model with 25 participants to assess acceptability and feasibility in HIV+ individuals taking HAART in BC.

METHODS: Between January and May 20125 each from 5 groups ('youth', 'mature', 'English as a second language', 'remote', and 'CD4 $<200$ ') were recruited from the Oak Tree Clinic. Participants were given a cell phone with unlimited texting if they did not have one, then received weekly text messages stating "How are you?" A clinic nurse responded to non- and negative responses. Demographics, CD4s, viral loads, adherence data, and weekly responses were collected throughout the study. Baseline and exit semi-structured qualitative interviews/questionnaires were conducted with participants and health care staff to assess the intervention. Interviews were audio-recorded and transcribed verbatim. Thematic analysis was conducted.
RESULTS: Median age 46 (range 16-60), 80\% female, mean nadir and baseline CD4 150 and 361 cells/mL respectively. Weekly response rates varied by targeted group, and were highest in those with low CD4. Participants and health workers perceived the intervention as feasible, acceptable, and an improvement in care. Of 20 exit interviews completed, all would recommend the intervention to a peer. Additionally, mean HIV VL declined by $0.28 \mathrm{log}$, mean CD 4 increased by 53 cells $/ \mathrm{mL}$ and physician recorded adherence increased in $29 \%$ of participants over the 6-month intervention.

CONCLUSIONS: Participants were very receptive to the intervention and believe it can improve care. A study of the intervention's efficacy (for improving clinical outcomes such as viral load etc.) is underway.

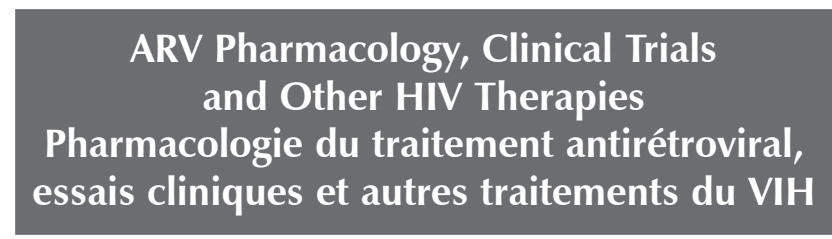

\section{P036}

\section{PLASMA NEVIRAPINE LEVELS FOLLOWING TREATMENT SWITCH FROM IMMEDIATE RELEASE TO EXTENDED RELEASE TABLETS}

McGovern, Rachel A; Lee, Guinevere; Dong, Weiyan;

Woods, Conan K; Yip, Benita; Lepik, Katherine J;

Harrigan, Richard

Vancouver, BC

BACKGROUND: Clinical trials demonstrate that extended-release Nevirapine (NVP-XR, $400 \mathrm{mg}$ ) taken once daily is non-inferior to immediate-release (NVP-IR, $200 \mathrm{mg}$ ) taken twice daily. Many patients in British Columbia take NVP-IR as a once-daily dose ( $2 \times 200 \mathrm{mg}$ tablets). To date there has been no comparison of plasma NVP concentrations in patients switching from NVP-IR once-daily to NVP-XR in British Columbia.

METHODS: Consenting patients having switched from NVP-IR oncedaily regimen to NVP-XR between April and June, $2012(\mathrm{~N}=76)$ were included in the analysis. Three plasma samples were collected for each patient, two collected during NVP IR once-daily therapy and one $\geq 32$ days after switching to NVP XR. Samples were untimed relative to dosing. NVP concentrations were measured using HPLC-tandem mass spectrophotometry.

RESULTS: Abacavir/lamivudine or emtricitabine/tenofovir were in the background regimen of $95 \%$ of patients. Prior to starting NVP-XR, 70/76 patients had undetectable $\mathrm{pVL}(<50$ copies $/ \mathrm{mL})$. After a median of 79 days NVP-XR exposure, 73 patients had undetectable pVL. Of patients with $>50$ copies $/ \mathrm{mL}$, the post-therapy median viral load was 58 copies/mL (IQR 56 to 173 ).

\begin{tabular}{|c|c|c|c|c|}
\hline & \multicolumn{3}{|c|}{$\begin{array}{l}\text { NVP Concentrations (ng/mL) } \\
\text { on NVP-IR } 2 \times 200 \mathrm{mg} \text { once daily }\end{array}$} & \multirow{2}{*}{$\begin{array}{c}\text { NVP Concentrations } \\
\text { (ng/mL) on NVP-XR } \\
400 \mathrm{mg} \text { once daily } \\
\text { Median } 79 \text { days on } \\
\text { NVP-XR }\end{array}$} \\
\hline & $\begin{array}{r}\text { Time point } 1 \text { (Median } 10 \\
\text { days before NVP-XR) }\end{array}$ & $\begin{array}{l}\text { Time Point } 2 \text { (Median } 11 \\
\text { days before NVP-XR) }\end{array}$ & $\begin{array}{l}\text { Average Before } \\
\text { Switch }\end{array}$ & \\
\hline Median & 5080 & 4810 & 4940 & 3930 \\
\hline IQR & $4060-6170$ & $3770-5650$ & $3920-5910$ & $3290-5020$ \\
\hline Minimum & 1610 & 1600 & 1605 & 1380 \\
\hline Maximum & 10900 & 11800 & 10350 & 10100 \\
\hline
\end{tabular}

There was no correlation between NVP concentrations during NVP-IR and NVP-XR treatment $\left(R^{2}=0.06\right)$. After switching to NVP-XR, median untimed NVP levels dropped by $23 \%$. Untimed NVP levels were lower after switching to NVP-XR in $68 \%$ patients, but still within the presumed therapeutic range. As an untimed study we cannot comment on peak and trough levels.

CONCLUSION: Despite an average decrease in untimed NVP levels after switching to NVP-XR in this short-term analysis, patients continue to suppress their viral load below detectable levels. 
P037

PH DEPENDENCE OF ATAZANAVIR INTESTINAL ABSORPTION AND ITS DRUG-DRUG INTERACTIONS WITH ACID-REDUCING AGENTS

Kis, Olena; Walmsley, Sharon; Bendayan, Reina Toronto, ON

BACKGROUND: Oral bioavailability of the HIV protease inhibitor, atazanavir, is highly sensitive to food intake and co-administration of acid-reducing agents such as proton-pump inhibitors (eg, omeprazole) and $\mathrm{H} 2$-receptor antagonists (eg, cimetidine). These interactions may be mediated by changes in the gastrointestinal $\mathrm{pH}$; however, to date little is known about the $\mathrm{pH}$-dependence of atazanavir intestinal absorption or contribution of other mechanisms, such as intestinal drug transport or metabolism, to these interactions.

METHODS: In vitro intestinal permeability (Papp) of $3 \mathrm{H}$-atazanavir across Transwell-grown Caco-2 monolayers was examined at different extracellular $\mathrm{pH}$ levels ( 4.5 to 8.5 ), in the presence or absence of acid reducers, or in a buffer simulating intestinal fluid in the fed state $(\mathrm{pH} 5.0$, $15 \mathrm{mM}$ sodium taurocholate, $3.75 \mathrm{mM}$ lecithin) or fasted state $(\mathrm{pH} 6.5$, $3 \mathrm{mM}$ sodium taurocholate, $0.75 \mathrm{mM}$ egg lecithin). In situ permeability (Peff) of atazanavir was investigated by single-pass perfusion of rat jejunum and ileum to further assess the effect of luminal $\mathrm{pH}$, food intake, and acid-reducing agents on atazanavir absorption.

RESULTS: In Caco-2 cells, atazanavir accumulation and apical-tobasolateral permeability were enhanced by acidic apical $\mathrm{pH}$, and were significantly higher in fed-state compared to fasted-state buffer. In situ permeability of atazanavir was also higher at acidic luminal $\mathrm{pH}$ compared to neutral or basic $\mathrm{pH}$. Omeprazole strongly inhibited efflux transport of atazanavir in Caco-2 cells. However, this effect was not observed in situ, suggesting that clinical drug-drug interactions between omeprazole and atazanavir are $\mathrm{pH}$-mediated.

CONCLUSIONS: Our in vitro and in situ data demonstrate that atazanavir intestinal permeability is strongly dependent on intestinal lumen $\mathrm{pH}$. Acid-reducing agents (ie, omeprazole, cimetidine) pharmacologically increase gastric and intestinal fluid $\mathrm{pH}$. Although some acid reducers such as omeprazole can also inhibit atazanavir metabolism and efflux in vitro, their effect on luminal $\mathrm{pH}$ appears to be primarily responsible for the clinical interactions observed between atazanavir and acid reducers. (Supported by CANFAR)

\section{P038}

\section{ASSESSMENT OF CHLOROQUINE AS A MODULATOR OF T CELL IMMUNE ACTIVATION TO IMPROVE CD4 RECOVERY IN HIV-INFECTED SUBJECTS RECEIVING ANTIRETROVIRAL THERAPY (CIHR/CTN 246 STUDY) \\ Routy, Jean-Pierre $^{1}$; Angel, Jonathan ${ }^{2}$; Patel, Mital ${ }^{1}$; Lee, Terry ${ }^{3}$; Singer, Joel ${ }^{3}$; Kanagaratham, Cynthia ${ }^{1}$; Radzioch, Danuta ${ }^{1}$; Kema, Ido4; Preziosi, Helen'; Ancuta, Petronela ${ }^{1}$; Jenabian, Mohammad-Ali ${ }^{1}$ \\ ${ }^{1}$ Montreal, QC; ${ }^{2}$ Ottawa, ON; ${ }^{3}$ Vancouver, BC; ${ }^{4}$ Groningen, Netherlands}

BACKGROUND: Chloroquine (CQ) inhibits endosomal Toll-like receptor 7 and 9 signaling in plasmacytoid dendritic cells (DCs) and is used as an anti-inflammatory drug for lupus erythematosus and rheumatoid arthritis. In patients with low CD4 count despite successful ART, we assessed whether CQ will improve CD4 recovery by decreasing immune activation.

METHODS: Nineteen adults with CD4 count $\leq 350$ cells $/ \mu \mathrm{L}$ and viral load (VL) $<50$ copies $/ \mathrm{mL}$ for at least 36 weeks, received $250 \mathrm{mg}$ of CQ orally, once a day for 24 weeks. Side effects, VL, CD4 and CD8 counts, D-dimer, CRP, T-cell activation, Kynurerine/Tryptophan ratio (the marker of IDO enzyme inflammatory activity in DCs) and 11 inflammatory cytokines were assessed before CQ, after week 24 of treatment and 12 weeks after drug discontinuation. Statistical analyses were assessed using Wilcoxon signed-rank test.

RESULTS: All subjects maintained a VL $<50$ copies/ml during the study and no grade 3 or 4 adverse events occurred although one subject discontinued due to a grade 2 GI side effect. Median (interquartile range) of CD4 and CD8 at baseline were 186 (127 to 278) and 541 (320 to 766) cells $/ \mu \mathrm{L}$, respectively. During the study period, CD4 (mean change=16.7, 95\% CI -6.1 to 39.5 ) and CD8 T-cell counts, D-dimer, CRP, percentage of CD38/HLA-DR on CD4 and CD8 T cells did not change. We then assessed CQ impact on IDO activity where Kynurerine levels decreased $(\mathrm{P}=0.06)$, but the Kynurerine/Tryptophan ratio remained unchanged $(\mathrm{P}>0.05)$. Levels of IL-17A and IFNa2 were increased after 24 weeks of treatment. Conversely, TNF $\alpha$, IL-7 and IL-8 were significantly decreased after 12 weeks of drug discontinuation.

CONCLUSION: Chloroquine at the dose of $250 \mathrm{mg}$ daily during 24 weeks was safe for patients with low CD4 T cells despite effective ART. No improvement in CD4 T-cell recovery and no decrease in immune activation were observed during therapy.

\section{P039}

\section{ELVITEGRAVIR/COBICISTAT/EMTRICITABINE/TENOFOVIR DF HAS DURABLE EFFICACY AND DIFFERENTIATED SAFETY COMPARED TO EFAVIRENZ/EMTRICITABINE/ TENOFOVIR DF AT WEEK 96 IN TREATMENT-NAÏVE HIV-1 INFECTED PATIENTS}

Zolopa, $\mathrm{A}^{1}$; Gallant, J' ; Cohen, $\mathrm{C}^{3}$; Sax, $\mathrm{P}^{3}$; deJesus, $\mathrm{E}^{4}$; Mills, $\mathrm{A}^{5}$; Wohl, $\mathrm{D}^{6}$; Borg, $\mathrm{P}^{7}$; Liu, $\mathrm{H} \mathrm{C}^{8}$; Rhee, $\mathbf{M ~ S}^{8}$; Szwarcberg, $]^{8}$ ${ }^{1}$ Palo Alto, California; ${ }^{2}$ Baltimore, Maryland; ${ }^{3}$ Boston, Massachusetts; ${ }^{4}$ Orlando, Florida; ${ }^{5}$ Los Angeles, California; ${ }^{6}$ Chapel Hill, North Carolina, USA; ${ }^{7}$ Mississauga, ON; ${ }^{8}$ Foster City, California, USA

PURPOSE OF THE STUDY: Week 96 interim data of an ongoing, non-inferiority, randomized, double-blind, double-dummy, activecontrolled Phase 3 trial of elvitegravir/cobicistat/emtricitabine/tenofovir DF (EVG/COBI/FTC/TDF) compared to efavirenz/emtricitabine/tenofovir $\mathrm{DF}(\mathrm{EFV} / \mathrm{FTC} / \mathrm{TDF})$ in treatment naïve patients.

METHODS: Key eligibility criteria included HIV-1 RNA $\geq 5,000 \mathrm{c} / \mathrm{mL}$ and eGFR $\geq 70 \mathrm{~mL} / \mathrm{min}$. Virologic success (HIV-1 RNA $<50 \mathrm{c} / \mathrm{mL}$ ) was assessed per snapshot algorithm. Adverse events and laboratory data were collected prospectively.

RESULTS: Seven hundred patients were randomized and treated. Virologic success of EVG/COBI/FTC/TDF was non-inferior to EFV/FTC/ TDF at Week 48 ( $88 \%$ vs $84 \%$,) and Week $96(84 \%$ vs $82 \%)$ and similar in patients with baseline HIV-1 RNA $>100,000 \mathrm{c} / \mathrm{mL}$ ( $81 \%$ vs $83 \%$ ). Mean CD4 cell increase (cells $/ \mathrm{mm}^{3}$ ) was 295 vs 273 . Emergent resistance was infrequent ( $3 \%$ vs $3 \%)$. Rates of study drug discontinuation due to adverse events ( $5 \%$ vs $7 \%$ ) and renal events ( $2 \%$ vs $0 \%)$ were low and comparable through Week 96. Two patients discontinued EVG/COBI/ FTC/TDF since Week 48 due to serum creatinine (Cr) increase without features of proximal renal tubulopathy. Rates of neuropsychiatric AEs were lower in EVG/COBI/FTC/TDF than in EFV/FTC/TDF (47\% vs $66 \%, \mathrm{P}<0.001)$, as were rates of rash ( $21 \%$ vs $31 \%, \mathrm{P}=0.006)$. Median changes in serum $\mathrm{Cr}(\mu \mathrm{mol} / \mathrm{L})$ at Week 96 in EVG/COBI/FTC/TDF vs EFV/FTC/TDF (11.5 vs 0.9) were similar to those at Week 48 (12.4 vs 0.9). EVG/COBI/FTC/TDF had smaller median increases in total $(0.23 \mathrm{mmol} / \mathrm{L}$ vs $0.47 \mathrm{mmol} / \mathrm{L}, \quad \mathrm{P}<0.001), \mathrm{LDL}(0.23 \mathrm{mmol} / \mathrm{L}$ vs $0.41 \mathrm{mmol} / \mathrm{L}, \quad \mathrm{P}=0.011)$, and $\mathrm{HDL}$ cholesterol $(0.18 \mathrm{mmol} / \mathrm{L}$ vs $0.21 \mathrm{mmol} / \mathrm{L}, \mathrm{P}=0.008)$, and similar increase in triglycerides $(0.05 \mathrm{mmol} / \mathrm{L}$ vs $0.09 \mathrm{mmol} / \mathrm{L}, \mathrm{P}=0.41$ ).

CONCLUSIONS: At Week 96, EVG/COBI/FTC/TDF demonstrated high rates of virologic suppression with low rates of resistance and a differentiated safety and tolerability profile relative to EFV/FTC/TDF. These results support the durable efficacy and long-term safety of EVG/ COBI/FTC/TDF in HIV-1 infected patients. 
P040

ELVITEGRAVIR/COBICISTAT/EMTRICITABINE/TENOFOVIR DF HAS DURABLE EFFICACY AND DIFFERENTIATED SAFETY COMPARED TO ATAZANAVIR BOOSTED BY RITONAVIR PLUS EMTRICITABINE/TENOFOVIR DF AT WEEK 96 IN TREATMENT-NAÏVE HIV-1-INFECTED PATIENTS

Chang, Benny ${ }^{1}$; Rachlis, Anita ${ }^{1}$; Murphy, Daniel ${ }^{2}$;

DeJesus, Edwin ${ }^{3}$; Rockstroh, Jurgen ${ }^{4}$; Henry, Keith ${ }^{5}$;

Retel, Andre E6; Wei, Xuelian ${ }^{7}$; Fordyce, Marshall7; Rhee, Martin ${ }^{7}$; Szwarcberg, Javier ${ }^{7}$

${ }^{1}$ Toronto, ON; ${ }^{2}$ Montreal, QC; ${ }^{3}$ Orlando, Florida, USA; ${ }^{4}$ Bonn, Germany; ${ }^{5}$ Minneopolis, Minnesota, USA; ${ }^{6}$ Vancouver, BC; ${ }^{7}$ Foster City, California, USA

PURPOSE OF THE STUDY: Week 96 interim data of an ongoing, non-inferior, randomized, double-blind, active-controlled, Phase 3 international trial of elvitegravir/cobicistat/emtricitabine/tenofovir DF (EVG/ $\mathrm{COBI} / \mathrm{FTC} / \mathrm{TDF})$ compared to ritonavir-boosted atazanavir $(\mathrm{ATV} / \mathrm{r})+$ FTC/TDF in treatment naïve patients.

METHODS: Key eligibility criteria included HIV-1 RNA $\geq 5,000 \mathrm{c} / \mathrm{mL}$ and eGFR $\geq 70 \mathrm{~mL} / \mathrm{min}$. Virologic success (HIV-1 RNA $<50 \mathrm{c} / \mathrm{mL}$ ) was assessed per snapshot algorithm. Adverse events and laboratory data were collected. Bone mineral density was assessed by DEXA scan in a subgroup of patients.

RESULTS: 708 patients were randomized and treated. Virologic success of EVG/COBI/FTC/TDF was non-inferior to ATV/r + FTC/TDF at Week 48 (90\% vs $87 \%)$ and Week 96 (83\% vs $82 \%)$, and similar in patients with baseline HIV-1 RNA >100,000 c/mL ( $82 \%$ vs $80 \%$ ). Mean CD4 increases were 256 vs 261 cells $/ \mathrm{mm}^{3}$. Emergent resistance was infrequent ( $2 \%$ vs $<1 \%$ ). Rate of study drug discontinuations due to adverse events $(4 \%$ vs $6 \%)$ and renal events $(0.8 \%$ vs $0.6 \%)$ were low and comparable through Week 96. Since Week 48, one patient in each group discontinued study drug due to serum creatinine $(\mathrm{sCr})$ increase without features of proximal renal tubulopathy. Median increases from baseline in $\mathrm{sCr}$ at Week $48(10.6 \mu \mathrm{mol} / \mathrm{L}$ vs $7.1 \mu \mathrm{mol} / \mathrm{L})$ and Week $96(10.6 \mu \mathrm{mol} / \mathrm{L}$ vs $7.1 \mu \mathrm{mol} / \mathrm{L}$ ) were similar. EVG/COBI/FTC/TDF continued to have smaller increases in triglycerides $(0.06 \mathrm{mmol} / \mathrm{L}$ vs $0.18 \mathrm{mmol} / \mathrm{L} ; \mathrm{P}=0.012)$; greater increases in total cholesterol $(0.36$ vs $0.21 \mathrm{mmol} / \mathrm{L} ; \mathrm{P}=0.046)$ at Week 96 only; similar changes were observed in LDL and HDL cholesterol. EVG/COBI/FTC/TDF had smaller mean decreases (\%) in BMD (hip: -3.16 vs -4.19 ; $\mathrm{P}=0.069$, spine: -1.96 vs $-3.54, \mathrm{P}=0.049$ ).

CONCLUSIONS: At Week 96, EVG/COBI/FTC/TDF demonstrated high rates of virologic suppression with low rates of resistance and a differentiated safety and tolerability profile relative to ATV/r + FTC/TDF. These results support the durable efficacy and long-term safety of EVG/ COBI/FTC/TDF in HIV-1 infected patients.

\section{P041}

\section{EFFICACY AND SAFETY OF ELVITEGRAVIR/COBICISTAT/ EMTRICITABINE/TENOFOVIR DF FROM AN INTEGRATED ANALYSIS OF PHASE 2 AND 3 CLINICAL TRIALS}

Laplante, Francois ${ }^{1}$; Wedgwood, Orson $\mathrm{M}^{2}$; Rhee, Martin ${ }^{3}$; Crofoot, Gordon ${ }^{4}$; Shamblaw, David ${ }^{5}$; Bellos, Nicholas ${ }^{6}$;

Ward, Douglas'; Kinder, Clifford ${ }^{8}$; Lui, Hui $\mathrm{C}^{3}$

${ }^{1}$ Montreal, QC; ${ }^{2}$ Mississauga, ON; ${ }^{3}$ Foster City, CA, USA;

${ }^{4}$ Houston, Texas; ${ }^{5}$ San Diego, California; ${ }^{6}$ Dallas, Texas;

${ }^{7}$ Washington, DC; ${ }^{8}$ Miami, Florida, USA

BACKGROUND: Elvitegravir/cobicistat/emtricitabine/tenofovir DF (EVG/COBI/FTC/TDF) demonstrated noninferior efficacy (margin: $-12 \%$ ) to efavirenz/emtricitabine/tenofovir DF (EFV/FTC/TDF) (Studies 102 and 104) and to ritonavir-boosted atazanavir plus truvada (ATV/ r+TDF/FTC) (Study 103) by snapshot analysis at Week (Wk) 48 in randomized, controlled trials of HIV-infected, treatment-naïve subjects. METHODS: Integrated analysis of efficacy and safety

RESULTS: Subjects had similar baseline characteristics (EVG/COBI/

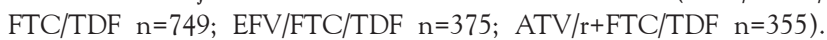
The rates of virologic suppression (HIV-1 RNA $<50 \mathrm{c} / \mathrm{mL}$ ) at Wk 48 in
EVG/COBI/FTC/TDF, EFV/FTC/TDF, and ATV/r+FTC/TDF groups were $88.8 \%, 84.0 \%$ and $86.8 \%$; the difference was $5.1 \%(95 \% \mathrm{CI}$ $0.7 \%$ to $9.4 \%$ ) between EVG/COBI/FTC/TDF and EFV/FTC/TDF groups; and $1.9 \%$ (95\% CI $-2.3 \%$ to $6.1 \%$ ) between EVG/COBI/FTC/ TDF and ATV/r+FTC/TDF groups. EVG/COBI/FTC/TDF efficacy was consistent across subgroups based on demographics, baseline HIV-1 RNA and CD4 cell count. The rates of adverse events (AEs) leading to study drug discontinuation were similar in the three groups (EVG/COBI/FTC/ TDF vs EFV/FTC/TDF vs ATV/r+FTC/TDF) (3.5\% vs $5.1 \%$ vs $5.1 \%$ ), as were serious AEs $(9.2 \%$ vs $6.7 \%$ vs $8.7 \%)$, and deaths $(0.1 \%$ vs $0.5 \%$ vs $0.8 \%$ ). Fewer EVG/COBI/FTC/TDF compared to EFV/FTC/TDF subjects reported neuropsychiatric $\mathrm{AEs}(42.9 \%$ vs $62.1 \%$; $<<0.001)$ and rash AEs $(17.5 \%$ vs $27.7 \%$; $<0.001)$. Small increases in serum creatinine (SCr) (median) were seen as early as Wk 2 in EVG/COBI/FTC/TDF $(+11.49 \mu \mathrm{mol} / \mathrm{L})$ and ATV/r+FTC/TDF $(+7.07 \mu \mathrm{mol} / \mathrm{L})$, but not in the EFV/FTC/TDF $(+0.88 \mu \mathrm{mol} / \mathrm{L})$ group. EVG/COBI/FTC/TDF had less increase (median) in total $(+0.26 \mathrm{mmol} / \mathrm{L}$ vs $+0.49 \mathrm{mmol} / \mathrm{L} ; \mathrm{p}<0.001)$ LDL $(+0.26 \mathrm{mmol} / \mathrm{L}$ vs $+0.44 \mathrm{mmol} / \mathrm{L} ; \mathrm{p}<0.001)$, and HDL cholesterol $(+0.13 \mathrm{mmol} / \mathrm{L}$ vs $+0.21 \mathrm{mmol} / \mathrm{L} ; \mathrm{p}=0.002)$ compared to $\mathrm{EFV} / \mathrm{FTC} / \mathrm{TDF}$ group; EVG/COBI/FTC/TDF also had less increase in triglyceride $(+0.09$ $\mathrm{mmol} / \mathrm{L}$ vs $+0.26 \mathrm{mmol} / \mathrm{L} ; \mathrm{p}=0.006)$ compared to $\mathrm{ATV} / \mathrm{r}+\mathrm{FTC} / \mathrm{TDF}$ group.

CONCLUSIONS: EVG/COBI/FTC/TDF demonstrated high rates of virologic suppression and was well tolerated, comparable to EFV/FTC/ TDF and ATV/r+FTC/TDF. Early small increases in SCr that stabilize are expected with EVG/COBI/FTC/TD due to COBI's inhibition of renal creatinine tubular secretion.

\section{P042}

\section{EARLY CANADIAN CLINICAL EXPERIENCE WITH COMPLERA $^{\text {TM }}$}

Shafran, Stephen; Christine, Hughes; Rosser, Stuart

Edmonton, AB

BACKGROUND: Complera ${ }^{\mathrm{TM}}$ is a single tablet antiretroviral regimen consisting of tenofovir disoproxil fumarate, emtricitabine and rilpivirine. Alberta began funding Complera ${ }^{\mathrm{TM}}$ in mid-August 2012. We present our initial clinical experience.

METHODS: We performed a chart review of all patients in our program who started Complera ${ }^{\mathrm{TM}}$ by December 1, 2012, focusing on patient demographics, reasons for Complera $^{\mathrm{TM}}$ use, tolerance and virologic response. RESULTS: Forty-seven patients started Complera ${ }^{\mathrm{TM}}$ between August 14 and December 1, 2012; one patient started it May 29 (compassionate release). Twelve were ARV naïve and 35 were ARV experienced.

ARV NAÏVE: Of the 12 ARV naïve patients, the median age was 37 years (range 29 to 61 ) and seven (58\%) were male. Median CD4 count was 385 cells $/ \mu \mathrm{L}$ and median viral load 27,000 copies $/ \mathrm{mL}$ (range 4200 to 100,000 ). Two patients discontinued Complera ${ }^{\text {TM }}$; one after seven days because of rash, and another after 30 days when he was placed on terminal compassionate care. The other 10 remain on Complera ${ }^{\mathrm{TM}}$ with falling or undetectable viral loads. The one ARV naïve patient with baseline RT K103N remains virologically suppressed.

TREATMENT EXPERIENCED: Of 35 treatment experienced patients, median age was 41 years (range 19 to 65$)$ and 22 (63\%) were male. Median CD4 count was 530 cells/ $\mu \mathrm{L}$. At the time Complera $^{\mathrm{TM}}$ was started, viral load was undetectable in 24 patients, from 35 copies $/ \mathrm{mL}$ to 170 copies $/ \mathrm{mL}$ in six, 470 copies $/ \mathrm{mL}$ in one (after four weeks of a prior ARV regimen) and $\geq 1900$ copies $/ \mathrm{mL}$ in three who were off ART. Antecedent ARV regimens were PI-based in 20 (19 boosted), EFV-based in 11 (10 Atripla $\left.{ }^{\mathrm{TM}}\right)$ and RAL-based in two. All 35 remain on Complera $^{\mathrm{TM}}$ without virologic failure to date. Updated results will be presented.

CONCLUSIONS: Complera $^{\mathrm{TM}}$ is well tolerated and effective in our clinic population with only one of 47 patients requiring discontinuation for toxicity or lack of efficacy. 
P043

COMPARISON OF HIV VIRAL LOAD SUPPRESSION BEFORE AND AFTER SWITCHING FROM $2 \times 200$ MG ONCE DAILY NEVIRAPINE IMMEDIATE RELEASE TO 400 MG NEVIRAPINE EXTENDED RELEASE TABLETS

Lepik, Katherine J; Barrios, Rolando; Yip, Benita; Ding, Erin; Lesovski, Dragan; Moore, David M; Toy, K Junine; Akagi, Linda; Harrigan, P Richard; Hogg, Robert S; McGovern, Rachel A; Montaner, Julio S

Vancouver, BC

BACKGROUND: Nevirapine $400 \mathrm{mg}$ extended-release tablets (nevirapine-XR) are a new product developed for once daily administration. Clinical trials demonstrate nevirapine-XR is non-inferior to $200 \mathrm{mg}$ twice daily immediate release tablets (nevirapine-IR); however, there is limited experience switching patients from the commonly prescribed dose of $2 \times 200 \mathrm{mg}$ nevirapine-IR once daily to nevirapine-XR. This observational study describes the virologic outcomes of British Columbia patients who switched from once daily nevirapine-IR to nevirapine-XR. METHODS: HIV positive patients aged $\geq 19$ years were included if they switched from an antiretroviral regimen containing once daily $2 \times 200 \mathrm{mg}$ nevirapine-IR ( $\geq 6$ months uninterrupted therapy) to nevirapine-XR between April 1, 2012 and September 30, 2012. Patients required at least one HIV plasma viral load measurement within six months before switching and $\geq 30$ days after switching to nevirapine-XR. This interim analysis describes results up to September 30, 2012. Patient demographic, clinical and viral load data were abstracted from BC Centre for Excellence in HIV/AIDS databases. The proportion of patients with suppressed viral load $(<40$ copies $/ \mathrm{mL})$ during nevirapine-IR and nevirapine-XR therapy was compared by $\chi^{2}$ test.

RESULTS: The 324 patients included were median (IQR) age 49 (44 to 57) years and $98 \%$ were male. Median (IQR) duration of nevirapine-IR use before switching and nevirapine-XR post-switch was 66 months (22 to 110 months) and 4.1 months (3.5 to 4.8 months), respectively. Concurrent antretrovirals included abacavir/lamivudine 56\%, tenofovir/emtricitabine or tenofovir/lamivudine $36 \%$ and other antiretrovirals $8 \%$. Median (IQR) number of qualifying viral load samples per patient was 2 ( 2 to 2 ) during nevirapine-IR and 1 ( 1 to 1 ) during nevirapine-XR treatment. The proportion of patients with all samples $<40$ copies/mL was $281 / 324$ (87\%) during nevirapine-IR and 298/324 (92\%) during nevirapine-XR therapy ( $\mathrm{p}=0.03)$. Most patients with detectable viremia during either nevirapine-IR or nevirapine-XR treatment had 'blips' of 40 copies $/ \mathrm{mL}$ to 200 copies $/ \mathrm{mL}$ : $38 / 43(88 \%)$ and $22 / 26(85 \%)$, respectively. No treatment failure was documented up to September 30, 2012.

CONCLUSION: Preliminary results suggest daily $400 \mathrm{mg}$ nevirapine-XR is not inferior to $2 \times 200 \mathrm{mg}$ nevirapine-IR. Follow-up continues.

\section{P044}

NEVIRAPINE EXTENDED RELEASE (XR) TABLETS: CLINICAL EXPERIENCE DURING THE FIRST SIX MONTHS OF POSTMARKETING USE IN BRITISH COLUMBIA

Lepik, Katherine J; Barrios, Rolando; Yip, Benita; Ding, Erin; Lesovski, Dragan; Moore, David M; Toy, K Junine; Akagi, Linda; Harrigan, P Richard; Hogg, Robert S; Montaner, Julio S

Vancouver, BC

BACKGROUND: Nevirapine $400 \mathrm{mg}$ extended-release tablets (nevirapine-XR) are a once-daily alternative to nevirapine $200 \mathrm{mg}$ immediate release tablets (nevirapine-IR). This observational study describes clinical experience with nevirapine-XR during the first six months of use in British Columbia.

METHODS: HIV positive patients age $\geq 19$ years were included if their first nevirapine-XR prescription was dispensed between April 1, 2012 and September 30, 2012. Patient demographic and clinical variables, HIV plasma viral load and adverse drug reactions reported during nevirapine-XR treatment up to September 30, 2012 were abstracted from the BC Centre for Excellence in HIV/AIDS databases and summarized using descriptive statistics.

RESULTS: The cohort included 538 patients who were $96 \%$ male with median (IQR) age 49 years ( 43 to 56 years). Median (IQR) duration of
nevirapine-XR use was 114 days (93 to 138 days). Of 399 (74\%) patients with viral load monitoring following $\geq 30$ days of nevirapine-XR use, $358 / 399$ (90\%) had virologic suppression ( $<40$ copies $/ \mathrm{mL})$. One patient with poor adherence developed virologic rebound with emergent drug resistance (mutations 181C, 184V, 118I, 69A, 219E).

Adverse drug reactions were reported in seven (1.3\%) patients (see Table). The nevirapine-XR tablet matrix is nondigestible and 23 (4.3\%) patients reported seeing apparently 'whole' tablets in their stool. No cases of treatment failure were associated with tablet remnants in the stool and six patients with nevirapine plasma concentration monitoring had therapeutic trough levels: median ( $\min$ to $\max ) 3100 \mathrm{ng} / \mathrm{mL}(2770 \mathrm{ng} / \mathrm{mL}$ to $3580 \mathrm{ng} / \mathrm{mL}$ ). However; observation of 'tablets' in the stool was the leading reason for nevirapine-XR discontinuation.

CONCLUSION: Results support nevirapine-XR as a safe, effective alternative to nevirapine-IR in clinical practice. Patients should be informed that tablet remnants may be visible in the stool.

\begin{tabular}{lc} 
Variable & $\mathrm{N}=538 \mathrm{n}(\%)$ \\
\hline Total patients treated with nevirapine $400 \mathrm{mg}$ XR tablets & $538(100)$ \\
Antiretroviral regimen prior to nevirapine-XR start & \\
Switch from nevirapine-IR 2x200 mg once daily & $444(83)$ \\
Switch from nevirapine-IR $200 \mathrm{mg}$ twice daily & $61(11)$ \\
Switch from other NNRTI (not nevirapine) regimen & $15(3)$ \\
Switch from other regimen (not NNRTI) & $12(2)$ \\
None- Restarting antiretrovirals after interruption & $4(0.7)$ \\
None- Antiretroviral treatment naive & $2(0.3)$ \\
Concurrent antiretroviral drugs & \\
tenofovir + emtricitabine/ lamivudine & $212(39)$ \\
abacavir + lamivudine & $272(51)$ \\
zidovudine + lamivudine & $9(2)$ \\
other antiretroviral combination & $45(8)$
\end{tabular}

HIV plasma viral load results $\geq 30$ days after Nevirapine-XR start

not measured

$139(26)$

all results $<40$ copies $/ \mathrm{mL}$

$358(67)$

one or more results $\geq 40$ but $\leq 200$ copies $/ \mathrm{mL}$

$33(6)$

one or more results $>200$ copies $/ \mathrm{mL}^{*}$

$8(1)$

*2/8 patients with >200 copies $/ \mathrm{mL}$ had declining viral loads following treatment start or restart

Adverse Drug Reaction (Any)

$7(1)$

Adverse drug reaction detail:

Hepatic transaminase, mild-moderate increase

5 (1)

Gastrointestinal upset, mild

$1(0.2)$

Skin rash (unspecified)

$1(0.2)$

Nevirapine-XR discontinued (Any Reason)

$26(5)$

Reason for discontinuation

Tablet remnants observed in stool

$11(2)$

Adverse drug reaction

$4(0.7)$

Treatment failure

$1(0.2)$

Reason not specified

$10(2)$

\section{P045}

VIROLOGICAL EFFICACY AND LIPID LEVELS IN HIVINFECTED ADULTS RECEIVING BOOSTED PROTEASEINHIBITOR (PI) COMPARED TO ATAZANAVIR ONLY: A META-ANALYSIS

Baril, Jean-Guy'; Conway, Brian2; Giguere, Pierre'3; Ferko, Nicole ${ }^{4}$; Hollmann, Sarah'; Angel, Jonathan ${ }^{3}$

${ }^{1}$ Montreal, QC; ${ }^{2}$ Vancouver, BC; ${ }^{3}$ Ottawa; ${ }^{4}$ Burlington, ON;

BACKGROUND: Treatment simplification involving induction with a regimen that includes a ritonavir (RTV)-boosted protease inhibitor (PI) replaced by a non-boosted PI (ie, atazanavir) is a viable option as shown in several studies of HIV-infected patients. To evaluate the cumulative 
evidence to date, we conducted a systematic review and meta-analysis of randomized controlled trials (RCTs) evaluating the efficacy and safety of this approach in patients with established virological suppression.

METHODS: PubMed, EMBASE, and Cochrane Library were searched without limits to time or language. Searches were also conducted of IAS, ICAAC and CROI conferences (2009 to present). RCTs were included if they compared a PI/RTV regimen to unboosted atazanavir, after an induction phase with PI/RTV in HIV-1 infected adults. The meta-analysis was conducted using a random effects model for outcomes including proportion with HIV-1 RNA levels $<50$ copies/mL through to study end, and change in mean lipid levels. Dichotomous outcomes were reported as risk ratios (RR) and continuous outcomes as mean differences (MD).

RESULTS: Five studies involving a total of 1249 patients met inclusion criteria. Results from the meta-analysis demonstrated that there was no statistically significant difference in virologic efficacy between PI/RTV and unboosted atazanavir regimens $(\mathrm{RR}=1.03$ [95\% CI 0.98 to 1.08$])$, with no heterogeneity observed across studies $\left(I^{2}=0\right)$. Findings were similar in a sub-analysis of studies where atazanavir/RTV was the only PI/RTV used during induction ( $R R=1.04$ [95\% CI 0.98 to 1.10]). A significant reduction in both total cholesterol $(\mathrm{MD}=-14.9 \mathrm{mg} / \mathrm{dL} ; 95 \% \mathrm{CI}-22.9$ to $-6.9 ; \mathrm{p}=0.0003)$ and triglycerides $(\mathrm{MD}=-38.0 \mathrm{mg} / \mathrm{dl}[95 \% \mathrm{CI}-59.4$ to -16.6; $\mathrm{p}=0.0005)$ was observed with unboosted atazanavir compared to $\mathrm{PI} / \mathrm{RTV}$, with no significant differences in HDL or LDL cholesterol.

CONCLUSIONS: The meta-analysis demonstrated that switching patients with virologic suppression from a RTV-boosted PI to unboosted atazanavir will lead to an improvement in total cholesterol and triglyceride levels without sacrificing virologic efficacy.

\section{P046 \\ PRELIMINARY RESULTS OF A RANDOMIZED CONTROLLED TRIAL (RCT) OF MAINTAINING RITONAVIR-BOOSTED ATAZANAVIR (ATZ/R) VS SWITCHING TO UNBOOSTED ATZ AMONG PATIENTS WHO ARE VIROLOGICALLY CONTROLLED ON TENOFOVIR (TDF)-BASED REGIMENS}

Ganase, Bruce; Hull, Mark; Guillemi, Silvia; Ding, Erin; Yip, Benita; Harris, Marianne

Vancouver, BC

BACKGROUND: Unboosting ATZ in TDF-containing regimens may be advantageous in selected patients.

METHODS: This ongoing open-label 48-week RCT is enrolling HIV+ adults with viral load (VL) $<40$ copies/mL while taking ATZ/r 300/100 mg with TDF and FTC/3TC, and no NRTI/PI resistance. Eligible consenting subjects are randomized 1:1 to continue ATZ/r (control) or switch to ATZ $400 \mathrm{mg}$. A preliminary analysis was conducted when 30 subjects reached 24 weeks. Categorical variables were compared using Fisher's Exact test and continuous variables using Wilcoxon's Rank Sum test. Lipid changes were not analyzed due to high variability and small sample size.

RESULTS: Of the first 30 subjects enrolled, 26 received TDF/FTC, four TDF/3TC; 28 were male; median age was 47 years. The arms (each $n=15$ ) were similar at baseline with respect to CD4 count (median $515 \mathrm{cells} / \mathrm{mm}^{3}$ ), total bilirubin (TBR, $43 \mu \mathrm{mol} / \mathrm{L})$, creatinine $(77 \mu \mathrm{mol} / \mathrm{L})$, eGFR $(94 \mathrm{~mL} / \mathrm{min})$, serum phosphate $(0.90 \mathrm{mmol} / \mathrm{L})$, urine albumin to creatinine ratio $(0.9 \mathrm{mg} / \mathrm{mmol})$, and time on ATZ/r (30 months). Subjects randomized to switch tended to have a shorter duration of pre-study $\mathrm{VL}<50$ copies $/ \mathrm{mL}$ than controls (median 8 months vs 17 months, $\mathrm{p}=0.054$ ). In the first 24 weeks, one subject was lost to follow-up and one virologic failure occurred (and subsequently resuppressed when ritonavir resumed), both in the switch arm. At week 24, the switch and control arms did not differ with respect to absolute values or changes from baseline in CD4, creatinine, eGFR, phosphate or UACR. Median change in TBR was $-14 \mu \mathrm{mol} / \mathrm{L}$ in the switch arm and $+3 \mu \mathrm{mol} / \mathrm{L}$ in the control arm ( $\mathrm{p}=0.045$ ); at week 24 , median TBR was $30 \mu \mathrm{mol} / \mathrm{L}$ and $45 \mu \mathrm{mol} / \mathrm{L}$ in the two arms, respectively $(\mathrm{p}=0.031)$.

CONCLUSION: In this preliminary 24-week analysis of subjects receiving TDF, virologic failure occurred in one patient $(1 / 14,7 \%)$ who switched to unboosted ATZ and none who stayed on ATZ/r. TBR decreased in those who switched as compared to controls. Changes in CD4 cell counts and renal parameters were similar in both arms.

\section{P047 \\ NOVEL KIVEXA-BASED REGIMENS IN EARLY COURSES OF TREATMENT FOR HIV INFECTION}

Tossonian, Harout; Alenezi, Osamah; Wong, Leala; Wang, Jeffrey; Qian, Christina; Conway, Brian

Vancouver, BC

BACKGROUND: As the long-term efficacy of antiretroviral therapy regimens is confirmed, there is a need to identify additional combinations that exhibit long-term safety and potency, while also favoring simplicity of administration. In this light, we have undertaken a review of the use of abacavir/lamivudine (Kivexa, KVX)-based regimens using integrase or CCR5 inhibitors as the third agent.

METHODS: A retrospective chart review was undertaken with informed patient consent. We identified all the patients in whom KVX was prescribed (following appropriate HLA-B5701 screening) with either raltegravir (RGV) or maraviroc (MVC) as initial therapy or as a switch from another regimen for reasons other than virologic failure. Virologic efficacy over four weeks was evaluated, along with specific drug-associated toxicity, adherence and regimen modifications.

RESULTS: A total of 38 patients were evaluated, 24 on RGV, 13 on MVC, one on RGV/MVC, as non-initial therapy in 35/38 cases. Switches included replacement of the third agent with RGV or MVC $(n=13)$, replacement of the NRTI backbone with KVX $(n=13)$ or both. Patients were predominantly MSMs ( $n=17)$ or IDUs $(n=12)$ with a median baseline CD4 cell count of 410 cells $/ \mathrm{mm}^{3}$, and plasma viral load of 66 copies $/ \mathrm{ml}$ (18 with full suppression at time of switch). At latest followup visit, 34/38 (89\%) achieved or maintained full suppression, with a median CD4 count of 465 cells/mm3. Virologic failure with the development of the M184V mutation was observed in 3/4 non-suppressed patients, and a loss of CCR 5 tropism and RGV resistance were observed in one case each, all in the context of reduced adherence. There were no treatment discontinuations for toxicity and no medication-associated series adverse events.

CONCLUSION: KVX-based regimens are safe and effective alternatives to more commonly used regimens in clinical practice, and offer the benefit of good long-term tolerability and little or no need to enhance follow-up for laboratory-based abnormalities. Consideration should be given to non-NNRTI and non-PI-based regimens to address issues of toxicity and simplification without apparent loss of efficacy.

\section{P048}

\section{SWITCHING TO DARUNAVIR OR ETRAVIRINE IN VIROLOGICALLY SUPPRESSED PATIENTS WHO ARE INTOLERANT OF THEIR CURRENT OR PRIOR COMBINATION ANTIRETROVIRAL THERAPY REGIMEN: A PHASE IV, OPEN-LABEL, MULTICENTRE OBSERVATIONAL TRIAL}

Conway, Brian ${ }^{1}$; Fraser, Christopher ${ }^{2}$; Tsang, Albert ${ }^{3}$; Smith, Graham ${ }^{3}$; Smaill, Fiona ${ }^{4}$; Camacho, Fernando ${ }^{3}$; Tossonian, Harout ${ }^{2}$; Hew, Huong ${ }^{1}$

${ }^{1}$ Vancouver; ${ }^{2}$ Victoria, BC; ${ }^{3}$ Toronto; ${ }^{4}$ Hamilton, ON

BACKGROUND: As patients achieve long-term virologic suppression on ARV regimens, strategies for continued management will focus on minimizing toxicities to enhance adherence and quality of life. Data have shown that either darunavir or etravirine with proven efficacy and minimal drug-associated toxicity could be considered for a single drug switch strategy. The current protocol is designed to evaluate this approach in a community-based setting.

METHODS: HIV-1 infected patients with an undetectable viral load who were experiencing significant symptoms (grade 1 or 2 drug-related toxicities) or who requested treatment simplification were included. While maintaining their current backbone regimen, a single agent was substituted with either etravirine (200 mg b.i.d.) or darunavir/ritonavir ( $800 \mathrm{mg} / 100 \mathrm{mg}$ q.d.). Clinically indicated safety and efficacy assessments 
were conducted at baseline and weeks 4,12 and 24. The primary endpoint (based on intention-to-treat analysis) was the change from baseline in the patient's total score on the HIV Symptoms Distress Module (HIVSDM).

FINDINGS: The study included 77 patients ( $91 \%$ male), with a median age of 48 years, and mean duration of infection and ARV treatment of 11.3 years and 7.6 years, respectively. 30 patients were switched to darunavir, 45 to etravirine and two patients received both agents. $76.3 \%$ reported one or more bothersome ("bothers me/bothers me a lot") symptoms on the HIV-SDM at baseline. Reasons for participating as reported by the patients included: desire to simplify current regimen $(50.6 \%)$; intolerance of current regimen $(40.3 \%)$; concern about long term side effects (32.5\%); and adherence issues (6.5\%). HIV-SDM data will be presented; the last study visit is scheduled for March 2013.

CONCLUSION: Despite medications that are able to suppress HIV virus, there may remain patients in whom a switch of ARV medication is warranted due to tolerability, simplification or long term side effect considerations

\section{P049 \\ USE OF POST-HAART DUAL THERAPY IN PATIENTS WITH LIMITED ARV OPTIONS}

MahMing, Jinell; Lee, Kathy; Krentz, Hartmut B; Gill, John

Calgary, AB

OBJECTIVE: To describe the clinical and pharmacological responses in patients with limited antiretroviral (ARV) options who have been switched to a dual ARV regimen after multiple HAART regimens.

METHODS: Observational descriptive analysis was conducted on patients who switched to nucleoside reverse transcriptase inhibitor (NRTI) sparing post-HAART dual therapy, and then maintained/ achieved undetectable viral loads for at least sox months. Data collection included: all ARV regimens including reasons for switching, current and nadir CD4 counts, HIV-1 RNA (viral load), cumulative months on NRTI and genotypic resistance.

RESULTS: As of December 31, 2012, 10 patients had been switched to post-HAART dual therapy. Reasons for switching to post-HAART dual therapy included long term NRTI toxicity, therapeutic change, and/or limited ARV options due to genotypic resistance. Median duration on post-HAART dual therapy was 44 months. Median time from HIV diagnosis date to switch was 16.1 years. Patients had a mean of 6.3 ARV regimens prior to their current dual therapy. Seven patients had genotypic testing: all showed NRTI resistance, four NNRTI resistance, two PI resistance, and one with all class resistance. Patients had received NRTIs for a cumulative median of 353 months. Post-HAART dual therapy regimens were: Etravirine/boosted Darunavir $(n=5)$; Efavirenz/Raltegravir $(\mathrm{n}=3)$; Lopinavir/ritonavir/Raltegravir $(\mathrm{n}=1)$; and Etravirine/Raltegravir $(\mathrm{n}=1)$. Median nadir and current CD4 counts were $32 \mathrm{cells} / \mathrm{mm}^{3}$ and 552 cells $/ \mathrm{mm}^{3}$, respectively. $80 \%$ of patients were undetectable (VL $<40$ copies $/ \mathrm{mL}$ ) for a median of six years before their switch and remained undetectable after their switch.

CONCLUSION: We found that a select group of highly ARVexperienced patients with limited ARV options were able to maintain/ achieve undetectable viral loads and immunologic responses with postHAART dual therapy for a median of almost four years. More research is required to determine if switching to a post-HAART dual therapy could be used as a long term strategy, however, our results appear promising.
Co-infections (including HCV, HBV, HPV) Co-infections (dont le VHC, le VHB et le papillomavirus)

P050

DELAYED SCREENING AND ADVANCED PRESENTATION OF TUBERCULOSIS IN HIV-INFECTED PRISONERS AND HOMELESS PERSONS IN A MULTI-CENTRE RUSSIAN COHORT STUDY

Rumman, Amir ${ }^{1}$; Sadeghi, Arash ${ }^{1}$; Zagdyn, Zinaida ${ }^{2}$; Hopman, Wilma ${ }^{1}$; Cox, David ${ }^{3}$; Rosenes, Ron ${ }^{3,4}$; Cooper, Curtis ${ }^{5}$; Frolova, Olga ${ }^{6}$; Balasanyants, Goar ${ }^{2}$; Wobeser, Wendy $\mathbf{L}^{1}$

${ }^{1}$ Kingston, ON; ${ }^{2}$ St Petersburg, Russia; ${ }^{3}$ Vancouver, BC; ${ }^{4}$ Toronto; ${ }^{5}$ Ottawa, ON; ${ }^{6}$ Moscow, Russia

AIM: Tuberculosis (TB) screening remains a substantial public health challenge in correctional facilities and the homeless population. In this cross-sectional study we evaluated TB screening of HIV-infected Russian prisoners and homeless persons (HPs) compared to the general population.

METHODS: Prospective data collection was conducted at TB treatment centres in three Russian regions (St. Petersburg, Vologda and Leningrad) between 2008 and 2011. Incident cases of active TB in HIV-infected individuals presenting for treatment were identified.

RESULTS: A total of 2809 patients were included in the analysis: $2268(80.7 \%)$ local residents, $404(14.4 \%)$ prisoners and $137(4.9 \%)$ homeless persons. There was no significant difference in baseline demographic characteristics (mean age 33.0 years, $74.8 \%$ male). Time between HIV and TB presentation was significantly shorter in prisoners and HPs ( 4.4 years and 3.5 years, respectively, vs 5.5 years; $p<0.01$ ). Most patients were referred with radiographic evidence only $(91.4 \%)$ and prisoners and HPs were less likely to have microbiological confirmation. Prisoners and HPs presented with a greater incidence of disseminated TB $(65.0 \%$ and $84.9 \%$ vs $38.4 \%$ with evidence of extra-pulmonary involvement; $\mathrm{p}<0.001$ ) and were more likely to report a longer symptomatic period $(14.7 \%$ and $19.8 \%$ vs $37.2 \%$ with symptoms lasting $>3$ years; $p<0.001$ ). Prisoners and HPs presented with more advanced HIV disease $(43.6 \%$ and $37.2 \%$ vs $27.5 \%$ with WHO Stage 3 or greater; $\mathrm{p}<0.05)$ and lower CD4 cell count (38.9\% and $48.2 \%$, respectively, vs $35.3 \%$ with CD4 $<200$; $<<0.05$ ). Resistance data was only available from the Leningrad Oblast. A total of 11 MDR-TB cases were detected in prisoners. All-cause mortality was greater in prisoners and HPs $(20.3 \%$ and $33.6 \%$ vs $11.1 \%$; $\mathrm{p}<0.01)$. CONCLUSIONS: HIV-infected prisoners and HPs were diagnosed with $\mathrm{TB}$ at a significantly more advanced disease stage and presented with greater morbidity and mortality. Targeted early screening and treatment interventions could improve outcomes in these populations.

\section{P051}

GONORRHEA CO-INFECTION AMONG GAY AND BISEXUAL MEN IN HIV CARE IN ONTARIO: TRENDS IN TESTING AND DIAGNOSIS, 2008-2011

Burchell, Ann N; Allen, Vanessa; Grewal, Ramandip;

Moravan, Veronika; Remis, Robert S; Gardner, Sandra;

Bayoumi, Ahmed M; Kaul, Rupert; Mazzulli, Tony; McGee, Frank; Millson, Peggy; Raboud, Janet; Rourke, Sean B; The OHTN Cohort Study Team

Toronto, ON

BACKGROUND: Reported gonorrhea cases have risen and antibiotic resistance is of concern. Testing for GC is recommended at least annually for sexually active gay/bisexual men. We report on the results of gonorrhea testing among HIV-positive men in care in Ontario.

METHODS: The OHTN Cohort Study is a multi-site cohort of persons in HIV care in Ontario. We obtained data from medical charts, interviews and data linkage at the Public Health Ontario Laboratories (PHOL). We analysed data from 2179 gay/bisexual male participants under follow-up in 2008-11 at clinics that submit gonorrhea tests to the 
PHOL (6013 person-years). We estimated the annual proportion tested, the positivity rate and prevalence. We identified risk factors for testing and positivity using GEE logistic regression.

RESULTS: Annual gonorrhea testing increased from $18.0 \%$ in 2008 to $31.7 \%$ in 2011 ( $\mathrm{p}<0.0001)$. Virtually all tests $(93.9 \%, 2,760 / 2,940)$ were urine-based nucleic acid amplification. Among those tested $(\mathrm{n}=858)$, only $4.1 \%, 5.6 \%$, and $7.0 \%$ were cultured using specimens from the genital, rectal or oral tract, respectively. Correlates of annual testing $(\mathrm{p}<0.05)$ were age $<30$; number of sex partners; attendance at a primary care clinic; Toronto residence; testing in the previous year; and more viral load tests in the current year. Positivity rates decreased over time (5.5\% in 2008; $3.6 \%$ in 2011). Conversely, the overall annual prevalence remained stable and was $1.1 \%$ (95\% CI 0.7 to 1.6$)$ in 2011. Gonorrhea risk factors were young age, detectable viral load and not being on cART. Among the 27 cultured cases, none had decreased susceptibility to the first-line oral therapy cefixime (defined as MIC $\geq 0.25 \mu \mathrm{g} / \mathrm{mL}$ ).

CONCLUSION: Annual gonorrhea testing increased, but remained much lower than recommended, while the prevalence of co-infection remained stable in 2008 to 2011. Specimens were rarely cultured, limiting our ability to identify and monitor antibiotic resistance. The infrequent collection of non-urine specimens suggests that oral and rectal cases may have been missed.

\section{P052}

\section{THE EPIDEMIOLOGY OF HIV AND CORRELATES OF HIV/ HCV CO-INFECTION IN A POPULATION-BASED CLINICAL COHORT FROM MANITOBA}

Shaw, Souradet Y; Becker, Marissa L; Campbell, Kristyn;

Keynan, Yoav; Pindera, Carla; Carnochan, Tara; Kasper, Ken

Winnipeg, MB

INTRODUCTION: There is a dearth of information on HIV epidemiology from the Canadian Prairie provinces. Overlapping epidemics of HIV and hepatitis $\mathrm{C}(\mathrm{HCV})$ have complicated care provision in Manitoba, although the extent of, and factors correlated with co-infection are not well-documented. This study describes recent trends in HIV epidemiology and correlates of co-infection in Manitoba.

METHODS: Data were from the Manitoba HIV Program (MHP), the single provider of HIV care in Manitoba, and included patients enrolled January 1, 2009 to December 31, 2011. Rates were age-standardised to the 2012 Canadian population, and $95 \%$ CIs were generated. Poisson models were used to calculate adjusted rate ratios (ARR) and 95\% CI between the sexes. Descriptive statistics and adjusted odds ratios (AORs) and $95 \%$ CI from logistic regression models examined the correlates of co-infection. Factors included sociodemographic, risk and clinical characteristics.

RESULTS: The total sample size was 281. HIV infections decreased for the total sample $(8.4$ per 100,000 to 6.6 per 100,000$)$ and by sex. HIV rates were significantly higher among males (ARR 1.7 [95\% CI 1.1 to 2.6; $\mathrm{p}=.014)$. HCV co-infection was $13 \%$ over the three years, increasing from $11 \%$ to $16 \%$. At the univariate level, age $(p=0.02)$, Aboriginal ethnicity $(20 \%$ vs $8 \%, p=0.003)$ and injection drug users (IDU, $p<0.001$ ) were associated with $\mathrm{HCV}$ co-infection; $80 \%$ of IDU were co-infected. The proportion of IDU decreased from $13 \%$ to $11 \%$. In multivariable models, adjusted for age, IDU status, sex and Aboriginal status, only age (AOR 1.06 [95\% CI 1.0 to $1.1 ; \mathrm{p}=0.02$ ) and IDU status remained statistically significant (AOR 90.6, 95\% CI 27.7 to 296.0; p<0.001).

CONCLUSIONS: While HIV rates have decreased, HCV co-infection has increased as a proportion of new HIV infections. HCV co-infection was associated almost entirely with IDU, and not with clinical features at presentation, such as opportunistic infections or CD4 count. IDUappropriate interventions are imperative for addressing the continuing HIV epidemic in Manitoba.
P053

TIME-DEPENDENT BIAS RELATED TO HEPATITIS C CLASSIFICATION: ISSUE ARISING FROM ANALYSIS OF TIME FROM ANTIRETROVIRAL THERAPY INITIATION TO MORTALITY

Gillis, Jennifer ${ }^{1}$; Cooper, Curtis²; Burchell, Ann';

Gardner, Sandra ${ }^{1}$; Manno, Michael ${ }^{1}$; Raboud, Janet ${ }^{1}$

${ }^{1}$ Toronto, ON; ${ }^{2}$ Ottawa, ON

BACKGROUND: In studies of HIV-hepatitis C virus (HCV) coinfection, participants are often considered HCV-positive throughout the entire study period. This classification was based on the assumption that HCV acquisition antedates that of HIV, and clearance of $\mathrm{HCV}$ is rare in HIV-positive individuals. Determination of HCV positivity is further complicated by the evolution of diagnostic tests. In time to event analyses, these issues may contribute bias that is influenced by individuals (1) acquiring HCV after time origin of interest, (2) clearing HCV during study period, and (3) having access to accurate diagnostic testing.

METHODS: We analyzed data from participants of the Ontario HIV Treatment Network Cohort Study (OCS) who had initiated ART. In a fixed variable analysis, individuals who ever tested positive for antiHCV antibodies and/or HCV RNA were classified as HCV-positive for the entire study period. For the time-updated analysis, individuals were classified as HCV-positive from the time of first positive to subsequent negative test. Left-truncated Cox proportional hazards (PH) models were used to estimate the impact of fixed and time-updated HCV status.

RESULTS: 4555 OCS participants initiated ART; the median (IQR) year of initiation was 1997 (1995-2004). Seven hundred forty-five individuals tested HCV-positive; 71 had prior negative serology results indicating seroconversion, and 39 had false positive antibody results. Seventy individuals cleared HCV. In multivariable $\mathrm{PH}$ models, the hazard ratio (HR) of death was 1.38 (95\% CI 1.07 to 1.77 ) for fixed HCV-positive status and 2.03 (95\% CI 1.54 to 2.67) for time-updated HCV-positive status.

CONCLUSIONS: Considerable time-dependent bias can cause underestimation of the relative risk of death when analysing HCV status as a fixed covariate. Previous assumptions on $\mathrm{HCV}$ acquisition and clearance may be appropriate in some analyses; however, if incidence of $\mathrm{HCV}$ acquisition and clearance are nontrivial, the use of time-updated HCV status may be more appropriate. Bias might be greater if probability of acquisition and clearance are higher than we observed. Further, with the availability of protease inhibitors, the evaluation of HCV clearance may become a clinically important outcome.

\section{P055}

INCIDENCE AND RISK FOR CARDIOVASCULAR DISEASE AMONG ANTIRETROVIRAL-TREATED HIV-HCV AND HIV-HBV CO-INFECTED PATIENTS

Raboud, Janet ${ }^{1}$; Gillis, Jennifer ${ }^{1}$; Smieja, Marek ${ }^{2}$; Cescon, Angela ${ }^{3}$; Cooper, Curtis ${ }^{4}$

${ }^{1}$ Toronto; ${ }^{2} \mathrm{Ham}$ ilton, ON; ${ }^{3}$ Vancouver, BC; ${ }^{4} \mathrm{Ottawa}, \mathrm{ON}$

BACKGROUND: It is well established that the risk for cardiovascular disease (CVD) is increased in HIV. Despite relatively high prevalence of viral hepatitis co-infection with HIV, there are few studies on the risk of CVD amongst antiretroviral-treated HIV-viral hepatitis co-infected patients.

METHODS: Ontario HIV Treatment Network Cohort Study participants who initiated ART and did not have prior CVD events were included in the analysis. HBV and HCV co-infected patients were identified by serology and RNA test results. CVD was defined as any of: coronary artery disease including bypass, myocardial infarction, congestive heart failure, cerebrovascular accident or stroke, angioplasty and sudden cardiac death. Competing risk models accounting for left truncation between ART initiation and enrolment into the OCS were used to assess the impact of HBV and HCV co-infection on time to CVD after adjustment for confounding variables and non-CVD death. 
RESULTS: A total of 3416 HIV mono-infected, 432 HIV-HBV and 736 HIV-HCV co-infected patients were followed for a median (IQR) of 2.32 years (1.36-8.02). Two hundred thirteen CVD events and 613 deaths were documented. The incidence (SE) of CVD (events per 1000 person-years follow-up) was 7.59 (0.70) for HIV, 8.70 (2.05) for HIVHBV and 9.62 (1.65) for HIV-HCV infected individuals. The median (IQR) year of initiating ART was 1997 (1995 to 2004), and median age was 36 years (31 to 43 years). Sixty-three per cent of HIV, 71\% of HIVHBV and $84 \%$ of HIV-HCV had ever smoked.

After adjustment for age, gender, race, year initiating ART, weight and smoking status, HBV was not associated with time to CVD onset $(\mathrm{HR}=1.05$ [95\% CI 0.63 to 1.75$] ; \mathrm{p}=0.84)$. A trend toward significantly increased risk of CVD for $\mathrm{HCV}$ co-infected individuals was observed $(\mathrm{HR}=1.44$ [95\% CI 0.97 to 2.13]; $\mathrm{p}=0.07)$. Current smoking at baseline $(\mathrm{HR}=1.50$, [95\% CI 1.00 to 2.25]; $\mathrm{p}=0.05)$, age $(\mathrm{HR}$ per 10 years $=1.99$ [95\% CI 1.70 to 2.34]; $\mathrm{p}=<0.0001$ ) and starting ART prior to 1990 were associated with increased CVD risk.

CONCLUSIONS: HIV-HCV co-infection may significantly increase the risk of CVD in HIV-positive individuals after accounting for confounding variables and competing causes of death.

\section{P056}

\section{TREATMENT OF HCV INFECTION IN THE SETTING OF HIV CO-INFECTION IN VULNERABLE POPULATIONS} Conway, Brian; Alenezi, O; Wang, J; Gallagher, L; Tossonian, H Vancouver, BC

BACKGROUND: Illicit drug users (IDUs) make up $75 \%$ of prevalent cases of HCV infection. Recent guidelines suggest that IDUs be considered for treatment of their infection in multidisciplinary care centres. About $10 \%$ of this group will also carry HIV infection, presenting a special challenge for both engagement and treatment. Little is known about response to treatment in this more complex population.

METHODS: We have conducted a retrospective analysis of treatment of $\mathrm{HCV}$ in IDUs in inner city Vancouver since 2003. All patients were viremic adults receiving combination therapy with ribavirin (RBV) and pegylated interferon (IFN), with all injections conducted weekly by study staff, at the time of clinic visits. Interventions included proactive toxicity management and access to extensive medical, psychiatric, addiction and counseling support. The primary endpoint was absence of viremia 24 weeks after the end of therapy (sustained virologic response, SVR, or "cure").

RESULTS: A total of 302 patients were treated, median age 53 years, $15 \%$ female, $14 \%(n=43)$ with HIV infection, $63 \%$ genotype 1, $83 \%$ treatment-naïve, $11 \%$ compensated cirrhotics, $70 \%$ on methadone maintenance therapy. Within the HIV co-infected subgroup, $86 \%$ were on suppressive ARV therapy, none with a median CD4 count $<200$ cells $/ \mathrm{mm}^{3}$. A total of $67 \%$ of patients completed therapy. Only $5 \%$ discontinued therapy due to a relapse in IDU, $11 \%$ due to medication toxicity, $17 \%$ due to lack of response. SVR rates were $53 \%$ (45\% in genotype $1,65 \%$ in genotype $2 / 3$ ), with rates of $47 \%, 42 \%$ and $60 \%$, respectively, in the setting of HIV infection.

CONCLUSION: In an optimized context, HCV infection can be successfully treated in IDUs, with HIV co-infection not being associated with any decrement in virologic response. Greater efforts need to be made to engage and treat $\mathrm{HCV}$-infected IDUs, irrespective of their HIV infection status.

\section{P057}

HIV CO-INFECTION DOES NOT APPEAR TO BE A BARRIER TO ACHIEVE A SUSTAINED VIROLOGICAL RESPONSE AFTER HCV TREATMENT

Vezina, Sylvie; Trottier, Benoit; Thomas, Réjean; Gallant, Serge;

Poliquin, Marc; Huchet, Emmanuelle; Gourde, Danielle;

Roy, Marie C; Machouf, Nima

Montréal, QC

BACKGROUND: HIV-HCV co-infection has always been associated with a lower Sustained Virologic Response (SVR). HCV-infected patients have been treated at Clinique médicale l'Actuel with a new model of care since 2008 (modified Directly Observed Therapy: mDOT). We analysed the determinants of a SVR and whether the patient's co-infection status was associated with SVR in an HIVspecialized clinic.

METHODS: L'Actuel's mDOT model consists of weekly visits to inject Peg-INF and treat adverse events, monthly monitoring for Ribavirin dosage, monthly monitoring of HIV-VL and CD4 (for co-infected patients). Data were analyzed using multivariate logistic regression analyses.

RESULTS: A total of 210 patients were included. They were mostly treated with Peg-INF+RBV: $79 \%$ were men (mean age 44 years) and $83 \%$ with IDU history ( $20 \%$ active). Twenty per cent were co-infected with HIV (83\% under ART). Co-infected patients had 537 CD4 at HCV-treatment initiation, and $47 \%$ reach a CD4 $<200$ during HCV treatment. We noticed less complications in HIV-HCV (76\%) than in HCV mono-infected patients $(92 \% ; \mathrm{p}=0.009)$ but both had similar Ribavirin adjustments ( $48 \%$ vs $54 \%$; $=0.278) .56 \%$ of patients $(62 \%$ of HIV-HCV and $54 \%$ of HCV) were infected with genotypes 1,4 while $44 \%$ (38\% of HIV-HCV and $46 \%$ of HCV) with genotypes 2,3. In univariate analyses, mono-infected patients appear to achieve a slightly higher but non-significant SVR (68\% vs 62\%; $\mathrm{p}=0.289$ ). After adjusting for treatment history, genotype, demographics, mono and co-infected patients did not differ in SVR (OR 0.69 [95\% CI 0.3 to 1.5$])$.

\begin{tabular}{lrrrr} 
SVR of patients on mDOT $(\mathrm{n}=210)$ & HIV-HCV $(\mathrm{n}=42)$ & HCV $(\mathrm{n}=168)$ & \multicolumn{1}{c}{ Total } & p-value \\
\hline Genotype 1,4 $(\mathrm{n}=117)$ & $16 / 26(62 \%)$ & $52 / 91(57 \%)$ & $68 / 117(58 \%)$ & 0.433 \\
Genotype 2,3 $(\mathrm{n}=91)$ & $10 / 16(63 \%)$ & $62 / 77(81 \%)$ & $72 / 93(77 \%)$ & 0.110 \\
Total & $26 / 42(62 \%)$ & $114 / 168(68 \%)$ & $140 / 210(67 \%)$ & 0.289
\end{tabular}

CONCLUSION: Under an mDOT model of care in an HIV-specialized clinic, HIV-HCV co-infected patients were as likely to achieve a SVR as HCV mono-infected patients.

\section{P058}

HPV GENOTYPE DISTRIBUTION AND ONCOGENE EXPRESSION IN HIV-POSITIVE ADULTS AND THE UNDERLYING RISK FACTORS FOR ANAL, ORAL AND GENITAL MALIGNANCY: AN ATLANTIC CANADA PROSPECTIVE COHORT STUDY

Aslanov, Rana; Mugford, Gerry

St John's, NL

BACKGROUND: Human papillomavirus (HPV) is the most common sexually transmitted infection. People with HIV or HIV-positive partners are at a higher risk of HPV-caused pre-cancerous lesions and malignancy. OBJECTIVES: To determine the prevalence of high risk (HR) HPV types in HIV-positive adults in Atlantic Canada; to correlate their prevalence with underlying pre-malignant lesions and malignancy; and to correlate these lesions with patients' demographics and risk factors.

METHODS: This study was designed for a three-year period subsequent to baseline screening. HIV-positive adults were approached by physicians to request participation in the study. Consented participants were required to complete a confidential questionnaire. Oropharyngeal and anal swabs were obtained from all participants and cervical specimen from females. All specimens were tested for cytologic abnormalities, HPV DNA and genotyping.

RESULTS: This analysis was based on 300 patients (91.7\% males): 
Halifax, 150; Moncton, 90; St. John's, 44; and Saint John, 16 participants. The mean age was 46.9 years. A total of $77.3 \%$ of participants tested positive for HPV infection, 54\% with multiple genotypes. Up to $46 \mathrm{HPV}$ genotypes were detected, of which $39 \%$ were HR. The most frequently detected HR types were: $16,11.8 \% ; 52,7.2 \% ; 45,5.6 \% ; 51,5.4 \%$ and $18 / 59,4.6 \%$ each. HR types $16,45,51,52,53$ and 59 were strongly associated with anal lesions: ASC-US in 12.3\%; ASC-H in 1\%; LSIL in 11.7\%; and HSIL in $1 \%$ of cases. The highest number of cytologic abnormalities was reported in anal specimens (26\%). Cytologic changes were significantly associated with patients' high-risk sexual behavior. The overall prevalence of the HR genotypes was $46.6 \%$ and of the cytologic abnormalities caused by them was $27.3 \%$ during the screening year.

CONCLUSIONS: Overall, 26\% of participants had abnormal anal cytology. All participants with precancerous lesions were referred to specialists for further investigation. The study results are comparable with the results from other studies.

\section{P059 \\ HPV INFECTION AND CERVICAL DYSPLASIA IN HAART- TREATED HIV POSITIVE WOMEN}

Moses, Erin ${ }^{1}$; Blitz, Sandra' ${ }^{2}$; Raboud, Janet ${ }^{2}$; Singer, Joel ${ }^{1}$;

Smaill, Fiona ${ }^{3}$; Loutfy, Mona ${ }^{2}$; Walmsley, Sharon²;

Money, Deborah ${ }^{1}$

${ }^{1}$ Vancouver, BC; ${ }^{2}$ Toronto; ${ }^{3}$ Hamilton, ON

OBJECTIVE: To determine the association between CD4 and HIV viral suppression on cervical dysplasia in HPV/HIV co-infected women

BACKGROUND: HPV infection and cervical dysplasia are common in immunocompromised HIV+ women, and progression to cervical cancer is more rapid in HPV/HIV co-infected women. In Canada, ready access to Highly Active Antiretroviral Therapy (HAART) permits assessment of HPV/HIV co-infection and cervical dysplasia in optimally treated HIV+ women.

METHODS: As part of an ongoing Canadian longitudinal study of the immunogenicity and safety of a quadrivalent HPV vaccine in HIV+ women, baseline data, along with genital HPV-DNA sampling and cervical cytology data, was collected prior to initial vaccination. HPV genotyping was conducted with the Linear array (Roche diagnostics) and cervical cytology data was centralized and reported by Bethesda criteria. RESULTS: Of 277 participants with cytology data, HPV-DNA typing has been completed on 254, with clinical information on 226. Clinical characteristics include: median age 37 years (IQR 31 to 44 years); ethnicity $38 \%$ white, $43 \%$ black, $11 \%$ aboriginal and $8 \%$ other; $74 \%$ and a total of $83 \%$ were on HAART. Cytology results were $82 \%$ negative, 2.9\% ASCUS, $11.6 \%$ LSIL and 3.6\% HSIL. In the subset of women with LSIL or HSIL ( $n=36$ ); HPV-DNA testing confirmed 97\% were infected with any HPV type, and $82 \%$ with an oncogenic strain compared to $69 \%$ and $43 \%$ respectively in those with no dysplasia $(n=190)$. Univariate comparisons showed that women who had LSIL and/or HSIL had more lifetime sexual partners (median 10 vs $5 ; \mathrm{p}=0.03$ ), lower CD4 counts (434 cells $/ \mathrm{mm}^{3}$ vs 526 cells $/ \mathrm{mm}^{3} ; \mathrm{p}=0.02$ ), lower CD4 nadirs ( 172 cells $/ \mathrm{mm}^{3}$ vs 230 cells $/ \mathrm{mm}^{3} ; \mathrm{p}=0.03$ ) compared to women with no dysplasia. However, in a multivariate logistic regression model, neither CD4 nor CD4 nadir were predictive of dysplasia after controlling for the number of oncogenic HPV types (OR 1.70; $\mathrm{p}=0.001$ ) and non-oncogenic HPV types (OR 1.68; p<0.0001).

CONCLUSION: High rates of HPV infection were observed in this cohort of HIV+ women. After controlling for the number of HPV types present at screening, no other clinical correlates were significant predictors of cervical intraepithelial neoplasia.

\section{P060}

RATES AND CORRELATES OF SELF-REPORTED PAP TESTING AMONG AFRICAN-CARIBBEAN HIV-POSITIVE WOMEN IN ONTARIO, CANADA

Andany, Nisha; Remis, Robert; Liu, Juan; Tharao, Wangari E; Kaul, Rupert; Loutfy, Mona R

Toronto, ON

BACKGROUND: PAP testing allows for early identification and treatment of high-grade cervical lesions and prevention of cervical cancer. Cervical dysplasia and cancer are more common and aggressive in HIVpositive women and guidelines recommend annual screening in this population. This study assessed prevalence and correlates of self-reported PAP testing among African-Caribbean (AC) HIV-positive women.

METHODS: This was a cross-sectional study consisting of a comprehensive questionnaire. Participants were recruited through a women's community health centre in downtown Toronto. PAP testing history was assessed by a single question asking when the last test was done (from the Canadian Community Health Survey). Baseline characteristics were summarized using proportions with confidence intervals for categorical variables and medians with inter-quartile ranges for continuous variables. Rates of PAP testing were summarized with frequencies and proportions. Multivariable logistic regression examined correlates of testing in the previous year.

RESULTS: Data were available for 126 women. Median age was 40 years (IQR 34 to 46 years); 53.2\% were East African and 16.7\% Caribbean; $96.0 \%$ were receiving antiretroviral therapy. $69.9 \%$ of women had received a PAP test in the previous year and $82.1 \%$ in the past three years; $10.6 \%$ had never been tested. In univariate analyses, women were more likely to have been screened in the past year if they were aged 35 to 49 years vs $>50$ years (OR 6.7 [95\% CI 1.7 to 25.1]), had been in Canada for $>2$ years (OR 4.6 [95\% CI 1.6 to 13.5]), had >2 sexual partners (OR 3.4 [95\% CI 1.1 to 10.7]) or had seen a family MD in the past six months (OR 2.6 [95\% CI 1.1 to 6.2]); no variables were significant in multivariable analyses.

CONCLUSIONS: In a clinic population of HIV-infected, AC women in Toronto, $70 \%$ had PAP screening in the previous year, which is higher than previously reported rates among the general population in Ontario. Women with HIV may be screened more often, but 30\% were not screened per guidelines. Larger studies are required to determine if these rates are representative of all HIV-infected women and to develop programs to increase screening to further reduce the burden of invasive cervical disease.

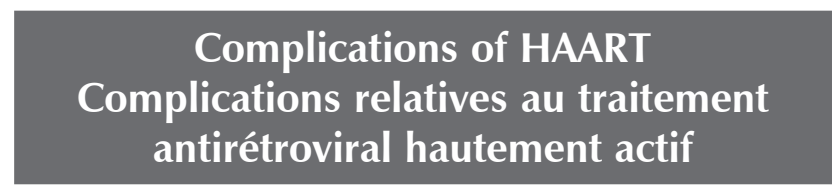

\section{P061 \\ MICROALBUMINURIA IS STRONGLY ASSOCIATED WITH INCREASED MORTALITY}

Kozai, Tsubasa; Harris, Marianne; Shurgold, Susan; Werb, Ron; Guillemi, Silvia; Montaner, Julio; Lima, Viviane Vancouver, BC

BACKGROUND: Kidney disease has increasingly been shown to be associated with increased mortality among HIV-infected population over the years. The present study was conducted to determine the impact of microalbuminuria (an important marker for kidney disease) on all-cause mortality in a large cohort of antiretroviral-naive HIV-infected patients with a median follow-up of 5.5 years (25th to 75 th percentile 3.0 to 9.0 years).

METHODS: Participants aged $\geq 19$ years, initiated antiretroviral therapy between January 1, 1996 and June 30, 2011 in British Columbia, Canada, and were followed until the last contact date, death or until June 30, 2012. The main outcome was all-cause mortality. Patients were classified as having microalbuminuria (ie, albumin to creatinine ratio ranging from $2.0 \mathrm{mg} /$ $\mathrm{mmol}$ in men, $2.8 \mathrm{mg} / \mathrm{mmol}$ in women to $30 \mathrm{mg} / \mathrm{mmol}$ ) if they tested positive in at least two consecutive urine samples that were six months apart, 
and within two years from the end of follow-up. Baseline and clinical factors were included in a multivariable Cox Proportional Hazards model. RESULTS: A total of 277 ( $\mathrm{n}=1204 ; 23 \%$ ) had confirmed microalbuminuria. A total of 37 deaths were documented, yielding an all-cause mortality rate of 4.98 per 1000 person-years, with 21 (10.7 per 1000 person-years) and 16 (2.9 per 1000 person-years) deaths in the microalbuminuria and no microalbuminuria groups, respectively. Patients with confirmed microalbuminuria had a hazard ratio 4.01 (95\% CI 1.81 to 8.88$)$ times higher than patients with unconfirmed microalbuminuria.

CONCLUSIONS: Microalbuminuria was significantly associated with an increased risk of mortality in a large cohort of antiretroviral-naive HIV-infected individuals as has been described in the general population. Our results support routine monitoring for the presence of microalbuminuria for early the identification of individuals at a higher risk of kidney disease and mortality. Further studies should be directed towards characterizing the specific causes of microalbuminuria, and whether these are amenable to therapeutic intervention.

\section{P062}

ESTIMATION OF GLOMERULAR FILTRATION RATE USING NOVEL SERUM BIOMARKERS CYSTATIN C AND BETATRACE PROTEIN IN HIV PATIENTS

Rumman, Amir; Gee, Katrina; White, Christine A;

Wobeser, Wendy L

Kingston, ON

AIM: Chronic kidney disease (CKD) is a prominent cause of morbidity in HIV-infected patients. Due to the limitations of serum creatinine ( $\mathrm{SCr}$ ) in this population, cystatin $\mathrm{C}$ and beta-trace protein (BTP) have been proposed as alternative markers of renal function. In this cross-sectional study, we compared estimated glomerular filtration rates (eGFR) using SCr versus cystatin C and BTP. We also evaluated the prevalence of CKD and a panel of inflammatory markers including IL-1, IL-6 and TNF-alpha.

METHODS: Cystatin C and BTP were measured nephelometrically and eGFR was calculated using five equations: MDRD for SCr, CKD-EPI for $\mathrm{SCr}$ and cystatin $\mathrm{C}$ (adjusted for age, sex and race) and the White equations for BTP (adjusted for gender and urea or SCr).

RESULTS: Thirty-two patients were included in the study, eight on abacavir- or zidovudine-based regimens (ABC/AZT), 12 on tenofovir-based regimens (TDF) and 12 untreated (mean age 48.9 years, $75 \%$ male, mean CD4 435 cells $/ \mathrm{mm}^{3}$, mean viral load $2.76 \log 10$ copies $/ \mathrm{mL}$ and $34 \% \mathrm{HCV}$ co-infected). Bland-Altman analysis showed only moderate agreement between SCr eGFR and cystatin C and BTP eGFR. Median eGFR derived from cystatin $\mathrm{C}$ and BTP equations was significantly lower compared to CKD-EPI SCr eGFR for all groups (TDF-based $80.3 \mathrm{~mL} / \mathrm{min} / 1.73 \mathrm{~m}^{2}$ and $91.4 \mathrm{~mL} / \mathrm{min} / 1.73 \mathrm{~m}^{2} \mathrm{vs} 101.1 \mathrm{~mL} / \mathrm{min} / 1.73 \mathrm{~m}^{2}$; untreated $85.3 \mathrm{~mL} / \mathrm{min} / 1.73 \mathrm{~m}^{2}$ and $94.9 \mathrm{~mL} / \mathrm{min} / 1.73 \mathrm{~m}^{2}$ vs $101.5 \mathrm{~mL} / \mathrm{min} / 1.73 \mathrm{~m}^{2}$ respectively, $\mathrm{p}<0.01$; and ABC/AZT-based $97.3 \mathrm{~mL} / \mathrm{min} / 1.73 \mathrm{~m}^{2}$ and $93.5 \mathrm{~mL} / \mathrm{min} / 1.73 \mathrm{~m}^{2}$ vs $103.0 \mathrm{~mL} / \mathrm{min} / 1.73 \mathrm{~m}^{2}$, respectively; $\left.\mathrm{p}<0.05\right)$. SCr-based equations failed to identify any patients with possible CKD, whereas cystatin C and BTP equations identified three and two possible CKD patients respectively. Inflammatory markers are pending.

CONCLUSION: Cystatin C- and BTP-based equations yielded a lower estimate of GFR and classified a larger proportion of patients with CKD. These novel biomarkers may be more sensitive for the early detection of HIV nephropathy and antiretroviral therapy-induced renal dysfunction.

\section{P063}

\section{NONINVASIVE SCREENING FOR HEPATIC FIBROSIS AND STEATOSIS IN HIV MONO-INFECTED PERSONS BY SIMPLE SERUM BIOMARKERS: PREVALENCE AND ASSOCIATED RISK FACTORS}

Sebastiani, Giada; Rollet, Kathleen C; Lalonde, Richard G; Pexos, Costas; Gilmore, Norbert; Klein, Marina B

Montreal, QC

BACKGROUND: Liver disease is a major contributor to mortality among HIV-infected persons. Nevertheless, relevant clinical data are scarce. We employed non-invasive biomarkers to screen HIV monoinfected persons for hepatic fibrosis and steatosis.
METHODS: A total of 974 consecutive HIV mono-infected persons $>18$ years of age (mean age 47 years, $69 \%$ men) followed in the last year in our unit were included. AST-to-platelet ratio index (APRI), Fib-4 and nonalcoholic fatty liver disease (NAFLD) fibrosis score were used to screen for hepatic fibrosis. Hepatic steatosis index (HSI) was applied to screen for hepatic steatosis. Risk factors associated with each serum biomarker were determined through multivariate logistic regression models. RESULTS: Overall, APRI, Fib-4 and NAFLD fibrosis score diagnosed liver fibrosis in $1.6 \%, 2.7 \%$ and $6.6 \%$ of cases, respectively. HSI was indicative of hepatic steatosis in $39.3 \%$ of cases. Multivariate analysis results are reported in the Table.

\begin{tabular}{|c|c|c|c|}
\hline & VARIABLE & OR & $\mathrm{p}$ \\
\hline \multirow[t]{10}{*}{$\overline{\text { APRI }}$} & Age & 0.943 & 0.12 \\
\hline & Female gender & 0.171 & 0.15 \\
\hline & Black ethnicity & 0.102 & 0.06 \\
\hline & Duration of HIV infection & 1.07 & 0.18 \\
\hline & CD4 count & 1 & 0.09 \\
\hline & HIV RNA load<50 & 0.94 & 0.95 \\
\hline & ALBUMIN & 0.78 & $<0.001$ \\
\hline & Glucose & 1.15 & 0.25 \\
\hline & Cholesterol & 0.99 & 0.96 \\
\hline & On antiretroviral therapy & 0.28 & 0.25 \\
\hline \multirow[t]{9}{*}{ FIB-4 } & Female gender & 0.29 & 0.09 \\
\hline & Black ethnicity & 0.45 & 0.2 \\
\hline & DURATION OF HIV INFECTION & 1.08 & 0.009 \\
\hline & CD4 count & 1 & 0.32 \\
\hline & HIV RNA load $<50$ & 0.49 & 0.24 \\
\hline & ALBUMIN & 0.84 & $<0.001$ \\
\hline & Glucose & 0.98 & 0.87 \\
\hline & Cholesterol & 0.91 & 0.68 \\
\hline & On antiretroviral therapy & 0.64 & 0.57 \\
\hline \multirow{8}{*}{$\begin{array}{l}\text { NAFLD FIBROSIS } \\
\text { SCORE }\end{array}$} & Female gender & 1.93 & 0.06 \\
\hline & Black ethnicity & 1.1 & 0.79 \\
\hline & DURATION OF HIV INFECTION & 1.06 & 0.008 \\
\hline & CD4 count & 1 & 0.42 \\
\hline & HIV RNA load $<50$ & 0.65 & 0.26 \\
\hline & GLUCOSE & 1.34 & $<0.001$ \\
\hline & CHOLESTEROL & 0.61 & 0.001 \\
\hline & On antiretroviral therapy & 1.5 & 0.49 \\
\hline \multirow[t]{9}{*}{$\mathrm{HSI}$} & AGE & 1.02 & 0.04 \\
\hline & FEMALE GENDER & 6.2 & $<0.001$ \\
\hline & BLACK ETHNICITY & 2.15 & $<0.001$ \\
\hline & Duration of HIV infection & 0.99 & 0.46 \\
\hline & CD4 count & 1 & 0.78 \\
\hline & HIV RNA load $<50$ & 0.81 & 0.38 \\
\hline & GLUCOSE & 1.35 & $<0.001$ \\
\hline & Cholesterol & 1.1 & 0.92 \\
\hline & On antiretroviral therapy & 1.34 & 0.34 \\
\hline
\end{tabular}

Importantly, hepatic fibrosis and steatosis by NAFLD fibrosis score and HSI, respectively, were significantly more prevalent in subjects with metabolic comorbidities as compared with the total cohort: $20.4 \%$ and $96.1 \%$ in obese subjects; $34 \%$ and $50 \%$ in diabetics; $20.4 \%$ and $51.1 \%$ in hypertensive subjects; $38.7 \%$ and $96.8 \%$ in subjects with metabolic syndrome.

CONCLUSION: Liver disease diagnosed by simple biomarkers appears frequent in HIV mono-infected persons, particularly in those with metabolic comorbidities. Prospective studies are needed to identify the best non-invasive tool for liver disease, to evaluate the prognostic impact of risk factors and to implement management strategies aimed at reducing the effects of insulin resistance/metabolic comorbidities on liver disease in this population. 
P064

PREVALENCE OF SEVERE LIVER DISEASE IN HIV MONOINFECTION: IT'S A MATTER OF HOW YOU LOOK FOR IT Sebastiani, Giada; Rollet, Kathleen C; Gilmore, Norbert; Lalonde, Richard G; Pexos, Costas; Klein, Marina B Montreal, QC

BACKGROUND: As HIV-infected adults age, their risk of chronic liver disease may increase, given their high rates of metabolic disease and alcohol use. We measured the prevalence of liver fibrosis and steatosis in HIV mono-infected persons using a panel of surrogate markers.

METHODS: We evaluated 974 HIV-mono-infected patients aged $>18$ years in active follow-up during 2011 and 2012 at a single centre. Liver fibrosis was estimated using biomarkers determined from routine blood tests and clinical parameters obtained at the last visit using literature defined cut-offs: AST-to-platelet ratio index (APRI; >1.5), AST-toALT ratio (AAR; $>1)$, FIB-4 (>3.25), and the non-alcoholic fatty liver disease (NAFLD) fibrosis score $(>0.676)$. Steatosis was estimated using the Hepatic Steatosis Index (HSI; >36).

RESULTS: Mean age was 47 years ( $n=581>45$ years), 68\% were men, $40 \%$ black; mean duration of HIV infection was 11.6 years, $92 \%$ were antiretroviral-experienced with $60 \%$ currently on PI-based and $35 \%$ on NNRTI-based therapy. Most recent median CD4 was 556 cells/ $\mu \mathrm{L}$ and $81 \%$ of patients had undetectable viral load. Information on alcohol use was not available. The estimated prevalence of significant fibrosis varied greatly ranging from $1.7 \%$ by APRI $(n=15), 2.7 \%$ by FIB- $4(n=26), 6.6 \%$ by NAFLD ( $\mathrm{n}=64)$ to $56.3 \%$ by AAR ( $\mathrm{n}=548) ; 383$ patients $(39.3 \%)$ met HSI criteria for hepatic steatosis. The majority of individuals identified as having fibrosis were among those $>45$ years (eg, $67 \%$ of those with APRI $>1.5 ; 85 \%$ of those with FIB-4>3.25).

CONCLUSIONS: The estimated prevalence of hepatic fibrosis can vary widely depending on the biomarker used. Regardless of the biomarker used, an important number of HIV mono-infected persons may be at risk for steatosis and advanced liver disease especially among those aged over 45 years. Prospective studies are needed to identify the best noninvasive diagnostic tool for liver disease and the long-term prognostic implications of these measures.

\section{P065}

\section{OUTILS DE TRAVAIL POUR LE DÉPISTAGE DES EFFETS INDÉSIRABLES LIÉS À LA PRISE DES ANTIRÉTROVIRAUX}

Therrien, Rachel; Dufault, Catherine; Desmarais, Manon

Montréal, QC

Outil de travail pour le dépistage des effets indésirables liés à la prise des antirétroviraux.

Manon Desmarais infirmière, Catherine Dufault infirmière et Rachel Therrien pharmacienne UHRESS du CHUM.

Une étude menée auprès de médecins Canadiens et leurs patients (Étude BEAHIV)1, a documenté que les effets indésirables, incluant les effets les plus incommodants, étaient signalés plus fréquemment par les patients que leur médecin traitant.

Afin d'améliorer la communication lors du questionnement sur les effets secondaires, nous avons élaboré un outil de travail.

Nous documenterons notre appréciation de l'outil, suite à son utilisation, dans le but de présenter les résultats obtenus.

L'outil a été utilisé auprès de patients VIH ambulatoires du CHUM, sélectionnés de façon aléatoire lors des cliniques médicales. Le questionnaire a été complété par le professionnel de la santé en présence du patient.

L'outil de travail a permis aux professionnels de la santé de communiquer avec le patient sur les effets indésirables de façon simple et structurée. L'outil a aussi permis d'identifier des effets indésirables qui autrement auraient pu passer inaperçus.

Au final, 120 questionnaires ont été complétés. De ces patients, 90\% se disaient satisfaits de leur thérapie antirétrovirale et $95 \%$ mentionnaient être fidèles à leur traitement, bien que $21 \%$ affirment oublier des doses. L'effet indésirable le plus fréquemment rapporté a été des troubles gastrointestinaux. Tous les autres effets secondaires évalués ont été rapporté chez moins de $10 \%$ des patients.

En conclusion, l'utilisation systématique d'un outil a permis de structurer la discussion et ainsi faciliter la communication sur les effets secondaires. Malgré une grande satisfaction face à leur traitement, les patients atteints du VIH souffrent toujours d'effets indésirables. Les résultats obtenus nous incitent donc à poursuivre l'utilisation de cet outil dans notre pratique quotidienne. Ainsi, la continuité dans la communication entre les professionnels de la santé et les patients sera favorisée et permettra d'assurer une meilleure gestion des effets indésirables.

Références :

1. Rachlis A, Gill J, Harris M et al. J Int AIDS Soc. 2010; 13(Suppl 4): P106.

\section{P066 \\ PREVALENCE AND CORRELATES OF ADVERSE EVENTS AND SYMPTOMS IN HIV-INFECTED WOMEN WITH VIROLOGIC SUPPRESSION ON COMBINATION ANTIRETROVIRAL THERAPY}

Andany, Nisha ${ }^{1}$; Blitz, Sandra ${ }^{1}$; Raboud, Janet $\mathbf{M}^{1}$; Walmsley, Sharon L'; ${ }^{1}$ Klein, Marina $\mathbf{B}^{2}$; Tseng, Alice ${ }^{1}$; Pick, Neora ${ }^{3}$; Conway, Brian ${ }^{3}$; Angel, Jonathon B ${ }^{4}$; Rachlis, Anita ${ }^{1}$; Gough, Kevin ${ }^{1}$; Cohen, Jeff ${ }^{5}$; Haase, David ${ }^{6}$; Burdge, David ${ }^{3}$; Smaill, Fiona ${ }^{7}$; de Pokomandy, Alexandra ${ }^{2}$; Loemba, Hugues ${ }^{4}$; Montaner, Julio ${ }^{3}$; El-Helou, Phillipe ${ }^{7}$; Trottier, Sylvie ${ }^{8}$; la Porte, Charles J ${ }^{4}$; Loutfy, Mona $\mathbf{R}^{1}$

${ }^{1}$ Toronto, ON; ${ }^{2}$ Montreal, QC; ${ }^{3}$ Vancouver, BC; ${ }^{4}$ Ottawa; ${ }^{5}$ Windsor, ON; ${ }^{6}$ Halifax, NS; ${ }^{7}$ Hamilton, ON; ${ }^{8}$ Laval, QC BACKGROUND: Women represent 22\% of HIV-infected Canadian adults. Some antiretroviral (ARV)-associated adverse events (AEs) are more common in women. This cross-sectional study assessed prevalence, severity and correlates of symptoms and possible AEs in HIV+ women on their first ARV regimen.

METHODS: At 14 Canadian centres, AEs were assessed by asking if women had side effects from ARVs. Symptoms were captured with the validated Symptom Index Score (SIS). Cmin and Cmax drug levels were drawn at three weekly visits. Demographics, prevalence and severity of symptoms were summarized using medians and inter-quartile ranges (IQR) (continuous variables) and frequencies and proportions (categorical variables). Logistic regression analyses assessed variables associated with reporting (a) side effects from ARVs, (b) >1 symptoms that bothered them "a lot/terribly".

RESULTS: Data was available for 82 women (median age 41 years [IQR 36 to 48 years], 56\% were Black); 57\% were taking a PI and $43 \%$ an NNRTI; median duration of ARV therapy was 20 months (IQR 9 to 45 months). $67 \%$ of women reported AEs and $73 \%$ of women reported $>1$ symptom that bothered them "a lot/terribly". The most common symptoms were fatigue $(79.3 \%)$, sleep disturbances $(72 \%)$ and depression $(70.7 \%)$. In univariate models, sqrt $\mathrm{Cmin}$ ratio was associated with reporting AEs (OR 5.01; $\mathrm{p}=0.02$ ), as was higher current $\mathrm{CD} 4$ count (OR 1.34/100 cells; $\mathrm{p}=0.04$ ); being menopausal decreased likelihood of reporting AEs (OR 0.28; $p=0.03$ ). Women from endemic countries were less likely to report $>1$ symptoms that bothered "a lot/terribly" (OR 0.28 ; $\mathrm{p}=0.01$ ).

CONCLUSIONS: Clinical symptoms are common in women on ARVs and are most commonly generalized rather than system-specific. Sqrt Cmin ratio, CD4 count and menopausal status affected reporting AEs in univariate analyses, while women from endemic countries reported fewer bothersome symptoms. Although the reported symptoms may not directly relate to ARV therapy, the frequency and severity of reported events needs to be better characterized to develop interventions that could be contemplated (including single drug switches) to improve the long-term adherence and quality of life of HIV-infected women on antiretroviral treatment. 
HIV and aging - including CVD, Osteoporosis, Neurologic effects Le VIH et le vieillissement - maladies cardiovasculaires, ostéoporose, effets neurologiques

\section{P067}

THE DEVELOPMENT OF A TOOL FOR PREVENTING AND MANAGING BONE DISEASE IN HIV-INFECTED ADULTS

Foisy, Michelle M; Hughes, Christine; Yuksel, Nese

Edmonton, $\mathrm{AB}$

OBJECTIVE: As the HIV-infected population ages, numerous comorbidities are emerging, including bone disease. HIV clinicians are faced with managing a variety of conditions that require additional knowledge and skills. The objective is to describe the development and content of a practical tool to assist clinicians in preventing and managing bone disease in HIV-infected adults.

METHODS: Development of the tool involved a group of local pharmacists with expertise in HIV and osteoporosis. The content was based on published literature, recent guidelines and an educational workshop facilitated by an experienced HIV endocrinologist. Input was also sought from our team dietician and an Infectious Diseases physician. The tool was then reviewed for content, readability and applicability by several other Canadian HIV/AIDS Network Pharmacists (CHAP). The final tool was published in the form of a 10-page fold-out pocket card.

RESULTS: The tool has four main sections: 1) Risk Factors for Fractures and Bone Loss: includes patient characteristics, diseases, nutrition, substances and medications, including certain antiretrovirals. 2) Patient Assessment: includes recommendations on initial screening, indications for bone mineral density testing, fracture risk assessment and diagnostic work-up. 3) Treatment of Osteoporosis: reviews who to treat based on risk assessment and treatment options, including drugs to treat osteoporosis. 4) Prevention: discusses non-pharmacologic and pharmacologic measures (ie, vitamin D and calcium supplementation). The pocket card was distributed to the local HIV interdisciplinary team and to CHAP members. Educational sessions are currently underway.

CONCLUSIONS: A clinical tool for the prevention and treatment of bone disease in HIV was developed by a group of expert pharmacists to provide practical guidance to clinicians and to standardize an approach to patient care. Follow-up study is needed to evaluate the clinical utility of the tool and impact on prevention and detection of osteoporosis and disease management in HIV-infected adults.

\section{P068}

\section{THE MONTREAL HIV AND AGING COHORT: RESULTS FROM A PILOT STUDY OF CORONARY CALCIUM MEASUREMENT}

Durand, Madeleine; Rau, Philip; Cyr, Lise; Matte, Stéphannie; Chamberland, Annie; Mansour, Samer; Chartrand-Lefebvre, Carl; Tremblay, Cécile L

Montréal, QC

BACKGROUND: Cardiovascular disease causes increasing morbidity and mortality in patients living with HIV. Although traditional risk factors are associated with cardiovascular disease, other risk factors (inflammation, virus and host factors) might drive this disease in patients living with HIV. We designed a large prospective cohort (the Montreal HIV and Aging cohort) to characterize cardiovascular disease in patients living with HIV. We report results of a pilot study aimed at measuring coronary artery calcium (CAC). METHOD: We prospectively enrolled HIV-positive patients with a Framingham cardiovascular risk score between 5 and 20\%. Participants underwent non-contrast ECG-gated cardiac computed tomography (CT) for CAC detection and quantification. Coronary calcium score (continuous score ranging from 0 to 1444) was analysed using non parametric equality of median test and linear regression.
RESULTS: Thirty-two patients (mean $[ \pm \mathrm{SD}]$ age $56.7 \pm 6.5$ years) were included. Twenty-eight (87.5\%) were males. Median duration of HIV infection was 17.1 years (range 3.3 to 26.8 years). All but two patients (30 [93.7\%]) were on antiretrovirals, and 26 (81.3\%) had suppressed viral loads. The median Framingham risk score was $11.5 \%$. Twenty-two $(68.8 \%)$ participants had coronary calcium (calcium score $>0$ ). The median calcium score was 8.3 , which was lower than expected for patients of the same age and sex. (Median age and sex percentile rank for calcium score $=32.5 \%$, expected $=50 \%$ ). In univariate analysis, no unique risk factor was found to be associated with increases in the calcium score.

CONCLUSION: Even if most patients (68.8\%) had coronary calcium, we found unexpectedly low calcium scores according to age and sex. The nature of coronary disease may differ in patients living with HIV (eg, less calcified plaques). Non-contrast cardiac CT can only detect calcified plaques. In the Montreal HIV and Aging cohort, further imaging techniques (CT coronarography, cardiac TEP scan, intracoronary ultrasound) will be performed to characterize cardiovascular disease and its drivers in HIV-positive patients.

\section{P069 \\ VIROLOGIC AND IMMUNOLOGIC RESPONSES TO HAART AMONG OLDER HIV-POSITIVE PATIENTS IN THE CANADIAN OBSERVATIONAL COHORT (CANOC) COLLABORATION}

Sok, Phan ${ }^{1}$; Chan, Keith²; Burchell, Ann N1; Cescon, Angela²;

Shurgold, Susan'2; Hosein, Sean ${ }^{1}$; Cooper, Curtis ${ }^{3}$; Klein, Marina B ${ }^{4}$; Loutfy, Mona R'; Machouf, Nima ${ }^{4}$; Montaner, Julio $\mathrm{S}^{2}$;

Raboud, Janet $\mathbf{M}^{1}$; Rachlis, Anita ${ }^{1}$; Tsoukas, Christos ${ }^{4}$;

Hogg, Robert $\mathbf{S}^{2,5}$; Rourke, Sean $\mathbf{B}^{1}$;

Canadian Observational Cohort, CANOC $^{2}$

${ }^{1}$ Toronto, ON; ${ }^{2}$ Vancouver, BC; ${ }^{3}$ Ottawa, ON; ${ }^{4}$ Montreal, QC;

${ }^{5}$ Burnaby, BC

BACKGROUND: Few observational studies have focused on virologic and immunologic responses among older HIV-positive patients. We aimed to characterize initial antiretroviral regimens and explore virologic and immunologic responses to HAART by age in a multisite Canadian cohort.

METHODS: CANOC is a multisite cohort study of antiretroviral-naive HIV-positive persons 18+ years who started HAART between 2000 and 2011 in British Columbia (BC), Ontario, or Quebec. The outcomes of interest were failure to rapidly suppress (first two measurements three to nine months after starting HAART not $<500$ copies $/ \mathrm{mL}$ ) and immunologic failure (12-month CD4 count change $<150$ cells $\left./ \mathrm{mm}^{3}\right)$. The primary variable of interest was older age (baseline $50+$ vs $<50$ years). We used logistic regression to model both outcomes.

RESULTS: A total of 6348 individuals were followed for a median of 46 months (IQR 23 to 78 months), with 5189 (82\%) males and 1148 persons $(18 \%)$ aged $50+$ years at treatment initiation. Compared to the younger group, older individuals were more likely to be male ( $89 \%$ vs $80 \%$; $\mathrm{p}<0.001)$, reside in $\mathrm{BC}(59 \%$ vs $48 \% ; \mathrm{p}<0.001)$, be on a regimen containing atazanvir $(25 \%$ vs $21 \% ; \mathrm{p}=0.016)$ and tenofovir/emtricitabine $(40 \%$ vs $36 \% ; \mathrm{p}<0.001$ ). Older individuals were less likely to have virologic failure ( $13 \%$ vs $16 \% ; \mathrm{p}=0.017)$, but no differences were noted by age in terms of immunologic failure ( $47 \%$ vs $45 \%$; $=0.281$ ). After adjusting for sex, province, HAART regimen, and baseline viral load, the older group remained less likely to have virologic failure (AOR 0.81 [95\% CI 0.65 to 1.00]; $\mathrm{p}=0.047$ ).

CONCLUSIONS: Older HIV-positive individuals in CANOC differ with respect to their initial HAART regimens, and are less likely to experience virologic failure. 
P070

PARTICIPATION AND SELECTION BIAS IN A PROPOSED HIV AND AGING STUDY

Szadkowski, Leah $\mathbf{M}^{\mathbf{1}}$; Raboud, Janet $\mathbf{M}^{\mathbf{1}}$; Tan, Darrell $\mathbf{H}^{\mathbf{1}}$; Smaill, Fiona $\mathbf{M}^{2}$; Smieja, Marek²; Klein, Marina B ${ }^{3}$;

Walmsley, Sharon $\mathrm{L}^{1}$

${ }^{1}$ Toronto; ${ }^{2}$ Hamilton, ON; ${ }^{3}$ Montreal, QC

BACKGROUND: Research on aging and age-related comorbidities is required to understand and treat complex health issues faced by an aging HIV-positive population. However, such research requires HIV-positive patients to participate in studies that can be time-consuming. Selection bias may occur if those willing to participate have more pre-existing comorbidities. This study investigated the willingness of patients to participate and the potential for selection bias in a proposed longitudinal HIV and aging study.

METHODS: Patients at four HIV clinics in Toronto (two), Hamilton, and Montreal were asked to complete a 30-item questionnaire regarding demographics, organ-specific health conditions (eg, involving kidney, heart or bone), and willingness to participate in a proposed longitudinal study involving lengthy questionnaires and a large set of physiologic evaluations. Demographics and organ-specific health conditions of those willing or not willing to participate were compared using Wilcoxon rank sum tests for continuous variables and $\chi^{2}$ or Fisher's exact tests for categorical variables.

RESULTS: A total of 246 patients completed the questionnaire. The median age was 54 years (IQR 49 to 59 years) and the median duration of $\mathrm{HIV}$ was 16 years (IQR 10 to 22 years). Sixty-eight per cent of patients reported known conditions with at least one organ system and $48 \%$ were on medications to treat conditions other than HIV. Seventy-five per cent (95\% CI 69\% to 80\%) were willing to participate in the proposed study. Patients willing and not willing to participate were not significantly different in terms of age, smoking status, years of HIV, and number and type of organ-specific problems.

CONCLUSIONS: A large proportion of HIV-positive patients who completed a feasibility questionnaire stated they were willing to participate in an age-related study involving a large set of evaluations. Those willing to participate were not more likely to have comorbidities. Similar evaluations need to be completed prior to initiating complex studies to ensure selection bias may not be a significant issue in enrollment.

\section{P071}

AGING WITH HIV: INTEGRATING A MODEL OF HEALTHY AGING WITH THE EXPERIENCE OF LIVING WITH HIV Hill-Mann, Alexandra $^{1}$; Lewellen, Denver ${ }^{2}$; Gahagan, Jacqueline ${ }^{1}$ ${ }^{1}$ Halifax, NS; ${ }^{2}$ Berkeley, California, USA

BACKGROUND: Advances in and access to antiretroviral therapies in Canada have increased life expectancy for persons living with HIV (PHAs). In 2009, 15\% of PHAs in Canada were 50 years of age and older. This demographic shift has implications for health care delivery and policies given that little is known about the intersection of HIV, long-term use of antiretrovirals, and 'normal' age-related health concerns. Concurrent to this shift, funding for AIDS-specific organizations (ASOs) is threatened by reductions in health spending. This research identifies gaps in the health system and in policy responses, which may preclude inclusion of PHAs among individuals who are considered to be 'aging healthily.'

METHODS: To address the lived experiences of PHAs in the face of non-inclusive conceptualizations of 'healthy aging' and reduced funding for ASOs, we employed a broad approach. A research paper examining Canadian 'healthy aging' documents provided a national context. Second, a cross-national scoping review of HIV and rehabilitation policies provided an in-depth look at gaps in policies and programs serving PHAs. Finally, pilot interviews with PHAs in Nova Scotia provided a context-rich perspective of the impact of changes to the health system on aging PHAs.

FINDINGS: Gaps in 'healthy aging' strategies and policies are identified, including the shortage of tangible health system approaches to complex chronic illness and inadequate support for PHAs. One barrier to the provision of PHA-appropriate services is the lack of inclusive frameworks of 'healthy aging'. Inspired by Hansen Kyle's core attributes of 'healthy aging', we constructed a preliminary model of 'healthy aging' for PHAs, encompassing historic and contemporary characterizations of HIV, including specialty health care, stigma, and ASOs.

CONCLUSIONS: Inclusive reconceptualizations of 'healthy aging' guidelines and policies, can provide health care frameworks through which the complex needs of the growing population of older PHAs can be better met across the lifespan.

\section{P072}

\section{UNCERTAINTY AS A KEY COMPONENT OF DISABILITY} EXPERIENCED BY OLDER ADULTS LIVING WITH HIV

\section{Solomon, Patricia ${ }^{1}$; O'Brien, Kelly ${ }^{2}$; Wilkins, Seanne ${ }^{1}$;}

\section{Gervais, Nicole ${ }^{1}$}

${ }^{1}$ Hamilton; ${ }^{2}$ Toronto, ON

BACKGROUND: Uncertainty is a core component of episodic disability and can have a negative impact on the health of people living with HIV (PHAs),

PURPOSE: To understand the contribution of uncertainty to the disability experienced by older adults living with HIV.

METHODS: We conducted a qualitative study using in-depth face-toface or telephone interviews with men and women 50 years of age or older living with HIV. We recruited participants from hospital clinics and AIDS service organizations in Toronto and the Hamilton area. We asked participants about their health-related challenges aging with HIV within a disability framework. Interviews were audio recorded, transcribed verbatim and analysed using a constructivist grounded theory approach. An Advisory Committee of PHAs and representatives from ASOs provided oversight to the process and assistance with data interpretation.

RESULTS: Twenty-nine men (59\%) and twenty women with a mean age of 56 years and a mean length since diagnosis of 13.5 years participated in an interview. Participants described sources of age-related uncertainty across six areas: source of health challenges, whether health providers had age-related knowledge and skills to manage their disability, financial uncertainty, transitioning to retirement, availability of ageappropriate housing and who would care for them as they got older. While not directly attributed to aging, the episodic nature of the illness left many worrying when their next episode of illness would occur and what the consequences of that episode might be.

CONCLUSIONS: Uncertainty is a key component of disability experienced by older adults aging with HIV. Results highlight the need to develop interventions that reduce uncertainty, which may subsequently reduce disability and enhance the overall health and successful aging of older adults with HIV.

\section{P073}

CAN CHANGES IN COGNITION BE QUANTIFIED WITH A FEW QUESTIONS? AN APPLICATION OF MODERN TEST THEORY

Brouillette, Marie-Josee; Forcellino, Laurence; Thomas, Réjean; Potter, Martin; Vézina, Sylvie; Harvey-Langton, Alexandra; Koski, Lisa; Finch, Lois; Fellows, Lesley; Mayo, Nancy Montreal, QC

BACKGROUND: While the ability to quantify a change in cognition is important clinically, available screening measures lack the psychometric properties required to provide a reliable change score. Our aim is to develop a measure that would reliably quantify cognitive ability with only a few questions, using modern psychometric methods. The objective of this study was to estimate the extent to which a measure of cognition, which combines responses to questionnaires and performance-based items, forms a hierarchical unidimensional construct.

METHODS: Cognition was assessed on three occasions in 100 consecutive non-demented HIV+ individuals using both performance-based measures (computerized battery), as well as a self-report inventory (Patient Deficit Questionnaire). We report here on baseline testing. All items were combined and Rasch analyzed to create a unique measure of 
"cognitive ability".

RESULTS: Characteristics of the sample are as follows: 95\% males; age $49 \pm 10.0$ years; education $18 \% \leq 12$ years, $47 \%$ University degree; $78 \%$ Caucasians. The final measure fit the Rasch model, and covered 11 logits (or s.d.) of the theoretical construct. It included several performancebased items as well as patient-reported items, demonstrating that both approaches measure the same underlying latent construct. Items covered the following cognitive domains: attention/working memory, executive function/abstraction, speed of information processing, language, memory.

CONCLUSIONS: The fit of the data to the Rasch model supports a straightforward approach to the measurement of cognition as a global construct, in which cognition is evaluated across an appropriate range of difficulty rather than across several distinct cognitive abilities, and rated on a scale that has the psychometric properties required to provide a reliable change score. It also paves the way to the development of an adaptive measure that could be administered in less than 15 minutes. Future research will document stability of the items over time.

\section{P074 \\ EFFECTS OF HIV AND AGING ON \\ NEUROPSYCHOLOGICAL PERFORMANCE AND RATES OF HAND IN CAUCASIAN MSM ON CART WITH SUPPRESSED PLASMA VIRAL LOAD: PROSPECTIVE RESULTS FROM THE OHTN COHORT STUDY}

Silverbrook, Max ${ }^{1}$; Gill, John ${ }^{2}$; Rachlis, Anita ${ }^{1}$; Carvalhal, Adriana ${ }^{1}$; Collins, Evan'; Arbess, Gordon'; Brunetta, Jason"; Pick, Neora ${ }^{3}$;

McCombe, Jennifer ${ }^{2}$; Letendre, Scott ${ }^{4}$; Marcotte, Thomas ${ }^{4}$; Bekele, Tsegaye ${ }^{1}$; Burchell, Ann ${ }^{1}$; Raboud, Janet ${ }^{1}$;

Ibanez-Carrasco, Francisco ${ }^{1}$; Thornton, Allen ${ }^{5}$; Bourgeois, Claire ${ }^{1}$; Rosenes, Ron ${ }^{1}$; Atkinson, Maggie ${ }^{1}$; Reinhard, Robert ${ }^{6}$;

McPhee, Paul ${ }^{1}$; McGee, Frank ${ }^{1}$; Rourke, Sean B $^{1}$; The Centre for

Brain Health in HIV/AIDS 1

${ }^{1}$ Toronto, ON; ${ }^{2}$ Calgary, AB; ${ }^{3}$ Vancouver, BC; ${ }^{4}$ San Diego;

${ }^{5}$ Burnaby, BC; ${ }^{6}$ San Francisco, California, USA

INTRODUCTION: There is continued debate about whether increased neuropsychological (NP) impairment occurs in those aging with HIV infection; minimal data is available on rates of HIV-Associated Neurocognitive Disorders (HAND) in older persons with HIV.

METHODS: 200 adult Caucasian men (89\% gay/bisexual) who were on cART with suppressed viral load $(<50$ copies $/ \mu \mathrm{l})$ at baseline completed brief NP battery testing psychomotor efficiency, working memory, and verbal learning/memory at baseline and one year later. To examine effects of aging, participants were grouped into 'younger' ( $\leq 45$ years, $n=72$; mean 41.0 years) and 'older' ( $50+$ years, $n=128$; mean 57.3 years) age subgroups.

RESULTS: At baseline, 57\% of participants had HAND (Antinori et al, 2007 criteria) with rates of $49 \%$ in 'younger' and 61\% in 'older' groups. Asymptomatic Neuropsychological impairment (ANI) was most common overall ( $62 \%$ of those with HAND), with $22 \%$ having Mild Neurocognitive Disorder (MND) and 16\% with HIV-Associated Dementia (HAD). Both age subgroups had similar MND and HAD rates but higher rates of ANI occurred in 'older' vs 'younger' subgroups (38\% and $11 \%$, respectively). MANOVA results indicated significant main effects of age on overall NP performance, $F(1,198)=25.2 ; p<0.01$, $\eta^{2}=0.113$, and significant interaction between age and time between the two age groups in overall NP performance over time $(\mathrm{F} 1,198=4.84$, $\left.\mathrm{p}=0.029, \dot{\eta}^{2}=0.024\right)$; performance improved by 0.09 standard deviation (SD) units among 'younger' participants but worsened by $0.08 \mathrm{SD}$ units among 'older' subgroup.

CONCLUSION: This is the first Canadian study to examine the rates of HAND and NP impairment in the context of aging. HAND is common in Caucasian MSM who are on cART and virologically suppressed, and increases in NP impairments and ANI occur as persons with HIV age. Neuro-behavioural interventions are critically needed to address these neurological complications and their associated effects on well-being and quality of life.

\section{P075}

INTERACTION OF GENDER AND RACE/ETHNICITY ON NEUROPSYCHOLOGICAL IMPAIRMENT RATES AMONG PEOPLE LIVING WITH HIV IN ONTARIO: FINDINGS FROM THE OHTN COHORT STUDY

Bourgeois, LC ${ }^{1}$; Carvalhal, Adriana ${ }^{1}$; Thornton, Allen $\mathrm{E}^{2}$;

Rachlis, Anita ${ }^{1}$; Burchell, Ann ${ }^{1}$; Collins, Evan ${ }^{1}$;

Ibanez-Carrasco, Francisco ${ }^{1}$; McGee, Frank ${ }^{1}$; Arbess, Gordon ${ }^{1}$; Loemba, Hugues ${ }^{3}$; Raboud, Janet ${ }^{1}$; Brunetta, Jason ${ }^{1}$;

McCombe, Jennifer ${ }^{4}$; Gill, MJ ${ }^{5}$; Atkinson, Maggie ${ }^{1}$;

Silverbrook, Max ${ }^{1}$; Pick, Neora ${ }^{6}$; MacPhee, Paul' ${ }^{1}$;

Reinhard, Robert ${ }^{7}$; Rosenes, Ron ${ }^{1}$; Letendre, Scott ${ }^{8}$;

Rourke, Sean $\mathrm{B}^{1}$; Marcotte, Thomas $\mathrm{D}^{\mathbf{8}}$; Bekele, Tsegaye ${ }^{1}$;

Tharao, Wangari ${ }^{1}$; Husbands, Winston ${ }^{1}$; The Centre for Brain

Health in HIV/AIDS ${ }^{6}$

${ }^{1}$ Toronto, ON; ${ }^{2}$ Burnaby, BC; ${ }^{3}$ Ottawa, ON; ${ }^{4}$ Edmonton;

${ }^{5}$ Calgary, AB; ${ }^{6}$ Vancouver, BC; ${ }^{7}$ San Francisco; ${ }^{8}$ San Diego,

California, USA

INTRODUCTION: Neuropsychological impairment (NPI) remains prevalent among persons with HIV. Results from multicenter US CHARTER study indicate that $52 \%$ of adults with HIV experience NPI in era of combination antiretroviral therapy (cART). Our study will begin to contribute critical data on rates of NPI and associated determinants for priority populations most affected by HIV in Canada.

METHODS: A total of 775 adults (58 Caucasian women, 490 Caucasian men, 118 African, Caribbean, and Black [ACB] women, and 109 ACB men) completed brief NP battery testing psychomotor efficiency, working memory, and verbal learning/memory. Mean age and years of education were 45.6 years and 13.6 years, respectively. Most were men (77\%), gay/ bisexual (60\%), born in Canada (61\%), on cART (83\%), and had suppressed plasma viral load $(71 \%)$. NP test scores were demographically corrected using published norms. Overall NPI was defined using Global Deficit Score approach (Carey et al, 2004).

RESULTS: Overall, $56 \%$ had NPI. There was no significant difference in NPI by race/ethnicity of participants (56\% in Caucasian and $57 \%$ in ACB populations; $\mathrm{p}=0.77$ ). A significant difference in NPI by gender was observed, with higher prevalence among women than men (69\% vs 52\%; $\mathrm{p}<0.01)$. The difference between women and men remained significant among ACB participants ( $72 \%$ vs $40 \%$; $<20.001$ ) but not among Caucasian women and men ( $64 \%$ vs $55 \% ; p=0.19)$. Significant predictors of NPI $(\mathrm{p}<0.05)$ across groups included: gender, English fluency, place of birth, current smoking, alcohol use in past year, current depression, CD4 nadir $<200$ and cART treatment.

CONCLUSIONS: Similar rates of NPI occur in Canada as in US but there are significant differences in rates of NPI by gender and within ACB populations in Canada. Further work is underway to tease out impact of comorbidities on NPI with goal of determining natural history of NPI in these populations and the appropriate interventions to address these neurologic complications.

\section{P076}

PREVALENCE OF A POSITIVE SCREEN FOR NEUROCOGNITIVE IMPAIRMENT, ANXIETY AND DEPRESSION IN HIV-1 INFECTED PATIENTS IN CANADA

Ackad, Nabil'; Gill, John²; Loutfy, Mona ${ }^{3}$; Baril, Jean-Guy; DeWet, Joss $^{4}$; Trottier, Benoit ${ }^{1}$; De Alvaro, Cristina ${ }^{5}$; Van Wyk, Jean ${ }^{6}$ ${ }^{1}$ St-Laurent, QC; ${ }^{2}$ Calgary, AB; ${ }^{3}$ Toronto, ON; ${ }^{4}$ Vancouver, BC; ${ }^{5}$ Madrid, Spain; ${ }^{6}$ Paris, France

BACKGROUND/AIM: HIV has been associated with neurocognitive impairment (NCI) ranging from asymptomatic NCI to HIV-associated dementia. Furthermore, anxiety and depression, previously associated with decreased quality of life and medication adherence, represent the most frequently observed psychiatric disorders. The aim of this analysis was to assess the prevalence of NCI, anxiety and depression in HIV-1 infected patients in a Canadian routine clinical care setting.

METHODS: Canadian patients enrolled in the Pan Western European \& Canadian multinational, cross-sectional, epidemiologic CRANIum 
study, were included in this sub-analysis. Neurocognitive function (NCF) was assessed with the Brief Neurocognitive Screen (BNCS); anxiety and depression were assessed with the Hospital Anxiety and Depression Scale (HADS). A positive NCI screen was defined as $\geq 1$ standard deviation (SD) below the population mean on two of the three BNCS tests or $\geq 2$ SD on one test. Based on average T-score accross the BNCS tests, NCF was classified as normal ( $\geq 40$ ), mild NCI (35 to 39), mildto-moderate NCI (30 to 34), moderate NCI (25-29), moderate-to-severe $\mathrm{NCI}(20$ to 24$)$, and severe NCI $(\leq 19)$. A positive screening for anxiety and depression was defined as a HADS score of $\geq 8$ for the individual components.

RESULTS: 124 patients (66.1\% male) were included with a mean ( \pm SD) age of $44.1 \pm 11.2$ years and mean $( \pm S D)$ time since HIV-1 diagnosis of $7.9 \pm 6.2$ years. Among these, $33.1 \%$ were HAART-naïve and $66.9 \%$ were HAART-experienced. At the time of assessment mean $( \pm \mathrm{SD})$ viral load was $45338.0 \pm 94630.4$ copies $/ \mathrm{mL}$ in HAART-naïve patients and $184.2 \pm 942.7$ copies $/ \mathrm{mL}$ in HAART-experienced patients, while CD4+ count was $538.0 \pm 196.9$ cells $/ \mathrm{mm}^{3}$ and $517.5 \pm 192.9$ cells $/ \mathrm{mm}^{3}$, respectively. The overall prevalence of a positive NCI screen was $35.5 \%$. Based on average T-score, $30.6 \%$ had NCI: $13.7 \%$ mild, $8.1 \%$ mild-to-moderate, $6.5 \%$ moderate and $2.4 \%$ moderate-to-severe. $38.7 \%$ and $16.1 \%$ of patients screened positive for anxiety and depression, respectively.

CONCLUSION: These data show that a significant proportion of HIV-1 patients seen in Canadian routine clinical care setting had a positive screen for NCI, depression and anxiety, highlighting the importance of screening for neuropsychiatric disorders.

\section{P077 \\ CORRELATES OF PSYCHOLOGICAL DISTRESS IN WOMEN LIVING WITH HIV PARTICIPATING IN THE OHTN COHORT STUDY IN ONTARIO, CANADA}

Benoit, Anita C' ${ }^{\text {; }}$ Light, Lucia ${ }^{1}$; Burchell, Ann N¹;

Gardner, Sandra ${ }^{1}$; Margolese, Shari ${ }^{1}$; Tharao, Wangari ${ }^{1}$;

Kwaramba, Gladys ${ }^{2}$; Loutfy, Mona ${ }^{1}$

${ }^{1}$ Toronto; ${ }^{2}$ Hamilton, ON

BACKGROUND: Women living with HIV experience many systemic inequalities which impacts psychological distress levels. Our objective was to determine the levels and correlates of psychological distress experienced by women living with HIV in Ontario.

METHODS: The OHTN Cohort Study is an observational, dynamic cohort of people with HIV. Data included chart extractions, database linkages, and structured interviews. Participants in this analysis selfidentified as women and completed the Kessler Psychological Distress Scale (K10) between 2009 and 2011 ( $\mathrm{n}=337)$. The K10 quantifies psychological distress as the frequency and severity of symptoms of anxiety and depression. It consists of 10 questions with 5-point scales where increasing values signify increased psychological distress. We carried out univariate and multivariable logistic regression analyses with a K10 score $>19$ as the outcome, and age and ethnicity were a priori exposures of interest.

RESULTS: Median age was 43 years (IQR 35 to 50 years) and $46.3 \%$ were white, $28.8 \%$ black or African, $16.9 \%$ Aboriginal, and $8.0 \%$ of multiple race. The median K10 score was 18 (IQR 13 to 25) and the overall distribution was $57.6 \%$ low psychological distress levels, $16.9 \%$ moderate, $10.4 \%$ high and $15.1 \%$ very high. Multivariable logistic regression revealed higher risk of psychological distress for disability (OR 2.7 [95\% CI 1.6 to 4.5]), living in a household without their children (OR 2.0 [95\% CI 1.2 to 3.3]), having an education level of some college or less (OR 1.9 [95\% CI 1.1 to 3.3]) and CD4 counts $>200$ cells $/ \mathrm{mm}^{3}$ (OR 2.3 [95\% CI 1.0 to 5.4]). Age and ethnicity were not associated with psychological distress.

CONCLUSIONS: Our findings suggest that social determinants such as education, physical determinants such as disability and CD4 count and familial determinants such as living without their children shape the experience of psychological distress for women living with HIV in Ontario. The contributing social, economical, and structural factors that modulate this psychological distress must be considered for its management.
P078

\section{AMYLOID AND TAU CEREBROSPINAL FLUID (CSF) BIOMARKERS IN PATIENTS WITH HIV ASSOCIATED NEUROCOGNITIVE DISORDER (HAND)}

Gil, Diana $\mathrm{M}^{1}$; Harris, Marianne ${ }^{1}$; Lee, Josephine Hoi Yan ${ }^{1}$; Guillemi, Silvia'; Hull, Mark'; Fok, Alice C ${ }^{1}$; Hogg, Robert ${ }^{1,2}$; Montaner, Julio ${ }^{1}$; Hsiung, G-Y Robin ${ }^{1}$

'Vancouver; ${ }^{2}$ Burnaby, BC

BACKGROUND: CSF beta-amyloid 42 (A $\beta 42)$, total tau ( $t$-tau), and phosphorylated 191-tau (P191-tau) are useful diagnostic tools for Alzheimer's Disease (AD). CSF A 42 levels are lower, and t-tau and P191-tau levels are higher in patients with AD than in patients without cognitive impairment. We examined levels of these biomarkers and their association with other HIV-related factors in HIV+ patients with HAND.

METHODS: Biomarkers were measured in CSF samples obtained from lumbar puncture (LP) performed in HIV+ adults ( $>18$ years) presenting with HAND to a Neurocognitive Disorder Clinic at St Paul's Hospital. CSF A 342 , t-tau, and P191-tau were measured with ELISA assays (Innogenetics, Ghent, Belgium). HIV clinical and laboratory data were obtained from the BC Centre for Excellence in HIV/AIDS Drug Treatment Program database. Associations between CSF biomarkers and HIV-related data were analyzed using Pearson's and Kendall's tau correlations.

RESULTS: Of 22 subjects, 21 were male, median age was 54 years (IQR 46 to 58 years), and all were on HAART at the time of LP. Median duration on HAART was 5.5 years (IQR 2.25 to 12 years).Median time since HIV diagnosis was 13 years (IQR 6 to 17.5 years), and eight had a history of opportunistic infections. Median CD4 nadir was 150 cells $/ \mathrm{mm}^{3}$ (IQR 50 to 210 cells $/ \mathrm{mm}^{3}$ ), and median CD4 prior to LP was 430 cells $/ \mathrm{mm}^{3}$ (IQR 352.5 to 652.5 cells $/ \mathrm{mm}^{3}$ ). At the time of the LP, VL was $<50$ copies/mL in plasma in 19/22 subjects, and in CSF in 15/18 (four did not have CSF VL measured).

CSF A 342 showed a strong positive correlation with current CD4 cell count $(\mathrm{r}=0.458 ; \mathrm{p}=0.032)$. CSF $\mathrm{t}$-tau $(\mathrm{r}=-0.473 ; \mathrm{p}=0.026)$ and P191-tau $(\mathrm{r}=-0.424 ; \mathrm{p}=0.049)$ were negatively correlated with HAART duration. When compared within HAND subtypes (asymptomatic/mild/dementia), a trend was observed of increased CSF $t$-tau $(p=0.114)$ in patients with greater cognitive impairment.

CONCLUSIONS: CSF biomarkers may show a similar pattern in HAND as in AD. Higher CD4 cell count and longer duration of HAART appear to have beneficial effects on these CSF biomarkers.

\section{P079}

\section{CURRENT SMOKING BUT NOT PREVIOUS SMOKING IS ASSOCIATED WITH SHORTER LEUKOCYTE TELOMERE LENGTH, A MARKER OF AGING, IN HIV+ AND HIV- ADULTS}

Zanet, DeAnna L; Thorne, Anona; Sattha, Beheroze;

Maan, Evelyn J; Gadawski, Izabelle; Murray, Melanie; Singer, Joel;

Money, Deborah M; Pick, Neora; Cote, Helene C; The CIHR

Emerging Team in HIV Therapy and Aging (CARMA)

Vancouver, BC

BACKGROUND: Smoking, a risk factor for many diseases, is highly prevalent in the Canadian HIV+ population. Smoking leads to inflammation, oxidative stress and leukocyte turnover. We investigated the effects of smoking and other relevant factors on leukocyte telomere length (LTL) in HIV+ and HIV- persons who currently, previously, or never smoked.

METHODS: Blood relative LTL was measured by qPCR in adults enrolled in the CARMA cohort study. Demographic and clinical variables univariately related to LTL $(\mathrm{p}<0.15)$ were used as candidates in the development of multivariate linear regression models.

RESULTS: LTL was measured in 194 (50\%) current- [median (range) age 40 years ( 20 to 76 ) years, $74 \%$ female, $56 \%$ HIV+]; 84 (21\%) previous- [34 years (20 to 75 years), $76 \%$ female, $65 \% \mathrm{HIV+}$ ]; and $112(29 \%)$ never-smokers [34 years (20 to 58 years), $78 \%$ female, $54 \% \mathrm{HIV+}$. 
Candidate variables for the multivariate model for all participants were: age, pack-years smoking, current and previous (vs never) smoking, illicit drug use ever, active and cleared HCV infection (vs never), HIV+ status, and income $<\$ 15 \mathrm{~K} /$ year. Due to missing data, parental ages were not included. Older age $(\beta=-0.25 / 10$ years; $p<0.0001), \mathrm{HIV}+$ status $(\beta=-0.13$; $\mathrm{p}=0.043)$, active $\operatorname{HCV}(\beta=-0.18 ; \mathrm{p}=0.042)$, and current smoking $(\beta=-0.19 ; p=0.029)$ were associated with shorter LTL. Among HIV+ participants, HIV-related parameters $(\mathrm{CD} 4, \mathrm{pVL})$ were not univariately related to LTL. In similar models developed for each smoking group, apart from older age ( $\leq 0.005$ in all groups), HIV+ status was only associated with shorter LTL among never-smokers $(\beta=-0.40 ; p=0.001)$ while active HCV showed some association among current-smokers $(\beta=-0.17$; $\mathrm{p}=0.063$ ). Pack-years smoking were not related to LTL. There was evidence of potential interactions as HIV status and low income were univariately associated with shorter LTL only among never-smoker participants.

CONCLUSIONS: Half of the cohort participants currently smoked. These preliminary analyses suggest that current smoking status rather than cumulative exposure, past smoking or HIV infection impacts LTL most, stressing the importance of smoking cessation interventions in this population.

\section{HIV in Women, Pregnancy and Children Le VIH chez les femmes, les femmes enceintes et les enfants}

\section{P080 \\ WHICH WOMEN ARE IN CONTINUOUS HIV CARE? AN EXAMINATION OF WOMEN PARTICIPATING IN THE OHTN COHORT STUDY (OCS)}

Light, Lucia'; ${ }^{1}$ Burchell, Ann N1; Gardner, Sandra1; Benoit, Anita $\mathrm{C}^{1}$; Margolese, Shari ${ }^{1}$; Tharao, Wangari $\mathrm{E}^{1}$; Kwaramba, Gladys ${ }^{2}$; Rourke, Sean $B^{1}$; Loutfy, Mona ${ }^{1}$ ${ }^{1}$ Toronto; ${ }^{2}$ Hamilton, ON

BACKGROUND: Continuous HIV care is an important component of the test and treat cascade. Our objective was to describe the proportion and characteristics of HIV-positive women in continuous HIV care in Ontario in 2007 to 2011.

METHODS: We analyzed data from 669 women participating in the multi-site OCS in 2007 to 2011. Data were obtained from medical charts, interviews, and linkage with databases at the Public Health Ontario Laboratories. We defined continuous HIV care as $\geq 2$ viral load or CD4 tests $\geq 90$ days apart in a given calendar year, among women who had at least 1 test in the preceding year. We identified predictors for being in continuous HIV care using multivariable GEE logistic regression.

RESULTS: At baseline, women were 41 years old (SE 0.4), living with HIV for 8 years (SE 0.2). Ethnicities were $36 \%$ white, $40 \%$ black/African, $11 \%$ Aboriginal, and $12 \%$ other. On average, $88.4 \%$ (95\% CI 86.6 to 90.1) were in continuous HIV care; annual rates were $87.2 \%, 87.2 \%$, $90.7 \%, 88.5 \%$ and $88.1 \%$ in 2007 through 2011. Adjusted factors for continuous care were: continuous care in previous year (OR 3.4 [95\% CI 2.3 to 5.1$]$ ), non-IDU (OR 1.5 [95\%CI 1.0 to 2.3]), age (OR 1.4 per $10+$ years [95\% CI 1.1 to 1.6$]$ ), nadir CD $4<200$ cells $/ \mathrm{mm}^{3} \leq 5$ years ago (OR 0.6 [95\% CI 0.5 to 0.9]), time since HIV diagnosis (OR 0.95 per year [95\% CI 0.93 to 0.98]). Continuous HIV care was not associated with socioeconomic status, family/marital status, region, or other clinical factors.

CONCLUSIONS: Most women who had contact with specialized HIV care in preceding year received continuous care. Nevertheless, it is troubling that $11.6 \%$ were in discontinuous care in 2007 to 2011 after being in care the prior year. This proportion is likely to be greater among all women diagnosed with HIV in Ontario, because OCS participants are likely to represent the upper spectrum of those receiving optimal HIV care by experienced physicians. Greater efforts are needed to understand and overcome barriers to continuous HIV care among women.
P081

A CASE SERIES OF MULTIPLE GESTATIONS IN HIVPOSITIVE PREGNANT WOMEN

Sovran, Laura D; MacGillivray, Jay; Yudin, Mark H

Toronto, ON

OBJECTIVE: To describe the number and clinical outcomes of multiple gestations in HIV positive mothers at one urban Canadian hospital over the past 10 years.

STUDY DESIGN: Descriptive single centered retrospective case series. RESULTS: From 2002 to 2012 four HIV positive mothers delivered twins at St. Michael's Hospital in Toronto, Canada. The average age was 31.5 years (range 24 to 37 years). Three sets of twins were dichorionic diamniotic while the remaining pair was monochorionic and diamniotic. All mothers received antepartum HAART to decrease viral load. The average gestational age at delivery was 36.9 weeks (range 36 to 37.9 weeks). Three patients had undetectable viral load at delivery. One patient had 3171 copies $/ \mathrm{mL}$ at the time of delivery, despite having undetectable loads previously. Three mothers were GBS positive and received intrapartum antibiotic prophylaxis. All patients received intravenous AZT prior to delivery (range $1 \mathrm{~h}$ to $12 \mathrm{~h}$ ). The types of delivery included a spontaneous vaginal delivery of Twin $A$ followed by a vacuum assisted delivery of Twin $\mathrm{B}$, an induction of labour resulting in a vaginal delivery of Twin A and a vacuum assisted delivery of Twin B and two planned cesarean sections. No mother developed post partum complications. Each infant was formula fed and treated with six weeks of AZT after birth. There were no NICU admissions or neonatal complications. All infants were HIV negative.

CONCLUSIONS: We managed a small number of HIV-positive women with multiple gestations over the past ten years. Their pregnancies were uncomplicated and resulted in the birth of HIV-negative children. More research is required on HIV-positive mothers with multiple gestations in the HAART era in high resource settings to better explore the pregnancy, delivery and neonatal outcomes associated with HIV infection in multiple gestations.

\section{P082 \\ ANGIOGENESIS AND ADVERSE PREGNANCY OUTCOMES IN WOMEN WITH HIV: THE AAPH STUDY}

Papp, Eszter; Loutfy, Mona; Yudin, Mark; Walmsley, Sharon;

Murphy, Kellie; Kennedy, Logan; Serghides, Lena

Toronto, ON

BACKGROUND: HIV-positive women experience higher levels of adverse pregnancy outcomes. The mechanisms responsible for this remain unknown. Alterations in angiogenic factors that push the balance towards an anti-angiogenic state have been associated with pregnancy complications such as low birth weight, pre-term birth and preeclampsia. Both HIV infection and HIV antiretrovirals have been reported to alter levels of some angiogenic factors. We hypothesised: that levels of angiogenic factors will differ between HIV-positive and uninfected women; and that changes in the levels of these factors will be predictive of adverse pregnancy outcomes. To test these hypotheses we have initiated a longitudinal cohort study of HIV-positive and HIV-negative pregnant women. This is an ongoing study.

METHODS: Recruitment of 100 HIV-positive women is underway at five sites in Toronto. HIV-positive pregnant women are being recruited in their first trimester or early in their second trimester. Four to nine blood samples are collected throughout pregnancy. At delivery maternal, placental, and cord blood, and placenta tissue are collected. Women are separated into three groups: those with uncomplicated full-term pregnancies, those who delivered a small for gestational age neonate, and those that delivered pre-term. One hundred HIV-negative pregnant women matched for parity, age, ethnicity and educational levels are being recruited as controls.

RESULTS: To date, 48 HIV-positive women (36 delivered) and $10 \mathrm{HIV}$ negative women (three delivered) have been recruited. Of these $36 \mathrm{HIV}$ positive women, six delivered pre-term $(16.7 \%)$, eight had small for gestational age babies (22.2\%), and one experienced a fetal demise at 12 weeks. Comparing to the 50th percentile for gestational age, HIV-positive 
women had significantly lower birth weight. Multiple placental abnormalities were observed in the HIV-positive group, including a 20\% rate of velamentous cord insertion.

DISCUSSION: Although recruitment is still ongoing, we have already observed a high incidence of adverse birth outcomes and placenta pathologies in the HIV-positive women.

\section{P084}

\section{MISSED OPPORTUNITIES FOR THE PREVENTION OF VERTICAL HIV TRANSMISSION (VT): EVIDENCE FROM THE CANADIAN PERINATAL HIV SURVEILLANCE PROGRAM (CPHSP)}

Bitnun, Ari' ${ }^{1}$; Lee, Terry $\mathrm{C}^{2}$; Singer, Joel ${ }^{2}$; Samson, Lindy $\mathrm{M}^{3}$; Brophy, Jason ${ }^{3}$; Kakkar, Fatima ${ }^{4}$; Lapointe, Normand ${ }^{4}$; Forbes, John²; Sauve, Laura ${ }^{2}$; Money, Deborah $\mathbf{M}^{2}$; Alimenti, Ariane'; ${ }^{2}$ The Canadian Pediatric and Perinatal HIV/AIDS Research Group (CPARG) ${ }^{5}$

${ }^{1}$ Toronto, ON; ${ }^{2}$ Vancouver, BC; ${ }^{3}$ Ottawa, ON; ${ }^{4}$ Montreal, QC; ${ }^{5}$ Canada

OBJECTIVES: To describe missed opportunities (MOs) for the prevention of vertical HIV transmission (VT) in Canada during the combination antiretroviral therapy (cART) era based on data from the Canadian Perinatal HIV Surveillance Program (CPHSP).

METHODS: All children born in Canada to HIV-infected mothers between 1997 and 2011 in the CPHSP database were reviewed. A MO was defined by failure to administer: (a) maternal cART $\geq 4$ weeks before delivery or; (b) intravenous zidovudine during labor or; (c) $\geq 4$ weeks of zidovudine to the infant.

RESULTS: Of 2551 children, 111 acquired HIV infection (4.4\%). Fortynine of 111 infected children (44\%) were identified after three months of age (median 1.4 years [0.25 to 10.6]); no preventive interventions were implemented in them. VT rate: $0.1 \%$ with maternal cART $\geq 4$ weeks; $4.6 \%$ with maternal cART $<4$ weeks; $4.0 \%$ with intrapartum and infant therapy only (no cART). One or more missed interventions (irrespective of VT) identified in 37\% ( $n=946)$ : no maternal cART $(13 \%$; $n=323)$; $<4$ weeks maternal cART $(17 \%$; $=434)$; no intravenous zidovudine during labor $(16 \% ; n=403)$; no/incomplete infant therapy $(8 \% ; n=204)$. On univariate analysis, receipt of no/ $<4$ weeks of cART was more likely for prairie provinces ( $45 \%$ vs $22 \%$ to $25 \%$; $p<0.01$ ), with intravenous drug use (IDU) as risk factor ( $47 \%$ vs $24 \%$; $<<0.01$ ), aboriginal ethnicity $(43 \%$ vs $22 \%$ to $32 \% ; p<0.01)$ and premature birth $(33 \%$ vs $25 \%$; $<0.01)$; in the multivariate analysis, geographic region $(\mathrm{p}<0.01)$ and IDU $(\mathrm{p}<0.01)$ retained significance. MOs by calendar year of birth declined from $93 \%$ in 1997 to $21 \%$ ( $10 \%$ for no/<4 weeks maternal cART) in 2011.

CONCLUSIONS: The reduction in VT attained since 1997 is threatened by the high rate of suboptimal implementation of standard-of-care preventive interventions in Canada. It is imperative that HIV-infected women be identified early so that timely cART can be initiated. Further exploration of the circumstances leading to MO's is planned.

\section{P085 \\ AWARENESS OF PERINATAL HIV TRANSMISSION AMONG THE GENERAL POPULATION AND WOMEN OF CHILDBEARING YEARS: RESULTS FROM THE 2011 SRC-CANFAR NATIONAL HIV/AIDS SURVEY}

Calzavara, Livianaa ${ }^{1,2}$; Worthington, Catherine2,3; Allman, Dan ${ }^{1,2}$; Tyndall, Mark ${ }^{4}$; Adrien, Alix ${ }^{5}$; White, Samantha ${ }^{1}$

${ }^{1}$ Toronto, ON; ${ }^{2}$ National; ${ }^{3}$ Victoria, BC; ${ }^{4}$ Ottawa, ON;

${ }^{5}$ Montreal, QC

BACKGROUND AND OBJECTIVES: In the absence of any interventions, rates of HIV transmission from an HIV-positive mother to her child during pregnancy, labour, delivery or breastfeeding is between $15 \%$ and $45 \%$ (WHO, 2012). The objectives are to examine awareness of perinatal-exposure risk among Canadians and women of childbearing years; and identify differences by socio-demographics and environmental factors (ie, prenatal screening policies, provincial HIV-prevalence).

METHODS: A bilingual survey of Canadians $(n=2,139) 16+$ years was conducted in May 2011. Participants were recruited from all provinces/ territories using random-digit-dialing with the option to self-complete online or be interviewed by telephone. The margin of error was \pm 2.1 percentage points at the $95 \%$ confidence level. Bivariate statistics were used to identify significant differences.

RESULTS: While most Canadians know HIV can be transmitted through unprotected sex and sharing needles $(99.1 \%$ and $98.9 \%$, respectively), fewer are aware of perinatal exposure $-82 \%$ through birth and only $36.1 \%$ through breastfeeding. Higher levels of breastfeeding-risk awareness are associated with younger age, women, being single, lower level of education, lower household income and living in a household without children. Geographic region, prevalence of HIV in the region, and prenatal screening policies are not associated with breastfeeding-risk awareness. Women of childbearing age (16 to 49 years of age) are slightly more likely than the general population to be aware of transmission through birth $(85.9 \%$ vs $81.1 \% ; \mathrm{p}=0.01)$ and breastfeeding $(39.7 \%$ vs $34.7 \% ; \mathrm{p}=0.03$ ); and awareness is greater among those who live in households with children and do not identify as a sexual minority.

CONCLUSION: While Canadian perinatal transmission rates are lower than global estimates, it is surprising that a high percentage of Canadians and women of childbearing age are unaware of the potential to transmit HIV through breastfeeding. At a minimum, results suggest we need to ensure that HIV+ women are appropriately informed, and women of childbearing years remain informed.

\section{P086}

\section{THE DEVELOPMENT AND IMPLEMENTATION OF A REGIONAL INTERDISCIPLINARY PERINATAL HIV PROTOCOL}

Foisy, Michelle M; Stadnyk, Maria; Hughes, Christine;

Nickel, Pam; Ho, Chantal; Houston, Stan

Edmonton, $\mathrm{AB}$

OBJECTIVES: The Northern Alberta Program operates out of Edmonton and cares for about 1900 patients. In the past decade, our program has experienced a significant increase in the number of pregnan cies in our women with overrepresentation of the aboriginal, black endemic and intravenous drug use population. In an effort to standardize management of these high risk deliveries between sites in northern Alberta, a regional perinatal protocol was developed to yield a comprehensive approach to care. The objective is to describe the development, content and implementation of this protocol.

METHODS: The protocol was coordinated by local pharmacists and a perinatal nurse with expertise in HIV, and input was sought from numerous disciplines and programs, including the interdisciplinary HIV team, adult and pediatric Infectious Diseases, the regional Women's Health program, the Provincial Laboratory and the Antimicrobial Subcommittee.

RESULTS: Sections of the protocol include: 1) Pre-Conception care; 2) Antenatal care and special considerations of antiretroviral therapy (ART) in pregnancy; 3) Intrapartum care; 4) Postnatal follow-up; 5) A care map algorithm; 6) Pre-printed maternal and infant orders; 7) Infant ART teaching sheets; 8) ART use in pediatrics (comparison tables with dosing, side-effects and special tips for administration); and 9) Checklists and contact information. Implementation of the revised 2012 protocol is currently underway and involves wide-spread regional distribution of the protocol, provision of educational in-services to relevant programs and coordination of antiretroviral stock to regional hospitals. The HIV team members, along with the designated perinatal HIV nurse, are key to ensuring ongoing adherence to the protocol and communication with other disciplines and institutions in our region.

CONCLUSIONS: A comprehensive perinatal protocol has been developed and implemented in our region for the past 12 years. This has resulted in a highly coordinated effort to deliver perinatal care in an often challenging and chaotic population. 


\section{P088}

CANADIAN PERINATAL HIV SURVEILLANCE PROGRAM (CPHSP): PERINATAL HIV TRANSMISSION, TREATMENT IN PREGNANCY AND DEMOGRAPHICS IN CANADA

Singer, Joel ${ }^{1}$; Sauve, Laura J1; Brophy, Jason ${ }^{2}$; Forbes, John ${ }^{1}$; Alimente, Ariane ${ }^{1}$; Bitnun, Ari ${ }^{3}$; Samson, Lindy ${ }^{2}$;

Money, Deborah ${ }^{1}$; Lapointe, Normand ${ }^{4}$; Tan, Ben ${ }^{5}$; Lee, Terry ${ }^{1}$; Kakkar, Fatima ${ }^{4}$; and the Canadian Pediatric and Perinatal HIV/ AIDS Research Group (CPARG) ${ }^{3}$

${ }^{1}$ Vancouver, BC; ${ }^{2}$ Ottawa; ${ }^{3}$ Toronto, ON; ${ }^{4}$ Montreal, QC;

${ }^{5}$ Saskatoon, SK

OBJECTIVES: To describe demographics of mother-infant pairs (MIP), treatment during pregnancy and vertical transmission (VT) rates in the Canadian perinatal HIV surveillance cohort of births to HIV+ mothers from 1990 to 2011

METHODS: Data are collected annually from 22 Canadian pediatric and HIV centres. VT rates are obtained from the "perinatally identified cohort" defined as MIP delivered in Canada and identified within three months of birth. Data collected include maternal characteristics, pregnancy ART and infant outcome.

RESULTS: Of the 229 identified HIV-positive women giving birth in Canada in 2011, 73\% had acquired HIV heterosexually, 20\% through injection drug use (IDU) and $0 \%$ perinatally; $54 \%$ of mothers were black and $21 \%$ were Aboriginal. Forty-four per cent of MIP were identified in Ontario, $14 \%$ in Alberta, $13 \%$ in BC, $12 \%$ in Québec, $10 \%$ in Manitoba and $7 \%$ in Saskatchewan. Among the $90 \%$ of mothers who received $>4$ weeks of HAART, there were no perinatal transmissions; among the $10 \%(n=21)$ who received no ART or suboptimal treatment, there were two perinatal transmissions resulting from one twin pregnancy in a mother who had discontinued HAART after four weeks of pregnancy. Proportion untreated has steadily decreased from $21.3 \%$ in 1997 to $6.1 \%$ in 2011. IDU (15\%) and aboriginal women (10\%) had higher rates of non-treatment in 2011. Among 2502 MIP identified perinatally in the HAART era (1997-2011), the overall VT rate was $2.5 \%$ but only $0.8 \%$ in women receiving HAART and $0.1 \%$ in women receiving $>4$ weeks of HAART.

CONCLUSIONS: VT rates of HIV in Canada remain low. Treatment uptake has improved overall but some populations are still not receiving adequate treatment, placing them at risk of HIV complications and their infants at risk of vertical transmission. Efforts to identify and support pregnant HIV-positive women to enhance their health and that of their infants must continue.

\section{P089}

\section{TRENDS IN LIVE BIRTH RATES AND ADVERSE INFANT OUTCOMES IN ONTARIO, 2002 TO 2009: A POPULATION-BASED STUDY}

Antoniou, Tony ${ }^{1}$; Zagorski, Brandon ${ }^{1}$; MacDonald, Erin' ${ }^{1}$; Bayoumi, Ahmed M ${ }^{1}$; Raboud, Janet ${ }^{1}$; Masinde, Khatundi'; Tharao, Wangari'; Brophy, Jason²; Yudin, Mark¹; Loutfy, Mona ${ }^{1}$; Glazier, Richard $\mathbf{H}^{1}$

${ }^{1}$ Toronto; ${ }^{2}$ Ottawa, ON

BACKGROUND: Population-based data describing trends in birth rates and adverse outcomes of pregnancy among Ontario's population of HIVpositive women are lacking. This study describes trends in HIV-related pregnancy outcomes for women in Ontario between 2002 and 2009.

METHODS: We conducted a population-based study using linked administrative healthcare databases to determine the annual rate of live births per 100 HIV-positive women in Ontario aged 15 to 49 years, and determine rates of low birth weight (LBW), pre-term birth (PTB) and small for gestational age (SGA) infants per 100 live births. We used generalized estimating equations with a log-link function to characterize trends over time and examine the association between pregnancy outcomes and sociodemographic characteristics, including age, region of birth and neighborhood income quintile.

RESULTS: Between 2002 and 2009, there were 551 live births during 15,610 person-years of follow-up. Crude rates of LBW, PTB, and SGA infants per 100 live births over this period were 3.6 (95\% CI 2.0 to 5.2), 17.4 (95\% CI 13.9 to 20.9) and 14.9 (95\% CI 11.7 to 18.1 ), respectively. National rates of LBW, PTB and SGA during this period ranged from 5.5 to $6.2,7.7$ to 8.2 and 7.8 to 8.2 and per 100 live births, respectively. Women aged 18 to 34 years had higher live birth rates than women aged 35 to 49 years (adjusted relative rate 5.6 [95\% CI 4.5 to 7.1]), as did women living in low relative to high income neighborhoods (adjusted relative rate 1.3 [95\% CI 1.1 to 1.7]. In addition, live birth rates (adjusted relative rate 1.3 [95\% CI 1.0 to 1.6]) were higher in women originally from Africa relative to women from Canada. Rates of adverse infant outcomes did not vary by maternal region of origin, year or neighborhood income.

CONCLUSION: Women with HIV have rates of preterm birth and small for gestational age infants that exceed national rates for these outcomes.

\section{P090}

\section{OUTCOMES OF HIV-EXPOSED UNINFECTED INFANTS REQUIRING NICU ADMISSION: A TEN-YEAR RETROSPECTIVE REVIEW}

\section{Taerk, Evan; Campbell, Doug; Caprara, Daniela; Yudin, Mark} Toronto, $\mathrm{ON}$

BACKGROUND: The highly effective nature of antiretroviral therapy (ART) and resulting reduction in the vertical transmission of HIV has given rise to a rapidly growing number of HIV-positive pregnant women with HIV-exposed, uninfected (HEU) infants. These children represent a unique clinical population but limited research exists to properly describe their neonatal outcomes, most of which has focused on HEU neonates within the developing world. The primary objective of this study was to describe the neonatal outcomes of HEU infants born over a 10 -year period within a large urban Canadian hospital, with a focus on those admitted to the neonatal intensive care unit (NICU).

DESIGN: A retrospective chart review, focusing on immediate neonatal outcomes, was performed of all neonates $(n=131)$ born to HIV-positive women at one Toronto teaching hospital between March 2000 and August 2011.

RESULTS: Of the $131 \mathrm{HEU}$ infants born during the study period, $23 \%$ required admission to the NICU. The overall rate of NICU admission for infants born at our institution is currently $9 \%$. The most common reason for admission were respiratory distress/support, preterm birth, hypoglycemia and inadequate maternal ART. Among those admitted, the mean gestational age at delivery was 36 weeks, with $50 \%$ of infants born preterm. The mean birth weight was 2989 grams, with 33\% weighing less than 2500 grams. $53 \%$ of neonates required some ventilatory support, and $25 \%$ required intubation. $53 \%$ of admitted neonates required triple ART, with a mean time to ART administration of 5.3 hours. The mean length of NICU stay was 10.5 days.

CONCLUSIONS: HEU neonates born at our centre required more frequent admission to the NICU than unexposed infants. Most of these infants were born to highly compliant mothers with well-controlled HIV It is unclear whether these differences in neonatal outcomes are secondary to ART exposure in utero, fetal development within a potentially immunocompromised maternal environment, or exposure to even minute levels of HIV.

\section{P091}

\section{BILIRUBIN AS A SURROGATE MARKER FOR ATAZANAVIR PLASMA CONCENTRATIONS IN A PAEDIATRIC POPULATION}

Wong, Alison Y1; Dayneka, Natalie²; Brophy, Jason $\mathrm{C}^{2}$;

Bowes, Jennifer ${ }^{2}$; la Porte, Charles J'; Samson, Lindy ${ }^{2}$

${ }^{1}$ Montreal, QC; ${ }^{2}$ Ottawa, ON

BACKGROUND: Atazanavir dose recommendations have not been extensively studied in paediatrics and present dosing may result in inadequate plasma concentrations. In adults, a positive association between bilirubin and atazanavir plasma levels has been shown. We describe paediatric atazanavir dosing and investigated plasma bilirubin concentration as a possible surrogate for atazanavir therapeutic drug monitoring (TDM). 
METHODS: TDM data of patients $\leq 18$ years were retrospectively analysed between Jan/2004-Jul/2011. Atazanavir minimum concentrations (Cmin) were estimated using a half-life of $10.10 \mathrm{~h}$ and $5.58 \mathrm{~h}$ with and without ritonavir respectively, for levels drawn more than $4 \mathrm{~h}$ post-dose. Cmin below $0.15 \mathrm{mg} / \mathrm{L}$ was considered subtherapeutic. Associations between dose-Cmin and bilirubin-Cmin were evaluated using linear regression and receiver-operator curve (ROC), respectively.

RESULTS: Fifteen patients, $67 \%$ female, 93\% black, underwent 35 TDMs. At the time of TDM, median age was 15 years (range 8 to 18 years), weight $49 \mathrm{~kg}$ (range 29 to $74 \mathrm{~kg}$ ), body surface area $1.47 \mathrm{~m}^{2}$ (range 1.00 to $1.88 \mathrm{~m}^{2}$ ). Median atazanavir dose was $4.42 \mathrm{mg} / \mathrm{kg}$ (range 3.40 to $10.49 \mathrm{mg} / \mathrm{kg}$ ) and $150 \mathrm{mg} / \mathrm{m}^{2}$ (range 125 to $299 \mathrm{mg} / \mathrm{m}^{2}$ ); $26(74 \%$ ) boosted with ritonavir. No association between Cmin and dose was found $\left(\mathrm{mg} / \mathrm{kg}, \mathrm{p}=0.81 ; \mathrm{mg} / \mathrm{m}^{2}, \mathrm{p}=0.75\right)$; similar results were obtained when adjusted for ritonavir use. Bilirubin concentrations of $18 \mathrm{mg} / \mathrm{L}$ were predictive of Cmin equal to or above $0.15 \mathrm{mg} / \mathrm{L}$ with $95 \%$ sensitivity and $80 \%$ specificity (ROC area under the curve 0.91 [95\% CI 0.76 to 1.00$]$ ). CONCLUSIONS: Though atazanavir dose is not predictive of $\mathrm{Cmin}$, bilirubin may be a surrogate marker for atazanavir $\mathrm{Cmin}$ in paediatric patients. These results should be confirmed in prospective studies.

\begin{tabular}{lccc} 
& $\mathrm{N}$ & Median (range) Cmin mg/mL & Subtherapeutic Cmin a n (\%) \\
\hline No ritonavir & $5 \mathrm{~b}$ & $0.05(0-0.25)$ & $3(60 \%)$ \\
With ritonavir & $19 \mathrm{c}$ & $0.79(0-1.85)$ & $2(11 \%)$
\end{tabular}

a. Subtherapeutic Cmin defined as Cmin $<0.15 \mathrm{mg} / \mathrm{L} ; b$. Cmin could not be calculated for 4 TDMs: 3 TDMs done $<4$ hours post-dose, 1 TDM unknown time post-dose; $c$. Cmin could not be calculated for 7 TDMs: 6 TDMs done $<4$ hours post-dose, 1 TDM unknown time post-dose. Abbreviations: Cmin (minimum concentration), TDM (therapeutic drug monitoring)

\section{P092 \\ PRELIMINARY OUTCOMES AMONG PERINATALLY INFECTED HIV PATIENTS TRANSITIONED FROM PEDIATRIC CARE TO THE OTTAWA HOSPITAL (2003- 2012)}

Clark, Michael; MacPherson, Paul; Brophy, Jason;

Angel, Jonathan; Samson, Lindy; Cameron, DW

Ottawa, ON

BACKGROUND: In the era of combined antiretroviral therapy (cART), children living with perinatally acquired HIV are now expected to survive into adulthood. Adolescents are at high risk for poor medication adherence, and retention in care can be challenging. Our objective was to summarize outcomes among perinatally infected youth at The Ottawa Hospital (TOH).

METHODS: We conducted a retrospective review of perinatally infected patients at $\mathrm{TOH}$, following their transition from pediatric care. Outcomes included retention in care, treatment failure (defined by two or more consecutive viral loads $>200$ copies $/ \mathrm{mL}$ following at least 48 weeks on cART), viral loads (VLs), and CD4 counts. Patients were considered not engaged in care if they met all of the following criteria: no visit in last six months; missed visit in last six months with no effort to reschedule (despite routine efforts by clinic nurse to contact them); and not known to have moved, transferred or received care elsewhere.

RESULTS: Eleven perinatally infected patients aged 18 years or more were engaged at $\mathrm{TOH}$ at some point during the 2003 to 2012 period. Three (27\%) are not currently engaged in care. Ten patients (91\%) were in care at $\mathrm{TOH}$ long enough to evaluate study outcomes. Of these, seven (70\%) experienced at least one treatment failure. Five (50\%) have never had virologic suppression while in care at $\mathrm{TOH}$. Nadir CD4 counts (at age 18 years or more) were $<200$ cells $/ \mu \mathrm{L}$ in five $(50 \%)$, and $>100$ cells $/ \mu \mathrm{L}$ in three $(30 \%)$.

CONCLUSION: These data show that perinatally infected youth at $\mathrm{TOH}$ are at high risk for treatment failure. Adherence to cART and retention in care are major issues to address in these patients. More research is needed to explore the determinants of these important outcomes, and inform new models of care for these high-risk youth.

\section{P093}

INCIDENCE AND PREDICTORS OF PREGNANCY AMONG A COHORT OF HIV-POSITIVE WOMEN AFTER INITIATING ANTIRETROVIRAL THERAPY IN MBARARA, UGANDA

Kaida, Angela $^{1}$; Matthews, Lynn T2 ${ }^{2}$; Kanters, Steve ${ }^{3}$;

Kabakyenga, Jerome ${ }^{4}$; Murooza, Conrad ${ }^{4}$; Mocello, AR $^{5}$;

Martin, Jeffrey N ${ }^{5}$; Hunt, Peter ${ }^{5}$; Haberer, Jessica ${ }^{2}$;

Hogg, Robert $\mathrm{S}^{1,3}$; Bangsberg, David $\mathrm{R}^{2}$

${ }^{1}$ Burnaby, BC; ${ }^{2}$ Boston, Massachusetts, USA; ${ }^{3}$ Vancouver, BC;

${ }^{4}$ Mbarara, Uganda; ${ }^{5}$ San Francisco, California, USA

BACKGROUND: Many people living with HIV in sub-Saharan Africa desire biological children. Implementation of strategies that support the reproductive goals of people living with HIV while minimizing HIV transmission risk to sexual partners and future children requires a comprehensive understanding of pregnancy in this population.

OBJECTIVE: To determine pregnancy incidence and predictors among HIV-positive women initiating antiretroviral therapy (ART) in a high HIV prevalence and fertility setting.

METHODS: We measured pregnancy incidence among women (18 to 49 years of age) enrolled in the Uganda Antiretroviral Treatment Outcomes (UARTO) cohort of HIV-positive individuals initiating ART in Mbarara between 2005-2011. Bloodwork (including CD4 cells $/ \mathrm{mm}^{3}$ and viral load) and questionnaires (including socio-demographics, health status, sexual behaviour, partner dynamics, and self-reported pregnancy) were completed at baseline and quarterly. Person-time methods measured pregnancy incidence after ART initiation. Multivariable Cox proportional hazards regression (with repeated events) identified baseline and time-updated predictors of pregnancy post-ART initiation.

RESULTS: Our analysis included 351 women. At baseline (pre-ART initiation), median age was 33 years [interquartile range (IQR) 27 to 37 years] and median prior livebirths was four [IQR 2 to 6]. 38\% were married with $61 \%$ reporting HIV-positive spouses. Seventy-three per cent of women had disclosed HIV status to a primary sexual partner. Median nadir CD4 was 137 cells $/ \mathrm{mm}^{3}$ (IQR 81 to 207). 9.1\% (31/342) reported current pregnancy. After ART initiation, 84 women reported at least one incident pregnancy (including 15 women reporting two pregnancies and three women reporting three pregnancies); totaling 105 pregnancies over 1117.6 Woman-Years (WYs) of follow-up (pregnancy incidence $=9.40 / 100$ WYs [95\% CI 7.68 to 11.4$]$ ). Three years post-ART initiation, cumulative probability of at least one pregnancy was $28 \%$ and independently associated with younger age (adjusted Hazard Ratio (aHR) 0.89/year increase [95\% CI 0.86 to 0.92 ]) and HIV disclosure to primary sexual partner (aHR 2.45 [95\% CI 1.29 to 4.63]).

CONCLUSIONS: Nearly one-third of women became pregnant within three years of initiating ART, highlighting the need for integrated services to prevent unintended pregnancies and reduce periconceptionrelated risks for HIV-positive women choosing to conceive. Association with younger age and disclosure suggests a role for early and couples-based safer conception counselling.

\section{HIV Prevention and Monitoring} Prévention et surveillance du VIH

\section{P094}

\section{HIV TRANSMISSION RISK: A SUMMARY OF THE EVIDENCE}

Paquette, Dana; Demers, Alain; Gale-Rowe, Margaret; Wong, Tom Ottawa, ON

BACKGROUND: Our knowledge of the risk of HIV transmission has evolved over the past decade as evidence on the impact of biological and behavioural co-factors, such as viral load, has come to light. We undertook a comprehensive review of the evidence on the risk of HIV transmission associated with sexual activities, injecting and other drug use and vertical transmission.

METHODS: A search was conducted for literature published between January 2001 and May 2012. The search focused on systematic, meta- 
analytic, and narrative reviews, where they existed. For topics where no reviews existed, primary research studies were included.

RESULTS: The risk estimates for the sexual transmission of HIV, per sex act, ranged from $0.5 \%$ to $3.38 \%$ (with mid-range estimates of $1.4 \%$ to $1.69 \%$ ) for receptive anal intercourse; $0.06 \%$ to $0.16 \%$ for insertive anal intercourse; $0.08 \%$ to $0.19 \%$ for receptive vaginal intercourse; and approximately $0.05 \%$ to $0.1 \%$ for insertive vaginal intercourse. For people who inject drugs, the risk of transmission from a contaminated needle, per injection, was estimated to be between $0.7 \%$ and $0.8 \%$. In the absence of any preventive intervention, the risk of vertical transmission ranges from $15 \%$ to $45 \%$ depending on whether breastfeeding alternatives are available. A number of factors impact the risk, including viral load, the presence of other sexually transmitted infections, and male circumcision.

CONCLUSIONS: Within each route of transmission, estimates of the risk of transmission varied widely, likely due to the role of behavioural and biological co-factors. Viral load (especially in plasma, but also in other relevant body fluids) appears to be an important predictor of transmission, regardless of the route of transmission. However, the evidence indicates that viral load is not the only determinant and that certain cofactors play a role in increasing (eg, STIs) or decreasing (eg, male circumcision) the risk of transmission.

\section{P095}

\section{REDUCING UNDIAGNOSED CASES OF HIV: NEW GUIDANCE FOR THE PROVISION OF HIV SCREENING AND TESTING IN CANADA}

Dodds, Jeff V; Wong, Tom; Gale-Rowe, Margaret; Brooks, James; Kim, John; Njoo, Howard; Paquette, Dana

Ottawa, ON

BACKGROUND: A new HIV screening and testing guide for care providers is being published by the Public Health Agency of Canada in 2013, reflecting advances in HIV testing and treatment, to help reduce the estimated $25 \%$ of people living with HIV in Canada who are undiagnosed.

METHODS: The Public Health Agency of Canada established an Expert Working Group to inform the development of a new clinical guide for HIV testing. The guide was further informed by a literature review, an online public consultation, and by input from laboratory and testing experts, provincial and territorial governments, public health professionals and clinicians.

RESULTS: The primary recommendation of the new guide encourages care providers to consider offering HIV testing more frequently and as a part of periodic routine medical care, moving away from an exclusive risk-based approach. This recommendation is based on good quality evidence reporting that individuals and care providers may underestimate the risk for HIV infection and evidence that those most at risk are also most likely to have been tested. The new guide highlights the potential individual and public health benefits associated with early detection of HIV, including decreased morbidity and mortality through engagement in care and early treatment, and reductions in the onward transmission of HIV through providing awareness of an individual's HIV status and by facilitating treatment as a means to prevention. The guide offers new streamlined approaches to risk assessments, pre- and post-test counselling, and consent, to help normalise HIV testing and to reduce barriers to HIV testing as identified in the literature.

CONCLUSIONS: Encouraging care providers to normalise HIV testing as part of periodic routine medical care can reduce the number of undiagnosed cases of HIV in Canada, improve individual and public health outcomes, and help reduce the stigma associated with HIV infection and testing.
P096

PREPARING FOR PREP: ASSESSING PERCEPTIONS, BELIEFS, AND READINESS OF CANADIAN PHARMACISTS FOR THE IMPLEMENTATION OF HIV PRE-EXPOSURE PROPHYLAXIS

Yoong, Deborah M; Naccarato, Mark; Sharma, Malika;

Wilton, James; Senn, Heather; Tan, Darrell

Toronto, ON

BACKGROUND: Pharmacists are well-positioned to educate on preexposure prophylaxis (PrEP). Our aim was to determine Canadian pharmacists' enthusiasm for PrEP and their opinions on PrEP implementation.

METHODS: We invited Canadian pharmacists with some experience in HIV care to complete a 27 -item online survey about their experiences and opinions regarding PrEP. The primary outcome measure was willingness to provide education positively supporting PrEP to high-risk patients. In exploratory analyses, chi-square tests and univariate logistic regression models were used to identify predictors of pharmacists' enthusiasm for PrEP.

RESULTS: Over three weeks in December 2012, surveys were completed by 43 pharmacists across Canada, of whom $70 \%$ were female $79 \%$ self-identified as having specialized HIV knowledge, and median (interquartile range [IQR]) number of years in practice was 13 (6 to 20). The majority (92\%) felt "very" or "somewhat familiar" with PrEP; 35\% had been questioned about PrEP more than once in the past year, and $12 \%$ knew patients that used PrEP. Half of respondents ranked efficacy as the most important issue related to PrEP implementation and 56\%, 14\% and 30\% believed Health Canada should, should not, or should "maybe" approve PrEP for use in Canada, respectively. Most (74\%) felt some of their patients would benefit from $\mathrm{PrEP}$, and the proportion who would provide education supporting PrEP use was $67 \%$ (95\% CI 53\% to $81 \%$ ). The principal barriers identified included drug coverage, not being familiar enough to educate and uncertainty of which patients would benefit. None of the respondent characteristics examined were found to be associated with pharmacists' enthusiasm for PrEP.

CONCLUSION: Many Canadian pharmacists with experience in HIV care would endorse PrEP for patients at high risk of HIV infection. Our study suggests that with wider dissemination of information and more affordable drug costs, PrEP may be more widely promoted as an effective HIV prevention strategy.

\section{P097}

HIV PREVENTION BASED ON PHYLODYNAMICS OF THE MONTREAL MSM EPIDEMIC

Brenner, Bluma G; Ibanescu, Ruxandra-llinca; Ohnona, Frédéric; Moisi, Daniela; Otis, Joanne; Rousseau, Robert; Moodie, Erica; Wainberg, Mark A; Roger, Michel

Montreal, QC

BACKGROUND: Phylodynamics is a vital tool for understanding the complex interplay of virus, host and environment on the evolution of individual HIV epidemics. This study integrates phylogenetic and epidemiologic data to explore the conundrum of rising rates of MSM infections in the post-HAART era.

METHODS: Phylogenetic analysis was performed on sequence datasets of primary/early stage infections ( $\mathrm{PHI}<6$ months) from the provincial genotyping program. Phylogeny was combined with SPOT and PHI cohort data to ascertain factors implicated in transmission and prevention.

RESULTS: Surveillance of primary infections (2002 to 2012) shows three characteristic patterns of onward spread of primary MSM infections. Although the mean viral loads $(4.7 \log$ copies $/ \mathrm{mL})$ in the three cluster groups were similar, viral variants associated with large clusters were more infectious, expanding over longer intervals with slower rates of genetic diversification. 
Cumulative growth of the Montreal MSM epidemic

\begin{tabular}{|c|c|c|c|c|c|c|}
\hline \multicolumn{4}{|c|}{ Viral networks } & \multicolumn{3}{|c|}{$\%$ PHI (number of variants) } \\
\hline \multirow{4}{*}{$\begin{array}{l}\text { Transmission } \\
\text { type }\end{array}$} & & Cluster & & \multirow[b]{4}{*}{$2005(n=497)$} & \multirow[b]{4}{*}{2009 (n=922) } & \multirow{4}{*}{$\begin{array}{c}2012 \\
\text { (n=1382) }\end{array}$} \\
\hline & Number of $\mathrm{PH}$ & duration in & $\%$ pol & & & \\
\hline & Cluster & months & Diversity & & & \\
\hline & (median) & (range) & (range & & & \\
\hline Unique PHI & 1 & - & $0.37[0-1]$ & $42 \%(207)$ & $35 \%(321)$ & $26 \%(391)$ \\
\hline Small clusters & $2-4(2)$ & $4.8[1-11.5]$ & $0.24[0-0.7]$ & $28 \%(53)$ & $22 \%(84)$ & $20 \%(104)$ \\
\hline Large clusters & $5-60(11)$ & $11[3.5-25.5]$ & $0.12[0-0.5]$ & $30 \%(16)$ & $43 \%(40)$ & $54 \%(64)$ \\
\hline
\end{tabular}

Timely diagnosis is necessary to avert the formation and expansion of transmission clusters. SPOT, an MSM community testing site, showed that only 52\% (869/1682) of HIV-negative and 28\% (10/36) of HIVpositive MSM had been tested in the previous year. Eight individuals $(25 \%)$ were diagnosed in primary infection ( $<3$ months to 6 months). Time of last test, cluster association, genetic diversity $(0.98 \pm 0.15)$ and X4 tropism $(n=6)$ identified 11 (30\%) "late" presenters. Overall, 25 of 36 identified infections (September 2009 to September 2012) were in recent cluster networks including 293 persons by the end of 2012 .

CONCLUSIONS: The success of "Treatment for Prevention" will be predicated on timely diagnosis. Research is ongoing to identify factors implicated in viral transmissibility and cluster formation.

\section{P098}

PRELIMINARY DATA FROM A PILOT PROGRAM OF NONOCCUPATIONAL POST-EXPOSURE PROPHYLAXIS IN VANCOUVER, BRITISH COLUMBIA

Pai, Jayaram; Michelow, Warren; Zhang, Wendy; Colley, Guillaume; Harris, Marianne; Montessori, Val; Guillemi, Silvia; Day, Irene; Montaner, Julio; Hull, Mark Vancouver, BC

BACKGROUND: In British Columbia (BC), publicly-funded antiretroviral (ARV) post-exposure prophylaxis (PEP) has been reserved for HIV exposures in occupational settings and sexual assault. Provincial coverage of PEP for non-occupational HIV exposures (NPEP) from consensual sex or needle-sharing has not been available. In July 2012, the BC Centre for Excellence in HIV/AIDS launched a PharmaCare-funded pilot program offering NPEP at six healthcare facilities serving populations at-risk for HIV in Metro Vancouver. We present preliminary data from the first six months of this program.

METHODS: Patients approved for NPEP received a seven-day ARV starter-kit containing Kaletra, Tenofovir and Lamivudine. Re-assessment of the exposure event after seven days determined the need to continue a full 28-day regimen. Standardized NPEP data forms were collected weekly during clinical follow-up. We examined patient data from July 2012 to January 2013. Among patients recommended 28 days of prophylaxis, characteristics of those who completed therapy were compared to those who discontinued early using Fisher's Exact and Wilcoxon tests.

RESULTS: A total of 104 individuals initiated NPEP. The majority were male $(91.7 \%$ ), median age was 30 (IQR 27 to 41 ) and unprotected anal intercourse (UAI) was the most commonly reported exposure event $(70.6 \%)$. Only $7.3 \%$ reported needle-sharing as their exposure event. Median exposure-to-assessment time was 16 hours (range 9 to 39). Overall, 62/104 patients were recommended the full 28-day course of NPEP. Patients completing therapy $(n=47)$ were more likely to be male $(p=0.004)$ and report UAI exposure $(p=0.001)$ and less likely to report needle-sharing $(\mathrm{p}=0.042)$ than those who discontinued early. Common side effects were nausea $(34.9 \%)$, diarrhea $(47.3 \%)$ and fatigue $(40.4 \%)$; however, no side-effect-related discontinuations were noted and only two patients changed regimens. No sero-conversions were reported during the four-week treatment period.

CONCLUSION: UAI was the most common risk event for patients initiating NPEP in this pilot program. Although NPEP was well-tolerated, $25 \%$ of patients failed to complete the full four-week course. Additional interventions to support NPEP adherence may be required.
P100

COMMUNITY VIRAL LOAD IS DECREASING AMONG HIV INFECTED PERSONS IN MONTREAL WITH BROADER USE OF ANTIRETROVIRAL THERAPY

Rollet, Kathleen C; Gilmore, Norbert; Lalonde, Richard G;

Routy, Jean-Pierre; Pexos, Costas; Klein, Marina B

Montreal, QC

BACKGROUND: Strong evidence regarding the role of viral load (VL) on HIV transmission exists. Community VL (CVL), the mean VL in a given population, has been used as a biomarker to monitor HIV burden. Recently, expansion of HIV testing, retention in care, and earlier initiation of antiretroviral therapy (ART) have been emphasized. Such efforts should reduce CVL and HIV transmission. We aimed to measure CVL in HIV-infected persons attending the CVIS and assess trends over time.

METHODS: A total of 1389 HIV-monoinfected patients residing in Montreal aged $>18$ years with available VL data were evaluated between 2007 and 2012. Mean and total CVL were calculated using most recent VL of each patient in each calendar year. Kruskal Wallis tests were used to investigate differences in CVL by sociodemographic and clinical characteristics. Geospatial analysis was done to map CVL by neighbourhood. RESULTS: Overall, the proportion of patients with suppressed VL increased over the past five years from $69 \%$ in 2007 to $79 \%$ in 2012 concomitant with an increase in the proportion of patients receiving ART from $82 \%$ to $87 \%$. Conversely, mean CVL decreased from 14,380 copies $/ \mathrm{mL}$ to 6876 copies/mL and total CVL from $9,807,420$ copies/mL to 6,401,181 copies/mL in 2007 and 2012, respectively. In 2011 to 2012, mean CVL was highest among males, MSM, and persons with CD4 $<200$ cells $/ \mu \mathrm{L}$, those not receiving ART and those previously diagnosed with AIDS. Clear geographic disparities were present with the north east and inner city, socioeconomically disadvantaged areas, showing higher CVL.

CONCLUSION: ART uptake and virologic suppression have increased over time and were associated with reductions in mean and total CVL. CVL may be a good indicator of a community's HIV burden and success of care and treatment. Geographic mapping of CVL may be useful in identifying hotspots for HIV transmission and disparities in care where public health interventions could be targeted.

\section{P101}

TIME FROM HIV DIAGNOSIS TO INITIATION OF ANTIRETROVIRAL THERAPY (ART) IN THE MONTREAL HIV COHORT

Rollet, Kathleen C; Vassal, Anne-Fanny; Machouf, Nima;

Thomas, Réjean; Dufresne, Serge; Klein, Marina B;

Tremblay, Cécile; Durand, Madeleine; Roger, Michel;

Sheehan, Nancy; Trottier, Benoît; Pexos, Costas; Baril, Jean-Guy

Montreal, QC

BACKGROUND: Current guidelines recommend ART initiation at different levels of CD4 and DHHS propose treating all patients with different levels of evidence for three defined strata of CD4: universal treat-

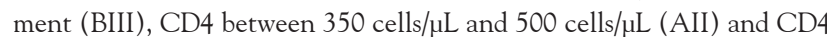
$<350$ cells/ $\mu \mathrm{L}$ (AI). Not all patients are treated accordingly to the recommendations for many reasons. The aim of this study was to assess the impact of rigorous application of the various treatment recommendations over time from a multicentre Montreal cohort.

METHODS: This descriptive analysis examines median time from HIV diagnosis to ART initiation. Additional person-years (PY) of treatment for the three defined strata were calculated for naive patients over time when compared to current practice.

RESULTS: During 2008 to 2010, 5525 actively followed HIV-infected patients aged $>18$ years with available laboratory measures were evaluated; 4234 had both date of HIV diagnosis and ART initiation available or were untreated on their last visit $(n=440[10 \%])$. Median follow-up time was 8.4 years and median time to ART initiation post diagnosis was 2.5 years. In untreated patients, median times to two consecutive CD4 values $<500$ cells/ $\mu \mathrm{L}(\mathrm{n}=1657)$ and $<350$ cells $/ \mu \mathrm{L}(\mathrm{n}=1195)$ were 1.8 and 2.3 years respectively. Over 36,497 person-years of follow-up (PYFU) 
rigorous application of universal treatment, treatment at the cut-off of CD4 $<500$ cells $/ \mu \mathrm{L}$ and CD4 $<350$ cells/ $\mu \mathrm{L}$ would represent respectively 19122,4041 , and 1438 additional PY of treatment. When limited to the 2008 to 2010 period, among 8458 PYFU, 1730 (+20.4\%), $912(+10.7 \%)$, $418(+4.9 \%)$ additional PY of treatment would be required for the three same strata respectively.

CONCLUSIONS: Over 8.4 median years of follow up, rigorous implementation of current guidelines would have represented an important increase in person-years of treatment. When limited to the 2008 to 2010 period, current practices better reflect more recent guidelines and universal treatment could represent a more achievable goal.

\section{P102}

\section{TRENDS IN HIV VIRAL LOAD TESTING IN ONTARIO, 1996 TO 2011}

$\underline{\text { Remis, Robert S}}^{1}$; Sullivan, Ashleigh $\mathrm{S}^{2,3}$; Wu, Keyi ${ }^{3}$;

Soliven, Dolores ${ }^{3}$; Maregmen, Jocelyn ${ }^{3}$; Allen, Marjorie ${ }^{3}$;

Mazzulli, Tony ${ }^{3}$

${ }^{1}$ Toronto; ${ }^{2} \mathrm{Ottawa} ;{ }^{3}$ Etobicoke, ON

BACKGROUND: HIV viral load testing is critical to the care of HIVinfected persons. It informs decisions to initiate anti-retroviral therapy (ART) and helps monitor its effectiveness. Viral load is also important from a public health perspective since reducing viral load through ART reduces the infectiousness of an HIV-infected person. We examined trends in viral load testing in Ontario.

METHODS: In Ontario, virtually all clinical viral load testing is performed at four laboratories coordinated by the Public Health Ontario Laboratories. Data are managed in an integrated laboratory information system. We examined viral load tests performed from September 1996 to December 2011.

RESULTS: 430,030 viral load tests were carried out during the study period, with about 34,000 tests annually in recent years. In $2011,34,547$ tests were carried out among 12,789 patients, for a mean of 2.70 tests per patient. $51.5 \%$ had at least three tests and $23.7 \%$ had at least four.

$91.5 \%$ of patients were 25 to 64 years of age, with a median age of 42.0 years. Women comprised $20.2 \%$ of patients and the annual number of tests was similar in males and females. However, those under 20 years of age had 3.49 tests annually compared to 2.70 in those 20 years and older $(\mathrm{p}<0.0001)$. Most viral load tests were among patients from Toronto $(57.1 \%)$ and Ottawa $(9.7 \%)$.

CONCLUSIONS: The number of patients having at least one viral load to December 2009 represented 98\% of cumulative patients diagnosed with HIV and alive since October 1996 when viral load testing began. A previous study found that most (82\%) persons have a viral load within six months following diagnosis; thus it appears that almost all others have a viral load test at some time following HIV diagnosis. The number of annual viral load tests is lower than the recommended interval of three months. Additional study is required to determine the reasons for this and to identify potential strategies to improve viral load testing in Ontario.

\section{P103 \\ HIV TESTING RATES IN CANADA: A REVIEW OF THE EVIDENCE}

Dodds, Jeff; Paquette, Dana; Tarasuk, Jill; Gale-Rowe, Margaret; Brooks, James; Wong, Tom

Ottawa, ON

BACKGROUND: In 2011, an estimated 25\% of those living with HIV in Canada were unaware that they were infected. The same year, of those newly diagnosed and not from a high HIV-prevalence country, $8.9 \%$ had no reported risk beyond heterosexual contact. We reviewed published data to determine HIV testing rates in Canada. This information will serve as a baseline to evaluate the impact of the Public Health Agency of Canada's new HIV Screening and Testing Guide.

METHODS: We searched multiple databases and the grey literature for articles on HIV testing rates published from 2001 to 2012 in Canada. Studies of testing rates among pregnant women for prenatal screening purposes and testing for immigration purposes were excluded.
RESULTS: In a preliminary search, 26 studies, conference abstracts and reports were located. This initial search found that among people who inject drugs (PWID), $\geq 88 \%$ had ever been tested, except in one study of Aboriginal PWID, where $73 \%$ had ever been tested. Regular testing (ie, in the previous year, or $\geq$ once per year) ranged from $42 \%$ to $63 \%$ among PWID. Most studies of gay men found that $\geq 81 \%$ had ever been tested, and regular testing ranged from $43 \%$ to $65 \%$. In studies of Aboriginal people, $42 \%$ to $60 \%$ had ever been tested. Among people from HIVendemic countries, $43 \%$ to $75 \%$ had ever been tested. Based on two studies of sex workers, $67 \%$ to $86 \%$ had ever been tested. Two recent Canadian surveys of the general population found that $29 \%$ and $37 \%$ had ever been tested for HIV.

CONCLUSIONS: Increasing the offer of HIV testing among the general population during medical visits and more regular testing of vulnerable populations may decrease the number of undiagnosed infections in Canada, facilitating earlier initiation of treatment and care and reducing the onward transmission of HIV.

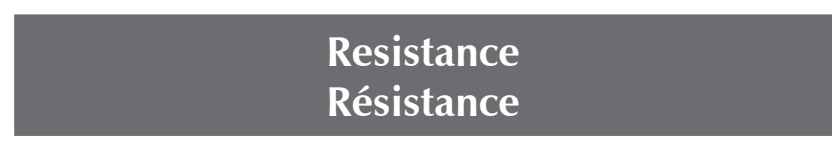

\section{P104}

\section{LARGE-SCALE PHYLOGENETIC ANALYSIS OF HIV SEQUENCES TO RECONSTRUCT HISTORICAL EPIDEMIC DYNAMICS AND TRANSMITTED DRUG RESISTANCE IN BRITISH COLUMBIA, CANADA}

Poon, Art F; Woods, Conan; Brumme, Chanson J; Harrigan, P Richard

Vancouver, BC

The rapid rate of HIV evolution presents a valuable opportunity to reconstruct the history of the epidemic, where similar virus sequences in different persons imply recent transmissions relative to sampling dates. We reconstruct the dynamics of the HIV epidemic and transmission of drug resistance in $\mathrm{BC}$ using doubly anonymized HIV sequences from the BC Centre Drug Treatment Program database.

HIV bulk sequences $(\mathrm{N}=27,144)$ of protease and the first 400 codons of reverse transcriptase (RT) were doubly anonymized and extracted from a secure database. These data, representing $7467 \mathrm{HIV}$-infected persons in BC from 1996 to the present, were used to generate a phylogeny. We extracted all pairs of tips in the phylogeny where: (1) the tip-to-tip branch length was below a cutoff of 0.02 expected substitutions per site, and (2) the tips represented sequences from different persons, of which at least one was the earliest sequence from that person (considered the putative recipient). The graph defined by these pairs comprised 718 "clusters" incorporating $4304(57.6 \%)$ persons, including a single large cluster of 488 persons that first emerged in June 1996 and grew at a mean rate of 31.1 persons/year. Rapidly-expanding clusters tended to emerge at early (1996) and more recent (2003 to 2006) stages of the epidemic. When one or more SDRM was present in the putative source, the same codon(s) were observed in the earliest HIV sequence from the recipient about $44 \%$ of the time. The apparent rate of transmitted drug resistance increased significantly with time of sampling $(\sim 2 \% /$ year, $\mathrm{P}=4 \mathrm{E}-5)$; however, this trend was not significantly different from increasing rates of transmitted synonymous mutations, which implies these results are primarily driven by increased sampling and sequencing of the epidemic over time.

Phylogenetic reconstruction from doubly anonymized HIV sequences can provide key insights into the historic and current dynamics of the HIV epidemic in BC. Further work will link non-identifying covariates such as age and risk groups to identify potential drivers of current HIV transmission rates. 
P105

\section{EFFECTS OF THE M50I AND R263K RESISTANCE MUTATIONS ON IN VITRO INTEGRASE STRAND- TRANSFER ACTIVITY}

Wares, Melissa; Mesplède, Thibault; Osman, Nathan;

Wainberg, Mark A

Montreal, QC

BACKGROUND: Dolutegravir, a second-generation strand-transfer inhibitor, appears to have a high genetic barrier to resistance in vivo. However, the emergence of the resistance mutations M50I and R263K have been observed using in vitro selection experiments with dolutegravir. Using biochemical cell-free assays measuring strand-transfer activity, we have been successful in characterizing these mutations alone and in combination in the subtype B integrase enzyme.

METHODS: Using site-directed mutagenesis, we introduced solubility and resistance mutations M50I, R263K and M50I/R263K into subtype B integrase. Integrase strand-transfer activity was measured using cell-free assays with varying concentrations of LTR, target DNA or varying concentrations of purified integrase protein. The 96-well plates were read using time-resolved fluorescence and the results were analyzed using the Michaelis-Menten equation for enzyme kinetics.

RESULTS: The solubility constant $(\mathrm{Km})$ of the subtype B integrase with the M50I mutation was decreased when compared with the wild-type with varying concentrations of LTR. In contrast, the $\mathrm{Km}$ for the mutation R263K was greater than the wild-type. Furthermore, when varying the concentration of target DNA the WT, M50I, and R263K integrase subtype B proteins followed the substrate inhibition model whereas the double mutant M50I/R263K followed the Michaelis-Menten model. Moreover, strand-transfer activity for M50I and the double mutant was maximal with an integrase concentration of $200 \mathrm{nM}$ while the WT was more active at $400 \mathrm{nM}$.

CONCLUSIONS: Overall, based on the Km values our results suggest that the M50I mutation in the subtype B integrase protein binds the LTR better than the wild-type. In addition, the double mutant M50I/R263K is not affected by increased concentrations of target DNA unlike the wildtype and the other mutants. This suggests that M50I/R263K mutation in subtype $B$ integrase results in a change in integrase-DNA binding that could indicate a change in the susceptibility to dolutegravir.

\section{P106}

\section{CLINICAL RELEVANCE OF HIV DRUG RESISTANCE TESTING AT LOW VIRAL LOAD}

\section{Gonzalez-Serna, Alejandro; Min, Jeong; Woods, Conan;}

Harrigan, Richard; Swenson, Luke C

Vancouver, BC

BACKGROUND: Low-level HIV viraemia (LLV; 50 to 1000 copies/mL) occurs frequently in patients receiving ART, but there is little or no data available demonstrating that HIV drug resistance testing at $\mathrm{pVL}<1000$ provides potentially useful information. Here we assess resistance testing at LLV and whether it is predictive of future virologic outcome in patients remaining on constant therapy.

METHODS: Genotypic resistance testing was attempted on 4893 plasma samples starting from $0.5 \mathrm{~mL}$ plasma. Analyses on virologic outcomes were restricted to previously ART-naive patients achieving undetectable viral loads ( $\mathrm{pVL}$ ) and rebounding to LLV $(\mathrm{N}=219)$. The genotypic sensitivity score (GSS) was calculated based on therapy and the resistance test results, and divided into four score categories corresponding to number of active drugs. Data were stratified by whether or not any nucleotide mixtures were observed during sequencing (suggesting $>1$ viral species amplified). Kaplan-Meier methods were used to monitor time to virological failure $\geq 1000$ copies.

RESULTS: A total of 4304 of 4893 LLV resistance assays (88\%) produced sequences. Overall, 18/219 (8\%) patients had resistance before therapy. 41/201 (20\%) patients without resistance before therapy evolved resistance to $\geq 1$ drug class after a median of 9.6 months (interquartile range 4.0 to 21 months), primarily to nRTIs $(15 \%)$ and/or NNRTIs $(10 \%)$. Patients with resistance mutations at LLV (GSS<3) had higher risk of virological failure $(\mathrm{p}=0.001)$. Progressively lower GSS scores at LLV were associated with a higher increase in $\mathrm{pVL}$ over time $(\mathrm{p}<0.001)$. All results were similar without or with virus "mixtures".

CONCLUSIONS: Routine HIV genotyping of LLV samples can be performed with a reasonably high success rate and predicts future virological outcomes on constant therapy, from even apparently single molecules (no mixtures) observed. The presence of resistance mutations at LLV significantly increases the risk of virological failure. $20 \%$ of participants beginning ART develop new class drug resistance mutations at LLV, mainly to nRTIs and NNRTIs.

\section{P107}

FACTORS ASSOCIATED WITH DEVELOPMENT OF K65R MUTATION AFTER FAILING ANTIRETROVIRAL THERAPY

Hull, Mark; Chan, Keith; Colley, Guillaume; Chau, William; Yip, Benita; Hogg, Robert; Montaner, Julio; Harrigan, Richard Vancouver, BC

BACKGROUND: The development of K65R mutation after failing antiretroviral therapy (cART) is uncommon, but is associated with compromise of future tenofovir and abacavir-based therapy. There is limited data regarding factors associated with this resistance pattern. We therefore evaluated factors associated with K65R mutation compared to M184V mutation following virologic failure.

METHODS: Data were analyzed from 606 individuals with no baseline resistance, initiating ART therapy in the British Columbia Drug Treatment Program between 1997 and 2009 with evidence of K65R or M184V mutation. Data were further restricted to those with resistance $>3$ months after ART initiation with virologic failure (two consecutive $\mathrm{pVL}>400$ copies/ $\mathrm{mL}$ ). Incidence rates of development of K65R mutation and M184V mutation were calculated for those failing NNRTI and PI therapy. Factors associated with the development of K65R compared to M184V mutation were assessed using multivariate logistic regression models adjusted for age, baseline IDU, HCV status, ART regimen, use of cART, duration of virologic failure, degree of viremia $(>1000$ copies $/ \mathrm{mL})$, adherence in the first six months of therapy and presence of additional mutations.

RESULTS: A total of 451 individuals were included in the analysis, 40 with K65R (8.8\%) and 411 (92.2\%) with M184V mutation. Overall $79 \%$ were male, $60 \%$ were $\mathrm{HCV}+, 58 \%$ reported IDU and the median age was 37 years (interquartile range [IQR] 32 to 43 years). The overall incidence of K65R was $0.21 / 100$ person-year (py) (95\% CI 0.15 to 0.27 ) vs $2.66 / 100$ py ( $95 \%$ CI 2.45 to 2.88 ) for M184V. In multivariable analysis, male gender (adjusted Odds Ratio [aOR] 3.04 [95\% CI 1.01 to 9.18]), third agent at time of mutation (aOR 3.25 [95\% CI 1.07 to 9.83] for efavirenz vs other agent) and NNRTI resistance at time of mutation (aOR 4.38 [95 CI 1.42 to 13.50]) were associated with development of K65R mutation vs M184V after adjusting for adherence.

CONCLUSION: Development of K65R is uncommon compared to M184V after failing ART, and is more commonly seen with NNRTIbased therapy. PI-based regimens in those with suspected poor adherence might decrease compromise of future regimens.

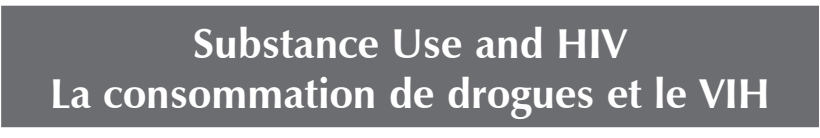

P108

SELF-MANAGEMENT OF PAIN AMONG PEOPLE WHO INJECT DRUGS IN VANCOUVER

Voon, Pauline; Callon, Cody; Nguyen, Paul; Wood, Evan;

Kerr, Thomas

Vancouver, BC

BACKGROUND: People who inject drugs (IDU) often contend with short-term and chronic pain due to various co-morbidities, including those associated with HIV disease. Health care providers may be reluctant to prescribe pain medications due to concerns about drug seeking by IDU. As a result, some IDU may resort to self-management of pain, although this phenomenon has received little attention. 
METHODS: We used logistic regression analysis to evaluate factors associated with self-management of pain among participants enrolled in two prospective cohort studies: the ACCESS study of HIV-positive IDU and the Vancouver Injection Drug User Study (VIDUS) of HIV-negative IDU. We also examined self-reported methods of pain management.

RESULTS: A total of 585 IDU were eligible for this analysis, of whom 281 (48.0\%) were HIV-positive. 404 (69.1\%) IDU reported self-management of pain. Variables independently and positively associated with self-managed pain included having been refused a prescription for pain medication (adjusted odds ratio [AOR] 2.01 [95\% CI 1.32 to 2.90]) and having a physical disability (AOR 2.09 [95\% CI 1.41 to 3.11]). Daily crack use was negatively associated with self-management of pain (AOR 0.58 [95\% CI 0.38 to 0.90]). Common methods of self-management of pain included injecting heroin $(41.0 \%)$ and obtaining diverted prescription pain medication from the street $(38.8 \%)$.

CONCLUSIONS: Self-management of pain was common among IDU in this cohort, particularly among IDU who have been refused a prescription for pain medication and those with physical disabilities. Selfmanagement of pain involved engagement in high-risk behaviors such as injecting illicit drugs or purchasing diverted pain medication off the street. These findings indicate the need for novel efforts to improve pain management to offset the risks associated with IDU self-managing their own pain.

\section{P109}

\section{ASSOCIATION OF SMOKING AND INJECTION DRUG USE ON HIGHLY ACTIVE ANTIRETROVIRAL THERAPY (HAART) OUTCOMES IN THE LONGITUDINAL INVESTIGATIONS INTO SUPPORTIVE AND ANCILLARY HEALTH SERVICES (LISA) STUDY}

Kozai, Tsubasa; Zhang, Wendy; Salters, Kate; Hogg, Robert; Montaner, Julio; Lima, Viviane

Vancouver, BC

BACKGROUND: The high prevalence of smoking (cigarette and marijuana) and injection drug use (IDU) has been well documented among people living with HIV/AIDS (PHA) but the extent in which these substances effect their disease progression is not clearly known. This present study evaluated the impact of both types of substance-use on treatment outcomes of PHA in British Columbia (BC), Canada.

METHOD: We obtained demographic and clinical information from LISA participants $(\mathrm{N}=917)$, which is a prospective study of hard-toreach, treatment-experienced PHA in BC. Two path analyses were conducted to understand the change in CD4 $(\triangle \mathrm{CD} 4)$ and in plasma viral load $(\Delta \mathrm{pVL})$ from baseline to the interview date. We built the model so that pharmacy refill adherence, measured at six months prior to the interview, would have a direct causal effect on both outcomes. Other variables such as age, gender, time on HAART, and the interaction between smoking and IDU were modeled to have a direct causal effect on adherence and an indirect causal effect on both outcomes.

RESULT: Adherence strongly affected $\triangle \mathrm{CD} 4$ (effect 0.294, ie, for a $1 \%$ change in adherence, we expect to see an increase in $\triangle \mathrm{CD} 4$ of 0.294 ; $\mathrm{p}<0.001$ ) and $\Delta \mathrm{pVL}$ (effect $-0.673 ; \mathrm{p}<0.0001$ ). Comparing to nonsmokers and non-IDUs, the only significant associations with adherence were seen among individuals who were only IDU ( $\triangle \mathrm{CD} 4$ effect -0.088 , $\Delta \mathrm{pVL}$ effect $-0.091 ; \mathrm{p}<0.002)$ and those who used both substances $(\Delta \mathrm{CD} 4$ effect $-0.182, \Delta \mathrm{pVL}$ effect $-0.184 ; \mathrm{p}<0.001)$.

CONCLUSION: Individuals who use both substances were at a significantly higher risk of having poor adherence, which in turn was associated with poor treatment response. Smoking, alone, had no effect on adherence, which can be true or an artifact of our data. Thus efforts should be given to increase individual's treatment adherence by successfully linking them to substance use cessation programs.
P110

\section{TOBACCO SMOKING TRENDS AMONG HIV+ CANADIAN GAY MEN}

Phillips, J Craig1; Ham, David²; Trussler, Terry²; Razao, David';

Ferlatte, Olivier²; Marchand, Rick²; Brondani, Mario $\mathrm{A}^{2}$;

Boomer, Jack ${ }^{2}$; Currie, Leanne $\mathbf{M}^{2}$

'Ottawa, ON; ${ }^{2}$ Vancouver, BC

There has been limited national level information about tobacco use among men having sex with men (MSM) because most national surveys do not gather information about sexual identity. Historically, tobacco marketing targeting MSM was perceived as evidence of society's acceptance of the gay community. Marketing to gay communities and perceived acceptance by tobacco companies outpaced public health and smoking cessation intervention strategies. In the absence of adequate evidence of the magnitude of tobacco use among gay men, there is little hope for developing effective smoking cessation strategies for gay men. It is well established, that tobacco smoking contributes to increased HIV-related morbidity and mortality. The purpose of this study was to describe tobacco smoking trends among HIV+ gay men $(n=667)$ from the Canadian Sex Now 2011 to 2012 survey of MSM ( $\mathrm{n}=8382)$. From this national sample, we observed that HIV+ gay men were 1.2 times (95\% CI 1.0 to 1.4 ) more likely to smoke than other MSM surveyed. Among HIV+ men, logistic regression analysis revealed that men who identified as being gay (OR 2.0 [95\% CI 1.1 to 3.8]; $\mathrm{p}=0.03$ ) or having income $\$ 30,000$ to $\$ 49,999$ (OR 2.0 [95\% CI 1.0 to 3.]; $\mathrm{p}=0.04$ ) were twice as likely to smoke. Other factors associated with smoking among HIV+ men, although not statistically significant, were living in a remote environment (OR $5.1[95 \% \mathrm{Cl}$ 0.5 to 53.7]; $\mathrm{p}=0.17$ ), not completing high school (OR 2.5 [95\% CI 0.9 to 7.3]; $\mathrm{p}=0.08$ ), being Métis (OR 2.3 [95\% CI 0.2 to 28.5]; $\mathrm{p}=0.53$ ), and being 20 to 24 years of age (OR 1.4 [95\% CI 0.4 to 4.5]; p=0.59). Each of the observed factors may have clinical relevance for development of smoking cessation interventions. Interprovincial, ancestry, age, and urban/rural differences must be considered in the development of these interventions. Tailoring smoking cessation interventions in collaboration with HIV+ gay men may be an effective strategy to reduce morbidity and mortality among this group of men.

\section{P111}

PREDICTORS OF CIGARETTE SMOKING INITIATION AMONG PEOPLE LIVING WITH HIV: FINDINGS FROM THE ONTARIO HIV TREATMENT NETWORK (OHTN) COHORT STUDY (OCS)

Bekele, Tsegaye $M^{1}$; Rueda, Sergio ${ }^{1}$; Gardner, Sandra ${ }^{1}$; Burchell, Ann N'; Kennedy, Rick'; Leahy, Bob ${ }^{1}$; Fletcher, David'; Cairney, John²; Bacon, Jean'; Rourke, Sean B'; The OHTN Cohort Study Team ${ }^{1}$

${ }^{1}$ Toronto; ${ }^{2}$ Hamilton, ON

BACKGROUND: People living with HIV have a much higher prevalence of cigarette smoking than the general population. Although several theories have been forwarded, little is known about why people initiate smoking in the first place. The objective of this study is to examine factors associated with smoking initiation behaviour among people in HIV care in Ontario.

METHODS: A total of 3790 individuals (1527 current smokers, 910 former smokers, and 1353 never smokers) provided baseline data on smoking status. Of these, people who identified as former smokers or never smokers at baseline were eligible for this study. Data on cigarette smoking, sociodemographic, substance use, and clinical data were collected at baseline and annual follow-up visits (median follow-up time $=$ 23 months). Logistic regression with Generalized Estimating Equations method (GEE) was used to examine predictor variables associated with initiation of cigarette smoking.

RESULTS: Data from 2263 individuals (5744 observations) were included in the analysis. Average age of the sample was 46.3 years. Most were men $(80 \%)$ and self-identified as gay, lesbian, or bisexual $(66 \%)$. More than half $(65 \%)$ were Caucasian, born in Canada $(61 \%)$, and employed (54\%). 
Over the follow-up period, 137 or 6\% (120 former smokers and 17 never smokers) individuals (re)initiated cigarette smoking. Compared to those who remained non-smokers, those who started smoking were significantly more likely to be male (OR 1.90 [95\% CI 1.07 to 3.35]) and use non-medicinal drugs (OR 1.97 [95\% CI 1.21 to 3.22]). Individuals who lived in smaller communities were more likely to initiate smoking than those who resided in large urban areas (OR 2.46 [95\% CI 1.23 to 4.90]). Individuals with longer duration of HIV infection (OR 1.03 [95\% CI 1.01 to 1.06$]$ ) were also more likely to initiate cigarette smoking. DISCUSSION: Despite its negative health effects, a considerable number of people living with HIV (re)initiate cigarette smoking or have difficulty with sustained cessation. Sustained smoking prevention and cessation interventions are needed, particularly, for men residing in smaller communities and with substance use issues.

\section{P112}

\section{WHO IS BUTTING OUT? SMOKING CESSATION AND ITS PREDICTORS IN PEOPLE LIVING WITH HIV: FINDINGS FROM THE ONTARIO HIV TREATMENT NETWORK (OHTN) COHORT STUDY (OCS)}

Bekele, Tsegaye $\mathrm{M}^{1}$; Rueda, Sergio ${ }^{1}$; Gardner, Sandra ${ }^{1}$;

Burchell, Ann N ${ }^{1}$; Kennedy, Rick'; Leahy, Bob ${ }^{1}$; Fletcher, David ${ }^{1}$; Cairney, John²; Bacon, Jean ${ }^{1}$; Rourke, Sean B'; The OHTN Cohort Study Team ${ }^{1}$

${ }^{1}$ Toronto; ${ }^{2}$ Hamilton, ON

BACKGROUND: The prevalence of cigarette smoking among people living with HIV is higher than in the general population and while many may want to quit smoking, little is known about factors that may influence smoking cessation in this population. Our objective was to examine factors associated with smoking cessation over time among people in HIV Care in Ontario.

METHODS: Study sample included 1527 individuals (1283 men and 244 women) who reported smoking cigarettes within 30 days prior to the baseline interview. Average age of participants was 44 years. Majority of the participants were born in Canada (81\%), Caucasian (75\%) and identified as gay, lesbian, or bisexual (64\%). Data on cigarette smoking, sociodemographic, substance use, and clinical markers were collected at baseline and annual follow-up visits. Logistic regression with Generalized Estimating Equations (GEE) method was used to examine predictors of smoking cessation using all available longitudinal data.

RESULTS: Over the study period (median follow-up time $=22$ months), $210(14 \%)$ persons quit smoking. Results of multivariate regression analysis showed that, compared to those who continued to smoke, those who quit smoking were more likely to be Caucasian (OR 4.89 [95\% CI 2.77 to 8.64])) and immigrants (OR 1.69 [95\% CI 1.23 to 2.32]), have a university level or higher education (OR 1.91 [95\% CI 1.19 to 3.09]), live in large urban areas (OR 2.30 [95\% CI 1.11 to 4.74]), and have longer duration of HIV infection (OR 1.05 [95\% CI 1.03 to 1.07]). They were, however, less likely to use non-medicinal drugs (OR 0.57 [95\% CI 0.39 to 0.84]) or consume higher quantity of alcohol per episode (OR 0.82 [ $95 \%$ CI 0.72 to 0.92$]$ ), and to have smoked for a longer period of time (OR 0.62 [95\% CI 0.52 to 0.73$]$ ).

DISCUSSION: Our findings suggest that smoking cessation interventions need to take into account sociodemographic factors, smoking history, and geographic variations. Interventions should also need to incorporate efforts to address other concomitant substance use issues.
P113

\section{A STEP-BY-STEP GUIDE TO DEVELOPING A NATIONAL, MULTI-SITE HIV QUIT SMOKING RANDOMIZED CLINICAL TRIAL TO HELP ADDRESS HIV CO-MORBIDITIES INCLUDING CARDIOVASCULAR DISEASE AND DEPRESSION}

Balfour, Louise' ${ }^{1}$; MacPherson, Paul1'; Smeija, Marek²; Cooper, Curtis ${ }^{1}$; Angel, Jonathan ${ }^{1}$; Pipe, Andrew ${ }^{1}$; Reid, Bob ${ }^{1}$; Tyndall, Mark ${ }^{1}$; Guigere, Pierre ${ }^{1}$; Holly, Crystal D ${ }^{1}$; Armstrong, Michael' ${ }^{1}$; James, Lawless ${ }^{1}$; Tasca, George' Corace, Kim ${ }^{1}$; Hafizi, Rita ${ }^{1}$; Cameron, Bill ${ }^{1}$ 1Ottawa; ${ }^{2}$ Hamilton, ON

BACKGROUND: Cardiovascular disease (CVD) has been recognized as an important cause of morbidity in HIV patients, such that they are two to four times more likely to have a myocardial infarction compared to the general population. Cigarette smoking has been shown to be the most significant predictor of CVD among HIV patients, which is especially worrisome since HIV patients have higher rates of smoking and are more nicotine dependent than their HIV negative counterparts. Furthermore, $40 \%$ to $60 \%$ of PHAs suffer from depression, which is another significant barrier to smoking cessation

METHODS: The four phases included (1) $707 \mathrm{HIV}+$ patients were screened for tobacco use; (2) A small pilot study with $47 \mathrm{HIV}+$ patients assessed the efficacy of a tailored cessation intervention; (3) Feasibility of program expansion across Canada was examined by meeting with HIV clinics across Canada; (4) Funding was obtained to conduct a Canada wide RCT HIV quit smoking intervention.

RESULTS: Of 707 participants, 34\% reported smoking in the past week, with the mean number of years of smoking being 24 years. At six months follow-up, $27 \%$ of participants who participated in a quit smoking pilot study remained smoke free. In terms of feasibility of expanding the program, HIV clinic sites across Canada expressed strong interest. A Canada-wide four arm, RCT smoking cessation (NRT vs Varenicline; standard care vs counselling) was developed, funded, and is currently underway.

CONCLUSION: Despite significant morbidity associated with cigarette smoking in HIV patients, smoking rates remain high. Few quit smoking interventions have been tailored to the needs of HIV patients including addressing the high rates of depression among HIV+ smokers. This study highlights the steps involved in the development of a large scale RCT investigating a novel smoking cessation program which could be used as a model for other vulnerable medical populations.

\section{P114}

\section{CORRELATES OF DRUG USE CESSATION AMONG} PARTICIPANTS IN THE CANADIAN HIV-HCV CO-INFECTION COHORT (CTN222): IMPROVING THE SOCIAL DETERMINANTS OF HEALTH MAY HELP

Cox, Joseph ${ }^{1}$; Maurais, Emilie; ${ }^{1}$; Moodie, Erica ${ }^{1}$; Walmsley, Sharon ${ }^{2}$; Cooper, Curtis ${ }^{3}$; Hull, Mark ${ }^{4}$; Pick, Neora ${ }^{4}$; Harris, Marianne ${ }^{4}$; Conway, Brian ${ }^{4}$; Klein, Marina $B^{1}$; For the Canadian Co-Infection Cohort (CTN222) ${ }^{1}$

${ }^{1}$ Montreal, QC; ${ }^{2}$ Toronto; ${ }^{3}$ Ottawa, ON; ${ }^{4}$ Vancouver, BC BACKGROUND: Although an issue of debate, many clinicians and sometimes patients view ongoing drug use as a barrier to starting HIV and HCV treatment. To facilitate treatment uptake, we identified correlates of drug use cessation in HIV-HCV co-infected drug users.

METHODS: This was a secondary analysis of patient-related data from CTN222 (1119 HIV-HCV co-infected participants). Data on sociodemographics, risk behaviours, and health information were collected using questionnaires, medical records and blood tests at each six-month study visit. HIV-HCV co-infected participants reporting injection drug use and/or smoking crack with at least two study visits $(n=447$ [40\%], 1425 visits) were included in the analysis. Associations between cessation (no drug use since last visit) and the measured characteristics were examined using non-linear mixed effects logistic regression models with random intercepts. 
RESULTS: A total of 241 (54\%) participants ceased injecting drugs/ crack use at least once. Of these, $36 \%$ relapsed during follow-up. Having a fixed address (adjusted odds ratio [aOR] 2.21 [95\% CI 1.23 to 3.97]) was positively associated with drug use cessation events. Living alone (aOR 0.52 [95\% CI 0.37 to 0.74), current tobacco use (aOR 0.43 [95\% CI 0.25 to 0.73 ) and having attended a greater cumulative number of addiction programs since cohort entry (aOR 0.81 per program initiated $[95 \% \mathrm{CI}$ 0.74 to 0.89 ) were negatively associated. Variables not associated include age, gender, education, monthly income, employment, incarceration, hazardous alcohol drinking, snorting drugs, duration of HIV/HCV infections, AIDS defining illness, and psychiatric diagnoses.

CONCLUSIONS: Drug use cessation among HIV-HCV co-infected persons is relatively common in CTN222. Improving social determinants of health, particularly housing and social support, could lead to better long-term abstinence and better health. Ongoing drug use despite more addiction treatment may reflect the extensive drug use histories in this cohort. The impact of drug use cessation on HIV/HCV treatment uptake, adherence and outcomes require further investigation

\section{P116 \\ PATTERNS OF IMMUNOLOGIC RESPONSE FOLLOWING INITIATION OF HIGHLY-ACTIVE ANTIRETROVIRAL THERAPY AMONG HIV-POSITIVE ILLICIT DRUG USERS IN VANCOUVER, CANADA}

Luma, Maxo; Milloy, M-J; Wood, Evan; Shoveller, Jean;

Shannon, Kate; Guillemi, Silvia; Hogg, Robert; Montaner, Julio;

Kerr, Thomas

Vancouver, BC

INTRODUCTION: Despite the availability of antiretroviral therapy (ART), HIV-seropositive illicit drug users continue to experience elevated levels of HIV/AIDS-associated morbidity and mortality. Evidence is needed to identify social- and structural-level barriers and facilitators of treatment success. Thus, we sought to investigate patterns of immunological response following the initiation of ART among a communityrecruited cohort of illicit drug users.

METHODS: We accessed data from the AIDS Care Cohort to evaluate Exposure to Survival Services (ACCESS) study, a long-running prospective cohort of illicit drug users linked to comprehensive HIV clinical monitoring and ART dispensation records in a setting of universal nocost HIV care. Using Cox proportional hazards modeling with a recurrent events framework we identified factors associated with an increase of $150 \mathrm{CD} 4+$ cells $/ \mathrm{mm}^{3}$ following ART initiation.

RESULTS: Between May 1996 and November 2009, 311 ART-naïve individuals initiated HIV treatment and contributed 1404 person-years of follow-up. During follow-up, we observed an immunologic response in $119(38.3 \%)$ individuals. The incidence density of immunologic response was 14.1 (95\% CI 12.1 to 16.4$)$ per 100 person-years. In a multivariate model, methadone maintenance therapy (MMT) was associated with higher rates of CD4+ recovery (adjusted hazard ratio [AHR] 1.55, [95\% CI 1.15 to 2.10]; $p=0.005)$, while individuals engaged in sex work (AHR 0.35 [95\% CI 0.17 to 0.72]; $\mathrm{p}=0.005$ ) had lower rates.

CONCLUSION: We observed low levels of immunologic response to ART among people who use drugs in Vancouver, especially those engaged in sex work. Meanwhile, MMT was strongly associated with higher rates of CD4+ recovery. These findings point to the need for increased supports to improve ART adherence among key vulnerable populations among illicit drug users.

\section{P117}

\section{CANNABIS USE ASSOCIATED WITH LOWER PLASMA HIV-1 RNA VIRAL LOAD AMONG RECENTLY INFECTED INTRAVENOUS DRUG USERS}

Milloy, M-J S'; Marshall, Brandon D²; Kerr, Thomas ${ }^{1}$;

Guillemi, Silvia'; Hogg, Robert ${ }^{1}$; Montaner, Julio' ${ }^{1}$; Wood, Evan ${ }^{1}$

${ }^{1}$ Vancouver, BC; ${ }^{2}$ Providence, Rhode Island, USA

BACKGROUND: A recent study of tetrahydrocannabinol (THC)

experimentally administered to simian immunodeficiency virus-infected rhesus macaques identified an antiviral effect for THC, supporting a hypothesized immunomodulatory pathway for cannabinoids. However, the possible effect of exposure to cannabinoids on plasma HIV-1 RNA levels among individuals living with HIV/AIDS (PLWHA) has not been evaluated.

METHODS: We used data from the Vancouver Injection Drug User Study (VIDUS), an ongoing community-recruited prospective cohort of injection drug users (IDU) in Vancouver, Canada. We included individuals who were HIV-seronegative at baseline who seroconverted during follow-up. The outcome of interest was plasma HIV-1 RNA copies/mL ( $\log 10$ transformed) in the first 365 days following the estimated date of seroconversion.

RESULTS: Between May 1996 and March 2012, 149 antiretroviral (ART)-naïve individuals acquired HIV infection and were included in these analyses. Median plasma HIV-1 RNA viral load (pVL) among at least daily cannabis use was $0.55 \log 10 \mathrm{c} / \mathrm{mL}$ lower compared to others (4.73 vs $4.18, p=0.003)$. In a multivariate linear mixed effects model, cannabis use remained associated with lower $\mathrm{pVL}(\beta=-0.47 ; \mathrm{p}=0.007)$.

CONCLUSION: Although medical and recreational cannabis use is common among PLWHA, we believe this is the first study to identify a potentially beneficial impact of cannabis on HIV disease state among ART-naive individuals. Our results support the need for the prospective study of the antiviral effect of THC in humans.

\section{P118}

\section{EMPLOYMENT INDEPENDENTLY PREDICTS SURVIVAL AMONG HIV-SEROPOSITIVE ILLICIT DRUG USERS IN A SETTING OF UNIVERSAL HIV CARE}

Richardson, Lindsey; Milloy, M-J; Wood, Evan; Kerr, Thomas

Vancouver, BC

BACKGROUND: Although employment is intrinsically linked to health, its effect on health outcomes among HIV-seropositive individuals who use illicit drugs remains largely unknown. We therefore sought to assess the impact of employment on mortality among a cohort of HIVpositive illicit drug users in Vancouver, Canada.

METHODS: We estimated the relationship between employment and mortality using proportional hazards survival analysis, adjusting for relevant behavioural, clinical, social and structural factors. The data was derived from a long-running prospective cohort of community-recruited drug users and is linked to comprehensive clinical data in Vancouver, Canada, a setting where HIV care is delivered without charge.

RESULTS: Among the 666 participants who contributed 2808 personyears (PY) of follow-up time, we observed 194 deaths, representing an incidence of 6.9 per $100 \mathrm{PY}$ (95\% CI 5.9 to 7.9$)$. In a multivariate survival model, a time-updated measure of full time, temporary or selfemployment compared to no employment was significantly associated with a lower risk of death (adjusted hazard ratio $=0.36$ [95\% CI 0.18 to $0.74]$ ). These results were robust to adjustment for relevant confounders, including age, baseline CD4+ T-cell count, antiretroviral therapy (ART) adherence (defined as $\geq 95 \%$ ART exposure in the last six months vs $<95 \%$ ART exposure), and daily or greater heroin injection or crack use.

CONCLUSION: Our results suggest that employment may be an important dimension in the overall mortality risk of HIV-seropositive illicit drug users. The potentially health-promoting impacts of labour market involvement warrant further exploration, particularly given widespread barriers to employment among this highly marginalized population and persistently elevated levels of preventable mortality among HIV-positive drug users. 
EPIDEMIOLOGY AND PUBLIC HEALTH SCIENCES / ÉPIDÉMIOLOGIE ET SCIENCES DE LA SANTÉ PUBLIQUE

Epidemiology and Surveillance of HIV/AIDS Epidémiologie et surveillance du VIH/sida

\section{P119}

DESCRIPTIVE FINDINGS FROM A NATIONAL ENHANCED HIV SURVEILLANCE SYSTEM, I-TRACK PHASE 3 (20102012): SEX-BASED ANALYSIS OF INJECTING, SEXUAL AND TESTING BEHAVIOURS AMONG PEOPLE WHO INJECT DRUGS

Tarasuk, Jill; Ogunnaike-Cooke, Susanna; Archibald, Chris P Ottawa, ON

BACKGROUND: I-Track, enhanced surveillance of HIV and hepatitis $\mathrm{C}$ risk behaviours among people who inject drugs in Canada, conducted Phase 3 data collection from 2010 to 2012 in 10 sentinel sites.

METHODS: An interviewer-administered questionnaire collected information regarding demographics, drug use, injecting and sexual risk behaviours, and HIV and hepatitis $\mathrm{C}$ testing. A biological sample was collected and tested for HIV and hepatitis C antibodies. Descriptive statistics were calculated and $\chi^{2}$ tests assessed sex-based differences with significance level set at $\mathrm{p}<0.05$.

RESULTS: A total of 1750 current injectors were interviewed between April 2010 and August 2012 in Whitehorse, Prince George, Edmonton, Regina, Thunder Bay, Sudbury, Toronto, London, Kingston and Halifax. Average age was 39 years and $63 \%$ were male. Overall HIV seropositivity was $8 \%$ (ranging from $0.5 \%$ to $16 \%$ across sentinel sites) and was significantly higher among females (10\%) than males (7\%). A slight overall downward trend in HIV prevalence was observed since Phase 1 (2003 to 2005); however, regional variations were noted. Awareness of HIV seropositivity was $71 \%$ and slightly higher among females (75\%) than males (68\%) ( $>00.05$ ). Lifetime exposure to hepatitis $\mathrm{C}$ was $66 \%$, ranging from $45 \%$ in Thunder Bay to $77 \%$ in London. Participants reported most frequently injecting cocaine (61\%), non-prescribed morphine (56\%), and hydromorphone $(48 \%)$ and both sexes reported similar proportions of these drugs. Significantly larger proportions of females than males reported injecting with used needles and with other used injection equipment. Condom use at last sex was 35\% and a significantly larger proportion of females than males reported two or more sex partners.

CONCLUSION: National enhanced surveillance findings from I-Track Phase 3 confirmed that HIV prevalence and lifetime exposure to hepatitis $\mathrm{C}$ remain high among people who inject drugs. These findings form the foundation for further analyses of factors associated with HIV, hepatitis $\mathrm{C}$ and risk behaviours.

\section{P120 \\ REGIONAL VARIATIONS IN CRACK INJECTION ACROSS THE SURVUDI NETWORK}

Leclerc, Pascale ${ }^{1}$; Roy, Elise ${ }^{1,2}$; Morissette, Carole ${ }^{1,3}$; Blanchette, Caty ; Alary, Michel ${ }^{3}$; Blouin, Karine ${ }^{3}$; The SurvUDI Working Group ${ }^{3}$ ${ }^{1}$ Montréal; ${ }^{2}$ Longueuil; ${ }^{3}$ Québec, QC;

AIMS: To describe the extent of crack injection and examine its temporal trend among injection drug users (IDUs) recruited from 2003 to 2010 in the SurvUDI network.

METHODS: IDUs who injected recently (past six months) are recruited in harm reduction and health programs across the province of Québec and in the city of Ottawa (three urban and six semi-urban/rural sites). Each visit includes completion of a structured questionnaire and collection of saliva samples for anti-HIV and anti-HCV antibody testing. Trend analyses were performed using generalized estimating equations. Some IDUs have multiple participations; for the descriptive analyses, their first interview was retained while for the trend analyses, their first interview per year was retained.

RESULTS: Of the 4088 IDUs recruited (2003 to 2010), 75.7\% were male and mean age was 35.5 years. Overall, 15.2\% (621) reported crack injection; large variations across sites were noted, from $0.3 \%$ in Québec City to $39.5 \%$ in Ottawa. The trend analyses were limited to Ottawa (449 crack injectors) and Montréal (121) since few crack injectors $(<22)$ were present in each of the other regions. For Ottawa, a significant decline was observed, from $52.2 \%$ to $37.6 \%$, with a prevalence ratio (PR) of 0.96 per year ( $95 \%$ CI 0.94 to 0.98). For Montréal, a significant rise was observed, from $5.1 \%$ to $16.9 \%$, with a PR of 1.31 per year $(95 \% \mathrm{Cl}$ 1.22 to 1.40 ).

CONCLUSIONS: Strong variations in crack injection exist through the SurvUDI network and reversed temporal trends have been observed in Ottawa and Montréal. These regional variations should be further examined along with the preparation practices for crack injection and their potential for HIV and HCV transmission. These data will be useful to local harm reduction programs to evaluate the need to distribute items required by crack injectors (including a dissolving agent) and develop prevention messages specific to crack injection.

\section{P121}

WHAT CAN 15 YEARS OF DATA COLLECTED AMONG WOMEN AND MEN IN OTTAWA WHO INJECT DRUGS TELL US AND HOW DO WE INTERPRET IT?

Leonard, Lynne E; Kom, Emily; Pelude, Linda Ottawa, ON

INTRODUCTION: Regionalized HIV and HCV prevalence data are essential to drive tailored region-specific development of prevention and treatment programs and policies. This paper reports on HIV and HCV prevalence rates among women and men in Ottawa who inject drugs (IDUs).

METHODS: From 1996 to 2011, more than 3000 active IDUs consented to structured interviews and the collection of saliva samples for $\mathrm{HIV}$ and HCV testing. Data were disaggregated into three five-year surveillance periods: 1996 to 2000; 2001 to 2005; and 2006 to 2011 (no data collected in 2007).

RESULTS: HIV prevalence among all participants decreased from $22 \%$ (1996 to 2000) to $10 \%$ (2006 to 2011), although in 2011 a prevalence rate of $12 \%$ was recorded. Among all participants who tested positive for $\mathrm{HIV}$, the proportion of women who tested positive increased from $28 \%$ to $30 \%$.

Overall rates of HCV prevalence also decreased ( $82 \%$ to $67 \%$ ). However, as with HIV prevalence, in 2011 this rate increased to $74 \%$. For each year of surveillance, HCV prevalence rates were higher among women.

Among people testing positive for HIV in the first surveillance period, $36 \%$ also tested positive for HCV. This rate subsequently increased dramatically to a co-infection rate of $92 \%$ in the last period of surveillance. CONCLUSIONS: Explanations for these declining rates include: a decrease $(78 \%$ to $37 \%)$ in the proportion of IDUs injecting stimulants (cocaine and crack) and a concurrent increase (16\% to 52\%) in the proportion injecting opiates (heroin, morphine, dilaudid and oxycontin); and a decrease (32\% to $15 \%$ ) in the proportion of IDUs reporting injecting with a previously-used needle. In addition to these individual factors, the contribution of the policy environment needs to be considered. For example, the initial one-for-one exchange policy and initial restriction on the total number of needles (three) staff were able to exchange at each visit eased over time and all restrictions were eventually lifted in 2005. These observations highlight the need for synthesis of individual HIVand HCV-related risk behaviours and those risk conditions imposed by region-specific policy. 


\section{P122}

FALLING THROUGH THE CRACKS? RE-ASSESSING TARGETED HIV TESTING STRATEGIES FOR INJECTION DRUG USERS AND STREET-BASED SEX WORKERS

Vasarhelyi, Krisztina; Wood, Evan; Deering, Kathleen; Carter, Allison; Chan, Keith; Simo, Annick; Colley, Guillaume; Gustafson, Reka; Montaner, Julio S; Shannon, Kate Vancouver, BC

BACKGROUND: Routine voluntary testing and targeted risk-based testing combined in comprehensive programs may be an effective strategy for concentrated HIV epidemics. We examined characteristics of undiagnosed HIV infections among injection drug users (IDU) and street-based sex workers (SW).

METHODS: Longitudinal cohort data for IDU (Vancouver Injection Drug User Study (VIDUS): 1996 to 2005), and for SW (MAKA: 2006 to 2007) were analyzed. Self-reported HIV status and test results at baseline were used to identify undiagnosed individuals, previously unaware of their infection but testing HIV-positive on enrollment. Diagnosed and undiagnosed HIV-positive groups were compared across sociodemographic, risk-behavior, risk-to-violence, and service-utilization variables. Chi-square and Fischer's exact tests were applied in bivariate comparisons. For IDU, a multivariate logistic regression model was developed, using statistically significant bivariate results.

RESULTS: A total of 311 IDU (258 diagnosed/53 undiagnosed) and 61 SW (50 diagnosed/11 undiagnosed) were compared. The proportion of undiagnosed infections at baseline was 17\% for IDU (decreasing over the 9-year period from $18.4 \%$ to $0 \%$ ) and $18 \%$ for SW in 2006 to 2007 . The table lists statistically significant differences in 35 bivariate comparisons for IDU: IDU with diagnosed HIV were more likely to have recognized risk factors, comorbidities and utilize services. In the multivariate model, female gender, Hepatitis-C infection, and binge injecting were significantly associated with diagnosed HIV. In 25 bivariate comparisons, SW with diagnosed HIV were significantly more likely to inject cocaine, have Hepatitis-C, report poor health, and live in a marginalized neighbourhood; while those undiagnosed were significantly more likely to experience physical violence, homelessness and sought health care more frequently.

CONCLUSIONS: We identified undiagnosed HIV cases even within recognized risk groups. Comprehensive testing programs could benefit from including routine voluntary testing. However, outreach testing remains essential in addressing structural barriers and emerging vulnerabilities.

\begin{tabular}{lccc} 
Variable & $\begin{array}{c}\text { Diagnosed (T=257) } \\
(\mathbf{N} / \%)\end{array}$ & $\begin{array}{c}\text { Undiagnosed (T=53) } \\
(\mathbf{N} / \%)\end{array}$ & p-value \\
\hline Female gender & $117 / 45.3$ & $14 / 26.4$ & 0.014 \\
Ethnicity & & & $<0.001$ \\
Caucasian & $147 / 57.2$ & $20 / 37.7$ & \\
Aboriginal & $92 / 35.8$ & $21 / 39.6$ & \\
Other & $18 / 7.0$ & $12 / 22.6$ & \\
Hepatitis C & $76 / 33.3$ & $5 / 9.8$ & $<0.001$ \\
Ever had mental illness & $60 / 23.3$ & $5 / 9.4$ & 0.025 \\
Binge injection in past 6 months & $150 / 58.6$ & $20 / 37.7$ & 0.006 \\
Used drug treatment facility in past 6 months & $97 / 37.7$ & $10 / 19.2$ & 0.011 \\
Accessed doctor in past 6 months & $228 / 88.4$ & $22 / 41.5$ & 0.004 \\
Accessed emergency room in past 6 months & $149 / 58.2$ & $22 / 41.5$ & 0.033 \\
Accessed hospital in past 6 months & $83 / 32.5$ & $10 / 18.9$ & 0.050 \\
Used needle exchange ever & $254 / 98.4$ & $48 / 90.6$ & 0.009 \\
Visite counsellor in past 6 months & $58 / 22.6$ & $1 / 1.9$ & $<0.001$ \\
\hline
\end{tabular}

P123

\section{RATES OF SEXUALLY TRANSMITTED AND BLOOD-BORNE INFECTIONS AND RELATED RISK BEHAVIOURS AMONG STREET YOUTH IN MONTRÉAL}

Leclerc, Pascale $^{1}$; Gallant, Serge ${ }^{1}$; Morissette, Carole ${ }^{1}$; Roy, Elise ${ }^{1,2}$ ${ }^{1}$ Montréal; ${ }^{2}$ Longueuil, QC

BACKGROUND: An enhanced surveillance system (E-SYS) targeting street youth (SY), a population vulnerable to sexually transmitted and blood-borne infections (STBBIs), was developed in 1998 by the Public Health Agency of Canada. In 2011 to 2012, Montréal was part of the Cycle 6 of E-SYS. The aim of this analysis is to describe the STBBI rates among SY along with associated risk and testing behaviours.

METHODS: Participants were aged 15 to 24 years and had been homeless for three consecutive days in the previous six months. They were recruited in SY services, namely in a medical clinic and in community organisations, and through word-of-mouth. A questionnaire was administered by a nurse and blood and urine samples were collected.

RESULTS: From October 2011 to April 2012, 182 participants were recruited (35\% at the SY-clinic and 20\% in SY-community organisations). Their mean age was 20.9 years and $70 \%$ were male. Overall, $98 \%$ had ever been sexually active; among those active in the previous three months, $78 \%$ reported no or inconsistent condom use during this period. A quarter (24\%) of participants had ever injected drugs; $36 \%$ of them ever injected with syringes previously used by someone else. Most participants had a history of STBBI testing: HIV (71\%), HCV (61\%), chlamydia (65\%), gonorrhea (66\%), and syphilis $(56 \%)$. Observed prevalence for HIV was $1.1 \%, 6.2 \%$ for chlamydia, $1.1 \%$ for gonorrhea, and $1.7 \%$ for syphilis; $6.3 \%$ had anti-HCV antibodies. Among those infected by one of those STBBIs, $65 \%$ felt they were at no or low risk for STBBI acquisition.

CONCLUSION: High rates of STBBIs and risk behaviours were observed among Montréal SY. This combined with poor self-evaluation of risk highlight the vulnerability of that population and the need for specific interventions around promotion of regular testing, self-evaluation of risk, and prevention of injection initiation. STBBIs among Montréal SY require close attention from public health authorities and SY services.

\section{P124}

HISTORY OF INCARCERATION AMONG HARDER-TOREACH PEOPLE LIVING WITH HIV AND THE IMPACT ON VIRAL SUPPRESSION

Salters, Kate A ${ }^{1}$; Cui, Zi-Shan ${ }^{1}$; Samji, Hasina ${ }^{1}$; Small, Will ${ }^{1,2}$; Chen, Yalin ${ }^{1}$; Montaner, Julio $\mathrm{S}^{1}$; Hogg, Robert $\mathrm{S}^{1}$

${ }^{1}$ Vancouver; ${ }^{2}$ Burnaby, BC

BACKGROUND: Research suggests that HIV-positive individuals may face greater obstacles in obtaining HIV treatment after being incarcerated and released from correctional facilities. We sought to examine the prevalence and correlates of incarceration among a cohort of people living with HIV/AIDS (PHA) in British Columbia (BC), as well factors associated with viral non-suppression.

METHODS: The Longitudinal Investigation into Supportive and Ancillary health services (LISA) cohort is a prospective study examining treatment and care experiences of harder-to-reach PHA in BC. Interviewer-administered surveys collected information regarding housing, drug use, sexual behaviour and other relevant socio-demographic factors. Longitudinal clinical variables, such as CD4 cell count and viral load, are obtained through linkages with the Drug Treatment Program at the BC Centre for Excellence in HIV/AIDS. The LISA cohort oversampled women, Aboriginal persons, and injection drug users. Bivariable and multivariable analyses identified factors associated with self-reported history of incarceration. Viral suppression was defined as having $\geq 2$ sequential $\mathrm{pVL}$ of $>50$ copies $/ \mathrm{mL}$ in the 12 months after interview. RESULTS: Of the 914 LISA participants included in this analysis, $476(52.1 \%)$ had ever been incarcerated, with 55 (6.0\%) reporting incarceration within the previous six months. Those who reported higher satisfaction of care from their physician (AOR 3.22 [95\% CI 1.49 to 6.94]), 
those who pick up their prescriptions daily (AOR 2.46 [95\% CI 1.61 to 3.77]), and reported ever being attacked (AOR 1.880 [95\% CI 1.234 to 2.864]) were more likely to report a history of incarceration. The 107 individuals with a history of incarceration who did not achieve viral suppression were more likely to be younger (AOR 1.03 [95\% CI 1.01 to 1.06]), female (AOR 1.56 [95\% CI 1.37 to 1.83]), have accessed the emergency room within the past three months (AOR 1.74 [95\% CI 1.50 to 2.10]), and report worsening health conditions compared to 12 months prior (AOR 1.59 [95\% CI 1.38 to 1.93$]$ ).

CONCLUSION: Individuals who are HIV-positive and have a history of incarceration may face more complex health challenges after being released from correctional facilities. Individuals who have been incarcerated may require more frequent and comprehensive care in order to achieve stability after incarceration.

\section{P125}

SEXUAL BEHAVIOUR IN GAY, BISEXUAL, AND OTHER MEN WHO HAVE SEX WITH MEN IN LONDON, ONTARIO: THE HEALTH IN MIDDLESEX MEN MATTERS (HIMMM) PROJECT SURVEY

Coleman, Todd A'; Pugh, Daniel'²; Aykroyd, Gloria ${ }^{1}$; Bauer, Greta R ${ }^{1}$; Fraser, Meredith ${ }^{1}$; Murphy, Kevin ${ }^{1}$; Newman, Rob ${ }^{1}$; Pierre Pitman, Lyn ${ }^{1}$; Powell, Leanne ${ }^{1}$ ${ }^{1}$ London; ${ }^{2}$ Toronto, ON

BACKGROUND: Information about sexual health and gay, bisexual, and other men who have sex with men (GB-MSM) from areas outside large metropolitan areas in Ontario is scarce. The Health in Middlesex Men Matters (HiMMM) Project is a community-based project based in Middlesex County, Ontario. HiMMM examines social determinants of health and other factors affecting health (physical, mental, social, sexual) and health care access for local GB-MSM.

METHODS: The HiMMM Project conducted an online survey containing original questions and questions from the Public Health Agency of Canada's M-Track questionnaire on sexual experiences and HIV-related risk. Respondents were recruited through online and in-person promotion, and through social networks. The survey was completed over 2011 to 2012 by adult GB-MSM living in Middlesex County ( $\mathrm{n}=202$ ).

RESULTS: Over half the sample was 18 to 34 years old, with $86.5 \%$ selfidentifying as white, $2.5 \%$ as Aboriginal and $11 \%$ with a non-Aboriginal racial group. Respondents were predominantly gay-identified $(87.1 \%)$ with fewer identifying as bisexual (8.5\%) and queer (9.0\%). Over $18 \%$ were currently in non-monogamous relationships. Over the past six months, $31.8 \%$ had one male sex partner, $25.4 \%$ had two to four, $18.9 \%$ had five to nine, and $16.9 \%$ had over 10 . Of those who had sex over this period, $35.3 \%$ had one anal sex partner, $23.4 \%$ had two to four, $10.0 \%$ had five to nine, and $7.5 \%$ had over 10 partners. Further, $53.2 \%$ had unprotected anal intercourse with another man over the past six months. Of these, $46.3 \%$ had it with a man who they knew to be HIV-negative, $7.6 \%$ with a man they knew to be HIV-positive, and $12.9 \%$ with a man whose status was unknown to them.

CONCLUSION: Results from this and future analyses will be compared to those from samples in larger metropolitan areas to better inform local sexual health promotion and HIV prevention programming and to instruct further predictive analyses.

\section{P126}

\section{RISKS FOR HIV AND OTHER SEXUALLY TRANSMITTED} INFECTIONS AMONG ASIAN MEN WHO HAVE SEX WITH MEN IN VANCOUVER, BRITISH COLUMBIA

Chen, Becky Biqi ${ }^{1}$; Maung Maung, Thiha ${ }^{2}$; Moore, David ${ }^{1}$;

Chan, Keith $^{1}$; Kanters, Steve ${ }^{1}$; Michelow, Warren ${ }^{1}$; Hogg, Robert ${ }^{1}$; Nakamura, Nadine ${ }^{1}$; Robert, Wayne ${ }^{1}$; Gustafson, Reka ${ }^{1}$;

Gilbert, Mark' ${ }^{2}$; ManCount Study Team ${ }^{1}$

${ }^{1}$ Vancouver; ${ }^{2}$ Burnaby, BC

BACKGROUND: Individuals of Asian heritage represent the largest ethnic minority in Canada and approximately $10 \%$ of the new HIV diagnoses in men in British Columbia occur among Asian-Canadians. However, the HIV risk patterns of Asian men who have sex with men (MSM) have not been extensively studied.
METHODS: Participants aged $\geq 19$ years were enrolled in a venue-based HIV serobehavioural survey of MSM in Vancouver, Canada. We compared the demographic characteristics, risk behaviours, and prevalence of HIV and other sexual and blood borne infections between Asian and non-Asian MSM using bivariate analysis and logistic regression confounder modelling.

RESULTS: Amongst 1132 participants, 110 (9.7\%) self-identified as Asian. Asian participants were younger than non-Asian participants (median age 29 vs 32 years; $p<0.001$ ), but otherwise did not differ in socio-demographic characteristics from other study participants. HIV prevalence was lower among Asian MSM compared to Non-Asian MSM ( $3.7 \%$ vs $19.0 \%$, $\mathrm{<}<0.001$ ). Among men who self-reported as HIV negative or unknown, we found no differences in reporting unprotected anal intercourse (UAI) with a discordant or unknown serostatus partner in the previous six months ( $11 \%$ vs $13 \%$; $p=0.503)$. However, Asian MSM were less likely to report ever using injection drugs (10.8\% vs $19.2 \%$; $\mathrm{p}=0.043)$ or using alcohol before having sex $(52 \%$ vs $64.4 \% ; \mathrm{p}=0.017)$. Confounder modeling demonstrated that Asian ethnicity was independently associated with not testing for HIV (adjusted odds ratio [AOR] 2.04 [95\% CI 1.08 to 3.86) and Hepatitis C (AOR 2.30 [95\% CI 1.27 to 4.19) within the past two years.

CONCLUSION: Asian MSM in our study reported similar rates of UAI as non-Asian MSM, but had a lower prevalence of HIV infection. Other factors which determine the HIV risk environment for Asian MSM such as the use of drugs and alcohol may partly explain these differences, however this requires further investigation.

\section{P127}

\section{NO ASSOCIATION FOUND BETWEEN YOUNGER MEN WHO HAVE SEX WITH MEN (MSM) AND UNPROTECTED ANAL INTERCOURSE WITH OPPOSITE OR UNKNOWN SEROSTATUS PARTNER IN A BRITISH COLUMBIA COHORT}

Forrest, Jamie I'; Carter, Allison ${ }^{1,2}$; Ding, Erin ${ }^{1}$; Shurgold, Susan ${ }^{1}$; Brown, Jesse ${ }^{1}$; Roth, Eric A ${ }^{3}$; Michelow, Warren ${ }^{1}$; Moore, David ${ }^{1}$; Hogg, Robert $\mathrm{S}^{1,2}$

${ }^{1}$ Vancouver; ${ }^{2}$ Burnaby; ${ }^{3}$ Victoria, BC

BACKGROUND: In 2011, MSM accounted for $57.8 \%$ of all new HIV diagnoses in $\mathrm{BC}$ with $22.2 \%$ of these being under 30 years of age. Since 2004, annual new HIV diagnoses among younger MSM born between 1980 and 1989 has increased, while this rate has remained relatively stable among older MSM born before 1980. The reasons these generational differences in rates of HIV infections remain unclear. In this study, we aimed to investigate the association between younger age ( $<30$ years) and unprotected anal intercourse (UAI).

METHODS: The Momentum Health Study is a longitudinal bio-behavioural cohort study among gay, bi and other MSM in greater Vancouver recruited using respondent driven sampling. This analysis was restricted to HIV-negative MSM enrolled at baseline $(n=126)$. Our main outcome measure was self-reported UAI with a partner of opposite or unknown serostatus in the past six months. Multivariable logistic regression was used to investigate whether being young ( $<30$ years) was associated with higher risk of self-reported UAI.

RESULTS: UAI with opposite or unknown serostatus partner in the past six months was reported by 39\% (16/65) of younger MSM and 61\% (25/61) of MSM 30 years and older. Among those who reported UAI, younger MSM were significantly more likely to report using Ecstasy/MDMA $(p=0.026)$ and LSD ( $p=0.048)$ in the past six months than older men; however, no significant age differences were observed for ethnicity, education, drugs in exchange for sex, and perceived risk of getting HIV. After adjusting for treatment optimism, party drug use and number of sexual partners, younger MSM were significantly less likely to report UAI (adjusted odds ration [AOR] 0.35 [95\% CI 0.15 to 0.80]) than older MSM.

CONCLUSIONS: These preliminary results suggest that younger MSM are not engaging in riskier sexual behavior than older MSM. 


\section{P128}

FORMATION OF A COHORT OF GAY, BISEXUAL AND OTHER MEN WHO HAVE SEX WITH MEN NEWLY DIAGNOSED WITH EARLY HIV INFECTION: RECRUITMENT OUTCOMES AND BASELINE DESCRIPTION

Gilbert, Mark; Steinberg, Malcolm; Kwag, Michael; Heath, Kate; Nohpal, Adriana; Pelzom, Dorji; Taylor, Darlene; Krajden, Mel; Patrick, David M; Ogilvie, Gina; Rekart, Michael

Vancouver, BC

OBJECTIVE: Acute HIV infection contributes disproportionately to HIV transmission, particularly among men who have sex with men (MSM), yet behavioural and psychosocial responses to acute infection are poorly understood. We describe the experience recruiting a cohort of MSM with acute HIV infection in Vancouver.

METHODS: Eligible men self-identified as MSM, were $>18$ years and newly diagnosed with acute HIV infection between April 2009 and June 2012. Providers at six clinic sites, where pooled nucleic acid amplification testing was used to enhance detection of acute HIV, referred eligible men to a Study Counsellor for support who informed about the study, or directly to the Study Coordinator who consented all participants. A message promoting availability of the Study Counsellor was added to all acute HIV test results in BC. Participants completed a self-administered questionnaire at baseline. Following initial slow recruitment we expanded eligibility to include newly diagnosed MSM with a negative HIV test in the past year (recent HIV).

FINDINGS: During the study 25 men consented (13 acute, 12 recent) and completed baseline data collection (median 35 days after diagnosis receipt; range 8 to 128 days). Participants were predominantly gay (96\%) and Caucasian (72\%); mean age was 39 years. The most common reasons for testing were risky sex (48\%), seroconversion symptoms $(32 \%)$, or regular routine $(32 \%)$. Twenty-three men were recruited from study clinic sites ( $23 \%$ of 101 eligible); two participants were recruited through the laboratory message ( $6 \%$ of 32 eligible).

DISCUSSION: Recruitment at the time of a new HIV diagnosis is challenging. We successfully recruited a cohort of MSM diagnosed with early HIV infection representing 19\% of all eligible men. Variation in time to baseline data collection may pose challenges when examining peri-diagnosis events. Working with providers from high incidence practices is critical to recruitment of MSM with early HIV infection.

\section{P129}

\section{ESTIMATED NATIONAL HIV INCIDENCE RATES AMONG} KEY SUB-POPULATIONS IN CANADA, 2011

Yang, Qiuying1; Halverson, Jessica'; Yan, Ping1; Zhang, Fan';

Remis, Robert ${ }^{2}$; Schanzer, Dena ${ }^{1}$; Archibald, Chris ${ }^{1}$;

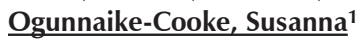

${ }^{1}$ Ottawa; ${ }^{2}$ Toronto, ON

OBJECTIVES: To estimate national HIV incidence rates among key sub-populations to inform the allocation of prevention and care resources.

METHODS: Multiple methods (workbook method, statistical modelling, and iterative spreadsheet model) were used to estimate the number of new HIV infections among key sub-populations in Canada in 2011. In order to calculate incidence rates, sub-population sizes (aged 15 and older) were approximated using Statistics Canada data (Aboriginal population and people from HIV-endemic countries) and available literature and survey data (Gay, bisexual, and other men who have sex with men (MSM) and people who inject drugs (IDU)).

RESULTS: The estimated number of new HIV infections in 2011 was 3175 (2250 to 4100); the corresponding incidence rate was 11.0 (7.8 to $14.2)$ per 100,000 among the general population aged 15 and older. MSM comprised the greatest proportion $(46.6 \%)$ of new infections; the estimated incidence rate among MSM was 504 per 100,000. Compared to other men, the MSM rate was 74 times as high. There were an estimated 435 (300 to 570) new infections among IDU. The incidence rate among IDU was 431 per 100,000; 46 times higher than the rate among non-IDU.
An estimated 390 (280 to 500) new HIV infections occurred in Aboriginal people; the incidence rate among Aboriginal people was 35.8 per 100,000 . Compared to non-Aboriginal people, the Aboriginal incidence rate was 3.5 times greater. An estimated 535 (370 to 700) new infections were attributed to people from HIV-endemic countries. The incidence rate among this population was 85.7 per 100,000; 9.1 times higher than the rate among other populations combined.

CONCLUSIONS: In spite of limitations to the denominator data, these calculations nonetheless indicated markedly different incidence rates among key populations, and particularly high among the MSM and IDU populations. Despite some uncertainties, these rate estimates provide important data that can guide programs and policy.

\section{P130}

\section{TRENDS IN HIV PREVALENCE, NEW DIAGNOSES AND MORTALITY IN ONTARIO, 1996 TO 2009: A POPULATION-BASED STUDY}

Antoniou, Tony; Zagorski, Brandon; Bayoumi, Ahmed M; Loutfy, Mona; Strike, Carol; Raboud, Janet; Glazier, Richard H Toronto, ON

BACKGROUND: Population-based estimates of HIV prevalence, rates of new HIV diagnoses and mortality rates among persons with HIV who have entered care are needed to optimize health service delivery and improve health outcomes of these individuals. However, these data are presently lacking for Ontario.

METHODS: Using a validated case-finding algorithm, we conducted a population-based study using linked administrative healthcare databases to determine the age- and sex-standardized prevalence of HIV and rates of new HIV diagnoses of HIV per 100,000 population of Ontario adults aged 18 years and older in Ontario between 1996 and 2009, as well as all-cause mortality rates per 1000 persons with HIV over this same period.

RESULTS: Between 1996 and 2009, the number of adults living with HIV increased by $98.6 \%$ and the age- and sex-standardized HIV prevalence increased by $51.9 \%(\mathrm{p}<0.001)$. Women and individuals 50 years and older accounted for an increasing proportion of persons with HIV, increasing from $12.8 \%$ to $19.7 \%(\mathrm{p}<0.001)$ and $10.4 \%$ to $29.9 \%$ $(\mathrm{p}<0.001)$, respectively, between 1996 and 2009. The proportions of newly diagnosed patients who were women or adults over the age of 50 increased from $15.4 \%$ to $24.7 \%(\mathrm{p}<0.001)$ and $10.7 \%$ to $15.6 \%$ $(\mathrm{p}=0.002)$, respectively, between 1996 and 2009. Rates of new diagnoses declined in all age-strata of men. Age and sex-standardized rates of new diagnoses and mortality rates among persons with HIV decreased $32.5 \%$ $(\mathrm{p}<0.001)$ and $71.9 \%(\mathrm{p}<0.001)$, respectively, during the study period. CONCLUSION: The prevalence of HIV has increased considerably in Ontario between 1996 and 2009, with a greater relative burden being assumed by women and individuals aged 50 years and older. In light of these trends, HIV-related health and support services will be required to adapt to the needs of an evolving and aging population of persons with HIV.

\section{P131}

A FRAMEWORK FOR THE E-TRACK: AN ENHANCED SURVEILLANCE SYSTEM DESIGNED TO MONITOR TRENDS IN THE PREVALENCE OF HIV AND ASSOCIATED FACTORS AND INFECTIONS AMONG PEOPLE IN CANADA FROM COUNTRIES WHERE HIV IS ENDEMIC

Ogunnaike-Cooke, Susanna ${ }^{1}$; McGuire, Marissa ${ }^{1}$; Husbands, Winston'; Remis, Robert S²; Tharao, Wangari²; McGee, Frank'; George, Clemon ${ }^{3}$; Adrien, Alix Venditti, Elizabeth'; Dare, Oluwayemisi ${ }^{1}$; Archibald, Chris $\mathbf{P}^{1}$ ${ }^{1}$ Ottawa; ${ }^{2}$ Toronto; ${ }^{3}$ Oshawa, ON; ${ }^{4}$ Montréal, QC

BACKGROUND: People born in countries where HIV is endemic comprise $2.2 \%$ of the Canadian population (Census 2006) but represented an estimated 15\% of people living with HIV in Canada in 2011. Enhanced (behavioural and biological) surveillance is integral to monitoring trends of HIV, other sexually-transmitted and blood-borne 
infections (STBBI) and associated behavioural and socio-demographic factors.

METHODS: The E-Track framework was developed from 2010 to 2012, led by the Public Health Agency of Canada, in collaboration with public health, research and community stakeholders from Ottawa, Toronto and Montreal. The framework was informed by the following: data needs at the federal, provincial and local levels; literature reviews on epidemiological, behavioural and social factors related to HIV risk and resilience; review of national and international behavioural surveillance indicators; HIV-focused behavioural studies in Canada; and consultations with community leaders, representatives and specific subject-experts (public health, service providers, policy, legal, immigration, etc.).

RESULTS: The E-Track framework defined core objectives, target population, survey methodology and implementation tools. As a surveillance system, E-Track will consist of repeated cross-sectional surveys at sentinel sites comprised of behavioural and biological components. The core behavioural component focuses on: socio-demographics; sexual behaviors; travel history; substance use; access to, and use of, health services; HIV testing, care and treatment; and HIV knowledge and beliefs. The biological component involves an optional finger-prick blood sample to be tested for HIV and other prioritized STBBI. Site principal investigators will have the option to include site-specific components to address local needs. Principles and strategies have been established to ensure the meaningful participation of local community leaders and members during development, implementation, results interpretation and dissemination. CONCLUSIONS: E-Track aims to provide valuable information that has not previously been available for this population and to identify behavioural and socio-demographic risk factors associated with HIV and other important STBBIs.

\section{P132 \\ EVALUATION OF THE A-TRACK SURVEILLANCE SYSTEM PILOT SURVEY CONDUCTED IN REGINA, SASKATCHEWAN}

Ogunnaike-Cooke, Susanna'; Tarasuk, Jill'; Zylak, Candace'; Faye, Rachel'2; Poitras, Margaret ${ }^{2}$; Lloyd, Kathy²;

Hennink, Maurice ${ }^{2}$

${ }^{1}$ Ottawa, ON; ${ }^{2}$ Regina, SK

BACKGROUND: A-Track was designed to monitor the prevalence of HIV and associated infections, behaviours and socio-demographic factors among Aboriginal populations in Canada. In order to determine the feasibility of conducting this surveillance system, a pilot survey was conducted in Regina, Saskatchewan from December 2011 to June 2012.

METHODS: Along with the surveillance-based objectives, the pilot survey aimed to determine: the feasibility (methodological, logistic and cost) of conducting enhanced surveillance focused on Aboriginal people; optimal questionnaire administration formats and methods; and optimal strategies to obtain a representative sample of Aboriginal people in Regina. Data sources for the evaluation included: document review and feedback from participants, survey staff, survey Elders, venue contacts and other community stakeholders.

RESULTS: The A-Track pilot survey was successfully implemented over the planned six-month timeline and recruited 1064 eligible participants ( $97 \%$ of the planned sample size). Electronic questionnaires were used by all survey participants and $99 \%$ consented to interviewer-assisted interviews. A representative sample of First Nations participants was obtained however the Métis population was under-represented. Virtually all survey participants completed the behavioral component of the survey $(>90$ questions), with variable-level non-response rate of $>10 \%$ for only four questions. Most (98\%) participants provided a specimen for HIV, hepatitis $\mathrm{C}$ and syphilis antibody testing. A dataset was available for analysis less than two months after completion of data collection. Participants, survey Elders, venue staff, and community leaders indicated positive support for and beneficial outcomes associated with the survey. Challenges included: ongoing implementation logistics (staff hiring and scheduling, interview venue setup), complex contractual agreements, substantial inkind time commitments from community and technical partners, terminology challenges for specific questions.
CONCLUSION: The evaluation demonstrated the feasibility of the A-Track surveillance system. Lessons learned from the evaluation of the A-Track pilot survey in Regina will inform future implementation of the A-Track surveillance system.

\section{P133 ANTIRETROVIRAL TREATMENT OUTCOMES AMONG HIV-INFECTED IMMIGRANT/REFUGEE PATIENTS IN NORTHERN ALBERTA}

Lefebvre, Megan; Hughes, Christine; Yasui, Yutaka; Saunders, L Duncan; Houston, Stan

Edmonton, $\mathrm{AB}$

INTRODUCTION: The HIV/AIDS epidemic disproportionately involves socially vulnerable populations. Since 2001, the proportion of immigrants/refugees among patients served by the Northern Alberta HIV Program (NAHIVP) has increased. Antiretroviral therapy (ART) has the potential to dramatically increase healthy life expectancy, however, requires life-long therapy and a high level of adherence. Despite the well documented benefits of ART, another disadvantaged group, Aboriginal HIV-infected patients experience less successful ART outcomes than non-Aboriginal patients. Immigrants/refugees may also experience poorer HIV treatment outcomes. Our study aimed to evaluate HIV treatment outcomes among HIV-infected immigrants/refugees to Canada.

METHODS: This study is a two-part retrospective cohort study using data collected by the NAHIVP. The NAHIVP currently cares for $\sim 1800$ HIV-infected patients in northern Alberta. We defined our exposure variable (immigrant/refugee status) as patients born outside Canada. Part 1 utilized logistic regression to compare the odds of experiencing initial virological suppression between immigrant/refugee and nonimmigrant/refugee patients. Part 2 used survival analysis to compare the rate of ART failure by immigrant/refugee status among patients who achieved initial virological suppression in Part 1.

RESULTS: Our study sample included 322 treatment-naïve patients (122 immigrant/refugees) who started ART in 2006-2012. For Part 1, 261 patients $(81 \%)$ achieved initial virological suppression within six months of initiating ART. After controlling for age, ART regimen, HIV risk exposure, and calendar year compared to non-immigrant/ non-Aboriginals, the odds of achieving initial virological suppression were statistically significantly lower for Aboriginal patients (odds ratio (OR) 0.44 [95\% CI 0.20 to 0.96]) but not for immigrants/refugees (OR 0.76 [95\% CI 0.33 to 1.73]). Part 2 included 261 patients who were followed for 635.1 person-years. Adjusting for age, sex, baseline CD4 cell count, and drug regimen, compared to non-immigrants/non-Aboriginals, Aboriginals and immigrants/refugees did not have statistically significant different rates of virological failure after achieving initial virological suppression (HR 1.54 [95\% CI 0.38 to 6.18]; HR 0.49 [95\% CI 0.11 to 2.20]; respectively). CONCLUSIONS: Our study sheds light on HIV treatment outcomes among Canadian immigrant/refugee patients. A better understanding of HIV treatment outcomes among immigrant/refugees is critical as the size of this population is growing.

\section{P134}

\section{RISK FACTORS ASSOCIATED WITH LOWER CD4+ COUNTS ( $\leq 200)$ AMONG HIV-POSITIVE ADULTS IN SASKATOON, SASKATCHEWAN}

Mondal, Prosanta K; Skinner, Stuart; Lim, Hyun

\section{Saskatoon, SK}

INTRODUCTION: It is crucial to identify the risk factors associated with lower CD4+ counts to slow disease progression and to prolong survival time. A high proportion of Saskatchewan's HIV infected patients is hepatitis $\mathrm{C}$ virus (HCV) co-infected and injection drug users (IDUs). OBJECTIVE: To determine the risk factors for CD4+ counts of 200 cells $/ \mathrm{mm}^{3}$ or lower in HIV-positive patients in Saskatoon.

METHODS: Retrospective longitudinal study of individuals diagnosed with HIV-positive between January 1, 2005 and September 1, 2011. Data was collected using medical charts from Royal University Hospital and from West Side Community Clinic in Saskatoon. A binary outcome of 
1 was designated for CD4+ counts $\leq 200$ cells $/ \mathrm{mm}^{3}$ ( 0 for CD4+ $>200$ cells $/ \mathrm{mm}^{3}$ ). Given the longitudinal outcome, the risk factors for CD4+ $\leq 200$ cells $/ \mathrm{mm}^{3}$ were estimated using Generalized Estimating Equation (GEE) model accounting for correlation between repeated measurements.

RESULTS: Of a total 388 patients, 186 (48\%) were female, 250 (72\%) were Aboriginals, 304 (82\%) were HCV co-infected and 294 (76\%) were IDUs. The mean age was 36 years $(\mathrm{SD}= \pm 10)$. In univariate analysis, co-infection of HCV, ethnicity (Aboriginals), ever use of ART, use of social assistance, and older age had significantly higher odds of CD4 $\leq 200$ cells $/ \mathrm{mm}^{3}$. In multivariate analysis, lower CD4+ count was associated with older age (adjusted odds ratio [aOR] 1.49 [95\% CI 1.20 to 1.84]), ever use of ART (aOR 2.74 [95\% CI 1.68 to 4.47]), and interaction of time and HCV co-infection (aOR 1.16 [95\% CI 1.05 to 1.29]). With one year of increment, odds of having CD4+ counts $\leq 200$ cells $/ \mathrm{mm}^{3}$ increased by $16 \%$ for HCV co-infected patients.

CONCLUSION: This study showed that patients co-infected with HIV/ HCV had a faster disease progression. Co-infection may complicate the treatment and management of HIV infection. Co-infected patients require earlier and more aggressive treatment of HIV and intensive follow-up.

\section{P135 \\ ENGAGEMENT IN CONTINUOUS HIV CARE BETWEEN 1997 AND 2009 AMONG ABORIGINAL PARTICIPANTS IN THE ONTARIO HIV TREATMENT NETWORK COHORT STUDY (OCS)}

Warren, Laura; O'Brien-Teengs, Doe; Zoccole, Art; Loutfy, Mona; Benoit, Anita; Gardner, Sandra; McGee, Frank; Millson, Peggy; Remis, Robert; Rueda, Sergio; Rourke, Sean B; Burchell, Ann N Toronto, ON

OBJECTIVE: To determine the patterns of engagement in continuous care among Aboriginal people compared to non-Aboriginal people in HIV care in Ontario.

METHODS: The OCS is an ongoing multi-site cohort of people in HIV care in Ontario. Data were obtained from medical charts, interviews, and linkage with databases at the Ontario Public Health Laboratories. We analysed data from 4472 persons with follow-up from 1997 to 2009, who had non-missing information on ethnicity (23,764 person-years). We defined continuous care as $\geq 2$ viral load and/or CD4 tests, $\geq 3$ months apart in a calendar year among participants who had $\geq 1$ viral load or CD4 test before July 1 st in the given year. We used multivariable longitudinal logistic regression to compare the proportion engaged in continuous care among 346 Aboriginal and 3911 non-Aboriginal participants.

RESULTS: The proportion engaged in continuous care was high but still lower among Aboriginal participants (91\%) than non-Aboriginal participants $(93 \% ; O R=0.8 ; p=0.01)$. Older participants and participants with a high CD4 count $\left(\geq 500\right.$ cells $\left./ \mathrm{mm}^{3}\right)$ had higher odds of being engaged in continuous care than younger participants $(\mathrm{OR}=1.02 ; \mathrm{p}<0.01)$ and participants with a low CD4 count $\left(\leq 200\right.$ cells $\left./ \mathrm{mm}^{3} ; \mathrm{OR}=1.3 ; \mathrm{p}=0.02\right)$, respectively. Participants residing in southwestern Ontario had a higher level of engagement in continuous care than participants residing in eastern Ontario $(\mathrm{OR}=1.5 ; \mathrm{p}<0.01)$ or the GTA $(\mathrm{OR}=1.5 ; \mathrm{p}<0.01)$. An interaction between ethnicity and injection drug use was present. NonAboriginal non-IDUs had a higher level of engagement in continuous care than Aboriginal non-IDUs (OR=1.5; $\mathrm{p}=0.02)$.

CONCLUSIONS: The majority of Aboriginal people with HIV who accessed HIV care at least annually were also engaged in continuous HIV care; we observed only minor differences between Aboriginal and nonAboriginal participants. Further research on a broader range of the population of Aboriginal people with HIV, including those not in care, is needed to fully evaluate whether inequities in access to health services exist.

\section{Evaluation of Interventions, Public Health Programs, and Policy Évaluation des interventions, des programmes et de la politique en matière de santé publique}

\section{P136 \\ MAPPING THE BRIDGES BETWEEN RESEARCHERS AND PUBLIC HEALTH PRACTITIONERS TO ADVANCE HIV PREVENTION RESEARCH IN CANADA}

Cheuk, Eve; Kettner, Joel D

Winnipeg, MB

The mission of the National Collaborating Centre for Infectious Diseases (NCCID) is to translate and exchange relevant knowledge (research evidence and experiential knowledge) for and with public health practitioners and policy-makers to reduce the burden of infectious diseases and health disparities. One challenge in bringing evidence to public health practitioners is insufficient amount of evidence that is relevant to frontline practice in Canadian settings. In the attempt to close this gap and to facilitate a knowledge translation and exchange process from knowledge generation to usage that is bi-directional, NCCID proposes to map out current loci of responsibility in the public health sector for HIV policysetting, and program development and implementation. A current and well-maintained map and database should complement CAHR's HIV Research Inventory. This initiative aims to assist researchers in navigating the labyrinth of Canadian HIV programs at all administrative and practice levels for the purpose of establishing connection and potential collaboration with public health practitioners to inform their research. Information about program parameters (e.g. target populations served, services offered, linkages with other services in the public health and non-public health domains, ways to address social determinants of health etc.) will be collected at the national, federal, provincial/territorial levels and from selected regions. The collected information will be searchable in a database using keywords identified and tested by public health practitioners and researchers. This presentation will discuss the concepts behind this proposed HIV prevention program inventory, our tentative plans for construction, and provide an opportunity to obtain feedback from attendees about elements of this inventory that would be important for inclusion.

\section{P137}

EFFICACY OF A WORK-SITE RANDOMIZED EDUCATIONAL INTERVENTION IN INCREASING HIV KNOWLEDGE AND REDUCING STIGMA AMONG WORKERS IN SHANGHAI, CHINA: IMPLICATIONS FOR THE CANADIAN CONTEXT

Calzavara, Liviana ${ }^{1,2}$; Kang, Laiyi3; Ren, Jinma ${ }^{3}$; Light, Lucia ${ }^{1,2}$; Myers, Ted ${ }^{1,2}$; Remis, Robert ${ }^{1,2}$; The Canada-China Team ${ }^{4}$ ${ }^{1}$ National; ${ }^{2}$ Toronto, ON; ${ }^{3}$ Shanghai, China; ${ }^{4}$ China

OBJECTIVE: One objective of the Canada-China Project was to evaluate interventions aimed to improve knowledge of HIV/STI transmission, decrease HIV/STIs, and reduce HIV-related stigma among migrant workers.

METHODS: In 2008, we conducted a randomized controlled trial among 1871 randomly selected workers in 18 construction sites in Shanghai. Baseline, three- and six-month post-intervention HIV/STI testing and interviews were conducted at the work-sites. We developed and implemented three HIV/STI educational interventions: low-level (pamphlets), intermediate (pamphlets, posters, DVD), and high (pamphlets, posters, DVD, group/individual counselling). Descriptive statistics examined changes following the intervention by level of intervention. RESULTS: Participants were mostly (94\%) male, mean age 38.7 years (range $=16-71$ ), $80 \%$ middle school or less, and $84 \%$ lived in onsite dormitories. At baseline, $99.7 \%$ agreed to be interviewed and $87 \%$ and $88 \%$ 
respectively provided blood and urine samples. Participants knew about ways HIV is transmitted (mean score 3.8/4 [95\% CI 3.75 to 3.84]) but frequently had misperceptions regarding transmission (i.e. mosquitoes, sharing food or toilet facilities, etc.) (mean score 2.63/5 [95\% CI 2.49 to 2.78]). Six months post-intervention, mean knowledge score significantly increased from 6.4 to 8.8 out of 10 (95\% CI 8.6 to 8.9 ; $p<0.0001$ ). Levels of HIV-stigma were reduced from mean $5.16 / 9$ to 3.9 ( $p<0.0001$ ). Those unsympathetic to HIV-positive persons decreased from 25\% to $13 \%$; those fearful from $39 \%$ to $13 \%$; and those repulsed from $29 \%$ to $8 \%$. Rates of infection at baseline were low [HIV 0.0\%, chlamydia 2.8\%, and gonorrhea $1.0 \%$ ]. The intervention did not reduce rates of new infection.

CONCLUSIONS: We observed dramatic improvements in HIV transmission knowledge and HIV-stigma. Even a low-level intervention, requiring minimal resources, was highly efficacious. Interventions administered at work-sites are a cost-effective way to reach and engage large numbers of workers who might otherwise ignore or not seek out such information. Similar trials should be conducted in Canadian worksettings.

\section{P139}

\section{THE IMPACT OF HIV TREATMENT AS PREVENTION IN THE PRESENCE OF OTHER PREVENTION STRATEGIES: LESSONS LEARNED FROM A REVIEW OF MATHEMATICAL MODELS SET IN DEVELOPED COUNTRIES}

Paquette, Dana; Schanzer, Dena; Guo, Hongbin;

Gale-Rowe, Margaret; Wong, Tom

Ottawa, ON

BACKGROUND: HIV treatment as prevention (TasP) is being considered in some jurisdictions as part of a combination HIV prevention approach. While the efficacy of TasP in a clinical trial setting has been well established, we know little about the potential effectiveness of TasP at the population level. We reviewed mathematical models of TasP set in developed countries to describe the potential prevention benefits, with a focus on the synergies of TasP with existing prevention strategies.

METHODS: Multiple databases were searched for mathematical models that examined the impact of scaling up treatment for the purposes of reducing transmission, set in developed countries, and published in the previous five years (July 2007 to July 2012). Nine models were found and were set in Canada, Australia and the United States.

RESULTS: The models predicted that the impact of expanding treatment rates could range from no decrease to a decrease of $76 \%$ in expected new infections. Models that found a reduction of $\geq 50 \%$ in expected new infections were characterised by rapid or immediate uptake, high treatment rates and high rates of testing and individuals in care. It remains unclear how current unknowns, such as the effectiveness of HAART in preventing all modes of transmission, adherence, drug resistance and risk compensation, could impact on the success of a TasP strategy. Increased testing, reducing STIs and programs to reduce risky practices were also found to be important strategies for reducing the number of new infections.

DISCUSSION: The results from evaluation studies of large scale pilots, such as the one currently taking place in one Canadian province, and from community randomised controlled trials will be useful in demonstrating how well this prevention strategy works in real world settings, and the factors that are needed to support its effectiveness.
P140

OUR YOUTH, OUR RESPONSE: AN ANALYSIS OF YOUTHFOCUSED HIV/HCV PREVENTION POLICIES IN ATLANTIC CANADA

Gahagan, Jacqueline ${ }^{1}$; Hill-Mann, Alexandra ${ }^{1}$; Dube, Anik ${ }^{2}$; MacDonald, Jo-Ann ${ }^{3}$; Harris, Gregory E ${ }^{4}$; Dykeman, Margaret ${ }^{5}$; Tucker, Maryanne ${ }^{4}$; Hudson, Pamela ${ }^{1}$; Jackson, Lois ${ }^{1}$; Proctor-Simms, Michelle ${ }^{1}$

${ }^{1}$ Halifax, NS; ${ }^{2}$ Moncton, NB; ${ }^{3}$ Charlottetown, PE; ${ }^{4}$ St John's, NL; ${ }^{5}$ Fredericton, NB

BACKGROUND: To date there has been no analysis of whether or not $\mathrm{HIV} / \mathrm{HCV}$ prevention policies focused on youth acknowledge and speak to gender-, sex- and diversity-based differences in the Atlantic Canadian context. The Our Youth, Our Response (OYOR) study brought together a team of interdisciplinary health researchers and students from the four Atlantic provinces to conduct an in-depth analysis of governmental (eg, department of health) and community-based (eg, needle exchange) policies focused on prevention of HIV/HCV among youth (15 to 24 years of age) in order to identify current policy gaps and provide recommendations to address these.

METHODS: Documents were drawn from academic and grey literature sources from a variety of databases (eg., Medline). In total, 425 documents were reviewed from New Brunswick (81), Nova Scotia (133), Newfoundland (149), and Prince Edward Island (62). Using an iterative approach, a coding structure was developed to capture if key determinants of health for youth and HIV/HCV prevention were included in the policy documents reviewed. Researchers also noted any 'novel' and 'wise practices'.

FINDINGS: In several documents key determinants of health were noted, however, not specifically in relation to youth. A distinct lack of sex-, gender- and diversity-based content, as well as low levels of inclusion and involvement of youth, was apparent. Novel and wise approaches were identified primarily in documents sourced from health and community-based organizations, with fewer emerging from the education sector. Very little reference to prevention of HIV/HCV for youth was found in the corrections sector policies reviewed.

CONCLUSIONS: Visibility and inclusion of youth, awareness of issues facing youth, and recognition of the impact of sex-, gender-, and diversity-based determinants are largely absent in the policies reviewed across the Atlantic region. There is an urgent need for government and community prevention polices aimed at youth to include recognition of diversity issues in order to better meet their needs.

\section{P141}

\section{HIV PREVENTION THROUGH MEDICINE SELLERS IN} PRIVATE-FOR-PROFIT PHARMACIES IN BANGLADESH

\section{Alam, Nazmul ${ }^{1}$; Alam, Anadil ${ }^{2}$}

\section{${ }^{1}$ Montreal, QC; ${ }^{2}$ Dhaka, Bangladesh}

Medicine sellers (MSs) in private-for-profit pharmacies in Bangladesh and many other low and middle income countries are important sources of medicines, advice, and referral for many people in low and middle income countries. Huge network of this cadre could be used for promoting safer sex education and other HIV prevention campaign. This study aims to assess if short training intervention improves MSs knowledge, skills and practice for prevention of HIV/STIs.

We used a quasi-randomized pretest protest with comparison group design to implement a set of intervention, which included a two day training session of the MSs, distribution of printed materials and establishment of local level referral linkages. A total of 269 MS participated in the baseline survey, 118 in the comparison area and 151 in the intervention area. In the endline survey conducted after six month of training, $116 \mathrm{MS}$ participated in the comparison area and 138 in the intervention area Knowledge of four correct methods of HIV transmission increased in both groups but significantly in intervention group (17\% to $28 \%)$. MS giving advice to clients with symptoms STI/HIV to use condoms ( $78 \%$ to $83 \%)$, partner treatment ( $1 \%$ to $20 \%)$ and use trusted partner $(40 \%$ to $64 \%)$ increased in the intervention group. Referral of clients with 
symptoms suggesting STI or HIV increased in both the comparison group (61\% to $69 \%$ ) and intervention group (59\% to $78 \%$ ) but the differences were not statistically significant.

Our study suggest that short training offers an effective means to increase knowledge and skills MS to act as change agent for HIV prevention in Bangladesh and other resource limited settings. Effort should be made to evaluate this intervention at larger scale along with cost effectiveness component to materialize larger impact at the community level.

\section{P142}

\section{PLANNING PHASES OF SIS IN MONTRÉAL:} DOCUMENTING THE HEALTH NEEDS OF INJECTION DRUG USERS (IDUS)

Leclerc, Pascale; Morissette, Carole; Panic, Mirna; Fall, Aissatou Montréal, QC

BACKGROUND: In December 2011, a study regarding the feasibility of supervised injection services (SIS) in Montréal concluded by a recommendation for their opening. In preparation for the opening of SIS, the regional health authorities are developing a surveillance system to document the health needs of IDUs, data stated as essential by the Supreme Court of Canada when requesting an exemption from the Ministry of Health. Overdoses are a key element to document through that system. METHODS: The first data analysed were from the "Bureau du coroner du Québec" (BCQ). Selected cases were deaths having occurred in Montréal from 2000 to 2009 that were due to accidental poisoning (primary ICD-10 codes X40-X44) or poisoning by undetermined intent (Y10-Y14). For each death, summary data, including one primary cause and one underlying cause (ICD-10 T codes), and the full report were provided. A two-step review process was used to identify deaths related to injection of illegal drugs and prescription drugs used for non-medical reasons. An initial key-word search was conducted to identify reports containing specific words related to injection; these pre-selected reports were reviewed manually.

RESULTS: Overall, 643 fatal overdoses were identified; $27.2 \%$ of them were injection-related, for an annual average of 17.5 deaths, with no significant changes over time. Most injection-related cases were male (82.9\%); the mean age was 36.6 years, with most aged 26 to 35 years (28.0\%) and 36 to 45 years $(30.9 \%)$. Most frequent contributing substances were heroin (T40.1: 34.9\%), cocaine (T40.5: 32.0\%), and other opioids (T40.2: 25.1\%).

CONCLUSION: The observed number of deaths demonstrates that Montréal IDUs would benefit from SIS. The BCQ has proved a valuable source of data on injection-related death. Other data on fatal and nonfatal overdoses will be obtained from the provincial death registry, paramedics, first responders, emergency departments, and hospital admissions. All these data will be critical when applying for an exemption to the Ministry of Health.

\section{P143}

INTEGRATING FULL HARM REDUCTION SERVICES INCLUDING SUPERVISED INJECTION SERVICES INTO A LICENSED 24-HOUR SPECIALIZED HIV/AIDS NURSING CARE FACILITY: A NECESSARY COMPONENT OF THE CONTINUUM OF HIV/AIDS HOUSING AND HEALTH CARE FOR PEOPLE LIVING WITH HIV/AIDS WHO USE ILLICIT DRUGS AND FACE OTHER PHYSICAL AND MENTAL HEALTH CHALLENGES

Baltzer Turje, Rosalind; Davis, Maxine; McDougall, Patrick; Payne, Martin

Vancouver, BC

ISSUE: While some Canadian jurisdictions have a broad range of supportive housing and care options for people living with HIV/AIDS, currently there is only one facility in one jurisdiction (the Dr. Peter Centre [DPC] Residence in Vancouver, BC) that incorporates supervised injection service into its wide range of harm reduction strategies and services to meet the complex needs of people living with HIV/AIDS who use illicit drugs and face other physical and mental health challenges.
CASE STUDY: The DPC Residence is a not-for-profit licensed HIV/ AIDS health care facility providing $24 \mathrm{~h}$ specialized nursing care for residents who have complex health issues in addition to HIV/AIDS including mental illness, active addiction, and cognitive impairment, as well as long standing issues due to experiences of social isolation, poverty and homelessness or substandard housing.

In 2002, the DPC Residence revised its harm reduction policy to incorporate supervised injection service after confirmation by the Registered Nurses Association of British Columbia (now the College of Registered Nurses of British Columbia) that the supervision of injections for the purposes of preventing illness and promoting health by registered nurses included nurses in the DPC Residence.

LESSONS LEARNED: This residential care model that incorporates a broad range of harm reduction strategies and services is effective in retaining individuals in care, sustaining their adherence to treatment (such as antiretroviral therapies), and improving health outcomes (such as CD4 counts and viral loads). This demonstrates the value of this residential care model as part of the necessary continuum of housing and care for this vulnerable population.

POLICY RECOMMENDATION: A continuum of housing and care for this vulnerable population needs to include $24 \mathrm{~h}$ specialized residential care that incorporates a broad range of harm reduction strategies and services, including supervised injection service. Ensuring the continuum includes this component could reduce the health and social inequities faced by this population, and improve acute health care utilization.

\section{P145}

\section{DOES STERILE NEEDLE DISTRIBUTION PROLONG INJECTION DRUG USING CAREERS?}

Werb, Dan; Kerr, Thomas; Buxton, Jane; Shoveller, Jean;

Richardson, Chris; Montaner, Julio; Wood, Evan

Vancouver, BC

BACKGROUND: Needle and syringe programmes (NSPs) have been shown to reduce HIV risk among people who inject drugs (IDU) However, concerns remain that NSPs delay injecting cessation.

METHODS: Individuals reporting injection drug use in the past six months in the greater Vancouver area were enrolled in the Vancouver Injection Drug Users Study (VIDUS). Analysis of variance (ANOVA) was used to generate annual estimates of the proportion of IDU reporting injecting cessation. Generalized estimating equation (GEE) analysis was used to assess factors associated with injecting cessation during a period of NSP expansion.

RESULTS: Between May 1996 and December 2010, the number of NSP sites in Vancouver increased from 1 to $29(\mathrm{P}<0.001)$. The estimated proportion of participants $(n=2710)$ reporting cessation increased from $0 \%$ in 1996 to $47.5 \%$ (95\% CI $34.8 \%$ to $60.2 \%$ ) in 2010 ( $\mathrm{P}<0.001)$. In a multivariate GEE analysis, the authors observed an association between increasing calendar year and increased likelihood of injecting cessation (adjusted odds ratio 1.04 [95\% CI 1.04 to 1.05]; $\mathrm{P}<0.001$ ).

CONCLUSION: The proportion of IDU reporting injecting cessation increased during a period of NSP expansion, implying that increased NSP availability did not delay injection cessation. These results should help inform community decisions on whether to implement NSPs.

\section{P146}

WILL A QUAD POINT-OF-CARE MULTIPLEXED ASSAY FOR HIV, HCV, HBV, SYPHILIS BE FEASIBLE, ACCURATE AND PREFERRED BY INJECTION DRUG USERS: A PILOT STUDY FROM MONTREAL, CANADA

Pant Pai, Nitika; Behlim, Tarannum; Landry, Genevieve; Savard, Pierrette; Joseph, Lawrence; Potter, Martin Montreal, QC

BACKGROUND: Injection drug users (IDU) carry a high burden of $\mathrm{HIV} / \mathrm{HCV}$ co-infection, and face a risk for contracting syphilis and HBV. Conventional laboratory-based screening is time consuming and expensive with potential loss to follow up of participants. Multiplexed point-ofcare (POC) assays that simultaneously screen four infections (HIV, HCV, 
HBV, syphilis) with one finger prick sample in $<15$ min offer a time, specimen and cost saving option but global data are limited.

METHODOLOGY/PRINCIPAL FINDINGS: A cross-sectional study was conducted in 100 outpatient IDU attendees presenting themselves to two Centre de recherche et d'aide pour narcomanes (CRAN) clinics in Montreal. A combined pre-test counseling session was followed by a multiplexed POC and a confirmatory lab test. Results were declared in a post-test counseling session with confirmatory laboratory tests. Linkages and referrals were arranged by the clinics.

The majority of the participants were male (69\%), white (93\%), 31 to 40 years of age $(36 \%)$, unemployed $(82 \%)$, with $67 \%$ admitting to sharing drug injecting equipment. Past screening history: HIV (98.9\%), HCV (96.8\%), HBV (83.9\%), and syphilis (58.1\%).

About 93\% (93/100) participants completed the study. Of 93, about $97 \%$ (90/93) of participants preferred multiplex based screening with $98 \%$ (91/93) willing to purchase it over-the-counter.

Specificity estimates for HIV, syphilis, HCV and HBV were as follows: $100 \%$ [ $95 \%$ CI $94.8 \%$ to $100 \%$ ], $97.7 \%$ [95\% CI $91.3 \%$ to $99.6 \%$ ], $96.9 \%$ [95\% CI $82.0 \%$ to $99.8 \%$ ] and $100 \%$ [95\% CI $94.8 \%$ to $100 \%$ ].

Sensitivity for HIV, Syphilis and Hepatitis C were 100\% [95\% CI 31.0\% to $100 \%$ ], $66.7 \%$ [ $95 \%$ CI $12.5 \%$ to $98.2 \%$ ], and $76.9 \%$ [95\% CI $60.3 \%$ to $88.3 \%$ ] respectively. Due to zero positives, sensitivity of Hepatitis B could not be calculated.

CONCLUSION/SIGNIFICANCE: Screening was found feasible to conduct, with high preference, high specificity of Miriad ${ }^{\mathrm{TM}}$. Improvements in sensitivity of Syphilis component is warranted. Its potential in expanded screening initiatives for co-infections in marginalized populations in Canada and the world remains unexplored.

\section{P147}

\section{"ANOTHER TOOL IN OUR TOOLKIT": TRAINING HEALTHCARE PROVIDERS IN POINT OF CARE (POC) "RAPID" HIV TESTING IN BRITISH COLUMBIA}

Fielden, Sarah ${ }^{1}$; Pedersen, Heather ${ }^{1}$; McAloney, Coleen ${ }^{1}$;

Tigchelaar, James'; Spencer, Daphne'; Keefe, Linda ${ }^{2}$;

Mackenzie-Clark, Tara ${ }^{2}$; Krajden, Mel'; Ogilvie, Gina ${ }^{1}$;

Gilbert, Mark ${ }^{1}$

${ }^{1}$ Vancouver; ${ }^{2}$ Prince George, BC

OBJECTIVES: In 2011, a province-wide Point of Care (POC) "rapid" HIV Testing Program was launched in BC. In addition to centralized distribution of POC test kits and quality assurance program components, a comprehensive training program targeting providers in healthcare settings was developed and rolled-out across the province.

METHODS: We present preliminary evaluation findings from the pilot phase of the Provincial POC HIV Testing Program, guided by a comprehensive program logic model and using both quantitative and qualitative methods. Training session summative evaluations were complemented by semi-structured qualitative interviews with key stakeholders $(n=20)$. Interviews were transcribed verbatim and managed using NVivo software. Analysis used descriptive statistics and thematic comparative coding techniques guided by qualitative inquiry and examining training-related aspects of program implementation.

RESULTS: To support over 60 sites joining the program in 2011 and 2012, 15 training sessions were conducted using the BCCDC curriculum. 173 professionals participated in the trainings, the majority (92\%) of whom were nurses including public and community health nurses, and nurse practitioners. 112 training evaluations were received showing high rates $(90-99 \%)$ of satisfaction with theoretical and practical training components. The qualitative program evaluation supported these results and further clarified programmatic training-related successes, challenges, and recommendations. Specifically, emergent themes illustrated facilitating influences of: provider experience with HIV testing; technical knowledge of POC tests and quality controls; and a lack of venipuncture skills. Also, connectedness to a central program contact was highlighted as a post-training benefit alongside challenging issues of staff turnover and long-term maintenance of competencies.

CONCLUSIONS: Results indicated that the BCCDC training model has been successful at improving provider comfort and competence with new POC HIV testing technology. Offering training sessions and ongoing support may improve client access to HIV testing and strengthen quality of care. This should be expanded through regional capacity-building, train-the-trainer and web-based approaches.

\section{P148}

IMPLEMENTATION OF A POINT OF CARE (POC) HIV TESTING PROGRAM IN BRITISH COLUMBIA: EVALUATION OF THE FIRST 18 MONTHS OF OPERATION Lindegger, Monika; Consolacion, Theodora B; Fielden, Sarah; McAloney, Coleen; Krajden, Mel; Isaac-Renton, Judy;

Ogilvie, Gina; Gilbert, Mark

Vancouver, BC

OBJECTIVE: POC HIV testing has been promoted as a strategy to expand HIV testing to increase test uptake and improve case detection. In April 2011, BC implemented a targeted POC HIV testing program using the INSTI ${ }^{\text {TM }}$ HIV-1/HIV-2 antibody test kit. Test and performance outcomes of the first 18 months of operation are reported.

METHODS: BC Centre for Disease Control Clinical Prevention Services Surveillance maintains a POC database with aggregate test volume data that is submitted monthly by test sites. Data were extracted for the period of April 1, 2011 to September 30th, 2012.Test sites were grouped according to testing site category and analyzed by overall test volume, specificity, percent positivity, positive predictive value (PPV) and use of test kits.

RESULTS: 61 sites used a total of 19,328 test kits of which 17,029 (88\%) were diagnostic tests, 1334 (7\%) quality control, $252(2 \%)$ training and 613 (3\%) wasted/expired. The median number of monthly diagnostic tests was 734 (range 437 to 2152). 80\% of tests were done in Public Health/STI clinics and outreach testing programs (26\% of all sites). POC tests made up $5.8 \%$ of all provincial HIV testing during this period. Preliminary results indicate a program percent positivity of $1.0 \% ; 99.9 \%$ specificity and 94.5\% PPV. Hospitals (2.0\%) and Mental Health and Addictions (2.0\%) were the testing site categories with the highest reported percent positivity. A total of 126 persons with newly diagnosed HIV were detected through POC testing, which was $30 \%$ of all newly diagnosed cases during this period. There were nine false positives (0.5 per 1,000 POC tests).

DISCUSSION: BC successfully implemented a targeted POC HIV testing program with low kit wastage, high case-finding, and a very low false positive rate. During our evaluation, $30 \%$ of newly diagnosed persons with HIV were detected through POC tests (comprising 5.8\% of all HIV tests in $\mathrm{BC}$ ).

\section{P149 \\ RAPID POINT-OF-CARE HIV TESTING IN A SMALL PROVINCE: POTENTIAL BENEFITS, LOGISTICS, AND POLICY IMPLICATIONS}

Lewis, Nathaniel; Gahagan, Jacqueline

Halifax, NS

BACKGROUND: Rapid point-of-care (POC) testing for HIV has been shown to increase the uptake of testing, rates of clients receiving test results, numbers of individuals aware of their status and timely access to care for those who test positive. In addition, several studies have shown that rapid POC testing for HIV is highly acceptable to clients in a variety of clinical and community-based health care settings.

RATIONALE: Most acceptability studies conducted in North America, however, have been conducted in large, urban environments where concentrations of HIV testing sites and testing innovations are greatest. Less has been done to explore the implications of rapid POC HIV testing outside of large, metropolitan, high-prevalence centres.

METHODS: First, we systematically compare the results of a recent survey on POC testing acceptability in Halifax, Nova Scotia to existing acceptability studies of rapid POC HIV testing in North America. Second, we assess the geographic landscape of rapid POC HIV testing in Canada (eg, numbers and locations of testing sites in each province). Third, we discuss the process of planning for a rapid POC testing pilot in 
a location where health professionals have not typically used this intervention.

CONCLUSIONS: Rapid POC testing may be of particular benefit to testing clients and potential clients in Nova Scotia and other regions of Canada that currently have few opportunities for anonymous HIV testing. Rapid POC testing appears to increase levels of testing and the frequency of receiving a result while reducing opportunity costs for clients who must travel to be tested. At the same time, the piloting process reveals logistical challenges and dissenting opinions surrounding rapid POC testing implementation in a region not traditionally considered an HIV epicentre.

CONCLUSIONS: Despite challenges, the introduction of rapid POC HIV testing in Nova Scotia and other Atlantic Provinces may address gaps in HIV prevention within this region.

\section{P150}

\section{TESTING THE LIMITS: INCREASING AWARENESS OF HIV RISK BEHAVIOURS AND PREVENTION THROUGH A PRISON-BASED HIV RAPID POINT-OF-CARE TESTING PILOT PROGRAM}

Globerman, Jason $\mathrm{M}^{1}$; Kiefer, Lori ${ }^{1}$; Bannan, Catherine ${ }^{2}$; English, Ken ${ }^{1}$; Bacon, Jean ${ }^{1}$; Gallaher, Sue ${ }^{2}$; Higeli, Adam² ${ }^{1}$ Toronto; ${ }^{2}$ Oakville, ON

BACKGROUND: In Ontario, HIV prevalence is significantly higher among persons in prison than in the general population. Because many individuals leave prison before standard HIV test results become available, rapid testing offers a unique opportunity for these individuals to learn their HIV status and seek information and care.

INTRODUCTION: This pilot study offered anonymous rapid point-ofcare (POC) HIV testing in an Ontario provincial prison for the first time. One objective of the pilot was to increase awareness of HIV risk behaviours and prevention.

METHODS: Promotional materials (posters, pamphlets, announcements) and face-to-face interactions with facility staff were used to offer anonymous rapid POC testing between October 2011 and March 2012. Individuals who volunteered to be tested were asked to complete a short, anonymous, self-administered survey afterwards. All testing and counseling was done by the Halton Region Public Health Department and not prison staff.

RESULTS: During the six month pilot, $\mathrm{n}=156$ individuals were tested for HIV, $42 \%$ of whom reported this as their first test. The voluntary survey was completed by $78 \%$ of participants. Following counseling, $98 \%$ of survey respondents said they "received the information they needed to know about how HIV is passed on"; $99 \%$ of respondents said they "got the information they needed about how to protect themselves and others from getting HIV"; and 98\% said they were "completely satisfied" with their HIV testing and counseling experience. Ninety-nine individuals offered feedback on ways to improve HIV testing and counseling in prisons.

CONCLUSIONS: This pilot project demonstrated increased awareness of HIV risk behaviours and prevention among those receiving testing and counseling. A high uptake of testing among those never tested was also observed. Rapid testing in prison provides an opportunity to increase the number of individuals testing for HIV which can result in better health outcomes while assisting HIV prevention efforts.

\section{P151}

\section{TESTING THE LIMITS: SURVEY RESULTS FROM A PRISON- BASED HIV RAPID POINT-OF-CARE TESTING PILOT PROGRAM}

Kiefer, Lori' ${ }^{1}$; Globerman, Jason $\mathbf{M}^{1}$; Bannan, Catherine ${ }^{2}$; English, Ken ${ }^{1}$; Bacon, Jean ${ }^{1}$; Qiyun Shi, Maggie ${ }^{1}$

${ }^{1}$ Toronto; ${ }^{2}$ Oakville, ON

BACKGROUND: In Ontario, HIV prevalence is significantly higher among persons in prison than in the general population. Because many individuals leave prison before standard HIV test results become available, rapid testing offers a unique opportunity for these individuals to learn their HIV status and seek information and care.
INTRODUCTION: This pilot study offered anonymous rapid point-ofcare HIV testing in an Ontario provincial prison for the first time. One objective of the pilot was to better understand facilitating factors for testing in prison.

METHODS: Between October 2011 and March 2012, a bi-weekly clinic was conducted by Halton Region Public Health Department (rather than prison) staff, who performed pre and post-test counseling, rapid HIV testing, and offered participants a voluntary, self-administered post-test survey which collected data on demographics and their HIV testing experience.

RESULTS: During the six month pilot, $\mathrm{n}=149$ male prisoners received rapid testing for HIV; post-test surveys were completed by 112 individuals (75\%). Of survey respondents, $44 \%$ reported this as their first HIV test, $62 \%$ identified as Canadian and $100 \%$ as heterosexual. A majority (76\%) reported previous incarceration, and $80 \%$ were currently remanded without sentencing. Mean $( \pm \mathrm{SD})$ age was $33.45 \pm 10.45$ years. The most common reasons for testing were "I had sex without a condom" (64\%) and "I have had many sex partners" (39\%). Top reasons for testing in prison were "I can get the HIV rapid/point-of-care (POC) test" (81\%) and "It's being done by Halton Region Public Health and not the prison" (34\%). CONCLUSIONS: This pilot demonstrated the unique value of both rapid testing in prisons as well as having non-prison staff perform tests. Rapid testing in prison offers opportunities to increase the number of individuals testing for HIV, which can result in better health outcomes while assisting HIV prevention efforts in a high-risk hard-to-reach population.

\section{P152 \\ "GET ON IT" MSM TESTING BLITZ: REFLECTIONS OF A TWO PHASE APPROACH}

Campbell, Diana ${ }^{1}$; Globerman, Jason $\mathbf{M}^{\mathbf{1}}$; Adam, Barry $\mathbf{D}^{\mathbf{1}, 2}$; Gardner, Sandra' ${ }^{1}$; Light, Lucia ${ }^{1}$; Major, Carol ${ }^{1}$; English, Ken ${ }^{1}$ ${ }^{1}$ Toronto; ${ }^{2}$ Windsor, ON

BACKGROUND: In Ontario, gay, bisexual and other men who have sex with men (MSM) continue to be greatly affected by HIV and syphilis. MSM represent the majority of people living with HIV and new syphilis cases in Ontario. Toronto is one of the most affected regions. To address increasingly high rates of HIV and syphilis infections among this population, the "get on it" campaign was established to educate MSM about issues relating to HIV and syphilis testing.

INTRODUCTION: The aim of the campaign was to provide MSM with the information they need to make an informed decision about whether or not they need to be tested for HIV and syphilis, and when they should test. By expanding MSM's knowledge about when they should be tested for HIV and syphilis, along with increased access to testing sites, the campaign was designed to reduce the number of MSM who have HIV and/or syphilis but don't know it, thereby increasing access to treatment and reducing further transmissions.

METHODS: A two-phase multimedia campaign ran in Toronto for six months, October to December 2011 and January to March 2012. Hassle Free Clinic ran numerous distinct drop-in clinics providing anonymous rapid point-of-care testing. Pre and post blitz surveys were distributed via gay men's websites to capture campaign awareness and uptake.

LESSONS LEARNED: Issues that arose in phase 1 around clearer messaging, clinic utilization and attracting more survey participants were addressed in phase 2 . Creative used for posters and print ads was altered slightly to emphasize additional drop-in service availability outside traditional clinics. Adjustments to scheduling of site locations and hours were done to ensure optimal utilization. Changes were made to the creative of the e-blast for the post-blitz survey to include an image to attract more survey responses. 
P153

"GET ON IT" MSM TESTING BLITZ: HIV TEST RESULTS

Gardner, Sandra'; Campbell, Diana ${ }^{1}$; Light, Lucia';

Adam, Barry D1,2; Globerman, Jason $\mathbf{M}^{1}$; Major, Caroli';

Sullivan, Ashleigh ${ }^{1}$; Fowler, Shawn ${ }^{1}$; Clarke, GB1 ; English, Ken¹;

McGee, Frank ${ }^{1}$

${ }^{1}$ Toronto; ${ }^{2}$ Windsor, ON

BACKGROUND: A comprehensive media campaign ran between October 2011 and March 2012. It targeted gay, bisexual and other men who have sex with men (MSM) in Toronto. One objective was to encourage high-risk MSM to test for HIV at one of the participating blitz clinics.

METHODS: Anonymous rapid point-of-care tests were performed. Blood samples were collected for reactive tests and submitted to the Public Health Ontario Laboratories (PHOL) for confirmatory testing. Chart data from the participating clinics were used for demographic summaries and to estimate the proportion reactive (PR) and exact binomial 95\% confidence intervals (CI). MSM test records from PHOL were used to compare number of records in the blitz period to the historical period of October 2010 to March 2011. Fisher's exact test was used to compare groups.

RESULTS: A total of 1,102 MSM were tested at eight Toronto blitz sites. Age: $43 \% 15$ to 29 years, $41 \% 30$ to 34 years, $12 \% 45$ to 54 years, $4 \% \geq 55$ years. $37 \%$ were non-white and $13 \%$ were first time testers (FTT). $72 \%$ had indication of sex without condom (SWC). Overall PR was $2.00 \%$ (CI 1.26 to 3.01 ). The FTT group had a higher PR $(2.72 \% \mathrm{CI}$ 0.75 to 6.82 ) than repeat testers (PR $1.80 \%$ [CI 1.05 to 2.87 ]); $\mathrm{p}=0.51$. The SWC group had a significantly higher PR (2.75\% [CI 1.71 to 4.18 ]) than the non-SWC group (PR 0.34\% [CI 0.01 to 1.86]); $\mathrm{p}=0.01$. There were $7951 \mathrm{MSM}$ tests reported by $\mathrm{PHOL}$ for Toronto during the blitz period, an increase of $20 \%$ over the historical period.

CONCLUSIONS: The "Get it on" campaign resulted in a large (20\%) increase in high risk MSM testing in Toronto primarily at the blitz clinics. The highest positivity rates were observed in first time testers $(2.72 \%)$ and MSM having sex without a condom (2.75\%).

\section{P154}

\section{"GET ON IT" MSM TESTING BLITZ: HIV KNOWLEDGE}

Gardner, Sandra ${ }^{1}$; Campbell, Diana ${ }^{1}$; Light, Lucia ${ }^{1}$;

Adam, Barry $\mathrm{D}^{1,2}$; Globerman, Jason $\mathrm{M}^{1}$; Major, Caroli';

Sullivan, Ashleigh ${ }^{1}$; Fowler, Shawn ${ }^{1}$; Clarke, GB ${ }^{1}$; English, Ken ${ }^{1}$;

McGee, Frank ${ }^{1}$

${ }^{1}$ Toronto; ${ }^{2}$ Windsor, ON

BACKGROUND: A comprehensive media campaign ran between October 2011 and March 2012. It targeted gay, bisexual and other men who have sex with men (MSM) in Toronto. One objective was to increase knowledge around HIV and syphilis transmission.

METHODS: Two web-based surveys were conducted using Survey Monkey in September 2011 (pre-blitz) and April 2012 (post-blitz). These anonymous responses could not be linked. Campaign awareness was measured in the post-blitz survey. Descriptive statistics and chi-squared tests are presented for demographic data and proportion correct (PC) for HIV and syphilis knowledge questions. Logistic regression was used to model respondent characteristics associated with good knowledge (GK), defined as answering $\geq 4$ out of 6 questions correctly. Adjusted odds ratios (OR) are reported.

RESULTS: A total of 408 pre-blitz and 871 post-blitz responses from the Toronto region were analysed. Age: $21 \% 15$ to 29 years, $37 \% 30$ to 34 years, $28 \% 45$ to 54 years, $14 \% \geq 55$ years. Orientation: $78 \%$ gay, $22 \%$ bisexual/other. HIV-positive: $12 \%$. For individual questions, PC ranged from very high - risk of unprotected anal sex (pre-blitz 99\%, post-blitz $98 \%, \mathrm{p}=0.42$ ) to low - common symptoms associated with acute HIV (pre-blitz 50\%, post-blitz 55\%, p=0.06). GK: $89 \%$ pre-blitz, $87 \%$ unaware of campaign post-blitz, 96\% aware of campaign post-blitz. GK was associated with campaign awareness post-blitz (vs pre-blitz, OR $2.5 ; \mathrm{p}<0.01$ ), having an HIV test within the last year (OR 2.1; $\mathrm{p}=0.02$, vs never tested, OR 1.8; $\mathrm{p}=0.02$, vs tested in past), gay identity $(\mathrm{OR} 1.7, \mathrm{p}=0.02)$, and higher education (OR 1.4 per level, $\mathrm{p}<0.01)$. All $\mathrm{OR}$ are adjusted for collector, age, region, immigration status, condom use, syphilis testing history.
CONCLUSIONS: Better overall knowledge was related to awareness of the "Get on it" campaign, having had a recent HIV test, higher education and gay identity. There are still gaps in knowledge related to acute HIV symptoms in survey respondents.

\section{P155 \\ FORMATIVE EVALUATION OF A MOBILE MSM SEXUAL HEALTH EDUCATION AND TESTING INITIATIVE IN VANCOUVER, BC: THE KNOW ON THE GO MOBILE HEALTH CLINIC}

Reiher, Geoffrey; Ford, Geoffrey; Compton, Miranda; Thumath, Meaghan; Brown, Jesse; Harrison, Scott

Vancouver, BC

BACKGROUND: Despite an overall decrease in the number of new cases of HIV in BC in 2011, the number of new HIV diagnoses in the MSM population has increased by $7 \%$ in the same period. Based on needs consultation recommendations, the VCH STOP HIV MSM outreach team has sought to expand current initiatives to address this increase by offering education, counselling, and testing opportunities in the social and sexual environments where this population meets. Through a collaboration with YouthCO (a community-based HIV organization) and the Vancouver STOP HIV Project, a mobile testing van was introduced to the larger LGBT community at the 2012 annual PRIDE event, followed by 15 outreach clinics occurring on the periphery of two identified MSM public sex environments. This evaluation examines the service uptake, testing profile of clients, and community acceptance of this new outreach initiative.

METHODS: A mixed method approach was utilized including chart review to examine client HIV risk behaviours and testing patterns. Qualitative and quantitative survey data was obtained to assess client satisfaction with the service provision at the introductory PRIDE event. RESULTS: Forty-eight clients (67\% MSM) were seen at the introductory PRIDE event, where 19\% had never tested for HIV and 31\% had not tested in more than one year. Consistently positive survey feedback on service provision was demonstrated. 49 clients (87\% MSM) were seen at the subsequent outreach clinics where $10 \%$ had never tested for HIV and $45 \%$ had not tested in more than one year. Testing uptake at these subsequent clinics occurred in the context of $59 \%$ of clients reporting inconsistent condom use.

CONCLUSION: Initial evaluation suggests that a mobile education, couselling, and testing service has community acceptance, and can provide an effective contribution to the expansion of current services offered by the STOP HIV MSM outreach team.

\section{P156}

INTENTION TO USE INTERNET-BASED TESTING FOR HIV AND SEXUALLY TRANSMITTED INFECTIONS IS HIGH AND WIDE-RANGING AMONG GAY, BISEXUAL AND OTHER MEN WHO HAVE SEX WITH MEN IN CANADA

Gilbert, Mark' ${ }^{1}$; Salway Hottes, Travis ${ }^{1}$; Trussler, Terry ${ }^{1}$; Marchand, Rick'; Taylor, Darlene'; Kerr, Thomas';

Fairley, Christopher ${ }^{2}$; Wong, Tom $^{3}$; Lester, Richard ${ }^{1}$; Ogilvie, Gina ${ }^{1}$; Shoveller, Jean ${ }^{1}$

${ }^{1}$ Vancouver, BC; ${ }^{2}$ Carlton, Victoria, Australia; ${ }^{3}$ Ottawa, ON

BACKGROUND: Despite reported use of internet-testing services by MSM and amenability to online interventions, to our knowledge no studies have examined the acceptability of internet-testing among a diverse range of MSM. We investigated intention to use internet-testing, perceived benefits, and drawbacks in the 2011-2012 Sex Now survey. METHODS: Participants were recruited for an online survey through dating/sex-seeking websites (76\%), community organizations (10\%), and word-of-mouth (9\%). Intention to use internet-testing was measured through a 5-point Likert scale. We restricted data to Canadian respondents and used multivariable logistic regression (comparing likely or very likely vs other options, combined) to identify factors associated with intent (spanning socio-demographics, sexual behaviours, healthcare access, and internet use). 
RESULTS: Among 7938 participants, 72\% intended to use internettesting with little variation across sub-groups. In multivariable analysis, intent to use was significantly higher $(\mathrm{p}<0.05)$ for: younger age, less formal education, not being 'out' about sexuality, finding sex partners/ searching for health information online, greater mobile phone use, no recent HIV test, avoiding or delaying testing because of privacy concerns or difficulty with access (e.g., long wait, limited hours), and dissatisfaction with healthcare services in general. Among men indicating intent to use the service, the most common perceived benefit was greater privacy (32\%), convenience $(24 \%)$, immediacy $(13 \%)$, and not needing to see a nurse/doctor $(12 \%)$. Among men not intending to use, the most common drawback was not seeing a nurse/doctor $(20 \%)$, wanting to talk to a provider in person about test results $(17 \%)$, not wanting online results $(18 \%)$, and low trust of service (15\%).

CONCLUSIONS: In this large online sample, all groups of Canadian MSM reported high intention to use internet-based testing. These findings speak to the potential for an internet-based testing program for HIV and STI under development in BC to reach men facing current barriers to HIV and STI testing.

\section{P157}

\section{POTENTIAL IMPACT OF A PROVINCIAL ELECTRONIC HEALTH RECORDS ON HIV AND STI TESTING AT STI CLINICS}

Taylor, Darlene; Lester, Richard; Gilbert, Mark; Achen, Melanie;

Ogilvie, Gina

Vancouver, BC

BACKGROUND: Clients who choose to attend sexually transmitted diseases clinics for care express a desire for anonymity and non-judgmental care. We surveyed a convenience sample of clients attending the $\mathrm{BC}$ Centre for Disease Control (BCCDC) Provincial STI clinic to assess the acceptability of electronic health records (EHR) containing sensitive sexual health information which may be available to healthcare professionals external to the BCCDC STI clinic.

METHODS: All clients attending the BCCDC STI clinic between JulyOctober 2012 were offered a 16-item questionnaire to determine if it was acceptable for their sexual health information to be made available to their family physician, pharmacist, medical specialist, and nurses at other STI clinics through the provincial EHR. Demographic information was also collected. Frequency analysis was conducted using SPSS-14

RESULTS: At total of 378 (227 [60\%] male) individuals completed the survey. Seventy-four per cent were 19 to 39 years old (range 14 to $>59$ years). Sixty-seven percent $(n=254)$ were Caucasian and 55\% $(n=209)$ had a university degree. The majority $(86 \%)$ of respondents stated they were satisfied with the current computer health record with closed access to external healthcare professionals. One hundred and one (27\%) respondents stated it would not be acceptable for their family physician $(n=101$ [27\%]), pharmacist $(n=197$ [52\%]), medical specialist $(n=106[28 \%])$, and nurses in other STI clinics $(\mathrm{n}=112$ [30\%]) to have access their BCCDC STI clinic record. 133 respondents (30\%) stating they would be less likely to get tested for HIV and STIs and if their BCCDC STI clinic record were made available as part of the provincial EHR.

CONCLUSION: The introduction of a provincial EHR for STI/HIV care information is not acceptable to a significant proportion of clients and may result in reduced screening, posing a threat to the control of sexually transmitted infections in British Columbia. Further monitoring and safeguards should be considered.

\section{P158 \\ ROUTINE HIV TESTING IN ACUTE AND PRIMARY CARE IN VANCOUVER}

Gustafson, Reka; Nathoo, Afshan; Demlow, Ellen; Chu, Tim;

Hall, David; Hull, Mark; Sandhu, Jat

Vancouver, BC

BACKGROUND: The benefits associated with early diagnosis of HIV infection are compelling. However, in Vancouver, the majority of new infections are diagnosed with CD4 counts $<500$ cells/ $/ \mathrm{L}$, well below thresholds for treatment initiation, and a significant proportion of these are diagnosed with advanced disease. In Vancouver, as in the rest of Canada, HIV testing has focused on those at known risk.

METHODS: In the absence of national or provincial recommendations, routine HIV testing in acute (hospital-based) and primary care in Vancouver was initiated as part of a pilot project. In both settings, broad and sustained healthcare provider-directed education was initiated, existing workflows were analyzed and robust pathways for follow-up of newly diagnosed patients were established. In acute care, data were collected through weekly chart audits to determine HIV test offer and acceptance rates. Hospital-level data were collected to assess the change in testing and diagnosis patterns from baseline. In addition, population level impact of the strategy was measured through monthly monitoring of HIV tests in the city.

RESULTS: During the first year, 10,102 HIV tests were performed in three hospitals. The offer rates were $46 \%, 34 \%$ and $64 \%$ respectively. Overall $92 \%$ to $94 \%$ of patients accepted an HIV test when it was offered. Diagnostic rates of new HIV infection varied from 3/1000 to $8 / 1000$ in acute care. Overall, testing increased four-fold in hospitals. At the community level, testing numbers increased from a mean of 17,073 (range 16,166 to 18,059 ) to 27,689 in the latest quarter of the pilot period, an increase of $62 \%$.

CONCLUSION: These results indicate that routine HIV testing in acute and community care is feasible and is well above the generally accepted cost-effectiveness threshold of 1/1000 in acute care. Patients are overwhelmingly accepting of routine HIV testing. Keys to success are broad and sustained education, clearly articulated recommendations, a robust follow-up protocol and the ability to measure and communicate results to providers.

\section{P159}

\section{RESULTS AND LESSONS FROM THE IMPLEMENTATION OF A TEST AND TREAT STRATEGY IN VANCOUVER, BRITISH COLUMBIA}

\section{Buchner, Christopher S; Harrison, Scott; Munroe, Val}

Vancouver, BC

BACKGROUND: Despite decades of prevention initiatives, the number of newly diagnosed HIV infections in Vancouver, British Columbia remains consistently high - around 200 per year. In 2009, the Provincial Government announced a pilot test and treat initiative aimed at changing the course of the epidemic. The Vancouver Coastal Health Authority and Providence Healthcare were provided $\$ 22.6 \mathrm{M}$ over four years to implement the initiative in the Vancouver pilot site.

METHODS: The project employed literature reviews, epidemiological analysis, patient journey mapping and workshops to identify priority gaps in the existing system of HIV care. These data were complemented by community engagement interviews and focus groups with patients to assess their needs and priorities. Forty pilot initiatives were identified and implemented focusing on targets related to HIV testing, diagnosis, timely linkage to care, treatment initiation, treatment adherence and social determinants of health.

A monitoring and evaluation framework was established to support six month reviews and revision of each pilot and associated targets.

RESULTS: The 40 pilots crossed the continuum of care from hospitalbased and primary care interventions to those delivered via peers and community-based organizations. Interventions included routine HIV testing, peer testing, social marketing, clinical outreach, housing supports, maximally assisted therapy, peer navigation, and a quality improvement collaborative. HIV testing volumes have increased by more than $50 \%$ with increases in HIV diagnoses, improvement in early diagnosis, and a decrease in community-level viral load.. Testing and care policies and structures have resulted in a transformed system of HIV care that will be sustained and spread regionally and provincially through knowledge exchange efforts.

CONCLUSIONS: This rapid ramp-up of an HIV test and treat strategy in a Canadian context is a complex undertaking that has generated knowledge regarding how to effectively plan and implement effective, sustainable strategies for improving HIV testing, diagnosis and treatment outcomes. 
P160

IMPACT OF OUTREACH TESTING AND INTENSIVE CASE MANAGEMENT ON LINKAGE AND ENGAGEMENT IN CARE AS PART OF A COMPREHENSIVE TREATMENT AS PREVENTION (TASP) PILOT IN VANCOUVER, BRITISH COLUMBIA

Tu, David; Thumath, Meaghan A; Demlow, Ellen; Chu, Tim; Heath, Kate; Yip, Benita; Colley, Guillaume; Gustafson, Reka; Sandhu, Jat

Vancouver, BC

BACKGROUND: Once a person is diagnosed with HIV, there are multiple factors that can delay linkage to care. Delayed linkage to care creates missed opportunities to prevent morbidity, and decrease HIV transmission risk. In Vancouver between 2008 and 2009, only 64\% of patients were linked to care within 30 days of their diagnosis. We examined the effectiveness of immediate case management on linkage and engagement to care.

METHODS: As part of the Seek and Treat to Optimally Prevent HIV (STOP) project, an interdisciplinary outreach case management team was created to promote early HIV diagnosis and rapid linkage to care among key populations. We conducted a retrospective comparative study of newly diagnosed patients case managed by the outreach team versus all other newly diagnosed patients in Vancouver over the same period.

RESULTS: Between January 2011 and July 2012, the team diagnosed a total of 27 people with HIV compared to 209 others diagnosed in Vancouver. Patients diagnosed by the outreach team were less likely to identify as MSM ( $52 \%$ vs $77 \%$; $=0.01$ ), five times more likely to identify as IDU $(37 \%$ vs $8 \% ; \mathrm{p}<0.001)$ and three times more likely to be from Vancouver's inner city ( $52 \%$ vs $18 \%, \mathrm{p}<0.001)$. Linkage to care within 30 days was not statistically different ( $91 \%$ vs $80 \%, p=0.26$ ), and $100 \%$ of patients in both groups were linked to care by six months. There were no differences in engagement in care at 12 months ( $96 \%$ vs $95 \%$ ), ART uptake ( $52 \%$ vs $65 \%$ ), percentage of patients with CD4 <200 cells $/ \mathrm{mL}$ ( $48 \%$ vs $41 \%$ ), or virologic suppression ( $35 \%$ vs $49 \%$ ).

CONCLUSIONS: Patients diagnosed by the outreach team were more likely to be from the inner city or use injection drugs. Despite these additional barriers, patients diagnosed by outreach had similar rates of linkage and engagement to care and clinical outcomes.

\section{P161 \\ IMMEDIATE STAGING AS A MECHANISM TO IMPROVE ENGAGEMENT IN HIV CARE IN LOW-THRESHOLD PUBLIC HEALTH HIV TESTING CLINICS SERVING GAY AND OTHER MSM IN VANCOUVER}

Lester, Richard; Sandstra, Irvine; Makaroff, Sylvia;

Brownrigg, Roberta; Barrios, Rolando; Doupe, Glenn;

Giffin, Cheryl; Ogilvie, Gina

Vancouver, BC

BACKGROUND: Public health-oriented STI clinics offer HIV testing, often non-nominally (ie, using pseudonym or initials), but clients who test positive may delay referral for follow-up care and treatment. The Bute Street and HIM clinics are low threshold STI/HIV testing facilities staffed by BC Centre for Disease Control nurses in downtown Vancouver that see predominately gay men and other MSM. These clinics diagnosed $18 \%$ of all new HIV cases in BC in $2011 \quad(n=53)$, yet confirming that clients were engaged in care was difficult. We proposed that offering onsite immediate staging, CD4 count and viral load (pVL), would aid in the transition to referral for nominal care and treatment.

METHODS: We began offering CD4 and pVL counts immediately upon new HIV diagnosis, at both clinics in August 2012 on a pilot basis. HIV staging results were communicated to patients during follow up, and supportive communication between nurses and clients at one and three months was offered.

RESULTS: From August to December, 2012, 20 clients were eligible to participate in the pilot. All were male, and 95\% were MSM. Of 20: 12 clients (60\%) had CD4/pVL testing done; 14 (70\%) had been followed by a nurse at one month post-diagnosis (four more clients are currently pending contact) and 15 (75\%) were engaged in the care of a physician or HIV specialty clinic.

Of four clients who chose non-nominal reporting initially, two decided to submit and had CD4/pVL blood work with their full name. Two did so non-nominally.

DISCUSSION: This pilot demonstrates that offering CD4/pVL at time of confirmatory testing and follow-up public health nursing support can assist in linkage to care at low-threshold HIV testing facilities. Early knowledge of HIV stage may enhance client desire for referral to an HIV care specialist and expedite initial HIV assessment following diagnosis.

\section{P162}

IMPROVING HEALTH OUTCOMES FOR PEOPLE LIVING WITH HIV/AIDS WHO FACE MULTIPLE BARRIERS TO CARE INCLUDING ADDICTION, MENTAL HEALTH ISSUES, HOMELESSNESS AND OTHER SOCIAL OR ENVIRONMENTAL FACTORS: RESULTS FROM A SEEK AND TREAT TO OPTIMALLY PREVENT (STOP) HIV/AIDS COLLABORATIVE PROJECT WITH VANCOUVER COASTAL HEALTH, PROVIDENCE HEALTH CARE AND THE DR. PETER CENTRE'S DAY HEALTH PROGRAM

Baltzer Turje, Rosalind; Compton, Miranda; Kille, Julie; Shergold, Jennifer; Macbeth, Allison; Payne, Martin; Simpson, Dianne; Dalgarno, Grace; McDougall, Patrick; Davis, Maxine

Vancouver, BC

BACKGROUND: In 2010, the Government of BC launched the Seek and Treat to Optimally Prevent (STOP) HIV/AIDS, a pilot project funded by the BC Ministry of Health Services to expand HIV testing, treatment and retention in care for clinically eligible individuals in BC; specifically individuals facing multiple barriers to care, including addiction, mental health issues, homelessness and other social or environmental factors.

In July 2011, Vancouver Coastal Health (VCH), Providence Health Care (PHC) and the Dr. Peter AIDS Foundation initiated a Project using the Dr. Peter Centre's (DPC) day health program for people living with HIV/AIDS (PHA) as the anchor for the Project. The day health program (funded by Provincial Health Services Authority and donation and fundraising revenue) promotes engagement in antiretroviral (ARV) therapy and adherence using a specific model of care.

INTERVENTIONS: The Project targets PHAs living in Vancouver's Granville corridor and West End who are vulnerable and require specific uninterrupted support to be adherent to ARVs. Working as a collaborative, the PHC Liaison Nurse, the DPC Project nurses, the DPC Project admission staff, the DPC day health program staff and the STOP HIV Clinical Outreach Team support individuals to ensure uninterrupted treatment. As individuals require less intensive ARV support, they remain in the DPC general day health program and new admissions are accepted into the Project to a maximum of 50 at any time.

RESULTS: For the reporting period ending Sept 2012: of the individuals who were in the Project at one time and who were on ARVs for six months or more by September $2012(n=37)$, the percentage who had a pVL of less than 200 copies $/ \mathrm{mL}$ went from $38 \%$ prior to intake into the Project, to $57 \%$ within three months of intake, to $81 \%$ at the most current measure. Satisfaction surveys administered throughout the Project demonstrated improvements in positive client-provider engagement scores from 50\% [n=10, March 2012] to 79\% [n=14, September 2012]. Overall satisfaction increased from $80 \%$ [n=10, March 2012] to $93 \%$ [n=14, September 2012].

\section{P163}

CHRONIC HIV MANAGEMENT: DESCRIPTION OF A NURSE AND PHARMACIST-MANAGED CLINIC FOR ROUTINE ASSESSMENT OF HIV+ PATIENTS

Kelly, Deborah V1' Burt, Kimberley A ${ }^{1}$; Bowmer, MI $^{2}$;

Missaghi, Bayan $^{1}$; Schulz, Cheryl' ${ }^{1}$; Lane, Charmaine ${ }^{1}$

'St John's, NL; ${ }^{2}$ Ottawa, ON

BACKGROUND: Individuals with HIV infection (PHA) are living longer, healthier lives. Chronic HIV care has shifted from managing OIs and 
AIDS-related complications to issues including long-term adherence, treatment complications, and health promotion. Increased HIV testing coupled with longevity of PHA presents a challenge for clinics to manage increasing numbers of patients. With limited resources, creative solutions are needed to ensure patients receive quality care from HIV-specialized health providers. Nurse and pharmacist-managed clinics are useful models to manage chronic conditions including diabetes, chronic pain, and cardiovascular disease, and may be useful for chronic HIV management as well.

DESCRIPTION: In 2009, the sole infectious disease specialist (ID) in Newfoundland and Labrador (NL) resigned. A locum ID returned for monthly HIV clinics, together with the HIV team nurse practitioner (NP), clinical pharmacist (PharmD), and social worker (SW). However, additional clinics were required to manage the patient waitlist. In February 2010, non-physician clinics (NPC) consisting of the HIV specialist NP and PharmD were added on a semi-regular, demand-driven basis ( 2 to 3/month). Patients were prioritized, with urgent/unstable patients seen in the locum clinic (LC), and stable patients seen in NPC. At each NPC, patients received physical exam, medication review, adherence and ART tolerability assessment, and review of pertinent test results to optimize HIV management, as well as other issues including dyslipidemia, hypertension, and osteoporosis. Emerging issues were managed through consultation with the locum ID via email/phone, with referrals as necessary. SW support was available to all patients, as required.

LESSONS LEARNED: Response to NPC has been positive, and the waitlist reduced dramatically. Informal feedback suggests patient acceptance of NPC. Team members and management feel the NPC is an effective, efficient means to provide care to stable patients. Even after an ID joined the HIV team, NPC continue to manage the patient waitlist in NL.

RECOMMENDATIONS: This poster describes an innovative model of providing care to chronic, stable HIV+ patients. Formal evaluation of NPC is needed to assess quality of care, patient outcomes and satisfaction

\section{P164}

\section{PHARMACIST-CONDUCTED MEDICATION RECONCILIATION IN HIV PATIENTS IN ONTARIO}

\section{Robinson, Linda J}

Tecumseh, ON

BACKGROUND: Pharmacists are trained to perform routine medication reconciliation to detect drug related problems (DRP) that may compromise treatment success in HIV and co-morbidities. Time constraints and insufficient knowledge have been identified as barriers to this act. A medication reconciliation tool was developed and endorsed by the Canadian HIV/AIDS Pharmacists (CHAP) to detect DRP's and collect valuable demographic and disease related data specific to the HIV population. Data collected using this form can identify the most common problems and co-morbidities and help HIV providers focus their discussions with PHA's to achieve the most time efficient impact.

PURPOSE: To conduct a pilot study to evaluate the results of pharmacist driven medication reconciliation in PHA's in Ontario.

METHODS: Pharmacists from three regions in Ontario were provided with copies of the Medsrec Tool. They were instructed to perform random medication reconciliations during PHA encounters beginning October 2012. The interim data was anonymously reported, collated and analyzed.

RESULTS: Interim data from 161 patients has been reported; $80 \%$ male, aged 20 to 80 with $79 \%$ older than 40 years of age. The average number of co-morbidities per patient was three; most related to cardiovascular and mental health. The average number of medications per patient was seven (range 1 to 19); 78\% prescribed. A total of 226 DRPs were identified, ranging from 0 to 4 per encounter, the majority surrounding issues of adherence, adverse drug reactions, and indications requiring treatment. CONCLUSION: Medication Reconciliation performed on PHA's by Ontario Pharmacists can reveal a number of DRP's. Understanding the prevalence and types of co-morbidities and drug related problems in this population can help members of the treatment team hone their skills and discussions to achieve the most time efficient impact. This qualitative research can generate similar studies in other provinces for national comparison and can lead to further education, hypotheses and epidemiological research.

\section{P165}

\section{ASSESSMENT OF SERVICES OF COMMUNITY PHARMACIES IN BRITISH COLUMBIA (BC) WORKING WITH HIV POSITIVE PATIENTS}

Hills-Nieminen, Cara; Olatunbosun, Caitlin D; Lee, Sarah; da Silva, Jack; Antic, Jelena; Yip, Benita; Toy, Junine Vancouver, BC

BACKGROUND: Antiretroviral (ARV) therapy is fully subsidized in BC and centrally distributed through St. Paul's Hospital Ambulatory Pharmacy (SPH) in Vancouver on behalf of the BC Centre for Excellence in HIV/AIDS Drug Treatment Program (BC-CFE-DTP). The majority of patients receive $A R V$ s through on-site visits with a trained HIV clinical pharmacist at SPH or at designated satellite pharmacies and clinics. For patients with geographical, social, or physical constraints, ARVs may be couriered to other healthcare sites including community pharmacies. Our objective was to create a database of these pharmacies and their services to facilitate appropriate patient referral and communication.

METHODS: Community pharmacies that received ARVs between January 1, 2012 and November 21, 2012 were identified through the BC-CFE-DTP. Between August and December 2012, these pharmacies were asked to complete a questionnaire by fax or telephone detailing distribution and clinical services. Results were summarized by descriptive statistics.

RESULTS: A total of 361 community pharmacies were identified Pharmacies were distributed throughout BC health authorities: Fraser (32\%), Vancouver Coastal (27\%), Vancouver Island (18\%), Interior $(14 \%)$, and Northern $(8 \%)$. The number of distinct patients per pharmacy ranged from one to 253 (median $=2$, IQR 1 to 3). Five pharmacies had over 50 patients. 357/361 (99\%) pharmacies were successfully contacted to complete the questionnaire of which 198/357 (55\%) responded (see Table)

TABLE: Number (percent) of survey respondents offering specific phar macy services

\begin{tabular}{lccc} 
N=198 & Yes (\%) & No (\%) & Unknown (\%) \\
\hline Distribution Services Offered & & & \\
Daily observed dispensing & $169(85.3)$ & $29(14.7)$ & 0 \\
Methadone dispensing & $164(82.8)$ & $34(17.2)$ & 0 \\
Blister packing & $193(97.5)$ & $5(2.5)$ & 0 \\
Medication Delivery & $176(88.9)$ & $21(10.6)$ & $1(0.5)$ \\
Open $\geq 12$ hours/day & $80(40.4)$ & $111(56.1)$ & $7(3.5)$ \\
Open 7 days/week & $135(68.2)$ & $56(28.3)$ & $7(3.5)$ \\
Clinical Services Available to HIV patients & & & \\
ARV drug interaction checks & $112(56.6)$ & $84(42.4)$ & $2(1.0)$ \\
ARV adherence checks & $76(38.4)$ & $117(59.1)$ & $5(2.5)$ \\
ARV counseling & $84(42.4)$ & $112(56.6)$ & $2(1.0)$ \\
Clinical notes (patient) & $79(39.9)$ & $92(46.5)$ & $27(13.6)$ \\
Laboratory reviews & $10(5.1)$ & $186(93.9)$ & $2(1.0)$ \\
Medication reviews & $163(82.3)$ & $9(4.5)$ & $26(13.1)$ \\
Other clinical services & $130(65.0)$ & $42(21.0)$ & $28(14.0)$ \\
Vaccinations & $120(60.6)$ & $51(25.8)$ & $27(13.6)$ \\
Diabetes Care & $56(28.3)$ & $115(58.1)$ & $27(13.6)$ \\
\hline
\end{tabular}

CONCLUSION: Numerous community pharmacies throughout BC receive ARV medications. The majority serve a small number of HIV positive patients. A high proportion of respondents offer many distribution services but limited clinical services to HIV positive patients. 
P166

ASSESSMENT OF HIV TREATMENT KNOWLEDGE OF COMMUNITY PHARMACISTS IN BRITISH COLUMBIA (BC) WORKING WITH HIV POSITIVE PATIENTS

Olatunbosun, Caitlin D; Hills-Nieminen, Cara; Lee, Harrison; da Silva, Jack; Toy, Junine

Vancouver, BC

BACKGROUND: Clinical HIV pharmacy services have shown to improve patient outcomes in hospital/clinic settings. Emerging data suggests community pharmacies also serve an important role. In British Columbia (BC), antiretroviral (ARV) therapy is distributed through a central hospital pharmacy and designated satellites with trained HIV pharmacists. However, approximately $40 \%$ of patients have ARVs couriered to another healthcare site, often community pharmacies without direct HIV pharmacist contact. We sought to identify HIV educational needs, willingness to provide clinical services and ways to strengthen links with community pharmacists.

METHODS: Community pharmacies that received ARVs in 2012 and expressed interest in ongoing partnership to serve HIV patients in a prior questionnaire were faxed or emailed an HIV educational needs assessment between December 12, 2012 and January 4, 2013. Results were analyzed using Microsoft Excel.

RESULTS: The survey was sent to 191 community pharmacies of which 77 pharmacists from 69 pharmacies responded (36.1\% response rate). Pharmacists rated their knowledge or skill in ten areas of HIV care (Table 2) and only two had recent training in HIV. Forty-six (59.7\%) pharmacists expressed interest in providing clinical services to HIV-positive patients and $70(90.9 \%)$ respondents were interested in additional education/training in HIV.

\begin{tabular}{|c|c|c|c|c|c|c|}
\hline Knowledge or skill area (1=low, $5=$ high) $(\%)$ & 1 & 2 & 3 & 4 & 5 & \\
\hline $\begin{array}{l}\text { Aware of where to find HIV treatment guidelines for } \\
\text { adults and adolescents }\end{array}$ & 23.4 & 27.3 & 26.0 & 13.0 & 7.8 & 2.6 \\
\hline $\begin{array}{l}\text { Skill in monitoring drug therapy (e.g. appropriate dos- } \\
\text { ages and combinations) }\end{array}$ & 36.4 & 33.8 & 22.1 & 3.9 & 2.6 & 1.3 \\
\hline Skill in recognizing side effects caused by HIV drug therapy & 23.4 & 42.9 & 22.1 & 9.1 & 1.3 & 1.3 \\
\hline Skill in managing side effects caused by HIV drug therapy & 31.2 & 40.3 & 22.1 & 3.9 & 1.3 & 1.3 \\
\hline $\begin{array}{l}\text { Skill in assessing adherence to HIV drug therapy and } \\
\text { making recommendations to improve adherence }\end{array}$ & 15.6 & 26.0 & 31.2 & 19.5 & 6.5 & 1.3 \\
\hline Knowledge of potential ARV drug/drug interactions & 23.4 & 37.7 & 26.0 & 9.1 & 2.6 & 1.3 \\
\hline $\begin{array}{l}\text { Knowledge of appropriate medication administration (e.g. } \\
\text { food requirements, required co-administration times) }\end{array}$ & 28.6 & 35.1 & 23.4 & 10.4 & 1.3 & 1.3 \\
\hline $\begin{array}{l}\text { Knowledge of immunizations recommended and con- } \\
\text { traindicated for HIV-positive patients }\end{array}$ & 23.4 & 28.6 & 31.2 & 9.1 & 5.2 & 2.6 \\
\hline $\begin{array}{l}\text { Knowledge of co-morbidities such as dyslipidemia, } \\
\text { osteoporosis, depression, substance abuse }\end{array}$ & 18.2 & 24.7 & 29.9 & 19.5 & 6.5 & 1.3 \\
\hline $\begin{array}{l}\text { Knowledge of treatment/prophylaxis of opportunistic } \\
\text { infections }\end{array}$ & 19.5 & 33.8 & 31.2 & 9.1 & 3.9 & 1.3 \\
\hline
\end{tabular}

CONCLUSION: Most community pharmacists who responded have not had recent training in HIV but are interested in increased education and providing more services. Patient care can be enhanced through an improved link between the clinical HIV pharmacists and community pharmacists.

\section{P167}

LEVEL OF HIV-SPECIALIZED OUTPATIENT PHARMACY SERVICE AND PATIENT OUTCOMES

Toy, Junine; Olatunbosun, Caitlin; Colley, Guillaume;

Chau, William; Cui, Zi-Shan; Lepik, Katherine; Harris, Marianne;

Hills-Nieminen, Cara; Da Silva, Jack; Akagi, Linda;

Hogg, Robert S; Montaner, Julio

Vancouver, BC

INTRODUCTION: In British Columbia (BC), antiretroviral therapy (ART) is provided through the BC Centre for Excellence in HIV/AIDS Drug Treatment Program (BC-CfE-DTP), with centralized distribution managed by St. Paul's Hospital Ambulatory Pharmacy. Patients pick up ART at health-care sites throughout BC; however, HIV-specialized pharmacy services vary between sites. This study examines the relationship between level of HIV pharmacy service and achievement of desired clinical standards and outcomes.

METHODS: BC-CfE-DTP participants aged $\geq 19$ years were included if they received ART between April 1, 2009 and March 31, 2010. Patients were classified by HIV pharmacy service category: Comprehensive (HIVspecialized pharmacist provides medication counseling, reviews ART safety, drug interactions, bloodwork), Intermediate (pharmacist at ART dispensing site provides medication counseling, checks drug interactions), or Basic (ART mailed to community pharmacy or physician office for pickup). Chi-square and Wilcoxon two-sample tests were used to test for differences between pharmacy service categories in demographic and clinical variables, ART adherence $\geq 95 \%$ by prescription refill, use of recommended ART regimens (IAS 2008-2010 guidelines), HIV plasma viral load (pVL) monitoring $\geq 2$ times/year, and $\mathrm{pVL}<50$ copies/mL at study end.

RESULTS: Of 4067 patients, $61 \%$ received Comprehensive, $8 \%$ Intermediate and 31\% Basic HIV pharmacy services (see Table). Relative to Comprehensive category, both Intermediate and Basic groups had a lower proportion meeting minimum recommended $\mathrm{pVL}$ monitoring frequency and receipt of recommended ART regimens. Patients receiving Basic services also had a significantly lower proportion achieving optimal adherence.

CONCLUSIONS: A higher proportion of patients receiving Comprehensive HIV pharmacy services achieved desired clinical standards of care and outcomes. The next steps will examine the association between HIV outcomes and pharmacy service adjusted for confounders to inform allocation of pharmacy services.

\begin{tabular}{ccccc} 
& \multicolumn{3}{c}{ Pharmacy Service Category } & $p$-value \\
\cline { 2 - 5 } Comprehensive Intermediate & Basic \\
Total & (A) & (B) & (C) \\
$\mathrm{n}=4067$ & $\mathrm{n}=2466$ & $\mathrm{n}=317$ & $\mathrm{n}=1284$ & A vs. B A vs. C B vs. C \\
\hline
\end{tabular}

Clinical and Demographic Variables at Study Start

Age, years (median, IQR) $39(33-46) \quad 39(33-46)$

Female gender $(n, \%) \quad 637(16) \quad 228(9)$

Injection drug use ever $(n, \%)$

No

Yes

Unknown $2278(56) 1656(67)$ $1360(33) \quad 475(19)$

CD4 >200 cells/uL (n, \%) $3603(89) \quad 2305(93)$

Duration of ART, years $\quad 8(3-12) \quad 8(3-13)$ (median, IQR)

MD experience by \# HIV $\quad 195(59-433) \quad 288(125-474) \quad 85(6-288) \quad 97(4-252)<0.001<0.001 \quad 0.588$ patients (median, IQR)

Statistical Area

Classification (Statistics

Canada) (n, \%)

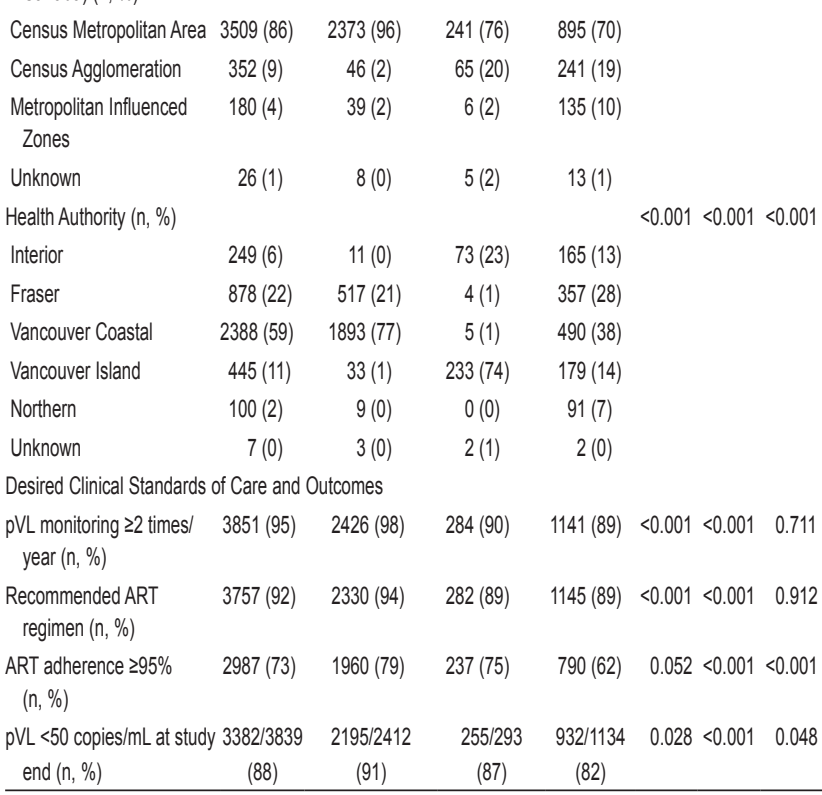

$41(35-47) \quad 39(33-46) \quad 0.04 \quad 0.455 \quad 0.024$

$43(14) \quad 366(29) \quad 0.015<0.001<0.001$

$210(66) \quad 412(32)$

$96(30) \quad 789(61)$

$11(3) \quad 83(6)$

$261(83) \quad 1037(81)<0.001<0.001 \quad 0.471$

$9(3-13) \quad 7(3-12) \quad 0.464 \quad 0.003<0.001$ $<0.001<0.001<0.001$ 


\section{P168}

USE OF NON-RECOMMENDED REGIMENS AS INITIAL ART IN BRITISH COLUMBIA IS ASSOCIATED WITH A REDUCED LIKELIHOOD OF VIROLOGIC SUPPRESSION

Moore, David ${ }^{1}$; Chan, Keith ${ }^{1}$; Nosyk, Bohdan ${ }^{1,2}$;

Colley, Guillaume'; Lepik, Katherine'; Barrios, Rolando';

Lourenco, Lillian ${ }^{1}$; Montaner, Julio ${ }^{1}$; Lima, Viviane ${ }^{1}$; Heath, Kate ${ }^{1}$; Gustafson, Reka ${ }^{1}$; Hogg, Robert ${ }^{1,2}$

1Vancouver; ${ }^{2}$ Burnaby, BC

BACKGROUND: Therapeutic guidelines for the use of antiretroviral therapy in British Columbia (BC) were developed in 1992 and have remained consistent with those published by the IAS-USA since 1996. However, these recommendations are not always followed by prescribers. We examined the effectiveness of non-recommended first-line ART regimens in BC.

METHODS: We analyzed data from all individuals aged $\geq 18$ years who initiated ART in BC from 2006 to 2010. Participants were ART naïve and were excluded if they had primary/baseline ART drug resistance. We compared characteristics of participants who received and did not receive a recommended ART regimen as their first prescribed therapy, based on contemporary therapeutic guidelines. We conducted multivariable logistic regression to determine the effectiveness of using a non-recommended ART regimen on virologic suppression $(\geq 2$ consecutive HIV plasma viral load measurements $<200$ copies $/ \mathrm{mL}$ ) within the first year of therapy. RESULTS: A total of 2390 individuals ( $80.9 \%$ male) initiated ART during the study period with 136 (5.7\%) initiating with a non-recommended regimen. The most commonly prescribed non-recommended regimens were: zidovudine/lamivudine/ritonavir/lopinavir (24\%); zidovudine/lamivudine/nelfinavir (16\%) and lamivudine/tenofovir/atazanavir/ritonavir (13\%). Individuals prescribed a non-recommended ART regiment were more likely to be younger (median age 37 vs 42 years; $\mathrm{p}<0.001$ ); female ( $62 \%$ vs $17 \%$; $\mathrm{p}<0.001)$; self-report as aboriginal $(18 \%$ vs $11 \%$; $\mathrm{p}=0.022)$; and have higher CD4 cell counts $\left(300\right.$ cells $/ \mathrm{mm}^{3}$ vs 240 cells $/ \mathrm{mm}^{3}$; $\mathrm{p}<0.001)$. Non-recommend regimens had a lower monthly cost than recommend regimens (median $\$ 1232$ vs $\$ 1291$ per month; $p<0.001$ ). The use of a non-recommended regimen was associated with reduced odds of achieving virologic suppression (AOR 0.52 [95\% CI 0.33 to 0.83$]$ ). However this effect became non-significant once prescription refill adherence was included in the model (AOR 0.78 [95\% CI 0.47 to 1.27]).

CONCLUSIONS: The use of non-recommended regimens was associated with a reduced likelihood of virologic suppression which appears to be mediated through reduced adherence to therapy.

\section{P169}

\section{CHARACTERISTICS OF PATIENTS RECEIVING} COMPASSIONATE SUPPLY OF ANTIRETROVIRAL MEDICATIONS AND ASSOCIATED MEDICATION COSTS IN A TORONTO HIV SPECIALITY CLINIC

\section{Yoong, Deborah M; Naccarato, Mark; Gough, Kevin A;}

Lewis, Jordan; Bayoumi, Ahmed M

Toronto, ON

BACKGROUND: Some patients do not adhere to therapy because the cost of medication is prohibitive. We quantified how frequently and why patients required compassionate supplies of antiretroviral therapy.

METHODS: We conducted a retrospective chart review of all patients requesting a compassionate supply of antiretrovirals at our centre from June 2011 to May 2012. We collected information about demographic characteristics, current or previous drug insurance, and the reason for interruption in drug coverage. We calculated the cost of supplied drugs using the amount paid by the Ontario Public Drugs Program.

RESULTS: Over one year, we provided 2886 days of antiretrovirals to 42 HIV-positive patients. Our sample consisted of 29 men (69\%), median age of 40.2 years (inter-quartile range [IQR] 31.8 to 47.3 years), diagnosed with HIV for 3.42 years (IQR 0.8 to 6.4 years), and of black (60\%), Caucasian (21\%), and other (9\%) ethnicity. Most requests (88\%) came from citizens or permanent residents of Canada; two patients had undocumented status. Thirty-four patients $(81 \%)$ were already taking antiretrovirals when they encountered a gap in their drug coverage, 13 of these patients (38\%) experienced a problem with Trillium activation or renewal and $12(35 \%)$ had a change in insurance status requiring enrollment into a new program. Most patients $(93 \%)$ received a compassionate medication supply that was the regimen of choice but $22(52 \%)$ did not receive the formulation of choice. Overall, the median value of supplied drugs was $\$ 1995$ per person (IQR $\$ 626$ to $\$ 6274$ ). The total value of all drugs supplied was $\$ 134,860$ for the year.

CONCLUSIONS: Many patients need a compassionate supply of antiretroviral medications, of whom the large majority are Canadian citizens or landed immigrants. The total value of supplied drugs is substantial. Our study suggests that Trillium Drug Program, Ontario's catastrophic drug insurance plan, leaves some HIV-positive patients with significant gaps in drug coverage.

\section{P170}

REGIONAL VARIATION IN NON-HAART DIRECT MEDICAL COSTS AMONG HIV-POSITIVE INDIVIDUALS IN BRITISH COLUMBIA

Nosyk, Bohdan; Lima, Viviane D; Colley, Guillaume; Yip, Benita; Heath, Kate V; Hogg, Robert S; Montaner, Julio S

Vancouver, BC

BACKGROUND: Through delayed HIV disease progression, Highly Active Antiretroviral Therapy (HAART) may reduce other direct medical costs, thus offsetting the costs of therapy. Recent findings regarding the secondary preventive benefits of HAART have necessitated closer consideration of efficiencies in care delivery across the province. Our objective is to compare non-HAART direct medical costs across the geographic regions of British Columbia (BC) throughout the HAART era.

METHODS: We considered the population of HIV-positive individuals ever accessing antiretroviral treatment in BC from 1996 to 2010. We employed linked health administrative databases capturing inpatient and outpatient care, diagnostic testing and medication dispensations. Multivariate analyses controlling for a range of demographic and clinical indicators were developed to identify differences in quarterly, non-ARV direct medical costs across BC's five health authorities (HA) (Fraser, Northern, Interior, Vancouver Island, Vancouver Coastal(VCH). Two-part models, estimated using generalized linear mixed modeling, were constructed to account for excess zeroes and skewness in cost data. The first stage modeled the probability of having a non-zero cost using a binomial distribution and logit link, while the second estimated the level of non-zero costs with gamma distribution and log link. Least square mean values were estimate by HA, evaluated at predicted means and adjusted for covariates. RESULTS: Our analysis included $\mathrm{n}=8571$ individuals with a mean follow-up of 8.56 years. Compared to clients in $\mathrm{VCH}$, those residing in the other four HAs had $22 \%$ to $65 \%$ decreased odds of having non-zero cost in a given quarter. Within quarters with non-zero costs, VCH clients accumulated costs of $\$ 1585$ (95\% CI $\$ 1542$ to $\$ 1630$ ) in 2009, compared to $\$ 1435$ ( $\$ 1365$ to $\$ 1508 ; \mathrm{p}<0.001$ ) in the Interior HA and $\$ 1167$ ( $\$ 1091$ to $\$ 1248 ; \mathrm{p}<0.001$ ) in the Northern HA.

CONCLUSION: There are substantial disparities in the costs of nonHAART medical care across BC HAs. Further analysis is needed to determine whether disparities in the quality of medical care follow similar patterns.

\section{P171}

\section{EVALUATION OF A PROVINCIAL HIV QUALITY} IMPROVEMENT COLLABORATIVE

Milligan, Robert S1; Nagi, Ashnoor²; Clarke, Christina²; Kerston, Paul $^{2}$; Barrios, Rolando ${ }^{2}$

${ }^{1}$ Prince George; ${ }^{2}$ Vancouver, BC

In 2010, a provincial HIV quality improvement collaborative began, aligning the efforts of 17 multidisciplinary primary and community care sites across British Columbia, to increase access to high-quality HIV care and treatment. All sites developed a common aim, shared measurement system, and applied the chronic care model within a quality improvement framework to improve quality indicators. These quality indicators showed improved client engagement and re-engagement in HIV care. Particular progress was reported in initiating ARVs in treatment-naive clients, 
re-engaging clients previously labelled as lost to care, and retaining clients currently in care. In January 2012, the Collaborative transitioned into a peer-driven learning Network to sustain and spread the gains. Increasing direction and leadership was provided by the "Response Team", a multidisciplinary team representing different scopes of practice and geographical areas in BC. Key to this collaboration was fostering networking, QI improvement, implementation of clinical best-practices, and putting the patient at the center of all conversations. This presentation will demonstrate a positive impact at a provincial level for five quality indicators that were sustained with Response Team support and facilitation. This presentation will also highlight selective unique initiatives from agencies across $\mathrm{BC}$ and the Provincial Collaborative series that have contributed to these successes.

\section{P172}

\section{PUBLIC VOICE SHAPES HIV SERVICE PLANNING AT VANCOUVER COASTAL HEALTH}

\section{Webster, Kath; Tolson, Margreth}

\section{Vancouver, BC}

PROBLEM: The STOP HIV pilot project was designed to improve HIV testing, treatment and support services for marginalized populations who face multiple barriers to care including addiction, homelessness, serious mental illness, and other social and environmental factors. From its initial stages, the STOP HIV Project identified community partnership and engagement as critical to an accountable, responsive and effective pilot project. But how could services best be shaped in order to reach those who face such severe challenges?

METHODS: For Vancouver Coastal Health Authority, it was clear that an ongoing Community Engagement (CE) strategy, conducted over the length of the project, would be essential. CE processes were used to meaningfully involve marginalized populations in shaping $\mathrm{VCH}$ pilot strategies, and to build trusting relationships with ASOs that would partner in service delivery. Our objectives were to:

- Provide opportunities for dialogue between $\mathrm{VCH}$ and the public it serves;

- Collect the experiences and recommendations of people living with and at risk for HIV, through a variety of methods, so that their perspectives would influence service-planning and decision-making;

- Strengthen partnerships between $\mathrm{VCH}$ leadership and community organizations.

RESULTS: This multi-phase, broad consultation strategy involved 510 individuals and 19 organizations from diverse communities that are affected by HIV and face significant stigma, and have multiple barriers to accessing services and influencing service planning. Community Engagement strategies increased community acceptance of the STOP HIV project services, and increased trust between community partners and the health authority. Recommendations from participants had direct and ongoing influence on service planning and decision-making.

\section{P173}

\section{THE BC HARM REDUCTION SUPPLY DISTRIBUTION PROGRAM: PREVENTING HIV AND HCV IN BRITISH COLUMBIA (BC)}

Buxton, Jane; Tzemis, Despina; Tang, Wrency; Yu, Amanda; Krajden, Mel

Vancouver, BC

BACKGROUND: Harm reduction (HR) and needle distribution programs have been found to reduce incidence of HIV and hepatitis C (HCV); consistent condom use can prevent sexual transmission of HIV. The BC Centre for Disease Control (BCCDC) oversees the distribution of HR supplies (including: safer sex, safer injection, and safer inhalation materials) for BC. BCCDC also monitors reported HIV by risk factor, and HCV sero-conversion.

OBJECTIVES: To describe needle/syringe and condom distribution, and newly reported HIV and $\mathrm{HCV}$ cases in $\mathrm{BC}$ associated with psychoactive substances use and risky sexual behaviour.

METHODS: HR supply data was extracted from the central ordering database; newly reported HIV and risk factors were obtained from the
BCCDC HIV 2011 annual report; 24-month HCV sero-conversion, determined by a positive anti-HCV following a negative result within 24-months, using BCCDC Public Health Microbiology and Reference Laboratory testing data. The correlation between annual harm reduction supply distribution and newly diagnosed HIV cases and 24-month HCV sero-conversion from 2006 to 2011 was assessed using Spearman's Rho. RESULTS: Between 2006 and 2011, needle/syringe distribution increased from 4,180,500 to 5,935,500(42\%) and condoms increased from $2,651,105$ to $4,160,292(57 \%)$. Overall HIV declined from 361 to 289; HIV associated with injection drug use also decreased; 24-month HCV sero-conversion decreased from 273 to 132. Spearman's Rho for the association between condom distribution and HIV is $-0.943(p=0.005)$; the correlation between needles/syringes distributed and 24-month HCV sero-conversion is $-0.963(\mathrm{p}=0.002)$.

DISCUSSION: Our findings demonstrate a statistical association between increased harm reduction supplies and a decreasing rate of newly reported HIV cases and 24-month HCV sero-conversion in BC. The decline is likely multi-factorial, affected by changing patterns of drug use and initiatives to address HIV transmission. However, programs facilitating safer sex and safer drug use play an important role in client engagement and contribute to reduced HIV and HCV transmission.

\section{P174}

EVALUATING A COORDINATED CARE NETWORK INCREASING ACCESS TO HCV CARE FOR VULNERABLE POPULATIONS BY LINKING COMMUNITY ORGANIZATIONS AND PRIMARY CARE TO SECONDARY AND TERTIARY SERVICES WITHIN AN HIV CLINIC

Yaphe, Sean; Cox, Joseph; Dugas, Manon; Morissette, Carole; Paquette, Nathalie

Montreal, QC

BACKGROUND: Hepatitis C viral infection (HCV) is a worrisome epidemic in Montreal, most prominent in vulnerable populations, including people who inject drugs and immigrants from endemic countries, groups that are traditionally difficult to reach. Given the multidisciplinary team necessary, and the opportunity to increase access to care, coordinated care networks between community organizations and primary, secondary, and tertiary care are imperative. The McGill University Health Centre (MUHC) and an outreach STD service of a health and social service centre (CSSS) established a network for HCV prevention, screening, and treatment using pre-existing HIV resources. An implementation evaluation of this network was performed to provide recommendations for improvement and to comprehend how to best develop future HCV coordinated care networks.

METHODS: Qualitative methods were used for the implementation evaluation. Questionnaires were developed for focus groups with medical professionals and administrators, and were based on the Organizational Design Framework. These discussed the development of the network, patient demographics, health and social services offered, medical professionals employed, inter/intra-organizational relationships established, and the process of implementation. Quantitative methods were used to report outcome indicators, such as number screened seropositive, and number currently on treatment

RESULTS: A model was described whereby outreach nurses from the CSSS performed screening in community organizations. Seropositive patients were accompanied towards the MUHC, where relationships were established with multiple departments to provide optimal care. Due to the demographics of the territory, the majority of the patients in this network were immigrants from endemic countries.

CONCLUSION: Utilizing HIV resources for HCV has proven successful in increasing access to care for vulnerable populations by linking community organizations with screening and specialized care. This evaluation has brought out recommendations for improvement, which include the establishment of primary care services to treat non-HCV related issues, establishing links to addictions services, and providing peer support. 


\section{P175}

OUTCOMES OF AN HIV PRECEPTORSHIP PROGRAM FOR FAMILY PHYSICIANS AND NURSE PRACTITIONERS (NPS) PROVIDED BY THE BRITISH COLUMBIA CENTRE FOR EXCELLENCE (BC-CFE)

Guillemi, Silvia A; Strehlau, Verena; Hall, David; Fung, Francesca; Barrios, Rolando; Montaner, Julio S

Vancouver, BC

BACKGROUND: HIV positive patients health outcomes are related to the level of training and expertise of their providers. Testing and treatment for HIV has significantly increased due to the Treatment as Prevention initiative in the province of British Columbia. This has led to an increased demand for healthcare providers trained in the management of HIV positive patients.

METHODS: The BC-CfE in collaboration with the Vancouver Coastal Health Authority developed a three-module compact training program targeted at primary care providers. In the first module, trainees completed an online HIV diagnosis and management course followed by a review of their main learning objectives with the BC-CfE program director. The second module consisted of a one-week clinical placement with HIV primary care and specialist clinics, an HIV ward and an HIV clinical pharmacy. Trainees attended focused daily noon seminars. Case reviews and program evaluations were done at the end of the clinical placements. The third module consisted of a three-month mentorship program. Changes in antiretroviral (ART) prescribing patterns were evaluated for up to 12 months upon completion of training.

OUTCOMES: A total of 26 family physicians and nurse practitioners were trained from September 2011 to September 2012. Final evaluations were received from 21 out of 26 participants. Of those 21 participants, $11(52.4 \%)$ were highly satisfied, and the remaining 10 (47.6\%) trainees were very satisfied or satisfied with the program. Except for one, none of the trainees prescribed ART prior to the training. A review of their ART prescription pattern showed that 12/26 initiated 45 patients on ARTs and 13/26 trainees had refilled 162 ART prescriptions.

CONCLUSIONS: We developed and evaluated an innovative HIV training program for primary care providers. This training led to an increase in the number of HIV positive patients receiving ART under their care.

\section{P176}

\section{EVALUATION OF THE CIHR CANADIAN HIV TRIALS}

\section{NETWORK PROGRAM}

Gunning, Jennifer Lㅜㄹ ; Anis, Aslam²; Cox, David²;

Dos Santos, Suzete ${ }^{1}$; Halloran, Danielle; ${ }^{1}$; Jones, Michelle ${ }^{2}$; Margolese, Shari ${ }^{3}$; McLean, Robert ${ }^{1}$; Mustoe, Heather ${ }^{1}$;

Pankovich, Jim²; Pater, Joseph ${ }^{3}$; Pendergraft, Kevin²; Sas, Jacquie²; Schechter, Martin ${ }^{2}$; Singer, Joel ${ }^{2}$; Tucker, Joanne ${ }^{1}$

${ }^{1}$ Ottawa, ON; ${ }^{2}$ Vancouver, BC; ${ }^{3}$ Kingston, ON

BACKGROUND: The Canadian Institutes of Health Research (CIHR) Canadian HIV Trials Network (CTN) is a partnership committed to developing treatments, vaccines and a cure for HIV disease and AIDS through the conduct of scientifically sound and ethical clinical trials (CTN web 2013). The CTN has been funded by the federal government for this purpose through a grant administered by the CIHR. CIHR, as a learning organization and an organization accountable to Canadian taxpayers, has undertaken a comprehensive evaluation of the CTN program to guide future research funding programs and policies.

METHODS: The evaluation employed multiple lines of inquiry including: targeted on-line surveys $(n=66)$; in-depth interviews with investigators, community members, CTN leadership, CIHR program staff, and subject area experts $(\mathrm{n}=32)$; document reviews; bibliometric analysis; and a comparative international network scan of best practices. The evaluation research was conducted in a collaborative fashion between evaluators, evaluation-users, and subject area experts.

RESULTS: The facilitation of collaboration between researchers, and with community and partner organizations is a key outcome of the network. Cross-country comparative bibliometric data indicates that CTN- affiliated investigators have produced high quality publications within Canada, and at the international level. Data indicate that high quality training is provided through the CTN Postdoctoral Fellowship Program. Exploratory survey and key informant interview data highlight that, overall, members are satisfied with the services offered by the CTN but there remain areas for improvement.

CONCLUSION: Results of the evaluation demonstrate both successes and limitations of the CTN program and its implementation. Multiple areas of learning emerge, including those pertaining to the research funder; investigator networking and knowledge creation; and governance of a research-focused network. The evidence uncovered in the evaluation will be used by CIHR and the CTN to make decisions regarding future program and network development.

\section{P177}

BUILDING BRIDGES BETWEEN CIHR/CTN AND UNIVERSITY OF PEKING FOR THE DEVELOPMENT OF CLINICAL TRIALS IN HIV-INFECTED SUBJECTS

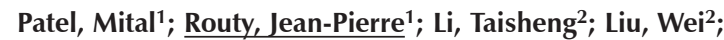
Massicotte, Angie ${ }^{1}$; Jenabian, Mohammad-Ali; ${ }^{1}$; Sas, Jacqueline ${ }^{3}$; Cox, David ${ }^{3}$; Panovich, Jim ${ }^{3}$

${ }^{1}$ Montreal, QC; ${ }^{2}$ Beijing, China; ${ }^{3}$ Vancouver, BC

BACKGROUND: Informal meetings between Canadian and Chinese physicians occurred and a common interest developed to join forces for HIV clinical trials and vaccine development in countries with different epidemics, populations and viral variants (clades).

OBJECTIVES: After receiving a planning grant from CIHR, a group of CTN members including the Chief Scientific Officer, the CTN Postdoctoral Fellowships and Committees Manager and the co-chair of the Vaccines and Immunotherapies Core, visited the Peking Union Medical College Hospital (PUMCH) in Beijing, China (October 2012). Exchanging knowledge and strategies to further develop effective clinical trials, we met with the PUMCH research team.

OUTCOME: Presentations and conferences were given by clinical investigators, research scientists, students and research nurses followed by an open discussion on the cultural perception of people living with HIV infection. We also visited an institution that has implemented a national blood banking for HIV samples from all over China and exchanged information about standard operating procedures and staff safety with scientists and technicians involved in quality control. From our perspective, we described the Canadian historical perspective on 20 years of HIV clinical trials and blood banking with a focus on Canadian regulations for phase I/II clinical trials involving experimental drugs. Based on the size of Chinese population, many issues were discussed regarding liability and global national management. Government regulations restrict the shipment of biological samples so we plan to perform HIV sample analyses using standardized methods in parallel in both countries to avoid shipping between our two centres.

CONCLUSION: After this first CIHR/CTN and PUMCH 2012 research meeting, plans are being made to have a student exchange and training for Chinese research nurses with a focus on quality control and strategies to overcome language issues for case report forms. Active discussions are ongoing for the implementation of clinical trials and cohort studies.

\section{P178}

\section{THE CANADIAN HIV VACCINE INITIATIVE - PROGRESS TO DATE}

Varughese, Nobel; Gaudreau, Marc-André; Jónsdóttir, Lilja; Cryan, Christine

Ottawa, ON

BACKGROUND: Through the Canadian HIV Vaccine Initiative (CHVI), a collaboration between the Government of Canada and the Bill \& Melinda Gates Foundation, a number of strategic investments have been made to contribute to global efforts to accelerate the development of an HIV vaccine.

METHODS: Consultations took place with Canadian and international stakeholders on the implementation of the CHVI Research and 
Development Alliance. Analysis was undertaken to ensure alignment with the priorities of the 2010 Global HIV Vaccine Enterprise's Scientific Strategic Plan and new programs were identified. A number of request for proposals were launched, open to researchers, community-based organizations, and the private sector

RESULTS: Since its inception, the CHVI has made important progress on a number of fronts. Some of the key successes include supporting: Canada-Africa research teams to build capacity for HIV prevention trials; large collaborative teams of Canadian and low- and middle-income country researchers through the development of integrated research programs; regulatory capacity building in low and middle-income countries; the Canadian private sector to develop an HIV vaccine and other technologies related to the prevention, treatment, and diagnosis of HIV; and domestic and international organizations to engage the community in HIV vaccine-related efforts.

The establishment of an expert Advisory Board ensures appropriate oversight and strategic direction of the CHVI, and the CHVI Alliance Coordinating Office facilitates collaboration and knowledge exchange. CONCLUSIONS: The launch and implementation of a number of CHVI initiatives since 2007, has ensured the involvement of Canadian expertise in exploring innovative solutions to the challenges facing HIV vaccine research and development.

\section{P179}

\section{EVALUATING CATIE'S NEW MANDATE}

\section{Challacombe, Laurel A; Rogers, Tim; Edmiston, Laurie}

Toronto, ON

BACKGROUND: In 2008 CATIE expanded its knowledge exchange (KE) programs and services to address all aspects of HIV prevention, care, treatment and support. In addition to maintaining treatment information products and services, CATIE launched a series of new services; expanded its promotion, networking and partnership base; expanded the range and scope of its publication development and distribution; expanded the range and scope of its educational program; and integrated KE practices across the continuum of prevention, care, treatment and support.

In 2012, CATIE sought to determine if these activities met the primary objectives of the Knowledge Exchange (KE) fund, specifically: 1) To increase knowledge of HIV/AIDS among front-line organizations, and 2) To enhance capacity of front-line organizations to plan and deliver programs.

METHODS: CATIE conducted many discrete evaluations of various programs and services. Each of these evaluations contained standard indicators that assessed whether these objectives were met. Key performance indicators were also collected to ascertain the type and quantity of work performed.

RESULTS: The evaluation highlighted that CATIE's multichannel model for KE has been very successful at reaching audiences. For example, 40,000 people attended workshops; Internet users made 7 million web visits; and 3.5 million publications were disseminated through CATIE's Ordering Centre.

CATIE's programs and services were rated as very effective at increasing knowledge of HIV ( $85 \%$ to $100 \%$ ). CATIE has also been very effective in increasing the capacity of front-line organizations to plan and deliver programs and services, particularly by providing information that frontline workers can use with their clients (96\%) and that responds to the needs of their communities (91\%). CATIE is also very effective at disseminating information that helps front-line organizations plan and deliver programs (85\%).

CONCLUSIONS: Evaluations identified a number of key factors for knowledge-building success including the ability to: communicate research findings in plain language, contextualize information for frontline workers and their clients, work effectively in partnership with organizations serving specific populations. CATIE has also been very effective at expanding networks for knowledge sharing.
Global Health Epidemiology Épidémiologie de la santé mondiale

\section{P180 \\ DISTANCE AND GEOGRAPHIC FACTORS ON HIV \\ TREATMENT OUTCOMES IN A KENYAN COHORT OF HIV PATIENTS ENROLLED IN AN SMS PROGRAM (WELTEL KENYA1)}

Luft, LeeAnne ${ }^{1}$; Memetovic, Jasmina ${ }^{1}$; Van der Kop, Mia ${ }^{1}$; Karanja, Sarah²; Kimani, Joshua²; Ngugi, Elizabeth²; Ritvo, Paul ${ }^{3}$; Mak, Sunny ${ }^{1}$; Lester, Richard ${ }^{1,2}$

${ }^{1}$ Vancouver, BC; ${ }^{2}$ Nairobi, Kenya; ${ }^{3}$ York, ON

BACKGROUND: The influence of geographic barriers to HIV care and delivery of antiretroviral therapy (ART) in sub-Saharan Africa has identified transport costs and distance as the most commonly cited factors associated with attrition.

METHODS: The WelTel Kenyal trial included a cohort of 538 participants receiving SMS intervention as part of their HIV ART initiation. Geographic factors including mode of travel, travel time, average time missed at home/work, actual distance to clinic via GIS mapping, urban vs rural home community, and return fare to and from clinic were analyzed with respect to association with self-reported adherence ( $\geq 95 \%$ of ART doses taken) and/or serologic viral load (VL) suppression (plasma HIV-1 RNA load $\leq 400$ copies/ $\mathrm{mL}$ ) at 12 months. Potential associations were determined using Fisher's exact test of association and a binary logistic regression model.

RESULTS: A significant relationship was found for travel cost and adherence $(p<0.05)$. No significant association was found between travel cost and viral load suppression. Amongst all users of an SMS-based support tool, the group of patients paying the most for travel to clinic $(\geq 700$ Kenyan shillings, $\mathrm{KSH}$ ) had $73 \%$ decreased odds (or 0.27 of odds) of reporting adherence. Comparator categories of low $(0-300 \mathrm{KSH})$ and intermediate ( 300 to $<700 \mathrm{KSH}$ ) paying patients did not demonstrate the same association. Other geographic variables examined did not correlate with adherence or VL suppression at 12 months.

CONCLUSION: Amongst of group of Kenyan HIV patients initiating ART with an SMS support intervention, travel costs were negatively associated with adherence whereas other geographic factors analyzed were not associated with either adherence or VL suppression at 12 months. One possible explanation is that SMS support helped to overcome many geographic barriers, however, more rigorous study is needed to validate if SMS is an effective intervention for factors other than travel cost.

\section{P181}

\section{MEDIUM-TERM PSYCHOSOCIAL OUTCOMES OF PATIENTS ON ANTIRETROVIRAL THERAPY IN RURAL WESTERN UGANDA}

Paulsen, Catherine ${ }^{1}$; Saunders, L Duncan ${ }^{1}$; Alibhai, Arif ${ }^{1}$;

Konde Lule, Joseph ${ }^{2}$; Kaler, Amy ${ }^{1}$

${ }^{1}$ Edmonton, $\mathrm{AB} ;{ }^{2}$ Kampala, Uganda

BACKGROUND: A study of patients after one year in a communitybased antiretroviral therapy (CBART) program in western Uganda found issues related to physical and psychological functioning. What is not known is whether these issues diminish over time.

OBJECTIVES: The purpose of this study is to explore medium-term changes in physical and psychosocial issues faced by CBART patients. METHODS: In-depth interviews were conducted in 2012 with 25 purposefully selected CBART patients with undetectable viral loads. Data were analyzed using content analysis.

RESULTS: Benefits observed during the first year of treatment, particularly decreased fear of death and increased hope for the future, have been maintained over time. Stigma has reduced considerably due to fewer visible signs of illness in patients. Physical functioning remains below preinfection levels, limiting participants' ability to work. Many have 
accepted and adapted to this reality. There were mixed attitudes towards sexual relations between married and single patients. Though most couples mention being fearful of having an HIV positive child, some couples mentioned they were actively trying to conceive.

CONCLUSIONS: Physical and psychosocial issues have reduced over time, which suggests treatment continues to improve quality of life. Some patients have found ways to adapt to their condition, which can provide lessons for others. Stigma is a broader social issue that is more difficult to address, though treatment has reduced symptoms that draw attention to someone's HIV status. Pressure to have children continues and more support is needed to counsel patients and help them find safer methods of conception.

\section{P182}

\section{BARRIERS, CHALLENGES AND CONCERNS REPORTED IN THE GLOBAL IMPLEMENTATION OF HIV RAPID AND POINT-OF-CARE TESTS: A SYSTEMATIC REVIEW (1996- \\ 2012)}

Deli-Houssein, Roni; Behlim, Tarannum; Wilkinson, Samantha;

Vadnais, Caroline; $\underline{\text { Pant Pai, Nitika }}$

Montreal, QC

OBJECTIVE: Barriers, challenges, and concerns operating at four levels of health care; health systems, providers, patients and test devices, have impeded the optimal global implementation of singleton HIV rapid and point-of-care (POC) tests. Understanding them is key to improving their integration and performance across all health care systems. We conducted a systematic review to synthesize them.

METHODS: For the period 1996 to 2012, two reviewers searched five databases (i.e., CINAHL, Embase, PsycINFO, BIOSIS and Medline [via PubMed]). A third reviewer resolved disagreements.

RESULTS: Across 94 studies, a recurrent pattern in barriers, challenges and concerns was observed. While test device related challenges $(44 / 94 ; 47 \%)$ dominated the bulk of the evidence, provider level challenges $(28 / 94 ; 30 \%)$, patient level concerns $(29 / 94 ; 31 \%)$ and health system level barriers $(25 / 94 ; 27 \%)$ followed closely. Test device related challenges $(n=44)$ were majorly related to: i) diagnostic accuracy $(38 / 44 ; 86 \%)$ and ii) issues in test conduct $(6 / 44 ; 14 \%)$. Provider related challenges $(n=28)$ were: i) complicated POC protocols $(14 / 28 ; 50 \%)$ and ii) challenges in clinical workflow $(14 / 28$; $50 \%)$. Patient level concerns were $(n=29)$ related to: i) a lack of awareness and misconceptions regarding POCs (13/29; 45\%), ii)privacy $(9 / 29 ; 31 \%)$, iii) difficulty in performing self tests $(4 / 29 ; 14 \%)$ and iv) high costs of the tests and additional cost of confirmatory testing $(3 / 29 ; 10 \%)$. Health system related barriers $(n=24)$ alluded to i) integration of POC within health systems $(11 / 24 ; 46 \%)$, ii) variation in POC protocols across facilities $(5 / 24 ; 21 \%)$, iii) a lack of quality control and assurance $(5 / 24 ; 21 \%)$, and iv) cost of implementing the test into the existing health system(3/24; $13 \%)$.

CONCLUSION: Improvements in diagnostic performance of tests, greater workflow integration of POC, standardization of test protocols, and improvements in costs will optimize the implementation of rapid and POC tests. Addressing these barriers, challenges and concerns is paramount to the future success of POC testing initiatives both nationally and internationally.

\section{P183}

\section{HIV TESTING AND COUNSELLING IN COLOMBIA: HOW TO REACH MOST-AT-RISK POPULATIONS?}

Galindo, Jaime $^{1}$; Montano, David ${ }^{1}$; Mueses-Marín, Hector $\mathrm{F}^{\mathbf{1}}$;

Pinzon-Fernandez, Maria $\mathrm{V}^{1}$; Tello-Bolivar, Ines $\mathrm{C}^{1}$;

Martinez-Cajas, Jorge $\mathbf{L}^{2}$; Alvarado, Beatriz $\mathrm{E}^{2}$

${ }^{1}$ Cali, Colombia; ${ }^{2}$ Kingston, ON

INTRODUCTION: HIV testing remains very low in Colombia with only $16 \%$ ever tested. This is related to poor access to health services and high levels of stigma. To approach this issue, the "Corporacion de Lucha Contra el Sida" (CLS) has implemented a multidisciplinary, providerinitiated, population-based HIV testing and counselling strategy named
BAFI. The experience of CLS reaching most-at-risk populations in 2008 2009 is described below.

METHODOLOGY: Two different approaches were used to reach those most at risk: one led by CLS and local health care providers (A1), and the other by CLS and community leaders (A2). Every campaign under the BAFI strategy included: HIV screening test after informed consent, a demographic questionnaire, self-reported HIV knowledge and behaviour questionnaires, pre and post-test counselling, confirmatory HIV tests when indicated, clinical follow-up and access to comprehensive care, including full access to antiretroviral treatment for those needing it.

RESULTS: A total of 2085 people were reached under approach A1 and 363 under A2. The indicators of effectiveness for HIV diagnosis, follow-up and access to care were, for approaches A1 and A2: Rate of HIV+ screening test $=1.2 \%$ and $4.4 \%$; HIV + confirmed frequency $=0.29 \%$ and $3.86 \%$; Rate of return for confirmatory results $=62.5 \%$ and $93.7 \%$; Rate of return to comprehensive care $=33.3 \%$ and $92.3 \%$; Rate of ART initiated $=20 \%$ and $53.8 \%$.

CONCLUSIONS: Although more people were reached with A1, the A2 approach which involves community leaders was more effective at reaching the most-at-risk population. The A2 approach found a population with higher HIV+ prevalence, and better follow-up and comprehensive care indicators in HIV+ individuals.

\section{P184}

\section{FACTORS ASSOCIATED WITH HIV TESTING IN TWO CITIES IN COLOMBIA}

Mueses, Héctor $\mathrm{F}^{1}$; Montano, David ${ }^{1}$; Galindo, Jaime ${ }^{1}$; Pinzon-Fernandez, Maria $\mathbf{V}^{1}$; Tello-Bolivar, Ines $\mathrm{C}^{1}$; Martinez, Jorge $\mathrm{L}^{2}$; Alvarado, Beatriz $\mathrm{E}^{2}$

${ }^{1}$ Cali, Colombia; ${ }^{2}$ Kingston, ON

INTRODUCTION: HIV testing in Colombia is very low as compared to North America. Among women in reproductive years, previous HIV testing (PHT) has been estimated in 16\%; among Men who have Sex with Men (MSM) in less than 30\%. Nevertheless, no data exist on factors related to the low prevalence of HIV testing. In the present study, the frequency and factors associated with HIV testing in women and men older than 18 years old from low income neighborhoods in two cities in Colombia was investigated.

METHODS: A cross-sectional study was conducted between 2008 and 2009, and included 2930 participants recruited as part of HIV voluntary counseling campaigns in three different populations: 1) people from low socio-economic status (SES) living in Popayan; 2) internally displaced population of low SES living in Popayan, and 3) a population of low SES living in Cali. Socio-demographic characteristics, history of sexually transmitted infections (STI), sexual/other related behaviours and self-reported HIV knowledge were assessed as factors related to no PHT. Bivariate and multivariate Poisson regression models were run to assess prevalence risk ratios and establish the main factors associated with PHT.

RESULTS: Mean age of participants was $36 \pm 12.9$ years, most of them were women, $72 \%$. The overall frequency of no PHT was $67.8 \%$ (95\% Cl 66.2 to 69.5$)$, being higher in Popayan (78\%) than in Cali (64\%). No PHT was also positively associated with low education levels, lack of access to health system, no having tattoos and piercings, no history of STIs, no history of anal sex, low condom use, and low HIV knowledge. CONCLUSION: Data from this population reveals a low percentage of previous HIV testing; and social, health system, and knowledge factors as main determinants of it. Free and accessible voluntary HIV testing implemented either by health providers or by the community should be enforced in Colombia. 


\section{Methodological Advances and Mathematical Models \\ Démarches méthodologiques avancées et modèles mathématiques}

\section{P186}

HIV TESTING STRATEGIES FOR IMPROVED TREATMENT AS PREVENTION OUTCOMES

Langille, Sarah ${ }^{1}$; Vasarhelyi, Krisztina ${ }^{1,2}$; Rutherford, Alexander²; Barrios, Rolando ${ }^{1}$; Gustafson, Reka ${ }^{1}$; Thumath, Meaghan ${ }^{1}$;

Tran, Lynn ${ }^{1}$; McPherson, Kendraa ; Nathoo, Afshan ${ }^{1}$;

Guillemi, Silvia ${ }^{1}$; Montaner, Julio ${ }^{1}$

${ }^{1}$ Vancouver; ${ }^{2}$ Burnaby, BC

BACKGROUND: Success of Treatment as Prevention (TasP) is contingent on early HIV diagnosis. Traditionally, routine testing, featuring low per-test cost and high population reach, has been recommended for highprevalence epidemics. Risk-based strategies, with higher diagnostic yield, are primarily employed in low-prevalence settings.

METHODS: In consultation with the Vancouver TasP pilot project (STOP HIV/AIDS) team, we developed an HIV transmission model coupled to a system dynamics model of the continuum of HIV care. We incrementally realigned existing resources between risk-based outreach testing and routine testing in acute care. Testing data were obtained from Vancouver Coastal Health/Providence Health Care and various STOP HIV/AIDS reports. We considered a conservative range of assumed cost ratios for outreach and routine testing of 1:1 to 7:1 (recent UK maximum estimate: 16:1). Treatment coverage, linkage and retention rates were fixed at published values.

RESULTS: The table shows five-year cumulative HIV incidence for resource allocation scenarios to outreach and routine acute care testing. Cost ratios higher than 1.26:1 increasingly favored routine testing as additional tests conducted compensated for the lower diagnostic yield. At the highest cost ratio, 11 new infections were averted over five years exclusively through realignment of testing resources.

CONCLUSIONS: We found that routine HIV testing - within a comprehensive testing strategy - can produce TasP benefits through reducing HIV incidence. Further gains are expected by optimizing testing with other components of the continuum of care. Populations served by riskbased and routine methods are partially overlapping but different, so combining approaches may extend reach for the overall strategy.

New HIV Infections Over 5 Years

\begin{tabular}{ccccccc}
\hline & & \multicolumn{5}{c}{ Relative Cost Ratio } \\
& & $1: 1$ & $1.26: 1$ & $3: 1$ & $5: 1$ & $7: 1$ \\
\cline { 3 - 7 } & & 1139 & 1140 & 1141 & 1142 & 1142 \\
\hline Distribution of & 0\% Acute & 1139 & 1140 & 1140 & 1140 & 1139 \\
Resources & $25 \%$ Acute & 1140 & 1140 & 1139 & 1138 & 1136 \\
& $50 \%$ Acute & 1140 & 1140 & 1138 & 1136 & 1134 \\
& $75 \%$ Acute & 1140 & 1140 & 1137 & 1134 & 1131 \\
\hline
\end{tabular}

\section{P187}

PARTNERSHIP CHARACTERISTICS, SEXUAL NETWORKS AND UNPROTECTED ANAL INTERCOURSE (UAI) AMONG MEN WHO HAVE SEX WITH MEN (MSM) IN SHANGHAI, CHINA: AN EVENT-SPECIFIC ANALYSIS OF A DAILY WEBADMINISTERED DIARY

Steele (nee Taleski), Sarah J ${ }^{1}$; Myers, Ted ${ }^{1}$; Ning, Zhen ${ }^{2}$;

Allman, Dan'; Moravan, Veronika'; Calzavara, Liviana';

Kang, Laiyi ${ }^{2}$

${ }^{1}$ Toronto, ON; ${ }^{2}$ Shanghai, China

OBJECTIVE: To utilise an event-specific analysis to explore variables associated with UAI among MSM.
METHODS: An interviewer-administered quantitative questionnaire collected information about socio-demographics, sexual history, social networks, masculinity and homophobia. Longitudinal data collection followed via a quantitative daily web-diary. Logistic regression models with generalised estimating equations were used to explore event-specific variables associated with UAI.

RESULTS: The 149 participants contributed 854 sexual events during the 28 days of follow-up. The median number of sexual events per participant was 4 (IR 2 to 6 ). The median age of participants was 27 years (IR 17 to 58 ) and $86 \%$ had at least some university or college education. Eighty-two per cent $(\mathrm{n}=122)$ identified as gay/homosexual and $17 \%$ $(n=26)$ as bisexual. Selected variables associated with UAI are shown in Table 1. In addition, as level of intoxication increased (10-point Likert scale) the odds of UAI decreased 0.69 (0.50 to 0.97) times. Some but not all variables related to mood (eg, stress) were associated with UAI.

CONCLUSIONS: This event-specific analysis suggests partner-type may be less important than other partnership characteristics in determining some sexual risk behaviour. Length of time that a partner is known, in combination with communication within partnerships may be critical to understanding risk behaviour. This may have implications for measurement in epidemiologic and surveillance studies. Here, shared partners within sexual networks suggest potential for transmission of HIV and STIs and highlight areas for education and prevention. Event-specific HIV diary studies are rare, and this was the first in China. With an increasing Chinese-Canadian population, lessons learned may inform study design and HIV prevention in Canada. Future analyses will investigate how event-specific risk behaviour varies with individual characteristics.

Table 1: Partnership characteristics and sexual networks in an event-specific analysis of unprotected anal intercourse among men who have sex with men in Shanghai, China.

\begin{tabular}{|c|c|c|c|c|c|}
\hline & & & \multirow[b]{2}{*}{$\begin{array}{l}\text { Odds } \\
\text { ratio }\end{array}$} & \multicolumn{2}{|c|}{$\begin{array}{c}95 \% \text { Confidence } \\
\text { interval }\end{array}$} \\
\hline & & & & $\begin{array}{l}\text { Lower } \\
\text { limit }\end{array}$ & $\begin{array}{l}\text { Upper } \\
\text { limit }\end{array}$ \\
\hline \multirow{8}{*}{$\begin{array}{l}\text { Partnership } \\
\text { characteristics }\end{array}$} & Talk with partner about personal matters & No & & Ref & \\
\hline & during sexual encounter & Yes & 0.58 & 0.37 & 0.90 \\
\hline & How long partner known & Just met & & Ref & \\
\hline & & Hours & 0.96 & 0.36 & 2.62 \\
\hline & & Weeks & 4.08 & 1.11 & 15.01 \\
\hline & & $1-6$ months & 3.13 & 1.29 & 7.60 \\
\hline & & $7-12$ months & 5.05 & 1.74 & 14.67 \\
\hline & & $\geq 1$ year & 3.51 & 1.41 & 8.73 \\
\hline \multirow[t]{2}{*}{ Sexual networks } & Partner had sex with $\geq 1$ of the & No & & Ref & \\
\hline & $\begin{array}{l}\text { participant's other partners during the } \\
\text { previous } 6 \text { months }\end{array}$ & Yes & 4.08 & 1.56 & 10.74 \\
\hline
\end{tabular}

\section{P188}

THE IMPACT OF QUESTIONNAIRE ADMINISTRATION MODE ON MISSING AND INACCURATE RESPONSES: RESULTS FROM THE 2011 SRC-CANFAR NATIONAL HIV/ AIDS SURVEY

Jones, Marcella ${ }^{1}$; Calzavara, Liviana ${ }^{1}$; Worthington, Catherine ${ }^{1,2}$; Allman, Dan'; Tyndall, Mark ${ }^{1,3}$

${ }^{1}$ Toronto, ON; ${ }^{2}$ Victoria, BC; ${ }^{3}$ Ottawa, ON

BACKGROUND: Bias may result from missing data and the differing accuracy of responses between modes of questionnaire administration. This analysis explores associations between mode of questionnaire administration and missing and inaccurate responses to different question types in an HIV/AIDS survey.

METHODS: A survey of Canadians aged 16 years and older $(n=2139)$ was conducted in May 2011 to explore public attitudes, knowledge and perceptions of HIV/AIDS. Participants were recruited from all provinces and territories using a random-digit dial (including land-lines and cellphones) and could select to be interviewed on the telephone or selfcomplete online. This analysis excluded $16-17$ year olds $(n=54)$, because 
of low online completion $(n=2)$. A regression analysis was conducted, adjusting for relevant socio-demographics.

RESULTS: 1690 participants (81\%) completed the questionnaire online and $395(19 \%)$ completed by telephone. Telephone respondents were older, reported less education, had lower incomes, and were more likely from Quebec. Of the selected stigma-related and sensitive questions, significant differences in responses were observed for two of six questions: fear of people living with HIV/AIDS and number of sexual partners. After adjusting for socio-demographic differences by administration mode, only the sexual partner question remained statistically significant, with telephone respondents having $75 \%$ significantly lower odds of reporting more than one sexual partner $(\mathrm{OR}=0.266)$. Of the selected non-sensitive and sensitive questions, significant differences in missing data were observed for two of nine questions: most concerning illness and HIV testing. These differences remained after adjusting for socio-demographics, with telephone respondents having significantly lower odds of missing responses for most concerning illness $(\mathrm{OR}=0.333)$ and HIV testing $(\mathrm{OR}=0.115)$.

CONCLUSIONS: While there are significant socio-demographic differences in respondents by questionnaire administration mode, for most questions there does not appear to be a difference in responses by mode. This is important in light of the increasing use of online questionnaires to reduce costs and increase response rates.

\section{P189}

\section{USING RESPONDENT-DRIVEN SAMPLING TO ACCESS PEOPLE WHO USE DRUGS IN OTTAWA-GATINEAU: PRACTICAL AND ETHICAL CONSIDERATIONS}

Schreiber, Yoko S; Beckerleg, WeiWei; Lin, Dolly M; Lazarus, Lisa; White, Ashley; Ramsay, Tim; Tyndall, Mark W

Ottawa, ON

BACKGROUND: Respondent-driven sampling (RDS) is a method designed to identify study participants from vulnerable or hidden populations, and improve response rates and eliminate biases associated with more traditional sampling methods. RDS uses incentives to encourage participants to recruit others for the study. This process of compensating peers for successful referrals has raised concerns of coercion. We evaluated the practical and ethical aspects of RDS in a cross-sectional study on migration among people using drugs in Ottawa-Gatineau.

METHODS: Starting with four seed participants, each respondent was asked to recruit three peers. Participants received a $20 \$$ store card for survey completion, and $5 \$$ store cards for each successful referral. Participants completed an "ethics checklist" assessing their motivation to participate and recruit, and any experience of harm or coercion as a result of participating in the survey.

RESULTS: Nine additional seeds were selected to boost enrolment, allowing recruitment of 410 participants in a three month period. 294 participants trace back to one seed. Only $10.7 \%$ recruited the maximum of three peers, while $39 \%$ recruited no one. Referral compensation was collected by $61 \%$ of participants. No participant reported coercion or experience of harm by a peer. Compensation for survey completion, desire to help the community and being asked by a relative motivated $32.9 \%, 33.9 \%$ and $38.9 \%$ of participants to complete the survey, respectively. Compensation for referrals was the most frequently cited motivation to recruit $(34.9 \%)$, followed by helping peers access opportunity (29.2\%) and helping the community in the long-term (21.7\%).

CONCLUSION: In this study, RDS allowed recruitment of a representative number of participants considered to be from a hidden or marginalized population. Compensation for survey completion and referral was an important, yet not the only motivator for recruitment. There was no evidence that the RDS process led to undue risk among the study participants.
P190

RESPONDENT DRIVEN SAMPLING (RDS) AND SEED RECRUITMENT BY GRINDR IN A BRITISH COLUMBIA COHORT OF MEN WHO HAVE SEX WITH MEN (MSM)

Forrest, Jamie I' ${ }^{1}$ Lal, Allan ${ }^{1}$; Chan, Keith ${ }^{1}$; Jollimore, Jody ${ }^{1}$; Shurgold, Susan ${ }^{1}$; Roth, Eric A ${ }^{2}$; Michelow, Warren ${ }^{1}$;

Fisher Raymond, Henry ${ }^{3}$; Moore, David ${ }^{1}$; Hogg, Robert ${ }^{1,4}$

${ }^{1}$ Vancouver; ${ }^{2}$ Victoria, BC; ${ }^{3}$ San Francisco, California, USA;

${ }^{4}$ Burnaby, BC

BACKGROUND: The Momentum Health Study, a longitudinal biobehavioural cohort study among gay, bi and other men who have sex with men (MSM) uses respondent driven sampling (RDS) to recruit its sample. RDS seeds are recruited through various channels and in one method we advertised on GRINDR, a MSM-specific location-based mobile phone app. This study descriptively reports Momentum RDS recruitment to date and further explores characteristics of GRINDR-recruited seeds and their recruits.

METHODS: Descriptive statistics are used to report characteristics of the Momentum Health Study sample to date. Chi-square test was used to explore characteristics of seeds recruited from GRINDR and their recruits compared to participants recruited from all other methods. Other methods of recruitment included community agency referral and social media advertising.

RESULTS: Fifty-seven seeds were recruited, completed study procedures and were offered the opportunity to recruit others. 28/57 active seeds produced a sample of 203 total participants. The median age of the sample, including seeds, is 37 (interquartile range [IQR] 26 to 48) and the median number of sexual partners in the past six months is 5 (IQR 3 to 12 ). $76 \%$ of the sample identified as Caucasian, $7 \%$ Aboriginal and $17 \%$ all other ethnicities. $95 \%$ of all respondents had ever received a test for HIV. The median age of GRINDR-recruited seeds and their recruits was 31 (IQR 26 to 39) compared to 45 (IQR 32 to 50) for participants recruited from other methods $(p=0.001)$. There were no differences in the number sexual partners in the past six months between GRINDR-recruited seeds and their recruits compared to all other seeds and their recruits.

CONCLUSIONS: GRINDR is a potentially powerful tool for recruitment of MSM in research studies using RDS, which may help penetrate populations to produce recruitment chains not otherwise sampled by other methods of seed recruitment.

\section{P191}

\section{MULTICENTER EVALUATION OF THREE NOVEL 4TH GENERATION HIV AG/AB COMBO ASSAYS: ABBOTT ARCHITECT, ROCHE ELECSYS AND SIEMENS ADVIA CENTAUR}

Serhir, Bouchra ${ }^{1}$; Vincelette, Jean ${ }^{2}$; Frost, Eric ${ }^{3}$; Bergevin, Marco ${ }^{4}$; Béliveau, Claire²; Phaneuf, Denis²; Sanfaçon, Roger²;

Poirier, André ${ }^{5}$; Doualla-Bell, Florence ${ }^{1}$; Tremblay, Cécile ${ }^{1,2}$

${ }^{1}$ Sainte-Anne-de-Bellevue; ${ }^{2}$ Montréal; ${ }^{3}$ Sherbrooke; ${ }^{4}$ Laval;

${ }^{5}$ Trois-Rivières, QC

BACKGROUND: Currently, 24 out of 39 laboratories in Quebec use the AxSYM HIV Ag/Ab Combo for HIV screening. This kit will be discontinued December 2013 and three novel 4th generation screening HIV assays (Architect, Roche Elecsys HIV Combi and ADVIA CENTAUR), approved by Health Canada, represent the alternative to AxSYM.

OBJECTIVE AND METHODS: To investigate the performance of these three novel screening HIV Combo assays, in six clinical sites from Quebec. A total of 150 samples from patients with documented acute infection, a panel of 25 Seracare HIV-1 specimens, three quality control specimens (HIV-1,2 Ab POS, p24Ag POS, HIV-1,2 NEG), and 5577 sera from routine diagnostic patients were tested.

RESULTS: Sensitivity assessment- The three novel combos demonstrated comparable $100 \%$ sensitivity. Confirmed positive samples on the Architect and Roche Elecsys presented much greater S/CO values than AxSYM

Specificity assessment- Each novel combo was compared separately to the AxSYM. Discordant results were confirmed using supplemental 
confirmatory assays. All 3 kits presented a high specificity (from 99.53\% to $99.9 \%$ ) comparable to AxSYM Combo. Amongst the 66 specimens that were reactive with novel and AxSYM combos, 52 (79\%) were confirmed positive. All discordant results were confirmed negative. The 5494 specimens that were negative with both kits (novel and AxSYM) demonstrated lower S/CO values on Architect and Roche than on AxSYM:

AxSYM

result ( $\mathrm{S} / \mathrm{CO}$ ) Architect ( $\mathrm{n}=1$ site) Roche Elecsys HIV Combi ( $\mathrm{n}=3$ sites) Advia Centaur $X p(n=2$ sites)

\begin{tabular}{|c|c|c|c|c|c|c|c|c|c|c|c|}
\hline & Reactive & $\begin{array}{c}\text { Non } \\
\text { reactive }\end{array}$ & Total & Reactive & $\begin{array}{c}\text { Non } \\
\text { reactive }\end{array}$ & $\begin{array}{l}\text { Indeter- } \\
\text { minate }\end{array}$ & Total & Reactive & $\begin{array}{c}\text { Non } \\
\text { ereactive }\end{array}$ & $\begin{array}{l}\text { Indeter- } \\
\text { minate }\end{array}$ & Total \\
\hline Reactive & 3 & 1 & 4 & 41 & 7 & 0 & 48 & 12 & 4 & 0 & 16 \\
\hline Non reactive & 0 & 1095 & 1095 & 10 & 3222 & 2 & 3234 & 2 & 1177 & 1 & 1180 \\
\hline Total & 3 & 1096 & 1099 & 51 & 3229 & 2 & 3282 & 14 & 1196 & 1 & 1196 \\
\hline $\begin{array}{l}\text { Western } \\
\text { Blot con- } \\
\text { firmation }\end{array}$ & 2 & 0 & 2 & 38 & 0 & nd & 38 & 12 & 0 & nd & 12 \\
\hline Specificity & & $\begin{array}{l}99 \\
90 \%\end{array}$ & & $99,53 \%$ & & & $99,75 \%$ & & & & \\
\hline $\begin{array}{l}\text { Concordance } \\
\text { with } \\
\text { AXSYM }\end{array}$ & $99,90 \%$ & & & $99,42 \%$ & & & $99,41 \%$ & & & & \\
\hline
\end{tabular}

CONCLUSIONS: The three novel HIV Ag/Ab Combo demonstrated good performance (sensitivity, specificity and concordance) with better segregation of positive and negative samples than AxSYM. All three kits represent a good alternative to the AxSYM.

\section{P192}

\section{DEVELOPMENT OF A DATA PROCESSING PORTAL IN} SUPPORT OF TAGGED POOLED PYROSEQUENCINGBASED HIV DRUG RESISTANCE ANALYSIS

Ji, Hezhao'; Liang, Binhua'; Knox, Craig²; Eisner, Roman²; Petkau, Aaron ${ }^{1}$; Enns, Eric ${ }^{1}$; Van Domselaar, Gary ${ }^{1}$;

Sandstrom, Paul ${ }^{1}$; Brooks, James ${ }^{1}$

${ }^{1}$ Ottawa, ON; ${ }^{2}$ Edmonton, $A B$

BACKGROUND: Cost-effective and with enhanced sensitivity for minor variant detection, tagged pooled pyrosequencing (TPP) has been shown to be a promising new HIV drug resistance (DR) testing method. Although pyrosequencing technology is becoming more accessible to laboratories, bioinformatics challenges hamper the practical applications of TPP. Current challenges include: (1) the requirement for high performance computing to process large scale TPP data sets; (2) the requirement for intelligent, HIV-specific quality control / error management procedures for cleaning and filtering raw pyrosequencing data; (3) the automation of TPP data analysis; and (4) reporting of end-user friendly HIV DR results. Here we describe a user-friendly, TPP data processing web portal designed to facilitate low cost HIV DR testing, with minor variant detection, suitable for all HIV genotyping laboratories especially labs with limited bioinformatics support.

METHODS AND RESULTS: A custom developed pipeline was developed to carry out the multi-step data processing workflow, from raw TPP data input (in .sff or .fna format) through to the generation of customizable HIV DR reports. A set of TPP quality control and error management modules are incorporated in the pipeline for optimizing the accuracy of HIV DR variant detection. All of the pipeline components are customizable allowing the user to control the analysis via a web interface. Users are able to upload data remotely, tune analysis parameters, display results, and retrieve customized HIV DR reports and related data sets. Links to external databases and other HIV DR resources are integrated into the interface.

CONCLUSIONS: Next generation sequencing offers considerable promise for reducing the expense and effort of HIV DR testing but the complexities in data analysis and information management are impediments to adopting these new technologies. We describe the development of a sophisticated, easy-to-use web-based bioinformatics analysis pipeline that facilitates HIV DR detection by TPP. Data analysis, error-correction and formatting of reports occur with minimal user intervention. The pipeline should be useful to laboratories conducting HIV genotyping and researchers investigating low abundance HIV drug resistance variants.

\section{P193}

HIV-1 DISEASE PROGRESSION DURING HIGHLY ACTIVE ANTIRETROVIRAL THERAPY: AN APPLICATION USING POPULATION-LEVEL DATA IN BRITISH COLUMBIA: 1996-2011

Nosyk, Bohdan; Min, Jeong; Lima, Viviane D; Yip, Benita; Hogg, Robert S; Montaner, Julio S

Vancouver, BC

BACKGROUND: Accurately estimating rates of disease progression in HIV is of central importance in developing mathematical models used to project outcomes and guide resource allocation decisions. Our objective was to specify a multivariate regression model to estimate changes in disease progression among individuals on HAART in British Columbia, Canada, 1996 to 2011.

METHODS: We used population-level data on disease progression and antiretroviral treatment utilization from the BC HIV Drug Treatment Program. Disease progression was captured using longitudinal CD4 and plasma viral load testing data, linked with data on antiretroviral treatment. The study outcome was categorized into $\left\{C D 4\right.$ count $\geq 500$ cells $/ \mathrm{mm}^{3}$, 500 to 350 cells $/ \mathrm{mm}^{3}, 350$ to 200 cells $/ \mathrm{mm}^{3},<200$ cells $/ \mathrm{mm}^{3}$, and mortality\}. A five-state continuous time Markov model was used to estimate covariate-specific probabilities of CD4 progression, focusing on temporal changes during the study period.

RESULTS: A total of 210,083 CD4 measurements among 7421 individuals with HIV/AIDS were included in the study. Results of the multivariate model suggested current HAART at baseline, lower baseline CD4 $\left(<200\right.$ cells $\left./ \mathrm{mm}^{3}\right)$ and extended durations of elevated plasma viral load were associated with accelerated progression. Immunological improvement was accelerated significantly from 2004 onward, with $27 \%$ and $42 \%$ increases in the probability of CD4 improvement from the 4th CD4 stratum $\{C D 4<200\}$ in 2004 to 2008 and 2008 to 2011 , respectively.

CONCLUSION: Our results demonstrate the impact of innovations in antiretroviral treatment and treatment delivery at the population-level. These results can be used to estimate a transition probability matrix flexible to changes in the observed mix of clients in different clinical stages and treatment regimens over time.

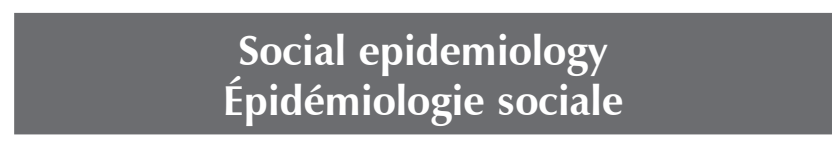

\section{P194}

THE RELATIONSHIP BETWEEN HIV-RELATED STIGMA AND HIV KNOWLEDGE IN AFRICAN, CARIBBEAN AND BLACK COMMUNITIES IN ONTARIO: REPORTS FROM THE KEEP IT ALIVE! (KIA) EVALUATION STUDY

Mbulaheni, Tola ${ }^{1}$; Husbands, Winston ${ }^{1}$; Shimeles, Helena ${ }^{1}$; Baidoobonso, Shamara ${ }^{2}$; George, Clemon ${ }^{1}$; Afzal, Arsalan'; Fenta, Haile ${ }^{1}$

${ }^{1}$ Toronto; ${ }^{2}$ London, ON

BACKGROUND: Keep It Alive! (KIA) was a social marketing campaign developed and implemented by the African and Caribbean Council on HIV/AIDS in Ontario from 2006-2009. The KIA evaluation study was implemented in 2009-2010, one of the objectives being to assess HIV-related attitudes, beliefs and knowledge of African, Caribbean and Black (ACB) communities.

METHODS: Survey participants responded to a self-administered questionnaire that included measures of HIV-related stigma and HIV knowledge. The scale measured participants' responses to statements related to stigma and knowledge. The stigma scale scored participants' responses from 1 (low stigma levels) to 8 (high stigma levels) and the HIV knowledge scale ranged from 1 (low) to 20 (high). 
RESULTS: The median stigma score (1) indicated low levels of stigma. However, $51.7 \%$ agreed that they would not share a cup previously used by someone living with HIV. While the overall median knowledge score (15) demonstrated high HIV knowledge, important gaps were also identified (e.g., 43.5\% of participants agreed that a person can get HIV from mosquitoes or other insect bites). High levels of stigma were significantly associated with low levels of HIV knowledge. Participants who were older, had higher levels of education, found the campaign's images appealing, and had tested for HIV in the past not only demonstrated lower stigma levels, but higher knowledge levels as well. Of note, less than $50 \%$ of participants reported that they would disclose an HIV diagnosis to extended family, religious leaders, sex partners or close friends. CONCLUSIONS: Better understandings of the relationship between HIV stigma and knowledge can inform more effective interventions. Exploring covert stigma experienced by PHAs and implementing HIV education campaigns targeting ACB youth may be beneficial. There is also a need for effective, culturally sensitive interventions addressing disclosure of HIV status.

\section{P195 \\ SOCIAL AND PROXIMATE PREDICTORS OF CONDON USE FREQUENCY AMONG AFRICAN, CARIBBEAN AND OTHER BLACK PEOPLE IN A SMALL CANADIAN CITY: RESULTS FROM THE BLACCH STUDY}

Baidoobonso, Shamara; Bauer, Greta; Speechley, Kathy N; Lawson, Erica; The BLACCH Study Team London, ON

BACKGROUND: Condoms are important biomedical tools for HIV prevention - they reduce the efficiency of HIV transmission during sexual intercourse. In Canada, condom use frequency among African, Caribbean and other Black people (ACBP) is under-researched, despite ACBP being a priority population for HIV prevention.

OBJECTIVES: To identify social determinants of health (SDOH) and proximate factors that predict condom use frequency, and proximate factors that mediate relationships between these $\mathrm{SDOH}$ and condom use frequency.

METHODS: Sexually active ACB adults $(n=126)$ in London, Ontario completed a self-administered questionnaire. The 2006 Census was used to weight the sample so that it reflected the local ACB population. Prevalence estimates were computed, and ordinal logistic regression was used to estimate prevalence odds ratios. This analysis included the following SDOH: sex/gender, poverty status, educational attainment, immigration experience, ethnicity and employment status.

RESULTS: In the past year, $58.9 \%$ of sexually active ACBP never used condoms during sexual intercourse, $20.6 \%$ used condoms sometimes, and $20.5 \%$ always used condoms. SDOH that were statistically significant predictors of condom use frequency in a multivariable model included: male sex $(p=0.0003)$, higher educational attainment $(p=0.0190)$, and unstable immigration class $(\mathrm{p}=0.0218)$. Proximate factors that were significant in the model and mediated the relationships between the $\mathrm{SDOH}$ and condom use frequency included: not having a cohabiting regular partner $(\mathrm{p}=0.0002)$, having a partner who did not want to use condoms $(\mathrm{p}=0.0024)$, history of forced or unwanted sex $(\mathrm{p}=0.0004)$, and lower number of lifetime sex partners $(\mathrm{p}=0.0030)$. Exposure to HIV prevention messages $(p=0.0775)$ and decreasing HIV knowledge score $(p=0.1362)$ approached significance but were not important mediators.

DISCUSSION: SDOH and partner-related factors were the strongest predictors of condom use frequency in the past year. Interventions to promote condom use among ACBP should target specific social groups identified by the $\mathrm{SDOH}$ and prioritize people with stable partners.
P196

SOCIAL AND PROXIMATE PREDICTORS OF PAST-YEAR HIV TESTING AMONG AFRICAN, CARIBBEAN AND OTHER BLACK PEOPLE IN AN URBAN-RURAL CANADIAN SETTING: RESULTS FROM THE BLACCH STUDY

Baidoobonso, Shamara; Bauer, Greta; Speechley, Kathy N; Lawson, Erica; The BLACCH Study Team

London, ON

BACKGROUND: HIV testing is important for primary and secondary HIV prevention. Although testing is promoted to African, Caribbean and other Black people (ACBP), a priority group for HIV prevention, it is estimated that many HIV-positive ACBP are unaware of their status. OBJECTIVES: To identify social determinants of health $(\mathrm{SDOH})$ that predict past-year HIV testing, explore if intersections between these SDOH impact past-year HIV testing, and identify proximate factors that mediate relationships between these SDOH and past-year HIV testing. METHODS: A convenience sample of 188 ACBP aged 18 and older in London, Ontario were recruited from 2010-2011. The sample was weighted to reflect the local ACB population, based on the 2006 Census. Population prevalences were estimated, and weighted prevalence ratios were calculated using modified Poisson regression. The SDOH explored were: sex/gender, poverty status, educational attainment, immigration experience, ethnicity and employment status.

RESULTS: An estimated 20.0\% of ACBP had tested for HIV in the past year. In a multivariable model, male sex $(\mathrm{p}=0.0145)$ was the only statistically significant SDOH predictor of past-year HIV testing. Predictors approaching significance included: not living in poverty $(\mathrm{p}=0.1479)$, stable immigration class $(p=0.1334)$, unstable employment $(p=0.0727)$, and the intersection between African ethnicity and not living in poverty $(p=0.1420)$. Lower number of lifetime sex partners $(p=0.0279)$, and increasing HIV knowledge $(\mathrm{p}=0.0002)$ were significant proximate predictors; and higher number of past-year sex partners approached significance $(p=0.1258)$. These proximate predictors mediated the relationships between past-year HIV testing and sex/gender, poverty status, ethnicity, and employment status.

DISCUSSION: ACBP were generally more likely to have tested for HIV in the past year than the broader Ontario population, but the frequency of past-year HIV testing among ACBP varied across groups. Interventions promoting yearly HIV testing among ACBP should target groups identified by the $\mathrm{SDOH}$ and proximate factors related to these $\mathrm{SDOH}$.

\section{P198}

THE CEDAR PROJECT: POLICE AND YOUNG ABORIGINAL PEOPLE WHO USE INJECTION AND NON-INJECTION DRUGS IN BRITISH COLUMBIA, CANADA

Pan, Stephen $W^{1}$; Christian, Chief $\mathbf{W}^{2}$; Pearce, Margo E ${ }^{1}$; Blair, Alden $\mathrm{H}^{1}$; Jongbloed, Kate ${ }^{1}$; Zhang, Hongbin ${ }^{1}$;

Teegee, Mary ${ }^{3}$; Thomas, Vicky ${ }^{4}$; Schechter, Martin Tㅜ; Spittal, Patricia $\mathbf{M}^{1}$; For the Cedar Project Partnership ${ }^{4}$ ${ }^{1}$ Vancouver; ${ }^{2}$ Enderby; ${ }^{3}$ Vanderhoof; ${ }^{4}$ Prince George, BC

BACKGROUND: Policing has profound health implications for people who use illicit drugs. Among Aboriginal communities, distrust of police is common, due in-part to legacies of colonial policing. In response to the paucity of research among Aboriginal people who use drugs, this study aims to: Describe the policing experiences of young Aboriginal people who use drugs and elucidate barriers to positive police relations.

METHODS: The Cedar Project is a cohort study involving young Aboriginal people in Vancouver and Prince George, British Columbia, who use illicit drugs. This mixed-methods study used period prevalence from 2007-2010 to describe policing experiences, mixed effects regression models to identify correlates of wanting a positive relation with police, and thematic qualitative analysis to assess attitudes about police relations.

RESULTS: Many participants were stopped by police (73\%), experienced police physical force (28\%), had drug equipment confiscated $(31 \%)$, and changed location of drug use because of police $(43 \%)$. 
Participants who reported dealing drugs $(40 \%)$ were significantly more likely to experience police engagement. Among participants in Prince George, $4 \%$ experienced non-consensual sex with members of the criminal justice system.

Due to personal experience, practical concerns, and intergenerational legacies of unfair policing practices, most participants did not want a positive relationship with police $(57 \%)$. Desire for a positive relationship with police was directly associated with being helped by police, and inversely associated with being stopped by police and experiencing physical police force.

CONCLUSION: Policing activities are may be impacting the wellbeing of Aboriginal people who use drugs. Due to focused prosecution of street-level drug dealing, some police may favor enforcement over harm reduction. Positive police engagement and less aggressive policing may enhance perceptions of police among young Aboriginal people who use drugs.

\section{P199 \\ PROGNOSTIC FACTORS ASSOCIATED WITH SHORT- TERM VIROLOGIC SUCCESS AMONG ILLICIT DRUG USERS INITIATING HIGHLY-ACTIVE ANTIRETROVIRAL THERAPY IN VANCOUVER, CANADA}

Le, Dan; Milloy, M-J; Wood, Evan; Simo, Annick; Guillemi, Silvia; Hogg, Robert; Montaner, Julio; Kerr, Thomas

Vancouver, BC

BACKGROUND: People who use illicit drugs (DU) continue to experience high rates of sub-optimal HIV treatment outcomes, including failure to suppress plasma HIV-1 RNA viral load ( $\mathrm{pVL}$ ) following initiation of highly-active antiretroviral therapy (HAART). Non-suppression of $\mathrm{pVL}$ is associated with disease progression as well as elevated risk of onward HIV transmission. We sought to identify factors that predicted short-term virologic suppression among illicit drug users (DU) starting HAART.

METHODS: We used data from the AIDS Care Cohort to evaluate Exposure to Survival Services (ACCESS) study, a long-running community-recruited cohort of HIV-seropositive DU linked to comprehensive HAART dispensation records and complete HIV clinical profiles. Our outcome of interest was the number of days in which plasma HIV-1 RNA $<50$ copies/mL within the 365 days following HAART initiation, dichotomized at the median. We identified clinical, behavioral and social factors in the period prior to HAART initiation associated with short-term pVL suppression using multivariate logistic regression.

RESULTS: From May 1996 to May 2012, 427 ART-naïve DU who initiated treatment were recruited for this analysis. The median number of days with suppressed pVL in the first year of treatment was 198 (interquartile range 0 to 308.) The proportion of individuals with more than 198 days of pVL suppression increased from $28 \%$ in 1996 to $73 \%$ in 2012 ( $\mathrm{p}<0.001$.) In a multivariate model, binge drug use had a strong negative association with $\mathrm{pVL}$ suppression (adjusted odds ratio 0.55; $95 \%$ CI 0.34 to $0.90, p=0.018$.)

CONCLUSION: Although rates of $\mathrm{pVL}$ suppression among DU initiating HAART have improved markedly in recent years, individuals reporting high intensity drug use were less likely to go on to achieve virologic control. These results reinforce the need to integrate addiction and HIV treatment programs to improve outcomes from HAART among DU.

\section{P200}

\section{DEVELOPMENT OF A SENSATION SEEKING SCALE FOR} USE AMONG VULNERABLE DRUG-USING POPULATIONS

Werb, Dan; Richardson, Chris; Wood, Evan; Kerr, Thomas

Vancouver, BC

OBJECTIVE: Injection drug use remains a primary risk factor for HIV transmission. To date, a variety of sociodemographic, drug-related, and structural factors associated with initiation of injecting have been identified. However, little is known regarding the impact of psychological factors, such as sensation seeking, on the risk of injecting initiation among street youth. We sought to adapt a measure of sensation seeking for use in a population of street youth and to assess the reliability, validity and predictive utility of this adapted sensation seeking scale (SSS) in predicting risky drug-related behaviours.

DESIGN: Data from street youth in the Vancouver-based At-Risk Youth Study were used to assess the adapted SSS. Exploratory factor analysis (EFA) using parallel analysis was undertaken to test scale essential unidimensionality. Confirmatory factor analysis (CFA) was undertaken to test scale internal validity using multiple methods. Predictive validity was tested by investigating the association between SSS score and injection drug use in a generalized estimating equation (GEE) model.

RESULTS: Overall, 226 street youth completed the SSS between October 2005 and May 2012. EFA results indicated that the SSS is essentially unidimensional. Reliability was moderate-to-good (Cronbach's alpha $=0.776)$. The results of the 1 -factor CFA were mixed with the comparative fit index $(\mathrm{CFI})$ suggesting an acceptable fit $(\mathrm{CFI}=0.914)$ and the RMSEA indicating moderate fit (RMSEA $=0.111[95 \% \mathrm{CI}$ 0.093 to 0.129$]$ ). In the GEE, sensation seeking was significantly associated with injection drug use (adjusted odds ratio [AOR] 1.04 [95\% CI 1.02 to 1.05$] ; \mathrm{p}<0.001$ ), corresponding to an AOR of 1.37 for SSS scores one standard deviation apart.

CONCLUSIONS: An adapted SSS for use among street youth may be reliable in predicting high-risk behaviours. Research should work to improve scale fit and reliability, in order to inform preventive interventions to reduce injecting and HIV transmission.

\section{P201}

\section{INCARCERATION LINKED TO USED NEEDLE LENDING AMONG INJECTION DRUG USERS WITH NON- SUPPRESSED PLASMA HIV-1 RNA VIRAL LOADS IN VANCOUVER, CANADA}

Milloy, M-J; Kerr, Thomas; Guillemi, Silvia; Hogg, Robert;

Montaner, Julio; Wood, Evan

Vancouver, BC

BACKGROUND: Informed by studies demonstrating the central role played by uncontrolled plasma HIV-1 RNA viral loads ( $\mathrm{pVL}$ ) on HIV transmission patterns, interventions to employ HIV treatment as prevention are underway. To optimize these efforts, evidence is needed to identify factors associated with both non-suppressed pVL and HIV risk behaviours. Thus, we sought to assess the possible role played by exposure to correctional facilities on pVL non-suppression and used syringe lending among HIV-seropositive active injection drug users (IDU).

METHODS: We used data from the AIDS Care Cohort to evaluate Exposure to Survival Services (ACCESS), a community-recruited prospective cohort of HIV-seropositive illicit drug users linked to comprehensive clinical monitoring data including regular $\mathrm{pVL}$ testing. We used longitudinal multivariate mixed-effects analyses to estimate the relationship between incarceration and plasma HIV-1 RNA viral load $>50 \mathrm{~mm} / \mathrm{mL}$ among antiretroviral therapy (ART)-exposed active IDU and, during periods of non-suppression, the relationship between incarceration and used syringe lending.

RESULTS: Between May 1996 and March 2012, 657 ART-exposed IDU were recruited and contributed 2923 person-years of follow-up. Incarceration was independently associated with higher odds of $\mathrm{pVL}$ non-suppression (adjusted odds ratio [AOR] 1.95, 95\% CI 1.44 to 2.63; $\mathrm{p}<0.001$ ). In a separate multivariate model restricted to periods of $\mathrm{pVL}$ non-suppression among active IDU, incarceration was independently associated with lending used syringes (AOR 2.20 [95\% CI 1.33 to 3.65]; $\mathrm{p}=0.002$ ).

CONCLUSION: In line with previous studies linking incarceration to non-adherence to ART, the current findings demonstrate how incarceration facilitates onward HIV transmission by increasing the odds of $\mathrm{pVL}$ non-suppression and syringe lending among HIV-positive IDU. In light of this, and given their refusal to implement effective prison-based harm reduction services for illicit drug-dependent individuals, Canadian correctional officials should urgently remove prison-associated barriers to optimal HIV treatment. 


\section{P202}

REDUCING HIV RISK OF WOMEN WHO USE DRUGS: HOW AND WHY WOMEN USE DRUGS DIFFERENTLY

White, Samantha; Bannerman, Molly; Francombe, Kate; Heath, Michele; Balian, Raffi; Millson, Peggy

Toronto, ON

BACKGROUND: To create more effective harm reduction research, policy and practice, drug use must be examined through a gender-based lens.

METHODS: The Gender and Drug Use Study (2012), a partnership between the University of Toronto and South Riverdale Community Health Centre (SRCHC) explored drug use patterns including prescribed and non-prescribed drugs, reasons for use, and gendered drug use practices, merging a quantitative research model with a community based participatory approach. Eight self-identifying drug users were hired and completed an extensive 8- session research training. They then conducted interviews with current drug users at SRCHC, 53 males and 49 females.

RESULTS: In preliminary analyses, women were significantly younger (mean 43.9 vs. $50, \mathrm{p}<0.01$ ), more likely to identify as a sexual minority ( $24.5 \%$ vs. $5.7 \%, \mathrm{p}<0.01)$ and to report government assistance as their primary income $(83.7 \%$ vs. $71.7 \%, \mathrm{p}<0.05)$. Alcohol, benzodiazepines, crack-cocaine, powder cocaine, codeine, oxycodone, sleeping pills and marijuana were the most commonly used drugs overall, with significantly more women using alcohol, codeine and benzodiazepines in the previous six months $(\mathrm{p}<0.05)$. Women and men reported similar overdose histories. For benzodiazepines and oxycodone, more women reported popping/ eating pills ( $96.4 \%$ vs. $82.4 \%$ for benzodiazepines; $64.3 \%$ vs. $38.5 \%$ for oxycodone) and more men injecting ( $0 \%$ vs. $5.88 \%$ for benzodiazepines; $25.0 \%$ vs. $38.5 \%$ for oxycodone), though these differences were not statistically significant, likely due to the small number of users ( $n=46$ benzodiazepine users, $\mathrm{n}=54$ oxycodone users). More men compared to women also identified their primary mode of cocaine use as injecting, but again this was not statistically significant $(\mathrm{n}=48)$.

CONCLUSIONS: Women presented different patterns of drug use, including frequency, mode of use and drug of choice. Findings can be used to examine the existing ways that drug policy and harm reduction reflects the gender dynamics of drug use and better tailor harm reduction services based on gendered use, as well as to inform future research.

\section{P203}

\section{LESBIAN AND BISEXUAL WOMEN AND SEX WORK: HIV} VULNERABILITIES, DRUG USE PATTERNS AND VIOLENCE $\underline{\text { Lyons, Tara }}^{1}$; Duff, Putu${ }^{1}$; Feng, Cindy²; Kerr, Thomas ${ }^{1}$;

Shannon, Kate ${ }^{1}$

${ }^{1}$ Vancouver, BC; ${ }^{2}$ Saskatoon, SK

OBJECTIVES: Lesbian and bisexual women are often overlooked in sex work and HIV research. Understanding the extent of lesbian and bisexual women's drug use patterns and risks for violence is important given the established links between these factors and heightened vulnerabilities for HIV. Therefore, this study examined the correlates of lesbian or bisexual identify among women engaged in street and hidden off-street sex work in Vancouver, Canada.

METHODS: Analysis drew on baseline data from women (transgender inclusive) participating in an open prospective cohort study of indoor and outdoor sex workers [SWs] in Vancouver (AESHA: An Evaluation of Sex Workers Health Access). Bivariate and multivariable logistic regression were used to identify independent relationships between individual-, sex work environment- and structural-level variables and lesbian or bisexual identity.

RESULTS: Of the 510 women in our sample, 95 (18.6\%) identified as lesbian or bisexual. In multivariable analysis, non-injection drug use (adjusted odds ratio [AOR] 3.16 [95\% CI 1.59 to 6.30]), youth $\leq 24$ years of age (AOR 2.39 [95\% CI 1.22 to 4.65]), and client-perpetrated physical and/or sexual violence (AOR 1.88 [95\% CI 1.17 to 3.02]) remained independently associated with identifying as lesbian or bisexual, after adjusting for potential confounders.
DISCUSSION: The sizeable number of participants who identified as lesbian or bisexual in our study highlights a major gap in our understanding of SWs' sexual identity and the health needs of non-heterosexual women engaged in sex work. Given the established links between client violence, non-injection drug use and HIV vulnerabilities, particularly among youth, these findings suggest an urgent need for HIV interventions tailored to lesbian and bisexual girls and women working in sex work.

\section{P204}

PREVALENCE AND CORRELATES OF UNPROTECTED VAGINAL SEX AMONG HARDER-TO-REACH WOMEN LIVING WITH HIV IN BRITISH COLUMBIA

Salters, Kate $\mathrm{A}^{1}$; Chan, Keith ${ }^{1}$; Cescon, Angela ${ }^{1}$; Chen, Yalin ${ }^{1}$; Patterson, Sophie²; Montaner, Julio S1; Hogg, Robert ${ }^{1}$;

Kaida, Angela ${ }^{2}$

${ }^{1}$ Vancouver; ${ }^{2}$ Burnaby, BC

BACKGROUND: Sexual behaviour of HIV-positive individuals, women in particular, has become a source for public health, social and political dialogue. We sought to investigate the prevalence and correlates of vaginal sex without a condom among a sample of non-pregnant sexually active women living with HIV.

METHODS: The Longitudinal Investigation into Supportive and Ancillary health services (LISA) cohort is a prospective study of treatment and care experiences of harder-to-reach people living with HIV over the age of 19 in British Columbia who are accessing antiretroviral therapy (ART). Interviewer-administered surveys collected information on sociodemographic, behavioural, healthcare utilization, and social factors while clinical variables were obtained through linkages with the Drug Treatment Program at the BC Centre for Excellence in HIV/AIDS. The outcome variable of interest was vaginal sex without a condom (unprotected sex) within the past six months. Covariates of interest included socio-demographic, psychosocial, behavioural, structural, and HIV clinical variables. Logistic regression was used to generate adjusted estimates of associations between unprotected sex and covariates of interest.

RESULTS: Of the 135 included women, median age was 40 (IQR 34 to 44), 62 (45.9\%) identified as Aboriginal, 44 (32.6\%) were currently in a relationship, and 77 (57.9\%) reported current use of illicit drugs. Overall, $71(52.6 \%)$ reported engaging in unprotected sex in the past 6 months with an median of 1 sexual partner (IQR 1 to 3 ). Women in a relationship (AOR 2.73 [95\% CI 1.20 to 6.22]), currently on ART (AOR 2.39 [95\% CI 1.02 to 5.59]) and those reporting being less likely to use a condom with an HIV-positive sexual partner (AOR 3.42 [95\% CI 1.50 to 7.78]) were more likely to engage in unprotected sex. Women who reported fearing they would face a hostile reaction from their sexual partner if they disclosed their HIV-status (AOR 5.35 [95\% CI 1.24 to 23.150]) were more likely to engage in unprotected sex. No associations with age, ethnicity, or injection drug use were observed.

CONCLUSION: Patterns of unprotected sex are greatly influenced by ART use, relationship status and perceived barriers to disclosing one's HIV-status. Social and structural inequality must be included in discussions of condom use.

\section{P205}

HISTORICAL RACIALIZED POLICIES INDEPENDENTLY CORRELATED WITH POLICE HARASSMENT OF INDIGENOUS SEX WORKERS: THE SOCIO-STRUCTURAL CONTEXT OF HIV RISK IN URBAN CANADA

Bingham, Brittany; Duff, Putu; Nguyen, Paul; Montaner, Julio;

Shannon, Kate

Vancouver, BC

BACKGROUND: Indigenous women in Canada remain highly overrepresented among new HIV infections and the most marginalized, visible aspect of the sex industry, and yet little is known the everyday violence and policing of Indigenous sex workers or how it relates to history of racialized polices. This study aimed to examine the prevalence and correlates of experiences of police harassment among Aboriginal sex workers. 
METHODS: Longitudinal analyses (2010-2012) draws on prospective cohort of street and off-street sex workers known as An Evaluation of Sex Workers Health Access (AESHA), in partnership with community organizations. Women (transgender inclusive) were recruited through outreach to street and hidden off-street venues (e.g. indoor, self-advertising) and participated in 6monthly interview questionnaires and confidential HIV/STI testing. Bivariate and multivariate logistic regression using generalized estimating equations (GEE) were used to examine independent correlates of experiencing police harassment without arrest among Indigenous sex workers.

RESULTS: Of a total 510, 202 (40\%) reported being of Aboriginal ancestry, of whom $45 \%$ experienced police harassment without arrest in the last 6 months, including threatened, detained against will, physically assaulted, property confiscated, and physically displaced. In multivariable GEE, having had a family member attend residential school (AOR 1.61 [95\% CI 1.09 to 2.40) and non-injection drug use (AOR 2.54, [95\% CI 1.20 to 5.38]) remained independently correlated with police harassment, while soliciting clients in off-street venues were correlated with reduced odds as compared to public spaces (AOR 0.23 [95\% CI 0.07 to 0.76 ).

CONCLUSIONS: Our findings suggest that criminalized sex work legislation gives police broad latitude to threaten or harass sex workers, and disproportionately affects Indigenous women and residential school survivors. This study underscores the critical need to examine Aboriginal SWs' HIV risk within a historical and ongoing structural violence lens. Policy reforms, including removal of criminal sanctions, and dialogue between Aboriginal communities, police and judicial system, remain necessary to an effective HIV response.

\section{P206}

PERCEIVED STIGMA AND DISCLOSURE OF HIV STATUS: THEIR ASSOCIATION WITH CLINICAL AND COMMUNICATION OUTCOMES IN THE WELTEL KENYA1 SMS RANDOMIZED CONTROLLED TRIAL

Memetovic, Jasmina ${ }^{1}$; van der Kop, Mia L'; Karanja, Sarah ${ }^{2}$; Kimani, Joshua ${ }^{2}$; Ngugi, Elizabeth N2${ }^{2}$; Ritvo, Paul3;

Lester, Richard $\mathrm{T}^{1,2}$

${ }^{1}$ Vancouver, BC; ${ }^{2}$ Nairobi, Kenya; ${ }^{3}$ York, ON

INTRODUCTION: Perceived stigma of HIV and disclosure status have been demonstrated to correlate with treatment management and psychosocial outcomes. The goal of this analysis is to examine the associations between perceived stigma and disclosure with treatment and communication outcomes, as well as their interaction with gender.

METHODS: In the WelTel Kenya1 trial, patients initiating antiretroviral therapy were randomized to either a short message service (SMS) interactive intervention or standard care $(\mathrm{n}=538)$. Clinical outcomes included self-reported ART adherence and plasma HIV-1 viral RNA load suppression $(<400$ copies $/ \mathrm{mL})$ at 12 months. At each follow-up visit, participants reported on their experience about using a mobile phone to communicate with health care providers, their perceived stigma of being HIV positive (ten-point scale recoded into "low", "moderate" and "high"), and the total number of people to whom status was disclosed. Univariate and multivariate analyses were conducted to examine associations between stigma and disclosure with sociodemographic data, communication and perceived care, and clinical outcomes using PASW 20.0.

RESULTS: The level of disclosure at 12 months was significantly associated with being female, married, and being unemployed. Perceived stigma at 12 months was associated with occupational status only. Participants with moderate levels of perceived stigma were less likely to feel comfortable discussing their treatment by phone compared to those with low stigma at baseline; this association was only significant in males (ARR 0.82 [95\% CI 0.74 to 0.91]; $\mathrm{p}<0.001$ ). Changes in perceived stigma over the course of the study was significantly associated with age, urban/rural residence and perceived quality of care at 12 months. Perceived stigma and disclosure was not significantly independently associated with the intervention vs. control arm, adherence, or viral load suppression. CONCLUSION: Perceived stigma and disclosure among HIV patients in Kenya are shown to correlate with sociodemographic characteristics and influence communication preferences with clinicians.
P207

INTERSECTING REPRODUCTIVE HEALTH AND HIV RISKS: CORRELATES OF UNINTENDED PREGNANCIES AMONG A COHORT OF YOUNG WOMEN SEX WORKERS WORKING IN BARS, TRUCK-STOPS AND LODGES IN POSTCONFLICT NORTHERN UGANDA

Duff, Putu ${ }^{1}$; Muldoon, Katherine $A^{1}$; Akello, Monika ${ }^{2}$; Simo, Annick ${ }^{1}$; Isiko, Sam ${ }^{2}$; Muzaaya, Godfrey ${ }^{2}$; Shannon, Kate ${ }^{1}$ ${ }^{1}$ Vancouver, BC; ${ }^{2}$ Gulu, Uganda

OBJECTIVES: More than two decades of conflict, displacement and deprivation in northern Uganda have created a large informal economic sector of exchanging sex for money in bars, truck-stops and lodges. Within post-conflict sub-Saharan African, little is known about the sexual, reproductive health and HIV risks of young women sex workers (SWs). This study was designed to examine the correlates of unintended pregnancies, a proxy for both reproductive health and HIV risks, among young women SWs in post-conflict northern Uganda.

METHODS: Data were drawn from the Gulu Sexual Health Study, a cross-sectional study of young women engaged in sex work. SWs were recruited using peer/sex work-led outreach to SW venues and community outreach to former internally displaced camps, in partnership with The AIDS Support Organization (TASO) and other Community Based Organizations. Bivariable and multivariable logistic regression was used to examine the prevalence and associations with ever having an unintended pregnancy.

RESULTS: Among 400 SWs (median age 20 years; IQR 19 to 25 years), $175(43.8 \%)$ reported at least one unintended pregnancy. Over one-third $(33.8 \%)$ were HIV sero-positive, with $46.8 \%$ having used hormonal or insertive contraceptives in their lifetime. In multivariable analysis, primarily serviced clients in lodges or brothels (adjusted odds ratio [AOR] 2.24 [95\% CI 1.03 to 4.84]), hormonal contraceptive usage (AOR 1.68 [95\% CI 1.11 to 2.59]) and substance use while working (AOR 1.64 [95\% CI 1.04 to 2.60]) were associated with increased odds of lifetime unintended pregnancy.

DISCUSSION/CONCLUSIONS: These findings highlight an urgent need for improved access to integrated reproductive health and HIV services, including family planning and non-judgmental sexual and reproductive health services catered to SWs in post-conflict settings. SW-led strategies, including peer-outreach, education, and harm reduction, should be considered, particularly for brothel/lodge-based SWs, together with structural strategies and education to reach brothel/lodge owners and managers.

\section{P208}

\section{DETERMINANTS OF RECENT SEXUAL PARTNERSHIP DISTRIBUTION AMONG VANCOUVER AREA MEN WHO HAVE SEX WITH MEN (MSM)}

Roth, Eric ${ }^{1}$; Michelow, Warren²; Forrest, Jamie ${ }^{2}$; Chan, Keith² Shurgold, Susan²; Rich, Ashleigh ${ }^{1}$; Schilder, Arn²; Moore, David ${ }^{2}$; Hogg, Robert ${ }^{2,3}$

${ }^{1}$ Victoria; ${ }^{2}$ Vancouver; ${ }^{3}$ Burnaby, BC

BACKGROUND: In $\mathrm{BC}$ in the past decade new HIV diagnoses have declined dramatically for all risk groups, except for MSM, where the numbers remain unchanged. Previous research indicates that having multiple sexual partners is strongly associated with HIV incidence and suggests large sexual networks may exacerbate the spread of drug-resistant HIV strains and other sexually transmitted infections. This study analyzed the distribution of MSM male sexual partners self-reported in the past six months and examined factors associated with this distribution. METHODS: The Momentum Health Study is a longitudinal biobehavioural cohort study among gay, bisexual and other MSM in the Greater Vancouver region. Participants were recruited using respondent-driven sampling and inclusion criteria required reporting same-sex sexual activity in the past six months. Multivariate analysis employed a negative binomial regression model with number of male sexual partners in the past six months as the continuously distributed dependent variable. 
RESULTS: There were 208 participants in the study. The distribution of sexual partners was highly positively skewed with a small number of respondents reporting a large number of sexual partners (total partners $=$ 3,172 , mean $=15.3$, median $=5$, standard deviation $=41.5$, range $=1$ to 500). Multivariable analysis revealed several independent factors strongly associated with larger number of partners including attending a group sex party (adjusted risk ratio $[A R R]=2.58$ ) and receiving money in exchange for sex (ARR=2.73). Scoring high on the Kalichman (1995) Sexual Sensation-Seeking Scale, using erectile dysfunction medication, and residing outside the City of Vancouver were marginally significant in the best-fitting model.

CONCLUSION: In this sample of Vancouver area MSM the distribution of recent sexual partners is highly positively skewed and strongly associated with sex party attendance and reporting sex for financial consideration. Further work will explore these factors in relation to the concepts of risk-seeking behaviour and positive deviance.

\section{P209 \\ EXPLORING HAART OPTIMISM AND SEXUAL ALTRUISM IN A COHORT OF MEN WHO HAVE SEX WITH MEN (MSM) IN VANCOUVER, BRITISH COLUMBIA}

Forrest, Jamie I' ${ }^{1}$ Rich, Ashleigh ${ }^{1}$; Chan, Keith ${ }^{1}$; Shurgold, Susan ${ }^{1}$; Roth, Eric A ${ }^{2}$; Michelow, Warren ${ }^{1}$; Schilder, Arn ${ }^{1}$;

Patterson, Thomas ${ }^{3}$; Moore, David ${ }^{1}{ }^{4}$; Hogg, Robert ${ }^{1,4}$;

Montaner, Julio; $\mathbf{S}^{1}$

${ }^{1}$ Vancouver; ${ }^{2}$ Victoria, BC; ${ }^{3}$ San Diego, California, USA;

${ }^{4}$ Burnaby, BC

BACKGROUND: Highly Active Antiretroviral Therapy (HAART) optimism and scepticism are important measures to understand how treatment innovations may result in riskier sexual practices. The Momentum Health Study, a bio-behavioural cohort study among gay, bi and other MSM in greater Vancouver, hypothesizes that HAART optimism and its antithetical measure, sexual altruism, may help explain why new HIV infections among MSM have not declined comparably to other at-risk populations in BC. This study aimed to characterize the association between these scales and explore factors independently associated with HAART optimism.

METHODS: One 14-item scale measures HAART optimism, while two scales measure sexual altruism: Personal (7 items) and Communal (6 items). Responses from 221 participants were included in this analysis. Pearson correlation coefficients were calculated for each pair of scales. Scale medians were used as cut-off points and multivariable logistic regression was used to determine factors independently associated with HAART optimism.

RESULTS: HAART Optimism is weakly correlated negatively with both sexual altruism - personal $(-0.23)$ and sexual altruism - communal $(-0.30)$, while the two sexual altruism scales are moderately correlated in the positive direction (0.76). HAART optimistic ( $>29$, median) MSM were significantly more likely to be $40+$ years of age (adjusted odds ratio [AOR] 3.42 [95\% CI] 1.40 to 8.33) and had heard of BC's STOP HIV/ AIDS program (AOR 2.99 [95\% CI 1.55 to 5.75]). HAART optimistic MSM were significantly less likely to have high sexual altruism - communal (>26, median) (AOR 0.39 [95\% CI] 0.21 to 0.71]). Differences in HAART optimism were observed across serostatus, number of sexual partners in past six months and number of HIV tests in the past two years; however, these factors were not selected in the final multivariate model. CONCLUSIONS: Our preliminary results show that HAART optimism and sexual altruism are important measures to consider in understanding HIV risk among MSM.
SOCIAL SCIENCES / SCIENCES SOCIALES

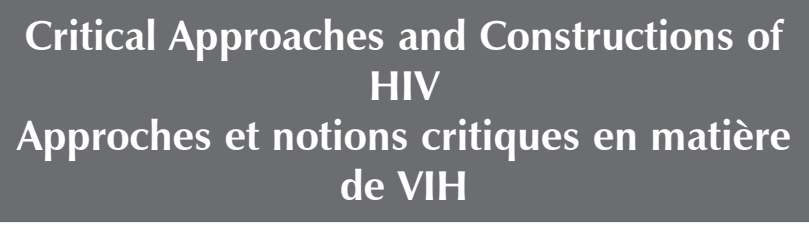

P210

THE RACIALIZATION OF DISEASE: A QUALITATIVE AND QUANTITATIVE ANALYSIS OF RACE AND ETHNICITY IN BIOMEDICAL RESEARCH

Small, Francine A

Montreal, QC

BACKGROUND: For several years there have been published guidelines covering the use of the term "race" and "ethnicity" in biomedical research. Because, when used inappropriately, the linkage of race or ethnic categories to cause and/or effect in biomedical research can have significant negative impacts on individuals and populations, biomedical research and genetic reject any strong biological (genetic) association/ difference, between/within races. This research will determine to what degree the recommendations for the use of "race" and "ethnicity" within biomedical publications has been followed.

METHODS: This research project retrieved publications from the Pub Med database. Papers were examined for their use of the terms "race" or "ethnicity" with regard to: title, journal, year of publication, the terms "race" or "ethnicity" in the title or abstract, the basis for assigning population to racial category, use of terms as independent or dependent variable, reasons for reference to the assigned or specific population, data source, disease by organ and finally was race defined, accounted for and which races were specifically mentioned within the articles.

RESULTS: The analysis of the publications when compared to the published recommendations revealed that the 205 surveyed papers not one defined race, and while still not defining race $2 / 3$ papers associated a medical outcome to race.

CONCLUSION: This research suggests that despite guidelines for the use of the terms "race" and "ethnicity" researchers and editors are neither using nor enforcing the use of them respectively. Further research should use alternative selection criteria and larger sample size to confirm these findings.

\section{P211}

\section{ASSESSING THE CONSTRUCT VALIDITY OF THE HIV DISABILITY QUESTIONNAIRE: A CONFIRMATORY FACTOR ANALYSIS}

$\mathrm{O}^{\prime}$ Brien, Kelly K ${ }^{1,2}$; Bayoumi, Ahmed $\mathbf{M}^{1}$; Solomon, Patricia $^{2}$

${ }^{1}$ Toronto; ${ }^{2}$ Hamilton, ON

BACKGROUND: The HIV Disability Questionnaire (HDQ) is a 69-item self-administered questionnaire that describes the presence, severity and episodic nature of disability experienced by people living with HIV (PHAs). We assessed the construct validity of the HDQ using confirmatory factor analysis.

METHODS: We recruited PHAs from hospital clinics, AIDS service organizations and a specialty hospital in Ontario and administered the HDQ followed by a demographic questionnaire. We hypothesized that domains in the HDQ represented six dimensions of disability, each represented by HDQ items: 1) physical symptoms and impairments (represented by 20 items); 2) cognitive symptoms and impairments (three items); 3) mental and emotional health symptoms and impairments (11 items); 4) uncertainty (14 items); 5) difficulties with day-to-day activities (nine items) and 6) challenges to social inclusion (12 items). We developed a measurement model to test these hypotheses using confirmatory factory analysis and used MPlus statistical software and maximum likelihood methods of estimation to determine model fit. We considered a 
threshold for the Root Mean Square Error of Approximation (RMSEA) of $<0.05$ as an indication of ideal overall model fit. We considered variables with factor loadings of $>0.30$ as representing a given domain of disability.

RESULTS: Of the 361 participants who completed the HDQ, $80 \%$ were men, $36 \%$ were 50 or older, $83 \%$ were taking antiretrovirals, and $77 \%$ reported living with at least two concurrent health conditions in addition to HIV. The overall goodness of fit measure was ideal, indicated by a RMSEA of 0.03 (95\% CI 0.028 to 0.033). All HDQ items represented our hypothesized dimensions of disability (factor loadings $>0.30$ ). Factor loadings ranged from 0.34 (item 2: 'I have diarrhea', loading on the physical dimension) to 0.90 (item 22: 'I have trouble thinking clearly', loading on the cognitive dimension). Dimensions of disability correlated with each other, ranging from $\mathrm{r}=0.51$ (between cognitive and uncertainty) to $\mathrm{r}=0.88$ (between mental-emotional health and social inclusion).

CONCLUSIONS: The six domain structure of the HDQ demonstrated construct validity when administered to PHAs living in Ontario.

\section{P212}

\section{HIV ORGANIZATIONS IN BRITISH COLUMBIA: STILL RELEVANT?}

\section{Medjuck, Melissa; Summers, Marcie; Barrett, Bronwyn} McIntyre, Catherine

Vancouver, BC

ISSUE: The climate of HIV social services under the current social service models in $\mathrm{BC}$ is changing. AIDS Service Organizations (ASO's) vary in their level of specificity of service. While some cater exclusively towards people living with HIV (PHAs), other organizations have mandates which include services to individuals living with Hepatitis $\mathrm{C}$, coinfection of Hepatitis $\mathrm{C}$, and other communicable diseases that have increased co-infection risks with HIV. Many organizations have gradually expanded programming to be more inclusive of co-infection issues and mono-infection of other non-HIV communicable diseases, mainly Hepatitis C. This change coincides with the gradual phasing out of the term AIDS in organizations' names. For many people, medical developments have made living with HIV more manageable than in the past and the rate of HIV developing into AIDS has declined with the introduction of HAART. Studies show co-infection with Hepatitis C and Tuberculosis present distinct challenges from mono-infection of either disease. With a changing medical reality, do ASO's need to change their model of service?

DESCRIPTION: Positive Women's Network (PWN) is a provincial organization providing a broad range of support and educational services to over 750 women living with HIV throughout British Columbia. Changes to service models will affect all aspects of service delivery, including implementing women-specific services. The authors conducted several interviews with ASO staff throughout BC on programming models.

LESSONS LEARNED: Concerns regarding a shift in service model include: Maintaining sufficient funding and changes to funding policies; increasing demands on staff; overstretching organizational capacity; impact on mono-infected PHAs and anti-stigma work unique to HIV.

NEXT STEPS: Research into which model is most appropriate (bloode-borne pathogens, communicable diseases, chronic diseases) is needed. Importantly, research into the needs of service consumers is needed as this change will have a huge impact on the health outcomes of PHAs in BC.

\section{P213}

3RD ONTARIO AFRICAN, CARIBBEAN \& BLACK RESEARCH THINK TANK - 2012: A STAKEHOLDER CONSULTATION TO SET PRIORITIES FOR HIV RESEARCH WITHIN AFRICAN, CARIBBEAN AND BLACK COMMUNITIES

Mbulaheni, Tola ${ }^{1}$; Tharao, Wangari ${ }^{1}$; Luyombya, Henry ${ }^{1}$;

Loemba, Hugues ${ }^{1,2}$; Karago-Odongo, Jane ${ }^{1,2}$;

Nleya-Ncube, Mercy ${ }^{1,3}$; Remis, Robert $\mathrm{S}^{1}$;

Baidoobonso, Shamara ${ }^{1,3}$; Pierre-Pierre, Valerie ${ }^{1}$;

Husbands, Winston ${ }^{1}$; Uddin, Zhaida ${ }^{2}$

${ }^{1}$ Toronto; ${ }^{2}$ Ottawa; ${ }^{3}$ London, ON

BACKGROUND: The 3rd Ontario African, Caribbean and Black (ACB) Research Think Tank was organized by the African and Caribbean Council on HIV/AIDS in Ontario in December 2012. The primary objective was to engage stakeholders in identifying research needs, interests and gaps for ACB communities and to set research priorities for the next 3-5 years.

METHODS: Current knowledge and past priorities were reviewed through epidemiologic, review and scientific track research synthesis presentations. These included an analysis of data from the OHTN Cohort Study and a review of research priorities from the 2009 Research Think Tank. Through a series of small group discussions, participants identified research priorities and dissected the issues and topics. Preliminary research planning was undertaken to consider potential research projects, partners, methodologies, timelines and other considerations.

RESULTS: The top six priority cross-cutting research themes were identified: 1) Best practices in HIV prevention programming; measuring effectiveness of intervention development/delivery, perceptions and implications of individual/community risk, role of culture. 2) HIV through life span; life span approach to clinical care research of PHAs, ACB youth transitioning to adult care, ageing with HIV, disclosure issues, motherhood, serodiscordant couples. 3) Policy analysis; impacts of changes to Immigration Act, criminalization of non-disclosure, Mental Health Act, Interim Federal Health. 4) Sexual behaviours; social epidemiology and qualitative exploratory questions, ACB youth, newcomers, MSM, influence of substance use. 5) Methodological issues; forms of 'knowledge', research design, mentorship, barriers to ACB participation in research. 6) Stigma; notions of stigma, violence and discrimination intersections.

CONCLUSIONS: The findings from this Research Think Tank will assist in revitalizing the ACB research agenda and strengthen an evidence-based response to HIV/AIDS in ACB communities. Systematic processes are being developed and implemented to ensure these priorities reflect the perspectives of stakeholders not in attendance, and to streamline the dissemination and uptake of the priorities.

\section{P214}

AM I INCLUDED? ENGAGING DIVERSITY WHEN IMPLEMENTING THE GREATER INVOLVEMENT OF PEOPLE WITH HIV/AIDS (GIPA) PRINCIPLES

\section{Michelow, Warren ${ }^{1,2}$; Whitbread, Jessica ${ }^{2}$; Sutton, Paul ${ }^{2}$}

${ }^{1}$ Vancouver, BC; ${ }^{2}$ Toronto, ON

BACKGROUND: The Greater Involvement of People with HIV/AIDS principles (GIPA) are important guiding principles for the AIDS movement, AIDS Service Organisations (ASOs) and research. Although first conceptualised in 1983 and subsequently formalised in 1994 at the Paris AIDS Summit, GIPA's principles are not universally implemented in the field and are arguably in need of updating for the emerging era of HIV as a chronic manageable illness. To this end, the CTAC recognizes GIPA as one of its Pillars of Treatment Access and consulted HIV-positive leaders nation-wide to assess the implementation of GIPA, its successes and failures.

METHODS: A full-day consultation with community members and high-level stakeholders from across Canada was held in Toronto in conjunction with CTAC's AGM and Council meetings. Community mapping, strengths and gaps analysis, and arts-based methods were used to 
elicit knowledge, opinions and experiences with GIPA successes, challenges and re-visioning. Activity products and discussion records were analysed inductively for common themes by CTAC staff and a professional researcher.

RESULTS: Sixteen leaders from the community as well as national, regional and local ASOs participated. 'Diversity and inclusion' was the key theme that emerged from the analysis. Social and physical location are fundamental in determining needs and access, yet people living with HIV/AIDS are frequently 'lumped' together in a way that erases centrally distinguishing and sometimes contradictory features informing their needs, capacities and locations. Implementing GIPA successfully is a difficult and ongoing process that can easily lead to inadvertent or even intentional exclusion and erasure. Tokenism is rife. Individual and community capacity building are key activities facilitating inclusion.

CONCLUSION: Organisational success, effectiveness and reach of programs and services, and fully attaining GIPA requires properly recognising and meaningfully engaging diversity in all areas of the HIV sector. GIPA principles can inform needed improvements in workplace and health systems.

\section{P215}

\section{COMMUNITY ENGAGEMENT AND OWNERSHIP IN THE DEVELOPMENT AND DESIGN OF PROUD, AN OTTAWA CBPR STUDY}

Lazarus, Lisa ${ }^{1}$; Marshall, Zack ${ }^{2}$; LeBlanc, Sean ${ }^{1}$; Shaw, Ashley ${ }^{1}$; Martin, Alana ${ }^{1}$; Dalton, Chris ${ }^{1}$; Proud Community Advisory Committee $^{1}$; White, Ashley ${ }^{3}$; Schreiber, Yoko ${ }^{1}$; Tyndall, Mark $W^{1}$ 1Ottawa, ON; ${ }^{2}$ St John's, NL; ${ }^{3}$ Hamilton, ON

BACKGROUND: The PROUD study is aimed at better understanding the HIV risk environment among people who use drugs in Ottawa, Ontario. This initiative incorporates community-based participatory research (CBPR) principles to actively engage a Community Advisory Committee (CAC) consisting of current and former drug users and their allies in all phases of a prospective cohort study, including the development of a quantitative survey tool.

METHODS: The research team recruited CAC members in collaboration with the Drug User Advocacy League, taking into consideration gender, ethnicity, age, drug use, HIV status, sex work experience, and francophone and Aboriginal perspectives. Eight people with lived experience, three allies and three ex-officio members were selected. The CAC met for 10 two-hour meetings from May-September 2012 to design the study and to develop the survey.

RESULTS: The first major activity of the committee was to develop the survey. The CAC selected research themes that they deemed important to their community including: drug use; harm reduction; health; sexual history; connections to community; housing; and law. Working groups of 2-3 members developed questions related to theme areas that most interested them. Questions were edited by the research team and the CAC, who suggested input on wording, flow of questions, and survey length. The CAC members, along with medical students, piloted the survey with 26 participants from the target population, who provided further feedback on the questionnaire.

CONCLUSION: This project provides a new opportunity to engage people who use drugs in community-based HIV research. By involving CAC members in every stage of survey development, academic and community partners are establishing an innovative framework that will inform all elements of this research project. This CBPR process will contribute new knowledge of successful approaches to the meaningful engagement and self-determination of people who use drugs and are most impacted by HIV infection.
P216

TAKE A PICTURE, IT WILL LAST LONGER: PEER-DRIVEN COMMUNITY ACTION THROUGH PHOTOGRAPHY

Nicholson, Valerie ${ }^{1}$; Wilson, Daniel; $\mathrm{G}^{2}$; Lamoureux, Robert ${ }^{2}$;

Hennan, Melvin'; ; Moors, Randy²; Bellrose, Lora²;

Parashar, Surita ${ }^{1,2}$

${ }^{1}$ Burnaby; ${ }^{2}$ Vancouver, BC

BACKGROUND: Until recently, the limited data capturing the impact of housing instability on the health of people living with HIV (PHA) in Vancouver, Canada, has been generated without meaningfully involving community; consequently, it does not reflect the experiences of affected populations. We sought out an ideal method to document and share our experiences as PHA who have experienced homelessness and unstable housing.

METHODS: Through funding from a CIHR Community Based Research (CBR) catalyst grant we were trained in the practice and ethics of CBR and Photovoice. Equipped with disposable cameras, we took photographs of our homes and neighbourhoods to illustrate our understanding of the relationship between HIV, health and housing. Through individual reflection and group discussion we developed narratives to accompany a selection of photos that we deemed to be representative of our community's strengths, capacities and needs in the realm of housing. We use our photos and stories to engage with policymakers and the general public. RESULTS: Between June and August 2011, we collectively took over 300 photographs, of which 50 were selected for display. To date we have organized three local photo exhibits, the first of which launched within 1 year of the project's start date. Members of our team have presented our work at local, national and international research conferences. Interest in our work has led to opportunities to present in different mediums, including video, print, radio and web-based interviews. Our events have attracted policymakers, service providers and members of the general public, many of whom are unfamiliar with the realities of living with HIV. Members of our own communities have also attended the exhibits and conveyed that the photos resonate with their realities.

CONCLUSIONS: We found Photovoice to be a dynamic and adaptable medium that allows for rapid knowledge exchange with diverse audiences. Through this project we have gained recognition of our ability to enact change, as well as a sense of accountability to our peers.

\section{P217}

\section{BRICK BY BRICK: BUILDING ON THE PRINCIPLES OF COMMUNITY-BASED RESEARCH}

Jones, Jennifer Evin ${ }^{1}$; Langlois, Andrea $\mathrm{M}^{1}$;

Worthington, Catherine ${ }^{2}$

${ }^{1}$ Vancouver; ${ }^{2}$ Victoria, BC

BACKGROUND: Community-based research (CBR) is rapidly gaining traction as the ethical and methodological benchmark for research on the social determinants of health related to HIV/AIDS. Much of the recent CBR literature focuses on how to increase community member participation and strengthen egalitarian-based partnerships. However, little research pays attention to the challenge of implementing the CBR principles of capacity-building and achieving tangible community benefits throughout the research process.

OBJECTIVE: In this analysis, we will use the case example of the development of the Positive Living, Positive Homes (PLPH) study (a prospective study on HIV and housing in British Columbia) to provide examples of issues in implementing CBR principles and concrete strategies for maximizing effective, multi-stakeholder partnerships that lead to tangible community benefits throughout the research process.

METHOD: Drawing upon documents from Phase I (catalyst) of the study (e.g., terms of reference, meeting minutes), and the report of an external evaluation conducted to assess the degree to which the study adhered to CBR principles in Phase I (participants included 21 community, policy, and academic team members), we, the study's community and academic lead partners, used a self-reflexive process to assess key challenges and supports for CBR community members to engage in the study.

RESULTS: Key themes and issues included: creating opportunities for team member training and capacity building, the challenges and benefits 
of consensus decision-making, increasing resource and time demands on community partners, building multi-stakeholder teams, and maintaining an action-oriented focus through all phases of a project.

CONCLUSIONS: Self-reflexivity is an important CBR principle to guide CBR practice and engage equitably with community in projects that have tangible community benefits. The results of this reflexive analysis will inform the PLPH study as it moves forward, and may also provide guidance to other HIV CBR studies as they are developed and implemented.

\section{P218}

\section{THE POLITICS AND PROCESS OF COMMUNITY- ACADEMIC RELATIONS IN COMMUNITY-BASED RESEARCH IN HIV: CASES FROM EMERGING RESEARCHERS}

Lachowsky, Nathan J1'; Baidoobonso, Shamara²; Coleman, Todd ${ }^{2}$; Marshall, Zack ${ }^{3}$; Grace, Daniel6; Scheim, Ayden I²;

Worthington, Catherine ${ }^{4}$; Ibáñez-Carrasco, Francisco ${ }^{5}$

${ }^{1}$ Guelph; ${ }^{2}$ London, ON; ${ }^{3}$ St John's, NL; ${ }^{4}$ Victoria, BC;

${ }^{5}$ Toronto, ON; ${ }^{6}$ Vancouver, BC

Community-based research (CBR) is championed as a best practice to meaningfully impact the HIV epidemic in Canada. Principles associated with CBR have been endorsed, adopted and integrated to varying degrees within research projects across the country, and have been shaped by discussions about the equitable involvement of specific communities (e.g., Greater Involvement of People Living with HIV/AIDS, Meaningful Involvement of People Living with HIV/AIDS and Ownership, Control, Access, and Possession). The objectives of our dialogical work are to convey potential scenarios in CBR for emerging HIV researchers from different disciplines who have divergent research foci and to highlight benefits and potential pitfalls in the practice of CBR. This work is based on peer consultation and de-briefing between six former CIHR University Without Walls Fellows. In our interdisciplinary dialogue, these threads are pulled together by the question of what the future of HIV CBR may resemble, particularly with regards to the experiences of emerging researchers, or researchers who are new to CBR.

This discussion provides an overview of CBR then delves into the politics of community-academic relations, particularly as experienced by emerging researchers, in a manner that honours CBR principles and sustains a professional future. If the inherent challenges and barriers to CBR are appropriately navigated then community members, community allies, health practitioners, academics, and emerging researchers may benefit from enriching experiences and produce research results that better impact the HIV epidemic in affected communities. However, several questions remain which we seek to elucidate, including: How will this type of research be funded in the future? What opportunities will exist if CBR goes increasingly mainstream? What other avenues exist for emerging HIV researchers?

\section{P219}

LOCALIZED PREVENTION: EXAMINING THE DISCURSIVE EFFECTS OF HISTORY AND CULTURE ON HIV AND GAY MEN IN NOVA SCOTIA FROM A COMMUNITY ACTIVIST PERSPECTIVE

\section{Numer, Matthew; Gahagan, Jacqueline}

Halifax, NS

INTRODUCTION: This presentation will examine how Nova Scotian culture influences the way HIV prevention aimed at gay men is designed and implemented from HIV community activists' perspectives. Geographical tools have been utilized by researchers and public health officials to track the incidence and prevalence of HIV, but seldom are the local culture and social conditions investigated to determine their impact on HIV prevention.

METHODOLOGY: This study employs an inductive exploratory approach to investigate the often unseen dimensions of HIV prevention among gay men. The 17 participants in this qualitative study were HIV/ AIDS activists currently living in Nova Scotia who have worked in the field for at least five years. This project employs a poststructural methodology to collect data through semi-structured one-on-one in-depth interviews. A critical discourse analysis is used to frame the findings of this research.

FINDINGS: HIV prevention efforts aimed at gay men are situated within the larger landscape of sexual subjectivity but are localized through experiences of social relations. These social relations have a historical context which is embodied by the broader gay community as well as the HIV activists who are primarily responsible for the design and implementation of prevention efforts. Findings indicate that participants frequently take into account the historical and social conditions which include religious influences, silencing of sex and sexuality, political restrictions on funding and more.

DISCUSSION: This research offers an analysis of the impacts of localized cultures on HIV prevention efforts through the experiences of community activists. These participants hold a unique place as both developers and recipients of HV messaging. Their experiences reveal the tensions in HIV prevention as there are often socially sanctioned public discourses which conflict with private practices. Exploring these dynamics contributes to the literature on how HIV prevention is experienced in the lives of gay men.

\section{P222}

LES MESSAGERS DE RUE; AMÉLIORER LA QUALITÉ DE VIE DES PERSONNES UTILISATRICES DE DROGUES PAR INJECTION ET INHALATION (UDII)

\section{Dubuc, Danièle}

Montreal, QC

Dans le but de prévenir la propagation des ITSS, les messagers diffusent de l'information en matière de prévention, réfèrent vers les ressources du quartier et distribuent du matériel.

La première équipe de messagers a débuté en mars 2010 et depuis d'autres organismes ont implanté le projet.

Les messagers de rue sont des pairs issus du milieu de consommation. Ils sont soutenus et encadrés pour effectuer des patrouilles rémunérées, dans lesquelles ils distribuent du matériel de protection et de prévention dans la rue. Le but est de réduire les comportements à risque de contracter les ITSS et d'améliorer la qualité de vie des personnes utilisatrices de drogues par injection et inhalation (UDII) des quartiers centraux de Montréal.

Les messagers bénéficient des formations : Sida 101, matériel d'injection à risques réduits et ramassage sécuritaire de seringues souillées, entre autres. Lorsqu'ils débutent les patrouilles, ils sont supervisés par un intervenant pour que les patrouilles se réalisent de façon sécuritaire.

Les personnes intéressées au projet sont rejointes par un affichage dans les différents organismes du quartier. Pour la sélection des candidats on priorise leur motivation ainsi que leur capacité à gérer leur consommation. Ils proviennent du milieu de la consommation, du travail du sexe et de l'itinérance.

Selon nos données la distribution de matériel d'injection et de protection a considérablement augmenté :

- 2010 : seringues 7378 / condoms 1238

- 2012 : seringues 25204/ condoms 12575

Le projet sert à diversifier et promouvoir les services existants (travail de rue, site d'échange de matériel) et à investir et explorer de nouveaux milieux de vie.

Par leur travail, les messagers sensibilisent leurs pairs sur l'importance de se protéger lors des relations sexuelles et de ne pas partager leur matériel de consommation. Ils agissent ainsi comme des agents multiplicateurs de comportements sécuritaires auprès de leurs pairs.

\section{P223}

\section{DOING INTERSECTIONALITY: RESEARCH TO ACTION}

Cameron, Ruth

Toronto, ON

BACKGROUND/OBJECTIVES: Studies incorporating intersectionality in HIV policy and the development of HIV-stigma related 
interventions have emphasized the need for practical applications of intersectionality. Intersectionality links inequities to historical and socioeconomic circumstances and identifies factors, concepts and perspectives particular to HIV/AIDS including vulnerability, risk, resilience, critical race theory, and $\mathrm{SDOH}$. Identifying how intersectionality can be practically applied to facilitate health equity will contribute to improve ASO services and address the dearth of evidence guiding providers on how to "do" intersectionality.

METHODS: A comprehensive literature review on intersectionality visa-vis health policy and HIV/AIDS summarizes two streams of current writing the subject: The first discusses the range of identified implications for health policy, health equity work and HIV/AIDS service delivery. The second suggests the qualitative approaches researchers might use to document intersectionality in service delivery and policy work. This data will form the deductive component of a pilot qualitative research study incorporating a thematic framework analysis of intersectionality-informed approaches to front-line ASO service delivery.

RESULTS: A preliminary scan of qualitative and mixed-methods intersectionality research related to health equity, HIV/AIDS and health policy was completed. The results highlighted that there is significantly more literature promoting the potential of intersectionality as a framework or theoretical approach, and calling for its practical application, than there is literature that discusses methods on how to do intersectionality effectively in policy, health-equity or service delivery settings. CONCLUSION: It is clear that more work on "doing intersectionality" is needed. Data from the literature review will inform application of intersectionality to front-line ASO service delivery. This consultation will support the goal of creating a practical intervention targeted to enhancing provider's skills in applying an intersectional perspective in their work, and could have significant impact on the front-line work of ASOs and implications for $\mathrm{KT}$ across applied research, policy and programmatic sectors.

\section{P224}

\section{PICTURE IT: A CONCEPTUAL FRAMEWORK MAPPING THE PATHWAYS BETWEEN HOUSING AND HEALTH}

\section{Parashar, Surita ${ }^{1}$; Wilson, Daniel $\mathrm{G}^{1}$; Nicholson, Valerie ${ }^{2}$} Lamoureux, Robert ${ }^{1}$; Hennan, Melvin ${ }^{1}$; Moors, Randy ${ }^{1}$;

Bellrose, Lora ${ }^{1}$

${ }^{1}$ Vancouver; ${ }^{2}$ Burnaby, BC

BACKGROUND: Until recently, the limited data capturing the impact of housing instability on the health of people living with HIV (PHA) in $\mathrm{BC}$ has been generated without meaningfully involving community; consequently, it does not reflect the experiences of affected populations. The way I see it: a photographic exploration of housing and health among people living with HIV in Vancouver is a Community Based Research initiative, which aims to develop an understanding of the housing-health nexus that is grounded in PHA's lived realities.

METHODS: This project adapts Photovoice, a research method used to assist people to reflect on their community's strengths, capacities and needs, engage with policymakers and encourage social change. Five men and four women were recruited through purposeful sampling at HIV/ AIDS Service Organizations and trained as Community Researchers (CR). CR generated and analyzed over 300 photographs, as well as transcripts of group and one-on-one discussions in order to identify emergent themes. In the preliminary phases of data analysis, CR identified key conceptual categories, which were subsequently mapped into a conceptual framework that hypothesized the mechanisms and pathways through which environment impacts health outcomes.

RESULTS: Four key categories emerged in the analysis. The proposed conceptual framework illustrates how individual-level characteristics[e.g. gender] interact with social and physical factors at the micro-environmental [e.g. dwelling] and meso-environmental levels [e.g. neighbourhood], all of which is influenced by the larger social, political and economic context [e.g. cost of living, eroding rental housing stock]. These interactions, in turn, impact intermediate and primary health outcomes at the individual and community level.

CONCLUSIONS: The determinants and impacts of housing instability amongst PHA in Vancouver occur within a complex multi-leveled system as modeled in this conceptual framework. This analysis is a first step towards building a comprehensive, community-informed conceptualization of the housing-health nexus as viewed through the eyes of people living with HIV in Vancouver, Canada.

\section{P225}

YOUNG EAGLES SOAR - ABORIGINAL YOUTH LEADERS IN COMMUNITY BASED RESEARCH

Konsmo, Erin $\mathrm{M}^{1}$; Danforth, Jessica ${ }^{1}$; Reading, Charlotte ${ }^{2}$;

Masching, Renee ${ }^{1}$; Robinson, Carrie'; McKay-McNabb, Kim ${ }^{3}$

${ }^{1}$ Toronto, ON; ${ }^{2}$ Victoria, BC; ${ }^{3}$ Regina, SK

BACKGROUND: "Young Eagles Soar" (YES) is a community based participatory research project lead by the National Aboriginal Youth Council on HIV and AIDS (NAYCHA). One goal of the National Aboriginal Youth Strategy on HIV and AIDS (NAYSHAC) was to increase community based research on HIV and AIDS in relation to Aboriginal youth for HIV and AIDS; and to support Aboriginal youth to be leaders in doing research, not just participants. YES was a direct way for NAYCHA to engage with that goal.

OBJECTIVES: Building on the implementation of NAYSHAC, the main objectives of the YES project were to: 1.) Facilitate stronger engagement of Aboriginal youth in HIV and AIDS research by involving Aboriginal youth in collaborative research relationships that build capacity and leadership; 2.) Initiate a short term research project that will serve as a 'hands on' opportunity to work through the research process and contribute to the improvement of a seminal youth peer education HIV resource; and 3.) Develop an HIV and AIDS program of research focused on Aboriginal youth education needs related to topics of prevention, screening, care, treatment and support.

METHODS: Using a participatory youth inquiry process that included working collaboratively as a National Indigenous Youth Council as well as returning to work in their home communities, NAYCHA examined the aforementioned research questions with the support of community based and academic Aboriginal researchers.

RESULTS: Two new questions arose for the need to develop an Indigenous youth research focus for HIV and AIDS: 1.) What role do relationships play in Indigenous youth lead research on HIV and AIDS; and 2.) What does youth empowerment look like within HIV and AIDS prevention, education, treatment and support.

CONCLUSION: NAYCHA looks forward to continuing the development of what specific research needs to be conducted with Aboriginal communities (rural and urban) in Canada, that is lead by Aboriginal youth themselves around peer education tools and how they are used by their peers to address HIV and AIDS.

\section{P226}

SHOULD THE MENTAL HEALTH NEEDS OF ADOLESCENTS BE CONSIDERED AS PART OF COMPREHENSIVE HIV PREVENTION PROGRAMS IN HIV ENDEMIC SETTINGS?

Davis, Jack; Nkala, Busisiwe; Dietrich, Janan; Kaida, Angela; Hogg, Robert; Gray, Glenda; Miller, Cari

Vancouver, BC

BACKGROUND: Adolescents and young adults (AYAs) are an important risk group accounting for almost half of the HIV prevalence worldwide. In South Africa, AYAs have been particularly affected by HIV, with many having lost one or more parent to HIV, others living with HIV, and many more at risk for transmission. We undertook this study to explore associations between a high depression score and risk behaviours among adolescents (aged 14 to 19 years) living in the urban South African community of Soweto.

METHODS: Study participants were recruited through Kganya Motsha Adolescent Centre (KMAC) and the Perinatal HIV Research Unit's Botsha Bophelo project. Eligibility criteria included age 14 to 19 years and living in Soweto. Targeted sampling was used to ensure representation from all 44 townships within Soweto, and to ensure age and gender balance was met. Logistic regression analysis modeled associations 
between independent variables and a depression score of 24 or more using the CES-D 9. Odds ratios (OR), hazard ratios (HR) and 95\% CIs were calculated.

RESULTS: In total, 827 individual adolescents (473 [57.2\%] young women and 354 young men) completed the depression scale and $287(34.7 \%)$ were found to have high depression scores. At the univariate level, we considered socio-demographic and risk associations including gender, type of housing, food security, sexual and physical violence, sexually active, self-reported STI, alcohol and drug use. When we considered all significant variables in the multivariate model, factors associated with depression included having an STI; (OR 2.66 [95\% CI 1.01 to 7.02]); being gay, bisexual, or MSM (OR 1.72 [95\% CI 1.01 to 2.92]); experiencing physical violence (OR 1.62 [95\% CI 1.20 to 2.20]); marijuana use ( $<1$ per month) (OR 1.61 [95\% CI 0.99 to 2.60]), and high food insecurity (OR 1.18 [95\% CI 1.08 to 1.30$]$ ).

CONCLUSIONS: Among adolescents in Soweto, there is a high prevalence of depression. Depression was associated with poverty markers and experiences of physical violence in addition to HIV risk factors including having an STI and being lesbian, gay or MSM. Depression was also associated with marijuana use, a finding postulated to be a potential coping mechanism for depression. Comprehensive HIV prevention programs for young people should consider depression as a potential barrier for risk reduction and provide treatment and counseling where necessary.

\section{HIV and Gender: Context, Experience and Response \\ Le VIH et le genre : contexte, expérience et intervention}

\section{P227}

\section{FACING FORWARD TO AGING WITH HIV - NEW POCKET GUIDE FOR WOMEN}

Madsen, Janet; Seatter, Erin; Desroches, Monique; Munro, Aretha Vancouver, BC

Positive Women's Network, (PWN - pwn.bc.ca) has been providing services to women with HIV in BC for over twenty years. As one of few organizations serving positive women exclusively, we are a leader within the HIV community. Programs and resources are developed in consultation with HIV+ members to meet the evolving needs of women with HIV.

Before the advances of antiretroviral therapy, HIV's outcomes were bleak. Death after diagnosis could come within weeks, or for the lucky, a few years. Thankfully, that has changed for those who have access to health care, treatment and support. Though HIV is still a serious disease, it can mean a life measured in decades, not months. Given this, women are more hopeful about their futures. But women want to know what the future might hold.

Consultation with positive women indicated a need for gender-specific resource information about HIV and aging. Interviews and discussion groups revealed that women want information about the physical and mental aspects of HIV and aging. This includes information on HIV progression, perimenopause and menopause, heart health, treatment, lipodystrophy, sexual well-being, violence against women and spiritual health.

The result is the development of a new print resource, The Pocket Guide on Aging for Women Living with HIV. This guide is for women who have been living with HIV for ten years or more; women with HIV going through perimenopause or who are post-menopausal; and women over 40 newly diagnosed with HIV. It will be distributed nationally through CATIE.ca.

Exciting developments in HIV treatment mean that women with HIV can live decades longer than they might have imagined. To support women in living with HIV as they age, The Pocket Guide on Aging offers much needed medical information and community support.

\section{P228}

ADVOCATING FOR WOMEN'S, TRANSWOMEN'S, AND GIRLS' HIV AND AIDS ISSUES: THE 2012 REPORT CARD FROM THE COALITION FOR A BLUEPRINT FOR ACTION ON WOMEN AND GIRLS AND HIV/AIDS

Stephenson, Leah $\mathrm{M}^{1}$; Gahagan, Jacqueline ${ }^{1,2}$; Medina, Claudia ${ }^{1}$; Margolese, Shari ${ }^{1}$; Muchenje, Marvelous ${ }^{1}$; Wertheimer, Sophie ${ }^{1}$; Price, Colleen ${ }^{1}$; Johnston, Christine ${ }^{1}$; Chu, Sandra K${ }^{1}$;

Skaling, Barby ${ }^{1,3}$; Binder, Louise D ${ }^{1}$;

Boulay-Coppens, Daniella R ${ }^{1,4}$; Frank, Peg1,5; Thomas, Kim ${ }^{1,6}$

${ }^{1}$ Toronto, ON; ${ }^{2}$ Halifax, NS; ${ }^{3}$ Prince George, BC; ${ }^{4}$ Montreal, QC;

${ }^{5}$ Victoria, BC; ${ }^{6}$ Ottawa, ON

DESCRIPTION: The Coalition for a Blueprint for Action on Women and Girls and HIV/AIDS (Blueprint) was founded in 2005 as a multisector collaborative of HIV-positive women and diverse Canadian HIV organizations and individuals, advocating for better prevention, services and supports for women, transwomen and girls infected and affected by HIV and AIDS. Coordinated by a National Steering Committee (NSC) of over a dozen volunteers, the Blueprint has updated and presented at every International AIDS Conference (IAC) since 2006 their Manifesto and national Report Card tools. These efforts have included a number of international partners (Zimbabwe and Nicaragua in 2008 and Norway in 2010) in shaping the global response to HIV and AIDS for, by and with women, transwomen and girls.

FINDINGS: Using an extensive bilingual survey disseminated to over 250 key informants across Canada (34.4\% response rate), including specific outreach to francophone women, remote women, young women, girls and transwomen, the 2012 Canadian Report Card was developed. It was launched through a media/social media campaign and a Women's Networking Zone (WNZ) event in July 2012 at Washington D.C.'s IAC. Six national media hits resulted, including CTV and CBC television and radio, plus public engagement via social media.

CONCLUSIONS: A variety of challenges have been observed throughout the Blueprint's evolution including the length of the survey (due to the Blueprint Manifesto's comprehensiveness), reaching francophone women, remote women, young women, girls and transwomen, resourcing advocacy initiatives in the current environment, structuring and resourcing a network of volunteers, and visibility within the WNZ. In addition, numerous lessons learned/opportunities include significant survey participation despite length, earlier engagement of francophone women, remote women, young women, girls and transwomen, broader, decentralized, participatory knowledge transfer exchange (KTE) of Blueprint tools, funding these activities, organizational renewal, and earlier, re-invigorated engagement in WNZ development.

NEXT STEPS: The key next step is the formation of a KTE working group and engaging women from diverse backgrounds to develop recommendations for the NSC on many of the identified opportunities.

\section{P229}

\section{EXPERIENCE TALKS, RESILIENCE SHAPES - REVISITING HISTORIC TRAUMA: IMPACT ON TREATMENT IN ABORIGINAL MALES LIVING WITH HIV/AIDS IN BRITISH COLUMBIA}

Chongo, Meck

Prince George, BC

INTRODUCTION: HIV incidence, prevalence and mortality rates are over-represented in the Aboriginal population. HIV eradication is not yet possible therefore persons must take highly active anti-retroviral therapy (HAART) regularly. However, many Aboriginal males do not adhere to therapy partly because they still have unresolved trauma secondary to the residential schools legacy. Most research on HIV/AIDS therapy has focused on supposedly maladaptive characteristics of Aboriginal males and less on their adaptive, life-maintaining characteristics or on how much historic trauma (HT) impacts one's choice to take up and adhere to therapy. HT in Aboriginal males living with HIV and AIDS therefore needs to be revisited to gain insight into why and how people make their choices, to explore areas of strength, and help inform policy. 
OBJECTIVES: 1) To explore the impact of HT on therapy in Aboriginal males living with HIV and AIDS in British Columbia (BC); 2) To explore the role of resilience in dealing with HT; and 3) To offer culturally-safe recommendations to better address HT, aimed at improving treatment-seeking behavior, adherence to therapy and reducing deaths due to HIV/AIDS.

METHODS: Aboriginal males from Vancouver, between the ages of 15 and 64, currently on HAART will be recruited through purposive sampling. Ethics approval will be sought from the University of Northern BC, and Tri-Council Policy Statement provisions followed. Qualitative interpretive description will be used, and a cultural safety lens applied, to explore participants' perspectives. The study will be explained and participant consent obtained. The services of a clinical counselor will be sought to help participants deal with painful emotions if they occur. Sixteen in-depth interviews and one focus group discussion will be conducted, transcribed and coded. Trustworthiness of data will be ensured and thematic analysis conducted enabling for comparisons between age groups, and between direct and indirect residential school survivors.

\section{P230 \\ SEXUAL HEALTH COMMUNICATION AMONG BLACK PARENTS AND CHILDREN: IMPLICATIONS FOR HIV PREVENTION AND HEALTH PROMOTION}

Davis, Antoinette ${ }^{1}$; Gahagan, Jacqueline ${ }^{1}$; George, Clemon $^{2}$; Lee-Foon, Nakia ${ }^{2}$; Hamilton-Hinch, Barb ${ }^{1}$; Patrick, Michelle ${ }^{1}$ ${ }^{1}$ Halifax, NS; ${ }^{2}$ Oshawa, ON

BACKGROUND: Black Canadian youth between the ages of 15 to 29 are at heightened risk for HIV and sexually transmitted infections, yet very few interventions exist to address these issues. The current study examined the role and experiences of Black parents in relation to sexual health communication with their children in two Canadian provinces. METHODS: This qualitative study used a community-based approach, including the engagement of community advisory committees, to conduct six focus group discussions $(n=26)$ in Nova Scotia (NS) and 17 key informants interviews ( $\mathrm{n}=10$ females, 7 males) in Ontario (ON) with parents, youth, health providers and educators. Discussions focused on issues of sex, sexual health, homosexuality and HIV and all interview and focus group transcripts were thematically coded and analyzed in Nvivo. RESULTS: In both NS and ON, gender had a significant impact on the quality and quantity of parents' sexual health discussion. In addition, in both provinces discussion of homosexuality was limited and when it did occur, it was focused on its association to HIV $(\mathrm{ON})$ and was acknowledged as another sexuality issue that children needed to be aware of (NS). In NS, the invisibility of HIV as a key health concern, fear and uncertainty as barriers to communication, the need for early sexual health communication, the effect of time constraints and technology, and the responsibility of community institutions also emerged as common themes. In $\mathrm{ON}$, fear of teenage pregnancy was both a hindrance and a focus of discussion and conversations were influenced by religious values. Finally, variations were observed in approaches to sexual health discussions amongst continental African, Caribbean Canadian, African Canadian, and Black Nova Scotian families.

CONCLUSION: Findings highlight the need for greater specificity in sexual health and HIV prevention education between Black parents and children. Additional consideration must also be given to non-schoolbased approaches to augment sexual health promotion and HIV prevention communication, including through community-engaged processes involving faith-based and youth-lead approaches.

\section{P231}

OPTIMIZING THE HEALTH OF ONTARIO WOMEN LIVING WITH HIV (WLWH) THROUGH KNOWLEDGE TRANSLATION AND EXCHANGE (KTE)

Morshed, Mubnii'; Tharao, Wangari'; Logie, Carmen²; Loutfy, Mona ${ }^{1}$

${ }^{1}$ Toronto, ON; ${ }^{2}$ Calgary, $\mathrm{AB}$

BACKGROUND: KTE in HIV research is a critical and participatory process that allows for decision makers and stakeholders to engage with timely and relevant data to impact future research, policy and practice. OBJECTIVE: To demonstrate the process of utilizing findings from a community based research (CBR) project to tailor effective and meaningful KTE strategies for diverse stakeholders, while simultaneously strengthening relationships and trust between researchers, service providers and HIV+ women to facilitate research participation.

METHODS: The WCBR project, "Involving Ontario HIV+ Women and their Service Providers in Determining their Research Needs and Priorities" utilized qualitative and quantitative methods to determine research needs and priorities of WLWH. Large amounts of data highlighting the needs and challenges facing WLWH was generated including: barriers to access due to $\mathrm{SDoH}$ and their intersection with multiple systems of oppression, research priorities to inform services and programs for better health outcomes, and strategies to support participation of WLWH as partners in research and practice.

RESULTS: Our KTE strategies effectively disseminate study findings and their translation into action among diverse audiences with increased transparency of the research process. They target five distinct but overlapping target audiences: service users, community leaders/advocates, service providers, researchers and policy makers. The activities for knowl edge to action are:

1) Convening regional knowledge mobilization, translation, and exchange meetings/fora bringing together all target audiences to discuss results from the study, and future steps in both research and practice

2) Development of a community report summarizing the CBR project disseminated both in print and electronically

3) Development of an educational film of the CBR process that captures the unique challenges and opportunities of involving Peer Research Assistants and WLWH throughout the study.

4) Manuscript publication in open access journals

CONCLUSIONS: Our KTE strategy utilizes multiple mediums, addresses barriers to knowledge uptake, and emphasizes the co-ownership of the research process. These strategies support the need for further engagement and research with WLWH, and mobilize marginalized communities to bridge the gap between HIV research and policy/practice.

\section{P232 \\ RHETORIC OR REALITY? BIOMEDICAL PREVENTION TOOLS AS A CATALYST FOR SEXUAL EMPOWERMENT}

\section{Patten, San}

Halifax, NS

BACKGROUND: There is great interest in biomedical prevention technologies which may empower individuals in sexual encounters to protect themselves, regardless of the actions and wishes of their sexual partners. An often touted promise of these technologies is that they will not only prevent HIV, but will also allow receptive partners to take control over their own sexual (and in some cases, reproductive) health decisions. However, these assumptions must be carefully tested to understand how these technologies make personal calculations of risk more complex and influence sexual autonomy.

METHODS: This critical analysis was undertaken by facilitating dialogue between experts in women's sexual health and HIV prevention and through literature review.

RESULTS: A noted limitation of the current dominant prevention approaches (e.g., condoms, abstinence, monogamy) is that they tend to be controlled by a male partner or are otherwise impacted by structural factors, such as gender inequality. Despite the rhetoric of empowerment offered by biomedical approaches, critical perspectives suggest that they may not be the panacea for women's equality and empowerment that they are purported to be. Important lessons can be learned from the implications of, and discourse around, reproductive technologies such as oral contraceptives. Like "the pill," NPTs may be a catalyst for long-term, broad structural change. However, this will only occur if female-con trolled interventions stimulate and are introduced within multi-level interventions addressing women's vulnerability around sexual autonomy, poverty, and reproduction. 
CONCLUSION: However promising, HIV prevention experts should not take for granted that biomedical prevention tools will necessarily directly translate into sexual empowerment for receptive partners. Critical social science helps to assess the gender implications of these technologies in terms of autonomy, covert use, partner negotiation and risk compensation, and to explore the social mechanisms by which the technologies could serve as a catalyst for broader structural changes in gender norms. True gender empowerment will only be achieved by acting at multiple levels of intervention: biomedical tools + dyadic sexual dynamics + structural equity.

\section{P233}

\section{INVOLVING HETEROSEXUAL MEN LIVING WITH THE HUMAN IMMUNODEFICIENCY VIRUS IN DEVELOPING A RESEARCH AND ADVOCACY AGENDA FOR THEIR COMMUNITY}

Pineau, David; Zuluaga, Jose; Tynan, Anne-Marie; Strike, Carol; Loutfy, Mona; Glazier, Richard H; Antoniou, Tony Toronto, ON

BACKGROUND: Heterosexual men living with HIV have been historically underrepresented in community-based research initiatives. Consequently, very little is known about the priorities of these men with respect to matters of research, programming and policy.

METHODS: A Summit for Heterosexual Men Living with HIV was planned by a collaboration of a Community Advisory Board comprised of four heterosexual men living with HIV and academic researchers. With the assistance of AIDS Service Organizations, twenty-five heterosexual men with HIV from across Ontario participated in the summit. The research team used the World Café approach to generate discussion of the following topics: priorities for research, programming, community advocacy and health care. The discussions were facilitated by the four Community Advisory Board members. Notes taken during World Café discussions and the large group debriefings underwent content analysis to identify the main domains of importance to participants.

RESULTS: Participants noted that their needs are often ignored by service providers and researchers. They identified several consequences of overlooking heterosexual men with HIV as a distinctive priority group, including a lack of power when funding for programs and research is at stake, lack of targeted programming, gaps in health care and stigma/low self-esteem. In addition, participants identified research priorities that could be grouped into the following domains: treatment of HIV and its complications, health and social support services utilization, social determinants of health, prevention, family planning, psychosocial research and barriers to research. The need to mobilize and challenge negative stereotypes imparted by the criminalization discourse was identified as being of critical importance. CONCLUSIONS: The Summit for Heterosexual Men Living with HIV identified several research and advocacy priorities specific to this group. Dissemination of these findings, expanding upon a group identity and translating the research priorities into fundable research projects were identified as important next steps by the participants.

\section{P235}

\section{TEA TIME AS PARTICIPATORY RESEARCH: MAPPING INFORMAL NETWORKS OF WOMEN LIVING WITH HIV} Whitbread, Jessica I

\section{Toronto, ON}

BACKGROUND: Across Canada it is estimated that women represent $25 \%$ of all people living with HIV (PLHIV). Despite the increase in these numbers, there is a lack of programs and services to address their unique health needs. As WLHIV continue to be even further marginalized, unsupported and separated from their peers, it has been challenging to determine how to move forward in the HIV response.

METHODS: The Tea Time method required WLHIV to be active participants in the organizing, promotion, and story sharing within the research process. Based in feminist and popular education frameworks, Tea Time shifts the power relations by enabling a collective learning experience as a means to create and explore community knowledge. This research asked:
How do community-based social support networks address the health needs of WLHIV? How can community-based research better engage WLHIV in ways that are respectful and useful to them? How can the Tea Time method facilitate research and network-building among WLHIV?

RESULTS: Thirty-seven women participated in eight tea parties in seven cities. Women's participation was represented through a teacup and letter to exchange that were photographed. Emerging from the research was the desire of WLHIV to build networks of much needed social support and to find innovative ways of connecting with peers. By having multiple avenues for connections, women were able to meet, maintain and foster relationships with other WLHIV, thereby increasing their access to peer support both locally and nationally.

CONCLUSION: Peer led, Tea Time was successful at creating space for participants to engage in critical dialogue about the issues that WLHIV face as a result of their HIV status. Tea Time was an active response to the increasing trend of eroding social programs as it aims to actively engage WLHIV in the process of rebuilding their social support networks.

\section{P236}

\section{GENDER DIFFERENCES IN HIV KNOWLEDGE, OPINIONS} AND ATTITUDES: RESULTS FROM THE 2011 SRC-CANFAR NATIONAL HIV/AIDS SURVEY

Calzavara, Liviana ${ }^{1}$; Montgomery, Robin ${ }^{1}$;

Worthington, Catherine ${ }^{2}$; Allman, Dan ${ }^{1}$; Tyndall, Mark ${ }^{3}$;

White, Samantha J'; Adrien, Alix ${ }^{4}$

${ }^{1}$ Toronto, ON; ${ }^{2}$ Victoria, BC; ${ }^{3}$ Ottawa, ON; ${ }^{4}$ Montreal, QC

BACKGROUND: HIV and AIDS in Canada: A National Survey explored trends in public attitudes, behaviours, knowledge and perceptions of HIV/AIDS. This analysis identifies gender differences in reported knowledge, attitudes, and perceptions of HIV.

METHODS: A survey of Canadians $(\mathrm{n}=2139) 16+$ years was conducted in May 2011. Participants were recruited from all provinces/territories using random-digit-dialing with the option to self-complete online or telephone-interview. Bivariate statistics identified significant differences $(\mathrm{p} \leq 0.05)$.

RESULTS: Participants included $\mathrm{n}=1103$ (52\%) women and $\mathrm{n}=1036(48 \%)$ men. Women were more likely than men $(\mathrm{p} \leq 0.05)$ to: describe themselves as "very knowledgeable" about HIV (28\%; 22\%); know a PHA who has died of AIDS (32\%; 27\%); perceive HIV as a 'somewhat/very serious' problem in Canada ( $82 \% ; 68 \%$ ); and less likely to say it was less of a problem today than 10 years ago $(14 \% ; 19 \%)$. No gender differences were found in HIV knowledge, with the exception that women were more aware of mother-to-child transmission via birth (85\%; $80 \%)$ and breastfeeding (39\%; 33\%). Major gender differences exist in attitudes toward PHAs. Women were more likely to say they were not afraid of PHAs ( $71 \%$; 63\%) and less likely to blame PHAs for getting infected. Women were more likely to say they were 'very comfortable' with family members/friends dating a PHA $(17 \% ; 14 \%)$, interacting with PHAs in a work context $(53 \% ; 45 \%)$ and public space $(47 \% ; 42 \%)$ but not with children attending school with HIV-positive students $(29 \%$; $28 \%)$. Women were more supportive of equal rights for PHAs $(93 \%$; $88 \%)$ and sexual rights (49\%; $41 \%)$, less aware of prosecution for nondisclosure $(83 \% ; 90 \%)$ and less supportive of imprisonment $(71 \% ; 78 \%)$ While more women reported charitable-giving/volunteerism, no gender differences were found in HIV-related giving/volunteerism - though women non-donors were more likely to consider doing so (52\%; 43\%). CONCLUSIONS: Despite no gender differences in overall HIV knowledge, significant differences were found across several HIV aspects, with women in general reporting greater awareness, concern and tolerance. 


\section{P237}

ESTABLISHING A COMMUNITY-BASED DEFINITION OF WOMEN-CENTRED CARE: A THEMATIC ANALYSIS FROM FOCUS GROUP DISCUSSIONS WITH WOMEN LIVING WITH HIV ACROSS CANADA

O'Brien, Nadia $^{1}$; Lewis, Johanna ${ }^{2}$; Carter, Allison; J J,4; Greene, Saara ${ }^{5}$; Bakombo, Dada ${ }^{1}$; Dayle, Janice ${ }^{1}$;

Kwaramba, Gladys ${ }^{2}$; Margolese, Shari²; Menard, Brigitte ${ }^{1}$;

Nicholson, Valerie J3 ; Smith, Stephanie'2; Rawson, Stephanie ${ }^{3}$;

Kaida, Angela ${ }^{3}$; de Pokomandy, Alexandra ${ }^{1}$; Loutfy, Mona $\mathbf{R}^{2}$;

Research Team, CHIWOS ${ }^{2}$

${ }^{1}$ Montreal, QC; ${ }^{2}$ Toronto, ON; ${ }^{3}$ Burnaby; ${ }^{4}$ Vancouver, BC;

${ }^{5}$ Hamilton, ON

BACKGROUND: Growing numbers of women living with HIV in Canada, and the gendered realities driving this trend, have evoked calls for tailored services that address women's specific needs. In response, the Canadian HIV Women's Sexual and Reproductive Health Cohort Study (CHIWOS) was created to study the health impacts of "women-centred care' (WCC). We explored definitions of WCC by women living with HIV towards developing a community-based definition of this emergent concept.

METHODS: Eleven focus group discussions (FGDs) were conducted with 77 women living with HIV in Quebec, Ontario and British Columbia (BC). FGDs were led by trained Peer Research Associates, audio recorded, and transcribed. Thematic analysis was conducted provincially, and themes were subsequently compared nationally.

RESULTS: Nationally, three themes regarding the definition of WCC emerged. These included care environments that: 1$)$ address social determinants of health (e.g., housing, employment, gender); 2) are cognisant of stigmatizing experiences and intersecting forms of oppression; and 3) encapsulate care for women's unique health needs (e.g., gynaecological health, reproduction, menopause). However, key features of WCC varied within and between provinces. In $\mathrm{BC}$ and Ontario, emphasis was placed on women's meaningful involvement in HIV care development and delivery through peer support and leadership. While in BC and Quebec, women prioritized the need for safe (physical and emotional) care environments. In Ontario, participants advocated for family-focused care. In Quebec, women highlighted the importance of long-term supportive services and permanent staff. Participants in each province expressed divergent perspectives on whether WCC must be delivered in womenonly spaces or exclusively by female healthcare providers.

CONCLUSION: Varying perspectives on the definition of WCC highlight the diversity of women with HIV in Canada. Care must therefore be flexible and adaptable to suit the needs of different communities and individuals. Nonetheless, women living with HIV identified strong commonalities, indicating that the shared reality of being a woman living with HIV in Canada is a sufficiently defining experience to warrant the development of a WCC approach to serve this growing population.

\section{P238}

\section{GENDER EXPECTATIONS LINK MASCULINITY AND HOMOPHOBIA AMONG MEN WHO HAVE SEX WITH MEN (TONGHZI) IN SHANGHAI, CHINA: AN ANALYSIS OF HOMOPHOBIA AND MASCULINITY SCALES}

Steele (nee Taleski), Sarah J1; Myers, Ted ${ }^{1}$; Allman, Dan' Ning, Zhen ${ }^{2}$; Moravan, Veronika ${ }^{1}$; Calzavara, Liviana ${ }^{1}$; Kang, Laiyi ${ }^{2}$ ${ }^{1}$ Toronto, ON; ${ }^{2}$ Shanghai, China

OBJECTIVE: To utilise factor analysis to explore homophobia and masculinity constructs among Tongzhi.

METHODS: An interviewer-administered quantitative questionnaire was used to collect information about socio-demographics, sexual behaviours, social networks, masculinity (21 items) and homophobia (two nine-item scales). Exploratory and confirmatory factor analyses (EFA and CFA) were conducted to determine and confirm factor structures prior to testing the theoretical measurement model.

RESULTS: The median age of the 817 participants was 26 years (IR 23 to 31$) .73 .3 \%(n=599)$ identified as gay/homosexual and $26 \%$ as bisexual $(n=210) .16 \% \quad(n=132)$ had ever been married to a woman. Among unmarried participants, $28 \%(\mathrm{n}=201)$ indicated they were likely/ very likely to get married. $16 \%(n=131)$ had at least one child. Of those without children $39 \%(n=270)$ indicated they were likely to have children. Preliminary EFA identified internalized (IH) and perceived communitylevel homophobia $(\mathrm{CH})$ as separate scales. EFA of the 9-item IH scale described an 8-item, three-factor solution: Unhappiness; Personal expectations; Weakness and shame. The 9-item $\mathrm{CH}$ scale identified two-factors: Views on normality; Perceptions of Tongzhi men. The 21-item masculinity scale resulted in a 13-item, three-factor solution: Alignment with traditional norms; External appearance; Social representations of masculinity. In individual CFA summary of fit indices were $>0.80$, suggesting adequate convergence for each scale.

The three scales were combined in a measurement model with a single secondary factor (Gender expectations). Summary of fit indices were $>0.80$ suggesting good model fit.

CONCLUSIONS: In this sample, analyses suggest gender expectations are shared components of both homophobia and masculinity, contributing to how these constructs are viewed in Chinese society. Further research is required to better understand the role of masculinity among Tonghzi and to investigate the association between gender expectations and sexual risk behaviour. In an increasingly diverse Canada, further understanding of how masculinity and homophobia intersect within the Chinese-Canadian context may inform HIV prevention programming.

\section{P239}

\section{THE SEX AND YOUTH PROJECT}

\section{Wilson, Ciann L ${ }^{1}$; Lee-Foon, Nakia $^{2}$}

${ }^{1}$ Brampton; ${ }^{2}$ Toronto, ON

INTRODUCTION: It is rare that KTE strategies place primacy on knowledge exchange between researchers and knowledge users in research; particularly in community-based research where the community are thought to already be contributors to the research process. Toward this end the Sex and YOUth (SY) project explores the use and importance of appropriate knowledge exchange strategies to promote sexual health discussions within African, Caribbean and Black (ACB) communities.

Funded by a CIHR KT supplement, the SY project led to the creation of a photo exhibit and four digital stories $(2 \mathrm{~min}$ to $5 \mathrm{~min}$ visual narratives that synthesize images, video, voice, music, and text to create compelling accounts of experience) from the findings of two community-based research studies in Toronto. The first study - "Let's Talk About Sex (LTAS)" collaborated with 15 young ACB women to explore the barriers and facilitators to making healthy sexual decisions through the use of interviews and photovoice. The second study - "The Black Parent-Youth Sexual Health Communication Study (BPYS)" interviewed 17 front line youth workers to explore how to best engage ACB parents and their youth in sexual health and HIV conversations.

METHODS: From June to August, 2012 four ACB youth (two males and two females) aged $15-20$ were selected through word of mouth and previous participation in the LTAS and BPYS studies to complete digital stories, images from which were also used for the photo-exhibit. The digital stories and exhibit were used as discussion starters at two CaféScientifiques also organized by the SY coordinators.

RESULTS: The digital stories and photo exhibit discussed parent-youth communication, religion, transactional sex, teen pregnancy, and healthy relationships. Both arts-based media were well received by attendees at the Cafés and audience members (who ranged in age) had much to contribute to these topics.

IMPLICATIONS: Creative KTE strategies can lead to interesting forms of triangulation of research findings; important reflections on taboo subjects such as sexual health and HIV in vulnerable populations; and uptake by knowledge users that can yield new research insight. 
P240

WOMEN-CENTRED CARE FOR WOMEN LIVING WITH HIV IN CANADA: TOWARDS MITIGATING LOSSES TO RETENTION IN THE CASCADE OF HIV CARE

Kaida, Angela ${ }^{1}$; Carter, Allison $\mathrm{J}^{1,2}$; O'Brien, $\mathrm{Nadia}^{3}$; Lewis, Johanna ${ }^{4}$; Nicholson, Valerie $\mathbf{J}^{2}$; Greene, Saara ${ }^{5}$; de Pokomandy, Alexandra ${ }^{3}$; Loutfy, Mona $\mathrm{R}^{4}$; Canadian HIV women's sexual and reproductive health cohort study, CHIWOS ${ }^{1,3}$ ${ }^{1}$ Burnaby; ${ }^{2}$ Vancouver, BC; ${ }^{3}$ Montreal, QC; ${ }^{4}$ Toronto; ${ }^{5}$ Hamilton, ON

INTRODUCTION: To realize the full benefits of antiretroviral therapy (ART), engagement throughout the 'cascade of HIV care' is essential. However, $>50 \%$ of individuals linked to care are not retained, a loss more common among women. This study explored women's reported use of women-centred HIV care (WCC) towards understanding whether WCC may improve retention-in-care.

METHODS: Self-administered intake questionnaires (in English or French) were collected from Focus Group Discussion (FGD) participants enrolled in the formative phase of the Canadian HIV Women's Sexual and Reproductive Health Cohort Study (CHIWOS) between August 2011 and April 2012. Participants were recruited through AIDS Service Organizations (ASOs), clinics, and peer outreach across multiple sites in BC, Ontario, and Quebec.

RESULTS: Seventy-seven women participated in 11 FGDs (28 women in BC, 25 in Ontario, 24 in Quebec). By age group, 7\% were $<30$ years, $68 \% 31$ to 50 years, and $26 \%>50$ years. $22 \%$ identified as Aboriginal, $30 \%$ black, and $44 \%$ Caucasian. $52 \%$ reported annual household income $<\$ 20,000.55 \%$ were diagnosed with HIV prior to 2000 and $85 \%$ were taking ART.

Women reported receiving HIV-related medical care from an HIV specialist $(58 \%)$ and/or family doctor $(38 \%)$ with expertise in women and HIV. Women accessed a median of 5.0 [IQR 3 to 7] additional HIVrelated services over the previous year, including ASOs (64\%), income support (34\%), ObGyn (34\%), counselling (21\%), and religious/spiritual (17\%) services. Overall, two-thirds reported currently accessing WCC. Of the one-third not accessing WCC, $83 \%$ reported wanting to. $77 \%$ of women accessing and $72 \%$ not accessing WCC reported that their HIVrelated care and outcomes would be better with improved access to WCC. Features of WCC reported as most important to improving access and retention-in-care included transportation support (66\%), childcare (52\%), accessibility (60\%), and peer support $(51 \%)$.

CONCLUSION: Findings suggest that women living with HIV access a range of medical and social services for their HIV-related care. Women's high desires and perceived benefits of WCC, suggest that WCC may play an important role in improving retention-in-care and, and ultimately, health outcomes for women.

\section{P242}

\section{TOWARDS A NATIONAL CONSENSUS STATEMENT ON HIV RESEARCH FOR, BY AND WITH WOMEN, TRANSWOMEN AND GIRLS}

Gahagan, Jacqueline ${ }^{1}$; Stein, Nicci ${ }^{2}$; Binder, Louise ${ }^{3}$; Margolese, Shari ${ }^{3}$; Muchenje, Marvelous ${ }^{3}$; Loutfy, Mona $\mathbf{R}^{3}$

${ }^{1}$ Halifax, NS; ${ }^{2}$ Ottawa; ${ }^{3}$ Toronto, ON

BACKGROUND: Women and girls now make up the majority of people living with HIV internationally (IAS, 2011). Current national HIV surveillance data indicate that women in Canada are continuing to become infected and yet their involvement in shaping HIV research, from prevention interventions to knowledge translation and exchange, remains limited.

METHODS: In keeping with the International AIDS Society (IAS, 2009) consensus statement, a Canadian-based collaborative, Gathering of Spirits, joined with a variety of national and regional HIV organizations to develop a National Consensus Statement on HIV Research for, by and with Women, Transwomen and Girls to ensure we are 'asking the right questions.' In consultation with national HIV stakeholders, a draft Consensus Statement was vetted and revised to take into consideration the diversity of gender-based HIV risk contexts that women in Canada continue to face.

FINDINGS: Although women and girls in Canada and internationally are disproportionately impacted by HIV both directly and indirectly, challenges remain in terms of ensuring that current evidence gaps in HIV knowledge to both prevent HIV and meet their unique needs. The development and uptake of a National Consensus Statement on HIV Research for, by and with Women, Transwomen and Girls can serve as an important touchstone for advancing the evidence base and closing existing research gaps.

CONCLUSION: The National Consensus Statement on Women, Transwomen and Girls and HIV Research is in keeping with the global observation from IAS and UNAIDS that without such guiding principles, the specific and complex prevention, care and treatment needs of these populations will remain at the margins of our responses.

HIV Positive Realities: Living with HIV, Stigmas, Stereotypes

La réalité des personnes séropositives : vivre avec le VIH, réprobation sociale, stéréotypes

\section{P243}

\section{SHIFTING THE FOCUS: IDENTIFYING THE ISSUES OF REFUGEE CLAIMANTS IN TRANSITIONAL HOUSING FOR PEOPLE LIVING WITH HIV/AIDS}

Ahluwalia, Amrita ${ }^{1}$; Aguinaldo, Jeffrey²; Hambly, Keith' Klusacek, Alan ${ }^{1}$; Koornstra, Jay ${ }^{3}$; Rankin, Bruce ${ }^{4}$; Rosslein, Kay ${ }^{1}$; Lopez, Atiba $^{1}$ ${ }^{1}$ Toronto; ${ }^{2}$ Kitchner; ${ }^{3}$ Ottawa; ${ }^{4}$ London, ON

BACKGROUND: For people living with HIV/AIDS (PHAs) with past experiences of housing instability and/or homelessness, transitional hous ing is an intermediary step that increases the likelihood of sustainability of future permanent housing. The transitional housing study is a longitudinal community-based study designed to identify factors related to housing-readiness, service use, and changes in support needs of PHAs. While the study aims to explore the impact of transitional housing on housing sustainability and health outcomes for PHAs more generally, this presentation compares the refugee and non-refugee residents' concerns around three key themes: disclosure (of HIV status), safety, isolation and re-integration.

METHODS: Qualitative data were collected through in-depth interviews with 25 PHAs who accessed the transitional housing in the only three transitional housing programs specifically for PHAs in Ontario. Four peer research assistants were trained in qualitative data collection methods and interviewing skills. Qualitative data were analyzed using thematic analysis. This presentation is based on a comparative analysis of themes collected from refugee and non-refugee residents in transitional housing.

FINDINGS: While there was considerable overlap in some of the needs of refugee and non-refugee residents, refugee claimants expressed a unique set of concerns about disclosure, safety, and isolation that stemmed from previous experiences of violence and trauma. Fears of disclosure and safety were based on past experiences of violence that precipitated their abscondment; isolation among refugee claimants was based on dislocation from family.

CONCLUSIONS: Two initial conclusions can be gleaned from the analysis. First, transitional housing programs may need to account for experiences of trauma and violence of refugee claimants during intake assessments and devise case management strategies sensitive to the specific needs. Secondly, partnerships with community organizations that are fully aware of and better equipped to address issues of dislocation and trauma, need to be developed in order to better serve this population. 
P244

\section{EMPOWERING PEOPLE LIVING WITH HIV THROUGH STRUCTURED MENTORSHIP AND CAPACITY BUILDING INITIATIVES: THE LEGACY PROJECT}

\author{
Luyombya, Henry; Komane, Gorata; Bisignano, Alex; \\ Owino, Maureen \\ Toronto, ON
}

BACKGROUND/PURPOSE: Newcomer PHAs to Canada are socially vulnerable and face service-access barriers that limit their pursuit of life goals. They face additional challenges of stigma and exclusion from traditional support from their own cultural communities. Committee for Accessible AIDS Treatment developed the Legacy Project to respond to mentoring needs for newcomers PHAs. The model innovates on traditional mentoring model by focusing on asset based/mutual learning approach that values lived experience.

METHODOLOGY/DESCRIPTION: To address challenges of serviceaccess, stigma and exclusion, the Legacy project was developed in partnership with local and provincial organizations to carry out different activities to support and empower newcomer PHAs. Activities included: 1) orientation sessions for knowledge transfer; 2) reflective practice sessions for ongoing info-sharing on mentorship relationship; 3) spotlight events to offer on-going support and to capture progress, and 4) experiential learning through dialogue. In addition, the project also developed and piloted a new training module for PHA workers. Qualitative and quantitative evaluations were carried out at all sessions.

RESULTS AND OUTCOMES: Since its inception in 2009, the project has successfully developed 55 pairs of mentors/mentees. Fifty are active to date. In orientation sessions participants benefited from building trust, career planning and developing life goals. Project evaluation data shows that reflective practice sessions enabled meaningful dialogue among mentors and mentees which increased their networks in the communities, and enabled effective communication/listening skills. Additional data shows that majority of participants indicated moderate or significant progress in taking more community leadership roles. Further, 55 developed life goals, 40 planned career changes while 45 increased volunteering in agencies.

CONCLUSION/RECOMMENDATION: The Legacy Mentorship project is a key innovative model that promotes health, capacity building and collective empowerment to address needs of diverse newcomer PHAs. The model can be integrated in broader settings for other refugee and newcomer populations.

\section{P245}

\section{BEYOND PREVENTION: OVERCOMING BARRIERS TO SEXUAL INTIMACY THAT INHIBIT QUALITY OF LIFE AMONG PEOPLE LIVING WITH HIV}

DePutter, Megan E; Hammond, Tom; Darisi, Tanya;

Murray, Sarah; Walker, Tim

Guelph, ON

HIV diagnosis introduces barriers that can complicate or inhibit sexual intimacy. In response to client and community needs, the AIDS Committee of Guelph \& Wellington County (ACG) led a communitybased research project to find innovative ways to support individuals to overcome barriers to sexual relationships. The project was guided by a working group and a multi-stakeholder advisory committee comprised of People Living with HIV \& AIDS (PHAs), researchers, and community agencies. Face-to-face interviews were held with 18 ACG clients about their experiences with finding sexual partners, maintaining long-term relationships, changing body image, stigma, disclosure and criminalization. A thematic analysis was completed in various collaborative sessions with working group members, the advisory committee, and participants. Findings revealed that the participants' desire for sexual activity and other forms of intimacy often went unmet; participants experienced lack of sex or celibacy associated with their HIV status, regardless of the length of time since diagnosis or relationship status. Five key themes were identified as prohibitive to sexual intimacy: fear of transmission, rejection, social isolation, challenges in meeting new partners and negative self-perception. Though the primary purpose was to use interview findings to develop programs and services that meet the needs of the PHA community in mid-sized urban and rural areas, the interviews also revealed the complexities involved with negotiating sex and sexuality as an individual living with HIV. This presentation will explore the complex ways in which trauma, stigma, aging and community development impacts sexual decision-making among PHAs, and how community and peer-support initiatives can contribute to building on existing coping strategies and resilience.

\section{P246}

RACISM, SEXISM, HIV-RELATED STIGMA AND QUALITY OF LIFE AMONG HIV-POSITIVE BLACK AFRICAN CARIBBEAN WOMEN IN ONTARIO, CANADA

\section{Logie, Carmen $\mathbf{H}^{1}$; James, Llana ${ }^{2}$; Tharao, Wangari²; Loutfy, Mona $\mathbf{R}^{2}$} ${ }^{1}$ Calgary, $\mathrm{AB} ;{ }^{2}$ Toronto, $\mathrm{ON}$

BACKGROUND: The deleterious impacts of racism, sexism and HIVrelated stigma on wellbeing are widely documented, yet most research has examined these forms of stigma separately. Rising HIV infection rates among Black African Caribbean women in Canada underscore the importance of understanding factors associated with quality of life (QOL). We used a feminist intersectional approach to examine the influence of racism, sexism and HIV-related stigma on QOL among HIVpositive Black African Caribbean women in Ontario, Canada.

METHODS: We conducted a community-based multi-method study triangulating qualitative and quantitative methods. Building on qualitative findings regarding stigma from 15 focus groups with HIV-positive women $(\mathrm{n}=104)$ in Ontario, we implemented a cross-sectional survey with HIV-positive Black African Caribbean women in three Ontario cities. Multiple linear regression (MLR) analyses were conducted to measure associations between independent (block 1: racism, sexism, HIV-related stigma; block 2: resilient coping, social support) and dependent (total QOL; QOL domains: physical; psychological; level of independence; social relationships; environment; personal beliefs) variables.

RESULTS: Survey participants $(n=163$; mean age $=40.7$ years, $S D=8.8$ ) reported frequent/everyday experiences of racism $(29.4 \%)$ and sexism (22.6\%) and high HIV-related stigma (disclosure: $84.4 \%$; personalized: 54.7\%; public attitudes 40.4\%; negative self-image: $27.6 \%$ ). In MLR analyses, racism, sexism and HIV-related stigma were associated with lower QOL scores (total; psychological; level of independence; social relationships; environment; personal beliefs). Resilient coping and social support accounted for a significant variance of higher QOL scores (total; psychological; social relationships; environment) after controlling for the effects of racism, sexism and HIV-related stigma.

CONCLUSIONS: HIV-positive Black African Caribbean women experience pervasive racism, sexism and HIV-related stigma associated with reduced QOL; social support and resilient coping were associated with higher QOL. Interventions tailored for HIV-positive Black African Caribbean women should aim to strengthen protective factors, such as resilient coping and social support, and challenge stigma and discrimination associated with HIV, race/ethnicity and gender.

\section{P247}

MY LIFE WITH HIV: A NATIONAL PROGRAM FOR NEWLY DIAGNOSE PHA'S STARTING OR THINKING OF STARTING TREATMENT

\section{Beaudry, Sylvain}

Montreal, QC

Even if it is 2013, knowing that you have HIV is still not a good news. Now that we have a lot more treatment options, it is more difficult to keep tracks on new and clear information with treatments.

At the same time, the same questions are showing up during the acceptation of this new reality of live with HIV.

My life with HIV is a serie of six workshops of $3 \mathrm{~h}$ each covering the most important topics and aspects on HIV treatments.

Through discussions in support groups, newly diagnosed participants will have the opportunity to identify challenges in their lives at both 
individual and broader levels (e.g. changing sense of identity, navigating new and complex treatment information, stigma, disclosure etc.), and to be linked to a community at similar stages to them in their relationships with the virus. By vocalizing their thoughts and sharing their experiences with others, they are able to develop their own strategies to cope and address these challenges.

This project seeks also to promote the health of PHAs by increasing their treatment knowledge and channelling their intention to explore the merits and drawbacks of starting treatment. This is done through informal 1:1 support and a workshop series where participants are encouraged to identify their own health needs, identify the barriers to achieve those needs, and develop the strategies necessary to surmount those barriers. In this regard, our activities provide the space and tools for PHAs to become active social agents in improving their health and overall wellbeing. Ultimately, the program will create a space for treatment-naive PHAs where they will develop their knowledge and confidence required to understand and address their own health needs.

\section{P248}

\section{EXPLORING THE CHALLENGES AND FACILITATORS OF LABOUR FORCE PARTICIPATION FOR PEOPLE LIVING WITH HIV/AIDS IN CANADA}

Oliver, Brent ${ }^{1}$; Worthington, Catherine ${ }^{2}$

${ }^{1}$ Calgary, AB; ${ }^{2}$ Victoria, BC

PURPOSE: Income support and labour force participation are critical issues for people living with HIV in Canada (PHAs). The objectives of this study were to identify factors that support or deter labour force participation, and to develop a conceptual framework to describe the processes and structures that shape PHAs' successful vocational experiences.

METHODS: Community-based research methods and a grounded theory framework were used to explore perspectives of PHAs in Canada who have sustained successful participation in the labour force. Purposive and theoretical sampling was conducted in the provinces of Ontario, Alberta, and British Columbia. Thirty-one semi-structured interviews were conducted with PHAs who were successfully engaged in the labour force for three months or more. Sampling was conducted concurrently with data analysis until saturation was achieved within emergent thematic categories. The data were transcribed and analyzed using grounded theory coding methods and NVIVO computer software.

FINDINGS: Eleven thematic categories emerged from participant narratives. Study results have been located within an ecological framework to describe the factors shaping participants' experiences of working successfully. At the centre of the framework are four psychosocial processes involved in working successfully: motivation for working, intrapersonal processes, interpersonal processes, and the benefits and rewards of working. Four key structures were found to shape and influence the overall process of working successfully: workplaces, community, public policy, and social structure. Three intersectional categories are embedded in, surround, and influence the processes and structures related to the phenomenon of working successfully. These are stigma and discrimination; societal attitudes regarding work, poverty, and income support; and the greater involvement of people living with HIV and AIDS principles.

CONCLUSION: Study findings contribute to an expanded contextual view of labour force participation for PHAs and highlight the importance of income support policy, access to medication, and the importance of community vocational support programs.
P249

CHRONIC STRESS: COMMON PART OF THE LIVED EXPERIENCES OF ABORIGINAL WOMEN LIVING WITH

HIV

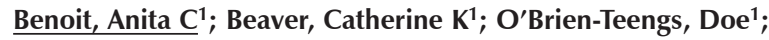
Greene, Saara ${ }^{2}$; Zoccole, Art ${ }^{1}$; Whitebird, Wanda ${ }^{1}$;

Masching, Renee ${ }^{3}$; Peltier, Doris ${ }^{4}$; Balfour, Louise ${ }^{5}$;

Raboud, Janet ${ }^{1}$; Loutfy, Mona ${ }^{1}$

${ }^{1}$ Toronto; ${ }^{2}$ Hamilton, ON; ${ }^{3}$ Dartmouth, NS; ${ }^{4}$ Montreal, QC;

${ }^{5}$ Ottawa, ON

INTRODUCTION: The complex realities of First Nations and Métis women in Ontario are multi-layered and are best described through their stories. As such with Aboriginal women residing in Ontario, this study aimed to characterize stressors, identify how stress is managed, and determine interest in learning new ways to manage stress.

METHODS: Eligible participants were 1) First Nations, Inuit, or Métis, 2) over 18 years of age, and 3) self-identified as a woman. Following consent, the women completed a questionnaire package including sociodemographic information and the perceived stress scale (score 0 to 40; cut-off $=14$ average stress, 15 to 24 high, $>25$ very high). Four focus group meetings (HIV-negative where $n=7, n=8 ; H I V$-positive where $n=2$, $\mathrm{n}=5$ ) and seven interviews (HIV-positive) were held to discuss stress using the medicine wheel to categorize stressors within the four directions with a Spiritual Leader providing support to the women. The discussions were audio-recorded and transcribed verbatim for thematic analysis.

RESULTS: High to very high stress was measured in HIV-negative Aboriginal women and those living with HIV (median score $=22.5$ and 22), respectively. Priority stressors identified by women living with HIV were housing instability, HIV-related stigma and disclosure whereas employment and education were higher priority concerns for HIVnegative women. Discrimination, body image, low income, and children (i.e. child protection agency involvement and caring for children) were stressors identified by both groups of women. Despite the all-consuming effect of stressors in their lives, an enthusiastic response to learning new ways to manage stress was expressed.

CONCLUSIONS: First Nations and Métis women participating in this study experience chronic daily stressors and are accessing support services within their community. However, for the women resolving certain stressors such as housing, caring for children, and employment takes precedence over their psychological, spiritual, and emotional well-being.

\section{P250}

THE IMPACT OF HIV STIGMA ON OLDER AND YOUNGER ADULTS LIVING WITH HIV: DOES AGE MATTER?

Emlet, Charles $\mathrm{A}^{1,2}$; Brennan, David J ${ }^{3}$; Brennenstuhl, Sarah ${ }^{3}$; Rueda, Sergio ${ }^{3}$; Hart, Trevor; $A^{3}$; Rourke, Sean; $B^{3}$ ${ }^{1}$ Tacoma, Washington, USA; ${ }^{2}$ Hamilton; ${ }^{3}$ Toronto, ON

BACKGROUND: While extensive research has been conducted on HIV stigma, few studies have examined HIV stigma across age groups. These studies have had small numbers of older adults and report divergent findings regarding aging and HIV stigma. Some suggest stigma is worse for older adults, while others show stigma diminishes with age.

METHODS: Cross-sectional data from the OHTN Cohort Study were selected based on adults aged $21+$ years at their most recent interview ( $\mathrm{n}=996)$. Total HIV stigma was assessed using the modified HIV Stigma scale (Berger, Ferrans, \& Lashley, 2001). Age groups were measured in quintiles ( 21 to 39,40 to 45,45 to 49,50 to $55,56+$ ). The youngest group was the reference category. The relationship between age and stigma was determined by hierarchical multiple regression. Covariates included socio-demographic (sexual orientation, gender, race, socioeconomic position, marital status), psychosocial (depression [CES-D], coping) and health-related (time since diagnosis, alcohol use) variables.

RESULTS: In a model including age only, each of the groups over age 40 had significantly less stigma than the youngest group. Only the oldest group (56+) had significantly less stigma when the socio-demographics were added into the model alone then together with the psychosocial and health-related variables. In the final model, female gender, heterosexual 
and bisexual orientation, Black race, depression and maladaptive coping predicted more stigma, while being in the oldest age group and longer time since diagnosis predicted less stigma. Independent variables accounted for $30 \%$ of the variation in stigma, $6 \%$ of which was explained by age alone.

CONCLUSIONS: Older age was associated with less stigma, but only the $56+$ group retained significance after controlling for socio-demographic, psychosocial and health-related variables. Older age may protect against stigma, even after accounting for other known stigma predictors (e.g., poor mental health). Further research is needed to better understand what characteristics associated with older age help decrease stigma.

\section{P251}

\section{HOUSING AND HIV IN QUEBEC (CANADA): BUILDING AN INNOVATIVE COMMUNITY-BASED RESEARCH PARTNERSHIP TO LEARN FROM THE POINT OF VIEW OF PLWHA FROM DIVERSE BACKGROUND}

Laperrière, Hélène ${ }^{1}$; Zuniga, Ricardo ${ }^{2}$; Légaré, Hélène ${ }^{2}$;

Blanchard, Michèle²; Richer, Thérèse ${ }^{3}$; Lamontagne, Bastien²;

Bernier, Mélina ${ }^{2}$

${ }^{1}$ Ottawa, ON; ${ }^{2}$ Montréal; ${ }^{3}$ Québec, QC

BACKGROUND: In Quebec, no study explores the impact of housing in terms of life trajectories of people living with AIDS (PLWHA). This project raises a strategy of partnership between HIV/AIDS communitybased organizations $(\mathrm{CBOs})$ and researchers actively engaged in supportive housing of the poorest PLWHA, such as sex workers, drug users, persons affected by mental health problems and homelessness. Ten $\mathrm{CBO}$ s members of the provincial Coalition of community groups (COCQAIDS) have defined a project on evaluating the impact of supporting housing on the life trajectories of this population.

METHODS: The project has begun with an experimental catalyst project in two regions of the provincial Coalition. The objectives were (a) to collect their expertise accumulated on supportive housing and (b) to document their impact of the community practices through the viewpoints of all the actors involved. We experimented a form of collaborative ethnography where we engaged eight PLWHA as peer research assistants from the poorest and most marginalized populations linked to four community housing organizations. They selected the popular education methods with which they felt most comfortable (storytelling, photographs and illustrations).

RESULTS: Over a period of eight months, the peer assistant researchers described and co-constructed collectively their experience with housing, care and visibility with their ethnological views. Each month, we explored together different themes, which linked HIV/AIDS community housing with life trajectories. The peers started to feel empowered to lead the discussion by themselves without the researchers and community partners. As their "vulnerable stage" changed with time (T1-T7), autonomy increased and disclosure appeared to be a main concern for them. CONCLUSION: One defining trait of Quebec governance is the participation of civil society organizations in the fabric of public health and social policies. The catalyst project tests research participation and some community-based evidences about the HIV/AIDS community housings and PLWHA's life trajectories.

\section{P252}

\section{DISCLOSURE COMPLICATES EVERYTHING: EXPERIENCES OF CANADIAN HIV-POSITIVE YOUTH}

\section{Landry, Tamara M; Orchard, Treena}

London, ON

BACKGROUND: HIV-positive youth experience multiple challenges, including stigma, sexual vulnerability, and a compromised immune system. The act of self-disclosure can enhance their disadvantage because of physical harm, rejection, and loss they experience upon sharing this sensitive and charged information. Currently, Canada does not have specific health promotion guidelines to assist HIV-positive youth in their decisions to disclose their HIV status, and very little research has been conducted on disclosure among this vulnerable group. Drawing on a study into disclosure and sexual health issues among HIV-positive youth in British Columbia and Ontario, this presentation focuses on the socioemotional and psychological factors that influence these youths' decisions regarding disclosure.

METHODS: Eighteen interviews were conducted with HIV-positive youth (14 males and four females), aged 21 to 29 years, who were recruited through AIDS Service Organizations. A phenomenological approach was employed, which allowed for an exploration and analysis of data that reflected the participants, "lived experience" relative to the complex issues of disclosure and sexual health.

RESULTS: This paper focuses on the two inter-connected themes of disclosure and non-disclosure, which are the most powerful, yet often contradictory sets of experiences that inform the youths' decisions regarding disclosure management. Being HIV-positive complicates relationships and sexual health. Youth who are ambivalent about disclosing their HIV status are still coming to terms with their HIV status, are afraid of physical harm and judgment and lack safer sex negotiation skills. Youth who disclose their HIV status have support from others, feel accountable and do not want to be criminalized.

CONCLUSIONS: Among both groups of youth the complex issues of who to disclose to, when to disclose, and how to disclose their HIV status play significant roles in the decision to disclose or not-disclose. The results demonstrate a need for tailored disclosure "scripts", including negotiation skills, and more substantive social and health-related supports, to assist youth with the complexities of HIV disclosure as well as living with the equally important decision to not-disclose.

\section{P253}

MEANINGFUL INVOLVEMENT OF PERSONS LIVING WITH HIV AND AIDS (MIPA) IN CLINICAL MENTORSHIP OF REGISTERED NURSES

Kerston, Paul ${ }^{1}$; Caine, Vera ${ }^{2}$; Udvardy, Steven ${ }^{2}$; Mill, Judy²;

Maina, Geoffrey ${ }^{2}$; Gahagan, Jaqueline ${ }^{3}$; $\mathrm{O}^{\prime}$ Brien, Kelly ${ }^{4}$;

Worthington, Catherine ${ }^{5}$; Solomon, Patricia ${ }^{6}$;

Dykeman, Margaret ${ }^{7}$; Rogers, Tim $^{8}$; dePadua, Anthony ${ }^{8}$

${ }^{1}$ Vancouver, BC; ${ }^{2}$ Edmonton, $\mathrm{AB} ;{ }^{3} \mathrm{Halifax}, \mathrm{NS} ;{ }^{4}$ Toronto, ON;

${ }^{5}$ Victoria, BC; ${ }^{6}$ Hamilton, ON; ${ }^{7}$ Fredericton, NB; ${ }^{8}$ Prince Albert, SK

BACKGROUND: A clinical mentorship model for Canadian nurses is a CIHR-funded study aimed at increasing knowledge and skills in HIV care. Using a transformative education approach, experienced PHAs mentored nurse mentees to increase knowledge about HIV care, treatment and prevention. The MIPA principle informed the integration of PHAs in the study.

METHODS: Mentors and mentees were recruited with the assistance of investigators, clinical facilities, and AIDS Service Organizations (ASOs) at each site. The 1-year intervention consisted of three two-day workshops at baseline, six months and twelve months at six sites (Edmonton, Vancouver, Prince Albert, Toronto, Halifax, and Sault Ste. Marie). Mentors and mentees continued to engage between workshops on a regular basis, primarily through face-to-face meetings. Data were collected using mixed methods, including surveys, interviews and observational data to assess the intervention. An inductive, iterative approach was used to analyze and identify themes and discover relationships among themes via systematic comparison.

FINDINGS: Sixty participants (10 nurse mentors, 10 PHA mentors, 40 nurse mentees) are currently enrolled or have completed the intervention. Barriers and facilitators for PHA involvement included perceptions, attitudes, readiness, and support for HIV and AIDS-related interventions which shifted over time, often leading to more meaningful and relevant engagement with PHAs. Regional differences across Canada were also found in current practice approaches of PHA involvement.

CONCLUSIONS: PHA involvement is critical to the mentorship of nurses in HIV care. Integration of PHAs must attend to regional and attitudinal differences, professional needs, and perceptions about the importance of HIV and AIDS in health care. Community-based organizations such as ASOs may serve a vital role in navigating the regional variations in preparedness for HIV and AIDS interventions among health care providers and PHAs. 
P254

MOTHER-CHILDREN EDUCATION TO SEXUALITY : PERSPECTIVE OF THE MOTHERS LIVING WITH HIV (MLHIV)

Fernet, Mylène; Racicot, Caroline; Blais, Martin;

Lapointe, Normand D; Samson, Johanne; Otis, Joanne;

Martin, Nancy

Montréal, QC

CONTEXT: Sexual education in families with mothers living with HIV (MLHIV) is often focused on unplanned pregnancy and HIV prevention, with limited discussion of emotional issues.

OBJECTIVE: Describe the perspective of MLHIV regarding their perceived role in sexual education, the themes discussed with their adolescents and the strategies used to talk about sexuality.

METHOD: Semi-directed individual interviews were conducted with 11 MLHIV (averaged age $=45.5$ years) recruited either in clinics $(n=8)$ and communities $(n=3)$. Participants were from various ethnocultural origins (Caucasian, Native American, Latin, African, Haitian) and had three children on average (children mean age $=16.6$ ). Seven were single mothers or unemployed and four gave birth to a children living with HIV. Thematic content analysis was performed.

RESULTS: Comfort regarding sexual education was the most common theme. Some MLHIV said they were not comfortable discussing sexuality with their children. The most common obstacles were: MLHIVs never received proper sexual education; are facing sociocultural taboos refraining discussions; are afraid of being questioned on how they got HIV; consider their children's level of development inappropriate for sexual education; feel their children are reluctant; or rely on schools and clinics to provide an education. Overall, discussions about sexuality were limited. Women claiming to be comfortable expected their children to ask questions or waited for opportunities (TV, Internet, etc.). All MLHIV felt responsible for protecting their children against STBBIs and unplanned pregnancies. Prevention and contraception are the most common themes discussed. CONCLUSION: MLHIV reported feeling comfortable discussing sexuality with their children. However, discussions were limited, generally initiated by children and focused on prevention. Emotional issues are often ignored.

\section{P255 \\ BIOGRAPHICAL RUPTURE AND CONTINUITY: PERINATALLY-INFECTED ADOLESCENTS' EXPERIENCE OF LEARNING ABOUT THEIR HIV DIAGNOSIS}

Proulx-Boucher, Karène ${ }^{1}$; Fernet, Mylène ${ }^{1}$; Blais, Martin ${ }^{1}$;

Lévy, Joseph J'; Otis, Joanne ${ }^{1}$; Lapointe, Normand $\mathrm{D}^{\mathbf{1}}$;

Samson, Johanne ${ }^{1}$; Morin, Guylaine ${ }^{1}$; Thériault, Jocelyne ${ }^{1}$;

Trottier, Germain ${ }^{2}$

${ }^{1}$ Montréal; ${ }^{2}$ Québec, QC

OBJECTIVE: Most studies on diagnosis disclosure to perinatally acquired HIV (PaHIV) concentrate on the motives and the concerns of the parents. Few examine the signification the PaHIV attributed to the diagnosis and the following identity and biographical transformations. The objective of this study is to explore the experience of the diagnosis disclosure from the PaHIV point of view.

METHODOLOGY: Semi-structured interviews were performed with 29 PaHIV (aged 10 to 18 years) recruited through the Centre Maternel et Infantile sur le sida of CHU Sainte-Justine (Montreal), followed by a qualitative content analysis.

RESULTS: Diagnosis disclosure follows a three-step trajectory: 1) a hidden reality where adolescents are aware of their fragile health, have daily medications and regular medical monitoring but ignore their HIV status; 2) a reality finally disclosed where they learn, toward the age of eleven, that they are infected with HIV, allowing them to make sense of their health status; and 3) a reality to integrate, the disclosure contributing to the personal and social identity building. The HIV disclosure is part of a biographical continuity that helps legitimizing HIV treatments administered. However, PaHIV contemplate a progressive biographical transformation with regard to their intimacy and sexuality.
DISCUSSION: PaHIV learn at an early age how to manage the chronic illness and the stigmatization associated with HIV. Learning their HIV diagnosis allow them to better understand their health status. While this event is not described as a biographical rupture at the time of the diagnosis, the learning of their HIV diagnosis still appears as a critical event in the construction of their identity. The way their sense of self is reshaping in relation to their first romantic and sexual experiences is discussed. Implications of the biographical issues and the stigmatization of the romantic and sexual life of PaHIV should be taken into consideration for intervention.

\section{P256}

\section{"HIV IS HIV": GAY MEN'S NARRATIVES OF THE DISCOVERY AND DIAGNOSIS OF AN ACUTE OR RECENT HIV INFECTION}

Grace, Daniel; Steinberg, Malcolm; Kwag, Michael;

Doupe, Glenn; Trussler, Terry; Rekart, Michael; Gilbert, Mark; CIHR Team in the Study of Acute HIV Infectioning Gay Men, The Vancouver, BC

BACKGROUND: Diagnosing HIV-positive MSM in active sexual networks earlier through enhanced testing technologies and historical testing data allows for the detection of presumptive acute HIV infection (AHI) or recent HIV infection. This provides opportunities for both individual and population health benefit. To date, no qualitative or mixed-methods analysis has been conducted to understand the experiences of persons newly diagnosed with acute HIV infection in Canada. METHODS: Study recruitment was conducted through six clinical sites in British Columbia, Canada (April 2009 - June 2012). All participants received an acute $(n=13)$ or recent $(n=12)$ HIV diagnosis and completed a series of self-administered questionnaires and semi-structured face-toface interviews over a one-year period. A thematic analysis of baseline qualitative interviews elicited data relevant to men's "discovery and diagnosis narratives" $(\mathrm{n}=25)$.

RESULTS: Three dominant themes emerged which are conceptually linked by tensions of serostatus knowledge, spatiality and temporality: (1) Technological innovations and testing protocols have shifted experiences of learning of one's diagnosis (e.g., many men "knowing before they knew" and reporting degrees of self-diagnosis); (2) Men have differing needs and report high degrees of psychosocial support at the time of diagnosis in clinical settings (e.g., having/not having social supports and networks of positive friends informed many men's diagnosis experience and the psychosocial support they sought); and (3) When first learning of their seroconversion, knowledge of acute infection had limited meaning for most participants (e.g., many reporting simply that "HIV is HIV").

CONCLUSION: These findings have important implications for providers delivering acute or recent HIV test results to patients whether over the phone or in-person. While knowledge of acute HIV infection status appears to have limited impact on the ways in which study participants construct their illness at the time of diagnosis, concerns of high viral load more generally and allied commitments to preventing onward transmission are expressed across the sample.

\section{P257}

\section{STIGMA MANAGEMENT OVER TIME IN PERINATALLY HIV- INFECTED YOUTH AND THEIR FAMILIES : A QUALITATIVE PERSPECTIVE}

Proulx-Boucher, Karène ${ }^{1}$; Blais, Martin ${ }^{1}$; Fernet, Mylène ${ }^{1}$;

Martin, Nancy ${ }^{1}$; Otis, Joanne ${ }^{1}$; Lévy, Joseph J ${ }^{1}$;

Lapointe, Normand $\mathrm{D}^{1}$; Samson, Johanne ${ }^{1}$; Morin, Guylaine ${ }^{1}$; Thériault, Jocelyne ${ }^{1}$; Trottier, Germain ${ }^{2}$

${ }^{1}$ Montréal; ${ }^{2}$ Québec, QC

INTRODUCTION: Perinatally HIV-infected youth (PHIVY) trajectories are influenced by family upheavals, stigmatization and stigma management strategies within the family. The objective is to explore the HIV-stigma management within the family from the perspective of PHIVY and their evolution over time.

METHOD: Within a qualitative longitudinal research framework, 18 PHIVY (11 girls and seven boys) aged 13 to 22 participated twice in 
individual semi-directed interviews within a three year interval at the Centre maternel et infantile sur le sida du CHU Sainte-Justine in Montreal. RESULTS: Initially, all participants used silence as a way of managing stigma and maintaining a family emotional balance. Two trajectories (estrangement and consolidation) emerged over time. 1) The estrangement trajectory is characterized by a separation, physical or emotional, from family, that is precipitated by family traumatic events (such as parental death) and that forced PHIVY to adapt to a new daily life context. In the absence of a safe and supporting environment, most of these PHIVY maintained silence as their main stigma management strategies, preventing them to accept the HIV diagnosis. 2) The consolidation trajectory is characterized by a strengthening of family ties that helps PHIVY in developing stigma management strategies based on experiences sharing and mutual support. Favorable conditions for the development of active stigma management strategies and the acceptation of the HIV diagnosis were: safe and stable familial environment, strengthening of family ties, emotional and social support, sharing experiences with other persons infected with HIV (usually their mother, also HIVinfected).

CONCLUSION: Supporting and stable relationships are of first importance for PVHIY in the process of accepting the HIV-infection and acquiring active stigma management strategies. Strengthening the ties with the medical staff and maintaining them beyond the transition to adult services could help in minimizing the impact of family upheavals on the PHIVY.

\section{P258}

\section{IMPACT OF FOOD INSECURITY AND HOUSING ON} HEALTH OUTCOMES AMONG PEOPLE LIVING WITH HIV FROM 2006-2009: LONGITUDINAL FINDINGS FROM POSITIVE SPACES, HEALTHY PLACES COHORT STUDY

Choi, Stephanie ${ }^{1}$; Globerman, Jason $\mathbf{M}^{1}$; Fielden, Sarah ${ }^{2}$;

Bekele, Tsegaye ${ }^{1}$; Koornstra, JJ (Jay) ${ }^{3}$; Hambly, Keith ${ }^{1}$;

Greene, Saara ${ }^{4}$; Sobota, Michael ${ }^{5}$; Watson, James ${ }^{1}$; Walker, Glen ${ }^{6}$;

O'Brien Teengs, Doe $^{1}$; Tucker, Ruthann ${ }^{4}$; Hwang, Stephen $W^{1}$;

Rourke, Sean ${ }^{1}{ }^{1}$; Positive Spaces, Healthy Places Team, The ${ }^{1}$

${ }^{1}$ Toronto, ON; ${ }^{2}$ Vancouver, BC; ${ }^{3}$ Ottawa; ${ }^{4}$ Hamilton; ${ }^{5}$ Thunder

Bay; ${ }^{6}$ St Catharines, ON

BACKGROUND: Studies of people living with HIV who are homeless or unstably housed show high prevalence of food insecurity $(>50 \%)$ and associated poor health outcomes. Most evidence to date, however, is in the form of cross-sectional studies. To better understand this issue, a longitudinal study was undertaken to examine the impact of food insecurity and housing on overall physical and mental health-related quality of life (HRQoL) among people living with HIV in Ontario.

METHODS: Six hundred and two participants living with HIV were enrolled in the Positive Spaces, Healthy Places (PSHP) study and followed from 2006 to 2009. Questionnaires were administered and generalized linear mixed effect models constructed to examine the longitudinal associations between food insecurity, housing and physical and mental HRQOL.

RESULTS: At baseline, 57\% were classified as food insecure using established criteria. These individuals were more likely to have poor physical and mental HRQoL and were more likely to use food bank services when compared to those who were food secure $(\mathrm{p}<0.001)$. Longitudinal analyses (using $R$ 2.13.1) revealed that food insecurity was significantly and negatively associated with both physical (effect size [ES] with 95\% CI: ES -2.0 [95\% CI -3.1 to -0.8$]$ ) and mental (ES $-2.6[95 \% \mathrm{CI}-3.9$ to -1.4]) HRQoL outcomes after adjustments for all potential confounders. In addition to food insecurity, several housing variables were also significantly related to HRQoL outcomes: (a) unstable livelihood (physical: ES -5.0 [95\% CI -3.9 to -1.4$]$; mental: ES -4.7 [95\% CI -5.3 to -2.2$]$ ), (b) past experience of difficulties in affording housing (physical: ES $-2.0[95 \% \mathrm{CI}$ -3.1 to -0.9 ]; mental: ES -2.0 [95\% CI -3.2 to -0.7$]$ ), and (c) housing discrimination (physical: ES -2.8 [95\% CI -4.3 to -1.3 ; mental: ES -2.9 [95\% CI -4.5 to -1.2$])$.

CONCLUSIONS: Food insecurity is highly prevalent among people living with HIV in Ontario and has strong negative impacts on their overall physical and mental health-related quality of life, particularly for those who face housing challenges. Changes in current housing programs, services and policies, and careful consideration of their unmet nutrition needs, are urgently needed for this vulnerable group of individuals.

\section{P259}

EXAMINING THE HEALTHCARE EXPERIENCES OF WOMEN LIVING WITH HIV AND PERCEIVED HIV RELATED STIGMA

\section{Elston, Dawn; Ion, Allyson}

\section{Hamilton, ON}

“...you likely won't remember all the details leading up to a diagnosis, but you'll remember how you were treated, always."

INTRODUCTION: Despite medical advances in HIV care and treatment that have improved longevity and quality of life for people living with HIV/AIDS (PLWHA), HIV-related stigma persists in healthcare settings when PLWHA access services for HIV testing and diagnosis, chronic disease management or obstetrical care. Increased rates of new HIV infections in women, particularly racialized or socially marginalized women, led to a study exploring healthcare challenges for women living with HIV. During face-to-face interviews women identified suboptimal healthcare as a barrier that they perceived as HIV-related stigma.

METHODS: During 17 semi-structured interviews women were asked what healthcare barriers they experience living with HIV. Thematic analysis was used to report women's healthcare experiences. Transcripts were read and coded for descriptions of healthcare experiences that women perceived as HIV-related stigma.

RESULTS: HIV-related stigma was perceived during HIV testing and diagnosis with healthcare providers who lacked HIV knowledge and did not obtain consent or offer pre- and post-test counselling. Moreover, women perceived that HIV-related stigma enacted stereotypes about HIV risk among healthcare providers. Women's healthcare experiences were interconnected with HIV disclosure in healthcare settings. Healthcare provider insensitivity to the importance of privacy and confidentiality was perceived as HIV-related stigma. Women perceived HIV-related stigma when healthcare providers, regardless of whether women were virologically suppressed, demonstrated fear of contagion or framed HIV as a terminal illness.

DISCUSSION: Women's experiences suggest that lack of up-to-date HIV knowledge by healthcare providers enacts HIV-related stigma during HIV testing, pre- and post-HIV test counselling and HIV disclosure, resulting in marginalizing healthcare practices. Women perceive healthcare that fails to contextualize HIV as a chronic health condition as stigmatizing. For women, contextualizing their HIV status was important to living an integrated life with the potential for more social support and improved health outcomes.

\section{P260}

\section{NEGOTIATING SAFETY, ACCESS, DISCLOSURE AND SELF CARE: RESILIENCY PATHWAYS OF ASIAN MSM PHAS}

Hui, Christian S; Li, Alan; Dandal, Jr Alvi; Poon, Maurice;

\section{Utama, Richard}

Toronto, ON

BACKGROUND/ISSUES: Asian men who have sex with men often face challenges and barriers related to cultural and family values opposing to their sexual identities. For Asian MSM who are HIV+, the added challenges of being sero-positive could make the needs of this group different from their sero-negative counterparts as they deal with health challenges, HIV-stigma, systemic discrimination, migration challenges, and the challenge to disclose to sex partners within a legal system which criminalizes HIV non-disclosure.

DESCRIPTION: Twelve HIV+ Asian MSMs participated in a PHAspecific focus group as part of an action research study conducted by Asian Community AIDS Services. Participants shared critical life events and their reflections on those experiences. The focus group interview transcript is then coded and analyzed thematically to identify the key challenges faced and the resiliency strategies used by participants in order to inform development of health promoting interventions. 
LESSONS LEARNED: Our focus group participants identified both internal and external factors that affected their abilities to negotiate the complex tasks of ensuring safety, acquiring service access, learning about self-care and disclosing their status to significant others. Disability support, linkages to culturally sensitive support services, relationships with trusted peers and professional service providers were identified to be key external factors supporting resiliency. Strong sense of social justice and cultural identity, belief in one's own abilities to overcome adversities, motivation to learn self care and being true to own self were identified as key facilitators that enable the journey to overcome complex challenges. RECOMMENDATION: Culturally appropriate programs that facilitate knowledge of self-care should be more readily available for Asian MSM PHAs. Skill development in disclosure will enable potential to connect to support and address concerns related to criminalization. In-depth analysis of the actual pathways undertaken by our participants will help inform resiliency interventions for further study.

\section{P261}

PUTTING CO-INFECTION ON THE MAP: RESULTS FROM CTAC'S NATIONAL CO-INFECTION PROJECT

Sutton, Paul; Price, Colleen; Beaver, Kerrigan; Diliso, Nicola; Gough, James; Hui, Christain; Lister, James

Toronto, ON

BACKGROUND: CTAC has a history of advocating for policy and program development for people living with HIV and viral hepatitis coinfection, including partnerships with the OHTN on a Think Tank (2007) and the first HIV/HBV/HCV Co-infection Summit (2010). Proceedings from the 2010 Summit led CTAC to obtain support from the Public Health Agency of Canada for a multi-phase project to build capacity of service providers, researchers and people living with co-infection across Canada.

METHODS: The project's four goals were to:

1) Produce an evidence-based training curriculum on co-infection research and best practices

2) Build capacity among people living with co-infection to facilitate and evaluate trainings and webinars

3) Deliver a national webinar series on co-infection research

4) Drive social change to meet the needs of people living with coinfection in Canada.

RESULTS: The project recruited and trained 10 facilitators, six of whom facilitated the national webinar series. A total of 64 different people have participated in in-person and/or webinar sessions from communities and organizations in each Canadian province. Preliminary analysis of program evaluation shows:

- Limited baseline knowledge of co-infection from a large majority of participants

- Moderate to high level of research literacy, especially amongst service providers

- Appetite for more advanced knowledge translation of co-infection research, additional opportunities to meet peers and colleagues working in co-infection and policy change, especially regarding supervised consumption facilities and prison harm reduction

CONCLUSION: CTAC is currently developing programming based on lessons learned from this project. In 2013, CTAC will bring together community organizations that are leaders in HIV and viral hepatitis service integration to assess research findings, generate a set of good practices and customize webinars to address the identified gaps.
P262

HEALTH CARE ENCOUNTERS AND ENGAGEMENT IN CARE: LESSON LEARNED FROM THE LIVED EXPERIENCES OF WOMEN LIVING WITH HIV IN ACCESSING HIV- AND NON-HIV-SPECIFIC CARE SERVICES

Ennabil, Nourane ${ }^{1}$; O'Brien, Nadia $^{1}$; Bakombo, Dada ${ }^{1}$; Dayle, Janice $^{1}$; Menard, Brigitte ${ }^{1}$; de Pokomandy, Alexandra ${ }^{1}$; Kaida, Angela ${ }^{2}$; Loutfy, Mona $\mathrm{R}^{3}$; Canadian HIV Women's Sexual and Reproductive Health Cohort Study, CHIWOS ${ }^{3}$

${ }^{1}$ Montreal, QC; ${ }^{2}$ Burnaby, BC; ${ }^{3}$ Toronto, ON

BACKGROUND: Given the increasing number of women living with HIV in Canada, healthcare services must adapt to fit their unique requirements. Furthermore, due to women's multiple intersecting needs (e.g., gynaecological, physical, psychological), and the long term nature of HIV, healthcare is not administered solely by HIV-specific clinics. Women also interact with emergency departments, family doctors, drop in clinics, and numerous specialists. In developing a range of services that adequately support women living with HIV, it is imperative to understand women's lived experiences of accessing HIV- and non-HIV-specific care to facilitate optimal engagement in care.

METHODS: Focus group discussions (FGDs) were conducted within the formative phase of the Canadian HIV Women's Sexual and Reproductive Health Cohort Study (CHIWOS). This analysis focuses on three FGDs with 24 women living with HIV from Quebec. FGDs were led by trained Peer Research Associates and conducted in collaboration with community organizations and medical clinics. FGDs were audio recorded, transcribed, translated and analysed in English and French using thematic analysis.

RESULTS: In describing supportive engagement in care, women living with HIV identified contrasting experiences within and outside HIVspecific healthcare settings. They underlined the significance of positive patient-provider relationships enabled by long-term interactions with HIV-specific clinics and HIV-providers. While in general healthcare settings, participants highlighted the lack of basic HIV knowledge, such as HIV transmission routes, leading to instances of stigmatization, humiliation, and involuntary HIV-disclosure. Participants also addressed the need for improved communication between HIV-specialists and general care providers to better support their engagement in care.

CONCLUSIONS: Various healthcare providers are essential to the provision of care for women living with HIV. Experiences described by participants, offer a cautionary note that despite decades of the epidemic, stigmatizing experiences still occur and a lack of HIV-knowledge still persists within the healthcare system, limiting the feasibility of decentralizing HIV-specific care. Finally, in addressing engagement in care amongst women living with HIV it is crucial that women be consulted to ensure that care is both effective and appropriate.

\section{P263}

BREAKING THE GLASS CEILING: THE MEANINGFUL INVOLVEMENT OF WOMEN LIVING WITH HIV IN THE DEVELOPMENT AND PROVISION OF WOMEN-CENTRED

\section{CARE}

Nicholson, Valerie ${ }^{1}$; Carter, Allison J'; Greene, Saara ${ }^{2}$;

Rawson, Stephanie'; Margolese, Shari ${ }^{4}$; Sanchez, Margarite; de Pokomandy, Alexandra ${ }^{3}$; Loutfy, Mona $\mathrm{R}^{4}$; Kaida, Angela ${ }^{1}$; Canadian HIV Women's Sexual and Reproductive Health Cohort Study, CHIWOS 4,1

${ }^{1}$ Vancouver, BC; ${ }^{2}$ Hamilton, ON; ${ }^{3}$ Montreal, QC; ${ }^{4}$ Toronto, ON; ${ }^{5}$ Salt Spring Island, BC

BACKGROUND: The Meaningful Involvement of Women Living with HIV/AIDS (MIWA) is a key feature of women-centred care. However, little is known about women's desires to become peer leaders in their care communities, the barriers they face, or the tensions that emerge from involvement. Identifying and mitigating potential consequences of MIWA must be considered as peer participation in health and supportive services gains prominence in Canada.

METHODS: Peer Research Associates conducted four focus groups with women living with HIV $(\mathrm{n}=28)$ in Vancouver, Victoria, and Prince 
George, BC. Focus groups were audio-recorded, transcribed, and thematically analysed.

RESULTS: Participants expressed strong desires to become involved in and provide expertise and leadership to the services they access. However, women identified a lack of clear organizational policies that recognize women living with HIV as not only recipients of services but as valued volunteers, employees, partners, and leaders within organizations. Additional structural barriers impeding peer involvement include: organizational prejudice against hiring their own clients; prevailing disempowering staff attitudes towards women's capacity to contribute; and a lack of remuneration and recognition for contributions. When women do become involved, several tensions emerge: being transparent about having women with HIV working in organizations yet wanting to protect confidentiality; securing funding through use of women's membership and volunteer efforts yet giving women no ownership or control over eventual policy/programming decisions; and having women contribute to an improvement of services to which they are then given limited access as staff members. Collectively, these unseen, yet seemingly unbreachable barriers/tensions yield an 'HIV peer engagement glass ceiling', which keeps many women with HIV from reaching their full leadership potential within their communities.

CONCLUSION: Organizations striving to operate within a womencentred model of care need to implement policies that include a genuine commitment to MIWA. However, there is an urgent need for successful strategies that mitigate barriers/tensions and facilitate women's involvement. Meaningful user engagement in care development and provision may contribute to more effective services and improved health outcomes for women living with HIV.

\section{P265}

\section{HIV/AIDS STIGMA AND KNOWLEDGE AMONG UNDERGRADUATE STUDENTS IN AFRICA}

\section{Shogbola, Rilwan T}

Lagos Mainland, Nigeria

BACKGROUND: The HIV/AIDS remains one of the greatest health challenges facing the World today. In Africa, HIV is predominant among young people for they constitute larger percentage of the society. This study examined stigmatizing attitudes toward HIV/AIDS among Undergraduate students in Africa. This study was specifically designed to

1) Examine whether HIV-related stigma and knowledge are related to one another and to gender, parents' education, and exposure to HIV/ AIDS education.

2) Assess stigmatizing attitudes toward HIV/AIDS and sexuality; HIV/ AIDS knowledge, and awareness of HIV-related health resources.

3) Suggest the way forward in minimizing the spread of HIV/AIDS in Africa.

4) Determine ways in which HIV/AIDS is contacted.

METHODS: In thirty (30) universities in Africa, 7,621 students: 15 universities from Nigeria, five universities from Ghana, two universities from South Africa, one university from uganda, two universities from Cotonou Benin, three universities from Cameroon, and two universities from Senegal completed a questionnaire assessing stigmatization of HIV/ AIDS, stigmatization of sexuality, knowledge of HIV/AIDS, HIV/AIDS education and resources, and demographic characteristics. Undergraduate Students varied in how much they stigmatized persons with HIV/AIDS. They generally lacked accurate knowledge about the disease and of related health resources.

RESULTS: However, those with greater exposure to HIV/AIDS education demonstrated significantly greater HIV/AIDS knowledge. Female undergraduate Students demonstrated significantly less knowledge about HIV/AIDS compared with male undergraduate students, while the males reported significantly greater exposure to HIV/AIDS education compared with the females.

CONCLUSION: Given the early stage of the HIV/AIDS epidemic in Africa, awareness and appropriate knowledge may play an important role in preventing the further spread of HIV/AIDS among the undergraduate students. HIV/AIDS continues to spread and affect the lives of millions of people, The human immunodeficiency virus (HIV), which causes acquired immunodeficiency syndrome (AIDS) principally, attacks CD4 T-cells, a vital part of the human immune system.

These results suggest a need for greater HIV/AIDS education and awareness of health resources, especially among female undergraduate students.

Education must directly address stigmatizing attitudes about HIV/AIDS, gaps in HIV/AIDS knowledge and awareness of HIV-related health resources.

\section{Social, Political, Ethical and Legal Research Recherche sociale, politique, éthique et juridique}

\section{P267}

\section{THE RENEWED LEADING TOGETHER - CANADA TAKES ACTION ON HIV/AIDS - A MULTISECTORAL DEVELOPMENT MODEL}

Pigeon, Terry $\mathrm{A}^{1}$; Gahagan, Jacqueline ${ }^{2}$; Lambert, Denise $\mathrm{T}^{3,4}$ ${ }^{1}$ Cowansville, QC; ${ }^{2}$ Halifax, NS; ${ }^{3}$ Edmonton; ${ }^{4}$ Onoway, AB

Leading Together provides a strategic blueprint which recognizes the strength of a collective, multi-sectoral and coordinated pan-Canadian response to HIV/AIDS.

Leading Together promotes a strategic, coordinated and collaborative approach to tackle both HIV and the determinants of health and health inequities that both influence and fuel new infections and which have significant effects on people infected, affected and at risk. It encourages effective and productive partnerships across jurisdictions, within the health care system and with other sectors, such as social services, education, housing and justice, to mitigate the impact of HIV and prevent new infections. It promotes empowerment of people living with and at risk of HIV, recognizing their strengths and encouraging meaningful participation in the design and delivery of programs and services that affect their lives.

The Leading Together Championing Committee (LTCC) conducted a gap analysis of the original 2005-2010 Leading Together call to action document. The LTCC led a literature review, key informant interviews and an online survey to identify any changes required in the call for action. An Editorial Board, composed of experts across all sectors and roles, including civil society groups and government, represented stakeholder views regarding the original document. The LTCC determined that the underlying principles and values of Leading Together had stood the test of time and are on the right path; however it must keep pace with new knowledge and emerging needs. The Leading Together renewal document considered new data, advances in biomedical science, expanded research categories including behavioural and social research, to propose specific activities for action to guide a coherent and complementary response in Canada.

Leading Together provides a bold vision of where Canadians can be effective in collectively managing the prevention and control of HIV. It sets out a hopeful, comprehensive, strengths-based approach to mitigate the impact of HIV - that focuses on underlying health issues and the social determinants of health. It further challenges Canadians involved in HIV to work together to develop a more effective, coordinated response to the epidemic.

\section{P268}

DIFFICULT DECISIONS: AN ETHICAL DECISION-MAKING TOOLKIT FOR COMMUNITY-BASED WORKERS CARING FOR HIV-AFFECTED CHILDREN AND FAMILIES IN KEY POPULATIONS

Miller, John; Ibarra, Kim; Wagner, Frank Toronto, ON

Carers working with children and families in stigmatized groups such as people living with HIV, people who use drugs and sex workers face difficult decisions--at least weekly and often daily, according to a global survey. In the absence of proper guidance, they can make decisions that are 
unethical, and often based on bias or influenced by stigma about who has the ability or right to parent. These decisions can do more harm than good.

This presentation will describe how an international working group is filling a major programmatic gap by developing a toolkit--adapted and expanded from a toolkit developed in Toronto--to help carers in community-based organizations to make better decisions when faced with competing choices or when the rights or interests of two people are in conflict. The third draft of the toolkit will be presented.

Anchored by a code of ethical values and principles and using a simple four-step tool, care workers will be guided in understanding how ethical decision-making differs from following the law, organizational policy, religion, culture or societal norms. They will then gather all facts, challenge stigma, identify ethical principles in conflict, make a decision, and document, debrief and self-evaluate. Managers will be encouraged to apply the tool consistently across staff teams.

The project working group comprises organizations representing people who use drugs, sex workers, transgender people, people living with HIV, MSM and other gay men, care worker organizations, ethicists, major NGOs and funders. This group undertook a global consultation and in May to August 2013 is pilot testing a third draft in five regions. An interactive multi-media version will be available as well as a PDF version. The toolkit will be translated into French, Spanish \& Russian, ready for dissemination in March 2014, and then evaluated and revised again in autumn 2014.

\section{P270}

\section{(M)OTHERING WITH HIV: HOW HIV-POSITIVE MOTHERS RECONSTRUCT EXPERIENCES OF HEALTH AND SOCIAL SURVEILLANCE}

Greene, Saara ${ }^{1}$; Ion, Allyson; ${ }^{1}$; Elston, Dawn ${ }^{1}$;

Kwaramba, Gladys ${ }^{1}$; Beaver, Kerrigan ${ }^{1}$; Loutfy, Mona R ${ }^{2}$

${ }^{1}$ Hamilton; ${ }^{2}$ Toronto, ON

INTRODUCTION: Identities of HIV-positive mothers are socially constructed before the baby's arrival and are influenced by conventional, long-established perceptions about 'healthy' babies and 'good' mothers. Macro systems, institutions and individuals (i.e. family, HIV community, service providers) enact these social constructions within the spaces that care and support are provided. Furthermore, HIV-positive mothers are uniquely impacted by social determinants of health including poverty, domestic violence, substance use and housing instability, which affects their access to health and social services while increasing their risk of health and social surveillance.

METHODS: A narrative methodological approach was used to interview 46 HIV-positive women in pregnancy and post-partum in the HIV Mothering Study from March 2011 to July 2012. Interviews were recorded and transcribed verbatim. Narratives regarding health and social surveillance emerged as a significant theme throughout the pregnancy to motherhood trajectories of HIV-positive mothers from across Ontario.

FINDINGS: Experiences of pregnancy and impending motherhood are complicated for HIV-positive women by imposed socially constructed identities of "motherhood" and external and internalized manifestations of HIV-related stigma. These tensions were highlighted in women's narratives of privacy and confidentiality concerns, disclosure of their HIV status and exclusion from health and social supports. HIV-positive women encountered surveillance when navigating systems of clinical and community-based care during pregnancy, childbirth and early motherhood, which reframed and challenged their experiences. In response to social constructions of motherhood and their experiences of policing and stigmatization, HIV-positive mothers engaged in acts of resistance, which will be further explored.

CONCLUSIONS: Narratives from the HIV Mothering Study exposed the spaces where HIV-positive mothers are under surveillance providing an alternative discourse about pregnancy and motherhood in the context of living with HIV. This work highlights how enacted stigma vis-à-vis institutionalized and everyday forms of health and social surveillance silence the ways of knowing and experiences of pregnancy and motherhood for HIV-positive women.

\section{P271}

\section{POPULATION AND PUBLIC HEALTH ETHICS OF HIV TESTING PRACTICES: A SCOPING REVIEW}

Knight, Rod E; Shoveller, Jean A; Greyson, Devon; Kerr, Thomas; Gilbert, Mark; Shannon, Kate

Vancouver, BC

BACKGROUND: Over the past decade, a number of theorists have called for a more robust population and public health ethics (PPHE) framework that expands upon traditional orientations of bioethics that focus primarily on the interests of individuals, often in clinical encounters. Instead, a PPHE focuses on a critical evaluation of questions concerning possible, actual and proposed public health measures, and considers issues such as health equity, social justice and how health and risk are distributed within and across populations.

OBJECTIVES: Our objective is to examine the breadth, range and foci of a variety of ethical issues pertaining to HIV testing approaches (e.g., voluntary testing and counselling; routine testing) within the peerreviewed literature, and how these issues address population and/or individual interests within three categories: HIV-related stigma; prioritising and targeting of testing efforts; and structural and agentic factors that influence HIV infection and testing experiences.

METHODS: We conducted a thematic analysis of peer-reviewed literature pertaining to the ethics of HIV testing since 2001 ( $n=35$ articles). We used a combination of inductive and deductive analytic approaches to identify patterns across the articles and to develop an overall interpretation of the literature from a PPHE perspective.

RESULTS: We identify potential tensions between individual and collective approaches as well as other concerns, including equity, justice and distribution of health and risk. Specifically problematic were consequentialist arguments that deem testing approaches as either morally permissible or impermissible without sufficiently robust empirical and/or theoretical underpinnings about how a particular approach would unfold and impact upon individuals and populations.

CONCLUSION: The current review underscores the need to continue to articulate an evidence- and theory-informed PPHE pertaining to HIV testing. Based on our review, we suggest that additional theoretical work and empirical research are required to inform more ethically robust debates related to population HIV testing practices.

\section{P272}

\section{L'ACCOMPAGNEMENT SOCIAL DES PERSONNES QUI TÉMOIGNENT PUBLIQUEMENT DE LEUR VÉCU AVEC LE VIH/SIDA : RÉSULTATS ET RETOMBÉES DU TRANSFERT DES CONNAISSANCES AUPRÈS DES INTERVENANTS SOCIAUX, DES ACTEURS MÉDIATIQUES ET DES CHERCHEURS SUR LES QUESTIONS ÉTHIQUES ET DE SOLIDARITÉ}

Mensah, Maria Nengeh; Bernier, Mélina; Gauvin, Marie-Eve; Légaré, René

Montréal, QC

CONTEXTE : Le projet de recherche-action VIHSIBILITÉ (www.vihsibilite.uqam.ca) a démontré qu'il existe un écart inquiétant entre les besoins des PVVIH qui désirent témoigner publiquement et les outils disponibles et connus des professionnels qui les accompagnent dans cette démarche. Qu'il s'agisse des pratiques de professionnels des médias ou de chercheurs qui recueillent leurs histoires personnelles en entrevue, ou d'intervenants sociaux qui offrent soutien et information, l'accompagnement social peut prendre différentes formes. Sachant que l'influence de l'accompagnateur social sur la qualité de l'expérience du témoignage peut être grande et l'absence d'accompagnement dévastatrice, notre projet prévoitle transfert des connaissances à ce sujet.

MÉTHODE : Un questionnaire permettant d'évaluer l'atteinte des objectifs de formation et l'appréciation des activités montre des résultats positifs. Plus de $90 \%$ des participants affirment que la formation leur a permis: 1) d'améliorer leurs connaissances sur les contextes à l'intérieur desquels se déroule le témoignage public de la séropositivité au VIH; 2) de prendre conscience des enjeux du témoignage pour les PVVIH; 3) de 
saisir les principaux éléments requis pour réaliser un accompagnement social de manière éthique; et de développer des habiletés pour agir avec solidarité auprès des PVVIH qui désirent témoigner publiquement.

RÉSULTATS : Notre communication présentera les résultats d'une série de 12 séances de formations d'une journée que nous avons dispensée dans 7 régions du Québec entre 2012 et 2013 auprès de 120 participants.

CONCLUSION : Le transfert des connaissances d'un tel projet de recherche-action constitue non seulement une occasion unique de partage interdisciplinaire entre trois milieux professionnels (intervention sociale, recherche, média), mais aussi une épreuve concrète de lutte contre la stigmatisation des PVVIH à travers le renforcement des pratiques d'accompagnement social éthiques et solidaires.

\section{P273}

\section{CRIMINAL LIABILITY FOR HIV NON-DISCLOSURE: THE} SUPREME COURT OF CANADA'S DECISIONS IN R. V. MABIOR AND R. V. D.C.

\section{Kazatchkine, Cecile; Elliott, Richard; Symington, Alison}

Toronto, ON

OBJECTIVE: Understanding the Supreme Court of Canada's decisions in two landmark HIV non-disclosure cases.

METHODS:

- Intervening as a coalition before the Supreme Court of Canada.

- Analyzing the court decisions from a legal and a public health perspective.

- Informing and mobilizing community members, health and legal professionals through workshops, accessible resources, and working group.

RESULTS: Contrary to suggestions in some of the media coverage, in its decisions in R. v. Mabior and R. v. D.C., the Supreme Court made Canada's criminal law regarding HIV non-disclosure harsher, not more lenient, for people living with HIV (PHAs). PHAs must now disclose their HIV-positive status before having sex that represents a "realistic possibility of HIV transmission" or they will face criminal prosecutions for aggravated sexual assault, imprisonment and registration as a sexual offender. Contrary to the rulings of several Canadian courts below including the Manitoba and Quebec Courts of Appeal in these cases the Supreme Court decided that neither using a condom nor having a low or undetectable viral load would alone be sufficient to preclude criminal liability for HIV non-disclosure. People would need to establish, at least in cases of vaginal sex, that they both used a condom and had a low viral load at the time they had sex. How the test set out by the Supreme Court will apply to oral sex and anal sex remains an open question and prosecutions for oral sex are already underway in Ontario.

CONCLUSION: These decisions represent major steps backwards. They contradict the science related to HIV and undermine the rights of PHAs as well as public health efforts to prevent HIV transmission. Research and advocacy efforts towards limiting the criminal law against PHAs in Canada, including through the development of prosecutorial guidelines, are needed more than ever.

\section{P274}

\section{IMPROVING ACCESS TO MEDICAL CANNABIS FOR PHAS THROUGH THE COURTS}

Burstein, Paul'1 ; Elliott, Richard ${ }^{1}$; Peck, Ryan'1; Belle-Isle, Lynne ${ }^{2}$

${ }^{1}$ Toronto, ON; ${ }^{2}$ Victoria, BC

OBJECTIVE: To improve PHAs' access to cannabis as medicine

METHOD: Appellate court intervention

BACKGROUND: Canada prohibits cannabis possession via the Controlled Drugs and Substances Act (CDSA), but ostensibly provides an exemption from prosecution for those authorized to possess it for medical purposes. Under the Marihuana Medical Access Regulations (MMAR), physicians act as gatekeepers for gaining access to a licence permitting legal possession and thereby as arbiters of patients' potential criminal liability. In 2011, an Ontario trial judge ruled the MMAR breach section 7 of the Canadian Charter of Rights and Freedoms: the regulations deprive seriously ill persons of their right to security of the person by denying them effective access to exemption from prosecution which deprivation does not accord with principles of fundamental justice. The federal government appealed. In 2012, three HIV organizations jointly intervened; the ruling is pending as of mid-January 2013. Meanwhile, the government has proposed MMAR reforms, but these will not resolve the concerns raised in this case.

CONCLUSIONS: The HIV groups endorsed arguments by civil liberties groups, and also argued that the MMAR are: (1) arbitrary in their effect by compelling "doctor-shopping"; and (2) lack adequate procedural safeguards to ensure a patient can obtain a full, fair and timely assessment of the merits of his or her application by an impartial decision-maker obliged to provide reasons for the decision. The lack of an alternative assessment mechanism for patients who lack access to a knowledgeable doctor unacceptably shifts consideration of some patients' claims for medical treatment to criminal courts. The government must create a viable alternative means for medical cannabis applicants to have their cases decided on the merits. Meanwhile, the court should order a moratorium on cannabis possession and production charges against anyone who can document an illness covered by MMAR.

\section{P275}

\section{PRINCIPLES INTO PRACTICE: LESSONS FROM A COMMUNITY-BASED RESEARCH PROJECT TO DEVELOP A REHABILITATION SERVICE DELIVERY MODEL}

Restall, Gayle; Carnochan, Tara; Roger, Kerstin; Sullivan, Theresa; Roddy, Pumulo; Etcheverry, Emily

Winnipeg, MB

BACKGROUND: The principles of greater and meaningful involvement of people living with HIV/AIDS (GIPA/MIPA) are grounded in the human rights of people to have a say in decisions that affect them. These principles are consistent with community-based research's emphasis on inclusion of people who are focus of the research in all stages of the research process.

PURPOSE: The purpose of this community-based research project was to identify important components of a rehabilitation service delivery model and priorities for future research while applying the principles of GIPA/MIPA.

APPROACH: Clients and staff of a community-based centre specializ ing in HIV prevention and care identified the need for supports and services to promote community participation. The centre and researchers partnered to develop a rehabilitation service delivery model and research priorities. People living with HIV were engaged in diverse ways throughout the process.

FINDINGS: The resulting service delivery model and research priorities were highly influenced by the expertise of people living with HIV. People living with HIV had opportunities to learn more about research, and utilize or develop research skills. Researchers benefited from greater understanding of the realities of living with HIV. The process highlighted several lessons around three pillars of engagement: 1) responsively to the ideas and goals of people living with HIV, 2) accessibility to research processes in diverse ways with attention to the creation of environments that promote engagement and equality, and 3) humility in the openness to mutual learning and transformation.

CONCLUSIONS: The actualization of the principles of GIPA/MIPA can be challenging for service providers and researchers. The lessons learned from the approaches within the three pillars of engagement used in this community-based research project can be applied to diverse opportunities to engage people living with HIV in decisions about research, services and policies that affect their lives. 
Research on Prevention and Support Recherche sur la prévention et le soutien

\section{P276}

WHAT ASIAN WOMEN IN CANADA THINK ABOUT HIV/ AIDS PREVENTION

Leonard, Lynne'; McWilliam, Sue ${ }^{2}$; Sutdhibhasilp, Noulmook ${ }^{3}$; Chikermane, Vijaya ${ }^{3}$; Loutfy, Mona ${ }^{3}$; Millson, Peggy ${ }^{3}$; Ramis, Robert ${ }^{3}$; Rowe, Janet ${ }^{3}$; Tharao, Wangari ${ }^{3}$; Yee, June ${ }^{3}$ 1Ottawa, ON; ${ }^{2}$ Halifax, NS; ${ }^{3}$ Toronto, ON

BACKGROUND: In Canada, women accounted for $23 \%$ of people living with HIV (2011). Yet there is little research on the HIV prevention needs of culturally diverse communities of women. The Ontario Women's Study (OWS) aims to address this gap in knowledge. The study includes a comprehensive qualitative analysis of women's attitudes towards HIV/ AIDS; their existing knowledge of risk; the individual and structural forces that add to risk; and, the strategies for prevention that are most relevant to their lives. This presentation is focused on the findings from the Asian women's components of the overall study in Ontario.

METHODS: Focus groups among East and Southeast Asian, and of South Asian women, facilitated by trained community-based researchers, were conducted separately. Participants were recruited through community-based organizations, members of the expert working group and through snowballing. Discussions were audio-recorded, transcribed verbatim, coded and analyzed thematically.

RESULTS: Twenty participants (mean age 40; range $=23$ to 69 years) spoke English, Tamil, Urdu, Chinese, Filipino, and other languages. From 2005 to 2010, the greatest proportion of women (45\%) never had an HIV test whereas 30\% had had an HIV test. They identified the following principal factors influencing HIV prevention:

1) Lack of discussion about HIV within Asian communities

2) Male-dominated or abusive relationships

3) Stigma surrounding HIV, sex and sexuality, perpetuating a lack of HIV-related education, resources and deterrence towards testing.

4) Gender norms infused with cultural and religious tradition, which hinder women to openly discuss their sexual health needs as related to HIV. Some see HIV as a 'moral' issue.

5) Pressure to maintain sexual innocence to preserve reputation and desirability.

6) Financial or social dependence on men, which increased HIV risk for heterosexual women with fewer options to ensure survival and safety. CONCLUSIONS: The women identified actionable strategies for HIV prevention for policy makers and service providers such as public and culturally-tailored health education embedded in more general health and well being programs to reduce community fear around HIV and stigma.

\section{P277 \\ HIV TESTING RATES AMONG THE ABORIGINAL POPULATION OF NOVA SCOTIA: A FIRST NATION PERSPECTIVE}

Barbour, Tammy L

Dartmouth, NS

BACKGROUND: Aboriginal peoples represent approximately 3\% of the Canadian population, and are over represented in the rate of Human Immunodeficiency Virus (HIV) infections where, 20\% of new HIV infections are among Aboriginal populations of Canada (Canadian Aboriginal AIDS Network, 2010). However, the rates of HIV infection among Atlantic-based Aboriginal populations are challenging to identify due to the small population size and issues of confidentiality. HIV infection rates in provinces with smaller populations are combined where, for example, Nova Scotia (NS) and Prince Edward Island (PEI) combine positive HIV test results to help ensure confidentiality. This creates challenges in understanding the true impact of HIV within the Atlantic Region, particularly given the undiagnosed fraction of the population.
METHODS: This exploratory qualitative study identifies knowledge and understanding about HIV, testing and barriers/opportunities within Nova Scotia's Aboriginal population. Data collected from NS Aboriginal communities: both on and off reserve, through an anonymous questionnaire distributed through a NS-based AIDS Service Organization identifies why or why not people have been tested for HIV. Data from focus groups, both on reserve and off reserve, identify perceptions of HIV testing within the Aboriginal communities; acceptability of HIV testing; the barriers to getting tested; and what would make HIV testing more accessible within the Aboriginal communities of NS.

FINDINGS: HIV testing among the Aboriginal population of NS remains a challenge. The stigma associated with HIV and the perceived negligible risk for HIV infection, remain barriers in the NS Aboriginal communities.

IMPLICATIONS: The findings of this study provide crucial information for developing culturally competent, realistic and effective health promotion initiatives for HIV testing within NS Aboriginal communities. Further, these findings will provide additional and contributing information about HIV among the Aboriginal population of Canada with the aim of augmenting the effectiveness and responsiveness of prevention, education and support initiatives in the Atlantic region.

\section{P278 \\ "SAFER ENVIRONMENT" INTERVENTIONS: A QUALITATIVE SYNTHESIS OF THE EXPERIENCES AND PERCEPTIONS OF PEOPLE WHO INJECT DRUGS}

\section{McNeil, Ryan; Small, Will}

Vancouver, BC

BACKGROUND: Safer environment interventions (SEI) have been increasingly identified as critical to mitigating the impacts of social, structural, and environmental forces on HIV transmission and other drug-related harms among people who inject drugs (PWID). To date, SEIs have been under-theorized, and how they minimize HIV and other health risks has not been systematically investigated. This review synthesizes qualitative studies exploring PWID's experiences with three types of SEIs (syringe exchange programs, supervised injection facilities and peerbased harm reduction interventions) to develop a comprehensive understanding of how these interventions minimize drug-related harms.

METHODS: A systematic literature search of nine academic databases was undertaken to identify eligible articles, and citation reports and reference lists were also reviewed. Only peer-reviewed articles reporting the direct experiences of PWID with SEIs were included. Articles were synthesized using a meta-ethnographic approach.

RESULTS: This synthesis included 29 papers representing 21 unique studies that included an aggregate of more than 800 PWID, and found that SEIs fostered social and physical environments that mitigated HIV and other risks as well as increased access to social and material resources. Specifically, the experiences of PWID indicated that SEIs: (1) provided refuge from street-based drug scenes; (2) enabled safer injecting by reshaping the social and environmental contexts of injection drug use; and, (3) mediated access to resources and health care services, including HIV treatment. Nonetheless, this synthesis underscored how the effectiveness of SEIs continues to be constrained by drug law enforcement in many settings.

CONCLUSION: These findings highlight the importance of situating SEIs in relation to the lived experiences of PWID, and in particular integrating broader environmental supports into these interventions. Given that existing drug laws limit the effectiveness of interventions, drug policy reforms are needed to enable SEIs to occupy a more prominent role in the response to injection drug use. 


\section{P279}

"I'VE LEARNED HOW TO SURVIVE, BUT NOT LEARNED HOW TO LIVE": NEEDS AND SERVICES IN TRANSITIONAL HOUSING PROGRAMS IN ONTARIO FOR PHAS

Aguinaldo, Jeffrey; P1; Ahluwalia, Amrita ${ }^{2,3}$; Hambly, Keith ${ }^{2}$; Klusacek, Allan ${ }^{2}$; Koornstra, Jay ${ }^{4}$; Rankin, Bruce ${ }^{4}$; Roesslein, Kay ${ }^{2}$ ${ }^{1}$ Waterloo; ${ }^{2}$ Toronto; ${ }^{2}$ Ottawa; ${ }^{4}$ London, ON

The Transitional Housing Study is a multi-site community-based pilot project. Focusing specifically on transitional housing programs in Ontario for PHAs, the pilot project aims to understand the individual and structural factors relevant to 'housing readiness' among PHAs, the range of support services and practices that best enhance 'housing readiness,' and the changing needs of PHAs throughout residency. In this paper, we report on initial findings of the project and, in particular, identify key services PHAs have accessed in transitional housing that potentially encourages housing readiness. We detail the range of needs these services address.

METHODS: In-depth qualitative interviews were conducted with twenty-five residents in the three transitional housing programs specifically for PHAs in Ontario (i.e., Fife House Foundation, John Gordon Home, and Bruce House). Phase I of the project involved qualitative interviews with participants at the time of their entry into transitional housing program and is the focus of the current presentation. Our analysis of the data is best characterised as thematic analysis.

FINDINGS: Participants entered into transitional housing with a variety of needs with regards to health care, financial support and assistance with activities of daily living. Although already connected with a variety of services from various AIDS service organizations, participants reported three key services provided by transitional housing programs that encouraged housing readiness among participants: management of HIV drug therapies, personal assistance with appointments, and service referral. CONCLUSIONS: We link these services to a broader discussion of health and social services and the needs they are designed to address. We distinguish between ameliorative needs, which require services that maintain 'normative' states of well-being with transformative needs, which require services that enable PHAs with new skills and, potentially, change the course and direction of their lives.

\section{P280 \\ EVIDENCE-INFORMED RECOMMENDATIONS IN REHABILITATION FOR OLDER ADULTS LIVING WITH HIV: A KNOWLEDGE SYNTHESIS}

$\mathrm{O}^{\prime}$ Brien, Kelly K ${ }^{1}$; Solomon, Patricia'; ${ }^{2}$ Baxter, Larry';

Casey, Alan ${ }^{1,3}$; Tynan, Anne-Marie'; Wu, Janet ${ }^{1}$; Zack, Elisse';

Knowledge Synthesis Team, and the HIV and Rehabilitation ${ }^{1}$

${ }^{1}$ Toronto; ${ }^{2}$ Hamilton, ON; ${ }^{3}$ Winnipeg, MB

OBJECTIVE: To develop evidence-informed recommendations on rehabilitation for older adults living with HIV.

METHODS: We conducted a knowledge synthesis, combining research evidence specific to HIV, rehabilitation and aging, with evidence on rehabilitation interventions for common comorbidities experienced by older adults with HIV. We searched for and included: a) highly relevant HIV-specific research addressing rehabilitation and aging and b) highquality evidence (systematic reviews and meta-analyses) on the effectiveness of rehabilitation interventions for comorbidities commonly experienced by older adults aging with HIV (bone and joint disorders, cancer, cardiovascular disease, mental health, neurocognitive decline, cardiopulmonary disease, diabetes). We extracted and synthesized relevant data from included studies to draft evidence-informed recommendations on rehabilitation for older adults aging with HIV. Draft recommendations were refined based on PHA and clinician values and preferences and then circulated to an interprofessional team for GRADE (quality) rating and further refinement.

RESULTS: This synthesis yielded 55 recommendations. Thirty-nine recommendations were derived from 109 moderate or high level research evidence articles (meta-analyses and systematic reviews) that described the effectiveness of rehabilitation interventions for adults living with health conditions that may be experienced by older adults with HIV. Recommendations address rehabilitation interventions specific to adults with HIV and across eight health conditions experienced by older adults with HIV: bone and joint disorders, cancer, stroke, cardiovascular disease, mental health, cognitive impairments, chronic obstructive pulmonary disease, and diabetes. Sixteen recommendations were derived from 42 research evidence articles specific to rehabilitation for older adults with HIV. The quality of evidence from which these recommendations were derived was either low or very low, consisting primarily of narrative reviews or descriptive studies with small sample sizes. Recommendations address approaches to rehabilitation assessment and interventions, and contextual factors to consider with rehabilitation of older adults living with HIV.

CONCLUSIONS: These evidence-informed recommendations provide comprehensive guiding principles for rehabilitation with older adults with HIV and those who may present with comorbidities. PHA and clinician values and preferences were integral in developing these recommendations.

\section{P281 \\ RETENTION OF INDIVIDUALS IN AN ON-LINE RANDOMIZED CONTROL PILOT STUDY}

Miranda, Joyal

Toronto, ON

INTRODUCTION: Men having Sex with Men (MSM) population, many individuals engage in risky sexual behaviors using the internet to meet their sexual partners. Developments of HIV prevention interventions using the internet have begun to emerge, yet many do not discuss the challenges particular to this medium of delivery. This study examines the retention challenges inherent in an on-line intervention to increase condom use among HIV-positive men having sex with men.

METHODS: The study used a two-armed randomized controlled pilot design. HIV-positive MSM who currently engage in unprotected anal sex were recruited for the study. Individuals were randomly assigned either to the Condom-HIM intervention (experimental) condition or to view a list of websites containing HIV/AIDS related information (control condition). Self-administered questionnaires were provided online prior to randomization and two weeks post intervention.

RESULTS: A total of 30 individuals were recruited for the study. Of those recruited, four were ineligible to participate in the study, six were loss to follow-up and twenty completed the study. The results demonstrated that of the twenty individuals who completed the study, fifteen individuals were given additional reminders to complete the two week questionnaire. On average, three additional reminders (one per week) were sent to individuals $(n=9)$. After three reminders many of the participants did not complete post-test measures. Participants that were loss to follow-up only one was due to an invalid e-mail address. The remainder of the individuals' reasons for loss is not available, as the only method of contact was through e-mail. Potential reasons for loss could have been participants' loss in interest in the study and e-mail reminders may have been filtered into the participant's junk-mail box.

CONCLUSION: Many lessons are still to be learnt in the particular strategies available for the retention if individuals participating in on-line studies.

\section{P282}

POTENTIAL BARRIERS AND FACILITATORS TO UPTAKE OF A FUTURE HIV VACCINE BY MARGINALISED COMMUNITIES: PERSPECTIVES OF FRONTLINE HEALTHCARE WORKERS IN KARNATAKA, SOUTH INDIA

McClarty, Leigh M ${ }^{1}$; Ramanaik, Satyanarayana ${ }^{2}$; Lorway, Robert ${ }^{1}$; Wylie, John'; Becker, Marissa ${ }^{1}$

${ }^{1}$ Winnipeg, MB; ${ }^{2}$ Bangalore, India

BACKGROUND: Approximately 2.4 million people in India live with HIV. The southern state of Karnataka has the nation's fifth-highest HIV prevalence at $0.63 \%$; its epidemic is largely driven by commercial sex work. HIV disproportionately burdens Karnataka's >100,000 female sex 
workers (FSWs) and 25,000 men who have sex with men (MSM) $16.3 \%$ and $17.6 \%$ prevalence, respectively. The best long-term strategy for managing the global HIV epidemic likely involves an effective vaccine. However, the availability of a vaccine will not guarantee its accessibility or acceptability. Therefore, a better understanding of the barriers and facilitators to delivery and uptake of a potential HIV vaccine among these key populations is required.

METHODS: We conducted a cross-sectional survey among healthcare workers (HCWs) in Karnataka who worked with MSM/FSWs in relation to HIV prevention, care and support for $>1$ year. Trained interviewers administered face-to-face structured interviews with open-ended, precoded questions. We used multistage, random sampling with stratification by district, target population, type of clinic/organization, and type of HCW. Data collection was conducted from September to December 2012.

RESULTS: In total, $379 \mathrm{HCW}$ sarticipated in the study; all refusals ( $<3 \%$ refusal rate) were by government-affiliated HCWs. More participants worked in urban $(58 \%)$ versus rural $(42 \%)$ districts. Fifty-seven per cent of participants worked at government-affiliated facilities, $29 \%$ at FSW-specific CBOs/NGOs, and 14\% at MSM-specific CBOs. Results addressing specific barriers/facilitators to vaccine uptake and delivery, HCWs' likelihood to recommend an HIV vaccine, and whether vaccine characteristics affect acceptability are pending.

IMPLICATIONS: This study contributes to the limited vaccine acceptability literature focusing on marginalized groups in low- and middle-income countries and the knowledge gap pertaining to HCWs' perspectives on future HIV vaccine acceptability amongst at-risk populations. Considering HCWs' perspectives on HIV vaccine acceptability might elucidate some stigma-related barriers to HIV vaccine uptake that MSM/ FSWs do not feel comfortable discussing. Information gleaned will also be useful for policy-makers involved in vaccine rollout and for developing advocacy strategies to influence Indian and international health authorities.

\section{P283}

WILLINGNESS TO PARTICIPATE IN HIV VACCINE TRIALS AMONG MEN WHO HAVE SEX WITH MEN IN CHENNAI AND MUMBAI, INDIA: A SOCIAL ECOLOGICAL

\section{APPROACH}

Chakrapani, Venkatesan ${ }^{1,2} ;$ Newman, Peter; A $^{3}$; Singhal, Neeti ${ }^{2}$; Jerajani, Jhalak'; Shunmugam, Murali ${ }^{1,2}$

${ }^{1}$ Chennai; ${ }^{2}$ Mumbai, India; ${ }^{3}$ Toronto, ON

BACKGROUND: India, with 2.4 million people living with HIV and 120,000 annual HIV-incident infections, has the highest disease burden of HIV in Asia, and a concentrated epidemic among men who have sex with men (MSM). Yet out of $>235$ HIV vaccine trials conducted globally, two phase-I trials were completed in India. Investigations of willingness to participate (WTP) in HIV vaccine trials have focused predominantly on individual-level determinants and high-income countries. We explored multi-level factors associated with WTP among MSM in India. METHODS: We conducted a qualitative investigation using a community-based approach; community-based organizations ( $\mathrm{CBO}$ s) in Chennai and Mumbai collaborated in study design, instrument development, data collection and interpretation. Twelve focus groups $(n=68)$ with lowsocioeconomic MSM and 14 key informant interviews with MSM community leaders/service providers were recorded, transcribed and translated into English. Two bilingual investigators conducted thematic analysis using line-by-line, axial and focused coding and a constant comparative method, with member-checking by community representatives.

RESULTS: Factors associated with WTP were evidenced across the social ecology of MSM - social-structural: poverty, HIV-, sexual- and gender non-conformity stigma, institutionalized discrimination; community-level: endorsement by MSM community leaders/organizations, fear of in-group discrimination; interpersonal: anticipated family discord, partner rejection, financially-dependent family members, sexual orientation disclosure; and individual-level: HIV vaccine trial knowledge/misconceptions, safety concerns, altruism and preventive misconception.
CONCLUSIONS: Decision-making about WTP was embedded in social-structural, community and familial spheres of influence. By focusing on individual-level determinants of WTP we risk misunderstanding and misconceptualizing barriers and motivators to participation, failing to mitigate ethical challenges and may thereby limit the effectiveness of interventions designed to support recruitment and retention in biomedical HIV prevention trials. Combatting stigma and discrimination against MSM and people living with HIV, supporting research literacy among low-socioeconomic communities and capacity-building for MSM CBOs may advance meaningful engagement of MSM in India in HIV vaccine trials.

\section{P284}

SYSTEMATIC CONDOM USE AND DATING VIOLENCE AMONG SEXUALLY ACTIVE ADOLESCENTS AGED 14 TO 20 IN THE PROVINCE OF QUEBEC: DATA FROM THE WAVE 1 OF THE YOUTHS' ROMANTIC RELATIONSHIPS SURVEY

Blais, Martin ${ }^{1}$; Hébert, Martine ${ }^{1}$; Lavoie, Francine ${ }^{1,2}$

${ }^{1}$ Montréal; ${ }^{2}$ Québec, QC

CONTEXT: Since 2000, the 15 to 29 year age group has accounted for $21 \%$ to $23 \%$ of annual HIV test reports and trends data suggest a slightly but constant increased in this rate (PHAC, 2010). As condom remains the best prophylaxis against HIV/STIs, monitoring its use and correlates is a priority.

OBJECTIVES: 1) To document systematic condom use among sexually active Quebec teenagers; 2) To identify psychosocial factors influencing condom use.

METHOD: Data were collected among 8194 high school students (aged 14 to 20 years) across the province of Quebec via a stratified, clustered sampling study. Condom use consistency and its correlates were explored among sexually active participants who reported a romantic relationship in the last 12 months $(41.6 \%$; $=3405)$.

RESULTS: Forty per cent of the participants reported systematic condom use when engaging in consensual vaginal or anal intercourse. Participants were less likely to report systematic condom use if they were female with male-only partners $(\mathrm{OR}=0.64)$, when compared to men with female partners only; older $(\mathrm{OR}=0.90)$; and if they reported experiencing emotional $(\mathrm{OR}=0.79)$ or physical abuse $(\mathrm{OR}=0.68)$ in dating relationships. Female with female-only partners, participants with both female and male, and male with male-only partners did not differ from male with female-only partners. Self-esteem and other forms of dating victimization (threatening behaviors, sexual coercion) were not associated with systematic condom use.

CONCLUSION: Adolescent girls with only male partners are less likely to report systematic condom use. Emotional and physical dating victimization appears to impair consistent condom use. Possible explanations are that they are more likely to delegate the choice of condom use to their male partners; dating violence may influence teenagers' sense of self-efficacy in regard to condom use, limiting the negotiation power of teenage girls that anticipate negative reactions from their partners. Other explanations and implications for condom promotion among adolescents are discussed.

\section{P285}

SEXUALITÉ SOUS INFLUENCE : LES INTERRELATIONS ENTRE LA CONSOMMATION PROBLÉMATIQUE DE SUBSTANCES PSYCHOACTIVES (SPA) ET LES COMPORTEMENTS SEXUELS À RISQUE CHEZ LES HOMMES AYANT DES RELATIONS SEXUELLES AVEC D'AUTRES HOMMES (HARSAH)

Flores-Aranda, Jorge; Bertrand, Karine; Roy, Élise Longueuil, QC

PROBLÉMATIQUE : Les HARSAH consomment davantage de SPA que les hommes hétérosexuels et leur consommation est associée à des comportements sexuels à risque. Toutefois, peu d'études décrivent qualitativement les interrelations entre ces comportements. 
OBJECTIF : Décrire les interrelations entre la consommation problématique de SPA et les comportements sexuels à risque du point de vue des HARSAH eux-mêmes.

MÉTHODOLOGIE : Nous menons une étude qualitative avec une perspective interactionniste symbolique. Des entrevues semi-dirigées sont effectuées auprès d'HARSAH ayant une consommation problématique de SPA (18 ans et plus, s'auto-identifiant comme gais/bisexuels, nés au Canada et habitant le grand Montréal). Le recrutement s'effectue par divers moyens (organismes communautaires, annonces dans des journaux, bouche-à-oreille). Une analyse thématique a été effectuée sur 16 entrevues.

RÉSULTATS : La plupart des participants accordent une grande importance à la sexualité. Chez eux, la consommation de SPA est très associée à la sexualité. La consommation leur permet de vivre plus librement leur sexualité, de chercher de nouvelles sensations, d'avoir un sentiment d'appartenance au groupe des HARSAH. Quelques-uns en viennent à se sentir incapables de renoncer à une sexualité sous l'effet de SPA. Certains rapportent des changements dans leurs comportements sexuels en lien avec leur consommation: exploration de nouvelles pratiques, multipartenariat, sexualité anonyme. Ces comportements parfois associés à la consommation de SPA, le sont aussi à une multiplicité de facteurs (difficultés personnelles, statut $\mathrm{VIH}+$, banalisation de comportements à risque). Toutefois, plusieurs participants rapportent aussi adopter spontanément des pratiques de réduction des risques sexuels.

DISCUSSION : Des interventions intégrées (sexualité-toxicomanie) devraient être mises en place afin de tenir compte des interrelations entre la consommation de SPA et la sexualité. Ces interventions devraient aussi tenir compte des stratégies de réduction des risques sexuels adoptées par les HARSAH.

\section{P286 \\ OPENING UP THE MODEL OF PEER WORK IN HIV- RELATED HARM REDUCTION PROGRAMS}

Penn, Rebecca A ${ }^{1}$; Strike, Carol J1; Mukkath, Sabin ${ }^{1}$;

Henschell, Calvin ${ }^{1}$; Andrews, John ${ }^{1}$; Danis, Carol' ${ }^{1}$;

Thompson, Marty' ${ }^{1}$; Thorpe, Mark¹; Gao, Yun²; Miller, Cherie ${ }^{1}$

${ }^{1}$ Toronto, ON; ${ }^{2}$ Montreal, QC

BACKGROUND: Peer work is a critical feature of HIV-related harm reduction programs at community health centres (CHCs). Designed to provide low threshold employment opportunities, peer work can spark and contribute to better health, increased self-esteem, greater stability, and development of new goals. Our goals were to understand how peer programs are designed and analyze benefits and challenges.

METHODS: Conducted in Toronto, Canada this community-based, mixed methods study was overseen by an advisory committee of peer workers, CHC staff members and researcher, graduate students and an academic. Data were collected during fourteen in-depth interviews with peer workers, CHC staff, and program clients, as well as two focus groups with peer workers $(n=12)$. All participants completed a demographic questionnaire. A thematic analysis was undertaken to describe the program model and analyze factors that contribute to role tensions for peer workers.

RESULTS: The positive outcomes of peer involvement including skill development provided an avenue through which the $\mathrm{CHC}$ expanded its peer program along a continuum ranging from a peer participation model to an employment model. This enables the engagement of a diverse group of peers including those who want the informality of a low-threshold, peer-participation model, and those who are looking for intensive training and employment opportunities. However, tensions emerge that pose a challenge to the sustainability and effectiveness of an approach that seeks to meet the needs and expectations of such a diverse population. IMPLICATIONS: In environments where demand for peer opportunities is high and resources are scarce, agencies may have to decide to focus on either a model of peer work that concentrates resources on a smaller group of experienced peers, or a model that offers opportunities for new people to experience that 'taste' of peer work that has spurred others to build significant changes in their lives.
P287

\section{SEXUAL COMPULSION IN A MULTIPLE TREATMENT SITE} SAMPLE OF SUBSTANCE ABUSE PROBLEM CLIENTS

Roth, Eric A; MacDonald, Scott; Martin, Gina

Victoria, BC

BACKGROUND: Sexual compulsion has been linked to high risk sexual behaviour and HIV/STI infection, particularly in gay/bisexual men. This study reports on high-risk sexual behaviour patterns associated with clients classified as sexually compulsive recruited from five treatment centres in Ontario and British Columbia.

METHODS: A sample of 408 people in-treatment for either cocaine or cocaine and alcohol substance abuse problems were analyzed with respect to sexual compulsion, STI history, and substance use and sexual behaviour patterns via univariate and multivariate methodologies.

RESULTS: The Sexual Compulsivity Scale developed by Kalichman and Rompa (1995) classified 15\% ( $\mathrm{n}=63)$ of the sample as sexually compulsive, exceeding levels found in the general population (3\% to $6 \%$ ). In bivariate analysis this group uniformly reported higher prevalence levels for a range of STIs, including HIV, featured significantly $(\mathrm{p}<0.05)$ different substance use patterns, with alcohol, ecstasy, crack/cocaine, marijuana/hashish strongly linked to sexual behaviour, and were significantly $(p<0.001)$ more likely to engage in commercial sex and have multiple sex partners. Multivariate logistic regression analysis showed that paying for sex $(A O R=2.97)$ and having multiple partners $(A O R=3.99)$, were significant $(\mathrm{p}<0.001)$ predictors of sexual compulsive group membership; age, gender and sexual orientation were all non-significant.

CONCLUSION: In this sample sexual compulsiveness occurs at elevated levels, is associated with higher STI prevalence, and is an indicator of high-risk sexual behaviour. While sexual compulsion's links to HIV risk are most commonly studied among gay and bisexual men, this study suggests it is important in other populations as well.

\section{P288 \\ PUTTING RESEARCH INTO ACTION: THE DEVELOPMENT OF A NEW HIV RISK COMMUNICATION TOOL FOR EDUCATION AND PREVENTION}

Manno, Michael; Burchell, Ann N

Toronto, ON

ISSUE: Communicating information on HIV transmission risk which incorporates information from disparate studies can be challenging Feedback from health educators identified the need for a comprehensive tool which would incorporate the most up-to-date information available and provide a measure of risk for a wide range of scenarios.

METHODS: Based on a literature review and a published Bernoull mathematical model (Fox, AIDS 2011; 25(8):1065-82), we piloted an application which provides evidence-based risk estimates for HIV transmission in serodiscordant couples using a defined set of model parameters. The application allows the user to create scenarios based on type of sex act and multiple risk/protective factors and to graphically see their impact on transmission risk (with error) in a single encounter and accumulated over multiple encounters. Risk/protective factors incorporated included: stage of HIV infection, presence of STIs, condom use, and ARV treatment among others. We made further modifications based on preliminary feedback from presentations to policy analysts, HIV service workers, and sexual health educators and counselors.

LESSONS LEARNED: Overall, feedback was very positive. Audience members found the tool novel and there was unanimous agreement that it would be useful for health educator training. Audiences were mixed in their opinions regarding appropriateness of such a tool for direct risk communication to clients, given fears that some may not understand the imprecision of statistical probabilities. The specification of parameter values was also a topic of debate.

RECOMMENDATIONS: Quantitative tools can help to combine HIV transmission risk information from multiple studies. Although the ability of any tool to incorporate all real-life scenarios is limited, their clinical relevance and compactness make for a good starting point. We believe that this approach has potential to be used as part of a prevention cam- 
paign or as an adjunct to safer sex counseling. Further evaluation in various formats and with larger audiences should be explored.

\section{P289 \\ ARE PUBLIC HEALTH AND HEALTH SERVICE AGENCIES PROVIDING USEFUL WEB CONTENT FOR TODAY'S INTERNET SAVVY YOUTH?}

George, Clemon; Higginson, Alyssa; Chartier, Lindsay; Service, Dorothea

Oshawa, ON

BACKGROUND: Provision of sexual health services for youth is a crucial prevention strategy. This abstract explores today's web connected youths' perception of sexual health services in the Durham region, Ontario.

METHODS: Focus group participants were assigned into one of four groups (urban, rural, a semi-rural and a group of gay, lesbian, bisexual and other sexually diverse youth (LGBT)), to reflect a diverse range of sexual health needs of youth in Durham Region. These groups met three times over the course of the 2011 summer to discuss their sexual health needs. Youth were consented before participating in the study. After having a light supper, the facilitator began meetings by introducing ice breakers before introducing the questions. Each meeting lasted for approximately two hours. All meetings were held at youth-friendly, accessible and safe locations. Discussions were recorded and were later transcribed verbatim. Using Nvivo, themes that arose in the transcripts were treated as outcomes.

RESULTS: Youth ranged in age from 15 to 24 years, with a mean age of 19. There were 32 participants - 19 females, 12 males, one transgender; 16 self-described as heterosexuals. All youth identified the Internet as a key source for information and the preferred medium for sexual health information. They used it to search for information that they may not have otherwise asked a friend, peer, parent, or health services provider. When they did ask, they first armed themselves with information through Google searches. Youth were aware of unreliable information on websites or in Chat forums and said that they would prefer to receive this information from credible websites such as school or a health agency. However, these sites were too clinical in content.

CONCLUSION: Youth want pragmatic information on sexual health from reliable web sites. Materials on sexual pleasure and intimacy are needed on public health websites or youth may be forced to search questionable websites for this information.

\section{P290}

\section{EXPERIENCES OF SEXUAL OBJECTIFICATION, BODY} IMAGE, \& SEXUAL RISK AMONG ETHNORACIALIZED GAY, BISEXUAL AND OTHER MEN WHO HAVE SEX WITH MEN IN TORONTO: COMPARISONS BETWEEN FOREIGNBORN AND CANADIAN-BORN PARTICIPANTS

Brennan, David J' ${ }^{1}$; Souleymanov, Rusty ${ }^{1}$; George, Clemon²; Hart, Trevor $A^{1}$; Newman, Peter $A^{1}$

${ }^{1}$ Toronto; ${ }^{2}$ Oshawa, ON

BACKGROUND: Immigrant ethnoracialized men who have sex with men (MSM) are at increased risk for HIV. This study compared the demographics, body image, experiences of sexual objectification and HIV risk among Canadian-born and foreign-born ethnoracialized MSM. METHODS: An online survey of 410 ethnoracialized MSM included socio-demographic, health, and psychosocial characteristics of 257 foreignborn and 124 Canadian-born participants. Body image was measured using the Drive for Muscularity Scale. Sexual objectification was measured using the Sexual Objectification Experiences Scale. HIV risk was measured as reported serodiscordant unprotected anal intercourse (SDUAI; unknown status was considered serodiscordant) in the last 6 months. Relationships between drive for muscularity, sexual objectification, demographic factors (age, income, time residing in Canada, sexual orientation) and HIV risk were examined using bivariate tests and logistic regression.

RESULTS: Analysis included 389 men who completed all items. Participants identified as: South Asian ( $\mathrm{n}=82 ; 21.1 \%)$, Black/African/
Caribbean $(\mathrm{n}=84 ; 21.6 \%)$, Latino/Brazilian $(\mathrm{n}=94 ; 24.2 \%)$, East/ Southeast Asian $(n=105 ; 27 \%)$, and mixed race $(n=24 ; 6.2 \%)$. The mean age was 33 years $(\mathrm{SD}=8.6)$. Among respondents, $64(16.9 \%)$ were HIVpositive, $284(75.1 \%)$ were HIV-negative, and 30 (7.9\%) were unsure of their HIV serostatus. Differences among the sample include foreign-born participants were more likely to be HIV-positive; SDUAI was less likely to be reported by East/Southeast Asian men (compared to other ethnoracial groups) and more likely to be reported by those with unsure HIV status (compared to HIV-positive or HIV-negative). The logistic regression analysis indicated that drive for muscularity predicted SDUAI among Canadian-born participants (OR 1.97 [95\% CI 1.23 to 3.16]). Among foreign-born participants, experiences of sexual objectification (OR 1.62 [95\% CI 1.08 to 2.41]) and lower income (OR 3.30 [95\% Cl 1.42 to 7.67$])$ predicted SDUAI.

IMPLICATIONS: Having lower incomes and being sexually objectified may impact the agency of foreign-born ethnoracialized men in relation to HIV risk. Development of culturally relevant supportive prevention services that address the social determinants of health for foreign-born eth noracialized MSM is critical.

\section{P291}

WELTELBC1: A QUALITATIVE ANALYSIS OF PARTICIPANT AND HEALTH CARE WORKER VIEWS OF FEASIBILITY AND ACCEPTABILITY OF AN MHEALTH INTERVENTION FOR IMPROVING CARE FOR HIV POSITIVE PATIENTS ON HIGHLY ACTIVE ANTIRETROVIRAL THERAPY (HAART) IN BRITISH COLUMBIA (BC), CANADA

Borek, Natasha V; Lester, Richard; Graham, Rebecca;

Maan, Evelyn J; Friesen, Karen; van der Kop, Mia L; Levine, Sarah; Abaki, Joshua; Pick, Neora; Maginley, Juanita; Ogilvie, Gina;

Money, Deborah M; Murray, Melanie C

Vancouver, BC

INTRODUCTION: HAART improves health and survival of HIV+ individuals. However, engagement in care and medication adherence are essential to prevent resistance, morbidity and mortality. In a randomized control trial in Kenya, WelTelKenya1, a weekly mHealth (mobile phone technology for health care) intervention improved HAART adherence and viral suppression. We conducted a six-month pilot study of a weekly interactive text-messaging intervention using the WelTel model with 25 participants to assess acceptability and feasibility in HIV+ individuals taking HAART in BC

METHODS: Five participants each from five groups ('youth', 'mature', 'English as a second language', 'remote', and 'CD4 <200') were recruited from the Oak Tree Clinic, from January-May 2012. Participants were given a cell phone with unlimited texting if they did not have one, then received weekly text messages stating "How are you?" Nurses responded to negative and late responses. Demographics, clinical data, and weekly responses were collected throughout the study. Baseline and exit semistructured qualitative interviews/questionnaires were conducted with participants and health care staff to explore intervention feasibility and acceptability. Interviews were audio-recorded and transcribed verbatim. Thematic analysis was conducted.

RESULTS: Perceived benefits included 'someone caring about you/your health', feelings of not being alone, appointment/test reminders, help with medication side effects/reordering, and help with crises. Challenges included language of text, difficulties texting responses, and service provider issues/reception. Recommendations included varying frequency and/or content of text messages, sending messages in preferred language, appointments /medication reminders, and receiving results via text message. Overall, participants and health workers perceived the intervention as feasible, acceptable, and an improvement in care. Of 20 exit interviews completed, all would recommend the intervention to a peer.

CONCLUSIONS: Participants were receptive to the intervention and believe it can improve care. An efficacy study of the intervention for improving clinical outcomes is underway. 


\section{P292}

FINDING A WAY: ADDRESSING INTANGIBLE BARRIERS THAT ARE CREATING TREATMENT AND CARE INEQUITIES, AND COMPROMISING THE HEALTH OUTCOMES OF VULNERABLE PHAS

Gurm, Jasmine ${ }^{1}$; Zhang, Wendy ${ }^{1}$; Zhu, Julia ${ }^{1}$; Parashar, Surita ${ }^{1,2}$; Samji, Hasina ${ }^{1}$; McNeil, Ryan ${ }^{1}$; Strike, Carol ${ }^{3}$; Pauly, Bernadette ${ }^{4}$; Salters, Kate ${ }^{1,2}$; Worthington, Catherine ${ }^{4}$; Milloy, M-J' ${ }^{1}$;

Kirkland, Susan ${ }^{5}$; Guillemi, Silvia ${ }^{1}$; Skinner, Stuart ${ }^{6}$;

Panessa, Ciro' ${ }^{1}$, McDougall, Patrick ${ }^{1}$; Baltzer Turje, Rosalind ${ }^{1}$;

Barrios, Rolando ${ }^{1}$; Hogg, Robert ${ }^{1,2}$

${ }^{1}$ Vancouver; ${ }^{2}$ Burnaby, BC; ${ }^{3}$ Toronto, ON; ${ }^{4}$ Victoria, BC; ${ }^{5}$ Halifax, NS; ${ }^{6}$ Saskatoon, SK

BACKGROUND: The availability of highly active antiretroviral therapy (HAART) has significantly decreased the incidence of mortality and morbidity for persons living with HIV/AIDS (PHAs); however, many PHAs still face substantial barriers in accessing and adhering to HIVrelated treatment and care. The Dr. Peter Centre (DPC), in Vancouver's west-end, seeks to address this inequity by providing integrated healthcare and support services, including a wide range of harm-reduction programming, and by facilitating access to other services in the community for PHAs facing barriers. This analysis serves to elucidate the nature, multiplicity and extent of the complex barriers experienced by PHAs who utilize the DPC.

METHODS: The LISA cohort is a cross-sectional study of persons on antiretroviral therapy in BC, interviewed between 2007 and 2010. Interviewer administered surveys captured information on housing, illicit drug use, mental health, trauma, sexual behavior and other socio-demographic characteristics. We used bivariate and multivariable analyses to compare between LISA participants who have and have not used the DPC.

RESULTS: Of 917 LISA participants, 11\% reported having ever used the DPC (DPC clients). Compared to non-DPC clients, DPC-clients were more likely to be male (adjusted odds ratio [AOR] 3.76 [95\% CI 1.99 to 7.10]), have been diagnosed with a mental health disorder (AOR 2.19; $95 \%$ CI 1.29 to 3.73]) and have experienced violence (AOR 2.32 [95\% CI 1.23 to 4.40]). DPC clients showed a greater likelihood of interrupted HAART $>1$ year (AOR 2.15 [95\% CI 1.34 to 3.43]) and of using supportive services on a daily basis (AOR 4.91 [95\% CI 3.03 to 7.98]). CONCLUSION: Results suggest DPC clients are more likely to experience psychosocial barriers leading to sub-optimal healthcare outcomes, such as HAART interruption. However, these PHAs also access and utilize supportive services at an increased frequency, indicating a potential need for integrative support services, such as those provided by the DPC, that serve to engage this population and reduce inequities to achieve improved health outcomes.

\section{P293}

\section{SPEAKING OUR LANGUAGE: REFLECTIONS FROM YOUTH ENGAGED IN A COMMUNITY-BASED PARTICIPATORY RESEARCH PROJECT}

Reid, Jesse; Spence, Heather; Grewal, Puneet; Coburn, Trevor; Cox, Kelsi; Tzemis, Despina; Smith, Annie; Buxton, Jane A Vancouver, BC

BACKGROUND: The Youth Injection Prevention (YIP) project is a collaborative study including academic researchers, experiential youth co-researchers and community partners. The YIP project sought to identify factors associated with preventing street-involved youth from transitioning to injection drug use (IDU), and thus, prevent $\mathrm{HIV} / \mathrm{HCV}$ infection among street-involved youth. Factors were identified through community-based, youth facilitated, focus groups (FGs) in the Lower Mainland. Youth co-researchers created a knowledge dissemination video highlighting the focus group results. In this next phase of the study, the FG findings were shared through youth-facilitated workshops at community organizations across BC.

OBJECTIVE: To describe the workshops and learn from the experiences of the youth co-researchers as they developed and facilitated the workshops.
METHODS: The youth co-researchers designed a knowledge exchange workshop to compare factors associated with risk and prevention of IDU among street-involved youth in each community, to those identified in the lower-mainland. Workshops included: developing a community agreement, risk and resiliency brainstorming, design your own youth centre, and discussions around key concepts seen in the knowledge dissemination video. Youth co-researchers' reflections were obtained through debriefing sessions after each knowledge exchange workshop and during team meetings.

RESULTS/DISCUSSION: Six workshops were held. Co-researchers reflected that to be research partners, they should be engaged from the beginning of the research process, allowed adequate time to develop skills, and ensure mutual respect between all involved. Authentic engagement of youth participants in the workshops includes: respect for participants, utilize team building activities, and defining terms youth may be unfamiliar with.

The YIP project takes innovative and groundbreaking approaches to community-based research with the engagement of experiential youth co-researchers at every stage of the initiative. Researchers and practitioners can learn much from the youth they work with. Working collaboratively can help all involved speak a language that respects the experiences of youth.

\section{P294}

\section{FINDINGS FROM THE YOUTH INJECTION PREVENTION (YIP) PROJECT: NEXT STEPS WORKSHOP}

Omura, John; Tzemis, Despina; Cox, Kelsi; Smith, Annie; Co-researchers, Youth; Funk, Anna; Martin, Ruth; Buxton, Jane Vancouver, BC

BACKGROUND: In 2011, 12\% of newly diagnosed HIV cases in BC were attributed to injection drug use (IDU), with $20 \%$ of all HIV cases reported in persons aged 15 to 29 years. The YIP Project is a collaborative study including academic researchers, experiential youth co-researchers and community partners that focuses on identifying resiliency factors associated with preventing the transition into IDU among street-involved youth. Such factors were previously identified for youth in BC's Lower Mainland through focus groups (FGs). In this next phase, the FG findings were shared through workshops across BC to identify similarities and differences of perceived risk and resiliency factors.

METHODS: In 2012, six youth-designed and facilitated workshops were held across BC's five regional health authorities. Fields notes were taken and four workshops were audio recorded. Analysis was conducted by constant comparative methods.

RESULTS: In total, 64 youth (38 female, 26 male; ages 15 to 24 years) participated. Perceived risk factors for IDU differed between the workshop settings and included: living situation, trauma, lack of familial support, boredom, media, drug availability and stigma. Common themes of resiliency factors were identified, factors included: desire for a better life, support from others, concerns for health and self-image, responsibility for others, goals, self worth and fear of needles. Factors relating to emotional and social capital may promote risk or resiliency depending on the severity and context. Youth voice emerged as a strong theme with youth expressing their desire to be heard.

DISCUSSION: Despite varying risk factors for initiating IDU, youth participants across BC identify similar protective factors. Efforts to prevent IDU among street-involved youth should focus on promoting common resiliency factors and seeking youth input. Such approaches will contribute to a comprehensive HIV prevention strategy in BC.

\section{P295}

CHILDREN WITH HIV: INTERSECTION WITH THE CHILD WELFARE SYSTEM FOR MEDICAL NON-ADHERENCE

Azzopardi, Corry; Salter, Robyn; Wade, Mark; MacDougall, Georgina M; Read, Stanley; Bitnun, Ari

Toronto, ON

BACKGROUND: Advancements in pediatric HIV treatment have brought new hope for improved health and survival for children living 
with HIV. However, illness chronicity and complex medical regimens combined with multifaceted psychosocial stressors pose substantial challenges associated with treatment adherence. Non-adherence has significant adverse health implications, sometimes meeting the theshold for medical neglect warranting mandated child welfare intervention.

OBJECTIVE: The primary objective of this study was to examine the nature and extent of medical non-adherence in children with HIV.

METHOD: Retrospective review of health records of perinatally HIV infected children followed at a pediatic hospital between 1997 and 2011 was conducted. Adherence to medical care (clinic attendance, medication compliance) was rated as excellent, moderate, or poor based on patient/caregiver self-report and healthcare team assessment. A range of demographic, psychosocial, and medical variables were analyzed with descriptive statistics and regressions.

RESULTS: The sample included 128 HIV infected children (54\% female) with a mean age of 4.4 years at referral $(\mathrm{SD}=4.35)$. Almost half $(49 \%)$ were categorized as having poor $(13 \%)$ or moderate $(36 \%)$ adherence to care. Regression analysis identified three clusters of psychosocial factors predictive of non-adherence: caregiver mistrust (disbelief of diagnosis, low confidence in treatment, poor relationship with medical team; $\beta=-0.48 ; \mathrm{p}<.001$ ); poor caregiver health (physical/mental health, substance use; $\beta=-0.39 ; \mathrm{p}<0.001$ ); caregiver under-involvement (poor understanding of diagnosis/treatment, lack of supervision, forgetfulness; $\beta=-0.29 ; p=.014) .43 \%$ had involvement with child welfare services, most due to neglect, with $17 \%$ being placed in out-of-home care, rates substantially higher than in the general population.

DISCUSSION: Children with HIV and their families are a particularly vulnerable group who face many challenges that impede capacity to fulfill critical treatment regimens, often resulting in child welfare intervention. An interprofessional model of care for healthcare and child welfare services working with children and families to optimize HIV treatment adherence is proposed.

\section{P296 \\ CHALLENGES IN THE CULTURAL ADAPTATION OF TWO HIV PREVENTION INTERVENTIONS FOR FRENCH SPEAKING MSM IN QUEBEC AND ENGLISH SPEAKING MSM IN ONTARIO}

Otis, Joanne $^{1}$; Hart, Trevor $A^{2}$; Adam, Barry ${ }^{2,3}$;

McFadyen, Amelie'; Willis, Amy C ${ }^{2}$; Stratton, Natalie ${ }^{2}$;

Greenspan, Nicole $\mathbf{R}^{2}$; Fadel, Riyas ${ }^{1}$; Bernier, Mélina ${ }^{1}$;

Blais, Martin ${ }^{1}$; Julien, Rick²; Simpson, Scott ${ }^{2}$; Rivas, Carlos ${ }^{2}$

${ }^{1}$ Montreal, QC; ${ }^{2}$ Toronto; ${ }^{3}$ Windsor, ON

BACKGROUND: MSM represent 46.7\% (including MSM who are IDU, 49.7\%) of all HIV-positive test results in Canada (PHAC, 2011). Quebec and Ontario represent the highest proportion of HIV-positive test results nationally. Therefore, there is a critical need for effective, evidence-based HIV prevention interventions for MSM in these two provinces. In response, two programs have been developed: the Phenix Program, a group sexual health education program using socio-behavioural approaches for MSM in Montreal and Gay Poz Sex (GPS), a group counseling program using motivational interviewing for HIV+ MSM in Toronto.

OBJECTIVE: The main goal is to bridge the gap between English and French speaking Canada regarding innovative HIV prevention programs for MSM.

METHOD: This study follows the CDC's methodology adaptation process published by McKleroy et al. (2006), and uses Tortolero et al's (2005) intervention mapping steps: 1) Identify theoretical foundation 2) Assess the populations 3) Review behavioral outcomes 4) Specify performance objectives 5) Prepare the adaptation 6) Pilot the adapted intervention and 7) Implement the adapted intervention.

RESULTS: A variety of challenges were encountered during the cultural adaptation process. The expected performance objectives and behavioural outcomes were outlined for each program's sessions. Core elements and key characteristics of each program were identified to ensure that program integrity was protected and maintained throughout the cultural adaptation process. Finally, there were challenges in identifying experts who were involved in community HIV prevention activities and who understood risk reduction strategies and/or motivational interviewing. CONCLUSIONS: The cultural adaptation process goes beyond simply translating a program. A thorough review of each program is necessary to ensure that theoretical foundations, performance objectives and behavioural outcomes are explicitly stated. Importantly, identifying the program's unchangeable core elements (such as type of counselling style) versus characteristics that can be more adapted for linguistic and cultural communities in Canada was key.

\section{P297 \\ HOW LIVING CONDITIONS IN THE STREET FRAME STIS RISK ASSESSMENT AND DOWNPLAY CONDOM USE AMONG STREET-INVOLVED YOUTH (SY)? A QUALITATIVE EXPLORATION}

Côté, Philippe-Benoit; Blais, Martin; Manseau, Hélène

Montréal, QC

CONTEXT: Street-involved youth (SY) face living conditions that challenge their sexual health. About $50 \%$ of SY report no condom use and rates of chlamydia and gonorrhoea among them are more than ten times those in the general population of youth (PHAC, 2006). Several studies have described living conditions and risk factors affecting condom use among SY (Marshall et al., 2009; Roy et al., 2003). Still, little is known about the psychosocial mechanisms through which these factors frame the STIs risk evaluation.

OBJECTIVES: 1) To describe factors framing the STIs risk assessment among SY; 2) To explore the role of street context on the risk assessment process among the SY.

METHOD: Individual semi-structured interviews were conducted with 42 SY ( 18 women, 24 men) in Montreal. They were aged 18 to 32 years (mean 23; SD 3.6). Qualitative material was coded following the de- and re-contextualization steps proposed by Tesch (1990).

RESULTS: Three main factors framing risk assessment were identified. 1) Taking care of the self loses its importance once in the street. 2) Risk deception strategies for selecting partners (based on appearance and assumed sexual lifestyle) and sexual practices with specific partners (based on trust) can reframe unsafe sexual practices into low- or no-risk practices. 3) Gains from unprotected sex (more money, pleasure or a potential pregnancy) make it appealing and outweigh the risks of STIs. Precarious life conditions play an important role in framing the way SY think about themselves, their partners and how they assess sexual behaviors and STIs risk.

CONCLUSION: Precarious life conditions in the street not only impacts the material living conditions of the SY by creating an unfavorable environment for condom use, but also triggers the reframing of the Self, the Other and the sexual behaviors in ways that reduce the perceived STIs risks. Implications for intervention are discussed.

\section{P298}

ONE IN SEVEN MEN REPORT HAVING BOUGHT OR SOLD SEX: RESULTS FROM THE MALE CALL STUDY WITH IMPLICATIONS FOR HIV RESEARCH IN CANADA

Allman, Dan ${ }^{1}$; Myers, Ted ${ }^{1}$; Alexander, Stephen ${ }^{2}$; Remis, Robert S$^{1}$; Blais, Martin ${ }^{3}$; Maxwell, John ${ }^{1}$; Calzavara, Liviana ${ }^{1}$;

Marchand, Rick ${ }^{4}$; Adam, Barry $\mathrm{D}^{5}$; Marlatt, Faria ${ }^{1}$;

Docter, Hamal ${ }^{1}$; Vernich, Lee $^{1}$

${ }^{1}$ Toronto, ON; ${ }^{2}$ Fredericton, NB; ${ }^{3}$ Montréal, QC; ${ }^{4}$ Vancouver, BC; ${ }^{5}$ Windsor, ON

OBJECTIVES: To examine the prevalence of, and demographic variables associated with gay men and other men who have sex with men providing or receiving, money, drugs or other goods and services in exchange for sex.

METHODS: Male Call Canada (MCC), a bilingual, nation-wide, toll-free telephone survey carried out in 2011/2012, gathered self-reported information on knowledge and attitudes related to HIV and health and wellbeing from gay men and other men who have sex with men 16 years-of-age or older. For this analysis, bivariate statistics were computed using SAS. 
RESULTS: Of the 1235 men who completed the telephone interview, $1085(87.9 \%)$ answered questions related to the buying and selling of sex. $7.7 \%(n=83)$ reported ever providing money, 2.2\% $(n=24)$ drugs and $3.2 \%(n=35)$ other goods or services in exchange for sex. In contrast, $7.4 \%(n=80)$ reported ever receiving money, $3.5 \%(n=38)$ drugs, and $4.7 \%(n=51)$ goods or services in exchange for sex. Combined, $9.1 \%$ $(n=112)$ reported ever providing and $9.2 \% \quad(n=114)$ ever receiving money, drugs or other in exchange for sex. $15.1 \%(n=187)$ reported some form of transactional sex. Older age was the only demographic variable significantly associated with ever providing resources for sex $(\mathrm{p}<0.005)$. A younger age $(p<0.0001)$, French as a first language $(p<0.001)$, European ancestry $(p<0.05)$, and being single $(p<0.0001)$ were significantly and positively associated with ever receiving resources in exchange for sex.

CONCLUSIONS: Male Call Canada provides a unique perspective on gay men and other men who have sex with men. Unlike many previous studies, MCC was not limited to core urban centres, and recruited a substantial proportion of participants from rural and peri-rural locations in all regions. More than one in seven respondents reported exchanging resources for sex. These findings contribute to our understandings of sexual exchange in this population in Canada. Further, these findings have important implications for the assessment, evaluation and study of sexual exchange in the next generation of surveillance studies in Canada.

\section{P299 \\ INTEGRATED HIV PREVENTION AND MENTAL HEALTH COUNSELLING FOR SOCIAL ANXIETY FOR HIV-NEGATIVE GAY AND BISEXUAL MEN}

Hart, Trevor $\mathbf{A}^{\mathbf{1}}$; Tulloch, Tyler $\mathbf{G}^{\mathbf{1}}$; $\mathbf{O}^{\prime}$ Cleirigh, Conall $^{2}$

${ }^{1}$ Toronto, ON; ${ }^{2}$ Boston, Massachussetts, USA

OBJECTIVE: To report a case series describing psychotherapeutic treatment of HIV-negative gay and bisexual men who report HIV risk behaviour and substance use in sexual situations due to high anxiety in social/ interpersonal situations. Social anxiety, or anxiety about being evaluated in interpersonal situations, is a reliable risk factor for unprotected anal intercourse among gay and bisexual men yet is modifiable via cognitivebehaviour therapy.

METHODS: The 10-session cognitive-behaviour psychotherapeutic treatment combines psycho-education regarding social anxiety, substance use, and sexual risk behaviour, cognitive restructuring to reduce social anxiety in sexual and dating situations, and exposure to situations that are social anxiety-inducing (e.g., meeting a new potential sexual partner). These are preliminary case series data for a novel HIV prevention intervention for gay and bisexual men. All cases reported social anxiety disorder, substance use in sexual situations, and unprotected anal intercourse with casual partners in the past three months.

RESULTS: At three-month follow-up post-therapy, all cases reported reductions in social anxiety, substance use in sexual situations, and in sexual risk behaviour. Two cases reported engaging in no unprotected anal intercourse, and a third case reported reducing sexual risk by engaging only in risky sex as the insertive partner. Reductions in social anxiety on the Liebowitz Social Anxiety Scale are reported in the figure.

\begin{tabular}{lccc} 
& Patient \#1 & Patient \#2 & Patient \# 3 \\
\hline Pre-treatment & 18 & 41 & 30 \\
Post-treatment & 7 & 30 & 34 \\
3 month follow-up & 0.1 & 26 & 28 \\
\hline
\end{tabular}

DISCUSSION: The treatment resulted in reductions in social anxiety, social anxiety-related avoidance, substance use in sexual situations, and unprotected anal intercourse. This novel approach suggests the benefit of integrating mental health counselling with HIV prevention for gay and bisexual men.
P300

EMPLOYMENT LOSS AND CHANGES IN EXPOSURE TO FACTORS ASSOCIATED WITH HIV INFECTION AMONG PEOPLE WHO INJECT DRUGS IN VANCOUVER, CANADA

Richardson, Lindsey; DeBeck, Kora; Wood, Evan; Montaner, Julio; Kerr, Thomas

Vancouver, BC

BACKGROUND: The social-psychological and mental health consequences of job loss in the general population often result in increases in substance use and related activities and exposures. These increases are potentially important among HIV positive and at-risk injection drug users (IDU), among whom increased substance use resulting from precarious labour market participation may increase the risk of HIV transmission. We therefore undertook the current study to explore dynamics surrounding employment cessation and drug use-related HIV risk.

METHODS: Using data from the Vancouver Injection Drug User Study (VIDUS), a series of adjusted and unadjusted generalized estimating equations (GEE) examined associations between employment cessation and increases in activities and exposures that have previously been associated with increased risk of HIV acquisition in this context.

RESULTS: Among the 1116 participants contributing 6791 observations from December 2005 to May 2010, 301 instances of employment cessation were documented. In multivariate GEE models adjusted for age, gender, ethnicity and addiction treatment enrollment, employment cessation was associated with the initiation or increased frequency of heroin injecting (adjusted odds ratio [AOR] 1.46 [95\% CI 1.02 to 2.10]); injection and non injection crack use (AOR 1.59 [95\% CI 1.18 to 2.16]); transitioning from stable to unstable housing (AOR 2.13 [95\% CI 1.53 to 2.96) and relocation to Vancouver's Downtown Eastside (AOR 1.86 [95\% CI 1.28 to 2.72]).

CONCLUSION: These results suggest that the social-psychological and socio-economic impacts of employment cessation may result in increased activities and exposures associated with elevated risk of HIV acquisition. Consideration of the consequences of labour market instability for IDU in general, and on supports that promote employment maintenance such as low-threshold employment or increased flexibility in particular, may therefore be of paramount importance to initiatives aimed at the prevention of HIV transmission among IDU.

\section{P302}

MOTIVATIONAL INTERVIEWING IN THE CONTEXT OF RAPID HIV TESTING AMONG MSM : LESSONS LEARNED FROM SPOT, MONTREAL, CANADA

Otis, Joanne

Montréal, QC

BACKGROUND: SPOT is an ongoing intervention-research project offering free, anonymous, rapid HIV testing to men who have sex with men (MSM). Between July 2009 and July 2012, the first 1184 participants have received a standard counselling, whereas the 555 last participants have received a counselling based on motivational interviewing (MI).

OBJECTIVE: To describe the challenges that occurred with the implementation of MI counselling in the context of rapid HIV testing among MSM.

METHODS: Mixed methods were used: 1) A logbook was completed after each meeting to collect the nurses' (NUR) and community worker' (COMW) perceptions in regards to having provided adequate services, having received the right tools for the task at hand and having been supported by the team; 2) A one-hour semi-structured interview was conducted with them $(\mathrm{N}=8)$ about their experience.

RESULTS: For all actors, the feeling of being supported is very high. Compared to the NUR, the COMW distinguish themselves by a higher perception of having offered the support needed to the participant with the use of MI and having received the right tools. Carrying out MI seemed more difficult for women, part-time workers, those who have practiced standard counselling before SPOT and those who had to intervene in a language that they were not used to. Most would have preferred a longer initial training, tighter individual coaching and more 
communication between them. They had more difficulty carrying out MI in some cases: when the participant did not present any risks or, on the contrary, took too many risks; when he had mental health problems, etc. CONCLUSION: These results have allowed to identify several gaps to be filled in order to improve the integration of $\mathrm{MI}$ in the context of rapid HIV testing among MSM. A randomized trial to evaluate the effectiveness of MI is underway.

\section{P303 \\ THE CEDAR PROJECT: UNDERSTANDING LIVED EXPERIENCES OF CHILDHOOD MALTREATMENT AND HIV VULNERABILITY AMONG YOUNG ABORIGINAL PEOPLE WHO USE DRUGS IN TWO CANADIAN CITIES THROUGH A MIXED-METHODS APPROACH}

Pearce, Margo $\mathrm{E}^{\mathbf{1}}$; Christian, Wayne $\mathbf{M}^{2}$; Blair, Alden $\mathrm{H}^{\mathbf{1}}$;

Schechter, Martin $\mathbf{T}^{\mathbf{1}}$; Spittal, Patricia $\mathbf{M}^{\mathbf{1}}$

${ }^{1}$ Vancouver; ${ }^{2}$ Enderby, BC

BACKGROUND: Childhood trauma is recognized as an early determinant of HIV vulnerability, however very little is understood regarding the pathways from childhood trauma to HIV risk among young, at-risk Aboriginal people in Canada, particularly in the context of historical or intergenerational trauma.

METHODS: Twenty-six participants (15 women) who are part of the Cedar Project, a cohort study of young Aboriginal people who use drugs in Vancouver and Prince George, BC, completed the childhood trauma questionnaire (CTQ) followed by an individual in-depth interview about personal and family history including residential school or foster care, transitions to drug use and sex work, and opportunities for healing and change. Using phenomenological approaches, researchers transcribed and analysed interview texts. Convergence of qualitative and quantitative data was achieved with descriptive analyses and comparison between groups.

RESULTS: All participants reported moderate to severe trauma in childhood. Most participants had extreme CTQ scores, particularly for physical neglect $(73.1 \%)$, sexual abuse $(46.2 \%)$, physical abuse $(46.2 \%)$ and emotional neglect $(46.2 \%)$. The majority $(65 \%)$ reported multiple types of extreme childhood traumas, most commonly a combination of physical and emotional neglect (35\%). Five (19.2\%) participants were HIV positive, of whom four reported extreme sexual abuse. A significantly higher proportion of men had extreme emotional neglect. Five major themes from the qualitative study emerged: (a) positive vs. negative outcomes after telling about abuse; (b) family disconnection through the child welfare system; (c) pain management with high-intensity drug use; (d) understanding intergenerational grief and pain within families; and (e) identifying sources of strength for survival.

CONCLUSION: The number of young Aboriginal men and women who experienced extreme childhood abuses is staggering. It is critically important for public health practitioners to recognize the associations between childhood and historical trauma on HIV risk and to support opportunities for culturally-safe healing and strength-based approaches to risk reduction.

\section{P304}

\section{HIV AND SUBSTANCE USE BY PEOPLE WHO USE ILLICIT DRUGS IN A SMALL, RURAL COMMUNITY}

\section{Peters, Heather; Wrath, Kathy}

\section{Quesnel, BC}

Although illicit drug use occurs in small Canadian communities, the extent and patterns of drug use as well as connections to HIV risk specific to populations in northern and rural communities are not well researched. One of the goals of this project is to better understand substance use in a small, rural community in northern $\mathrm{BC}$ and determine implications for the prevention of HIV. Results are based on almost 200 surveys conducted with people using illicit drugs in Quesnel, BC. Drugs used the most frequently were: marijuana (59\% of participants), cocaine $(55 \%)$, ecstasy $(41 \%)$, crack $(35 \%)$, and illegally obtained narcotics $(33 \%)$. Marijuana and cocaine were the drugs most likely to be used on a regular basis, while magic mushrooms, mescaline, or peyote and ecstasy were the drugs most likely to be used casually. The most preferred drugs include marijuana (52\%), cocaine (38\%), crack (28\%), ecstasy (25\%), and magic mushrooms, mescaline, or peyote (20\%). $70 \%$ of participants have been tested for HIV and 59\% tested for Hep C, with 14\% stating they were Hepatitis C positive and 3\% stating they were HIV positive. However, more than half of the respondents did not believe that their drug use puts them at risk for HIV. Illicit drugs were used in a variety of locations, most commonly at a friend's $(69 \%)$ or their own place $(51 \%)$. Although $46 \%$ of participants were aboriginal, the majority used only off-reserve $(66 \%)$ or a combination of both on and off-reserve (24\%). Illicit drugs were most often purchased within the community (79\%). Both regular and casual illicit drug use occurs in this small, rural community in northern $\mathrm{BC}$, as do risk behaviours, indicating that efforts to prevent the spread of HIV need to also take place in these rural communities with drug using populations.

\section{P305}

APPLICATION OF THE SELF-EXPLORATION AND SELFREGULATION MODEL TO ENHANCE SELF-ESTEEM IN AFRICAN, CARIBBEAN \& BLACK (ACB) YOUTH LIVING WITH HIV

\section{Ndung'u, Mary; Moyo, Stanley}

Toronto, ON

This study aimed to develop intervention strategies leading to increased self-esteem in a sample of 62 African, Caribbean \& Black (ACB) Youth living with HIV in Toronto, Canada. The youth group (members aged 16-30yrs) consisted of newcomers newly diagnosed with HIV, long-term HIV positive survivors, those born with HIV and LGBTQ youth. These youth faced a variety of psychosocial challenges that including the lack of cultural adaptation in Canada, weak negotiation skills, lack of assertiveness, discrimination (in jobs or services), weak language skills, history of abuse, homelessness, lack of medical adherence, lack of social support, HIV disclosure issues, immigration challenges and more significantly lack of self-esteem.

The study sought to determine the role that self-exploration (defined as the process of examining one's personal resources such as strength or weakness in order to measure the potential for possible change) and selfregulation (defined as the act of planning \& implementing actions in order to bring desired change) could play in addressing self-esteem and other challenges faced by the youths. Specific techniques used in selfexploration and regulation included journaling, the use of expressive arts, value-belief system recognition, and training in goal-setting.

The self-exploration $\&$ self-regulation approaches together with work shop presentations led to increased self-confidence, increased assertiveness, increased self-empowerment, increased trust in own values $\&$ beliefs as well as improved self-esteem.

Significantly, increased self-esteem led to clients accessing support services more consistently and an improved quality of life associated with improved adherence to anti-retroviral medications, improved skills for self-advocacy, return to school, active job-searching, successful immigration applications and smoother cultural-adaptation. This model offered the possibility to address the significant challenges faced by the HIV positive youth in an effective largely peer-driven intervention. Furthermore, the intervention was also appropriate for this segment of the ACB community because of the manner in which it was derived. 


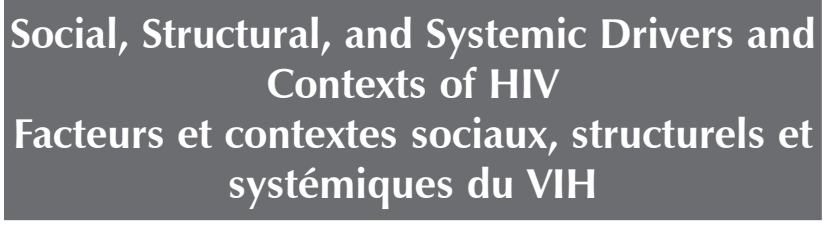

\section{P306}

UNIVERSAL ACCESS TO HIV/AIDS TREATMENT: HOW DO PHYSICIANS PROVIDING CARE TO HIV/AIDS PATIENTS IN RESOURCE LIMITED SETTINGS IN CARIBBEAN STATES?

\section{Reddock, Jennifer}

Hamilton, ON

The commitment to universal access to HIV/AIDS treatment and care has not resulted in equitable access for resource-poor nations or socioeconomically disadvantaged patients in the six Independent countries of the Organization of Eastern Caribbean States (OECS). The governments of the OECS countries currently provide antiretrovirals, diagnostic and monitoring tests to patients free of charge through donor assistance. Inequity in the provision of treatment and care for HIV/AIDS patients is perpetuated by both structural factors traceable to asymmetries between international commitments, and the organization of local health care delivery systems. A focus on the provision of medical services, and not on the contextual factors of access including the structure and capacity of health systems and the socio-economic status of patients is traceable to the global response to the disease which has been predominantly biomedical.

This paper presents the perspectives of OECS physicians on the ability to provide optimal care for HIV/AIDS patients in the context of global policies and local delivery arrangements. Physicians report not having access to vital resources that optimize patients' chances of survival at critical stages of their HIV/AIDS diagnosis. Through qualitative interviews with physicians in the OECS, this paper emphasizes that (i) OECS countries have not benefited from the highest aspirations of universal access if because of the countries' economic capacity, only some treatments and monitoring technologies are deemed affordable and(ii) even when treatment and care is provided free of charge, service delivery arrangements in small societies where HIV/ AIDS is stigmatized often results in socioeconomically-challenged patients receiving care in a way that sometimes forces them to forfeit privacy. Access to health care must be understood as a composite of the impact of global standards for access to medicines and the conditions for the delivery and receipt of care at the local and individual level.

\section{P307}

EARLY SEX WORK INITIATION INDEPENDENTLY ELEVATES ODDS OF HIV/STIS AND POLICE ARREST AMONG ADULT SEX WORKERS IN VANCOUVER

Goldenberg, Shira; ${ }^{1}{ }^{1}$; Chettiar, Jill'1; Simo, Annick'1;

Silverman, Jay G²; Strathdee, Steffanie A $^{2}$; Montaner, Julio ${ }^{1}$; Shannon, Kate ${ }^{1}$

\section{${ }^{1}$ Vancouver, BC; ${ }^{2}$ San Diego, California, USA}

OBJECTIVES: To explore individual, interpersonal, and social-structural factors associated with early sex work initiation, and to model the independent effect of early initiation on HIV/STI infection and prostitution arrests among adult sex workers (SWs).

DESIGN: Baseline data (2010-2011) were drawn from a cohort of female (including male-to-female transgender) SWs who had exchanged sex for money within the last month and were recruited through timelocation sampling in Vancouver, Canada. Analyses were restricted to adults $\geq 18$ years old.

METHODS: SWs completed an interviewer-administered questionnaire and HIV/STI testing. Using multivariate logistic regression, we fit an explanatory model to identify associations with early sex work initiation ( $<18$ years old $)$ and constructed confounder models examining the independent effect of early initiation on HIV/STIs and prostitution arrests among adult SWs.

RESULTS: Of 508 SWs, 193 (38.0\%) reported early sex work initiation, with $76.9 \%$ among primarily street-involved SWs and $22.0 \%$ among offstreet SWs. The combined prevalence of any STI/HIV was $20.9 \%$, which was $33.2 \%$ among early initiates. Early initiates were more likely to be Canadian-born (Adjusted Odds Ratio [AOR]: 4.3 [95\% CI 1.6 to 11.3]), inject drugs (AOR 1.5 [95\% CI 1.1 to 2.0]), and report having worked for a manager (AOR 1.3 [95\% CI 1.1 to 1.6$]$ ). Early initiation retained an independent effect on increased risk of HIV/STIs (AOR 1.83 [95\% Cl 1.3 to 2.7]) and police arrest for sex work-related charges (AOR 1.51 [95\% CI 1.1 to 2.1]) in adulthood.

CONCLUSIONS: Adolescent sex work initiation is concentrated among marginalized, drug and street-involved SWs. Moreover, early initiation holds an independent increased effect on both HIV/STI infection and criminalization of adult sex workers. Findings suggest the critical need to shift to evidence-based approaches to reduce harm among adults and youth in Canada's sex industry, while undertaking measures to prevent youth sexual exploitation.

\section{P308}

\section{HIV AND AGING}

Roger, Kerstin; Kirkland, Susan; Mignone, Javier

Winnipeg, MB

Little research can be found describing social aspects of aging with HIV/ AIDS in Canada, despite an overall increase in our aging population and the increased numbers of those aging with HIV. It is well-known that social aspects of health and aging such as social networks and social support, social and gender roles, caregiving, and access to services can dramatically shape physical wellness and emotional well-being. Yet, we know little about this for the population aging with HIV in Canada. A literature review was conducted with a selective focus on social aspects related to older adults living with HIV and older adults at risk of HIV, relevant to the Canadian context. Key sub-themes that emerged in the literature include: gender and sexual orientation; ageism and stigma; psychosocial function and mental health, and social networks and supports. Recommendations for future research, theoretical models and programming are presented.

\section{P309}

CLASSIFIED: FACTORS ASSOCIATED WITH USE OF SELFADVERTISEMENT IN THE SOLICITATION OF CLIENTS BY FEMALE SEX WORKERS IN A CANADIAN CONTEXT

Chettiar, Jill M; Morris, Jennifer; Deering, Kathleen N;

Simo, Annick; Leo, Diane; Shoveller, Jean; Shannon, Kate

Vancouver, BC

OBJECTIVES: Criminalization of sex work in Canada, in particular provisions in the criminal code which prohibit public communication for purposes of prostitution, has subjected sex workers (SWs) to heightened police surveillance and harassment, and many SWs have sought alternative means of soliciting clients. This study investigated the prevalence and characteristics of SWs using self-advertisement (including online and paper-based classifieds, 1-800 phone chat services) to solicit clients in Vancouver, Canada.

METHODS: Baseline data (Jan 2010 to July 2011) were used from an open prospective cohort study of street and off-street female (transgender inclusive) SWs in Metro Vancouver (AESHA: An Evaluation of Sex Workers' Health Access). Multivariate logistic regression was used to identify factors associated with use of self-advertisement to solicit clients in the past 6 months. Adjusted odds ratios and $95 \%$ confidence intervals were reported (AOR [95\%CIs]).

RESULTS: Of 510 women, 95 (18.6\%) reported using self-advertisement to solicit clients in the previous 6 months. In multivariate analyses, SWs using self-advertisement were significantly younger (0.94 [0.91 to 0.98]), more likely to identify as members of sexual and/or gender minorities (lesbian, gay, bisexual, transgender, transsexual, or two-spirit versus heterosexual and non-transgender) (AOR 3.06 [95\% CI1.78 to 5.25]); to 
have worked with a manager (AOR 6.60 [2.25 to 19.35]); and to have used a mobile phone for self-protection (AOR 3.50 [95\% CI 2.05 to 5.97]). SWs who used self-advertisement were significantly less likely to be HIV-positive (AOR 0.24 [95\% CI 0.08 to 0.74]), and to provide services for clients in indoor sex venues (massage/beauty parlours, microbrothels) (AOR 0.47 [95\% CI 0.23 to 0.97$]$ ) versus public/outdoor spaces (street, park).

CONCLUSIONS: This study suggests that self-advertisement is being used by more marginalized SWs (younger, sexual and/or gender minority) who often experience barriers accessing services, and who are working in environments with less infrastructure and support (informal indoor spaces, public outdoor spaces). This points to the need to develop novel HIV prevention interventions that are tailored to meet the needs of SWs who use self-advertisement (online/paper classifieds).

\section{P310}

\section{STICK IT TO THE STRUCTURES! NEW APPROACHES TO SOCIAL AND STRUCTURAL DRIVERS OF HIV AMONG MSM IN CANADA}

\section{Tooley, Len}

\section{Toronto, ON}

BACKGROUND: New, holistic ways of conceptualizing the HIV response are arising as the distinctions between HIV treatment and prevention - and biomedical, behavioural and social interventions - are being blurred. Emerging biomedical tools have begun to show partial efficacy at preventing HIV transmission while the evidence demonstrating the fundamental importance of structural and social drivers has never been stronger. Where do we go from here?

METHODS: A narrative literature review was conducted exploring "social-structural drivers" approaches to HIV (as conceptualized by the aids2031 Social Drivers Working Group) among gay, bisexual, two-spirit, and other cis and trans MSM in Canada. This approach builds on earlier models of social determinants of health and how to better integrate them into research and practice.

DISCUSSION: Blurring distinctions between biomedical, behavioural and structural interventions makes explicit the need to address structural change in every intervention, and could ultimately result in an increase in the number of prevention options available to gay/bi/two-spirit MSM. Reconceptualising the "complex, fluid, non-linear and contextual" (Auerbach et al., 2011) ways structural drivers impact HIV epidemics aligns with established understandings of gay men's health including lifecourse approaches, syndemics, and minority stress. Emphasizing community-level social change continues a history of community-led behaviour change and activism evidenced among gay men's communities early in the epidemic. Finally, cultivating AIDS resilience by creating and supporting AIDS-competent communities, fostering individual agency, and building health-enabling environments, resonates with strengths-based approaches to gay men's health. These concepts capitalize on pre-existing resiliencies to tailor appropriate, effective interventions.

NEXT STEPS: Many successful examples of operationalizing social structural drivers approaches already exist in Canada. Moving forward some priorities include: an enhanced surveillance system that includes sociological assessment; increased ownership over funding and priority setting by communities; a renewed and expanded fiscal commitment; utilizing legal structures to assure human rights; and growing synergistic intersectoral partnerships.

\section{P311}

\section{A DOSE-DEPENDENT RELATIONSHIP BETWEEN} EXPOSURES TO POLICING AND SYRINGE SHARING AMONG PEOPLE WHO INJECT DRUGS IN BANGKOK, THAILAND

$\underline{\text { Hayashi, Kanna }}^{1}$; Ti, Lianping1'; Kaplan, Karyn²; Suwannawong, Paisan²; Wood, Evan'; Kerr, Thomas ${ }^{1}$ ${ }^{1}$ Vancouver, BC; ${ }^{2}$ Bangkok, Thailand

BACKGROUND: Thailand has implemented aggressive police crackdowns on illicit drug use. While intensive drug law enforcement is increasingly recognized as a social-structural driver of human immunodeficiency virus (HIV) epidemics among people who inject drugs (IDU) in many settings, the effect of direct encounters with police has not been well investigated. Therefore, we sought to identify the impact of exposures to specific policing tactics on HIV risk behavior among IDU in Bangkok, Thailand.

METHODS: Data were derived from a community-recruited sample of IDU in Bangkok participating in the Mitsampan Community Research Project between July and October 2011. Using multivariate log-binomial regression, we examined the relationship between syringe sharing and exposures to policing, which were measured in four levels: (1) reporting having ever been beaten by police and tested for illicit drugs by police in the past six months; (2) reporting drug testing by police only; (3) reporting police beating only; and (4) reporting neither of the events (our reference category).

RESULTS: In total, 435 IDU participated in this study, with 75 (17.2\%) participants reporting having shared syringes in the past six months. In multivariate analyses, after adjustment for potential social, demographic, and behavioral confounders, syringe sharing was independently associated with reporting both police beating and drug testing by police (adjusted prevalence ratio [APR] 2.40), reporting drug testing by police only (APR: 2.00), and reporting police beating only (APR 1.82) (all $\mathrm{p}<0.05)$

CONCLUSION: We found that a substantial proportion of a sample of IDU in Bangkok reported recent syringe sharing, and exposures to policing had independent and dose-dependent relationships with this form of HIV risk behavior. The impact of specific encounters with police on HIV risk found in the present study highlights the importance of addressing the policy and social environment surrounding IDU as a means of preventing HIV transmission.

\section{P312}

"WE'RE MORE THAN YOU SEE": ENABLERS AND BARRIERS TO MORE MEANINGFUL INVOLVEMENT IN AN ASO

Duffy, Lynne $^{1}$; Amirault, Marni ${ }^{2}$

${ }^{1}$ Moncton, NB; ${ }^{2}$ Halifax, NS

PROJECT OVERVIEW: The majority of research about GIPA (Greater Involvement of Persons Living with HIV/AIDS) has been carried out in large urban centres with little understanding of its operationalization in rural areas and more specifically with further marginalized, at-risk groups such as drug users and women. A team of academic and community researchers in the three Maritime Provinces is carrying out a staged and mixed qualitative study to better understand and address this gap. This presentation describes Stage 2; a photovoice project involving a small number of AIDS Service Organization (ASO) volunteers in Cape Breton, Nova Scotia, an area with a high incidence of poverty, illiteracy, and drug use.

METHOD: Photovoice is a qualitative, community-based research approach in which participants as co-researchers take photographs of the study focus. Following a five-hour training session participants were provided with a digital camera and asked to visually document their understanding and experiences of meaningful involvement with their local AIDS Service Organization (ASO). When they returned a month later, participants chose which photos to present through a facilitated a discussion and analysis. In total there were three photo assignments.

RESULTS: It is clear that the ASO plays an important role in the lives of these participants. A strong connection has been established at the ASO by welcoming staff, while participants give of their time and energy, receiving support and a sense of belonging in return. Presently, their involvement centers on tasks of everyday organizational maintenance and fund raising. While they have aspirations for greater involvement in the ASO, both personal and structural barriers hinder this.

This session briefly introduces photovoice method and its use with a specific group of at-risk participants, and, through their photos and stories, describes enablers and barriers to more meaningful involvement in an ASO. 
P313

BUILDING COMMUNITY-BASED HIV AND STI PREVENTION PROGRAMS ON THE TUNDRA: DRAWING ON INUIT WOMEN'S STRENGTHS AND RESILIENCIES

Rand, Jenny $\mathbf{R}$

Victoria, BC

BACKGROUND AND OBJECTIVES: This presentation will discuss the preliminary findings of an innovative study that combines the art and science of Inuit knowledge with community based, participatory research to reduce inequitable access to HIV/STI prevention programs for Inuit women and their families. The objective of the study was to address the lack of information available for the basis of programming to improve sexual and reproductive health of Inuit women in isolated communities in the Canadian Arctic. In addition to addressing an issue clearly articulated by Inuit women, the utilization of an Indigenous model of community-based research demonstrates respectful and active collaboration with the community.

METHOD AND RESULTS: Nine story sharing sessions took place with 21 Inuit women 18 to 60 years of age. These stories have created a space for dialogue among and with Inuit women that can inform the development of future Inuit-specific HIV and STI prevention initiatives. Participants identified several key determinants of sexual health within their community such as housing, education, early childhood experiences, among others. Participants also shared various ideas of innovative approaches that they believe will work as prevention efforts within their community.

DISCUSSION: Western, inductive techniques form the analysis of the data; yet, Inuit knowledge and ways of understanding informed the basis of the theoretical framework upon which interpretations were formed, conclusions drawn, and recommendations offered. Likewise, local Inuit forms of information sharing represent a critical component of the dissemination process. Women know best how to look after themselves and their families, and through dissemination of these findings, the voices of Inuit women may be centralized in informing future HIV prevention efforts. These research results build upon the limited knowledge currently available about perceptions of HIV among Inuit women living in the remote north.

\section{P314}

\section{PREJUDICE AND STRUCTURAL DRIVERS OF HIV AMONG MSM IN CANADA}

\section{Trussler, Terry}

\section{Vancouver, BC}

Our objective was to measure the extent to which social prejudice against gay men affects HIV related health indicators within a national sample of men who have sex with men (MSM). We constructed a questionnaire to probe experience with lifetime prejudice events, employment history and social participation along with a broad range of health and sexuality indicators. The survey was conducted online between September 2011 and February 2012.

A total of 8607 men responded and 8497 were eligible for further analysis. The sample included men from all provinces and territories, covering approximately $72 \%$ of all Canada Post Forward Sortation Areas. Self reported sexual orientation was $65 \%$ gay, $32 \%$ bisexual, $2 \%$ straight, and $1 \%$ other. About $21 \%$ were married or partnered with a woman.

We queried employment discrimination defined as "dismissed, rejected or restricted" from work or advancement resulting from sexual prejudice. Employment discrimination was reported by $17 \%$, however, it was unevenly distributed: $22 \%$ among gay men; $5 \%$ bisexual men; 3\% married men.

Logistic regression analysis, comparing men reporting employment discrimination against those not - controlling for age and income - showed that gay men (OR 4.408 [95\% CI 3.738 to 5.198]) under 40 (OR 1.786 [95\% CI 1.488 to 2.366 ) were at substantially greater risk than other men. Those who had disclosed their sexual orientation to anyone in the workplace (OR 2.845 [95\% CI 2.385 to 3.395]) or who worked in unsupportive settings (OR 1.515 [95\% CI 1.299 to 1.767]) were at increased risk. Key health outcomes of employment discrimination were: perceived careerbased status damage (OR 7.310 [95\% CI 6.098 to 8.763]); suicidality (OR
1.782 [95\% CI 1.576 to 2.223]) and increased unprotected sex with partner(s) of unknown-HIV-status (OR 1.252 [95\% CI 1.095 to 1.431]). Employment discrimination is an important social variable toward understanding health determinants operative among MSM and an indicator of significant structural inequity underlying HIV outcomes.

\section{P315}

GETTING TO WORK: CREATING A LEARNING RESOURCE FOR INDIVIDUALS AND ORGANIZATIONS FACILITATING LABOUR FORCE PARTICIPATION FOR PEOPLE LIVING WITH HIV

Worthington, Catherine ${ }^{1}$; Solomon, Patricia ${ }^{2}$; Sinclair, Lynne ${ }^{3}$; Mangion, Martine ${ }^{3}$; Popiel, Melissa ${ }^{3}$; Salbach, Nancy ${ }^{3}$; $\mathrm{O}^{\prime}$ Brien, Kelly ${ }^{2}$; Rueda, Sergio ${ }^{3}$; Oliver, Brent ${ }^{4}$; Mollison, Ashley ${ }^{1}$ ${ }^{1}$ Victoria, BC; ${ }^{2}$ Hamilton; ${ }^{3}$ Toronto, ON; ${ }^{4}$ Calgary, AB

BACKGROUND: We conducted a scoping study and produced a conceptual framework called The HIV and Employment Framework (The Framework) that was summarized in a booklet and series of fact sheets to highlight labour force participation issues for people living with HIV (PHAs) in Canada. The objectives of this follow-up study were to promote and assess uptake of these knowledge tools, and use this feedback to create The "Getting to Work" Learning Resource for People and Organizations Engaged in Employment Work with People Living with HIV.

METHODS: The research team carried out activities to evaluate and assess the knowledge tools produced in the scoping study. The feedback from this phase of the study contributed to the development of The "Getting to Work" Learning Resource. To develop The Learning Resource, the research team held a focus group with participants from the target audiences $(n=8)$ to assess their learning needs. Problem-based educational modules were produced and two focus groups conducted $(n=16)$ to assess the appeal and applicability of the content. An advisory committee that included PHAs, vocational rehabilitation professionals, employers and service providers guided this research.

RESULTS: We produced a problem-based learning resource for people engaged with PHAs on employment issues. The Learning Resource is comprised of four modules that use case examples to highlight the barriers and facilitators to work for PHAs, and highlight employment experiences unique to HIV. The modules are designed to be completed in small groups and include objectives, case studies, general and discussion questions, key content and "advice from the experts" from focus group and advisory committee participants. Response to the materials was very positive. CONCLUSIONS: The "Getting to Work" Learning Resource may be used to help increase knowledge about labour force participation issues for PHAs. These knowledge tools are available for dissemination in French and English and are available online.

\section{P316}

MISTRUST, MISUNDERSTANDINGS, AND CORRUPTION: EXPLORING THE SOCIO-CULTURAL AND POLITICAL FACTORS OF HIV VACCINE ACCEPTABILITY IN KENYA du Plessis, Elsabe'; Kaddu, Ronniem P2; Doshi, Monika ${ }^{3}$; Gichuhi, Mary'; Kimani, Joshua ${ }^{2}$; Avery, Lisa ${ }^{1}$; Lorway, Robert ${ }^{1}$ ${ }^{1}$ Winnipeg, MB; ${ }^{2}$ Nairobi, Kenya; ${ }^{3}$ Washington, DC, USA BACKGROUND: An efficacious HIV prevention vaccine would provide vulnerable groups like female sex workers (FSW) and men who have sex with men (MSM) access to a prevention method within their control. As such it could significantly affect the HIV pandemic but many factors could reduce uptake. In Kenya, MSM and FSW are highly stigmatized and experience social and legal oppression. Although promising research is underway at the Kenya AIDS Vaccine Initiative (KAVI), involving the MSM and FSW communities, the social context may limit eventual access to the vaccine. The research presented here forms part of a larger research program funded by the CIHR that examines social and cultural issues related to introducing future HIV vaccines among MSM and FSW in China, India and Kenya.

METHODS: We employed peer ethnography and fieldnote writing pertaining to "informal chatting". The purpose of this participatory approach 
was to document perceptions and attitudes towards the state and western biomedicine as well as knowledge of vaccines and diseases of public health importance. The information presented here is based on thematic analysis of 71 transcripts ( 36 MSM, and 35 with FSW) from three districts in Kenya.

RESULTS: MSM and FSW's mistrust of the government and health care system is a major barrier to accessing health services. Private and public facilities are viewed as poorly regulated, corrupt and lacking trained staff and supplies. Although most individuals were familiar with vaccines there is some confusion between vaccines as prevention and treatment. Additionally, suspicion of the true intentions of the HIV vaccine (a method of population control) persists.

CONCLUSIONS: Targeted messaging regarding the HIV vaccine and careful consideration of where vaccines will be made available are important for successful vaccine campaigns. Community involvement in planning campaigns and selecting the venues for vaccination may alleviate some of these challenges.

\section{P317}

\section{EXPLORING EVOLVING CRYSTAL METHAMPHETAMINE USE AMONG A POPULATION OF STREET-ENTRENCHED YOUTH IN VANCOUVER: IMPLICATIONS FOR HIV PREVENTION}

Fast, Danya; Small, Will; Wood, Evan; Kerr, Thomas

Vancouver, BC

Among youth who live and use drugs "on the streets," transitions into more harmful forms of illicit drug use have been identified as important foci for research and intervention. In many settings, crystal methamphetamine (meth) use among youth has been associated with numerous sexand drug-related HIV risk behaviours. In order to examine young people's experiences and understandings of meth use over time, we conducted over 100 in-depth interviews and ongoing ethnographic fieldwork with 54 young people who used meth (including 33 who injected meth) in greater Vancouver over a four-year study period. Consideration of meth use in relation to the broader risk trajectories experienced by youth over time revealed positive understandings of meth in relation to other forms of drug addiction and untreated mental health issues. Young people were aware of the numerous health harms and social costs associated with heavy meth use, even during the period of time when they were initiating meth use. As their meth use escalated, youth indicated that continued use allowed them to "function" on the streets in the context of homelessness, material deprivation and boredom. Over time, positive understandings of meth may be increasingly contradictory to a lived reality in which meth use is a factor in further marginalizing youth, although this may not foster cessation of meth use. In order to address HIV risks among this population, our analysis underscores the need to move beyond efforts to educate young people about the dangers of meth use, towards a focus on developing novel and accessible addiction treatment programs and mental health services targeting the needs of street-entrenched youth. In addition, interventions are urgently needed to address the broader social structural contexts of meth use (e.g., homelessness, lack of employment opportunities), and systemic issues of alienation from available programs and services across the lifecourse.

\section{P318}

INTERPERSONAL ENVIRONMENTS OF HIV RISK: PARTNER-LEVEL FACTORS ASSOCIATED WITH INCONSISTENT CONDOM USE WITHIN INTIMATE AND NON-PAYING PARTNERSHIPS OF STREET AND OFFSTREET SEX WORKERS

Deering, Kathleen N ${ }^{1}$; Strathdee, Steffanie ${ }^{2}$; Simo, Annick ${ }^{1}$;

Montaner, Julio $\mathrm{S}^{1}$; Shannon, Kate ${ }^{1}$

${ }^{1}$ Vancouver, BC; ${ }^{2}$ San Diego, California, USA

BACKGROUND: The role of intimate or other non-paying partners (NPPs) in shaping HIV-related vulnerability of sex workers (SWs) remains unclear, although globally, condom use is often substantially lower than within commercial sex transactions. Our study investigated the individual and interpersonal factors associated with inconsistent condom use by NPPs of SWs in Vancouver, Canada.

Baseline (January/10-February/12) data were used, including an interviewer-administered questionnaire and HIV/STI testing from an open prospective cohort of street and off-street SWs (self-identified women; trans inclusive)(An Evaluation of Sex Workers' Health Access[AESHA]). Multivariable logistic regression using generalized estimating equations was used to identify individual and interpersonal factors (partner/dyadlevel factors) associated with inconsistent condom use $(<100 \%)$ with up to three NPPs of SWs in the last six months. Multivariable (adjusted) odds ratios and 95\% confidence intervals were reported (AOR:[95\%CIs])

RESULTS: Of 579 street/off-street SWs, 301 (51.9\%) reported having $\geq 1 \mathrm{NPP}$ (mean $1.2 \mathrm{NPPs}$ per SW). Across multiple partners, inconsistent condom use was high $(68.3 \%)$, with $11.2 \%$ and $14.1 \%$ of NPPs having sexual relationships with other SWs or other non-commercial female partners and $16.3 \%$ and $61.7 \%$ of NPPs using injection and non-injection drugs, respectively. In multivariable analysis, the following factors were significantly associated with increased odds of inconsistent condom use: SW HIV sero-negativity (5.88 [2.32 to 14.29 ); having a regular/cohabiting relationship (7.90 [3.43 to 18.27 ) or a regular/non-cohabiting relationship (2.87 [1.46 to 5.67]) versus a casual relationship; NPPs' dependence on SWs for financial support (3.96 [1.45 to 10.82); SWs' dependence on NPPs for material support (2.39 [1.26 to 4.54); and NPPs' injection drug use (2.39 [1.25 to 4.59]).

CONCLUSIONS: Our results highlight low condom use and important potential routes of HIV acquisition to SWs from their NPPs, and to NPPs' other partners, and identify gender-based power relations as having a primary role in HIV risk. Empowerment strategies for SWs and malefocused interventions should be incorporated into strategies to reduce HIV risk to SWs.

\section{P319}

\section{HIV AND RELIGION IN THE DEMOCRATIC REPUBLIC OF THE CONGO}

Hawkes, Michael ${ }^{1}$; Sivasivugha, Eugenie $\mathrm{S}^{2}$; Ngigi, Simon $\mathrm{K}^{2}$;

Masumbuko, Claude $\mathrm{K}^{3}$; Brophy, Jason $\mathrm{C}^{4}$.

Kibendelwa, Zacharie $\mathrm{T}^{5}$

${ }^{1}$ Toronto, ON; ${ }^{2}$ Nairobi, Kenya; ${ }^{4}$ Ottawa, ON; ${ }^{3}$ Butembo,

Democratic Republic of the Congo (Kinshasa); ${ }^{5}$ Goma,

Democratic Republic of the Congo (Kinshasa)

BACKGROUND: Religious affiliation, by modulating behaviours related to HIV transmission, may be a significant determinant of HIV positivity among individuals in sub-Saharan Africa, yet few empiric data are available on the relationship between religion and HIV.

METHODS: We conducted a cross-sectional survey of individuals attending voluntary counseling and testing clinics in Eastern Democratic Republic of the Congo, and examined relationships between their HIV serostatus and risk factors, with attention to religious affiliation as a potential risk factor.

RESULTS: 380 individuals attending six clinics for voluntary HIV testing were enrolled. The median age was 25 years (range 18 to 68 years) and $54 \%$ were female. 369 participants (97\%) self-identified as Christian (44\% Catholic; 53\% non-Catholic Christian). Twenty-eight patients (7.4\%) tested positive for HIV. We observed statistically significant differences in patient age, education level, marital status and religious affiliation between HIV positive and HIV negative individuals. Further analysis indicated that age $>30$ years (adj OR 47 [95\% CI 2.9 to 770]; $\mathrm{p}=0.007]$ ), married status (adj OR 3.7 [95\% CI 1.1 to 13$] ; p=0.037]$ ), and Catholic religion (adj OR 2.7 [95\% CI 1.1 to 6.8]; $\mathrm{p}=0.030$ ]) were independent risk factors for HIV seropositivity in a multivariable logistic regression model in our cohort. Rates of HIV were higher among Catholic than non-Catholic Christian participants in both single and married participants. Among non-married respondents, the primary HIV prevention modality was condom use $(47 \%)$ and abstinence $(28 \%)$. Among married respondents, being faithful to one partner (62\%) and condom use $(22 \%)$ were the most common prevention modalities reported. The proportion of participants reporting condom use as a 
primary prevention modality did not differ significantly between religious groups; however, within both Catholic and non-Catholic Christian groups, increasing church attendance was associated with decreased use of condoms $(\mathrm{p}=0.011)$.

CONCLUSIONS: In this cross-sectional survey, Catholic (relative to non-Catholic Christian) religious affiliation was associated with an increased risk of HIV. Increasing dialogue and interaction between biomedical practitioners and religious leaders may strengthen HIV prevention efforts in sub-Saharan Africa.

\section{P320}

\section{WHAT AFRICAN, CARIBBEAN AND BLACK WOMEN IN ONTARIO, CANADA HAVE TO SAY ABOUT HIV PREVENTION: IMPLICATIONS FOR POLICY AND} PROGRAMMES FROM THE ONTARIO WOMEN'S STUDY

Tharao, Wangari E1; McWilliam, Sue ${ }^{2}$; Leonard, Lynne ${ }^{3}$; Millson, Peggy'; Benoit, Anita ${ }^{1}$; Remis, Robert ${ }^{1}$; Loutfy, Mona ${ }^{1}$; Sutdhibhasilp, Noulmouk ${ }^{1}$; Chikermane, Vijaya ${ }^{1}$; Rowe, Janet ${ }^{1}$ ${ }^{1}$ Toronto, ON; ${ }^{2}$ Halifax, NS; ${ }^{3}$ Ottawa, ON

BACKGROUND: At the end of 2011, women constituted approximately $23 \%$ of people living with HIV in Canada. In Ontario, an estimated $45 \%$ of women living with HIV are from African, Caribbean and Black (ACB) communities. Our study explored Ontario women'sunderstanding of, and factors influencing acquisition and transmission of HIV. We report here findings involving $\mathrm{ACB}$ women.

METHODS: Participants for a focus group with heterosexual/straight women and one with queer ACB women were recruited through: networks of community-based researchers on the team; posting promotional materials at organizations accessed by women; and snowballing. The discussion(s) were recorded, coded and analyzed thematically.

RESULTS: Seventeen women (heterosexual/straight $(n=10)$ and Queer $(n=7))$ participated and identified the following as factors influencing HIV prevention among ACB women:

a) Silence and misconceptions/misinformation about HIV, complacency, view of HIV as a 'gay' disease and not an issue in Canada.

b) Intersection of migration and settlement processes (lack of legal status, limited access to employment, services and inability to speak official languages, HIV not being prioritized and use of prevention approaches different from those in countries of origin).

c) Gendered cultural and social norms that allowed men to be "players" and reduced women to "second class" citizens, limiting ability to negotiate and/or say no to risky sex.

d) Homophobia.

e) Support networks (friends, family, faith and ethnic communities) that had both positive and negative impacts on HIV acquisition.

CONCLUSIONS: This study highlights the importance of focusing on individual and systemic factors to help ACB women respond to HIV. Breaking the silence surrounding HIV, providing accurate information on HIV within ACB communities in Canada and empowering women to support individual action are critical to reducing HIV transmission. The intersection of social identities and inequities increases ACB women's vulnerability, affects ability to mount effective responses and should be integrated in HIV prevention interventions.

\section{P321}

\section{COSTS AND BENEFITS OF EMPOWERMENT: THE IMPACT ON ACCESS TO SUPPORT AND SELF-CARE WHEN PHAS BECOME SERVICE PROVIDERS}

Wales, Joshua; Li, Alan; Owino, Maureen; Miao, Andrew;

Maseko, Precious; Wong, Josephine; Perreault, Yvette;

Guiang, Charlie

Toronto, ON

BACKGROUND: Community capacity building initiatives have facilitated greater and more meaningful involvement of PHAs (GIPA/MIPA) in the workforce. Research and frontline experience have identified concerns about the impact of role transitions of PHAs from service users to service providers within the HIV sector. Particularly, there are concerns about their access to peer support, mental health care and self-care. A community research partnership was developed to study key concerns, address these challenges, and enhance resiliencies.

METHODS: The study recruited 27 Toronto-based PHAs who have transitioned from clients to service providers within the HIV sector. The participants were diverse with respect to age, gender, ethno-racial backgrounds, and professional roles. To elucidate the contextual experiences of PHAs from different social ethno-racial backgrounds, we conducted three focus groups: one with racialized minority PHAs, one with nonracialized PHAs, and one with PHAs of any ethno-racial identity. Three individual interviews were also conducted. Transcripts were coded by team members based on thematic codes identified collaboratively. Key findings will be communicated to target stakeholders to inform intervention development and knowledge translation strategies.

RESULTS/FINDINGS: Preliminary results identified major changes and challenges in self-care and support-seeking behaviours of PHAs who have transitioned to service providers. Key challenges included: altered self and peer expectations in terms of support needs; boundary challenges due to role changes; loss of social support from former peers who have become clients, and loss of access to former service providers who have become colleagues. Alternative sources of support included new networks of peer PHA service providers, mentorship, and access to care outside the HIV s sector care among others.

CONCLUSION/IMPLICATIONS: The study identified critical emerging issues affecting the health and well-being of PHAs in service provider roles. Further analysis and stakeholder engagement is underway to facilitate identification of individual and community level strategies for further research and evaluation.

\section{P322}

THE FUTURE IS ALREADY HERE: SCENARIO PLANNING AS A TOOL FOR STRATEGIC FORESIGHT

\section{Patten, San ${ }^{1,2,3}$; LeBlanc, Marc-Andre ${ }^{1}$}

${ }^{1}$ Halifax, NS; ${ }^{2}$ Toronto, ON; ${ }^{3}$ Sackville, NB

BACKGROUND: In 2010, the Chief Public Health Officer asked the Ministerial Advisory Council (MAC) on the Federal Initiative to Address HIV/AIDS in Canada to provide its thinking on the future of HIV/AIDS. The MAC commissioned the authors to develop a Foresight Document on the future of HIV/AIDS in Canada over the next 25 years as a basis for long term HIV/AIDS policy advice.

METHODOLOGY: Scenario planning is a technique used for long-term strategic analysis and planning. Scenarios are narratives that describe how the world might look in the future if certain trends were to strengthen or diminish, or if various policy choices are made. The Foresight Document was developed through an iterative process of evidence-gathering, synthesis and consultation. We used the "two axes" method of scenario development and then created evidence-based narratives of the four resulting scenarios.

RESULTS: We will provide an overview of the scenario planning process we underwent for the MAC, as well as the subsequent development of Aboriginal-specific companion resources for the National Aboriginal Council on HIV/AIDS (NACHA). We found that scenario planning can assist decision makers in assessing their assumptions and reviewing policy options. Scenarios are a useful means of identifying early indicators of trends or patterns that may signal a shift towards a certain future outcome, raising awareness of current worldviews about the HIV epidemic, and creating a common language about the dilemmas faced and choices that need to be made. Although scenarios appear to be simple, their implications can be difficult to understand, and thus require careful engagement of stakeholders in their development and interpretation. CONCLUSION: The main value of scenario planning is as a tool for diagnosis, not prescription. Scenario planning broadens the perspective of the HIV/AIDS response in Canada to frame it within the broader health care system and within society as a whole. It provides a robust and innovative framework to think about how the future will evolve and how to position ourselves, in order to influence the future. 
P323

KEY CHALLENGES IN MEETING THE NEEDS OF PEOPLE WHO USE DRUGS: THE PERSPECTIVES OF PEOPLE WORKING IN SHELTERS IN ATLANTIC CANADA

Jackson, Lois'; ${ }^{1}$ Dingwell, Julie ${ }^{2}$; McWilliam, Susan'; Gahagan, Jacqueline'; LeVangie, Dolores ${ }^{1}$

${ }^{1}$ Halifax, NS; ${ }^{2}$ St John, NB

BACKGROUND: The services of shelters are often required by people who use drugs (PWUD), especially the most marginalized sub-population of PWUD. However, little is known from the perspective of those who work in shelters, about the challenges associated with providing services to PWUD. Understanding these challenges is critical to ensuring shelters can address the unique needs of this population, many of whom are living with HCV and/or at risk for HIV.

OBJECTIVE: To explore, from the perspective of people working in shelters, the key challenges in providing services/supports to PWUD.

METHODS: One-on-one voluntary semi-structured qualitative interviews were conducted with 14 people working in shelters in the Atlantic provinces (NFLD, NB, NS \& PEI). When permission was granted, interviews were audio-taped $(n=12)$ otherwise notes were taken $(n=2)$. A qualitative software package (Atlas $\mathrm{Ti}$ ) was used to code the data. Thematic analysis was conducted to determine key themes and subthemes across and within the interviews.

FINDINGS: Key structural challenges were conceptualized in terms of two themes: 1) challenges internal to shelters; and 2) challenges external to shelters. Internal challenges varied across shelters given the diversity in shelter sizes, organizational policies, etc. Nevertheless, for a number of shelters, limited staff resources for responding to the complex needs of PWUD was a key challenge (e.g. the time required to speak with PWUD about their drug issues and other health, social and/or legal concerns). External challenges center on ensuring PWUD have access to needed health and social services. For example, in some communities there are limited methadone and/or detoxification programs, wait times are lengthy and/or admission criteria cumbersome.

CONCLUSIONS: This research documents significant resource challenges for shelters attempting to respond to the complex social, health and harm reduction needs of PWUD, including HIV/HCV prevention. More harm reduction services are urgently needed to ensure shelters can provide appropriate services, supports and referrals for this at-risk population.

\section{P324 \\ KNOWLEDGE AND ATTITUDES RELATED TO HIV/AIDS - SHAPING THE CONTEXT OF HIV PREVENTION IN CANADA}

Dowd, Courtney; Dodds, Jeff; Tremblay, Genevieve;

Galley, Susan; Nelson, Vanessa; Boodram, Chris

Ottawa, ON

BACKGROUND: The Public Health Agency of Canada (the Agency) contracted EKOS Research to conduct a study measuring awareness, knowledge, attitudes and behaviours related to HIV/AIDS. This is the third study in a cycle that identifies trends over time (previous cycles in 2003 and 2006) and by demographics. The 2012 survey also included, for the first time, similar measures related to hepatitis $\mathrm{C}(\mathrm{HCV})$.

OBJECTIVES: This research measures knowledge, attitudes and behaviours related to HIV/AIDS in Canada over time to assist in the identification of priorities for awareness, surveillance, research and program activities including guideline development.

METHODS: A total of 2,000 randomly selected respondents were interviewed; aboriginal, foreign-born, and youth respondents were over-sampled to allow for more detailed analysis of these populations. The margin of error for the overall sample is $\pm 2.2 \%, 19$ times out of 20 .

RESULTS: Overall, compared to previous study cycles, knowledge levels and awareness of modes of HIV transmission and HIV infection are on the decline, however provincial variance does exist. Only 63\% of respondents are able to identify unprotected sexual intercourse as a mode of HIV transmission and only $31 \%$ could identify needle sharing $(84 \%$ and
$45 \%$ respectively in 2003). Furthermore, findings indicate that public attitudes are improving with $71 \%$ of respondents exhibiting low levels of stigma (64\% in 2006), however, HIV remains stigmatized (29\% of respondents exhibited either medium or high levels of stigma) which may be a barrier to disclosure, testing and access to key services.

CONCLUSIONS: Knowledge and awareness of HIV/AIDS are key components influencing the context of HIV prevention efforts in Canada. The Agency will use these findings to develop and deliver community-based social marketing and awareness training to stakeholders, implement public awareness activities and to inform priorities for communicable disease funding solicitations.

\section{P326}

PERCEPTIONS OF "RISK" IN GAY, BISEXUAL, AND OTHER MEN WHO HAVE SEX WITH MEN IN LONDON, ONTARIO: A QUALITATIVE ANALYSIS FROM THE HEALTH IN MIDDLESEX MEN MATTERS (HIMMM) PROJECT

Pugh, Daniel $^{\text {; }}$; Gamble, Kathleen ${ }^{2}$; Coleman, Todd A2 Aykroyd, Gloria ${ }^{2}$; Bauer, Greta $\mathbf{R}^{2}$; Fraser, Meredith ${ }^{2}$; Murphy, Kevin²; Newman, Rob²; Pierre Pitman, Lyn²; Powell, Leanne ${ }^{2}$

${ }^{1}$ Toronto; ${ }^{2}$ London, ON

BACKGROUND: Health in Middlesex Men Matters is a communitybased project examining how various factors impact the health of local gay, bisexual and other men who have sex with men (GB-MSM). Limited information exists on GB-MSM health for those living outside metropolitan areas in Ontario - including how GB-MSM understand/define HIV risk, sexual health, and 'risky' behaviours.

METHODS: Twenty individual, in-depth interviews were conducted with local men, 17 to 76 years old. Interviewees answered questions about knowledge related to HIV/AIDS, sexual health, and risk factors/behaviours. Data from these interviews were coded and organized using NVivo 10. Data were analyzed using critical discourse analysis (CDA) - an approach focusing on how social and political relationships are reproduced and formed within text and speech. Here, CDA is used to gain a better understanding of how risk is conceptualized by GB-MSM and how it factors into sexual practices and decision-making.

RESULTS: The predominant theme from the data was 'risk' and it's relation to participants' knowledge of sexual health and HIV/AIDS. Participants felt that sex was important to their overall health/well-being. Monogamy and condom use were identified as ways of practicing 'safer sex'. 'Risky behaviours' were identified as having multiple sexual partners, having unprotected anal sex and substance/alcohol abuse. Participants felt these practices were more likely to occur (or be encouraged) in particular spaces (i.e. bathhouses and clubs). Most participants indicated some history with risk taking. HIV testing was only likely to occur if sexual practices were self-identified as 'risky'. Participants also used various decision-making methods to assess risk before engaging in 'risky behaviours'.

DISCUSSION: Data on definitions of HIV risk provided information on current levels of sexual risk taking and how risk is assessed before participants engage in 'risky behaviours'. Results will be shared with community and will help guide local HIV prevention efforts.

\section{P327}

STIGMA, DISCRIMINATION, MENTAL HEALTH AND HIV/ STBBI VULNERABILITY

Boodram, Chris; Lary, Tanya; Jackson, Clare; Soon, Christine Ottawa, ON

ISSUE: Emerging research shows a complex relationship between stigma and discrimination, mental health and vulnerability to HIV and other sexually transmitted and bloodborne infections (STBBIs) for certain key populations. The Public Health Agency of Canada has developed a series of population and issue-specific reports and guidance documents examining the links between these factors and STBBI vulnerability for key populations.

METHODS: Agency publications examined include Population-Specific HIV/AIDS Status Reports on people from countries where HIV is 
endemic, Aboriginal Peoples, women, gay and other men who have sex with men, and people living with HIV/AIDS; as well as three questions and answers style documents on sexual orientation, gender identity, and sexual health education for youth with physical disabilities.. Literature examined for these publications included evidence regarding the relationship between stigma and discrimination, mental health and STBBI vulnerability.

FINDINGS: For all key populations examined, evidence indicates an important relationship between the experience of stigma and discrimination, mental health and STBBI vulnerability. Experiences of institutional and interpersonal stigma and discrimination in forms such as racism, sexism, homophobia, HIV-phobia and ableism have an important impact on the ability to form and maintain social support networks, access to safe and appropriate health care, and ultimately on self-esteem, mental health outcomes, risk of suicidality and likelihood of engaging in risk behaviours. Experiences of stigma and discrimination in childhood and adolescence were strongly associated with increased STBBI vulnerability over the lifecourse. Conversely, safe and supportive social environments were generally associated with resilience against STBBIs.

CONCLUSIONS: Addressing stigma and discrimination at institutional and interpersonal levels is key to preventing and addressing HIV and other sexually transmitted and bloodborne infections. Childhood and adolescence are key intervention periods for establishing supportive social environments and providing comprehensive sexual health education, to develop healthy relationships and behaviours, and thus mitigate STBBI vulnerability over the lifecourse.

\section{P328}

\section{REACHING YOUNG PEOPLE WITH INFORMATION ON HIV/AIDS AS AN INFECTIOUS DISEASE IN LAGOS STATE \\ NIGERIA}

\section{Abati, Samuel A}

Mainland, Nigeria

BACKGROUND: Young people constitute the largest section of any country's population. The prevalence rate of HIV is highest among young people of between 18 and 24 years of age.

There have been little or no efforts in engaging the young people on issue of HIV/AIDS information.

Young people are fun of loving, having sex, in so far condom use reduce the risk of HIV infection, based on this we need to engage young people in like manner by organizing edutainment activities like concerts and musical shows for awareness to be able to inform, educate and sensitize them on issues of sexually infection disease like HIV and how it affecting them.

METHODS: In order to carry young people in Lagos Mainland along with HIV/AIDS information. Batula youth movement organization a youth led and youth focus organization working in the field of HIV/AIDS organized a musical concert to educate and sensitize the young people about the basic facts on HIV, the current development on HIV/AID prevention, sexual rights and dangers of sex. The event had in attendance professional in area of HIV/AID, who presented papers on HIV on young people.

RESULT: Most of the participants were hearing about HIV is an infectious disease for the first time. Over $85 \%$ of the participants believe that there is a need for new ways in engaging young people in issues affecting them. More than $70 \%$ of the participant agreed to be a change agent who would advocate for young people involvement in HIV/AIDS campaign. CONCLUSION: If we must make any meaningful or successful head way in passing correct and factual information on HIV/AIDS and any other development as regard HIV/AID as an infectious diseases prevention. 
A

Abaki J.......................... P291

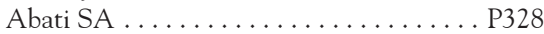

Abbas Z . . . . . . . . . . . . . . . . . .0013,0014

Aboud L......................... O057

Aboud LG . . . . . . . . . . . . . . . . . . P015

Achen M . . . . . . . . . . . . . . . . O048,P157

Ackad N. . . . . . . . . . . . . . . . P076

Adam B . . . . . . . . . . . . . . . . . . . P296

Adam BD . . . . . . O094,P152,P153,P154,P298

Adams A E. . . . . . . . . . . . . . . . . P026

Adrien A . . . . . . . . . . . P085,P131,P236

Afzal A....................... P194

Aghaeepour N . . . . . . . . . . . . . . P010

Aguinaldo J . . . . . . . . . . . . . . . . . P243

Aguinaldo JP . . . . . . . . . . . . . . . . . . P279

Ahluwalia A. ................P243,P279

Ahmed A . . . . . . . . . . . . . . . . . . . . .0082

Akagi L. . . . . . . . . . . . . . . . P043,P044,P167

Akello M ................. O071,P207

Alam A .................... P141

Alam N ...................... P141

Alary M . . . . . . . . . . . . . . . . . . P120

Albert A.................... .0009,0037

Albert A Y . . . . . . . . . . . . . . . . . O035

Alberts A ...................... O036

Alenezi O . . . . . . . . . . . . . . . . . P047,P056

Alexander S. . . . . . . . . . . . . . . . O094,P298

Al-Ghazawi FM . . . . . . . . . . . . . . P021

Alibhai A. . . . . . . . . . . . . . . . P181

Alimente A . . . . . . . . . . . . . . . . P088

Alimenti A. . . . . . . . O O015,0036,0037,P084

Allam $\mathrm{O} \ldots \ldots \ldots \ldots \ldots \ldots \ldots \ldots . . \ldots 082$

Allen M . . . . . . . . . . . . . . . . . P102

Allen V . . . . . . . . . . . . . . . O O047,P051

Allman D . . . . . . . . O094,P085,P187,P188, P236,P238,P298

Alonso M...................... O001

Altenberg J. . . . . . . . . . . . . . . . . . . . . . . О069

Alvarado BE. . . . . . . . . . . . . P183,P184

Amirault M .................. P312

Amram O. . . . . . . . . . . . . . . . O016

Ancuta P . . O027,O028,O030,P002,P004,P038

Andany N . . . . . . . . . . . . . . . P060,P066

Anderson K . . . . . . . . . . . . . . . . O051

Anderson S . . . . . . . . . . . . . . . O112

Andrews J. . . . . . . . . . . . . . P286

Angel J . . . . . . . . O063,P038,P045,P092,P113

Angel J B . . . . . . . O026,O029,O081,P021,P066

Anis A ................... P176

Anmole $G \ldots \ldots \ldots \ldots \ldots \ldots \ldots \ldots$. . . . . . . . . . . . . . . .

Antic J . . . . . . . . . . . . . . . . . . P165

Antoniou T . . . . . . . . . . . P089,P130,P233

Appellaniz B. . . . . . . . . . . . . . . . . P018

Apuzzo L. . . . . . . . . . . . . . . . . . . . . . O096

Ara A ................... O062,P023

Arbess G. . . . . . . . . . . . . . O011,P074,P075

Archibald C . . . . . . . . O013,О014,О093,P129

Archibald CP .................P119,P131

Armstrong M . . . . . . . . . . . . . . . . P113

Asad S . . . . . . . . . . . . . . . . . . O003

Aslanov R .................... P058

Atim S . . . . . . . . . . . . . . . . . O018

Atkinson M . ........... O011,P074,P075

Auyeung K................... O032

Avery L........................ P316

Ávila-Ríos S. . . . . . . . . . . . . . . . . . . O060

Aykroyd G . . . . . . . . . . . . . . P125,P326

Azzopardi C . .................. P295

\section{B}

Bacon J. . . . . . . . O . O 73,P111,P112,P150,P151

Bahlji A ................... 0036

Baidoobonso S . . . P194,P195,P196,P213,P218

Baig T. . . . . . . . . . . . . . . P022

Bajunirwe F . . . . . . . . . . . . . . P234

Bakombo D ................... $237, \mathrm{P} 262$

Balasanyants G. . . . . . . . . . . . . . . P050

Balfour L. . . . . . . . . . . . . . . . . . . P113,P249

Balian R . . . . . . . . . . . . O069,P202

Ball B . . . . . . . . . . . . . . . . O057,P015

Ball TB. . . . . . . . . . . . . . . . . . O001

Balneaves L . . . . . . . . . . . . . . . . . . P034

Baltzer ........................ P292

Baltzer Turje R . . . . . . . . . . . . P143,P162

Bam RA ......................... 0005

Bangsberg DR. . . . . . . . . O . . . . . . P087,P083,P093

Bannan C. . . . . . . . . . . . . . . . P150,P151

Bannerman M .................. P202

Baraki B . . . . . . . . . . . . . . . . . .0087

Barbour TL. . . . . . . . . . . . . . . . . P277

Baril J-G. . . . . . . . . O103,P045,P076,P101

Barr SD . . . . . . . . . . . . . . . . . . 0083

Barrett B. . . . . . . . . . . . . . P212

Barrios R. . . . . . O097,O100,P031,P043,P044, . P161,P168,P171,P175,P186,P292

Barry C. . . . . . . . . . . . . . . . . . . O001

Bauer G . . . . . . . . . . . . . . . P195,P196

Bauer GR . . . . . . . . . . . . . . . . P125,P326

Baxter L . . . . . . . . . . . . . . . . . P280

Bayoumi AM . . O047,P051, P089, P130 P169,

......................... P211

Beaudry S................... P247

Beaver CK . . . . . . . . . . . . . . . . . . . P249

Beaver K.................P261,P270

Becker M ..................... P282

Becker M L. . . . . . . . . . . . . . . . . . . . . . P052

Beckerleg WW. . . . . . . . . . . . . . P189

Behlim T . . . . . . . . . . . . . . P146,P182

Bekele T. . . . . . . . . . . . . P074,P075,P258

Bekele TM . . . . . . . . . . . O011,P111,P112

Béliveau C . . . . . . . . . . . . . . . . P191

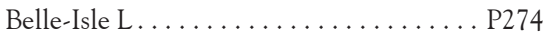

Bellos N . . . . . . . . . . . . . . . . . . . . . P041

Bellrose L . . . . . . . . . . . . . . . . P216,P224

Bendayan R . . . . . . . . . . . . O090,0091,P037

Bennett M T . . . . . . . . . . . . . . O0008

Benoit A. . . . . . . . . . . . . . . . P135,P320

Benoit AC . . . . . . . . . . P077,P080,P249

Bergevin M. . . . . . . . . . . . . . . . . P191

Bernard N. . . . . . . . . . . . . . . . . . O027

Bernier A . . . . . . . . . . . . . . . . . 0030

Bernier M.......... O077,P251,P272,P296

Berry N. . . . . . . . . . . . . . . . . P234

Berthoux L. . . . . . . . . . . . . . . . . . . . . O085

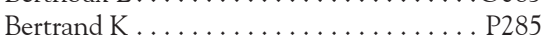

Bielawny T. . . . . . . . . . . . . . . . . . . O001

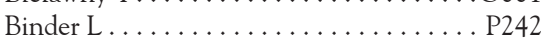

Binder LD . . . . . . . . . . . . . . . . P228

Bingham B .................. P205

Birungi J . . . . . . . . . . . . . . . O0 71

Bisignano A . . . . . . . . . . . . . . . O052,P244

Bisignano C . . . . . . . . . . . . . . . . 0053

Bitnun A . ......... O015,P084,P088,P295

Bitnun S A. . . . . . . . . . . . . . . . O068

BLACCH Study Team. . . . . . . . . . . P195,P196

Blair AH. . . . . .......... O049,P198,P303

Blais M...... O021,O094,P254,P255,P257,

............P284,P296,P297,P298,P301

Blanchard M .............. P251
Blanchette C . . . . . . . . . . . . . . P120

Blitz S. . . . . . . . . . . O068,P059,P066

Blouin K. . . . . . . . . . . . . . . . . . P120

Bondy GP. . . . . . . . . . . . . . . . . . 0008

Boodram C...................P324,P327

Boomer J. . . . . . . . . . . . . . P110

Borek N V . . . . . . . . . . . . . P035,P291

Borg P. . . . . . . . . O005,0063,0064,P039

Boucher M . . . . . . . . . . . . . . . . О039

Boulassel M-R . . . . . . . O028, О027, 0030

Boulay-Coppens DR . . . . . . . . . . . P228

Bourassa C . . . . . . . . . . . . . . O013, 0014

Bourgeois C . . . . . . . . . . . . . . . . . . . . O011,P074

Bourgeois LC . . . . . . . . . . . . . . . P0 P 75

Bowes J. . . . . . . . . . . . . . . . . . . P091

Bowmer MI .................. P163

Bozorgzad A . . . . . . . . . . . . . . . . O0888

Brakier-Gingras L. . . . . . . . . . . . . . . . . P025

Brennan DJ ............ O023,P250,P290

Brennenstuhl S . . . . . . . . . . . . . P250

Brenner B................ O103,P020

Brenner BG . . . . . . . . . . . . . . . . . P019,P097

Brinkman R . . . . . . . . . . . . . . P010

Brockman M . . . . . . O O060,0061,0086,P007

Brockman MA................. . 0087

Broliden K . . . . . . . . . . . . . . . . . . . 0057

Brondani MA................... P110

Brooks J . . . . . . . . . O O093,P095,P103,P192

Brophy J . . . . . . . . O015,P084,P088,P089,P092

Brophy JC. . . . . . . . . . . . . . . P091,P319

Brouillette M-J. . . . . . . . . . . . . . . . . P073

Brown J. . . . . . . . . . . . . . . . . . P127,P155

Brown M ................... O078

Brownrigg B . . . . . . . . . . . . . . . . . . . . . . . . . P1646

Brownrigg R.................. P161

Brumme C . . . . . . . . . . . . . . . . . O006

Brumme CJ. . . . . O060,0087,0089,0092,P104

Brumme Z. . . . . . . . . O061,0086,P011,P013

Brumme ZL ............. O059,0060,0087

Brunetta J. O011,O044,O063,O064,P074,P075

Bubela T..................... O106

Buchbinder $\mathrm{S} \ldots \ldots \ldots \ldots \ldots \ldots \ldots \ldots 61$

Buchner C S. . . . . . . . . . . . . . . . . . P159

Burchell A . . . . . . . . . . . . P0 P053,P074,P075

Burchell AN. ..... . O011, O017, O047, O101, .0102, P051,P069,P077,P080,P111,P112,

..................... P135,P288

Burdge D . . . . . . . . . . . . . P066

Burgener A. .............. O057,P015

Burstein P. . . . . . . . . . . . . . . . . P274

Burt KA . . . . . . . . . . . . . . P163

Buxton J . . . . . . . . . . . . . . P145,P173,P294

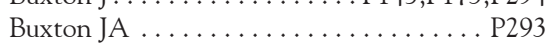

Byakwaga H.............. O087,P083

\section{$\mathrm{C}$}

Cadar A E . . . ................. P025

Caine V . . . . . . . . . . . . . . . . . P253

Cairney J . . . . . . . . . . . . . P111,P112

Callon C. . . . . . . . . . . . . . . . . . . . P108

Calzavara L. . . . . . . . . O094,P085,P137,P187,

P188,P236,P238,P298

Camacho F. . . . . . . . . . . . . P0 P0 8

Cameron B................ O090,P113

Cameron DW. . . . . . . . . . . . . . . . P092

Cameron R. . . . . . . . . . . . . . P223

Campbell D . . . . . . . . . P090,P152,P153,P154

Campbell K . . . . . . . . . . . . . . P052

Canada-China Team The ........... P137

Canadian Co-Infection 
Cohort (CTN)

Canadian HIV women's sexual and

reproductive health

cohort study (CHIWOS).... . P240,P262,P263

Canadian Observational Cohort

(CANOC) . . . . . . . . O017,O101,0102,P069

Canadian Pediatric AIDS

Research Group . . . . . . . . . . . . . . . . . O015

Canadian Pediatric and Perinatal HIV/AIDS

Research Group (CPARG) . . . . . . . .P084,P088

Cantin R . . . . . . . . . . . . . . . . O103

Capina R .......................0001

Caprara D. . . . . . . . . . . . . . . . . P090

Carlson J. . . . . . . . . . . . . . . . . .0061

Carlson JM. . . . . . . . . . . . . . . . . . . O060

Carnochan T ...............P052,P275

Carrasco-Medina L . . . . . . . . . . . . P003,P021

Carsone S C . . . . . . . . . . . . . . . . . O055

Carter A . . . . . . . . . . . . . . . .P122,P127

Carter AJ . . . . . . . . . . O033,P237,P240,P263

Carter R . . . . . . . . . . . . . . . . . . . P054

Carvalhal A ............. O011,P074,P075

Casey A . . . . . . . . . . . . . . . . . . P280

Cattaneo J S. . . . . . . . . . . . . . . . . . . . .0055

Cedar Project Partnership . . . . . . . . . . . P198

Centre for Brain Health

in HIV/AIDS . . . . . . . . . . O011,P074,P075

Cescon A . . . . O007,O017,,0034,O101,O102,

................... P055,P069,P204

Chaban B..................... 0035

Chakrapani V..................... P283

Challacombe L A. . . . . . . . . . . . . P179

Chamberland A................. P068

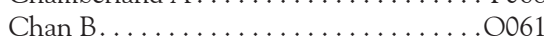

Chan JK...................... P005

Chan K. ..... O007, O017, O100,O101,P069,

.P107,P122,P126,P168,P190,P204,P208,P209

Chang B . . . . . . . . . . . . . . . . . . . . P040

Charbonneau J.................... P025

Charest H. . . . . . . . . . . . . . . . . . . . O103

Chartier L. . . . . . . . . . . . . . . . . . . . . . P289

Chartrand-Lefebvre C . . . . . . . . . . . . P068

Chau W ............... O097,P107,P167

Chaworth-Musters T . . . . . . . . . O0036,0037

Chelico L . . . . . . . . . . . O062,P022,P023,P024

Chen BB. . . . . . . . . . . . . . . P126

Chen Y.............. O080,P124,P204

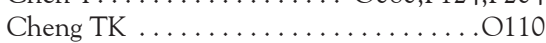

Cherid H . . . . . . . . . . . . . . O025,P001

Chettiar J . . . . . . . . . . . . . . . . O016,O . . . . . P312,P307

Chettiar J M. . . . . . . . . . . . . . . . . P309

Cheuk E...................... P136

Cheung W-F. . . . . . . . . . . . . P016

Chikermane V . . . . . . . . . . . . . . .P276,P320

Chinski L. . . . . . . . . . . . . . . . . . . . O097

Chiu C G....................... . O034

Choi S . ................. O073,P258

Chongo M . . . . . . . . . . . . . . . . . . . P229

Chopera D . . . . . . . . . . . . . . O061,P011

Chown S . . . . . . . . . . . . . . . . O020

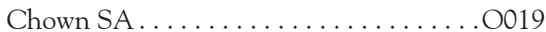

Christian CW .................. P198

Christian WM . . . . . . . . . . . . . O049,P303

Christine H . . . . . . . . . . . . . . . . . P042

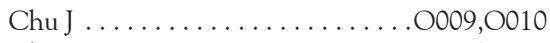

Chu SK. . . . . . . . . . . . . . . . O105,P228

Chu T. . . . . . . O072,O097,O098,P158,P160

Chui CK. . . . . . . . . . . . . . . . . . . . . O0889

Cihlar T . . . . . . . . . . . . . . . . . . O005

CIHR Emerging Team in HIV Therapy and Ag-

ing (CARMA) ........... O012,P079

US GROUP INC.

CIHR Team in the Study of Acute HIV Infection in Gay Men . . . . . . . . . . . . O020,P256 Clark B E . . . . . . . . . . . . . . . . . . . .0032 Clark M . . . . . . . . . . . . . . . . . . P092 Clarke C...................... P171 Clarke GB ................... .P153,P154 Clayton K. . . . . . . . . . . . . . . . . О031 Cleret A. . . . . . . . . . . . . . . . . . . . . . P004 Cleret-Buhot A . . . . . . . . 0027,0028,0030 Coburn T . . . . . . . . . . . . . . . . . . P293 Cockerill R.................... P138 Cohen C. . . . . . . . . . . . . . . . . P039 Cohen J . . . . . . . . . . . . . . . . . P066 Coldman A . . . . . . . . . . . . . . . . . . . O034 Coleman T. . . . . . . . . . . . . . . . . . P218 Coleman TA . . . . . . . . . . . . P125,P326 Colley G. . . . . . . . . O O007,0072,0089,0097, O100,O102,P031,P098,P107, .P122,P160,P167,P168,P170

Collins E. . . . . . . . . . . . . . . O O O11,P074,P075

Compton M........... O072,P155,P162

Connick E . . . . . . . . . . . . . . . . . . . . O086

Consolacion T B . . . . . . . . . . . O O 48,P148 Conway B. . . . . . . . O064,0096,P045,P047, P048,P056,P066,P114 Cooper C . . . . O017,O067,O101,O102,P050, .P053,P054,P055,P069,P113,P114,

Cooper C L . . . . . . . . . . . . . . P003 Corace K . . . . . . . . . . . . . . . . . . P113 Co-researchers Y . . . . . . . . . . . . . . P294 Costiniuk C T . . . . . . . . . . . . . . P021 Côté H . . . . . . . . . . . . O009, O010,0012 Cote H C . . . . . . . . . . . . . . . . . . . P079 Côté P-B. . . . . . . . . . . . . . . . . . . . . P297 Cote S C . . . . . . . . . . . . . . . P021 Cotton LA . . . . . . . . . . . . . . . . . . . . O061 Coutlee F . . . . . . . . . . . . . . . . . O068 Cox D. . . . . . . . . . . . P050,P176,P177 Cox J . . . . . . . . . . O067,P114,P174 Cox K. . . . . . . . . . . . . . . . . . . P293,P294 Crawley AM. . . . . . . . . . . . . . . . . O026,P003 Crofoot G. . . . . . . . . . . . . . . . . . P041

Cryan C . . . . . . . . . . . . . . . . P178

Cui Z-S..................P124,P167

Currie LM .................. P110

Cyr L . . . . . . . . . . . . . . . . . P068

Czarnecki C.................... . O001

Czarnecki C K . . . . . . . . . . . . . . P012

\section{$\mathrm{D}$}

Da Fonseca S . . . . . . . . . . . . . O027 da Silva J . . . . . . . . . . . . P165,P166,P167 Daar ES ..................... O086 Dalgarno G. . . . . . . . . . . . . . . P162

Dalton C . . . . . . . . . . . . . . P215

Dandal A ...................... O022

Dandal J A . . . . . . . . . . . . . . . . . . . . P260

Danforth J . . . . . . . . . . . . O051,P225

Daniel CA .......................... .0070

Danis C . . . . . . . . . . . . . . . . . P286

Daniuk C . . . . . . . . . . . . . . . . . . O001

Danroth R . . . . . . . . . . . O086, O0887,P013

Dare O . . . . . . . . . . . . . . . . P131

Darisi $\mathrm{T} \ldots \ldots \ldots \ldots \ldots \ldots \ldots \ldots . \ldots . \ldots . \ldots . \ldots 245$

Dave C....................... O081

Davidson A . . . . . . . . . . . . . . . . . . . O026

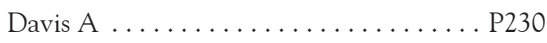

Davis J . . . . . . . . . . . . . . . . . . P013,P226

Davis M . . . . . . . . . . . . . . P143,P162

Day I. . . . . . . . . . . . . . . . . . . . . . . P098

Dayle J ................P237,P262
Dayneka N . . . . . . . . . . . . . . . . . . . P091

De Alvaro, Critina. . . . . . . . . . . . P076 de Pokomandy A ........ O077,P066,P237,

P240,P262,P263

De Rosa MF . . . . . . . . . . . . . . . . . . . O091

DeBeck K.................. O110,P300

Deering K..................... P122

Deering KN . . . . . . . . . O O 16, P309,P318

DeGrado W . . . . . . . . . . . . . . . . P018

deJesus E..................P039,P040

Deli-Houssein R. . . . . . . . . . . . . P182

Demers A . . . . . . . . . . . . . . . . P094

Demlow E. . . . . O072,0097,0098,P158,P160

De-Oertel S . . . . . . . . . . . . . O063,0064

dePadua A ..................... P253

Depatureaux A................. P019

DePutter M E................... P245

Desmarais, Manon.................. P065

Desroches M. . . . . . . . . . . . . . . . . P227

DeWet, Joss .................... P076

Dewey CE. . . . . . . . . . . . . . . . . . . . . O045

Dickson N . . . . . . . . . . . . . . . . O045

Dietrich J . . . . . . . . . . . . . . P226

Diliso N . . ................... P261

Dilley L. . . . . . . . . . . . . . . . . . O107

Ding E . . . . . . . . P031,P043,P044,P046,P127

Ding S . . . . . . . . . . . . . . . 0084

Dingwell J. . . . . . . . . . . . . . . P323

Dobrer S.........................0071

Docter H . . . . . . . . . . . . O094,P298

Dodds J. . . . . . . . . . . . . . . . . P103,P324

Dodds J V . . . . . . . . . . . . . . . . . . . P095

Donahue D A. . . . . . . . . . . . . . . . . . . O004

Dong W . . . . . . . . . . . . O006,0089,P036

Dos Santos S . . . . . . . . . . . . . . P176

Doshi M......................... P316

Doualla-Bell F . . . . . . . . . . . . . O103,P191

Doupe G. . . . . . . . . . . . . O048,P161,P256

Dowd C . . . . . . . . . . . . . . . . . P324

Dowdall-Smith S . . . . . . . . . . . . . O093

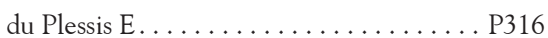

Dube A................... P140

Dubuc D.................... P222

Ducandas X .................. 0056

Ducruet T. . . . . . . . . . . . . 0039,0040

Dufault, Catherine. . . . . . . . . . . . . P065

Duff P . . . . . . . . . . . P203,P205,P207

Duffy L . . . . . . . . . . . . . . . . . P312

Dufresne $\mathrm{S} \ldots \ldots \ldots \ldots \ldots \ldots \ldots \ldots . . \ldots$. . . . . . . . . . .

Dugas M.................... P174

Durand M. . . . . . . . . . . . . P068,P101

Dushoff J. . . . . . . . . . . . . . . . . P185

Dykeman M........... O054,P140,P253

\section{$\mathrm{E}$}

Edmiston L. . . . . . . . . . . . . . . . . . P179

Eisner R . . . . . . . . . . . . . . P192

El-Helou P . . . . . . . . . . . . . . . . . O096,P066

Elliott R . . . . . . . . . . O O105,P273,P274

El-Salfiti A...................... 025

Elston D . . . . . . . . . . . . . . . .P259,P270

Embree $J \ldots \ldots \ldots \ldots \ldots \ldots \ldots \ldots$. . . . . . . . 12

Emerging Team Grant in HIV Therapy

and Aging (CARMA) and the CIHR. ....0009

Emlet CA................... P250

English K . . . . . . P150,P151,P152,P153,P154

Ennabil N................... P262

Enns E ...................... P192

Erikson S .................. P234

Etcheverry E.................. P275 
Fadel R...................... P296

Fairley C. . . . . . . . . . . . . . . . . P156

Falkenhagen A................. O003

Fall A....................... P142

Faller E....................... O025

Farr C . . . . . . . . . . . . . . . P017

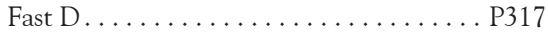

Faye R. . . . . . . . . . . . O013, O014,P132

Fellows L . . . . . . . . . . . . . . P0 073

Feng C . . . . . . . . . . . . O110,P203

Feng CX................... 016

Feng Y .................... P024

Fenta H .................... P194

Ferbeyre $G \ldots \ldots \ldots \ldots \ldots \ldots \ldots . \ldots . \ldots 25$

Ferko N .................... P045

Ferlatte O............... O041,P110

Fernandes J. . . . . . . . . . . . . . . . . . . . . . O029

Fernandes J R. . . . . . . . . . . . . . . . O08 81

Fernet M. . . . . . . . . . . . P254,P255,P257

Fielden S . . . . . . . O099,P147,P148,P258

Finch $\mathrm{L} \ldots \ldots \ldots \ldots \ldots \ldots \ldots \ldots \ldots$. P0 . . . . . . . . . .

Fisher M.....................0064

Fisher Raymond H. . . . . . . . . . . . . P190

Flavia N ..................... P234

Fletcher D . . . . . . . . . . . . P111,P112

Flicker $S \ldots \ldots \ldots \ldots \ldots \ldots \ldots \ldots$. . . . . . . . . 051

Flores-Aranda J . . . . . . . . . . . . . . P285

Foisy M M . . . . . . . . . . . . P067,P086

Fok AC . . . . . . . . . . . . P078

Fongmoon D . . . . . . . . . . . . 0058

Forbes J. . . . . . . O015,О036,О037,P084,P088

Forcellino L . . . . . . . . . . . . . P073

Ford $G \ldots \ldots \ldots \ldots \ldots \ldots \ldots$. P155

Fordyce M .................. P040

Forrest J . . . . . . . . . . . . . . O042,P208

Forrest JI. . . . . . . . . . . O043,P127,P190,P209

Fowler S . . . . . . . . . . . O095,P153,P154

Fralich T. . . . . . . . . . . . . .063,0064

Francombe K . . . . . . . . . . . . P202

Frank P.................. P228

Fraser C . . . . . . . . . . . . . O096,P048

Fraser M ................P125,P326

Friesen K . . . . . . . . . . . P035,P291

Frolova $\mathrm{O} \ldots \ldots \ldots \ldots \ldots \ldots \ldots \ldots$. 050

Frost $\mathrm{E} \ldots \ldots \ldots \ldots \ldots \ldots \ldots \ldots \ldots$ P191

Fuchs J . . . . . . . . . . . . . . O061

Fung F. . . . . . . . . . . . . P175

Fung K .................... 0053

Funk A ...................... P294

\section{$\mathrm{G}$}

Gadawski I . . . . . . . . . . . . . . . . . P079

Gahagan J. . . . . . O054,P071,P140,P149,P219, . P228,P230,P242,P253,P267,P323

Gale-Rowe M. . . . . . . . P094,P095,P103,P139

Galindo J . . . . . . . . . . . . . . . . P183,P184

Gallagher L . . . . . . . . . . . . . . . . . . . . P056 . . . P P1

Gallaher S . . . . . . . . . . . . . . . P150

Gallant J. . . . . . . . . . . . . . . . . P039

Gallant S . . . . . . . . . . . . O066,P057,P123

Galley S . . . . . . . . . . . . . . . . . P324

Gamble K.................. P326

Gamble M ..................... O044

Ganase B ..................... P046

Gao Y.................... P286

García-Morales C. . . . . . . . . . . . . . . O060

García-Téllez T . . . . . . . . . . . . . . 0060

Gardner S. . . . . . O047,P051,P053,P077,P080, P111,P112,P135,P152,P153,P154

Garrido-Rodríguez D . . . . . . . . . . . . . 0060

Gaudreau M-A................ P178
Gaudreau M-C. . . . . . . . . . . . . . . . . . . . P004

Gauvin M-E.................. P272

Gee K....................... P062

Gendron K.................. P025

George C . . O023,P131,P194,P230,P289,P290

Germain A. . . . . . . . . . . . . . . . . P115

Gervais N................... P072

Ghazawi F. . . . . . . . . . . . . . O025,P001

Gichuhi M................. P316

Giesbrecht C . . . . . . . . . . . . . . O012

Giffin C . . . . . . . . . . . . . . . P161

Giguere P . . . . . . . . . . . . P029,P045

Gil DM..................... P078

Gilbert C . . . . . . . . . . . . .P006,P014

Gilbert E. . . . . . . . . . . . . . . . . . . . . P016

Gilbert M. . . . . . . . O020,0046,О048,0099, .O100,P126,P128,P147,P148, P156,P157,P256,P271

Gill J. . . . . . . . . . . . O011,P049,P074, P076 Gill MJ . . . . . . . . . . . . . . P033,P075,P099

Gillis J . . . . . . . . . . . . . . . P053,P055

Gilmore N . . . . . . . . . P002,P063,P064,P100

Glazier RH . . . . . . . . . . . . P089,P130,P233

Globerman JM . . P150,P151,P152, P153, P154,

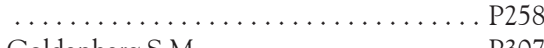

Gonzalez-Serna A . . . . . . . . . . . P106

Gosselin A . . . . . . . O027,0028,0030,P004

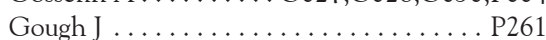

Gough K. . . . . . . . . . . . . . . P066

Gough K A. . . . . . . . . . . . . . . . . P169

Goulet J-P . . . . . . . . . . . . . . . . 0030

Gourde D . . . . . . . . . . . . . . O066,P057

Grace D . . . . . . . . . . . . . O020,P218,P256

Graham R. . . . . . . . . . O012,P035,P291

Gray $G \ldots \ldots \ldots \ldots \ldots \ldots \ldots \ldots . \ldots \ldots 226$

Greene S . . P237,P240,P249,P258,P263,P270

Greenspan NR . . . . . . . . . . . . . .P138,P296

Grewal P. . . . . . . . . . . . . . . . P293

Grewal R . . . . . . . . . . . . . . . P051

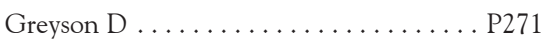

Gu L....................... P028

Guiang C . . . . . . . . . . . . . . . P321

Guigere P................... P113

Guillemi S . . . . . P P034,P046,P061,P078,P098, P116,P117,P186,P199,P201,P292

Guillemi SA . . . . . . . . . . . . . . . . . . . P175

Guimond T . . . . ..............0069

Guirguis-Younger M. ............... O107

Gulzar N. . . ................ P018

Gunning JL ............... P176

Guo H ..................... P139

Gurm J . . . . . . . . . . . . . . . . . . . P292

Gustafson R . . . . . . O072,О097,О098,О100, P122,P126,P158,P160,P168,P186

\section{$\mathrm{H}$}

Haase D . . . . . . . . . . . . . . . . . . . P066 . . . . . . . P083,P093

Haberer J . . . . . . . . . . . . . . . . . P083,P093

Haddad E . . . . . . . . . . . . . . . . . 0030

Hafizi R . . . . . . . . . . . . . . . P113

Hagen BN ................. P030

Haidara A. . . . . . . . . . . . . . . . . . P014

Haig T . . . . . . . . . . . . . . . . O021

Hall D. . . . . . . . . . . . . . . P158,P175

Hall JC . . . . . . . . . . . . . . . . . . P016

Halloran D . . . . . . . . . . . . . P176

Hall-Patch C . . . . . . . . . . . . . . . . O012

Halpenny R . . . . . . . . . . . . . . . . . . . . O044

Halverson J. . . . . . . . . . . . . . . . O093,P129

Ham D . . . . . . . . . . . . . . . . P110

Hambly K............. P243,P258,P279
Hamilton-Hinch B. . . . . . . . . . . . . P230

Hamm J ...................... 0034

Hammond T. . . . . . . . . . . . . . . P245

Han Y............... O002,P027,P028

Hannigan B . . . . . . . . . . . . . P018

Haq K..................... P005

Hardy I . . . . . . . . . . . . . . . . . . O103

Harrigan PR . . . . . .0059,0089,0092,0093,P043,

P044,P104

Harrigan R . . ..... O006,O0087,P036,P106,P107

Harris G . . . . . . . . . . . . . . . . . O054

Harris GE . . . . . . . . . . . . . . . P140

Harris M. . . . P046,P061,P078,P098,P114,P167

Harrison S . . . . . . . . . . . . P155,P159

Hart TA . . . . . . O023, P250,P290,P296,P299

Harvey-Langton A. . . . . . . . . . . . . . P073

Hasselrot K. . . . . . . . . . . . . . . . . . 0057

Hassounah S. . . . . . . . . . . . O002,P027

Hawkes M . . . . . . . . . . . . . . . P319

Hayashi K................... P311

Heath K . . . . . . . O072,O0097,P128,P160,P168

Heath K V . . . . . . . . . . . . . . O100,P170

Heath M. . . . . . . . . . . . . . . . . P202

Hébert M.................... P284

Hemmingsen S. . . . . . . . . . . . . O035

Hennan M . . . . . . . . . . . . . . . . . P216,P224 . . . OO13,O014,P132

Hennink M ........... O013,O014,P132

Henrick B M .................. P008

Henry K . . . . . . . . . . . . . . . . . O063,P010,P040

Henry KA. . . . . . . . . . . . . . . . . . . . P009

Henschell C.................... P286

Hernández-Juan R . . . . . . . . . . . . . . . 0060

Hew H . . . . . . . . . . . . . . . . . . . P0 P 48

Higeli A . . . . . . . . . . . . . . P150

Higginson A. . . . . . . . . . . . . . . P289

Hill J. . . . . . . . . . . . . . . . . . . 0035

Hill-Mann A .......... O054,P071,P140

Hills-Nieminen C . . . . . . . P165,P166,P167

Ho C. . . . . . . . . . . . . . . P086

Hogg R. . . . O080, P078,P107,P109,P116,P117,

.... . P126,P168,P190,P199,P201,P204,P208,

P209,P226,P292

Hogg RS . . . . . O007,,0017,О033,О034,О042

.. O043,O074,O100,O101,O102,O104,P031, .034P043, P044,P069,P093,P124,P127, P167, P170,P193

Hollmann S . . . . . . . . . . . . . . . . P045

Holly CD . . . . . . . . . . . . . . . . . P113

Homer J . . . . . . . . . . . . . . O 112

Hongbin Z . . . . . . . . . . . . . . . O 111

Hopman W ................... P050

Hoque T. . . . . . . . . . . . . . . . . 0090,0091

Horwitz MS . . . . . . . . . . . . . . P017

Hosein SR . . . . . . . . . . . O101,0102, P069

Hottes T. . . . . . . . . . . . . . O041,0046

Houston $\mathrm{S} \ldots \ldots \ldots \ldots \ldots \ldots \ldots$. P086,P133

Howard T. . . . . . . . . . . . . . . 0043

Hsiung G-YR . . . . . . . . . . . . . . . . P078

Huang W . . . . . . . . . . . . . . . P027

Huarte N . . . . . . . . . . . . . . . . . . P018

Huchet E ................ O066,P057

Hudson P . . . . . . . . . . . . . . . P140

Hudson P L . . . . . . . . . . . . . . . . . . 0054

Hughes A . . . . . . . . . . . . . . . . . 0045

Hughes C . . . . . . . . . . . . . . . P067,P086,P133

Hui C . . . . . . . . . . O022,0052,О053,P261

Hui C S . . . ............... P260

Hull M . . . . . . . O0667,P046,P054,P078,P098,

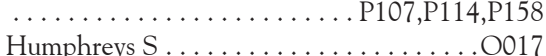


Hunt $\mathrm{N}$ . .0069

Hunt P . . P083,P093

Hunt PW

Husbands W

Husbands WC

$\mathrm{P} 138, \mathrm{P} 194, \mathrm{P} 213$

Hwang SW

$\mathrm{O} 075, \mathrm{P} 221$

Hyshka E

$\mathrm{O} 107, \mathrm{P} 258$

......................

I

Ibanescu I. . . . . . . . . . . . . . . . . . . . . P020

Ibanescu R-I . . . . . . . . . . . . . . . . . . P097

Ibáñez-Carrasco F. . . . . O011,P074,P075,P218

Ibarra K . . . . . . . . . . . . . . P268

Ion $\mathrm{A} \ldots \ldots \ldots \ldots \ldots \ldots \ldots$. P259,P270

Irving A . . . . . . . . . . . . . . . . . . . . . . . P054

Isaac-Renton J . . . . . . . . . . . . . . . P148

Ishida T . . . . . . . . . . . . . . . P028

Isiko S. . . . . . . . . . . . . . . . . . . P207

Iwajomo $\mathrm{OH} \ldots \ldots \ldots \ldots \ldots \ldots \ldots$. . . . . . . . . . .

Iwamoto A . . . . . . . . . . . . . . P028

$\mathrm{J}$

Jackson C.................... P327

Jackson L . . . . . . . . . . . . . . . P140,P323

James L....................P113,P246

Jenabian M-A . . . O082,P002,P006,P038,P177

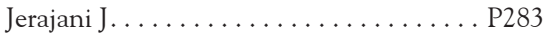

Jessen $\mathrm{H} \ldots \ldots \ldots \ldots \ldots \ldots \ldots$. . . . . . . . . . 0086

Ji H. . . . . . . . . . . . . . . . . . . . P192

Jia J...................... O112

Johns K W . . . . . . . . . . . . . . . O008

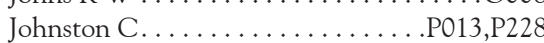

Jollimore J . . . . . . . . . . . . O020,P190

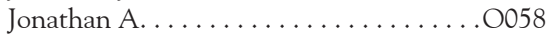

Jones B . . . . . . . . . . . . . . . . P007

Jones JE . . . . . . . . . . . . . . . P217

Jones M . . . . . . . . . . . . . P176,P188

Jongbloed K . . . . . . . . . . . . . O111,P198

Jónsdóttir L . . . . . . . . . . . . . . . . P178

Joseph L.................... P146

Joshi S . . . . . . . . . . . . . . . . . . . O0003

Julien R . . . . .............. P296

\section{$\mathrm{K}$}

Kabakyenga J . . . . ................ P093

Kaddu RP . . . . . . . . . . . . . . . . P316

Kagan RM . . . . . . . . . . . . . . . . . O092

Kaida A . . O033,O077, O080,O104,P083,P093, .P204,P226,P234,P237,P240,,P262,P263

Kain K C ................... 0065

Kakal J . . . . . . . . . . . . . . . . . . . . O025

Kakkar F. . . . . . . . O O039,O040,P084,P088

Kaler A. . . . . . . . . . . . . . . . . . . . . . P181

Kanagaratham C . . . . . . . . . . . P002,P038

Kang L . . . . . . . . . . . . . . . P137,P187,P238

Kanters S . . . . . . . . . . . . P083,P093,P126

Kaplan K . ..................... P311

Karago-Odongo J . . . . . . . . . . . . . P213

Karanja S . . . . . . . . . . . . . P180,P206

Karapita S. . . . . . . . . . . . . . . . . . . O055

Kasper K. . . . . . . . . . . . . . . . . . O096,P052

Kastner J. . . . . . . . . . . . . . . . . . . . . P234

Katherine R . . . . . . . . . . . . . . . . . . O069

Kaul R ........ O044,O047,O065,P051,P060

Kazatchkine C . . . . . . . . . . . O O105,P273

Keefe L....................... P147

Kelleher A . . . . . . . . . . . . O061,0086,0087

Kelly DV ...................... P163

Kema I . . . . . . . . . . . . . . . . . . . . P038

Kema IP . . . . . . . . . . . . . . . . . . . . . P002

Kembabazi A ................... P083

Kendall PR. . . . . . . . . . . . . . . . . . O046
US GROUP INC.

Kennedy L . . . . . . . . . . . . . . . . . . . . . . . P0 P082

Kennedy R ........... O073,P111,P112

Kerr T. . . O076,O108,O109,O110,P013,P034, P108,P116,P117,P118,P145,P156,P199, P200,P201,P203,P271,P300,P311,P317

Kerston P . ................P171,P253

Kesler M A. . . . . . . . . . . . . . . . . . . . O044

Kettner JD . . . . . . . . . . . . . . . . P136

Keynan Y . . . . . .............. P052

Kibendelwa Z T . .................. P319

Kiefer L. . . . . . . . . . . . . . . . P150,P151

Kille J....................... P162

Kim J . . . . . . . . . . . . . . . . . P095

Kimani J................ P180,P206,P316

Kinder C. . . . . . . . . . . . . . . . . . . P041

King K . ........................ . . . . . . 555

Kingsley L. . . . . . . . . . . . . . . . . . . . . . . P009

Kirkland S . . . . . . . . . . . . . . . . .P292,P308

Kis $\mathrm{O} \ldots \ldots \ldots \ldots \ldots \ldots$. . . . . . . . . O091,P037

Kiwanuka N. . . . . . . . . . . . . . . . . . О018

Klaric K-A . . . . . . . . . . . . . . . . P018

Klein MB . . . . . O017 O067,O101,O102,P054, P063,P064,P066,P069,P070,P100, ......................P101,P114,

Klusacek A.................P243,P279

Knight R E.................. P271

Knowledge Synthesis Team HIVR . . . . . P280

Knox C. . . . . . . . . . . . . . . . . . . P192

Kobinger G. . . . . . . . . . . . . . . . . . . . . . O001

Koblin B...................... O061

Kolla G. . . . . . . . . . . . . . . . . . . . . . . 0069

Kom E. ..................... P121

Komane G . . . . . . . . . . . . . . . P244

Konde Lule J . . . . . . . . . . . . . . . . P181

Kongmanas K. . . . . . . . . . . . . . . . .0058

Konsmo E M .................... O051

Konsmo EM . . . . . . . . . . . . . . . . . P225

Koornstra J. . . . . . . . . . . . . . . . . P243,P279

Koornstra JJ(J) . . . . . . . . . . . . . P258

Koski L.......................... P073

Kozai T....................P061,P109

Krajden M . . O068,O099,P128,P147,P148,P173

Krentz H B . . . . . . . . . . . . . . P033,P049,P099

Krusi A.................... O112

Kuang XT. . . . . . . . . . . . . .0061,0086,0087

Kumar S . . . . . . . . . . . . . .....0065

Kwag M . .......... O020,P128,P256

Kwaramba G ........ P077,P080,P237,P270

$\mathrm{L}$

La D . . . . . . . . . . . . . . . . . . . 0001

la Porte C........................0090

la Porte CJ . . . . . . . . . . . . . . .P066,P091

Lacap P. . . . . . . . . . . . . . . . . . O001

Lachowsky NJ . . . . . . . . . . O019, O045,P218

Lakor S. . . . . . . . . . . . . . . . . . O018

Lal A ..................... O042,P190

Lalonde R G. . . . . . . . . . . . . . P063,P064,P100

Lamarre V . . . . . . . . . . . . . O015,0039,0040

Lambert DT . . . . . . . . . . . . . . . . . . P267

Lamontagne B . . . . . . . . . . . . . . P251

Lamoureux R . . . . . . . . . . . . . . P216,P224

Landry $G \ldots \ldots \ldots \ldots \ldots \ldots \ldots \ldots \ldots$. P146

Landry T M . . . . . . . . . . . . . . . . P252

Lane C . . . . . . . . . . . . . . . . . . . . . P163

Langille S..................... P186

Langlois AM .................. P217

Laperrière H. . . . . . . . . . . . . . . P251

Laplante F . . . . . . . . . . . . . . . . . O064,P041

Lapointe N. . . . . O015,O039,O0040,P084,P088

Lapointe ND . . . . . . . . . P254,P255,P257

Lapointe R . . . . . . . . . . . . . . . P002
Larkan F. . . . . . . . . . . . . . . . P030

Lary $\mathrm{T} \ldots \ldots \ldots \ldots \ldots \ldots \ldots \ldots . \ldots . \ldots . \ldots 327$

Latendre-Paquette J . . . . . . . . . . . . . . . . O093

Lavoie F.................... P284

Lavoie S . . . . . . . . . . . . . . . O066,P301

Lawson E . . . . . . . . . . . . . . . . . P195,P196

Lazarus L. . . . . . . . . . . . . . . . . . P189,P215

Le A ................ O061,0086,P013

Le AQ ...................... 0087

Le D . . . . . . . . . . . . . . . . . . P199

Leahy B .................P111,P112

LeBlanc M-A.................... P322

LeBlanc S.................... P215

LeBlanc Z . . . . . . . . . . . . . . . .......... P016

Leclerc P. . . . . . . . . . . . . P120,P123,P142

Lee $G \ldots \ldots \ldots \ldots \ldots \ldots \ldots$. . . . . . . . . . . . . . . . . . . .

Lee GQ. . . . . . . . . . . . . . . . . . . . . . . O0087

Lee $H \ldots \ldots \ldots \ldots \ldots \ldots \ldots \ldots \ldots$. . . . . . . . . . . . . . . . PO 66

Lee JHY . . . . . . . . . . . . . . . . . . . P078

Lee K ...................... P033,P049

Lee $\mathrm{S} . \ldots \ldots \ldots \ldots \ldots \ldots \ldots \ldots \ldots \ldots \ldots$

Lee $\mathrm{T} \ldots \ldots \ldots \ldots \ldots \ldots \ldots \ldots$. . . . . . . . . P088

Lee TC . . . . . . . . . . . . . . O015,P084

Lee-Foon N . . . . . . . . . . . . . . . . P230,P239

Lefebvre M.................. P133

Légaré $H \ldots \ldots \ldots \ldots \ldots \ldots \ldots \ldots$. . . . . . . . . . . . . . . . . . . . . .

Légaré R.................... P272

Legault J . . . . . . . . . . . . . . . . . P014

Lemieux L . . . . . . . . . . . . . . . . . . . . O103

Leo D . . . . . . . . . . . . . . . . . . . P309

Leonard L. . . . . . . . . . . . . . P115,P276,P320

Leonard LE . . . . . . . . . . . . . . . P121

Lepik K. . . . . . . . . . . . . . . . .P167,P168

Lepik K J . . . . . . . . . . . . . P036,P043,P044

Lesovski D . . . . . . . . . . . . . . . . . . P043,P044

Lester R . . . . . . . . . . . . . . . . . P46,O048,P035,P156,

$\mathrm{P} 157, \mathrm{P} 161, \mathrm{P} 180, \mathrm{P} 291$

Lester RT . . . . . . . . . . . . . . . . . . P206

Letendre S . . . . . . . . . . O011,P074,P075

LeVangie D . . . . . . . . . . . . . . . . P323

Levine S. . . . . . . . . . . . . . .P035,P291

Lévy JJ . . . . . . . . . . . . . . . P255,P257

Lewellen D. . . . . . . . . . . . . . . . . . P071

Lewis J . . . . . . . . . . . P169,P237,P240

Lewis N . . . . . . . . . . . . . . P149,P325

Li A . . . . . . O022,0052,O053,P260, P321

Li T. . . . . . . . . . . . . . . . .P028,P177

Li Y....................... P028

Liang B. . . . . . . . . . . . . . O001,P192

Liang C. . . . . . . . . . . . . . . . . . . . . . O08

Liang RH . . . . . . . . . . . O006,0059

Light L . . . . . P077,P080,P137,P152,P153,P154

$\operatorname{Lim} H \ldots \ldots \ldots \ldots \ldots \ldots \ldots \ldots$. . . . . . . . . . . . . .

Lima V . . . . . . . . . P031,P061,P109,P168

Lima VD O007,O033,O074,O100, O102,P170,

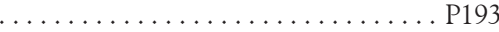

Lin D M . . . . . . . . . . . . . . . P189

Lindegger M. . . . . . . . . . . . . O048,P148

Lisle C . . . . . . . . . . . . . . . . . . . P054

Lister J . . . . . . . . . . . . . . . . . . P261

Little S . . . . . . . . . . . . . . . . . . O086

Liu F. . . . . . . . . . . . . . . . . . P016

Liu HC. . . . . . . . . . . . . . . . . . . . . . . P039

Liu J . . . . . . . . . . . . . . . . . О44,P060

Liu W . . . . . . . . . . . . . . . . . P177

Lloyd K. . . . . . . . . . . . . . . . O013,O014,P132

Lo $\mathrm{E} \ldots \ldots \ldots \ldots \ldots \ldots \ldots \ldots \ldots \ldots . \ldots \ldots$

Loemba H. . . . . . . . . . . . P066,P075,P213

Logie C. . . . . . . . . . . . . . . . . . . P231

Logie $\mathrm{CH} \ldots \ldots \ldots \ldots \ldots \ldots \ldots$. . . . . . . . .

Lopez A . . . . . . . . . . . . . . . . . . . . . . P243 
Lorway R . . . . . . . . . . . . . . P282,P316

Lourenco L. . . . . . . . . . . . . . P031,P168

Loutfy M. . O044,O068,O096,P059,P076,P077, P080,P082,P089,P130,P135, .P231,P233,P249,P276,P320

Loutfy MR . . . O017,O070,O077,O101,O102, P060,P066,P069,P237,P240, P242,P246,P262,P263,P270

Love RP . . . . . . . . . . . . . . O062,P022

Lu S ....................... P018

Luft LA. . . . . . . . . . . . . . P180

Lui HC . . . . . . . . . . . . . . . . . P041

Luma M . .................... P116

Luo M................. O001,P012

Lush J . . . . . . . . . . . . .......... О073

Luyombya H. . . . . . . . O052,O053,P213,P244

Lyons T. . . . . . . . . . . . . . . O076,P203

\section{$\mathrm{M}$}

Maan EJ . . . . . . . . .0009, O010,0012,0036, . P035,O037,P079,P291

Macbeth A.................... P162

MacDonald E. . . . . . . . . . . . . . . . . . . . . P089

MacDonald J-A . . . . . . . . . . . O054,P140

MacDonald KS. . . . . . . . . . . . . . . . P005,P016

MacDonald L. . . . . . . . . . . . . . . . 0097,0098

MacDonald S . . . . . . . . . . . . . . . . . P287

MacDougall G M. . . . . . . . . . . . . P295

MacGillivray J . . . . . . . . . . . . . . . . P081

Machouf N. . . . . . . . . O . . . . . . P17,0066,O101,O102,

....................P057,P069,P101

Mackenzie-Clark T . . . . . . . . . . . P147

MacPhee P............... O011,P075

MacPherson P . . . . . . O025,P001,P092,P113

Madsen J. . . . . . . . . . . . . . . . . . . P227

Maginley J . . . . . . . . . . . . . P035,P291

Mah Ming J . . . . . . . . . . . . . . . . . . . P033

Mahal D..................... O035

Maher L . . . . . . . . . . . . . . . . . . . . . . O O . . . . . P0412

MahMing J. . . . . . . . . . . . . . . . . . P0 P0 49

Mai A. . . . . . . . . . . . . . . . . . .0009, 0010

Maina G.................... P253

Major C . . . . . . . . . . . . P152,P153,P154

Mak S................... P180

Makaroff S ................... P161

Makoroka L . . . . . . . . . . . . . . . . . . .0055

Malm M.....................0003

ManCount Study Team . . . . . . . . . . . . P126

Mangion M ................... P315

Manhas S..................... O032

Mann J . . . . . . . . . . . . . . . . . . . . . . O0087

Manno M............. O047,P053,P288

Manseau H. . . . . . . . . . . . . . . . . . . . P297

Mansour S . . . . . . . . . . . . . P068

Marchand R . O0041,O046,O094,P110,P156,P298

Marcotte T................ O011,P074

Marcotte TD .................... P075

Maregmen J . . . . . . . . . . . . . . . . P102

Margolese S . . . . . . . . P077,P080,P176,P228,

$\mathrm{P} 237, \mathrm{P} 242, \mathrm{P} 263$

Margolick J. . . . . . . . . . . . . . . . . . . 0096

Marit MR . . . . . . . . . . . . . . . . . . . P016

Markle T ....................0086

Markowitz M . . . . . . . . . . 0061,0086,0087

Marlatt F . . . . . . . . . . . . . . . . . . . . . . . P94,P298

Marshall BD .................... P117

Marshall Z . . . . . . . . . . O054,P215,P218

Martel M .................... O056

Martin A . . . . . . . . . . . . . . . . . P215

Martin E. . . . . . . . . O061, O087, P007,P011

Martin G . . . . . . . . . . . . . . . . . . P287

Martin JN. . . . . . . . . . O0887,P083,P093
Martin N . . . . . . . . . . . . . . . .P254,P257

Martin R. . . . . . . . . . . . . . . . . . P294

Martinez JL. . . . . . . . . . . . . . . . . . . . P184

Martinez-Cajas JL . . . . . . . . . . . P183

Masching R ... O O013,O014,O050,P225,P249

Maseko P . . . . . . . . . . . . . . P321

Masinde K .................... P089

Massaquoi N. . . . . . . . . . . . . . . . . . . 078

Massicotte A . . ................ P177

Masumbuko CK . . . . . . . . . . . . . . . . P319

Mathias S..................... O110

Maticka-Tyndale E. ..................0053

Matte S. . . . . . . . . . . . . . . . . . . P068

Matthews L . . . . . . . . . . . . . . . . P234

Matthews LT . . . . . . . . . . . . . . . P083,P093

Maung Maung T . . .............. P126

Maurais E...................... P114

Maxwell J . . . . . . . . . . . . . . . . . . O094,P298

Maver K . . . . . . . . . . . . . . . . . . . O061

Mayo N . . . . . . . . . . . . . . . . . . . P073

Mazzulli T. ............ O047,P051,P102

Mbave EM ...................... P266

Mbulaheni T . . .............P194,P213

McAloney C . . . . . . . . . . . . O099,P147,P148

McCandless L. . . . . . . . . . . . . . . . . . O104

McClarty LM . . . . . . . . . . . . . . . . . . P282

McCloskey R . . . . . . . . . . . . . . . . 0006,0087

McCloskey RM . . . . . . . . . . . . . . . . O059

McCombe J . . . . . . . . . . . O O011,P074,P075

McCully M. . . . . . . . . . . . . . . P018

McDougall P . . . . . . . . . . P143,P162,P292

McFadyen A. . . . . . . . . . . . . O021,P296

McGee F. . . . . . . . . O011,0047,O073,P051, P074,P075,P131,P135,P153,P154

McGovern R A . . . . . . . . . . . . . . . . . . . . . P036,P043

McGuire MA ................. P131

McIntyre C. ................. P212

McKay-McNabb K. . . . . . . . . . . . . . . P225

McLean MD . ................... P016

McLean R. . . . . . . . . . . . . . . . . . . P176

McNeil R . . . . . . . . . O107,O109,P278,P292

McPhee P. . . . . . . . . . . . . . . . P074

McPherson K . . . . . . . . . . . . . . . . . . P186

McWilliam S . . . . . . . . P P276,P320,P323

Medina C.....................P228,P269

Medjuck M.................. P212

Memetovic J. . . . . . . . . . . . . .P180,P206

Menard B . . . . . . . . . . . . . . . . P237,P262

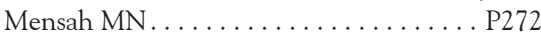

Mesplède T. . . . . . . . P019, P105,P020,P027

Mesplède T R.................. O002

Miao A..................... P321

Michelow W ....... O042,O043,P098,P126,

........P127,P190,P208,P209,P214

Mignone J. . . . . . . . . . . . . . . . . . P308

Mill J . . . . . . . . . . . . . . . . . . . . P253

Miller C . . . . . . . . . . . . O017,P226,P286

Miller J . . . . . . . . . . . . . . . . P268

Milligan RS . . . . . . . . . . . . . . P171

Milloy M-J . . . . . O108,P011 P013,P034,P116,

............ P118,P199,P201,P292

Milloy M-JS . . . . . . . . . . . . P117

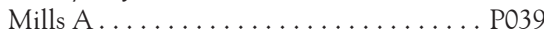

Millson P . . . . . . . O047,O069,P051,P135,

..................P202,P276,P320

Min J . . . . . . . . . . O 074,O102,P106,P193

Min JE . . . . . . . . . . . . . . . . . . . . . . . . . . .

Miranda J . . . . . . . . . . . . . . . . . . . P281

Missaghi B ....................... P163

Miura T .....................0086

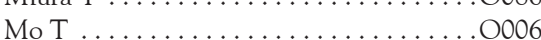

Mocello AR ....................... P093

Moir S . . . . . . . . . . . . . . . . . P010

Moisi D. . . . . . . . O O103,P019,P020,P097

Mollison A..................... P315

Mondal P K . . . . . . . . . . . . . . . . P134

Money D . . . . . O009,0010,0012,0015,0034,

$\mathrm{O} 035, \mathrm{O} 036, \mathrm{O} 068, \mathrm{O} 080, \mathrm{O} 104$

......................P059,P088

Money DM. . . . . . O037,P035,P079,P084,P291

Montaner J. . . . . O108,O110,P034,P061,P066, .P078,P098,P107,P109,P116,P117, . . . . . . P145,P167,P168,P186,P199, $\mathrm{P} 201, \mathrm{P} 205, \mathrm{P} 300, \mathrm{P} 307$

Montaner JS. ...... O007, О017, О074,О033, . O034,O100O101,O102,O104,P031,

P043,P044P069,P124,P122,P209P170, $\mathrm{P} 175, \mathrm{P} 193, \mathrm{P} 204, \mathrm{P} 318$

Montano D. . . . . . . . . . . . . . P183,P184

Monteiro P. . . . . . . . . . . . . . . O028,0030

Monteith K . . . . . . . . . . . . . . . . . . 0077

Montero M. . . . . . . . . . . . . . . . . . . . . P018

Montessori V . . . . . . . . . . . . . . . . . . . . P098

Montgomery R. . . . . . . . . . . . O050,P236

Moodie E . . . . . . . . . . . . . . . . . . .P097,P114

Moore D . . . . . . . . . O042,O043,P031,P126,

......P127,P168,P190,P208,P209

Moore D M .................P043,P044

Moors R ..................P216,P224

Moqueet N. . . . . . . . . . . . . . . . . P054

Moravan V............ P051,P187,P238

Morin G. . . . . . . . . . . . . . . P255,P257

Morissette C. . . . . . . P120,P123,P142,P174

Morris J. . . . . . . . . . . . . . . . . P309

Morshed M. . . . . . . . . . . . . . O048,P231

Moses E . . . . . . . . . . . . . . . . . . . . . P059

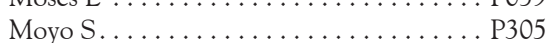

Muchenje M. ..............P228,P242

Mueses HF . . . . . . . . . . . . . . . . . . . . . P184

Mueses-Marín HF . . . . . . . . . . . . . P183

Mugford G . . . . . . . . . . . . . . P058

Mujib S. . . . . . . . . . . . . . . 031,0088

Mukkath S . . . . . . . . . . . . . . . . . P286

Muldoon KA ................ O071,P207

Munro A .................... P227

Munroe V. . . . . . . . . . . . . . . . . . P159

Murira A . . ................... P010

Murooza C . . . . . . . . . . . . . . . . P083,P093

Murphy D. . . . . . . . . . . . . . P040

Murphy DG . ................... O103

Murphy K. . . . . . . . . . . . P082,P125,P326

Murray M . . . . . . . . . O009,,O012,О034,P079

Murray MC . . ......... O010, P035,P291

Murray S. . . . . . . . . . . . . . . . . P245

Murzin K . . . . . . . . . . . . . . . . . . O055

Mustoe H.................... P176

Muzaaya G . . . . . . . . . . . . . . . O071,P207

Muzoora C . . . . . . . . . . . . . . . . . . . 0087

Mwimanzi P . . . . . ...........0086,0087

Myers T . . . . . . O094,P137,P187,P238,P298

\section{$\mathrm{N}$}

Naccarato M . . . . . . . . . . . . . . . .P096,P169

Nagi A . . . . . . . . . . . . . . . . . P171

Nakamura N. . . . . . . . . . . . . . P126

Nathoo A. . . . . . . . O097,O098,P158,P186

Ndayikengurukiye G . . . . . . . . . . . . 0052

Ndung'u M. . . . . . . . . . . . . . . P305

Ndung'u T . . . . . . . . . . . . . . . 0087

Nelson V . . . . . . . . . . . . . . . . . . . . P324

Newman P . . . . ............. O078

Newman PA. . . O023,O024,O070, P283,P290

Newman R................ P125 
Newman R..................... P326

Ngigi SK. . . . . . . . . . . . . . . P319

Ngugi E. ..................... P180

Ngugi EN . . . . . . . . . . . . . . . . P206

Nguyen P . . . . . . . . . O016,P108,P205

Nicholson V............ P216,P224,P263

Nicholson VJ . . . . . . . . . . . . .P237,P240

Nickel P . . . . . . . . . . . . . . . . . . . P086

Nieva J . . . . . . . . . . . . . . . . . P018

Ning Z . . . . . . . . . . . . . .P187,P238

Njoo H. . . . . . . . . . . . . . . . . P095

Nkala B .................... P226

Nleya-Ncube M . . . . . . . . . . . . . . . P213

Nohpal A..................... P128

Nomellini J F . . . . . . . . . . . . . . . P017

Nosyk B . . . . . . . O074,O100,P168,P170,P193

Numer M . . . . . . . . . . . . . . . . .P219,P220

\section{$\mathrm{O}$}

O’Brien K. . . . . . . . . . . . . . P072,P253,P315

O'Brien KK . . . . . . . . . . . . P211,P280

O'Brien N. . . . . . . . . O077,P237,P240,P262

O'Brien-Teengs D . . . . . . . . P135,P249,P258

O'Cleirigh C . . . . . . . . . . . . . . P299

Oakes W......................... O075

Ochieng C . . . ................ О056

Ogilvie G . . . . . O046,O048,,O099,P035,P128, P147,P148,P156,P157,P161,P291

Ogilvie G S . . . . . . . . . . . . . . O034

Ogunnaike-Cooke S...... O013,O014,P119,P129

P131,P132

Ohnona F. . . . . . . . . . . . . . . . . . . . . P097

OHTN Cohort

Study Team ......... O047,P051,P111,P112

Olatunbosun C. . . . . . . . . . . . . . P167

Olatunbosun C D. . . . . . . . . . . . .P165,P166

Oliveira M . . . . . . . . . . . . . . P019,P020

Oliver B . . . . . . . . . . . . . . . . . P248,P315

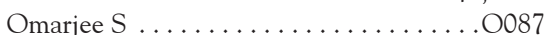

Omura J . . . . . . . . . . . . . . . . . . . P294

Orchard T . . . . . . . . . . . . . . . P252

Osman N . . . . . . . . . . . . . . . O002,P105

Ostrowski M. . . . . . . . . . . . . . . . O088,,P007

Ostrowski MA ................... О031

Otis J . . . . . . . . O021,P097,P254,P255,

$\mathrm{P} 257, \mathrm{P} 296, \mathrm{P} 301, \mathrm{P} 302$

Oviedo-Joekes E. . . . . . . . . . . . . . . O111

Owino M . . . . . . . . . . . O052,P244,P321

\section{$\mathrm{P}$}

Pai J ............................ P098

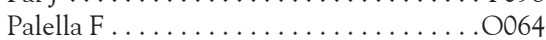

Palmer AK....................... O017

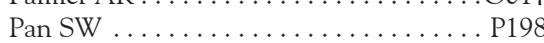

Panessa C. . . . . . . . . . . . . . . . . . . . P292

Panic M ..................... P142

Pankovich J ................P176,P177

Pant Pai N . . . . . . . . . . . . . . . . P146,P182

Pantophlet R . . . . . . . . . . . . . . O032

Papp E . . . . . . . . . . . . . . . O038,P082

Paquette D . . . . . . . . P094,P095,P103,P139

Paquette N.................... P174

Parashar S. . . . . . . . . . . . P216,P224,P292

Parfyonov M. .................. P018

Parmar P. . . . . . . . . . . . . . . . . . . . O025

Parry R . . . . . . . . . . . . . . . . O020

Patel M. . . . . . . . . . . . . P002,P038,P177

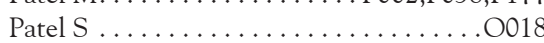

Pater J. . . . . . . . . . . . . . . . . P176

Patocs A E . . . . . . . . . . . . . . . P185

Patrick D M ................. P128

Patrick M ...................... . P230

US GROUP INC.

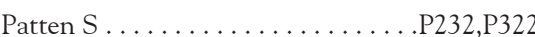

Patterson S. . . . . . . . . . . . O080,P204

Patterson T. . . . . . . . . . . . . . . . . . . P209

Paulsen C...................... P181

Pauly B . . . . . . . . . . . . . . . . . . P292

Pawlica P . . . . . . . . . . . . . . . . . . O0855

Payne M. . . . . . . . . . . . . . . . P143,P162

Pearce ME .............. O049,P198,P303

Peck R . . . . . ................ P274

Pedersen H. . . . . . . . . . . . . . . . . . O099,P147

Peltier D. . . . . . . . . . . . . . . . . . P249

Pelude L . . . . . . . . . . . . . . . . . . P121

Pelzom D . . . . . . . . . . . . . . . . P128

Pendergraft K................... P176

Penn R . . . . . . . . . . . . . . . . . . . . . . . O069

Penn RA . . . . . . . . . . . . . . P286

Penney K . . . . . . . . . . . . . . . . . O061,P011

Pequeno P . . . . . . . . . . . . . . . P032

Pereyra F. . . . . . . . . . . . . . . . . . O0886

Perreault Y .................. P321

Peters H........................ P304

Petkau A . . . . . . . . . . . . . . . . . P192

Petronela A . . . . . . . . . . . . . . . . . P014

Petropoulos CJ . . . . . . . . . . . . . . . . . . P027

Pexos C . . . . . . . . . . P063,P064,P100,P101

Phaneuf D . . . . . . . . . . . . . . . . P191

Phillips JC . .................... . P110

Pichette A . . . . . . . . . . . . . . P014

Pick N . . O009,,0010,0011,0012,0033,0034, $.0035,0036,0037,0067,0104$ P035,P066,P074,P075,P079,P114,P291

Pierre Pitman L . . . . . . . . . . . . P125,P326

Pierre-Pierre V . . . . . . . . . . . . . . P213

Pigeon TA ................... P267

Pillet S . . . . . . . . . . . . . . . . . . . O001

Pilon R.................. O001,P005

Pindera C................... P052

Pineau D. . . . . . . . . . . . . . . . . . . P233

Pinto JC . . . . . . . . . . . . . . . . . O001

Pinzon-Fernandez MV . . . . . . . . . . .P183,P184

Pipe A . . . . . . . . . . . . . . . . P113

Pitman L. . . . . . . . . . . . . . . . . . . . . P125

Plantier J-C . . . . . . . . . . . . . . . . . . . P019

Platt R . . . . . . . . . . . . . . . . P054

Plummer F . . . . . . . . . . . O057,P012,P015

Plummer FA. . . . . . . . . . . . . . . O001

Poirier A. . . . . . . . . . . . . . . . . . . . . P191

Poitras M . . . . . . . . . . . O O 13,0014,P132

Poliquin M. . . . . . . . . . . . . . . O066,P057

Poncia A . . . . . . . . . . . . . . . . . . . P115

Poon A. . . . . . . . . . . . . . . . . 0006, 0061

Poon AF. . . . . . . . . . . . . . . . . . . . O059,P104

Poon M. ................ O022,P260

Pooyak S. . . . . . . . . . . . . . . . . 0050

Popiel M. . . . . . . . . . . . . . . P315

Porter D . . . . . . . . . . . . . . . . . . . 0063,0064

Positive Spaces HPTT . . . . . . . . . . . . . P258

Potter M. . . . . . . . . . . . . . . . . P054,P073,P146

Powell L . . . . . . . . . . . . . . . . . . . . . . P125

Powell L . . . . . . . . . . . . . . . . . . . P326

Prego C........................ O001

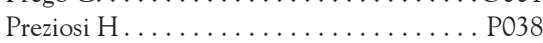

Price C . . . . . . . . . . . . . . . . .P228,P261

Proctor-Simms M. . . . . . . . . . . . . . P140

Proud Community Advisory Committee. . .P215

Proulx S . . . . . . . . . . . . . . . . . . P006

Proulx-Boucher K . . . . . . . . . . P255,P257

Pugh D . . . . . . . . . . . . . . . .P125,P326

\section{Q}

Qian C . . . . . . . . . . . . . . . . . . . P047

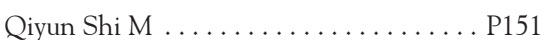

Quashie P.................... P020

Quashie PK ............ O002,P019,P027

\section{$\mathrm{R}$}

Raboud J. . O011,O047,,O065,O068,P051,P053 $\mathrm{P} 055, \mathrm{P} 059, \mathrm{P} 074, \mathrm{P} 075, \mathrm{P} 089, \mathrm{P} 130, \mathrm{P} 249$

Raboud JM ...... O017,O101,O102,P032,P066,

............................ $069, \mathrm{P0} 70$

Rachlis A. . O011,0063,0064,О067,0096,O101

......... O102,P040,P066,P069,P074,P075

Racicot C . . . . . . . . . . . . . . . . . . . P254 . . . . POO2,

Radzioch D. . . . . . . . . . . . . . . . . P002,P038

Rajwans N ................... 0065

Ramanaik S . . . . . . . . . . . . . . . P282

Ramdahin S . . . . . . . . . . . . . P012,P015

Ramis R . . . . . . . . . . . . . . . P276

Ramsay $\mathrm{T} \ldots \ldots \ldots \ldots \ldots \ldots \ldots \ldots \ldots$. . . . . . . . . . . . . . P31

Rand JR ...................... P313

Rank C. . . . . . . . . . . . . . . . . . O0993

Rankin B ................... P243,P279

Rankin J . . . . . . . . . . . . . . . . O051

Rau P ...................... P068

Rawson S . . . . . . . . . . . . P237,P263

Razao D . . . . . . . . . . . . . . . . P110

Read S . . . . . . . . . . . . . . . . P295

Read S E. . . . . . . . . . . . . . . . . . O003

Reading C . . ................. P225

Rebbapragada A................... O044

Reddock J. . . . . . . . . . . . . . . . . . . P306 . . . . . . P113

Reid B...................... P113

Reid J . . . . . . . . . . . . . . . . . . . P293

Reiher G.................... P155

Reinhard R. . . . . . . . . . . . O O O 11,P074,P075

Rekart M . . . . . . . . . . . O020,P128,P256

Remis R . . . . . . P060,P129,P135,P137,P320

Remis RS ... . O044, O047, O093,O094,P051,

..............P102, P131,P213,P298

Ren J.................... P137

Research Group Oak Tree Clinic ....... O037

Research Group Vogue . . . . . . . . . . . . O035

Research Team CHIWOS........... P237

Restall G . . . . . . . . . . . . . . . . . . P275

Retel AE. . . . . . . . . . . . . . . . . . . . . . . O063,P040

Reyes-Terán G................. 0060

Rhee M. ....................P040,P041

Rhee MS .................... P039

Rich A . . . . . . . . . . . . . O043,P208,P209

Richardson C...............P145,P200

Richardson L . . . . . . . . . . . . . . . P118,P300

Richer T.................... P251

Richman D. . . . . . . . . . . . . . . . . P009

Rinaldo Jr CR ... . . . . . . . . . . . . . . . P009

Ringlein, M . . . . . . . . . . . . . . . . 0079

Ritvo P................... P180,P206

Rivas C. . . . . . . . . . . . . . . . . P296

Robert W . . . . . . . . . . . . . . P126

Robillard KR .................... 0090

Robinette J...................... O044

Robinson C . . . . . . . . . . . . . . . P225

Robinson J . . . . . . . . . . . . . . . . . P029

Robinson LJ . . . . . . . . . . . . . . . . . . P164

Rockstroh J. . . . . . . . . . . . . . . P040

Roddy P . . . . . . . . . . . . . . . . . P275

Roesslein K. . . . . . . . . . . . . . . . . . P279

Roger K . . . . . . . . . . . . . . P275,P308

Roger M . . . . . . . . . . . . O103,P097,P101

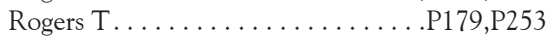

Rollet K ........................ O067

Rollet K C . . . . . . . . P063,P064,P100,P101

Rosenberg E................. O086

Rosenes R. . . . . . . . O011,P050,P074,P075 


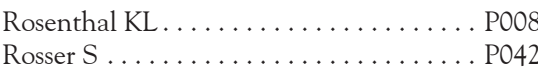

Rosslein K . . . . . . . . . . . . . . . . . . P243

Rossnagel E . . . . . . . . . . . . . . P012

Roth E . . . . . . . . . . . . O042,P208

Roth EA . . . . . . . O043,P127,P190,P209, P287

Rotondi M .......................0069

Rotondi NK . . . . . . . . . . . . . . . . . . . O0699

Rouleau J . . . . . . . . . . . . . . . . . . P016

Roungprakhon S . . . . . . . . . . . . O024

Rourke SB . . . O011,O047,O073,O077,O101, P051,P069,P074,P075,P080,P111,

$\ldots \ldots \ldots \ldots \ldots \ldots$ P112,P135,P250,P258

Rousseau R. . . . . . . . . . . . . O021,P097

Routy J-P . . . . . O027,O028,О030,O082,P002,

......... P004,P006,P028,P038,P100,P177

Rowe J . . . . . . . . . . . . . . . P276,P320

Roy E . . . . . . . . . . . . . . . . . . P120,P123

Roy É . . . . . . . . . . . . . . . . . O O069,P285

Roy M C. . . . . . . . . . . . . . . . . O066,P057

Rueda S . . . . . . . P111,P112,P135,P250,P315

Rumman A. . . . . . . . . . . . . . . . . P050,P062

Rutherford A . . . . . . . . . . . P026,P186

Rwigema MJ. . . . . . . . . . . . . . . . . O078

\section{$\mathrm{S}$}

Sadeghi A. ...................... P050

Salbach N. .................... P315

Salter R . . . . . . . . . . . . . . . . P295

Salters K. . . . . . . . . . . . . . . .P109,P292

Salters KA . . . . O034,O080,O104,P124,P204

Salway ......................0041,0046

Salway Hottes T. . . . . . . . . . . . . O048,P156

Samarani S. . . . . . . . . . . . . . . . . . O082

Samji H . . O007,O100,O102,P031,P124,P292

Samson J. . . . . . . . . . . . P254,P255,P257

Samson L . . . . . . . . . . . . . . . . . . . . . . P015,P088,P091,P092

Samson LM . . . . . . . . . . . . . . . . . P084

Sánchez A . . . . . . . . . . . . . . . . . . . . O019

Sanchez M . . . . . . . . . . . . . . . . . . P263

Sandhu J. . . . . . . O072,O097,O098,P158,P160

Sandre R. . . . . . . . . . . . . . . . . . . .0093

Sandstra I . . . . . . . . . . . . . . . . . P161

Sandstrom P. . . . . . . . . O001,P005,P192

Sanfaçon R...................... P191

Sas J ......................P176,P177

Sattha B . . . . . . . . . . . . . . . . P079

Saunders LD . . . . . . . . . . . . . . . .P133,P181

Sauve L. . . . . . . . . . . . . . . . . . . . P084

Sauve LJ . . . . . . . . . . . . . . . O015,P088

Savard P.................. P146

Sax P . . . . . . . . . . . . . . . P039

Saxton P. . . . . . . . . . . . . . . . . . . . O045

Schanzer D.................P129,P139

Schechter M. . . . . . . . . . . . . . . . . P176

Schechter MT . . O018,O049,O111,P198,P303

Scheim AI . . . . . . . . . . . . . . . . P218

Schilder A .................P208,P209

Schreiber YS . . . . . . . . . . . . . P189,P215

Schulz C......................... P163

Schwartz J A ................... O0 . . . 031

Scott J. . . . . . . . . . . . . . . . . . . . . P010

Scott JK . . . . . . . . . . . . . . P009,P018

Seatter E. . . . . . . . . . . . . . . . . P227

Sebastiani G. . . . . . . . . . . . . . . . P063,P064

Sekaly R-P . . . . . . . . . . . . . . . . . . . . O030

Senn H. . . . . . . . . . . . . . . . . . . . O095,P096

Serghides L................ O038,P082

Serhir B . . . . . . . . . . . . . . . . . . . P191

Serrano....................... O019

Service D . . . . . . . . . . . . . . . . . . . . P289

Sewankambo NK ............... . 018
Shafran S . . . . . . . . . . . . . . . . . . P042

Shahid A . . . . . . . . . . . . . O060,P011

Shamblaw D. . . . . . . . . . . . . . . P041

Shannon B....................0065

Shannon K. . . O O016,O071,O076,O109,O112, P116,P122,P203,P205,P207, $\ldots \ldots \ldots \ldots \ldots$ P116,P122,P203,P205,P207,

Sharma M. . . . . . . . . . . . . . . . . O095,P096

Shaver L. . . . . . . . . . . . . . . . . . . . . . O109

Shaw A....................... P215

Shaw S Y .................... P052

Shea R .......................0056

Sheehan N. . . . . . . . . . . . . . . . . P101

Shergold J. . . . . . . . . . . . . . . . . P162

Shi C-F. . . . . . . . . . . . . . . . . . . . . . P185

Shimeles H. . . . . . . . . . . . . . . . . . . . P194

Shindler S ......................0079

Shogbola RT .................... P265

Shore R ..................... . P144

Shoveller J . . . . . . . . . P116,P145,P156,P309

Shoveller J A . . . . . . . . . . . . . . . . P271

Shunmugam M. . . . . . . . . . . . . . . P283

Shurgold S . . . . . . . . . P031,P061,P069,P127,

.................... P190,P208,P209

Silver R(B) . . . . . . . . . . . . . . . . O069

Silverbrook M . . . . . . . . . . . . . . . P074,P075

Silverman GJ . . . . . . . . . . . . . . . P009,P307

Simo A . . . . . . . . . P013,P034,P122,P199,

$\mathrm{P} 207, \mathrm{P} 307, \mathrm{P} 309, \mathrm{P} 318$

Simpson D . . . . . . . . . . . . . . . . P162

Simpson S . . . . . . . . . . . . . . . . P296

Sinclair L . . . . . . . . . . . . . . . . P315

Singer J. . . . . . . . O015,O0068,O096,P038,P059,

............... P079,P084,P088,P176

Singh J . . . . . . . . . . . . . . . . . O003

Singhal N. . . . . . . . . . . . . . . . P283

Singhroy D N. . . . . . . . . . . . . . . O002

Sivasivugha ES.................. P319

Skaling B . . . . . . . . . . . . . P228

Skinner S . . . . . . . . . . . . . . . . . P134,P292

Skinner SJ . . . . . . . . . . . . . . . . . . P030

Slawson G . . . . . . . . . . . . . . . . . . P034

Sloan R D. . . . . . . . . . . . . . . . . . . . . O0004

Smaill F . . . . . . . . . . O068,P048,P059,P066

Smaill FM. . . . . . . . . . . . . . . . . . . P070

Small FA . . . . . . . . . . . . . . . P210

Small W. . . . . . . . . O043,O076,O107,O108,

................ O109,P124,P278,P317

Smieja M . . . . . . . . . . . P055,P070,P113

Smit J . . . . . . . . . . . . . . . . . P017

Smith A .................... P293,P294

Smith D . . . . . . . . . . . . . . . . . . . O056

Smith $G \ldots \ldots \ldots \ldots \ldots \ldots \ldots$ P0 $4 . \ldots \ldots$

Smith S . . . . . . . . . . . . . . . . . P237

Smith V . . . . . . . . . . . . . . . . . 0056

Sobota M . . . . . . . . . . . . . . . . . . P258

Sok P . . . . . . . . . . . . . . . . . . . P069

Sokalski K . . . . . . . . . . . . . . . . . . O009

Sokalski K M . . . . . . . . . . . . . . . . .0010

Soliven D . . . . . . . . . . . . . . . P102

Solomon P . . . . . P072,P211,P253,P280,P315

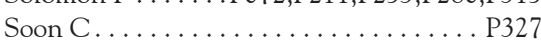

Soudeyns H . . . . . . . . . . . . . . . . .0039,0040

Souleymanov R . . . . . . . . . . . . O023,P290

Sovran L D . . . . . . . . . . . . . . . . P081

Speechley K N . . . . . . . . . . . . . . . . .P195,P196

Spence H . . . . . . . . . . . . . . . . . . P293

Spencer D. . . . . . . . . . . . . . . . . P147

Spira B . . . . . . . . . . . . . . . . P020

Spittal PM . . . . O018,O049,O111,P198,P303

SPOT Study Group . . . . . . . . . . . . . . . . . . O021
Stadnyk M . .

Steele (nee Tales

Stein N. ................................ 242

Steinberg M .......... O020,P128,P256

Stephenson LM . . . . . . . . . . . . . . P228

Stone $\mathrm{S} \ldots \ldots \ldots \ldots \ldots \ldots \ldots \ldots$. . . . . . . . . . . . 30

Strathdee SA ................P307,P318

Stratton N . . . . . . . . . . . . . . . . . . . P296

Strehlau V . . . . . . . . . . . . . . . . P175

Strike C . . . . . . . . . O069,P130,P233,P292

Strike CJ. .................. P286

Subra C . . . . . . . . . . . . . . . P006

Sugden S . . . . . . . . . . . . . . . . . . 0025

Sullivan A . . . . . . . . . . O093,P153,P154

Sullivan AS . . . . . . . . . . . . . . . P102

Sullivan T . . . . . . . . . . . . . . . P275

Summerlee AJ ...................0045

Summers M ................. P212

SurvUDI Working Group . . . . . . . . . . P120

Sutcliffe P. . . . . . . . . . . . . . . . . . 0093

Sutdhibhasilp N.............P276,P320

Sutton P................P214,P261

Suwannawong P............... P311

Sweezey A . . . . . . . . . . . . . . . . . . . O054

Swenson L . . . . . . . . . . . . . . . . . ○006

Swenson LC . . . . . . . . . . . . . . O092,P106

Symington A . . . . . . . . . . . . . . O . . . . . O105,P273

Szadkowski L . . . . . . . . . . . . . . . . .0065

Szadkowski L M . . . . . . . . . . . . . . . . . P032

Szadkowski LM .................. P070

Szwarcberg J . . . . . . . . . . . P039,P040

\section{$\mathrm{T}$}

Taerk E. . . . . . . . . . . . . . . . . . . . . . . P090

Tan B . . . . . . . . . . . . . . O $015, \mathrm{P} 088$

Tan D . . . . . . . . . . . . . . . . . . O095,P096

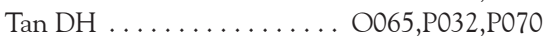

Tang D . . . . . . . . . . . . . . . . . . O001

Tang W...................... P173

Tanphaichitr N . . . . . . . . . . . O0 58

Tarasuk J. . . . . . . . O013,O014,P103,P119,P132

Tasca G. . . . . . . . . . . . . . . . P113

Taylor D . . . . . . . . . . . . P128,P156,P157

Teat J . . . . . . . . . . . . . . P016

Teegee M . . . . . . . . . . . . . . . . . . . . P198

Teengs D. . . . . . . . . . . . . . . . . 0050

Tello-Bolivar IC. . . . . . . . . . . . . . . .P183,P184

Tepjan S.................... O024

Tessier P . . . . . . . . . . . . . . . . . . . . . . P006

Tharao W. . . . . . . . . P075,P077,P089,P131,

Th.......... P213,P231,P246,P276

(W...........P060,P080,P320

Thébault P..................... P002

Thériault J . . . . . . . . . . . . . . . P255,P257

Therrien R.... . . . . . . . . . . . . . . P065

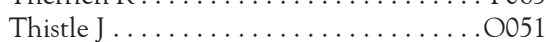

Thomas K. . . . . . . . . . . . . . . . . P228

Thomas R. . . . . O0056,O066,P057,P073,P101

Thomas V. . . . . . . . . . . . . . . . O111,P198

Thompson M . . . . . . . . . . . . . . . . P286

Thorne A................... P079

Thornton A ................. O011,P074

Thornton AE ............... O012,P075

Thorpe M................... P286

Thumath M . . . . . . . . . O097,P155,P186

Thumath M A . . . . . . . . . . . . O072,P160

Ti L....................... P311

Tigchelaar J . . . . . . . . . . . . . P147

Tolson M .................. P172

Tooley L . . . . . . . . . . . . . . . . . . . . P310

Tossonian H. . . . . . . . O096,P047,P048,P056

Toy J . . . . . . . . . . . . . P165,P166,P167

Toy KJ. . . . . . . . . . . . . . . P043,P044 
Tran L. . .

Tremblay C. . . . . . . . O . . . P27, O030,0082,O103, $\mathrm{P} 002, \mathrm{P} 004, \mathrm{P} 101, \mathrm{P} 191$

Tremblay CL . . . . . . . . . . . . . P068

Tremblay G . . . . . . . . . . . . . . . P324

Trottier B . O056,0063,0066,P057, P076,P101

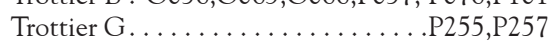

Trottier S . . . . . . . . . . . . . . . O02068,P066

Trussler T ........ O020,0041,O046,P110,

..................P156,P256,P314

Tsang A . . . . . . . . . . . . . . P048

Tse K. . . . . . . . . . . . . . . P007

Tseng A ................. P066

Tsoukas C. . . . . . . . . O017,O101,O102,P069

Tu D . . . . . . . . . . . . . . . O072,P160

Tucker J . . . . . . . . . . . . . P176

Tucker M .................... P140

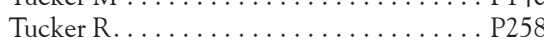

Tuff J. . . . . . . . . . . . . . . . . . . . O001

Tulloch TG ................... P299

Turje R . . . . . . . . . . . . . . . P292

Tyler $\mathrm{S} \ldots \ldots \ldots \ldots \ldots \ldots \ldots \ldots \ldots . \ldots . \ldots . \ldots 001$

Tynan A-M . . . . . . . . . . . . P233,P280

Tyndall M...... O067,P085,P113,P188,P236

Tyndall MW................P189,P215

Tzemis D .............. P173,P293,P294

\section{$\mathrm{U}$}

Uddin Z . . . . . . . . . . . . . . . . . . P213

Udvardy S . . . . . . . . . . . . . . . P253

Ueno T. . . . . . . . . . . . . . . . . O0866

Ulas G . . . . . . . . . . . . . . . . . . . P018

Utama R. . . . . . . . . . . . . . . . . . . . P260

Utama R B . . . . . . . . . . . . . . O022

\section{V}

Vadnais C......................... P182

Valenzuela-Ponce H. . . . . . . . . . . . . . 0060

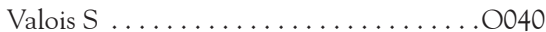

Van der Kop M. . . . . . . . . . . . . . . P180 van der Kop ML. . . . . . . . . P P035, P206,P291

Van Domselaar G. . . . . . . . . . . . . . . P192

van Schalkwyk J. . . . . . . . . . . . . . 0036,0037

Van Wyk, Jean . . . . . . . . . . . . . . . . P076

Vancouver Injection Drug

Users Study (VIDUS) . . . . . . . . . . . . . . P013

Varughese N. . . . . . . . . . . . . . . . P178

Vasarhelyi K. . . . . . . . . . . .P122,P186

Vassal A-F . . . . . . . . . . . . . . P101

Veillette-Bourbeau L . . . . . . . . . . . . . . . . O021

Veldhuis L . . . . . . . . . . . . . . . . . . . . P016

Venditti E................... P131

Veres T....................... P027

Vernich L. . . . . . . . . . . . . . . O094,P298

Vezina S . . . . . . . . . . . . . . . O O066,P057

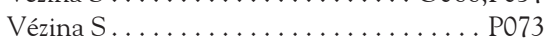

Vicol L . . . . . . . . . . . . . . . . . . . . . . . O035

Vincelette J ................... P191

Voon P.................... P108

W

Wacleche V ................... P014

Wacleche VS . . . . . . . . . . . . . . . . P004

Wade M . . . . . . . . . . . . . . . . . . . . P295

Wagner E . . . . . . . . . . . . . . . . .0037

Wagner E C ..................... O035

Wagner F . . . . . . . . . . . . . . . P268

Wagner T..................... 0061

Wainberg M............. O103,P020

Wainberg MA ..... O002,0004,O021,P019,

...................P2027,P097,P105

Wales J . . . . . . . . . . . . . . . . . . P321

Walker B . . . . . . . . . . . . . . . O061,0086

Walker BD ..................... 0087
US GROUP INC

Walker G..................... P258

Walker T ................... P245

Wallach I . . . . . . . . . . . . . . . . . . . . O056

Walmsley S. . . . . . . . . . O065,,0067,0068,

..........P037,P054,P059,P082,P114

Walmsley SL . . . . . . . . . . P032,P066,P070

Wang C . . . . . . . . . . . ....... P009

Wang H . . . . . . . . . O063,O064,P016,P028

Wang J . . . . . . . . . . . . . . . . . P047,P056

Wang S. . . . . . . . . . . . . . . . . . P018

Wang X ................. O104,P009

Ward D...................... P041

Wares M. . . . . . . . . . . . . . . . . . P105

Wares M A......................0002

Warren L . . . . . . . . . . . . . . . . . . . P135

Watson J...................... P258

Weaver J. . . . . . . . . . . . . . . . . . . . . . O078

Webster K..................... P172

Wedgwood OM . . . . . . . . . . . . . O064,P041

Wei X........................ . P040

Wei Z . . . . . . . . . . . . . . . . . . P007

Werb D......................P145,P200

Werb R...................... P061

Wertheimer S. . . . . . . . . . . . . . . P228

Whitbread J ................P214,P235

White A...................P189,P215

White C A.................... P062

White S . . . . . . . . . . . . . . . P202

White SJ.....................P085,P236

Whitebird W . . . . . . . . . . . . . P249

White-Jones K . . . . . . . . . . . . . . . . P115

Wild TC..................... O106

Wilkins S ................... P072

Wilkinson S . . . . . . . . . . . . . . . . P182

Willer D . . . . . . . . . . . . . . . . . . P016

Willer DO ...................... P005

Williams C...................... O078

Willis AC ....................... P296

Wilmot JM .................... P030

Wilson CL ...................... P239

Wilson DG..................P216,P224

Wilton J . . . . . . . . . . . . . . . . . . O095,P096

Wiseman S M. . . . . . . . . . . . . . . . . O0034

Wittenberg R . . . . . . . . . . . . P026

Wobeser WL . . . . . . . . . . . . . . . P050,P062

Wohl D. . . . . . . . . . . . . O063,P039

Wong AY . . . . . . . . . . . . . . . . . . . . P091

Wong G . . . . . . . . . . . . . . . . . . . . O001

Wong J . . . . . . . . . . . . . . . O053,P321

Wong L. . . . . . . . . . . . . . . . . . . . . . . . P047

Wong T . . . . . . P094,P095,P103,P139,P156

Wood E. . . O108,O110,P013,P034,P108,P116,

$\ldots \ldots \ldots \ldots$ P117,P118,P122,P145,P199,P200,
$\ldots \ldots \ldots \ldots \ldots \ldots \ldots$ P201,P300,P311,P317

Woods C. . . . . . . . . . . . . O006,P104,P106

Woods CK . . . . . . . . . . . . . . . . . . . P036

Woods M W. . . . . . . . . . . . . . . . O0083

Worthington C . . P085,P138,P188,P217,P218,

.....P236,P248,P253,P292,P315

Wrath K................... P304

Wu J . . . . . . . . . . . . . . . . . . . . P280

Wu K ................ O093,P102

Wu S ..................... P009

Wylie J . . . . . . . . . . . . . . P282

\section{$\mathrm{X}$}

Xie J .

$\mathrm{Y}$

Yan $\mathrm{P}$

\section{$Y$}

Yant SR

.0005
Yao X ....................... P008

Yaphe S .................... P174

Yasui Y . . . . . . . . . . . ......... P133

Yee $\mathrm{J} \ldots \ldots \ldots \ldots \ldots \ldots \ldots \ldots \ldots . . \ldots \ldots \ldots$

Yeung $\mathrm{M} \mathrm{W} \ldots \ldots \ldots \ldots \ldots \ldots 67$

Yi TJ ..................... 0065

Yip B. . . O007,О072,О074,О089,О097,О100, . . . . . . . . P031,P036,P043,P044,P046,

.P107,P160,P165,P170,P193

Yoong D M. . . . . . . . . . . . . . . . P096,P169

Young C D . . . . . . . . . . . . . . 058,P021

Yu A ....................... P173

Yuan X-Y . . . . . . . . . . . . . . . . . 0001

Yudin M . . . . . . . . . . . . . P082,P089,P090

Yudin $\mathrm{MH} \ldots \ldots \ldots \ldots \ldots \ldots \ldots \ldots$. . . . . . . . . . . . .

Yuksel N. . . . . . . . . . . . . . . . . P067

\section{$\mathrm{Z}$}

Zack E. ................... P280

Zagdyn Z. . . . . . . . . . . . . . . . . P050

Zagorski B. . . . . . . . . . . . . . P089,P130

Zanet DA L . . . . . . . . . . . . . . . . . . P079

Zhang F . . . . . . . . . . . . . . P129

Zhang G...................... 0090

Zhang H. . . . . . . . . . . . . . . . P198

Zhang W . .O034,O080,O104,P098,P109,P292

Zhu J. . . . . . . . . . . . . . . . . . . . . . P292

Zoccole A...................P135,P249

Zolopa A .................... P039

Zuluaga J. ...................... P233

Zuniga R................... P251

Zúñiga-Pflücker J C . . . . . . . . . . . . . . . 0003

Zurba N ...................... P144

Zylak C. ............. O013,0014,P132 


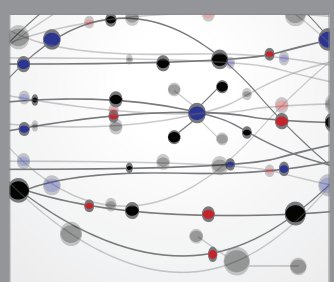

The Scientific World Journal
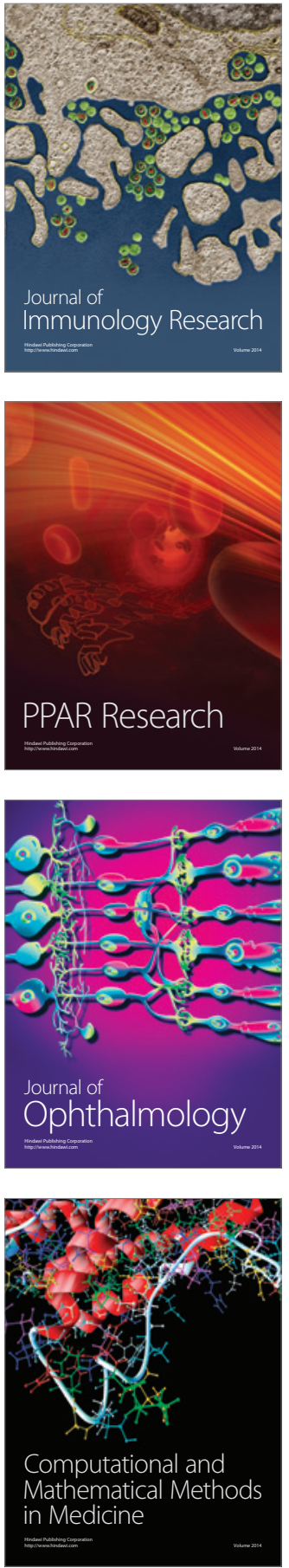

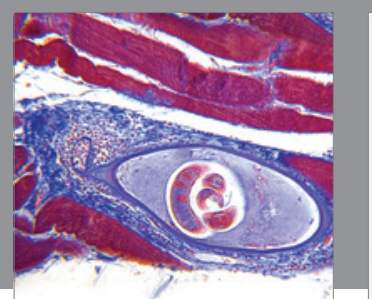

Gastroenterology Research and Practice

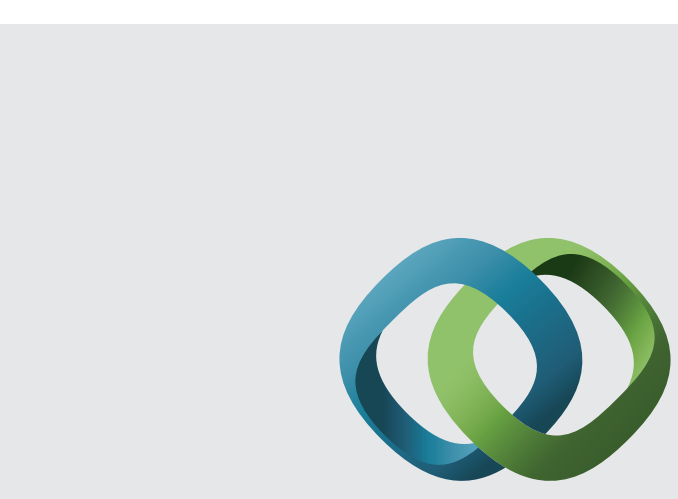

\section{Hindawi}

Submit your manuscripts at

http://www.hindawi.com
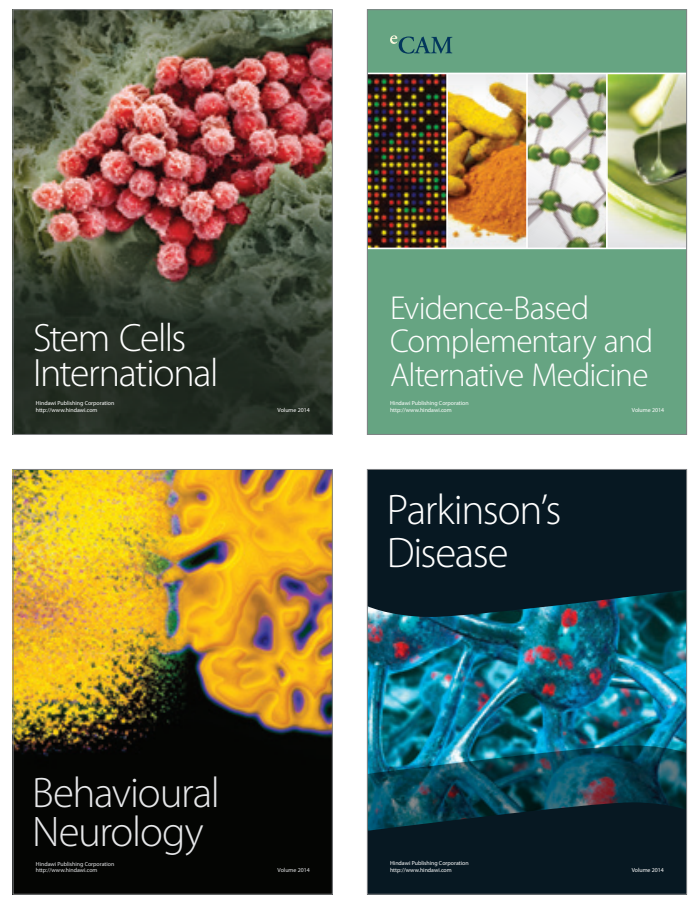
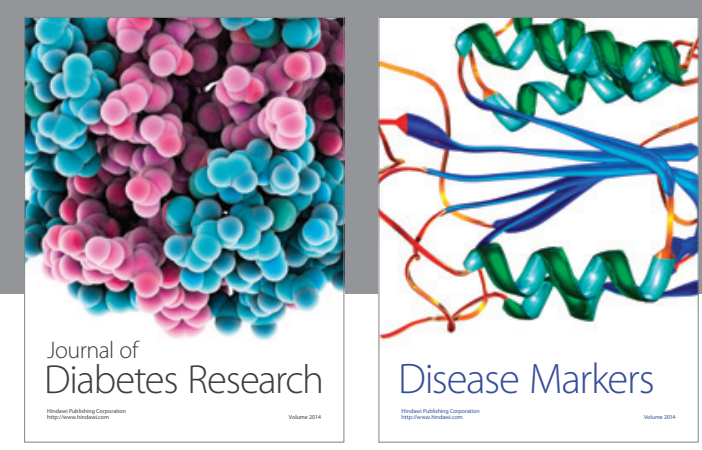

Disease Markers
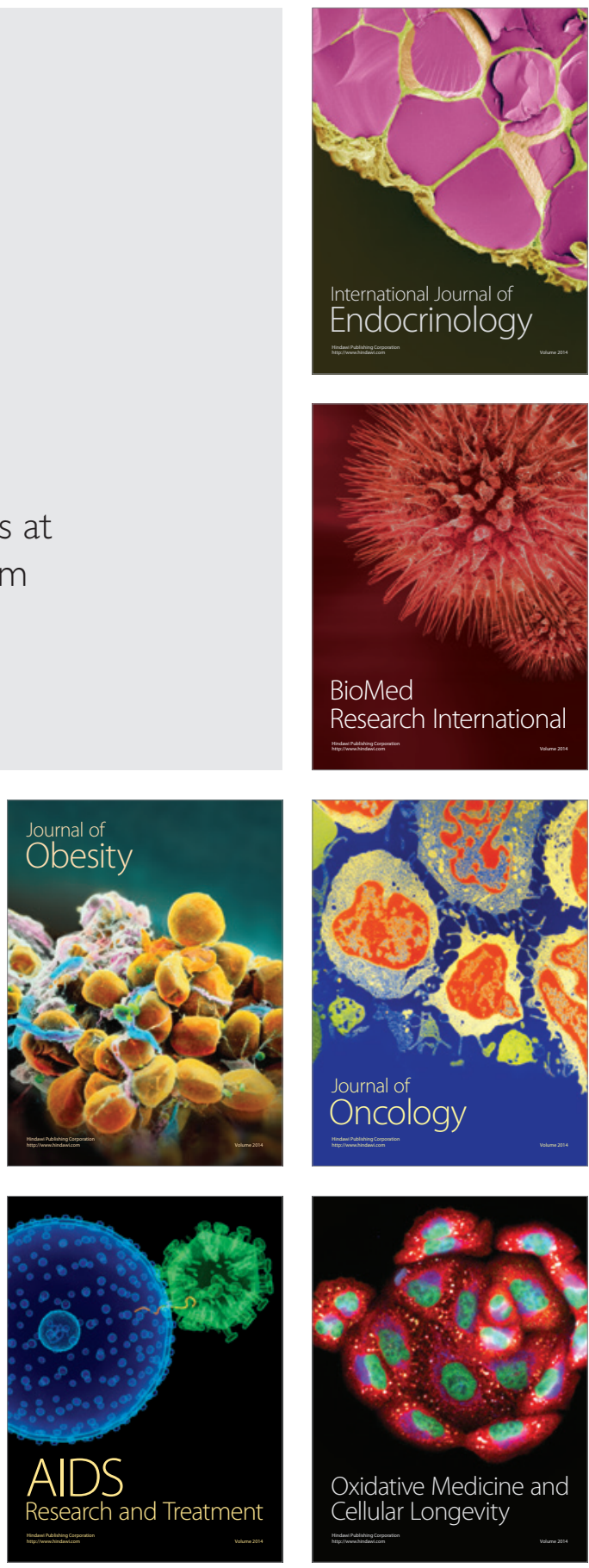